\title{
Injury Experience in Metallic Mineral Mining, 1989
}

U.S. Department of Labor Elizabeth H. Dole, Secretary

Mine Safety and Health Administration

William J. Tattersall, Assistant Secretary

Information Report

IR 1197

1800

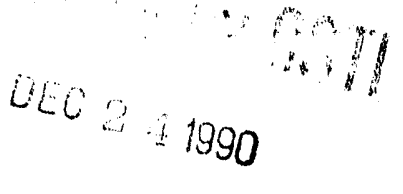


This publication has been reviewed and approved for distribution to the mining piblic by the office of the Assistant Secretary for Mine Safety and Health. 
Figure 1. Scheduled charges in 1ost workdays for loss or 1oss of

Figure 2. Quse involving hand or foot- mine employment and coal production report f:orm-

Figure 3. Mine accident, injury, and illness report form- -

TABLES FOR METALLIC MINERAL OPERATIONS IN THE UNITED STATES, 1989 (OPERATOR DATA)

1. General statistics on injury experiance and worktime--

2. Injury experience by degree and worktime for work location--...-

3. Number of injuries, 1ckl- workdays, and average severity by degree for work location-

4. Injury experience by degree and worktime for work location and employment size group-

5. Number of injuries, 10st workdays, and average severity by degree for work location and employment size group- -

6. Injury experience by degree and worktime for type of mineral. work location, and State-

7. Number of injuries, lost workdays, and average severity by degree for type of mineral, work location, and State-- -

8. Number of injuries, incidence rates, and severity measures by degree for work location and part of body injured-_..............

9. Number of injuries. 1ost workdays, and average severity by degree for work location and part of body injured-..................

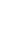
(1) 
TABLES FOR METALLIC MINERAL OPERATIONS IN THE UNITED STATES, 1989

(OPERATOR DATA) --Continued

Page

18. Number of injuries, 1ost workdays, and average severity by degree for work location and accident classification-.......-.-

19. Number of injuries, 10st workdays, and average severity by degree for accident classification and part of body injured-.-.

185

20. Number of injuries, 10st workdays, and average severity by degree for accident classification and nature of injury-a.- 194

21. Occurrences reported to MSHA as occupational illnesses- 200

22. Related noncoal mining summary reference tabulation, number of injuries, incidence rates, average number of workers, and employee-hours by work location and mineral industry-

23. Related coal mining summary reference tabulation, number of injuries, incidence rates, average number of workers, employee-hours, and production by type of coal mined and work location

TABLES FOR METALLIC MINERAL OPERATIONS IN THE UNITED STATES, 1989 (CONTRACTOR DATA)

Table reference guide (contractor data) 210

1. General statistics on injury experience and severity

3. Number of injuries, 1ost workdays, and average severity by

7. Number of injuries, lost workdays, and average severity by

9. Number of injuries, lost workdays, and average severity by

degree for work location and part of body injured--- Number of injuries, 10st workdays, and average severity by
11.

degree for work location and nature of injury
12. Number of injuries, 10st workdays, and average severity by

13. Number of injuries by accident clasaification and degree for work location and State- accident classification and degree

14. Number of 10 st workdays by accident classification and degree of injury for work location and State-

15. Number of injuries by accident classification and degree for

16. Number of lost workdays by accident classification and degree of injury for work 1 ocation and type of mineral-

17. Number of injuries, lost workdays, and average severity by
degree for work location and occupation at time of injury

17. Number of injuries, lost workdays, and average severity by
degree for work location and occupation at time of injury----

18. Number of injuries, lost workdays, and average severity by degree for work location and accident classification--

19. Number of injuries, 10st workdays, and average severity by degree for accident classification and part of body injured--- 
TABLES FOR METALLIC MINERAL OPERATIONS IN THE UNITED STATES, 1989 (CONTRACTOR DATA)--Continued

$\underline{\text { Page }}$

20. Number of injuries, 1ost workdays, and average severity by

$\begin{array}{ll}\text { degree for accident classification and nature of injury-a-n- } & 289 \\ 21 \text {. Occurrences reported to MSHA as occupational illnesses-a-n } & 293\end{array}$

22. Related noncoal mining summary reference tabulation, number of injuries, incidence rates, average number of workers, and employee-hours by work location and mineral industry--_-_- 294

23. Related coal mining summary reference tabulation, number of injuries, incidence rates, avrage number of workers, employee-hours, and production by type of coal mined and work location- 
INJURY EXPERIENCE IN METALILIC MINERAL MINING, 1989

by

Staff, Division of Mining Information Systems

Safety and Health Technology Center

Technical Support, Denver, Colorado

\begin{abstract}
This Mine Safety and Health Administration (MSHA) informational report reviews in detail the occupational injury and illness experience of metallic mineral mining in the United States for 1989 . Data reported by operators of mining establishments concerning work injuries are summarized by work location, accident classification, part of body injured, nature of injury, occupation, and principal type of mineral. Related information on employment, worktime, and operating activity also is presented. Data reported by independent contractors performing certain work at mining locations are depicted separately in this report. For ease of comparison with other metal and nonmetallic mineral mining industries and with coal mining, summary reference tabulations are included at the end of both the operator and the contractor sections of this report.
\end{abstract}

\title{
INTRODUCTION
}

Data used in compiling this report were reported by operators of metallic mineral mines and preparation plants on a mandatory basis as required under the Federal Mine Safety and Health Act of 1977. Public Law 91-173 as amended by Law 95-164. Since January 1, 1978, operators of mines or preparation plants or both which are subject to the Act have been required under $30 \mathrm{CFR}$. Part 50, to submit reports of injuries, occupational ilinesses, and related data.

Only data reported by the operators and contractors themselves are utilized in this report. No estimates are made of employment, employeehours, or injuries for nonrespondent operations except as necessary to meet: statistical processing requirements by providing minimum employment data for reported unmatched injury data.

Beginning with calendar year 1978, statistical teasures for injury experience in mining were changed from the measures used from 1930 through 1977. The statistical measures for injury experience adopted by MSHA in 1978 compare closely with the measures used in the Office of Occupational Safety and Health Statistics, Bureau of Labor Statistics, U. S. Departmert. of Labor. Therefore, beginning with 1978 data, the mining industry can be compared on a standard basis with other United States industries reporting under the Occupational Safety and Health Act of 1970.

Occurxences identified as occupational illnesses by mine operators were reported to MSHA and were processed in accordance with 30 CFR PART 50.20-6. The reported illnesses are summarized in seven categories for each work location, type of mineral, and State. Incidence rates and severity measures are not computed for occupational ilinesses. 
Because of the modifications in reporting and processing procedures that became effective January 1, 1978, injury rates as currently computed are not precisely comparable to those of previous years, except for fatality rates in which the "incidence rate" (the term used after 1977) is one-fifth of the "frequency rate" (the term used prior to 1978) for otherwise similar groupings of fatal injuries and worktimes. Because of this disparity, MSHA annual pub1ications concerning injuries, illnesses, and operating activity will not contain data for years prior to 1978 .

\section{SCOPE OF STATISTICS}

The statistical data in this report cover the work experience of all personnel engaged in mine exploration, development, production, maintenance, repair, and construction work, including supervisory and technical personnel, working partners, and onsite officeworkers. The classification of industries is in close general agreement with the Standard Industrial Classification. $1 /$

It should be noted that amendments to legislation that funded MSHA and other government programs from December 16, 1981, through July 18, 1982 , prohibited MSHA from administering or enforcing the Federal Mine Safety and Health Act of 1977 (Mine Act) or MSHA standards and regulations with respect to any person engaged in the surface mining or milling of stone, clay, feldspar, colloidal phosphate, or sand and gravel. They also provided that MSHA could not administer or enforce the Mine Act or MSHA standards and regulations on any State or political subdivision thereof. Further, MSHA could not administer or enforce the Mine Act or MSHA standards or regulations with respect to any independent construction contractor who was engaged by an operator for the construction, repair or alteration of structures, facilities, utilities or private ways or roads located on (or appurtenant to) the surface areas of any coal or other mine, and whose employees worked in a specifically demarcated area, separate from actual mining or extraction activities. No data on exempted activities are included for that period of time in Table 1 of this publication.

MSHA issued guidelines which additionally reduced the reporting requirements of independent contractors. Reporting of employment, hours worked, and injury/iliness data by independent contractors is 1 imited to the following types of work performed: mine development, including shaft and slope sinking: construction or reconstruction of mine facilities, including building or rebuilding preparation plants and mining equipment, and building additions to existing facilities; demolition of mine facilities; construction of dams: excavation or earthmoving activities involving mobile equipment; equipment installation, such as crushers and mills; equipment service or repair done underground or in the pit for a pexiod exceeding five

1/ Executive Office of the President, Office of Management and Budget. Standard Industrial Classification Manual. 1972 revision. 
consecutive days at a particular wine; material handling within mine property, including haulage of coal, ore, refuse, etc.. unless for the sole purpose of direct removal from or delivery to mine property; drilling and blasting. Also, data collected for employment and hours worked in those activities reflect the contractor's total metallic/nonmetallic work locations and total coal work locations and not the individual mine sites. For more detailed information concerning these changes, see the guidelines accompanying MSHA Policy Memoranda Nos. 82-6A MM and 81.-35A C.

\section{DEFINITION OF TERMS}

Occupational Injury.--An occupational injury is an injury to a mine worker which occurs at a mine and for which medical treatment is administered, or which results in death or 10 ss of consciousness. restriction of work or motion, inability to perform all job duties on any day after an injury, 1ost workdays, temporary assignment to other duiles, transfer to another $j o b$, or termination. The injury usually results from a recognizable single incident. For example, an explosion of a battery which splattered a worker with sulfuxic acid would be a single incident and the worker would be considered to have suffered an injury. Another example would be an injury where a worker was overcome by hydrogen sulfide gas released from an exploding vessel.

Occupational illness.--An occupational illness is an illness or disease of a mine worker which may have resulted from work at a mine or for which an award of compensation is made. To be classified as an occupational illness, the disability must result from repeated exposure to the condition or substance which caused the disability. A classic illness example is a pneumoconiosis disability which may have been induced by repeated exposure to the causative dusty condition. Dermatitis resulting from repeated exposure to 1 ime dust or other material in the work environment also should be reported as an illness. In cases where the time of onset of illness is in doubt, the day of diagnosis of illness will be considered as the first day of illness.

Number of Operations and Associated Work Locations.--Number of operations is the number of individual respondents with a unique Federal identification number that have reported worktime data. The count and kind of operation are based on the lowest numbered (primary) subunit on employment report Form 7000-2 (see figure 2, p. 10) that contains worktime data. An associated work 1 scation is a subunit reported with a primary subunit, e.g., an "office" is almost always an associated work location. Independent contractors working on mine property performing the types of work described under "Scope of Statistics" are assigned an identification number, report to MSHA, but are not included in the count of operations. Contractor data are depicted separately in this report.

Worktime.-- Worktime includes the number of workers and the number of employee-hours worked. "Average number of workers" is a summary of the average number of persons working at individual establishments during calendar quarters of active operations. "Employee-hours" is a summary of employee-hours reported. 
Employment Size Group.--Reporting opexations are classified into size groups according to the average number of workers in specified work locations.

Degree of Injury.--Degree of injury indicates the seriousness of injuries. FATAL injuries are those occurrences resulting in death. NFDL (NonEata1 with Days Lost) injuries are nonfatal occurrences that result in days away from work, statutory days charged, or days of restricted work activity. NDL (No Days Lost) injuries are occurrences having no 10 st workdays; that is, nonfatal injury occurrences resulting only in temporary loss of consciousness or medical treatment other than first aid.

A permanent total disability is caused by any work injury or illness other than death that permanently and totally incapacitates a worker from following any gainful occupation, or that results in the loss, or the complete loss of use, of both or any combination of: hands, arms, legs, feet, or eyes.

A permanent partial disability is caused by any work injury or illness other than death or permanent total disability that results in the 1oss, or the complete loss of use, of any member or part of a member of the body, or any permanent impairment of functions of the body or part thereof, regardless of any preexisting disability of the injured member or impaired body function.

Incidence Rate.--Incidence rate is defined as the number of injuries per 200,000 employeemhours, rounded to two decimal places. The highest rate given is 99.99. If an incidence rate is greater than 99.99 , then 99.99 is printed. If no data were reported, then a hyphen is printed. If a computation is less than 0.01 after rounding, then an asterisk inside parentheses $(*)$ is printed. The following is the standard incidence rate furmula:

$$
I R=\frac{\text { number of injuries } \times 200.000}{\text { number of employee-hours }}
$$

Lost Workdays (LWD).--Lost workdays consist of days away from work (DAW) and days of restricted work activity (DRA), or statutory days charged as prescribed from a table of standard charges (see p. 7), e.g., 6,000 days for a fatality or permanent total disability.

Severity Meacure.--Severity measure is the number of 10 st workdays per 200,000 employee-hours, rounded to whole numbers. The highest rate given is 99.999. If the computed rate is greater than 99,949 , then 99,999 is printed. If no data were reported, then a hyphen is printed. If the computation results in a zero after rounding, then an asterisk inside parentheses ( $*$ ) is printed. The lollowing is the standard severity measure formula:

$$
S M=\frac{\text { number of 10st workdays } \times 200,000}{\text { number of employee-hours }}
$$


Average Severity.--Average severity is the average number of $10 \mathrm{t}$ workdays per injury and is computed only for injuries clasified as EATAL or NEDL. Lost workdays are selected on the same basis as that for computing the severity measure. The following is the atandard average severity formula:

$$
\text { AS }=\frac{\text { number of logt workdays }}{\text { number of injuries contributing the 10st workdays }}
$$

Type of Mineral.-- The type of mineral is determined from the Standard Industrial Classification (S.I.C.) code assigned to an uperation based on information provided by the mine operator. Data for all work locations in the operation are crediled to the assigned S.I.C.

Classification of Accident.--Accident classification identifies circumstances which contributed most directly to the resulting accident. The 21 classifications in the following 1ist are designed to categorize all accidents, whether injury-producing or not.

Electrical.--Accidents in which the electric current is most directly responsible for the resulting accident.

Entrapment.--Accidents involving entrapment of persons.

Exploding Vesse1s Under Pressure.--Accidents involved with bursting of air hoses, air tanks, hydraulic 1 ines, hydraulic hoses, stand pipes, etc.. due to internal pressure.

Explosives and Breaking Agents.--Accidents involving the detonation of manufactured explosives; includes Airdox and Cardox.

Falling, Rolling, or Sliding Rock or Material of Any Kind.-Accidents caused directly by faliing material other than material from the roof or face. Or, if material was set in motion by machinery, by haulage, by handtools, or while being handled or disturbed, etc., the force that set the material in motion determines the classification. For example, where a rock was pushed over a highwal1 by a bulldozer and the rock hit another rock which hit and injured a worker-the accident is classified as machinery. Machinery (a bulldozer) most directly caused the resulting accident.

Fall of Face, Rib, Pillar, Side, or Highwall (from in place).-Accidents in this classification include falls of material while barring down or placing props, also pressure bumps and bursts. Not included are accidents in which the motion of machinery or haulage equipment caused the fall ej.ther directly or by knocking out support.

Eal1 of Roof, Back, or Brow (from in place).--Underground only Accidents which include falls while barring down or placing props. al so pressure humps and bursts. Not included are accidents in which the motion of machinery or haulage equipment caused the fall either directly or by knocking out support. 
Fire.--Accidents related to ul.controlled burning of material or mineral in the mine environment. Not included are fires initiated by electricity or by ignition or explosion of gas or dust.

Handling Material.--Accidents related to handling packaged or loose material while lifting, pulling, pushing, or shoveling.

Handtools.--Accidents related to nonpowered tools.

Nonpowered Haulage.--Accidents related to the motion of nonpowered haulage equipment. Included are accidents involving wheelbarrows, manually pushed mine cars, timber trucks, etc.

Powered Haulage.--Accidents related to the motion of powered haulage equipment. Included are accidents involving conveyors, front-end loaders, forklifts, shuttle cars, load-haul-dump units, locomotives, rail road cars, haulage trucks, pickups, automobiles, and personnel carriers.

Hoisting.--Accidents involving cages, skips, ore buckets, and elevators. The accident results from the action, motion, or failure of the hoisting equipment or mechanism. Included are equipment such as cranes and derricks only when used in shaft sinking: also suspended work platforns in shafts. Not included is equipment such as chain hoj.sts, come-alongs, and winches.

Ignition or Explosion of Gas or Dust.--Accidents resulting as a consequence of the ignition or explosion of gas or dust.

Impoundment.--Accidents caused by an unstable condition or failure of an impoundment, refuse pile, or culm bank requiring emergency preventative action or evacuation of an area.

Inundation.--Accidents caused by inundarion of a surface or underground mine by a liquid (or semi-solid) or a gas.

Machinery.--Accidents related to the motion of machinery. Included are all electric and air-powered tools and mining machinery such as drills, tuggers, winches, slushers, draglines, power shovels, loaders, and compressors.

Slip or Fall of Person (from an elevation or on the same level).-Accidents include slips or falls while getting on or of machinery and haulage equipment which is not moving, and slips or falls while servicing or repairing equipment or machinery.

Stepping or Kneelirg on Object.--Accidents are classifled in this category only where the object stepped or kneeled on contributed most directly to the accident.

Striking or Bumping.--This classification is restricted to those accidents in which an individual, while moving about, strikes or bumps an object but is not handling material, using handtools, or operating equipment. 
Other.--Accidents not elsewhere classified.

Abbreviations.--Space 1imitations in column headings and stub entries of standard-format tabulations require occasional adaptation of terminology. In this report PLNTS should be interpreted as "plants."

Statutory Days Charged.--Some injuries or illnesses are of such a degree of severity that a standard time charge of 10 st workdays has been adopted by MSHA as follows:

\section{Disability}

Lost Workdays

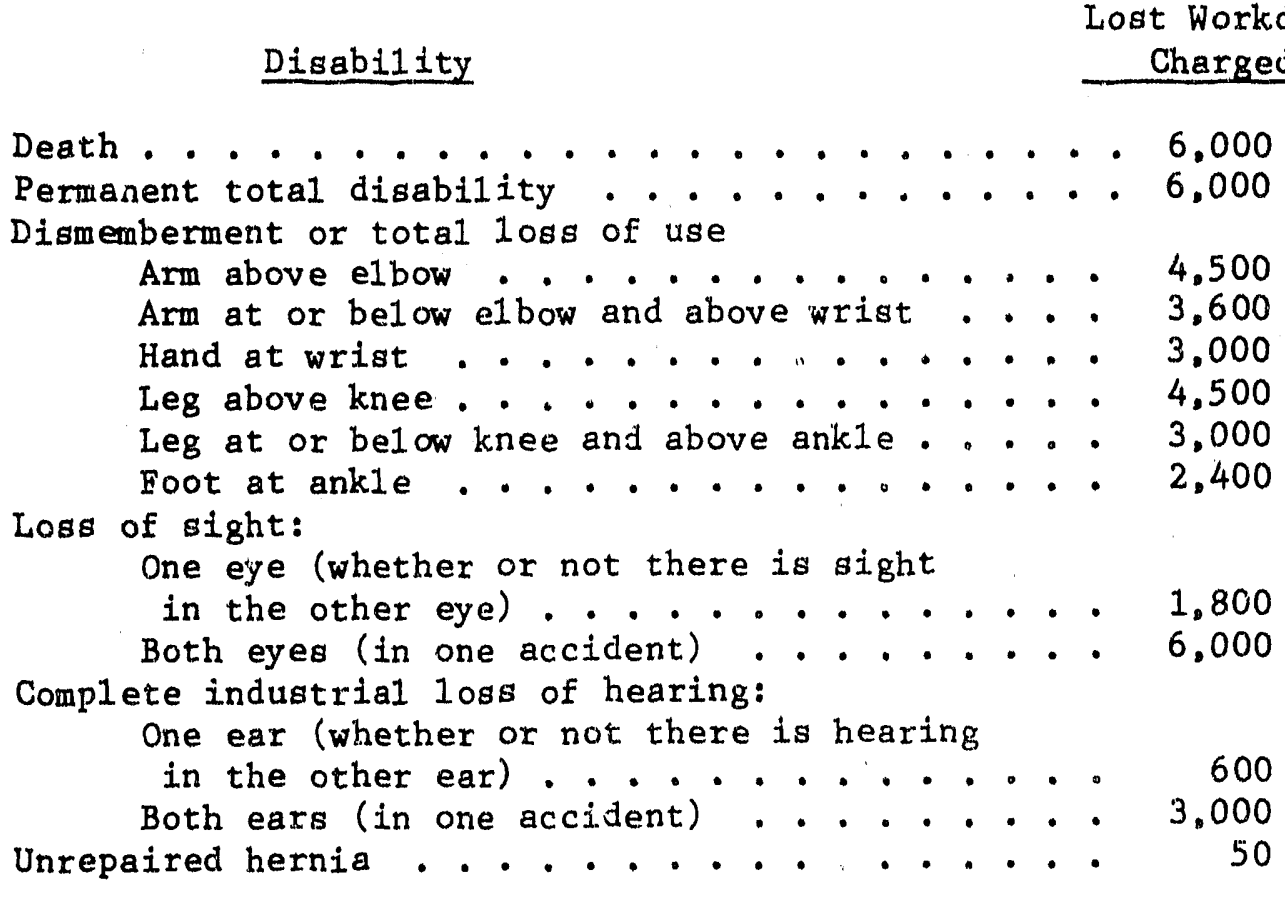

3/ Not more than 6,000 10st workdays will be charged in a single accident that results in multiple injuries to a mine worker. (See definition of permanent total disability on page 4. )

\section{TABLE REFERENCE GUIDE (OPERATOR DATA)}

The following reference guide facilitates locating particular data elements contained in the annual statistical tables for operators. A comparable guide precedes the contractor section of this report. The number appearing in each cell indicates the table number in which that particular element may be found. All elements 1 isted under the stub headings appear as vertical headings in the tables. Elements 1isted under column headings appear as horizontal headings. The only exception to this is table 1, in which the headings have beent reversed.

To use this guide, read down or across the elements 1 isted in both the stub and column headings to determine which combinations are of interest. For example, incidence rates by work location appear in seven different tables, numbers $2,4,6,8,10,22$, and 23 . However, only one, table 10 , provides a further division by nature of injury as well. The occupation of the injured is found in table 17 and, as seen in the column headings. provides the number of injuries, 10st workdays, and average severity. 
morerance GUIDE to getallic statistical tables (OPERATOR DATA)

\begin{tabular}{|c|c|c|c|c|c|c|c|c|c|c|c|c|c|}
\hline STUE UEADINGS & 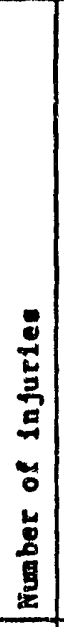 & 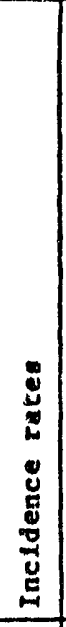 & 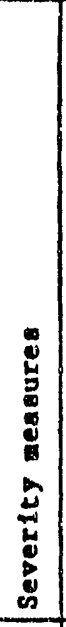 & $\begin{array}{l}0 \\
0 \\
3 \\
8 \\
8 \\
0 \\
0 \\
\end{array}$ & 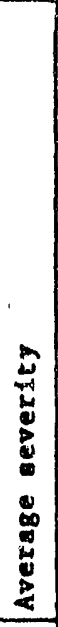 & 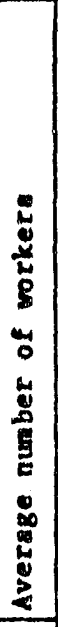 & 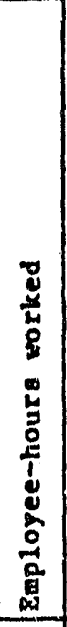 & 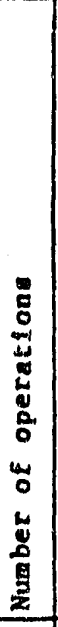 & 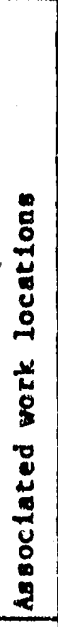 & 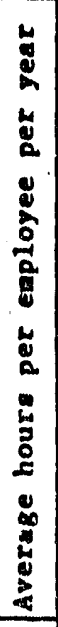 & 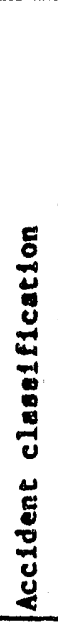 & 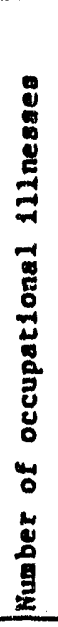 & 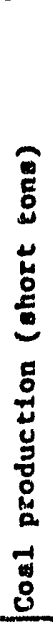 \\
\hline $\begin{array}{l}\text { PFior year data } \\
\text { weh and without off } 1 \text { ce } 1\end{array}$ & 1 & 1 & 1 & & 1 & 1 & 1 & 1 & 1 & 1 & & & \\
\hline \multirow{19}{*}{ Work Locac1on } & 2 & 2 & 2 & & & 2 & 2 & 2 & 2 & & & & \\
\hline & 3 & & & 3 & 3 & & & & & & & & \\
\hline & 4 & 4 & 4 & & & 4 & $\frac{4}{4}$ & 4 & 4 & & & & \\
\hline & 5 & & & 5 & 5 & & & & & & & & \\
\hline & 6 & 6 & 6 & & & 6 & 6 & 6 & 6 & & & & \\
\hline & 7 & & & 7 & $\underline{T}$ & & & 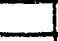 & & & & & \\
\hline & 8 & 8 & 8 & & & & & & & & & & \\
\hline & 9 & & & 9 & 9 & & & & & & & & \\
\hline & 10 & 10 & 10 & & & & & & & & & & \\
\hline & 11 & & & 11 & 11 & & & & & & & & \\
\hline & 7,3 & & & & & & & & & & 13 & & \\
\hline & & & & 14 & & & & & & & 14 & & \\
\hline & 15 & & & & & & & & & & 1.5 & & \\
\hline & & & & 46 & & & & & & & 16 & & \\
\hline & 17 & & & 17 & 17 & & & & & & & & \\
\hline & 18 & & & 18 & 18 & & & & & & & & \\
\hline & & & & & & & & & & & & 21 & \\
\hline & 22 & 22 & & & & 22 & 22 & & & & & & \\
\hline & 23 & 23 & & & & 23 & 23 & & & & & & 23 \\
\hline \multirow{2}{*}{ Employwent 1ze group } & 4 & 4 & 4 & & & 4 & 4 & 4 & 4 & & & & \\
\hline & 5 & & & 5 & 5 & & & & & & & & \\
\hline \multirow{5}{*}{ Kind of uinaral } & 6 & 6 & -1 & & & 6 & 6 & 6 & 6 & & & & \\
\hline & 1 & & & $\Gamma$ & 7 & & & & & & & & \\
\hline & 15 & & & & & & & & & & IS & & \\
\hline & & & & 16 & & & & & & & 16 & & \\
\hline & & & & & & & & & & & & 21 & \\
\hline \multirow{5}{*}{ State } & 6 & 6 & 6 & & & 6 & 6 & 6 & 6 & & & & \\
\hline & 7 & & & 7 & 7 & & & & & & & & \\
\hline & 13 & & & & & & & & & & 13 & & \\
\hline & & & & 14 & & & & & & & 14 & & \\
\hline & & & & & & & & & & & & 21 & \\
\hline \multirow{4}{*}{ Part of body injured } & 8 & 8 & 8 & & & & & & & & & & \\
\hline & 9 & & & 9 & 9 & & & & & & & & \\
\hline & 12 & & & 12 & 12 & & & & & & & & \\
\hline & 19 & & & 19 & 19 & & & & & & & & \\
\hline \multirow{4}{*}{ Heture of Injury } & 10 & 10 & 10 & & & & & & & & & & \\
\hline & 11 & & & 11 & 11 & & & & & & & & \\
\hline & 12 & & & 12 & 12 & & & & & & & & \\
\hline & 20 & & & 20 & 20 & & & & & & & & \\
\hline $\begin{array}{l}\text { Occupation at tine of } \\
\text { Iufury }\end{array}$ & 17 & & & 17 & 17 & & & & & & & & \\
\hline \multirow{3}{*}{ Accident clasesflcation } & 18 & & & 18 & 18 & & & & & & & & \\
\hline & 19 & & & 19 & 19 & & & & & & & & \\
\hline & 20 & & & 20 & 20 & & & & & & & & \\
\hline Kineral Induetry & 22 & 22 & & & & 22 & 22 & & & & & & \\
\hline Type of coal & 23 & 23 & & & & 23 & 23 & & & & & & 23 \\
\hline
\end{tabular}

1/ Por table 1 only, the headings have been reversed to aloplify this gulde; that in: the data elements indicated 1n the columa headings for this table actually appen as vertical headings. 

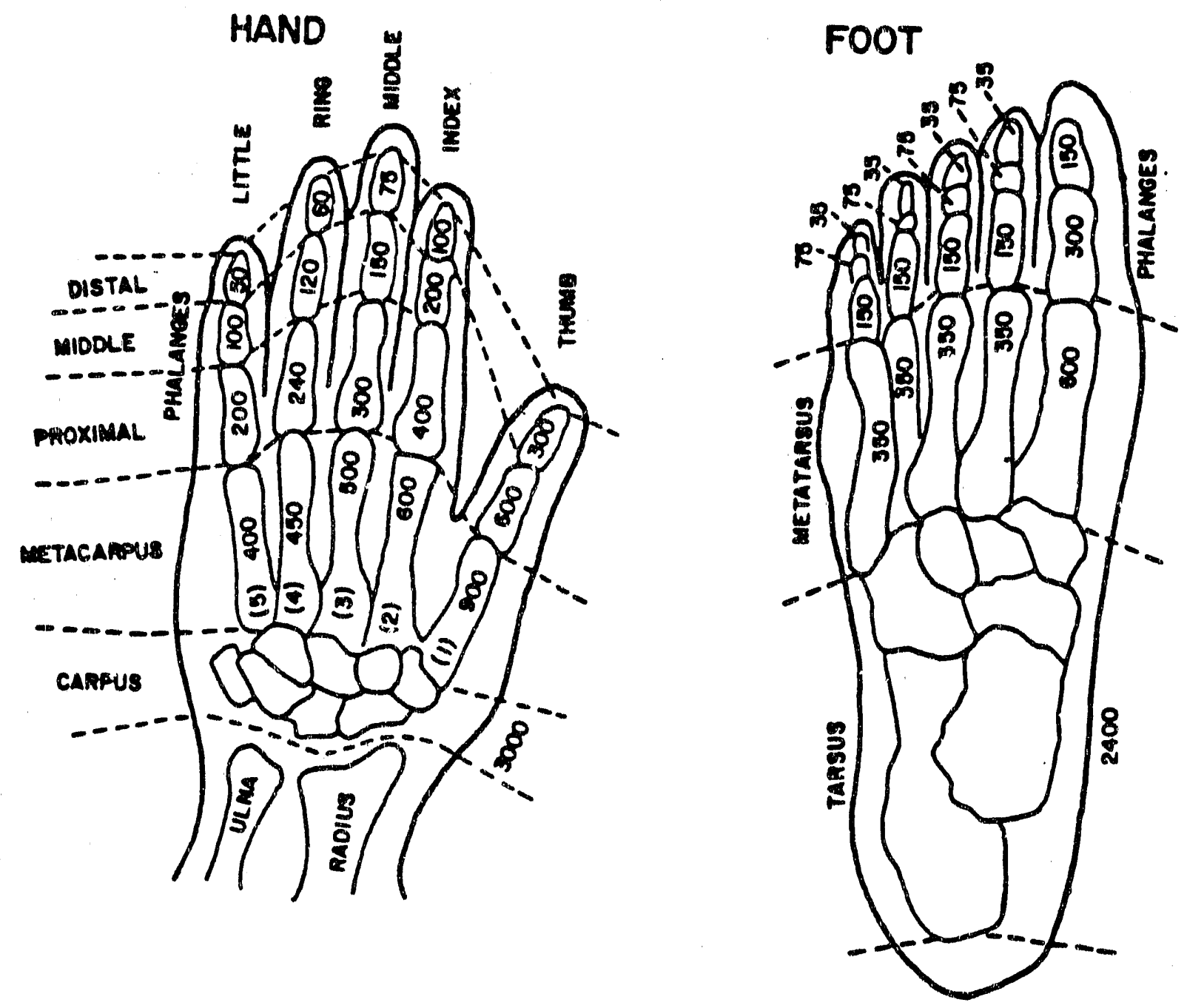

Note:

Numbers shown on diagrams are statutory charges of lost workdays for loss of all or part of bone.

Proportional charges may be computed based on medical determination of percentage loss of use.

FIGURE I. Scheduled charges in lost workdays for loss or loss of use involving hand or foot. 


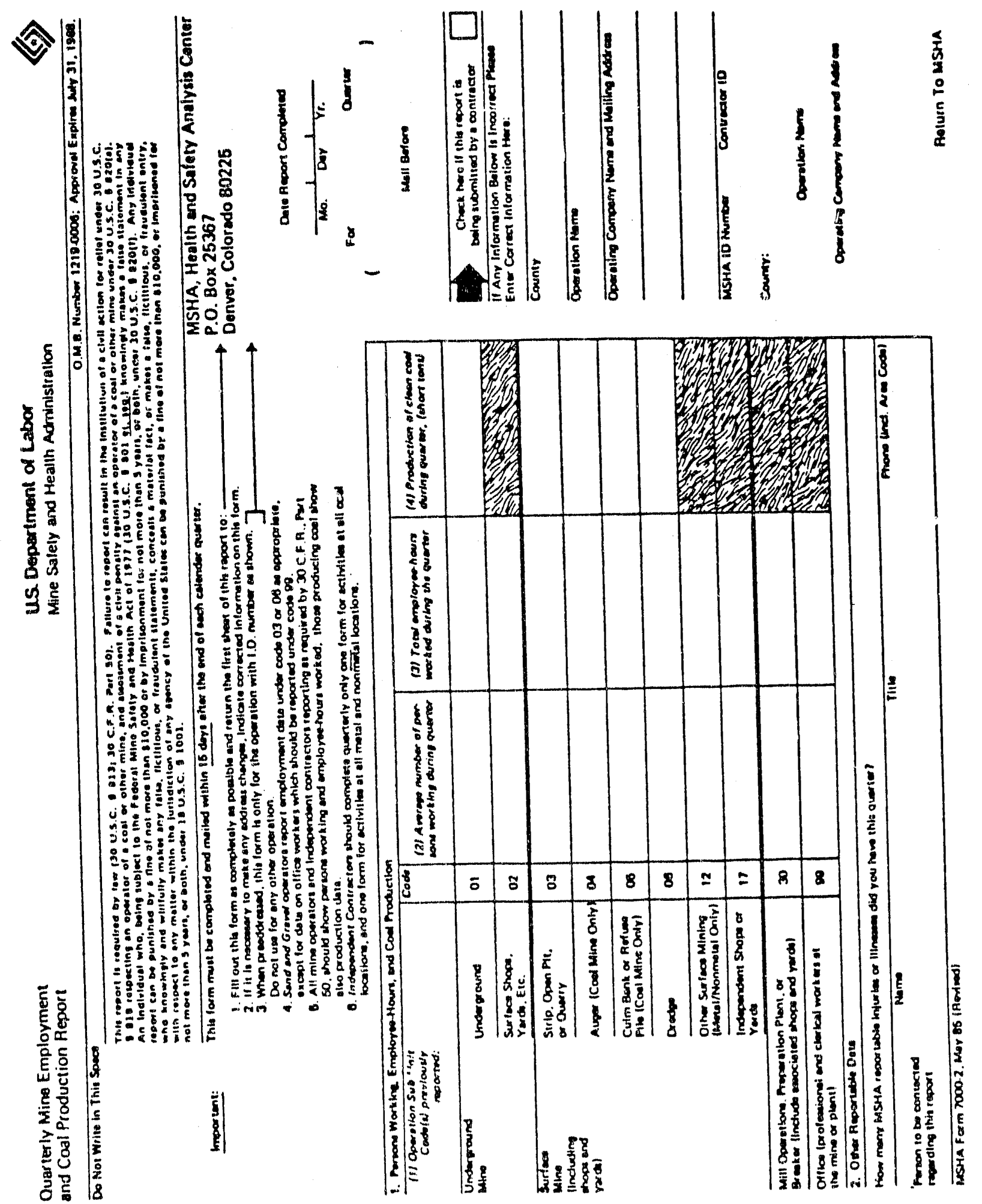

FIGURE 2. Quarterly miat employment and coal production report form. 
Mine Accident, Injury and IIIness Feport

\section{US. Department of Labor Mirve Safery and Mealth Administration}

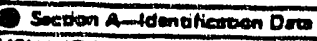

MSHA ID Number Conmeceor ID

Min Nem

\section{Aecort Catipary}

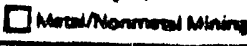

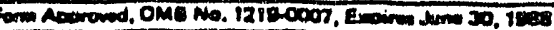

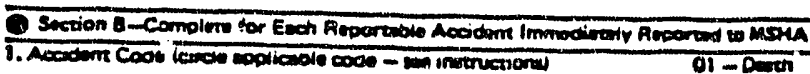

04 - inuadation

Q0 - Miro Fine

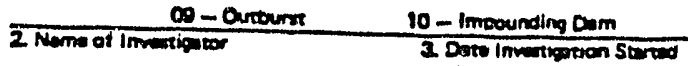

11 - Moiraing

Co-Senow Impry

Creat new it noort pertion be comerewer.

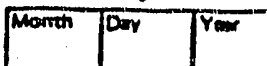

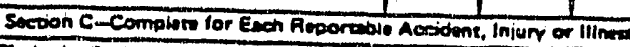

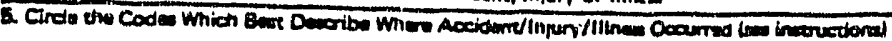

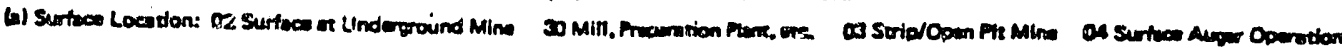

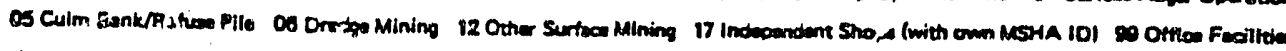

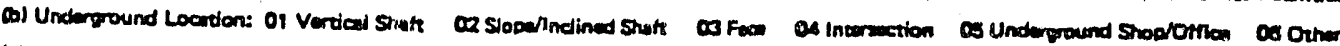

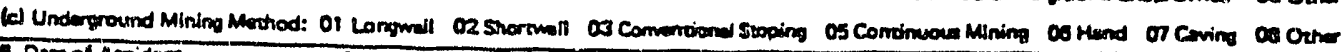

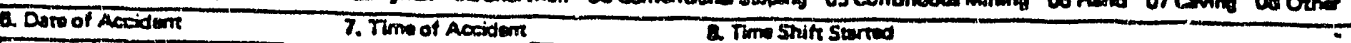

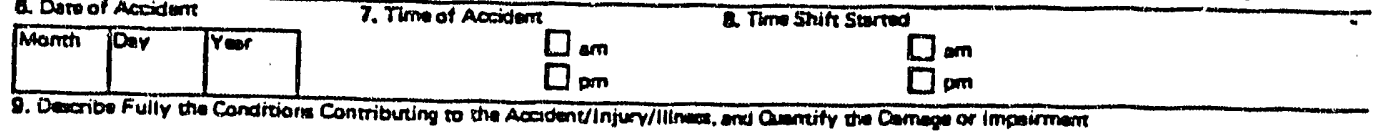

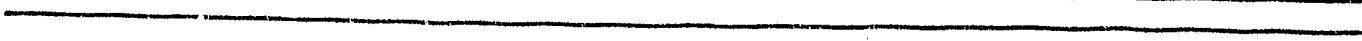

$\longrightarrow$

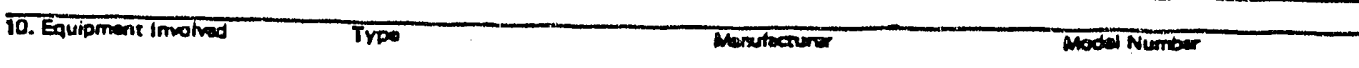

11. Nome of Witnese to Acosident/injurv/lilines

12 Nuinow of Acporable Injurie or

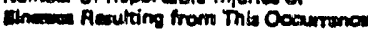

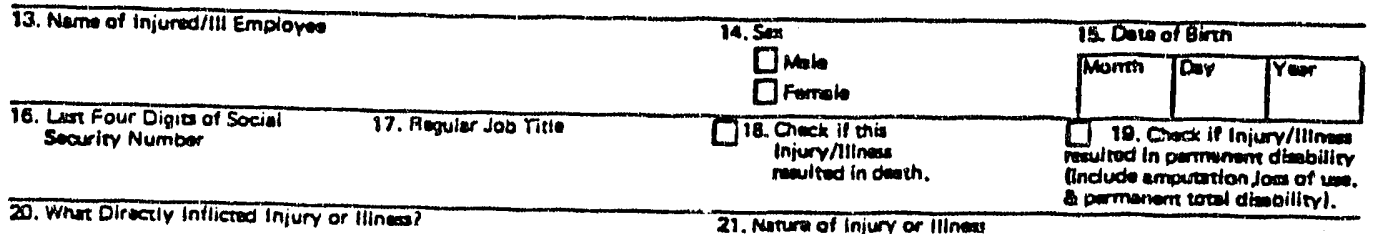

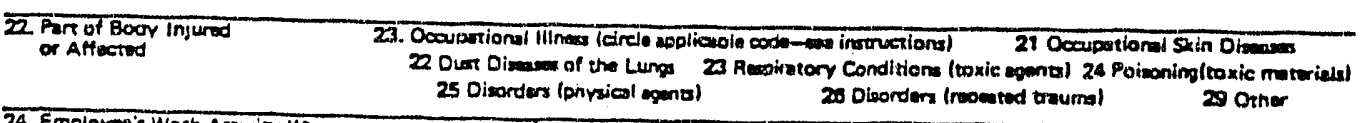

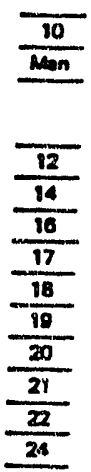

24. Employou's Work Acivily When Injury or llinew Deaured

Section D-Aerum to Dutr intormerion

Exourience

25. Exarimas in mus too Titlo

26. Experionow at Tha Nine

27. Toal Mining Excorienca 20 Other

口28. Permpnantly Tromierred or $\boldsymbol{\alpha}$. Termitusted lif crecked complete iterrs 9.30 . \& 311

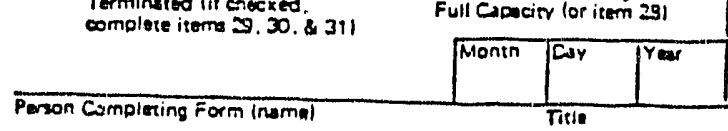
14nner 30831 miom can is claved

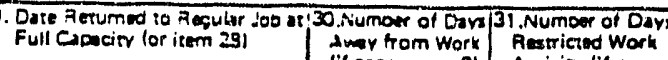
til nome, enter 0 Activin

Dare Tha Report Prearad Imonth, day. Yeor Are 6xe ins Ption Nunow

MSHA Form iDCO-1, FEO JU (revised)

MAIL THIS PAGE TO THE HEALTH ANO SAFETY ANALYSIS CENTER, MINE SAFETY ANO HEALTH ADMINISIAATION, P.O. BOX Z387, DENVEA, COLOAADO 80225

FIGURE 3. Mine accident, injury, and illness report form.

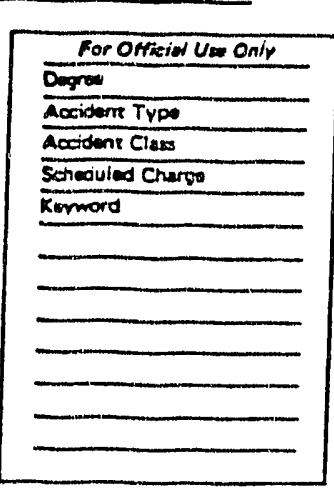




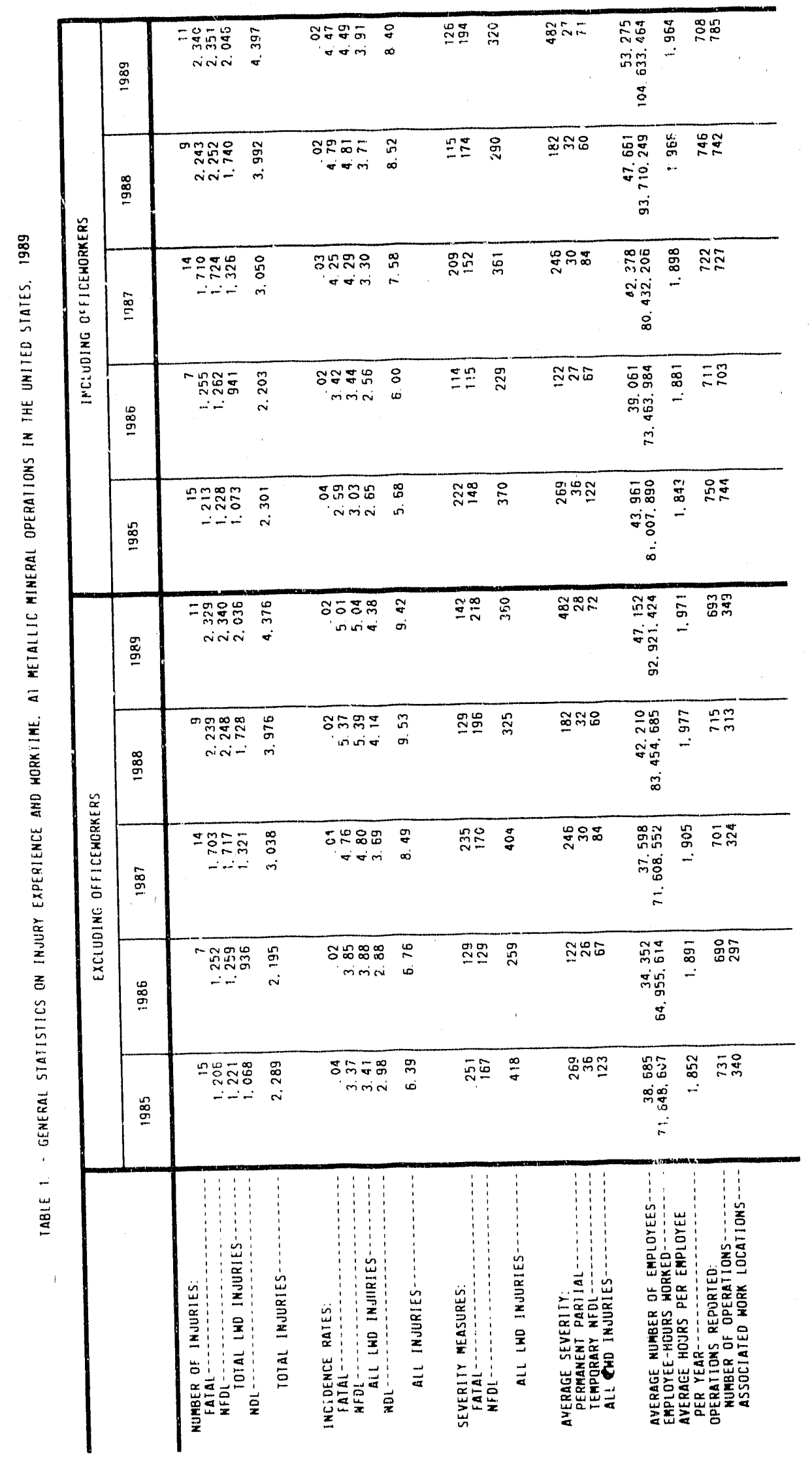




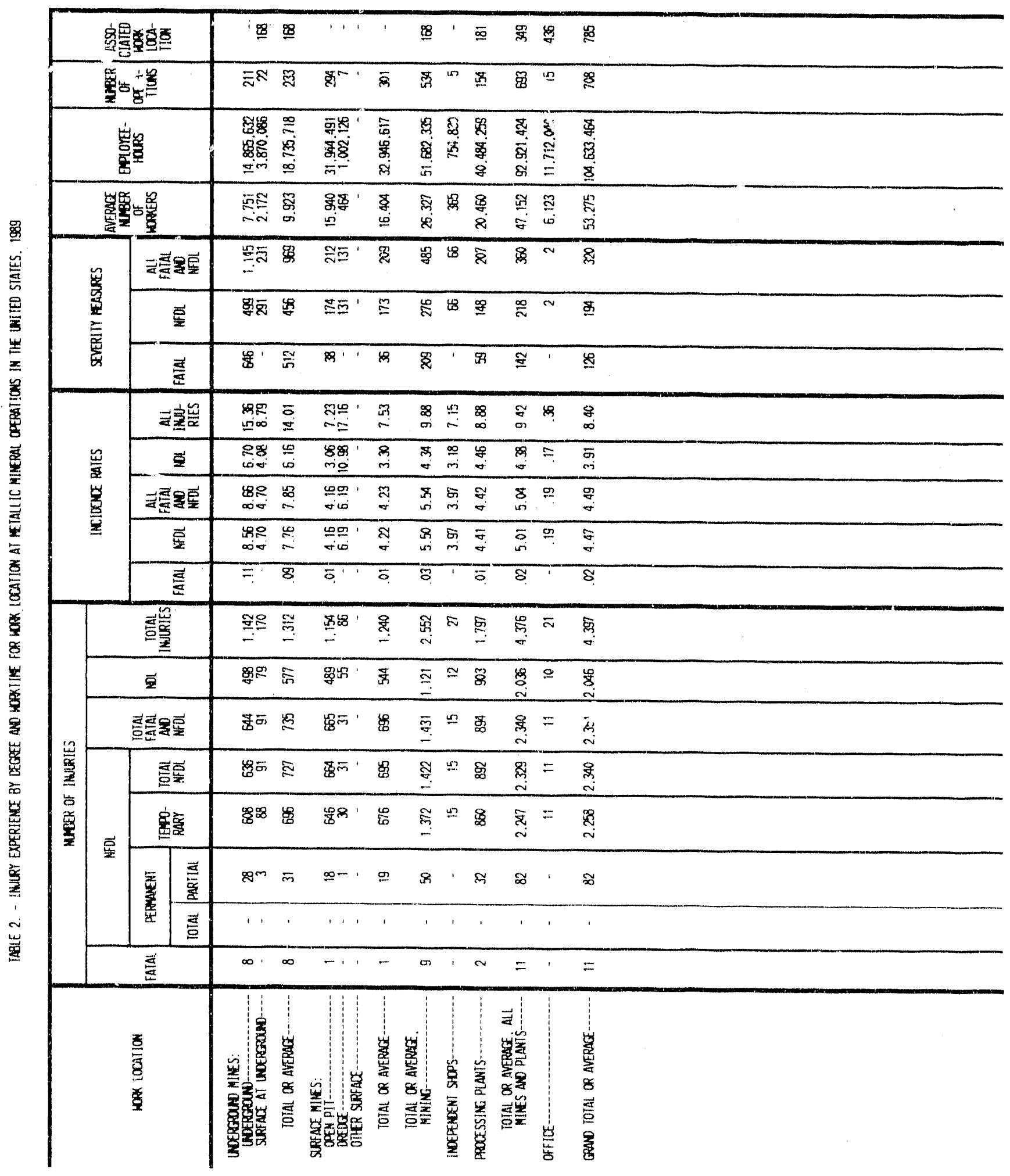




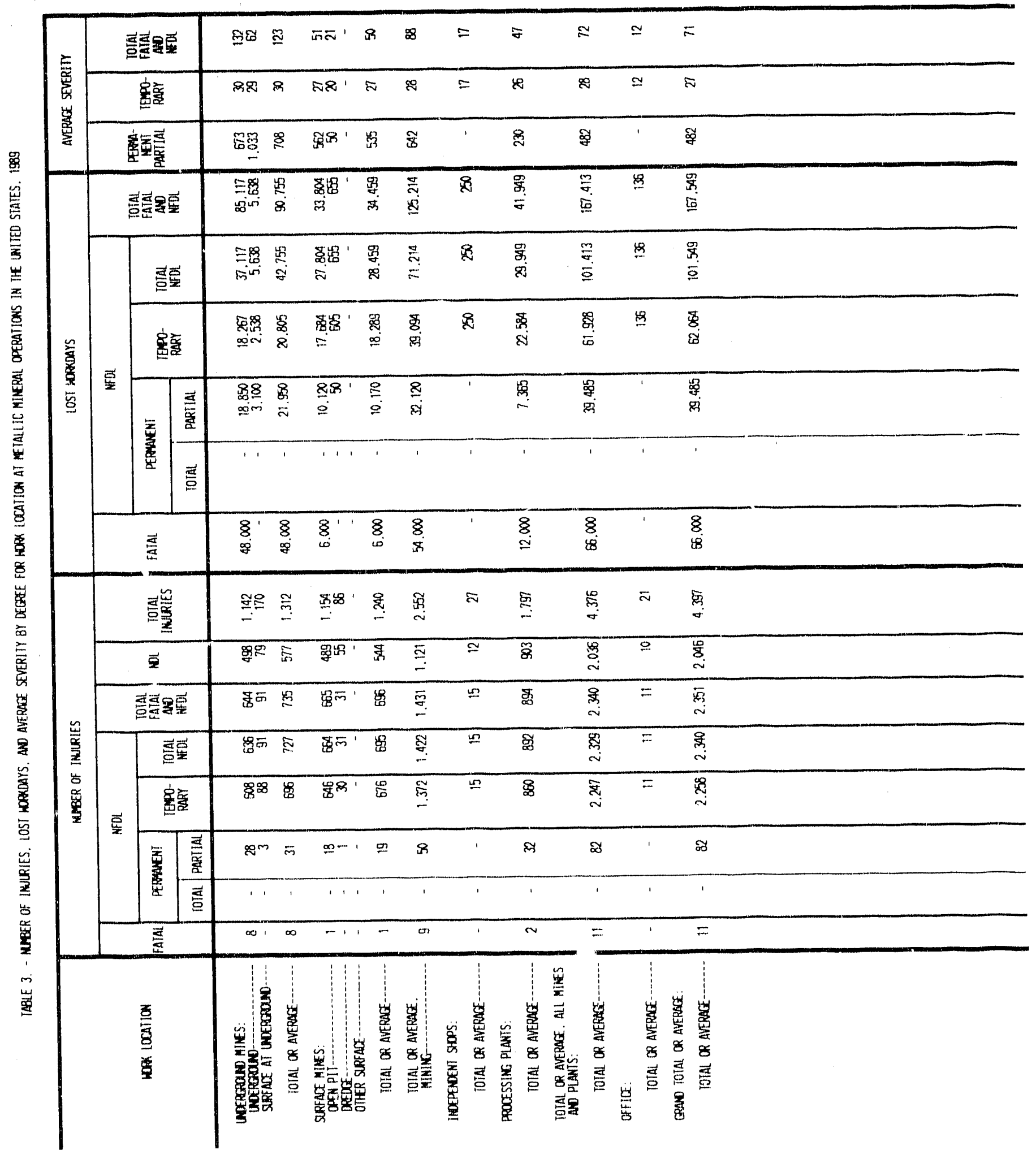




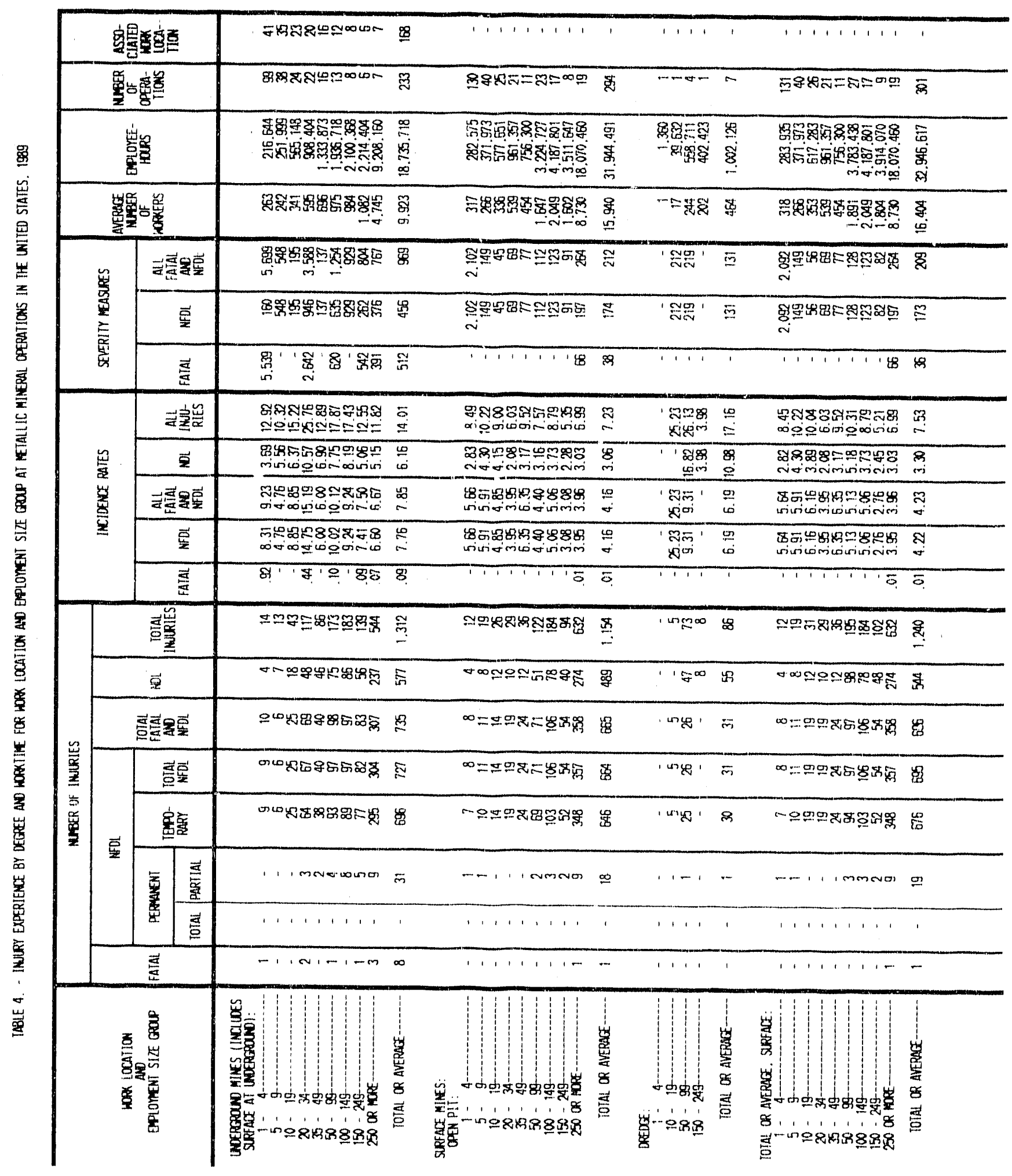




\begin{tabular}{|c|c|c|c|c|c|c|c|c|}
\hline \multicolumn{3}{|c|}{ 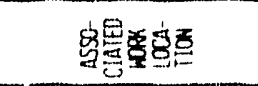 } & 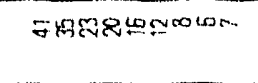 & & 1, & ' & 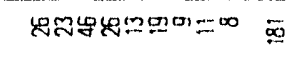 & \\
\hline \multicolumn{3}{|c|}{ 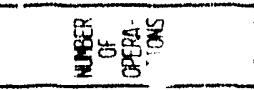 } & 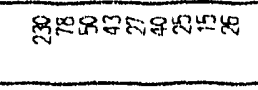 & 范 & $\sqrt{2} \cdots$ & 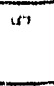 & 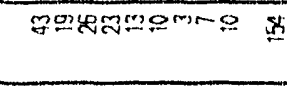 & \\
\hline \multicolumn{3}{|c|}{ 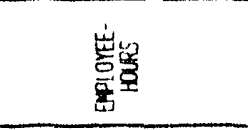 } & 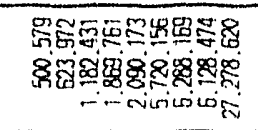 & 幽 & 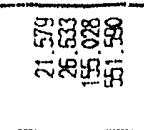 & $\begin{array}{l}8 \\
8 \\
0 \\
0\end{array}$ & 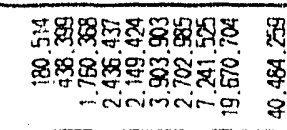 & \\
\hline \multicolumn{3}{|c|}{ 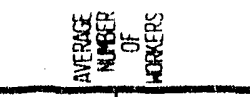 } & 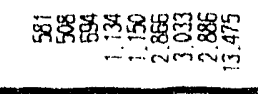 & 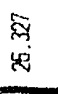 & wm的苏 & 8 & 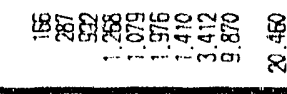 & \\
\hline \multirow{3}{*}{ 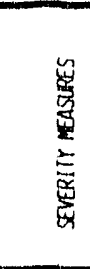 } & \multirow{3}{*}{\multicolumn{2}{|c|}{ 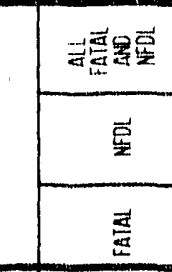 }} & 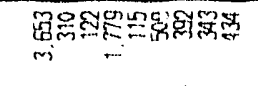 & 岁 & 8.68 & 88 & 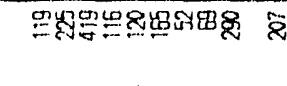 & \\
\hline & & & 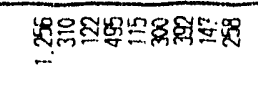 & $\dddot{\varpi}$ & 1.848 & 88 & 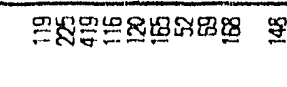 & \\
\hline & & & 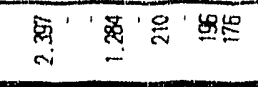 & 욜 & & & $111 . \$ \$$ & \\
\hline \multirow{5}{*}{$\breve{s}$} & & 容夋 & 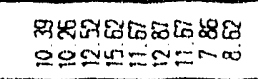 & $\mathscr{\infty}$ & 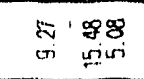 & $\stackrel{\square}{=}$ & 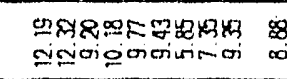 & \\
\hline & & $\underline{\underline{z}}$ & 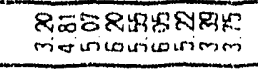 & कु & $\begin{array}{l}E \\
0 \quad \frac{9 \pi}{n i n} \\
\end{array}$ & 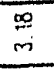 & 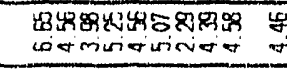 & \\
\hline & 表 & 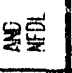 & 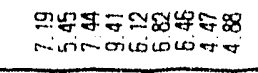 & 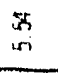 & 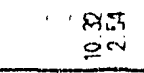 & Ei & 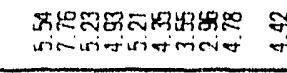 & \\
\hline & & $\overline{\underline{z}}$ & 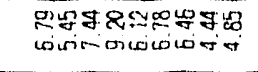 & 旾 & ' & हू & 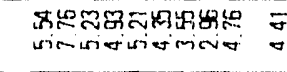 & \\
\hline & & 吾 & 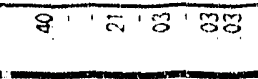 & लू & & ' & 111185 & \\
\hline \multirow{8}{*}{ 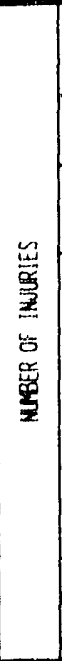 } & 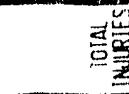 & & 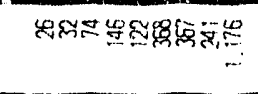 & 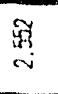 & $\cdots \div \square$ & $E$ & $=\curvearrowleft \bar{\alpha}$ & \\
\hline & 堂 & & "xing, & $\Xi$ & $m$ & $\cong$ & 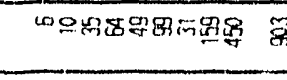 & \\
\hline & 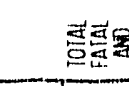 & & 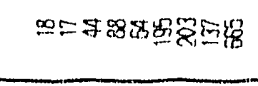 & 字 & , wor & $\dddot{2}$ & 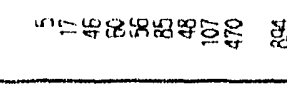 & \\
\hline & \multirow{4}{*}{$\overrightarrow{\vec{p}}$} & $\overrightarrow{\underline{c} \overrightarrow{\underline{R}}}$ & 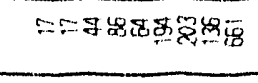 & $\underset{్}{ }$ & $1.00 \mathrm{~m}$ & 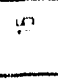 & 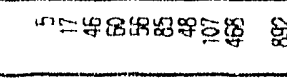 & . \\
\hline & & 䔡意 & 응 & 总 & $\cdots$ & 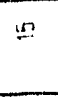 & 두 때 & \\
\hline & & 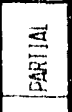 & 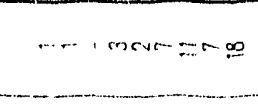 & 8 & 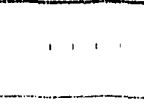 & ' & 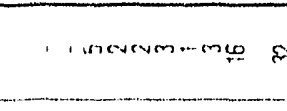 & \\
\hline & & 咅 & , . . , , & . & , . & & $1, \ldots 1,1,1$ & \\
\hline & \multicolumn{2}{|l|}{ 売 } & 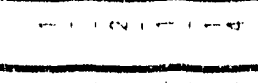 & $\theta$ & & & $\ldots \ldots \ldots$ o & \\
\hline \multicolumn{3}{|c|}{ 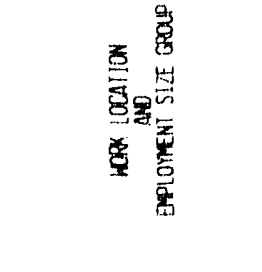 } & 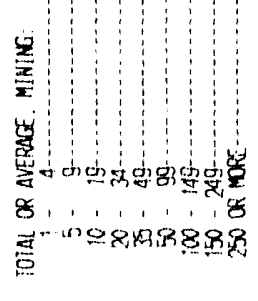 & 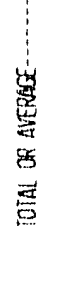 & 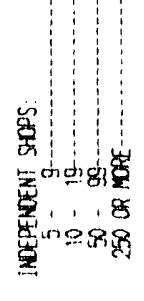 & 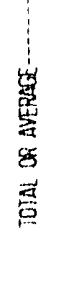 & 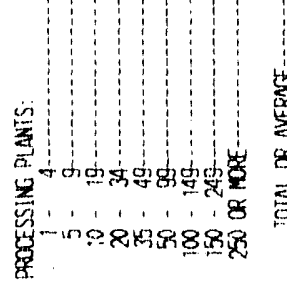 & \\
\hline
\end{tabular}




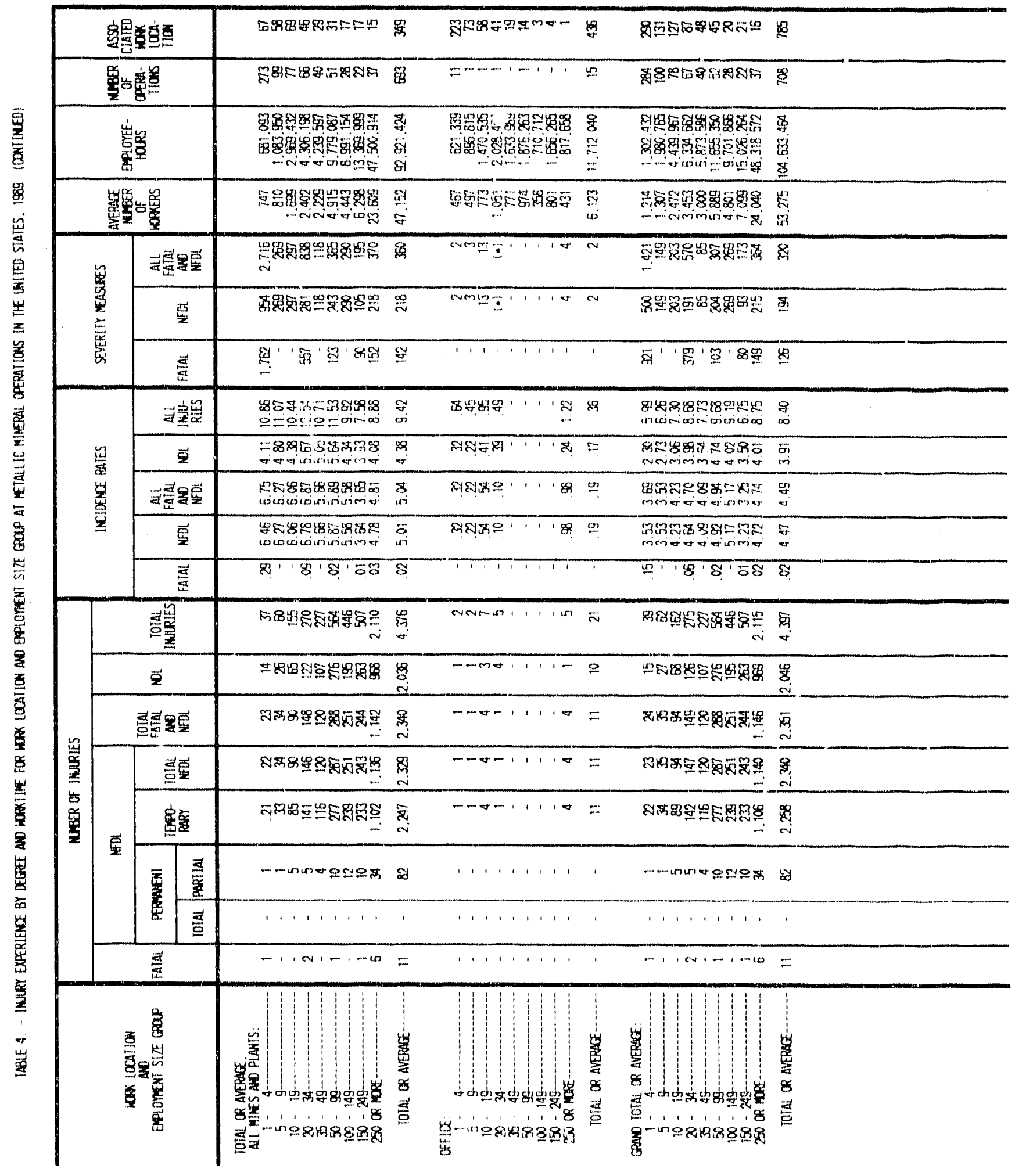




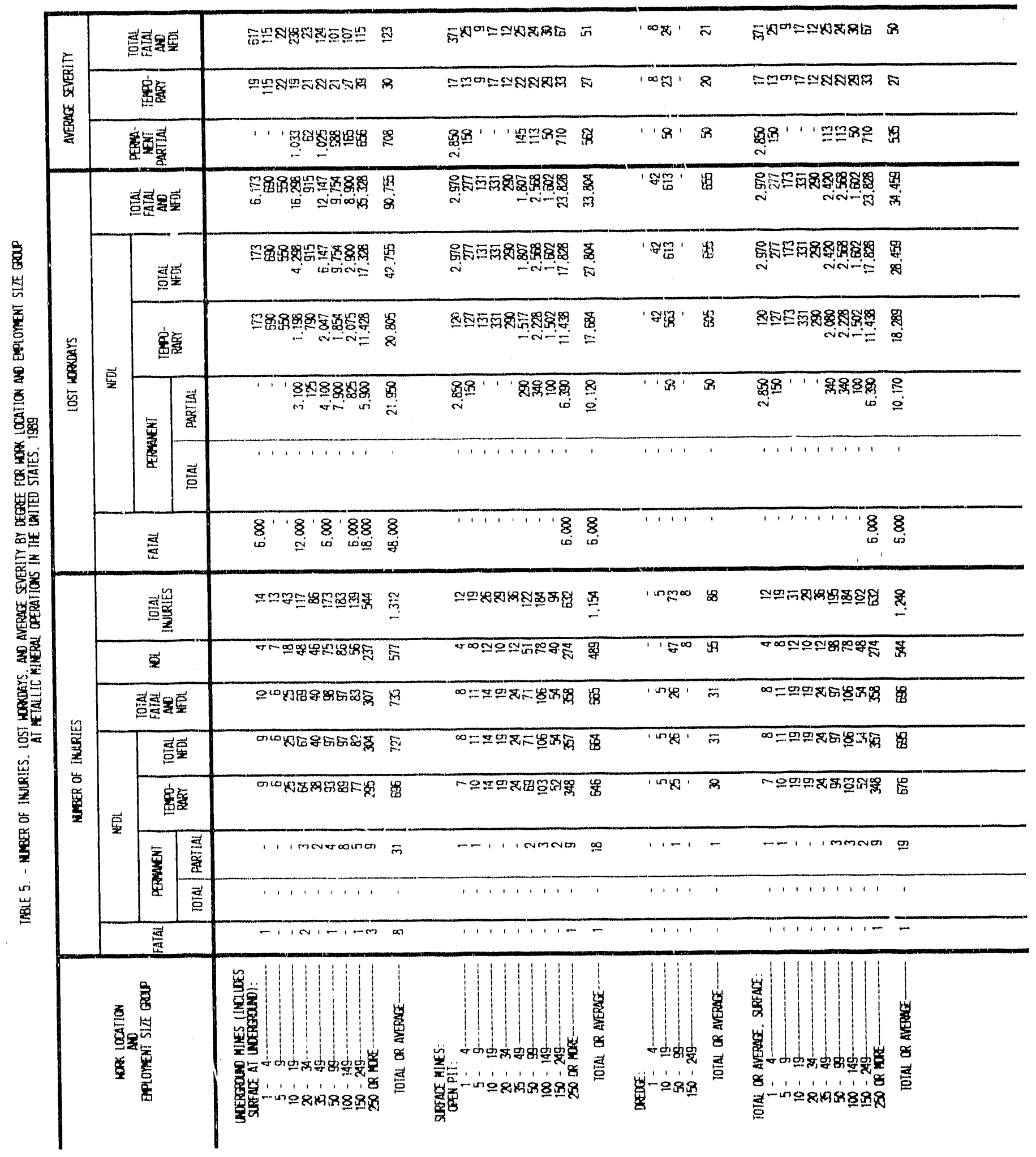




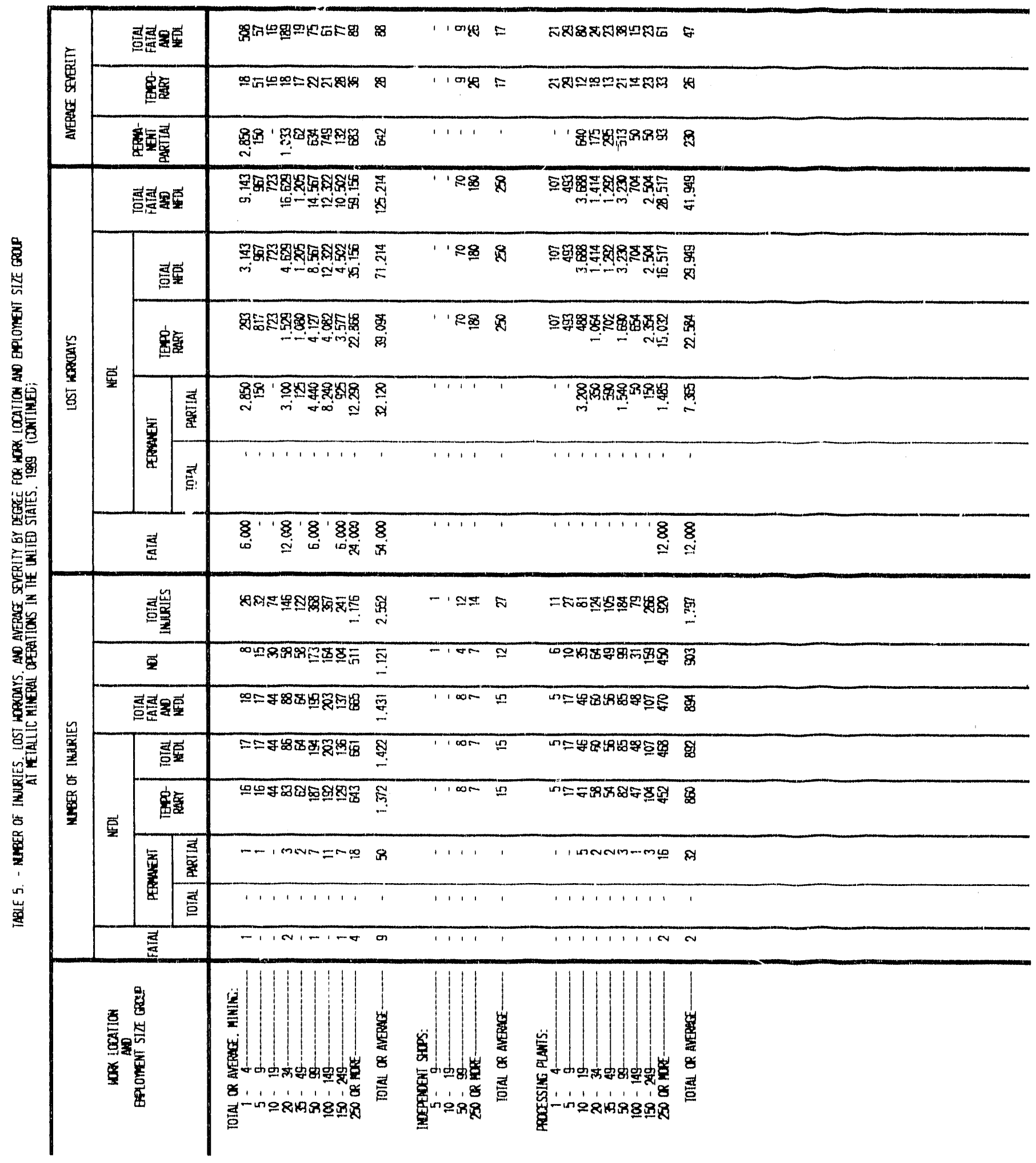




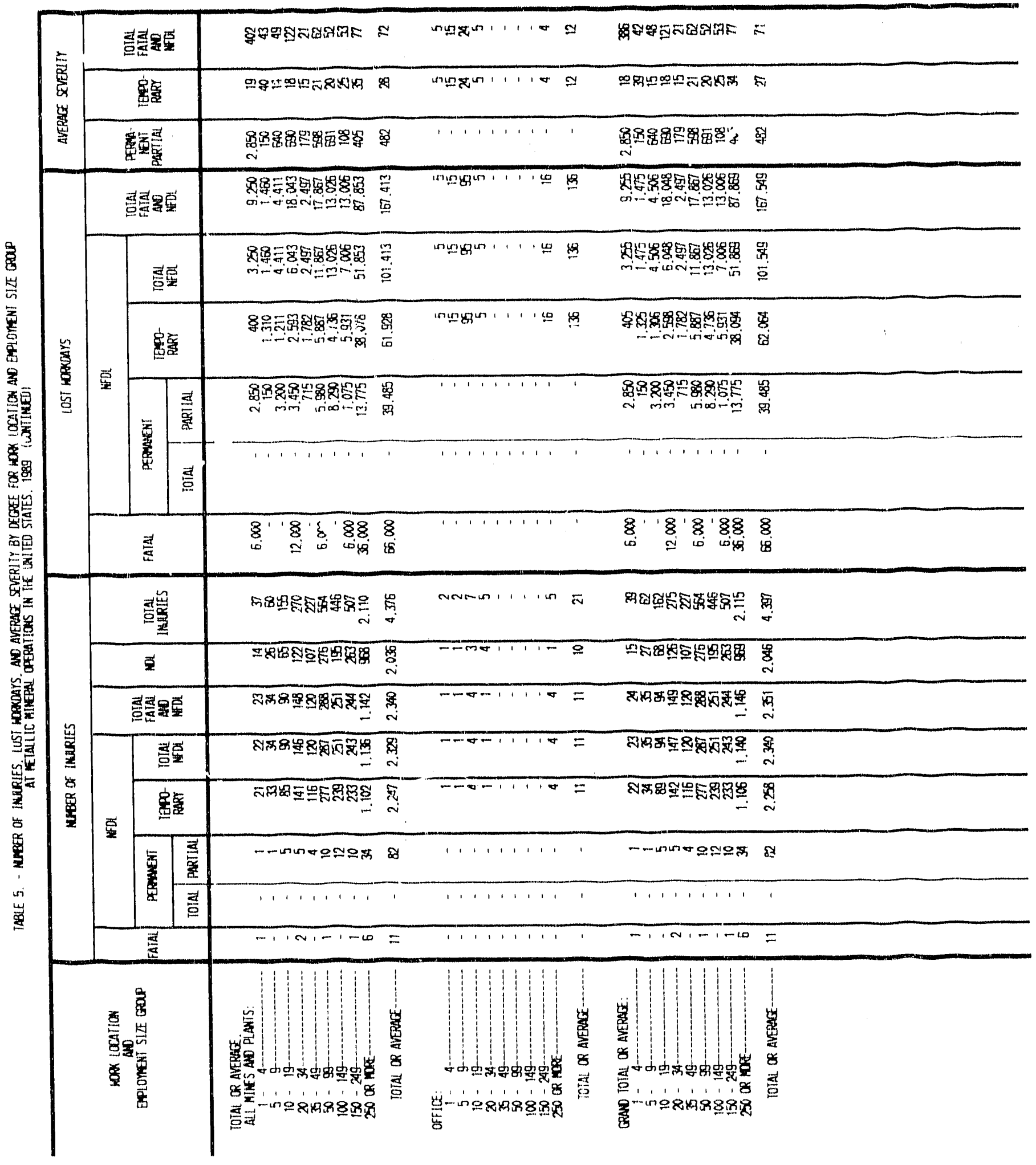




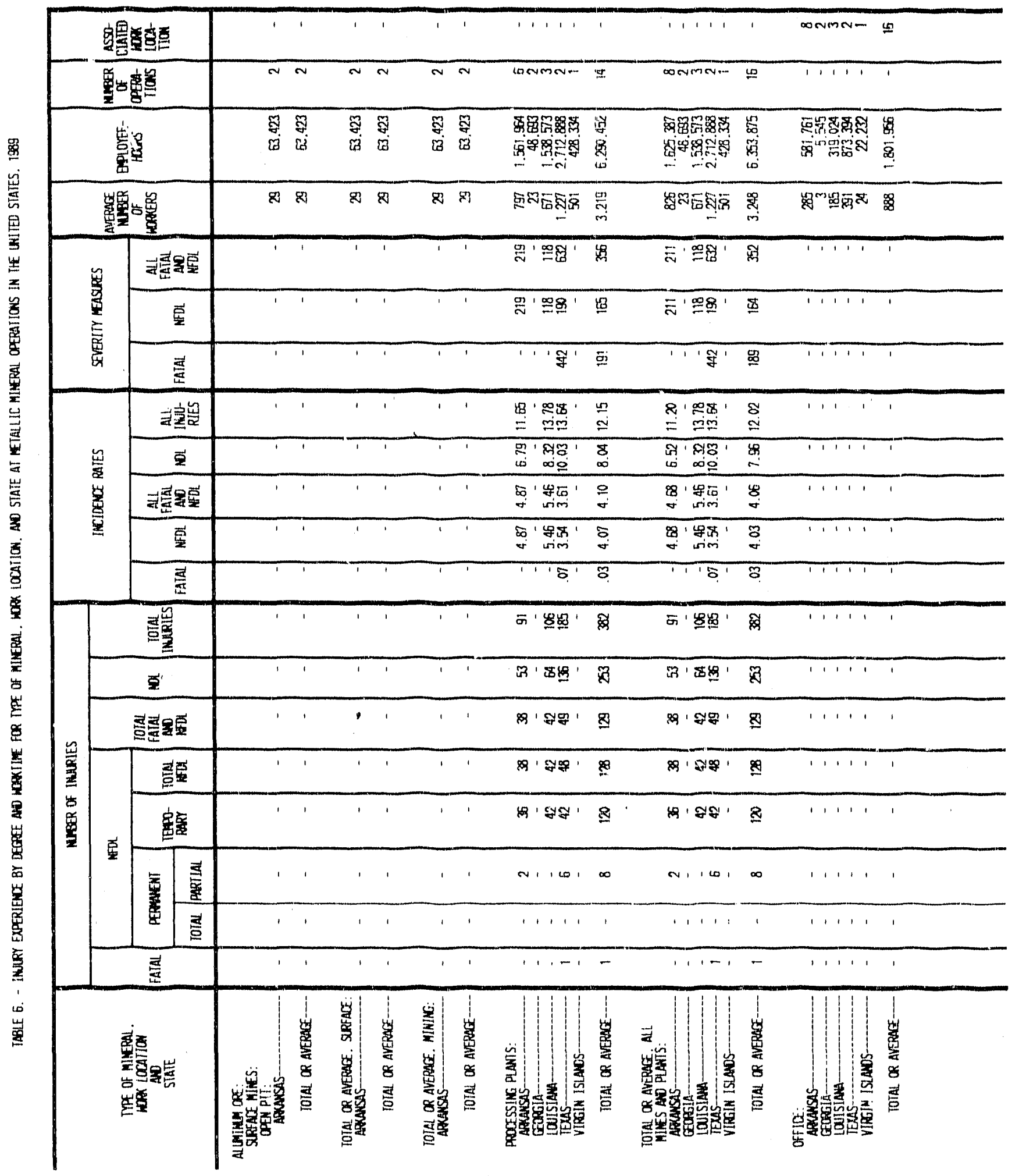




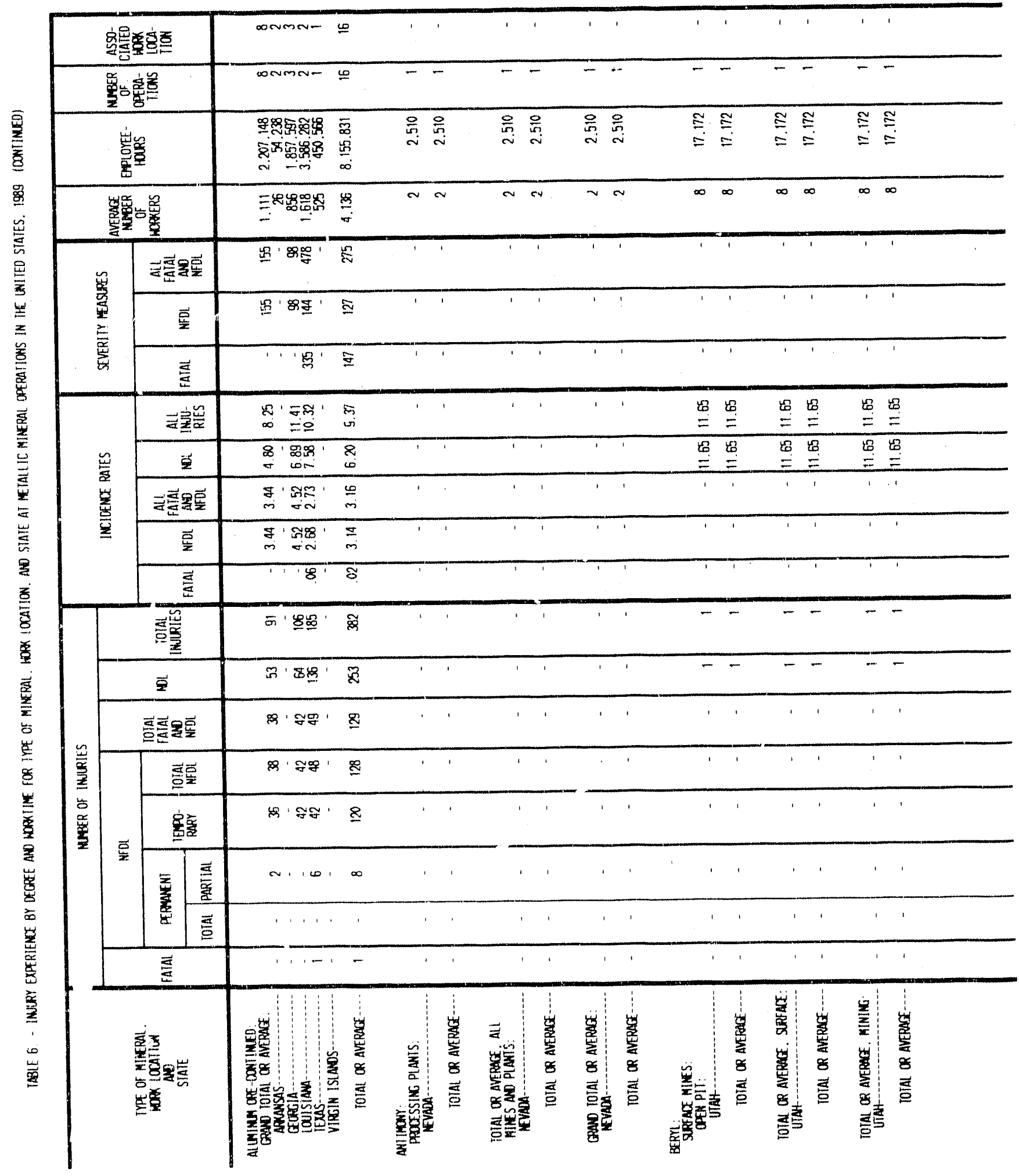




\begin{tabular}{|c|c|c|c|c|c|c|c|c|c|c|c|c|c|c|c|c|c|c|c|c|}
\hline & \multicolumn{3}{|c|}{ 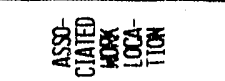 } & & ' & ' & & $\sim$ & $\sim$ & $\sim$ & $\sim$ & ' & ' & & ' & & & & 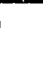 & \\
\hline & \multicolumn{3}{|c|}{ 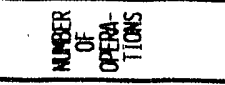 } & - & - & $\sim$ & $1 \sim$ & ' & ' & $\sigma$ & $\approx$ & - & - & - & - & - & - & & - & \\
\hline & \multicolumn{3}{|c|}{ 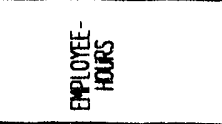 } & & $\begin{array}{l}\text { 罗 } \\
\stackrel{8}{0}\end{array}$ & $\begin{array}{l}\bar{g} \\
\underline{\tilde{g}}\end{array}$ & $\begin{array}{l}\bar{g} \\
\underline{B}\end{array}$ & $\begin{array}{l}: \\
\$ \\
q 8\end{array}$ & 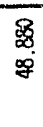 & $\begin{array}{l}\overline{\mathbf{s}} \\
\stackrel{\sim}{\sim}\end{array}$ & 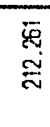 & 8 & 8 & 8 & s & $\underset{0}{\circ}$ & 을 & & : & \\
\hline & \multicolumn{3}{|c|}{ 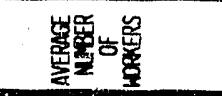 } & B & $B$ & $\approx$ & $\approx$ & 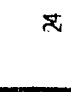 & $\pi$ & $\Phi$ & $\Phi$ & - & - & - & - & $m$ & $m$ & & $r$ & \\
\hline \multirow{3}{*}{\multicolumn{2}{|c|}{ 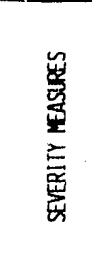 }} & 球 & 晴走 & $\bar{m}$ & $\bar{m}$ & $\approx$ & $\approx$ & & ' & $\mathbb{A}$ & $\mathbb{E}$ & 1 & 1 & & 1 & & 1 & & 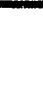 & \\
\hline & & & 总 & $\bar{m}$ & $\bar{m}$ & $\mathscr{2}$ & $\mathscr{D}$ & ' & ' & $\approx$ & $\mathbb{1}$ & ' & ' & & ' & 1 & ' & & 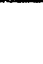 & \\
\hline & & & 랄 & ${ }^{\circ}$ & ' & ' & ' & 1 & ' & ' & ' & ' & 1 & & ' & ' & 1 & & 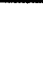 & \\
\hline & \multirow{5}{*}{ 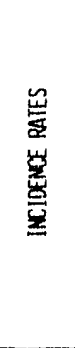 } & & 春岂 & $\vec{\forall}$ & Fi & $\cong$ & $\underset{0}{\simeq}$ & ' & ' & $\bar{\sigma}$ & $\bar{\sigma}$ & ' & ' & & ' & ' & ' & & . & \\
\hline & & & $\overrightarrow{\underline{2}}$ & $\stackrel{\circ}{\circ}$ & $\stackrel{\circ}{\circ}$ & $\stackrel{8}{-}$ & 8 & $T$ & ' & $\begin{array}{l}E \\
m\end{array}$ & $\underset{m}{E}$ & ' & & & ' & ' & 7 & & 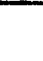 & \\
\hline 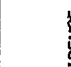 & & 球 & 是豆 & 5 & ह5 & શ્ & $\stackrel{\simeq}{=}$ & ' & ' & क & का & ' & ' & & ' & ' & ' & & & \\
\hline & & & 鬲 & 5 & $\stackrel{5}{-2}$ & ฐ & $\Xi$ & ' & ' & के & $\%$ & 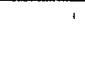 & $T$ & & I & 1 & ' & & 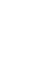 & \\
\hline & & & $\frac{\vec{k}}{\underline{E}}$ & r & I & & r & & & & 1 & & & & ' & ' & ' & & & \\
\hline \multirow{8}{*}{ 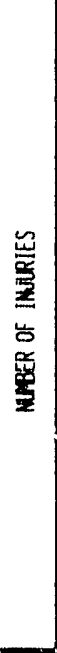 } & & 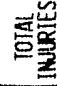 & & $\sigma$ & $\sigma$ & n & in & & ' & in & in & ' & ' & ' & I & ' & ' & & 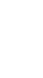 & \\
\hline & & $\overrightarrow{\underline{x}}$ & & $m$ & $m$ & $\sigma$ & $\pi$ & ' & ' & $\sigma$ & $\sigma$ & ' & & ' & ' & ' & ' & & 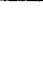 & \\
\hline & & 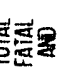 & & - & - & - & - & ' & ' & - & - & ' & ' & & ' & ' & ' & & 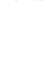 & \\
\hline & \multirow{4}{*}{$\overrightarrow{\underline{\Gamma}}$} & \multicolumn{2}{|c|}{ 駧要 } & - & - & - & - & ' & ' & - & - & ' & ' & & 1 & ' & ' & & & \\
\hline & & \multicolumn{2}{|c|}{ 鱼蜜 } & - & - & - & - & & ' & - & - & ' & & ' & ' & ' & ' & & & \\
\hline & & \multirow{2}{*}{ 要 } & 总 & ' & ' & & ' & ' & ' & ' & ' & ' & ' & ' & ' & ' & ' & \multicolumn{2}{|c|}{ ' } & \\
\hline & & & 홍 & ' & 1 & & ' & & ' & ' & ' & ' & ' & ' & + & ' & ' & & & \\
\hline & \multicolumn{3}{|c|}{$\frac{\vec{d}}{\underline{a}}$} & & 1 & & ' & ' & ' & ' & ' & . & ' & ' & ' & ' & , & \multicolumn{2}{|c|}{ ' } & \\
\hline \multicolumn{4}{|c|}{ 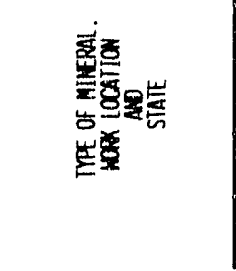 } & 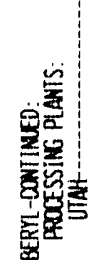 & 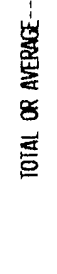 & 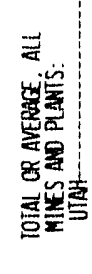 & 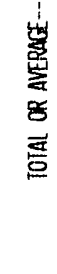 & 嘿嗀 & 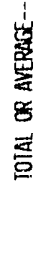 & 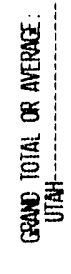 & 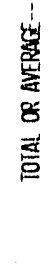 & 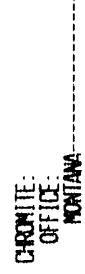 & 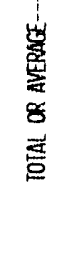 & 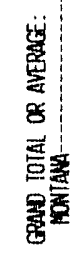 & 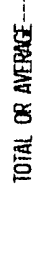 & 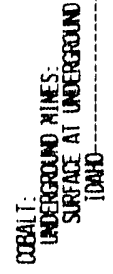 & 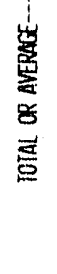 & 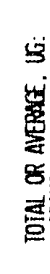 & $\begin{array}{l}\xi \\
8 \\
8\end{array}$ & \\
\hline
\end{tabular}




\begin{tabular}{|c|c|c|c|c|c|c|c|c|c|c|c|c|c|c|c|c|}
\hline \multicolumn{3}{|c|}{ 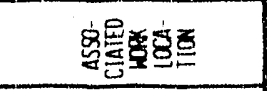 } & & & & & & - & - & - & & & simer- = & $=$ & $\sin \operatorname{lon}^{-1}$ & $=$ \\
\hline \multicolumn{3}{|c|}{ 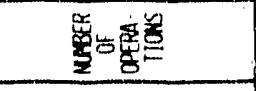 } & 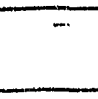 & - & - & - & & & 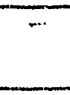 & - & mind & $=$ & -11. & - & comn. & $\Xi$ \\
\hline \multicolumn{3}{|c|}{ 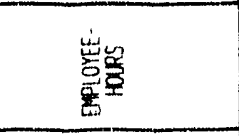 } & 高 & $\begin{array}{l}\text { O응 } \\
\text { si }\end{array}$ & $\frac{9}{5}$ & $\stackrel{8}{i}$ & 量 & $\frac{5}{c}$ & $\Xi$ & ह & 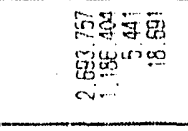 & 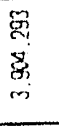 & 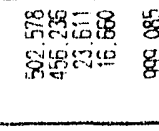 & 要 & 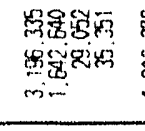 & 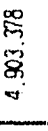 \\
\hline \multicolumn{3}{|c|}{ 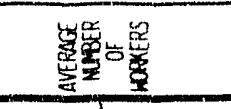 } & $m$ & $m$ & $m$ & $m$ & $\cdots$ & $\cdots$ & 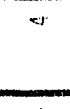 & $\sigma$ & $s_{n}$ & $\stackrel{8}{8}$ & 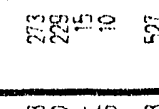 & تัن & 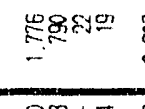 & 蒫 \\
\hline \multirow{3}{*}{ 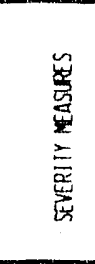 } & 清案 & & & ' & ' & & & & & . & E. & $\bar{\xi}$ & 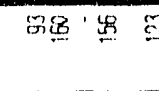 & 9 & $\frac{89}{6 \pm}$ & 斀 \\
\hline & 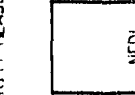 & 兽 & & & & & & 1 & & , & 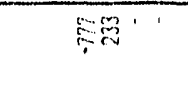 & 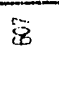 & 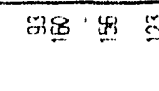 & $\underset{2}{2}$ & 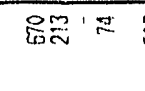 & 恕 \\
\hline & & 迨 & & ' & & & & & & & & ' & & & & ' \\
\hline \multirow{5}{*}{ 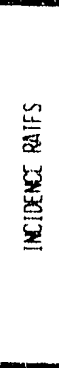 } & & 䒠密 & & & & ' & & & & $\therefore$ & 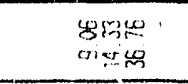 & $\stackrel{8}{2}$ & 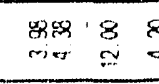 & 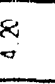 & 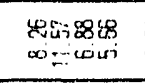 & 旁 \\
\hline & & $\bar{z}$ & & & & ' & & & & 1 & 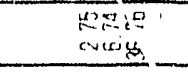 & $\begin{array}{l}8 \\
8 \\
\end{array}$ & 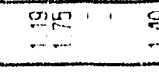 & 8 & 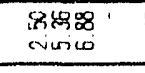 & 学 \\
\hline & 找我 & 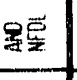 & & & & ! & & ' & $\cdot$ & ' & 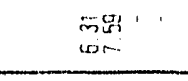 & $\begin{array}{c}\Psi \\
w \\
0\end{array}$ & 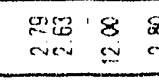 & $\underset{8}{i}$ & 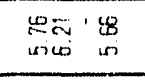 & $\begin{array}{c}\infty \\
\infty \\
i n \\
i n\end{array}$ \\
\hline & & 缟 & & & & & & & T & ' & $\begin{array}{l}\text { wis ' } \\
\text { wom } \\
\text { wom }\end{array}$ & $\begin{array}{l}88 \\
6 \\
\end{array}$ & 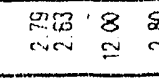 & $\underset{ن}{8}$ & 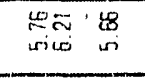 & 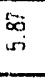 \\
\hline & & $\underline{\underline{E}}$ & & & & & & & & & & & & & & \\
\hline \multirow{8}{*}{ 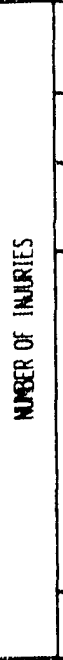 } & 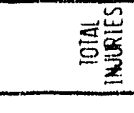 & & & & ' & & ' & 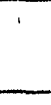 & & & $\sum_{0}$ & 嵒 & 음 & $\pi$ & $58-$ & \& \\
\hline & 포 & & ' & ' & & ' & ' & & & ' & $58^{-}$ & $\cong$ & me्ध 1 & $m$ & $89-1$ & 8 \\
\hline & 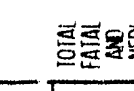 & & ' & ' & & ' & & & & ' & 마욤 & 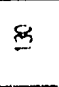 & .6o $=$ & \pm & fisin ' - & 车 \\
\hline & \multirow{3}{*}{ 蒙 } & 业总 & & ' & & ' & & & . & ' & mi 1 & 8 & $-\infty \cdot=$ & \pm & รक्ष - & 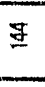 \\
\hline & & 蜜家 & & ' & . & ' & & & ' & & $\operatorname{gng} 1$ & \pm & w I - ? & $=$ & $\mathscr{\infty} \mathbf{n}^{--}$ & E \\
\hline & & 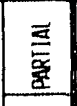 & & , & & ' & & ' & & & & $\infty$ & - & - & & - \\
\hline & & 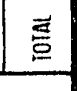 & & , & & & & . & & & & & & ' & & ' \\
\hline & \multicolumn{2}{|c|}{$\overline{\frac{z}{x}}$} & & . & & & & ' & . & & & ' & & $\cdot$ & & ' \\
\hline \multicolumn{3}{|c|}{ 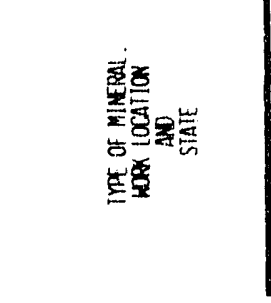 } & 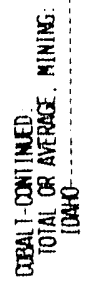 & 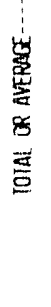 & 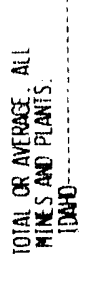 & 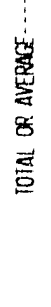 & & 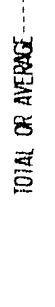 & 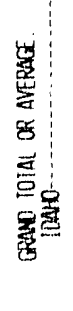 & 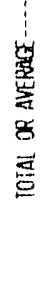 & 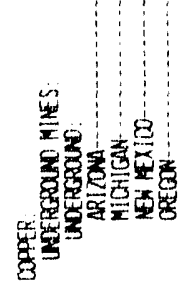 & 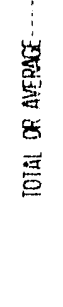 & 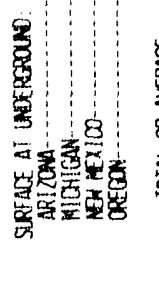 & 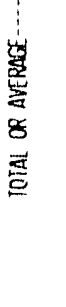 & 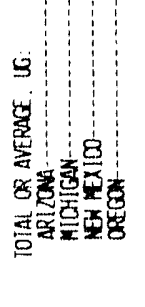 & 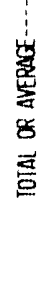 \\
\hline
\end{tabular}




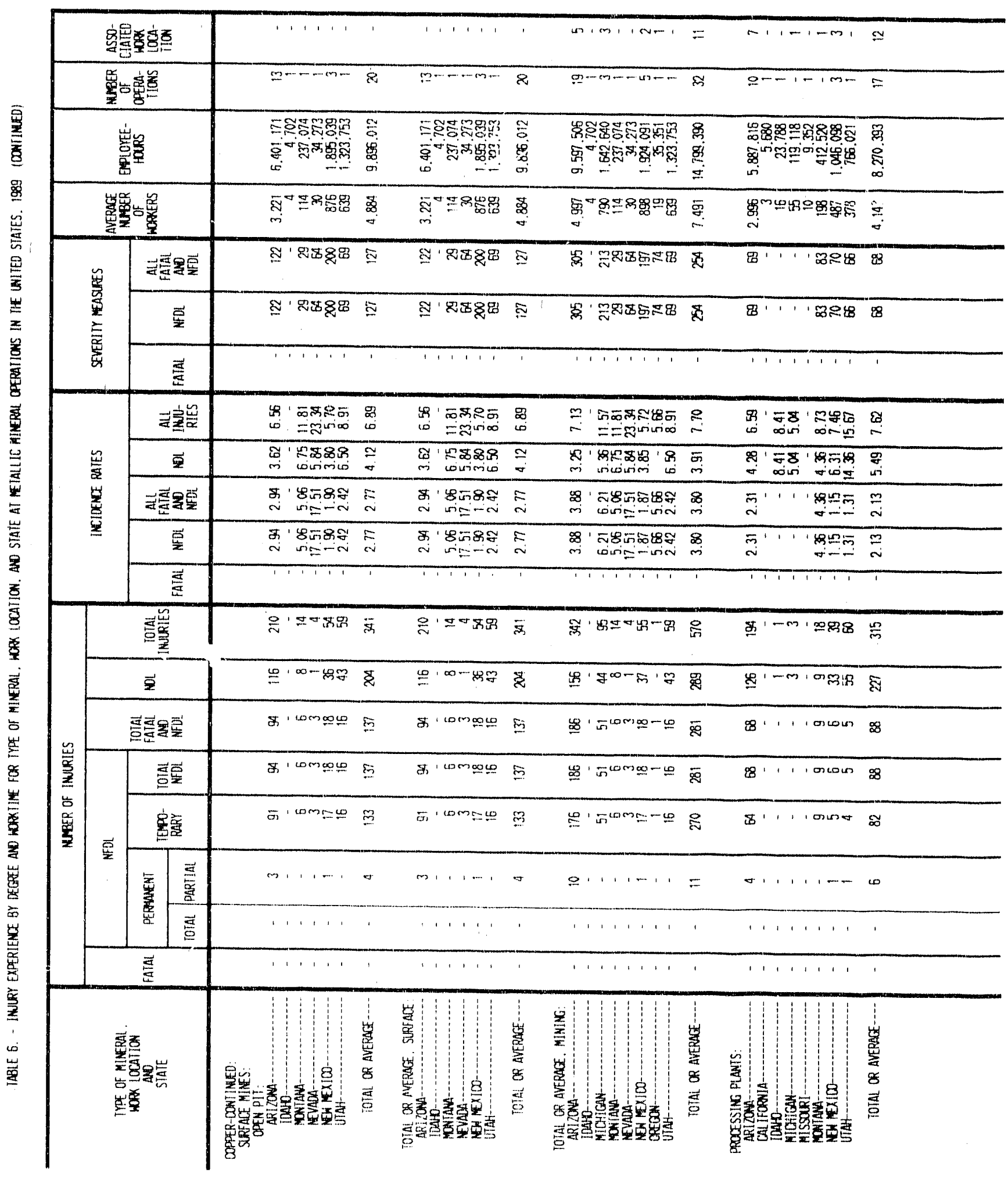




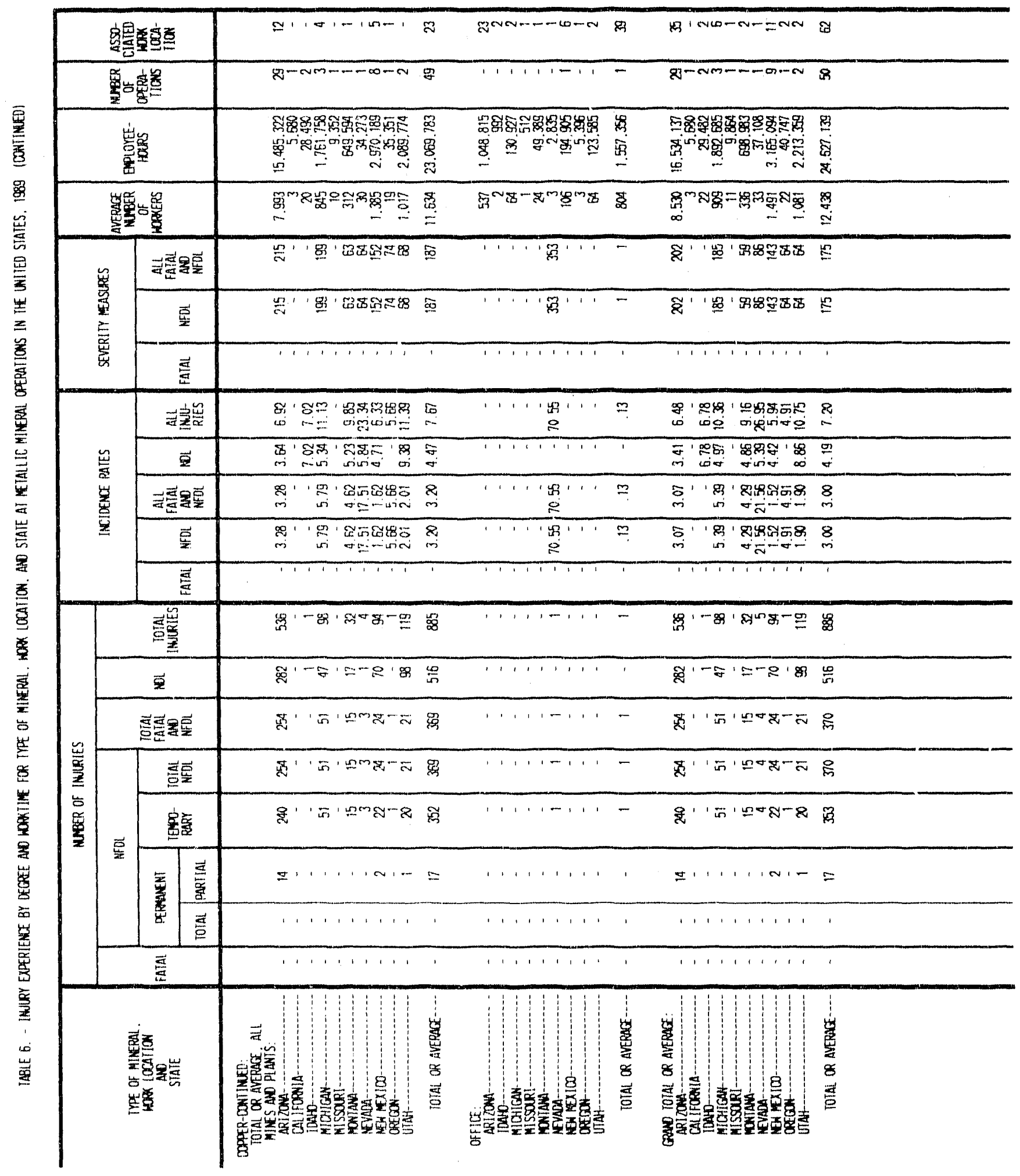




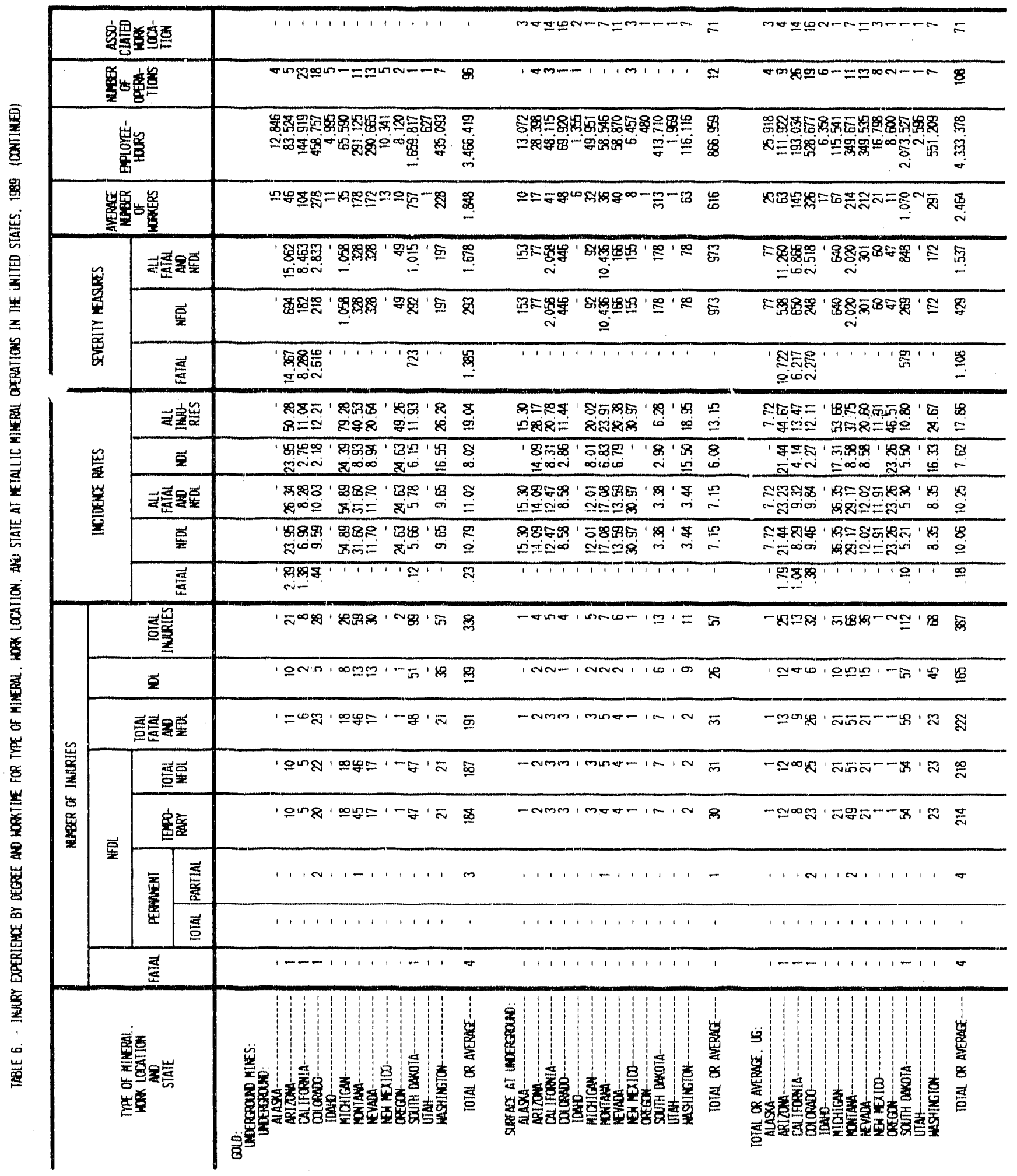




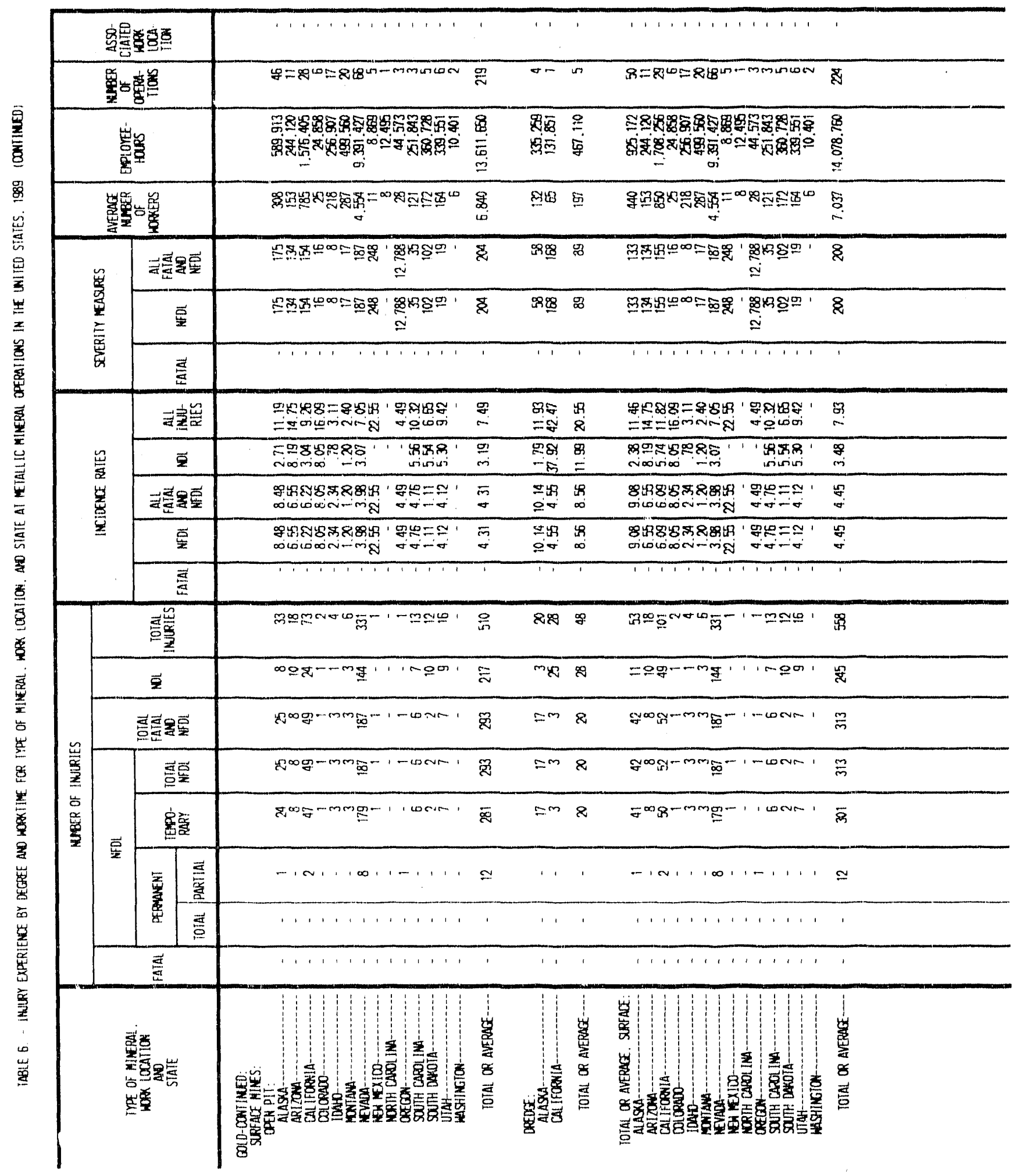




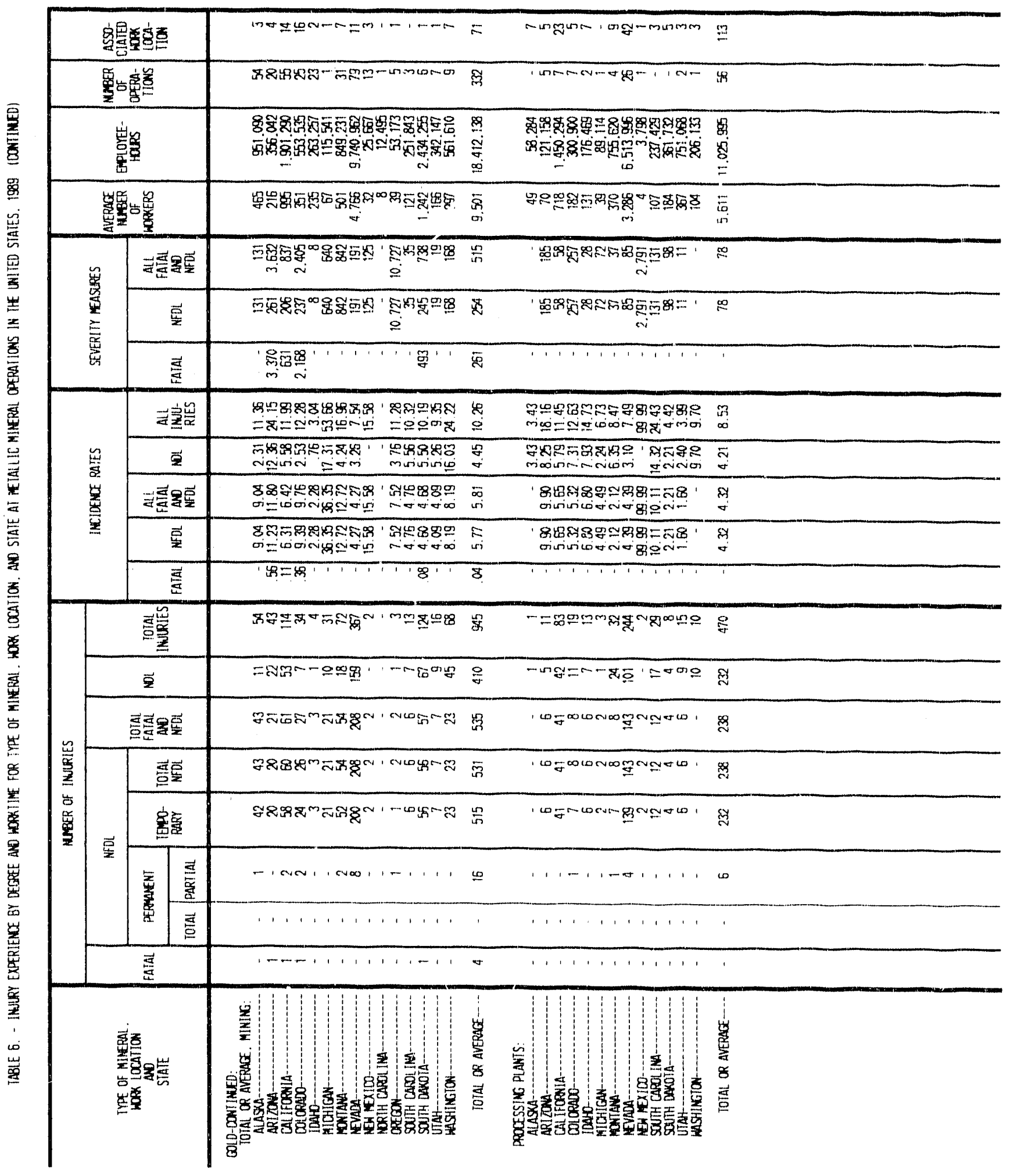




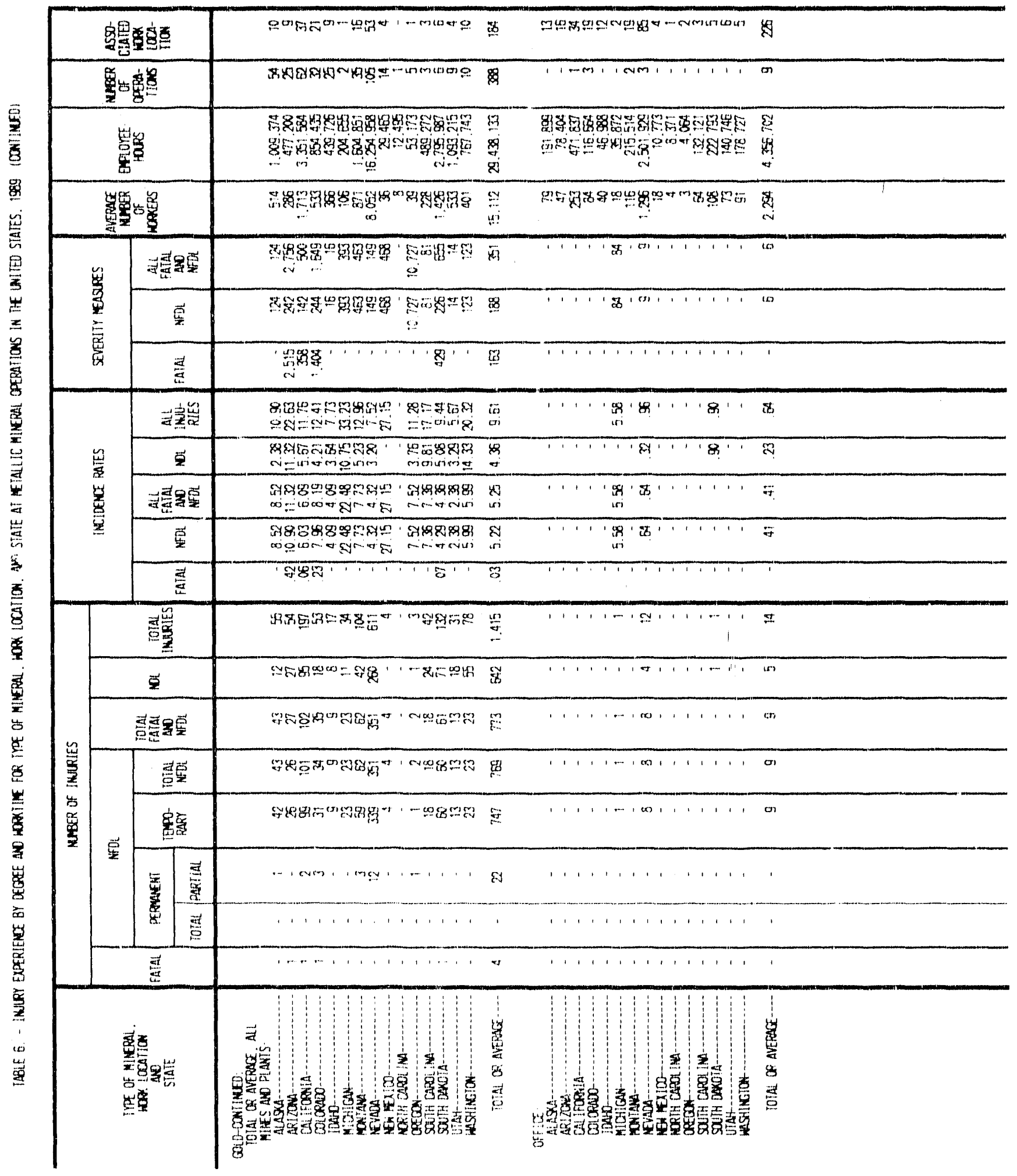




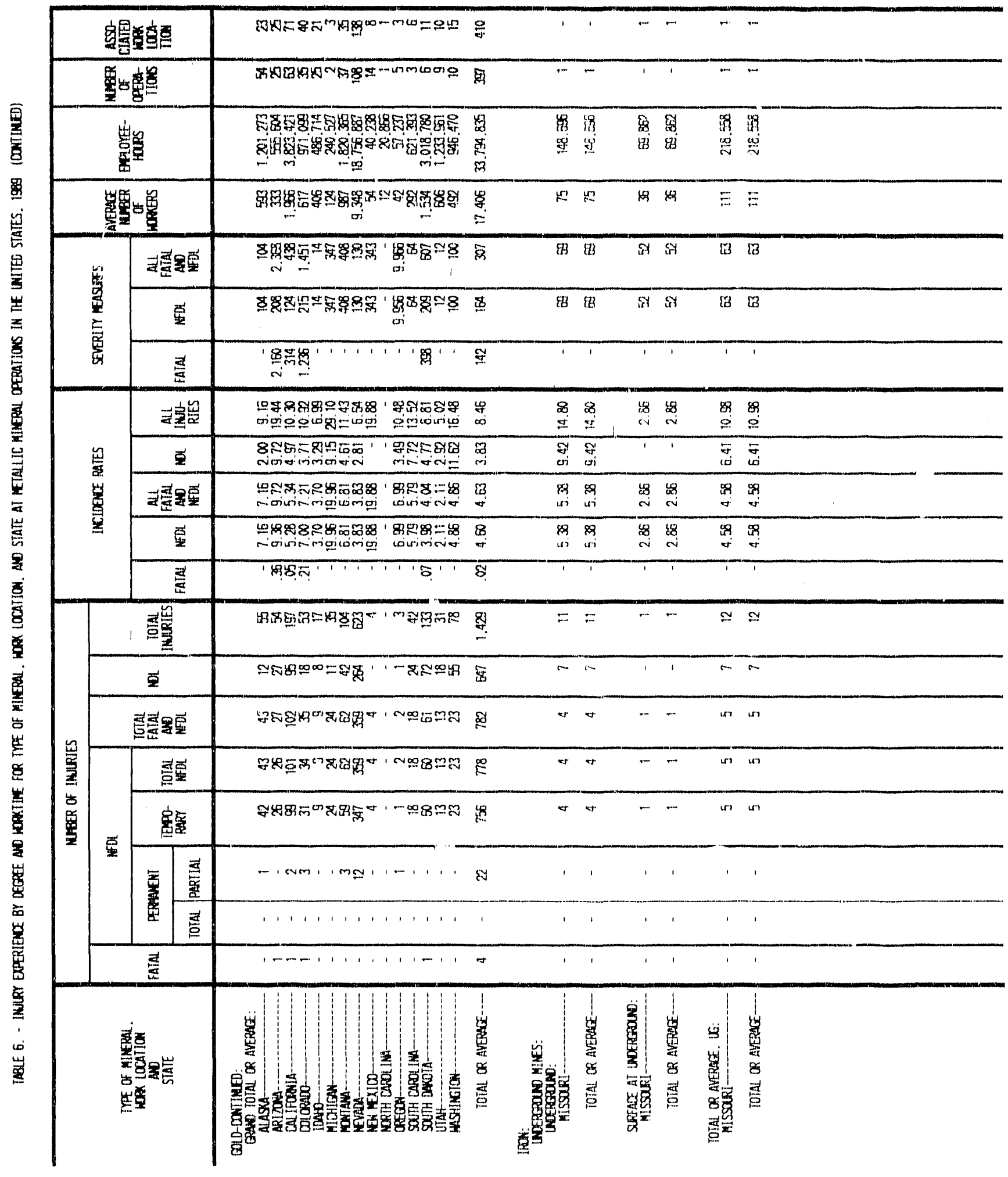




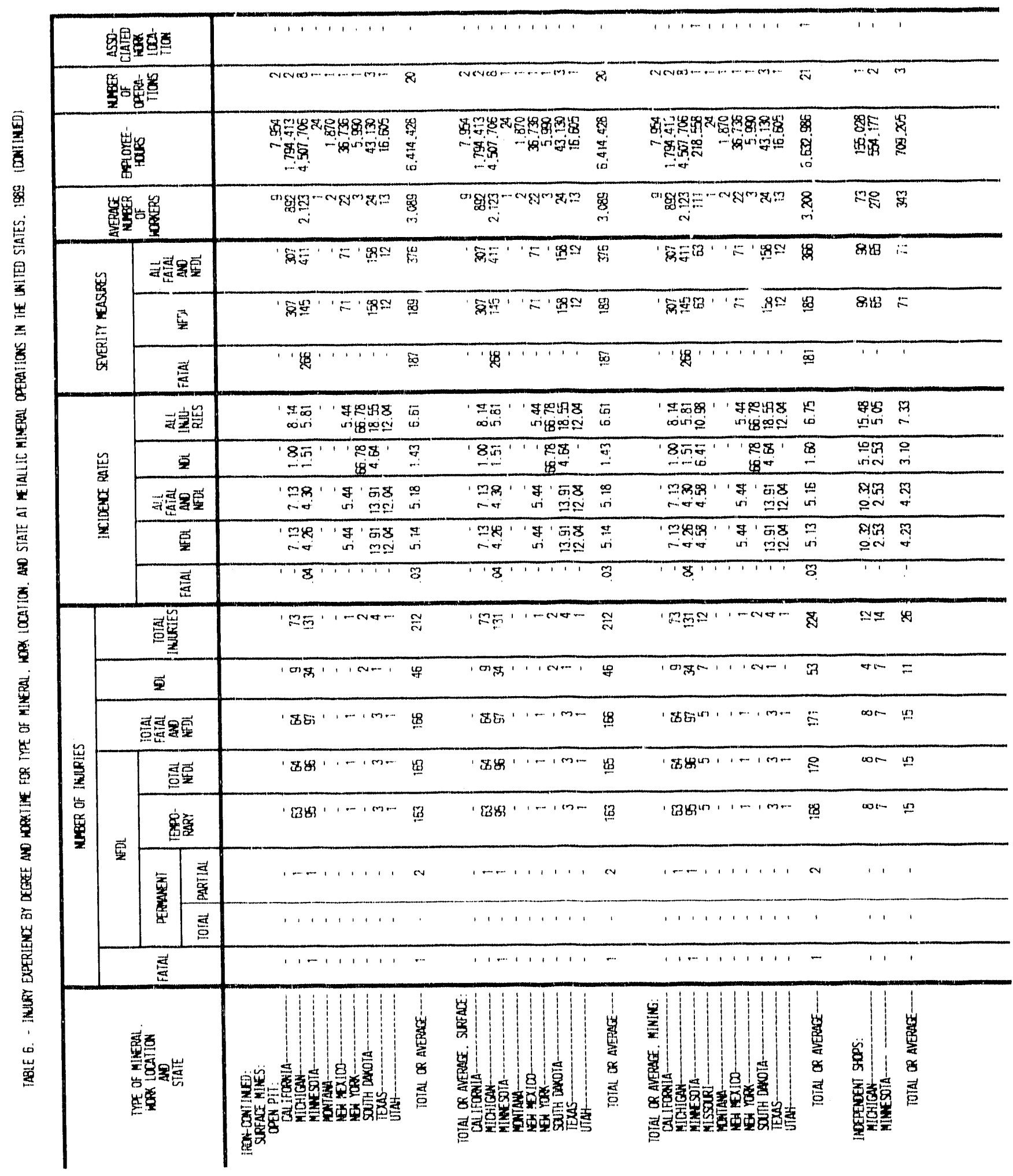




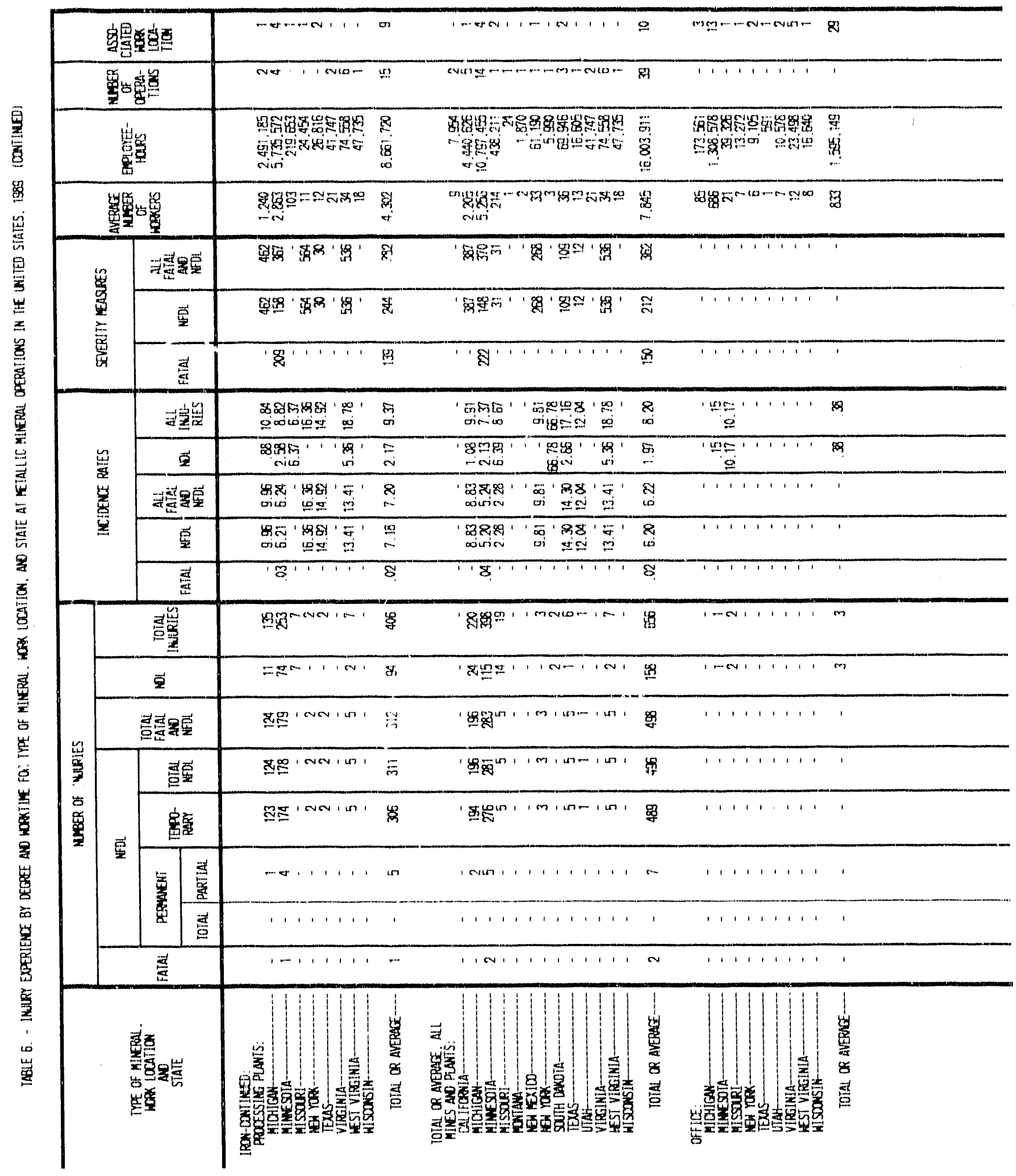




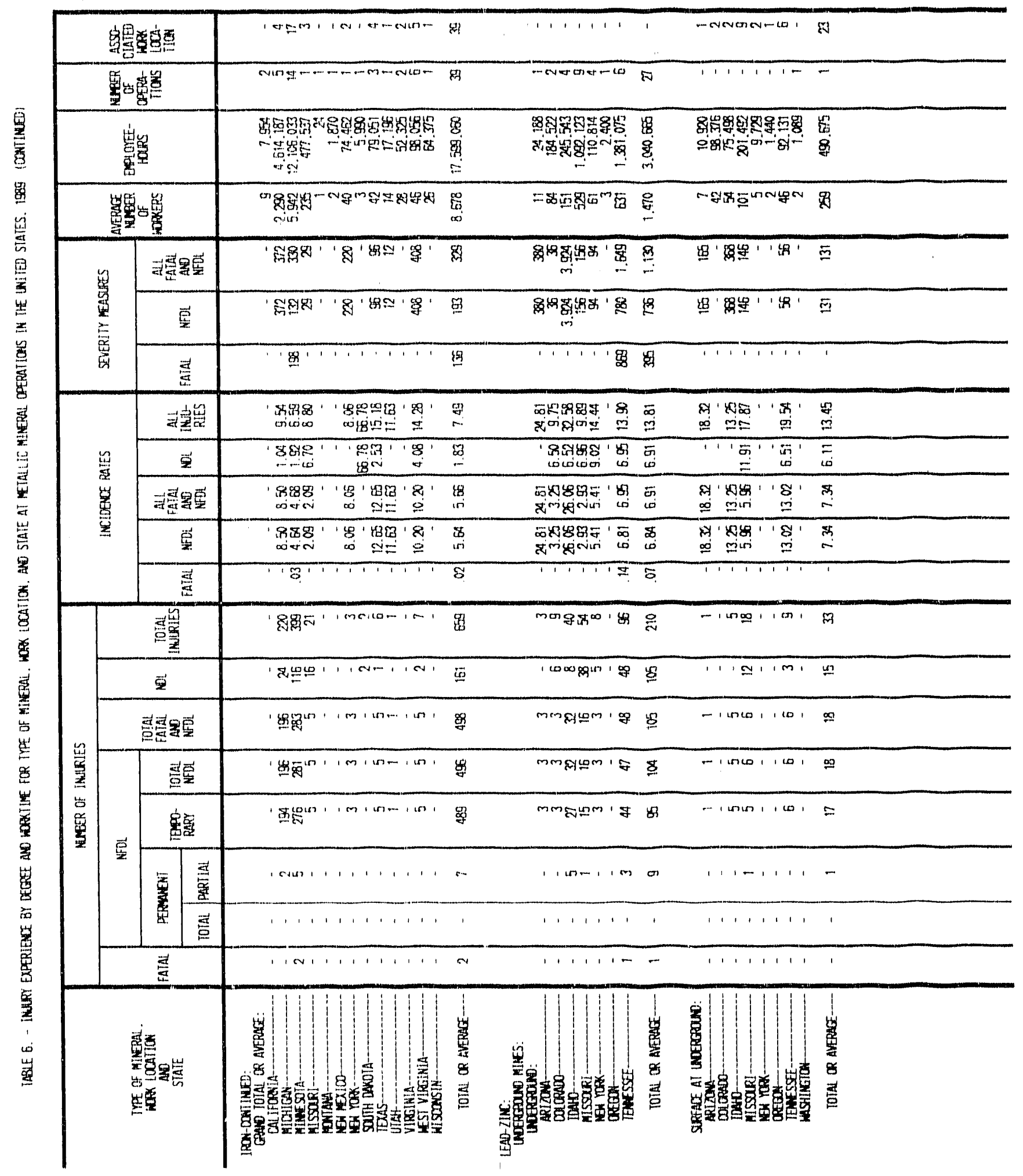




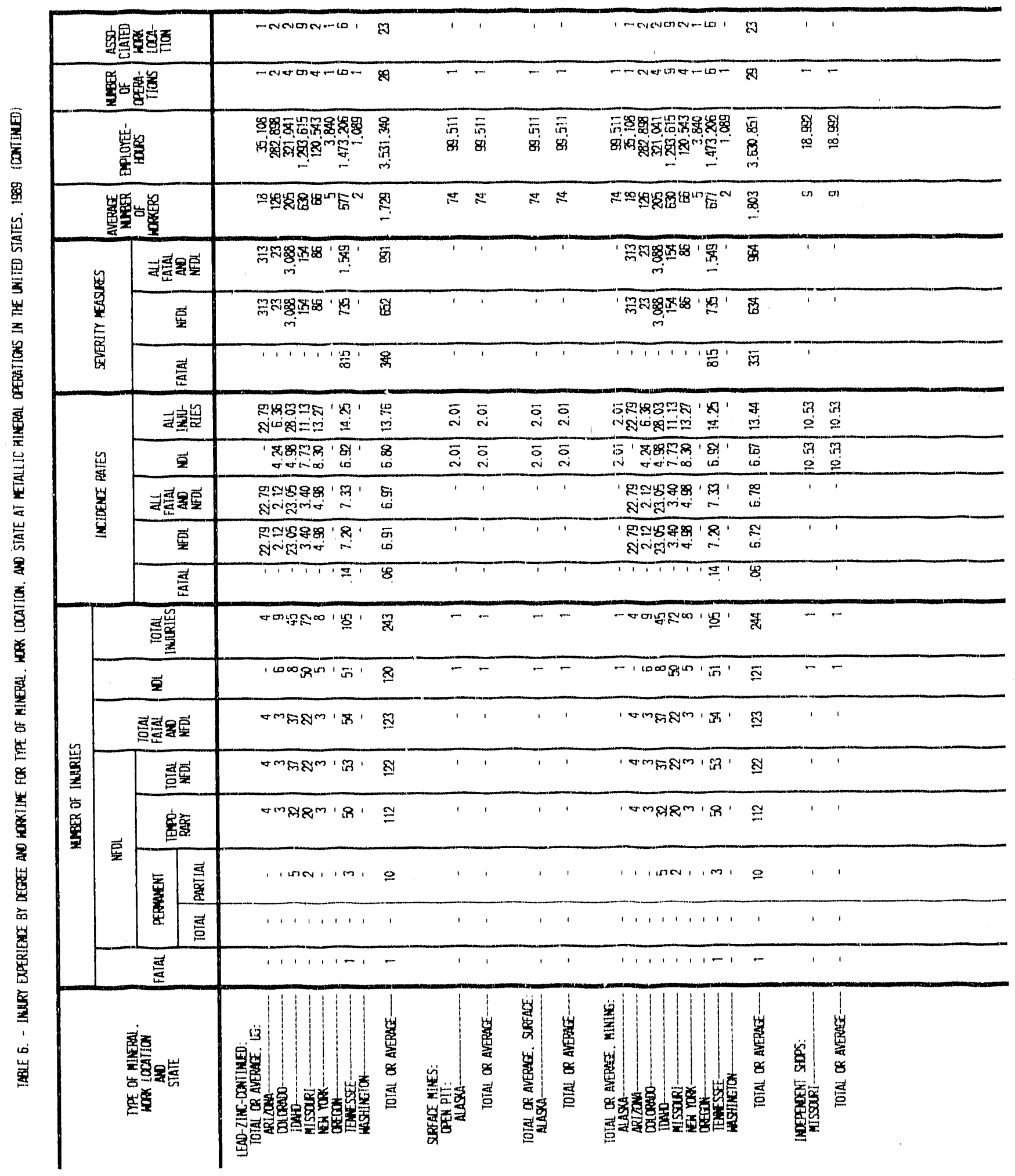




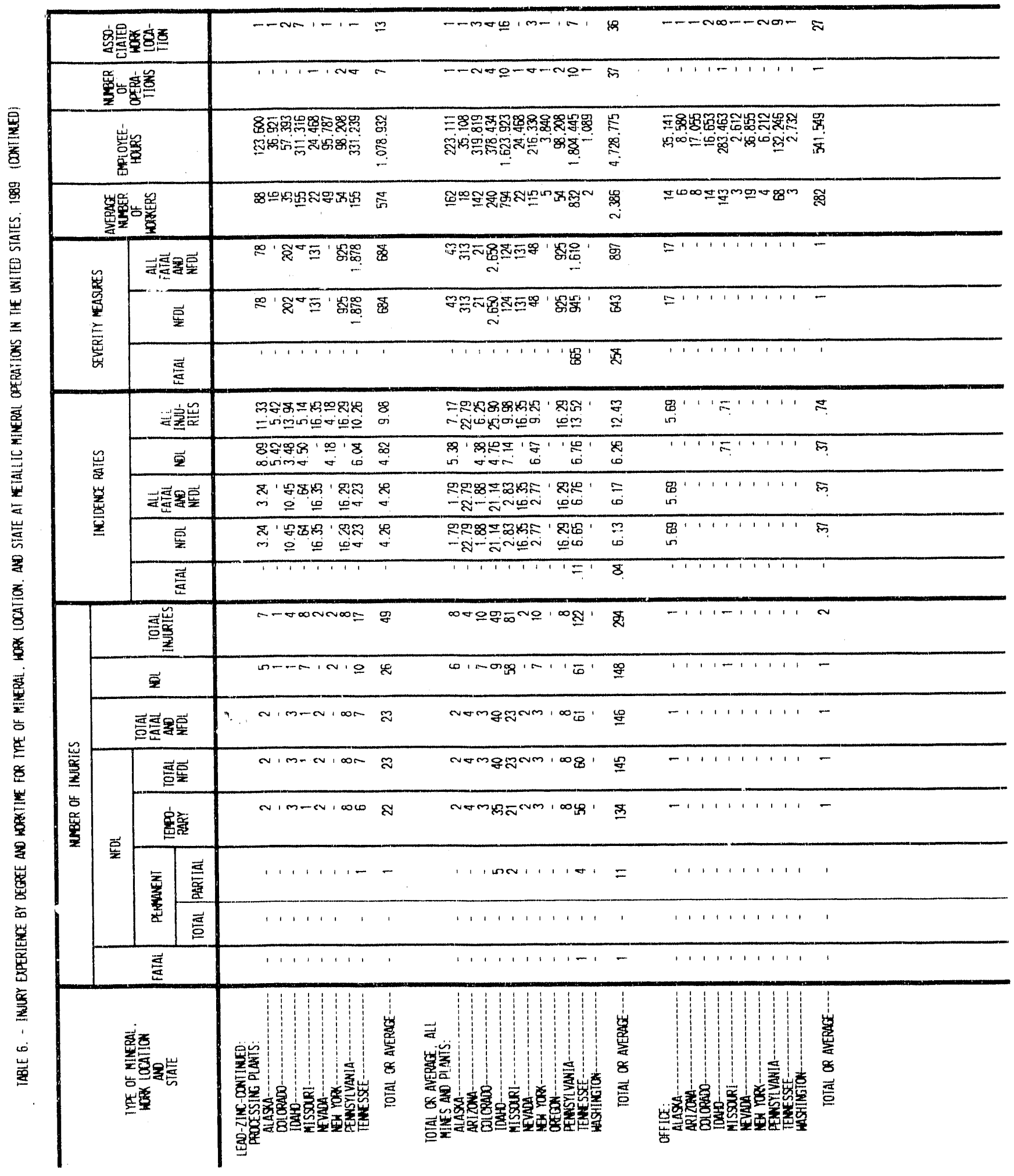




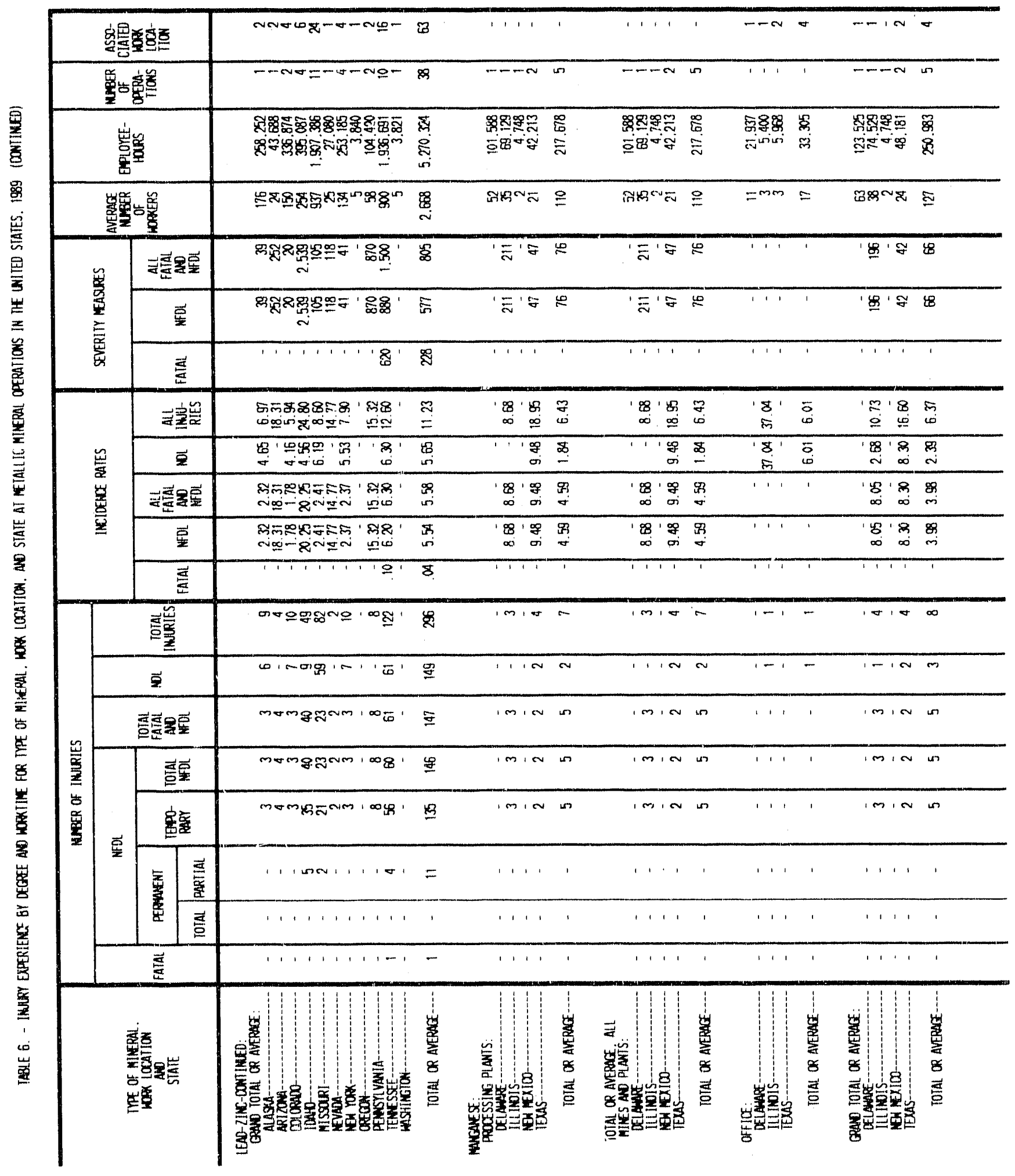




\begin{tabular}{|c|c|}
\hline 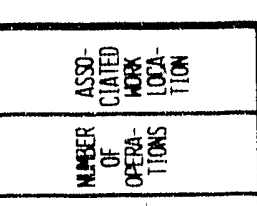 & \\
\hline 䤃 & 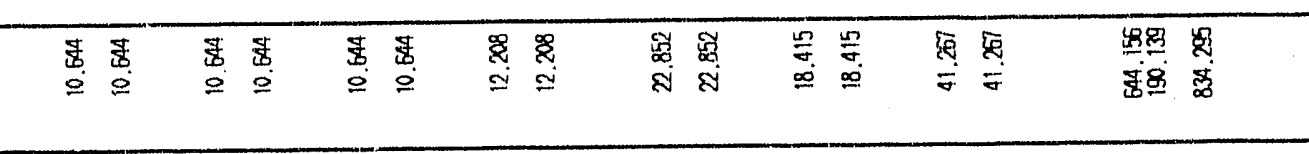 \\
\hline 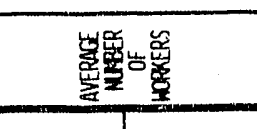 & 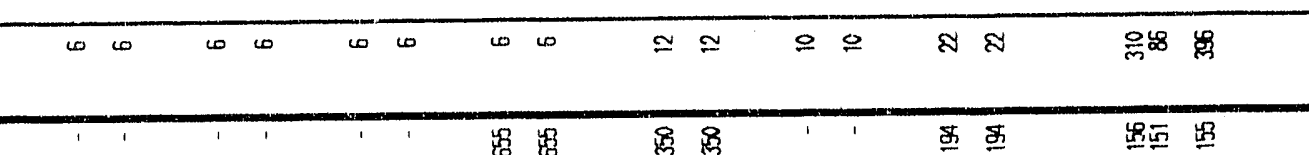 \\
\hline$\frac{1}{8}$ & 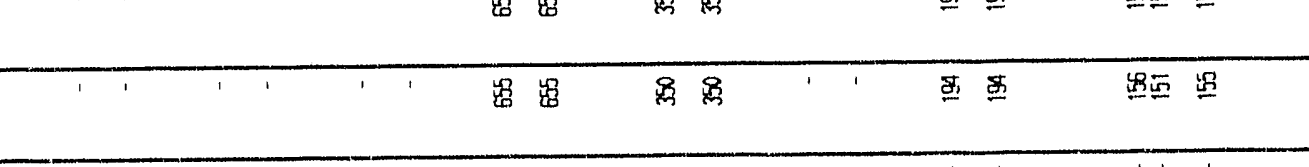 \\
\hline & 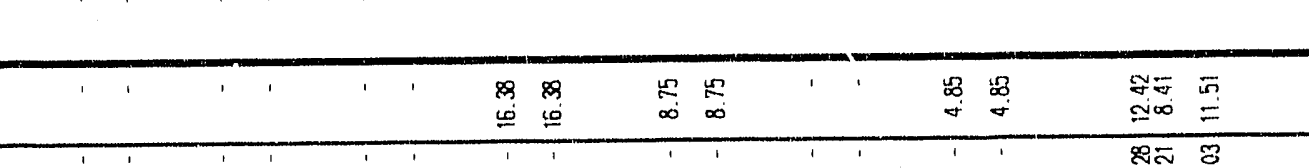 \\
\hline$\frac{138}{5}$ & 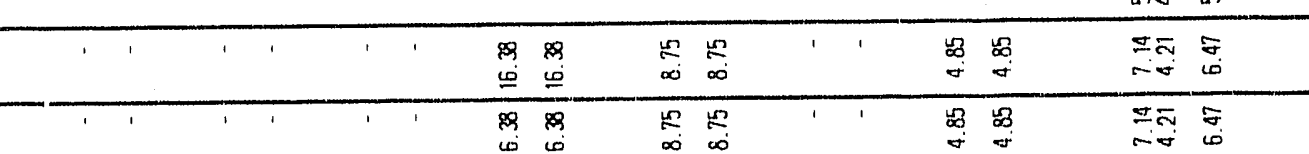 \\
\hline 政 & 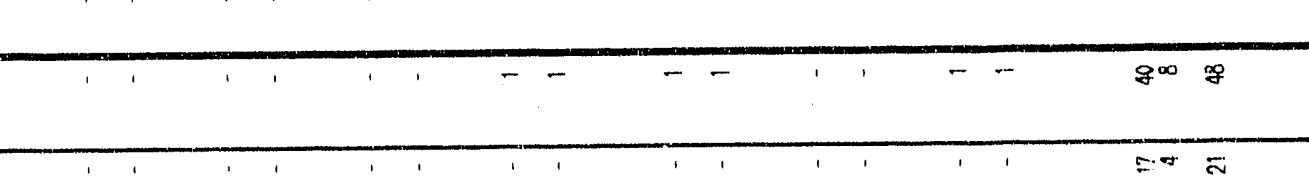 \\
\hline 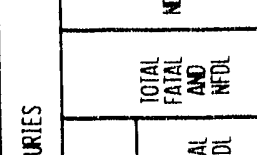 & $s=5$ \\
\hline 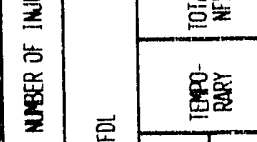 & \\
\hline 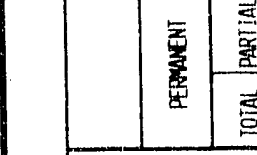 & 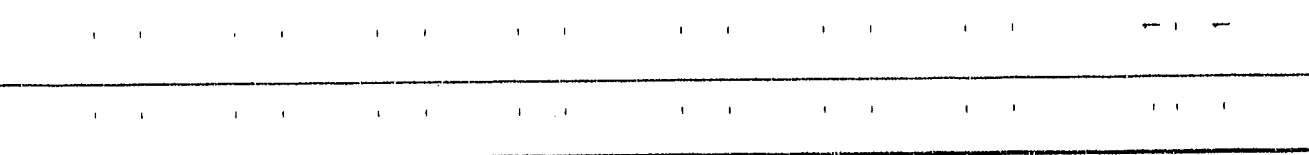 \\
\hline & 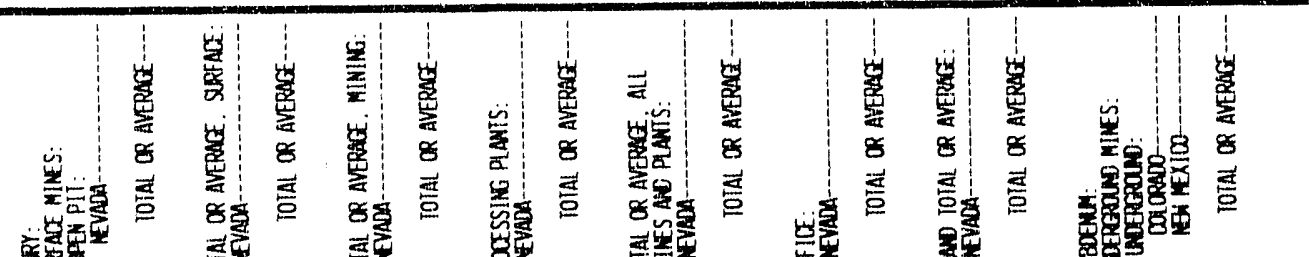 \\
\hline
\end{tabular}




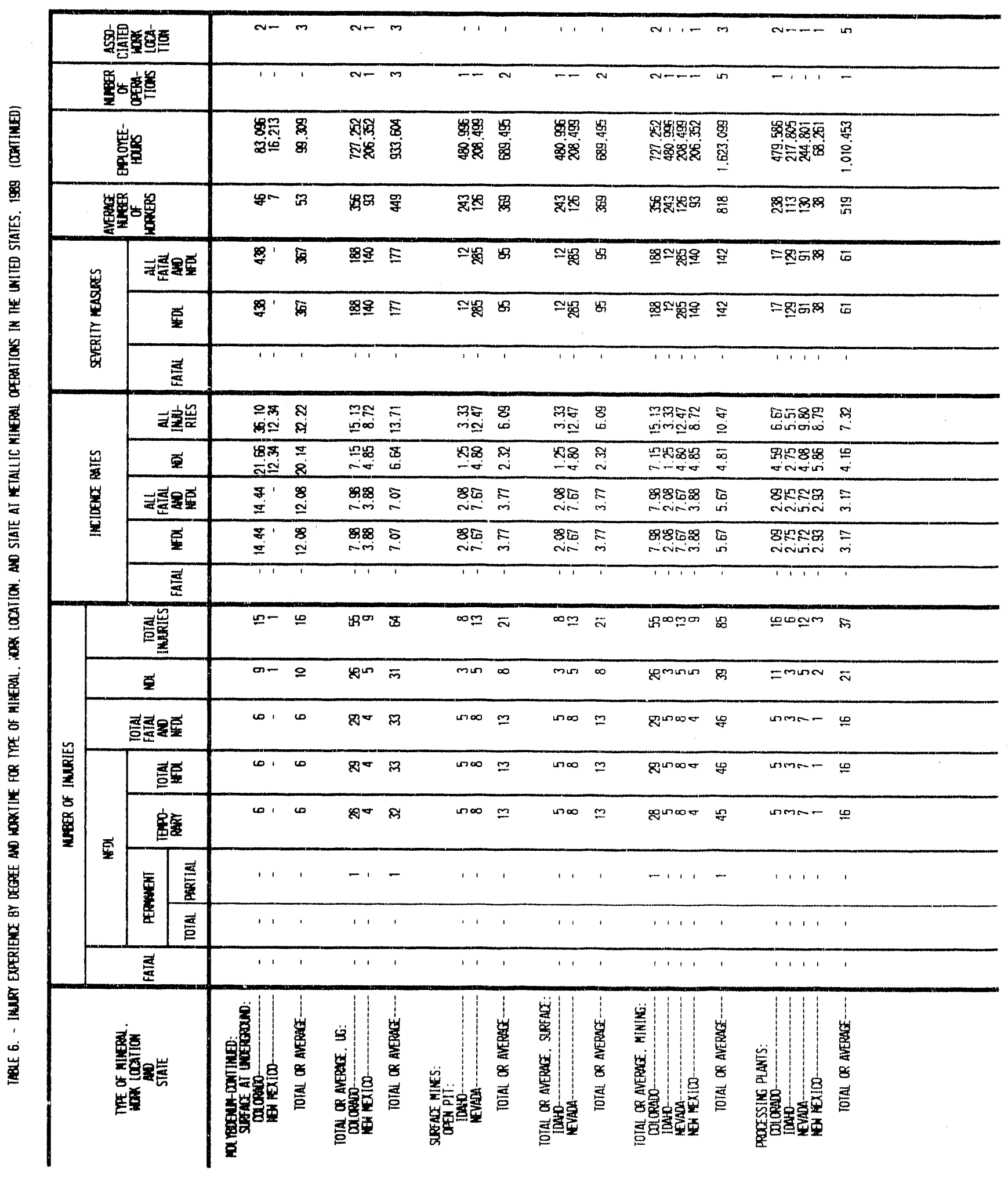




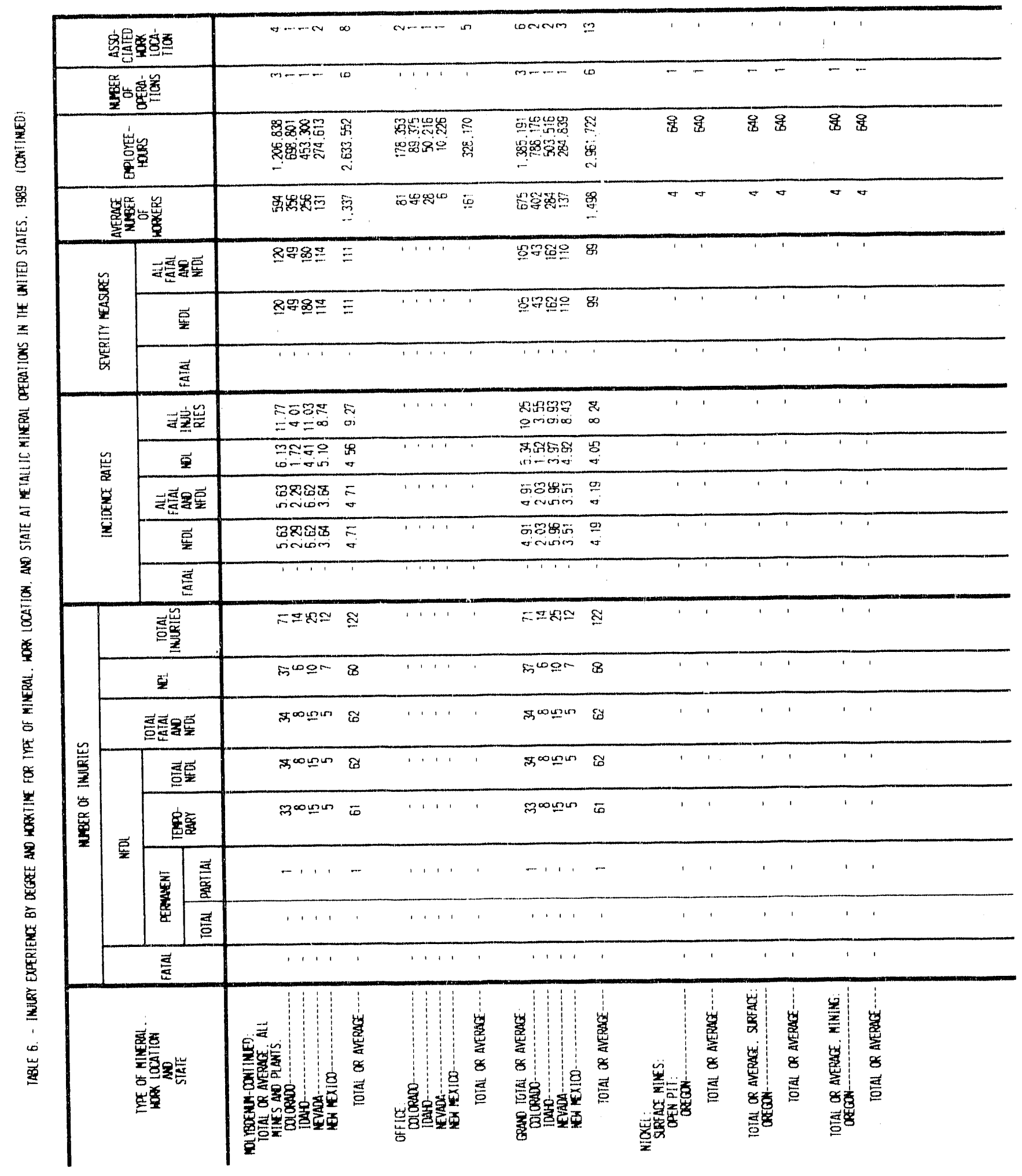




\begin{tabular}{|c|c|c|c|c|c|c|c|c|c|c|c|c|c|c|c|c|c|c|}
\hline \multicolumn{3}{|c|}{ 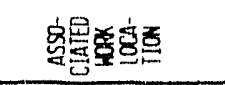 } & & & & ' & & ' & - & $\cdots$ & -- & $\cdots$ & & & ' & ' & $1-$ & - \\
\hline \multicolumn{3}{|c|}{ 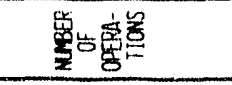 } & $\cdots$ & $\ldots$ & 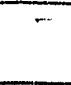 & $\cdots$ & 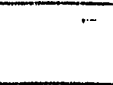 & $\cdots$ & ' & ' & $\sim$ & - & - & $\cdots$ & - & - & $\cdots$ & 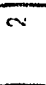 \\
\hline \multicolumn{3}{|c|}{ 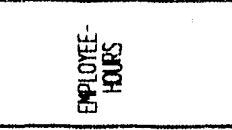 } & 8 & 8 & 8 & 8 & $\begin{array}{l}\text { 总 } \\
\text { 品 }\end{array}$ & $\begin{array}{l}\text { 吾 } \\
\text { 出 }\end{array}$ & $\begin{array}{l}\text { 兽 } \\
\check{\Phi}\end{array}$ & $\begin{array}{l}\text { 岁 } \\
\stackrel{0}{0 .}\end{array}$ & $\underset{\substack{8 \\
i}}{\stackrel{\infty}{i}}$ & $\underset{\substack{\mathcal{R} \\
\stackrel{\infty}{*}}}{\stackrel{\infty}{*}}$ & $\stackrel{\text { g. }}{-}$ & 第 & 学 & 舀 & 留 & 管 \\
\hline \multicolumn{3}{|c|}{ 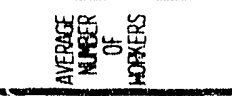 } & $\sigma$ & o & $\theta$ & $\sigma$ & 㞼 & 哭 & $s$ & 心 & ह & 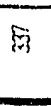 & $r$ & ᄀ & $m$ & $m$ & ${ }^{3}$ & 果 \\
\hline & स्ड & 军菖 & & & & & 喿 & $\underset{\sim}{\stackrel{\overbrace{}}{~}}$ & & ' & ? & 品 & ' & ' & ' & ' & '星 & 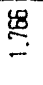 \\
\hline & E & $\overrightarrow{\underline{\Sigma}}$ & I & ' & 1 & T & g & g & ' & ' & 8 & 8 & 1 & $i$ & 7 & 7 & 8 & 8 \\
\hline & 妾 & 譬 & . & ' & ' & . & $\stackrel{\frac{m}{i}}{i}$ & $\stackrel{p}{i p}$ & ' & & $\stackrel{8}{0}$ & $\stackrel{8}{\circ}$ & ' & ' & & & '萑 & $\underline{E}$ \\
\hline & & 崖宸 & ' & ' & & ' & $\stackrel{g}{\underline{g}}$ & $\stackrel{9}{9}$ & ' & ' & $\underset{\infty}{6}$ & $\begin{array}{l}x_{3} \\
\infty \\
\infty\end{array}$ & ' & ' & ' & ' & , & $\begin{array}{l}\aleph_{3} \\
\infty\end{array}$ \\
\hline & $\frac{2}{3}$ & $\bar{\Sigma}$ & & & & 1 & $\begin{array}{l}\infty \\
\infty \\
\infty \\
\infty\end{array}$ & $\begin{array}{c}\infty \\
0 \\
0 \\
0\end{array}$ & T & & י & פ্ম & & 7 & 7 & 7 & : & is \\
\hline 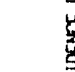 & 接 & 是菖 & & & ' & ' & $\underset{m}{\$ 8}$ & $\underset{m}{8}$ & & 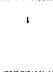 & $\underset{\mathrm{m}}{\mathscr{g}}$ & $\underset{m}{8}$ & ' & ' & ' & ' & ' & $\underset{m}{\mathscr{g}}$ \\
\hline & & $\overrightarrow{\vec{p}}$ & 1 & . & 7 & ' & $\underset{m}{88}$ & 8 & ' & . & $\begin{array}{l}\infty \\
\stackrel{\infty}{i v} \\
i v\end{array}$ & $\stackrel{\infty}{\sim}$ & ' & 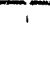 & T & 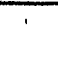 & $\stackrel{\infty}{i \infty}$ & $\stackrel{\infty}{\stackrel{\circ}{\sim}}$ \\
\hline & & 莣 & & 1 & T & T & फ़ & $\mathscr{m}$ & $T$ & & व्व & 8 & & & 1 & 1 & क्ạ & 29 \\
\hline \multirow{8}{*}{ 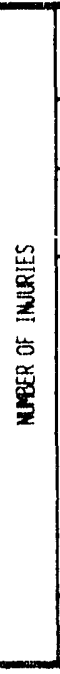 } & 這営 & & I & ' & ' & . & 8 & 8 & . & ' & s & 8 & 1 & 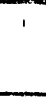 & ' & ' & 18 & 8 \\
\hline & $\overrightarrow{\underline{z}}$ & & ' & ' & I & ' & $\underline{\underline{n}}$ & o & I & 1 & 의 & ? & 1 & 1 & 1 & 1 & 9 & 마 \\
\hline & 营產 & & ' & ' & ' & ' & $=$ & $=$ & ' & ' & $=$ & $=$ & ' & ' & ' & ' & $'=$ & $=$ \\
\hline & \multirow{4}{*}{ 营 } & 홍밀 & ' & . & ' & ' & 은 & 요 & 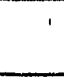 & . & 으 & 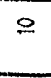 & ' & ' & ' & ' & 10 & $\cong$ \\
\hline & & 鱼衰 & & ' & ' & & 인 & 0 & ' & ' & o & 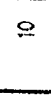 & & ' & ' & ' & 19 & 으 \\
\hline & & $\begin{array}{l}\text { 폴 } \\
\text { 葢 } \\
\end{array}$ & , & ' & ' & ' & . & , & . & ' & ' & ' & ' & ' & ' & ' & , ' & ' \\
\hline & & $\underline{\underline{t}}$ & , & I & I & ' & , & , & ' & ' & ' & I & & ' & , & ' & 1. & ' \\
\hline & \multicolumn{2}{|l|}{ 童 } & & I & I & . & $\ldots$ & - & . & ' & - & -- & & & , & ' & $1-$ & - \\
\hline & \multicolumn{2}{|c|}{ 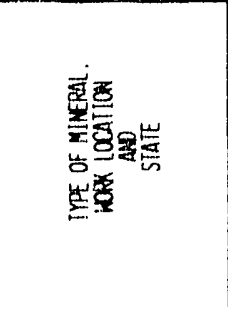 } & 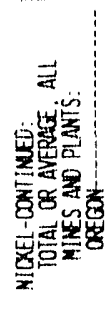 & 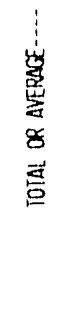 & 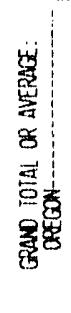 & 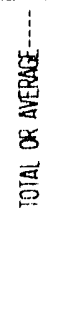 & 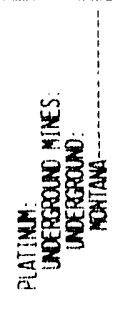 & 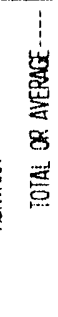 & 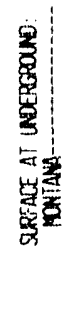 & 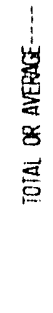 & 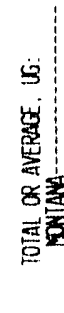 & 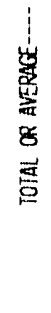 & 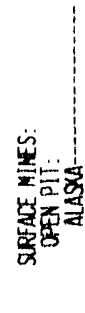 & 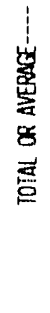 & 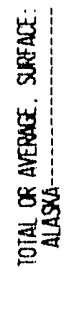 & 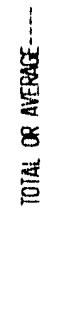 & 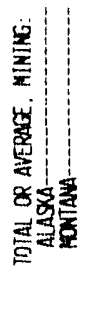 & 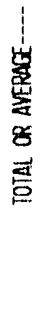 \\
\hline
\end{tabular}




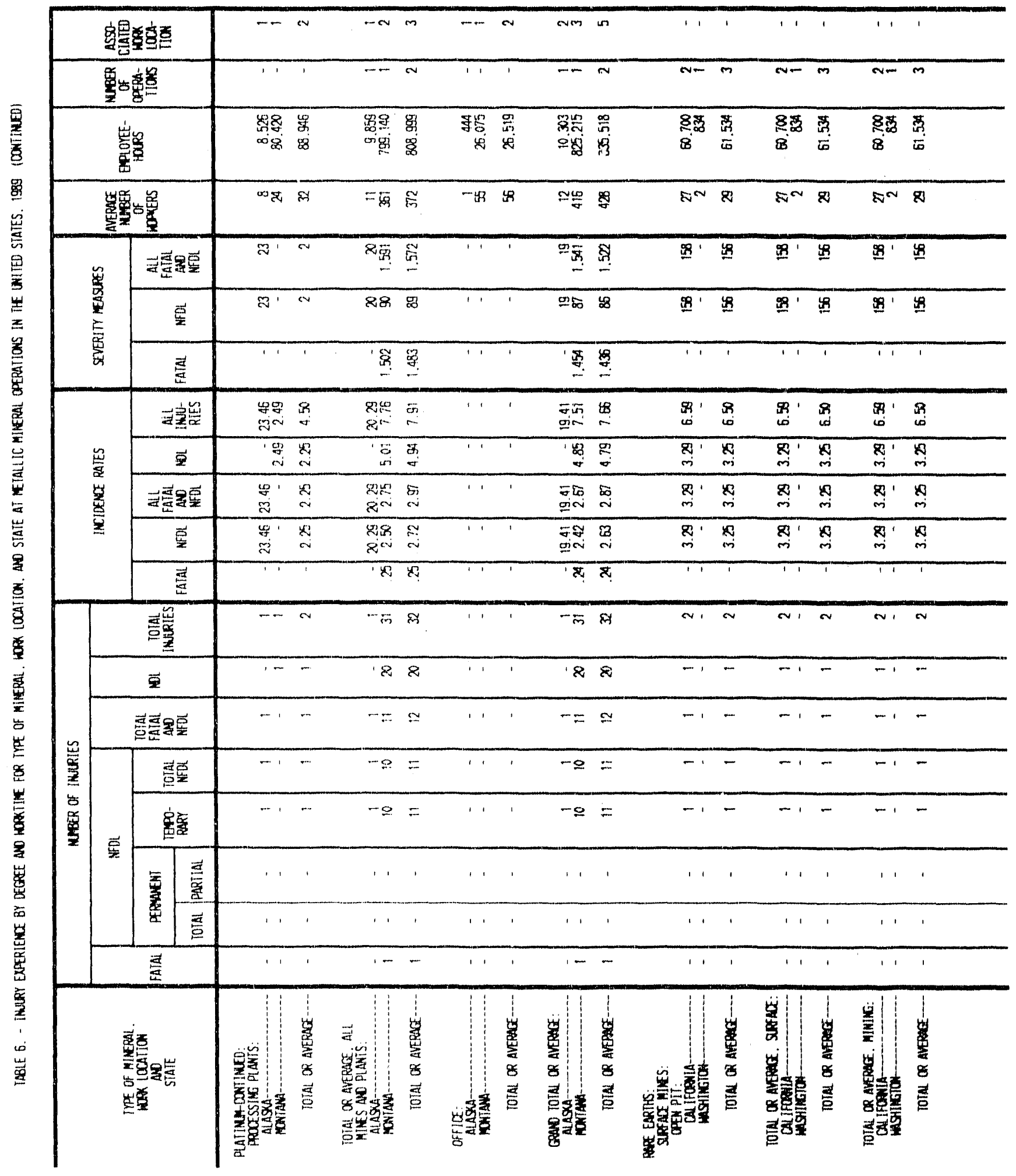




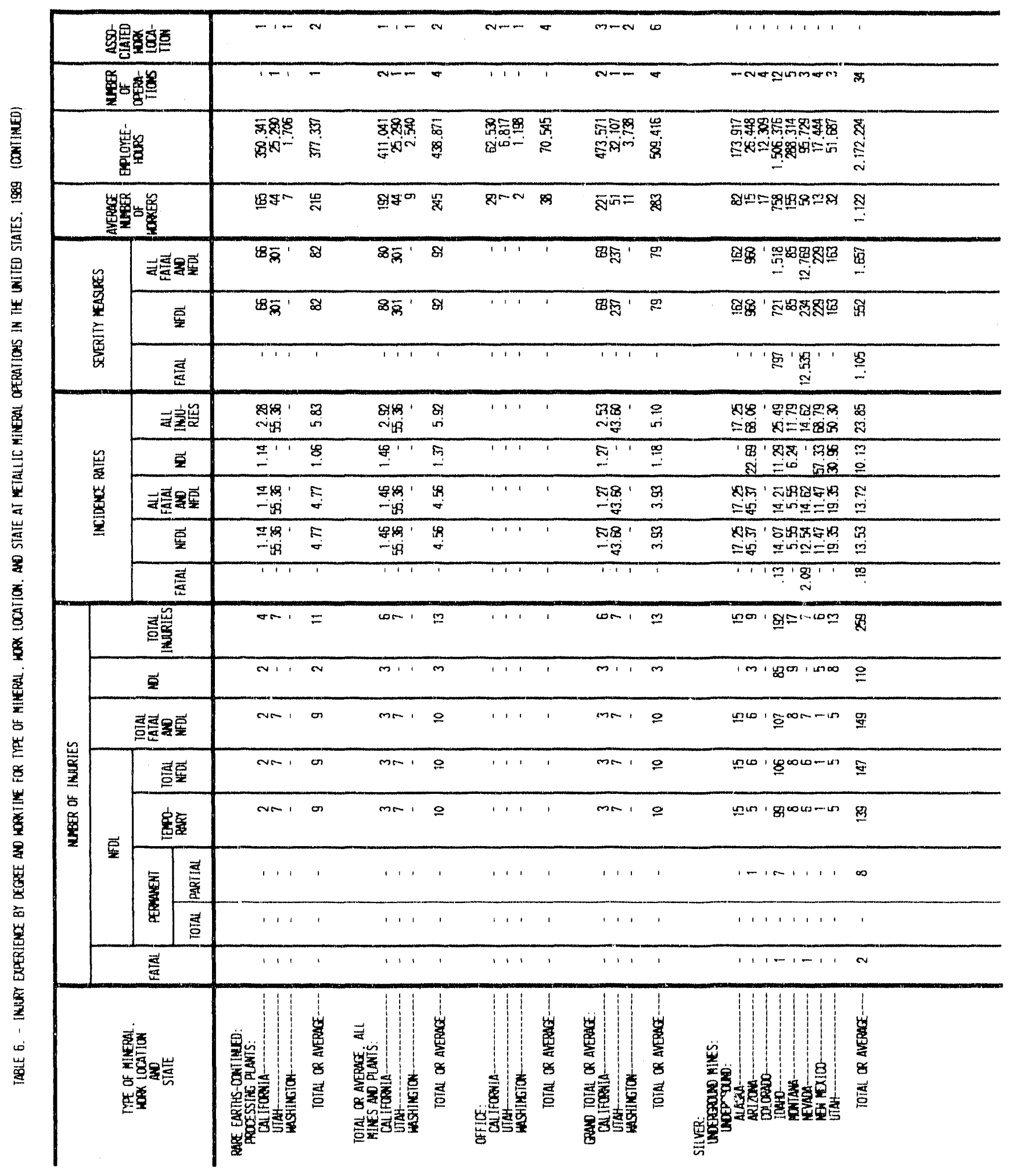




\begin{tabular}{|c|c|c|c|c|c|c|c|c|c|c|}
\hline \multicolumn{3}{|c|}{ 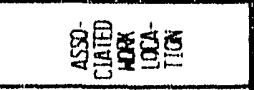 } & - Nawermam & $\infty$ & - mertorventer & $x$ & 111 & 1 & 111111 & 1 \\
\hline \multicolumn{3}{|c|}{ 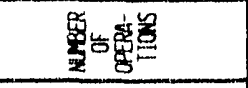 } & $1-1-11$ & $\sim$ & -mognman & 69 & -nw-men & 으 & -namenar & 요 \\
\hline \multicolumn{3}{|c|}{ 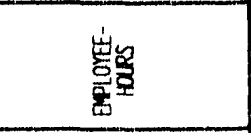 } & 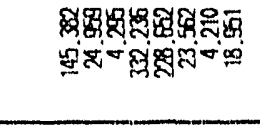 & ఫ્d & 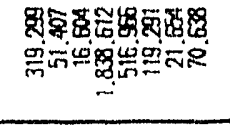 & 悹 & 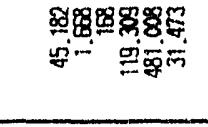 & 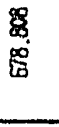 & 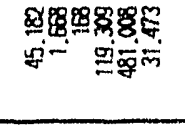 & 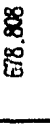 \\
\hline \multicolumn{3}{|c|}{ 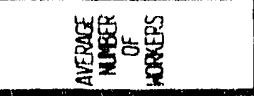 } & LR= & E్ & 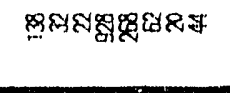 & 若 & 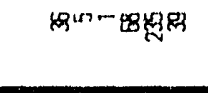 & 幽 & 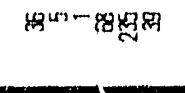 & 嵒 \\
\hline \multirow{3}{*}{\multicolumn{2}{|c|}{ 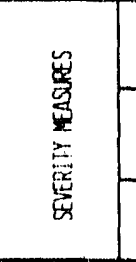 }} & & 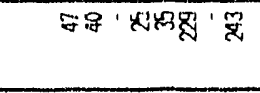 & $F$ & 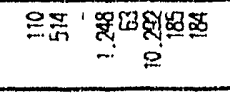 & 扆 & का '尊名' & $\bar{d}$ & (क) ' '管绐' & 胥 \\
\hline & & 悹 & 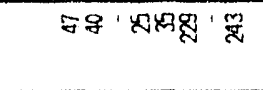 & F & 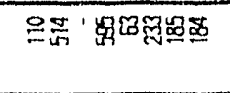 & $\Xi$ & m' '爵品' & 䒽 & 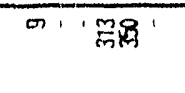 & 落 \\
\hline & & 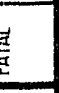 & 111 & ' & ' ' '留'贸' & $\cong$ & 1111 & T & ' & ' \\
\hline & & 它 & 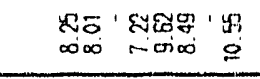 & $\underset{\infty}{\infty}$ & 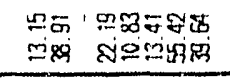 & $\stackrel{?}{\underline{Q}}$ & $\begin{array}{l}\infty \\
\infty \\
\infty \\
\infty\end{array}$ & $\begin{array}{l}R \\
\underline{\underline{c}} \\
\end{array}$ & 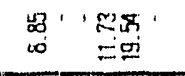 & $\begin{array}{l}R \\
\underline{6} \\
\end{array}$ \\
\hline & & 3 & 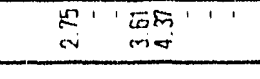 & ल্ & 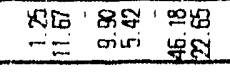 & लि & 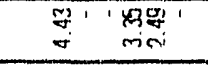 & $\stackrel{\leftrightarrow}{\sim}$ & 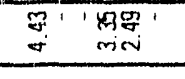 & 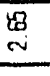 \\
\hline & 起焉 & & 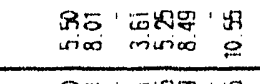 & $\stackrel{8}{8}$ & 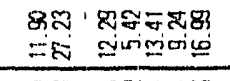 & $\stackrel{D}{=}$ & $\begin{array}{l}F^{\prime} \\
\vdots \\
\end{array}$ & $\stackrel{\mathbb{2}}{\stackrel{m}{=}}$ & 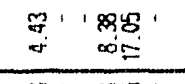 & $\stackrel{m}{m}$ \\
\hline & & $\overrightarrow{\mathrm{z}}$ & 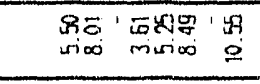 & $\stackrel{8}{+}$ & 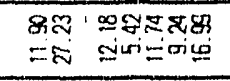 & $\stackrel{\Xi}{=}$ & 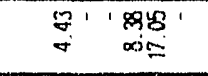 & 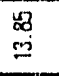 & 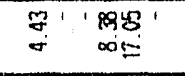 & 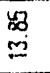 \\
\hline & & Et & 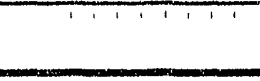 & 1 & $7,1=181$ & $\underline{I}$ & 1.111 & ' & 11111 & 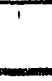 \\
\hline \multirow{8}{*}{ 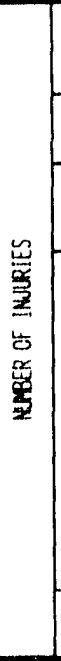 } & 运墨 & & $w-1 \simeq=-1-$ & $\mathscr{7}$ & $\therefore$ 어 ' & $\overline{8}$ & $w+125$ & 48 & $\sim, m$ & 48 \\
\hline & $\underline{z}$ & & $2+1600011$ & 9 & 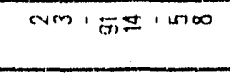 & $\stackrel{\Xi}{\Xi}$ & -1 1 cover & क & - ino: & on \\
\hline & 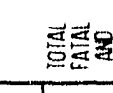 & & $\sigma+1 \cos 0 . .1-$ & 무 & $g m, \cong \pm \infty-\infty$ & $\not 8$ & $-11 x=1$ & $F$ & - & 8 \\
\hline & \multirow{3}{*}{ 昰 } & 营否 & $\theta-1 \cos \theta+\cdots$ & 미 & 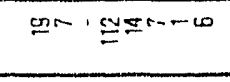 & 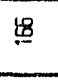 & -11的手 & $\xi$ & $-1,1 \mathrm{~s} F$ & $\%$ \\
\hline & & 象哀 & 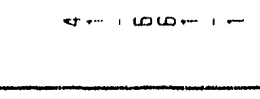 & 이 & 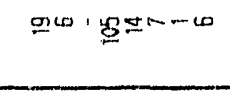 & 98 & - 'l in & $F$ & 一、牥 & $F$ \\
\hline & & 总 & $1 \ldots 1,1$, & , & $1-1$, & $\infty$ & $\cdots, 1$, & ' & 1, & ' \\
\hline & & $\stackrel{\vec{a}}{\underline{a}}$ & & ' & & ' & $1,11.1$ & & $\cdots$ & ' \\
\hline & \multicolumn{2}{|c|}{$\frac{3}{3}$} & $1,1,1$, & 1 & $1, \ldots, 1,1$ & $\sim$ & $1,1,1$ & & 11 & ' \\
\hline \multicolumn{3}{|c|}{ 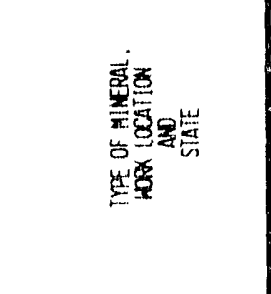 } & 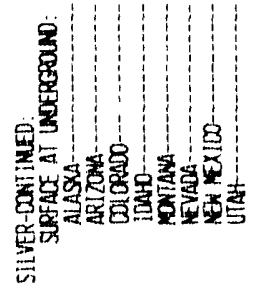 & 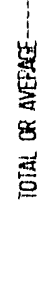 & 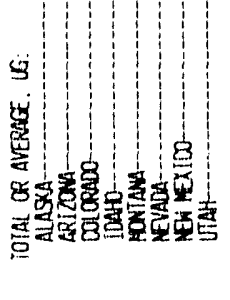 & 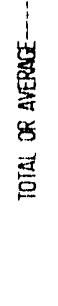 & 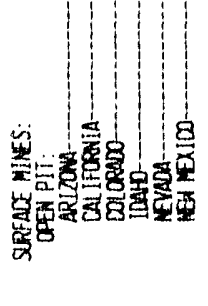 & 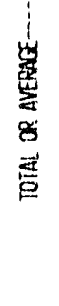 & 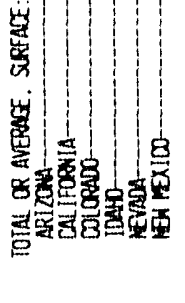 & 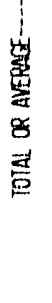 \\
\hline
\end{tabular}




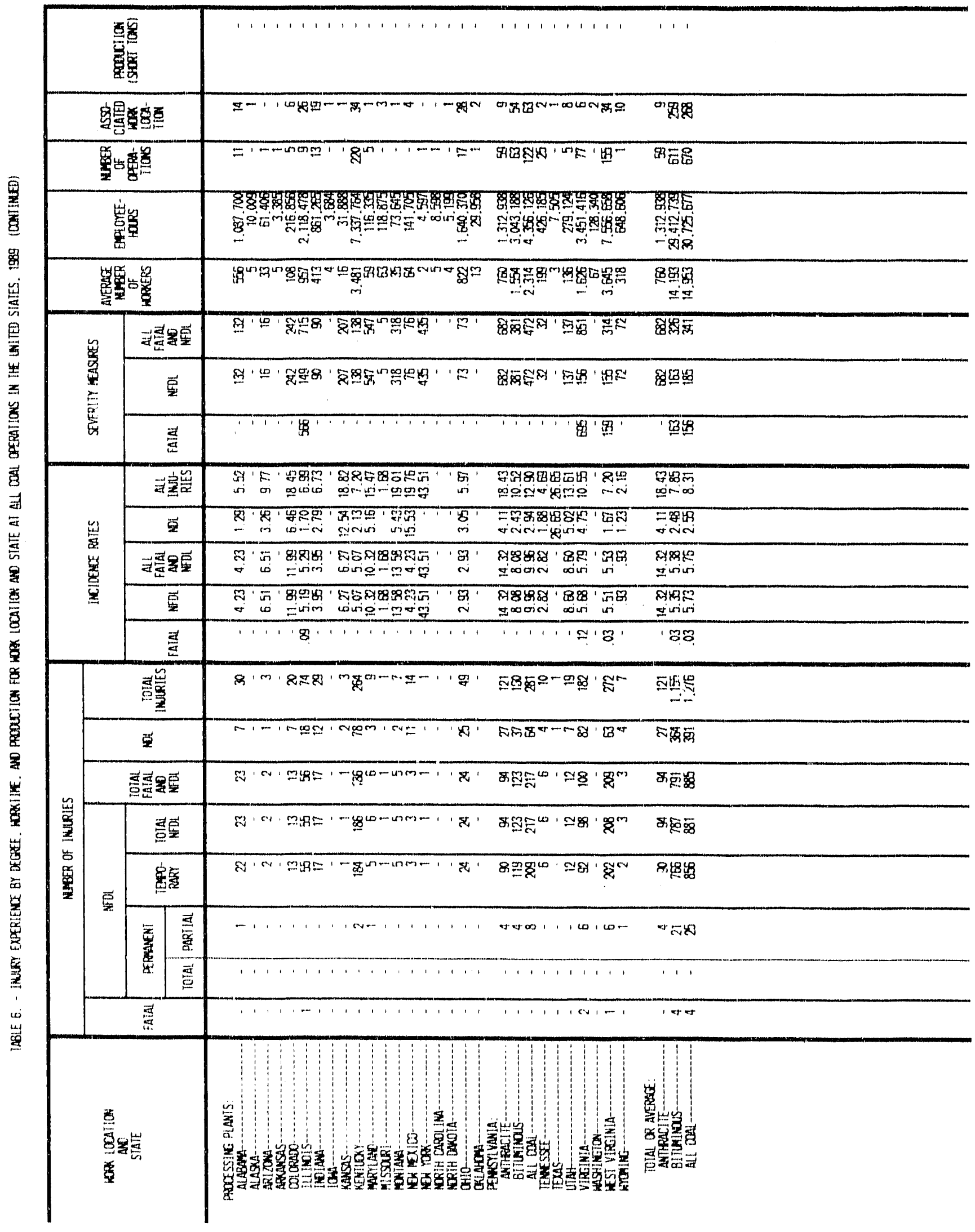




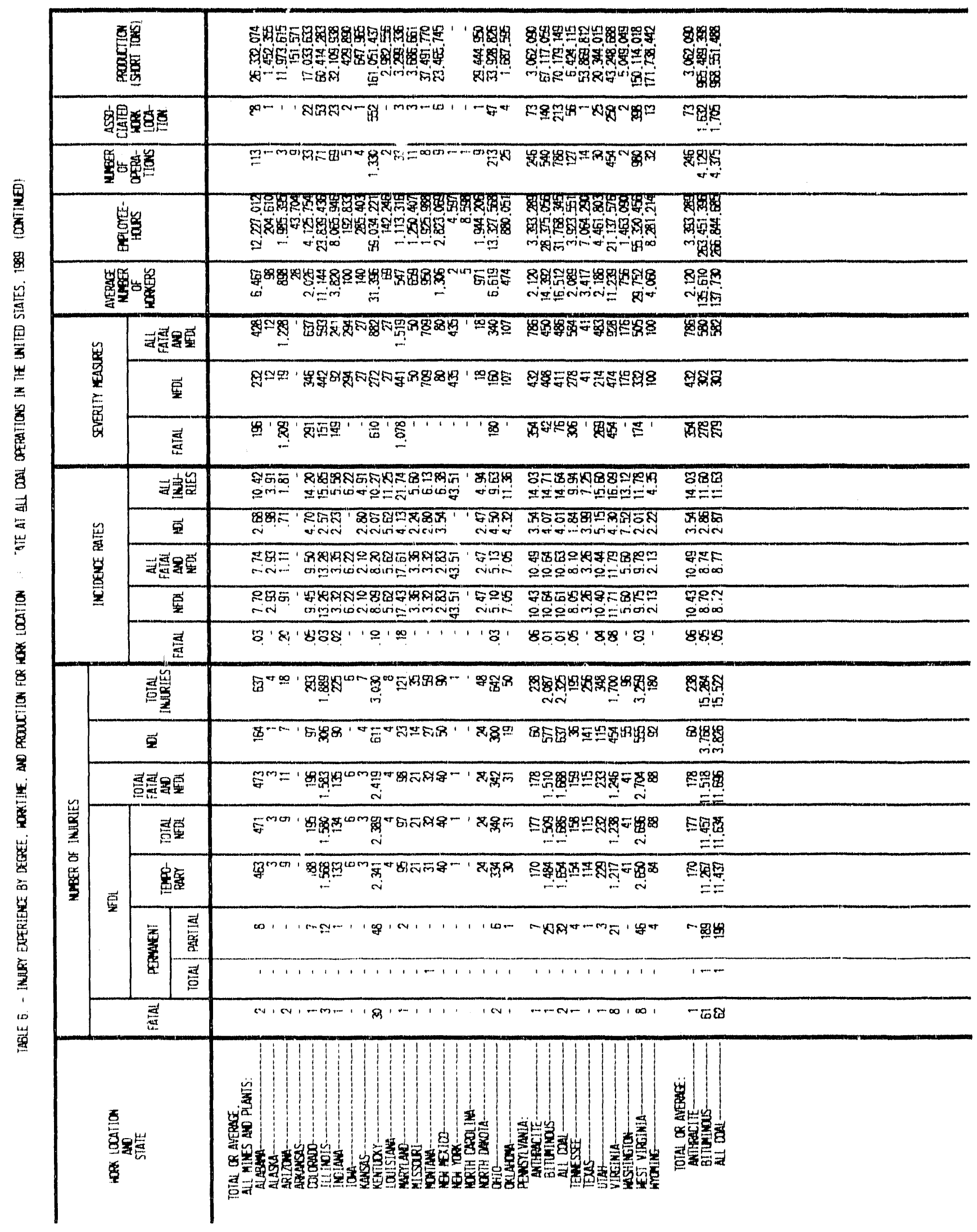




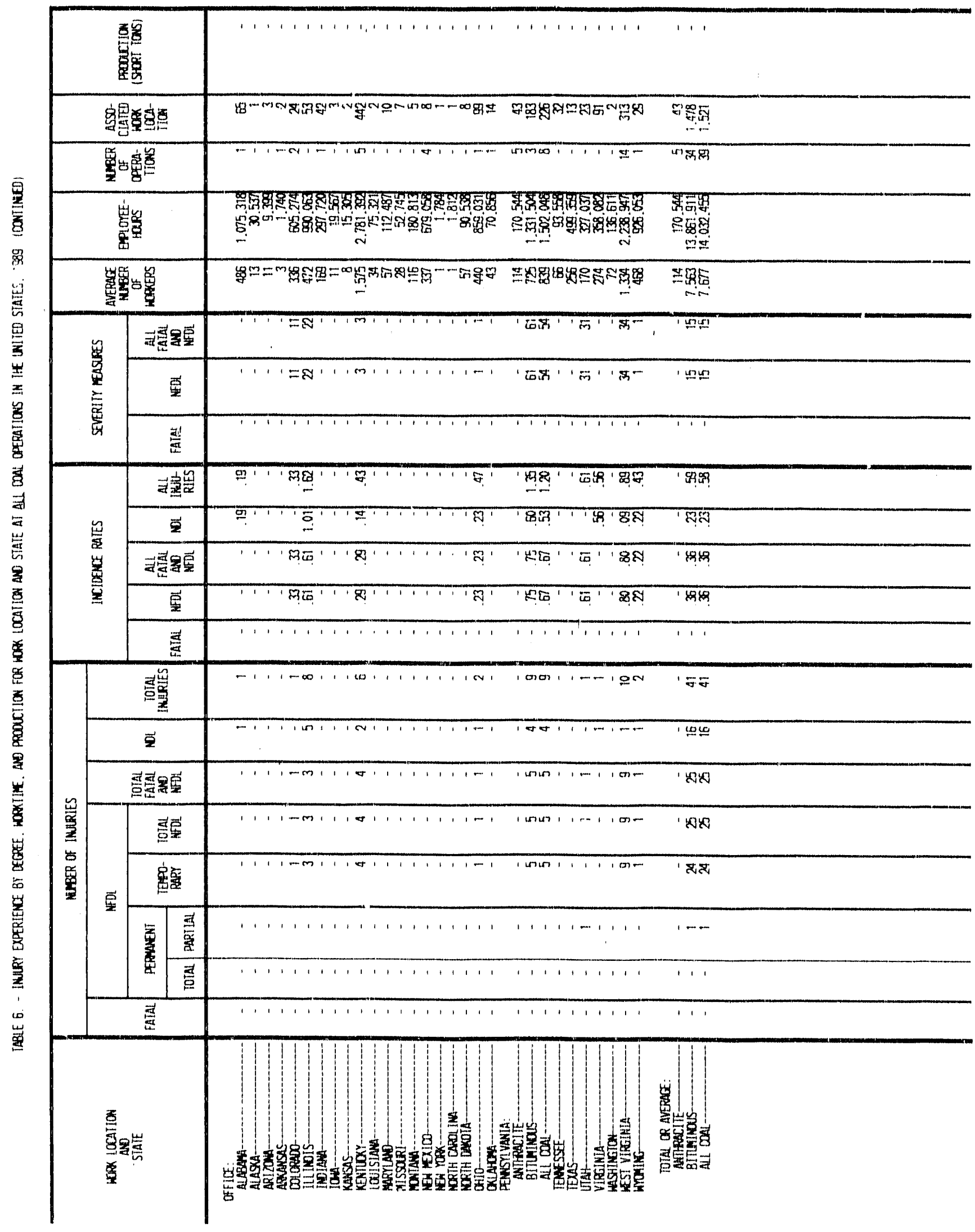




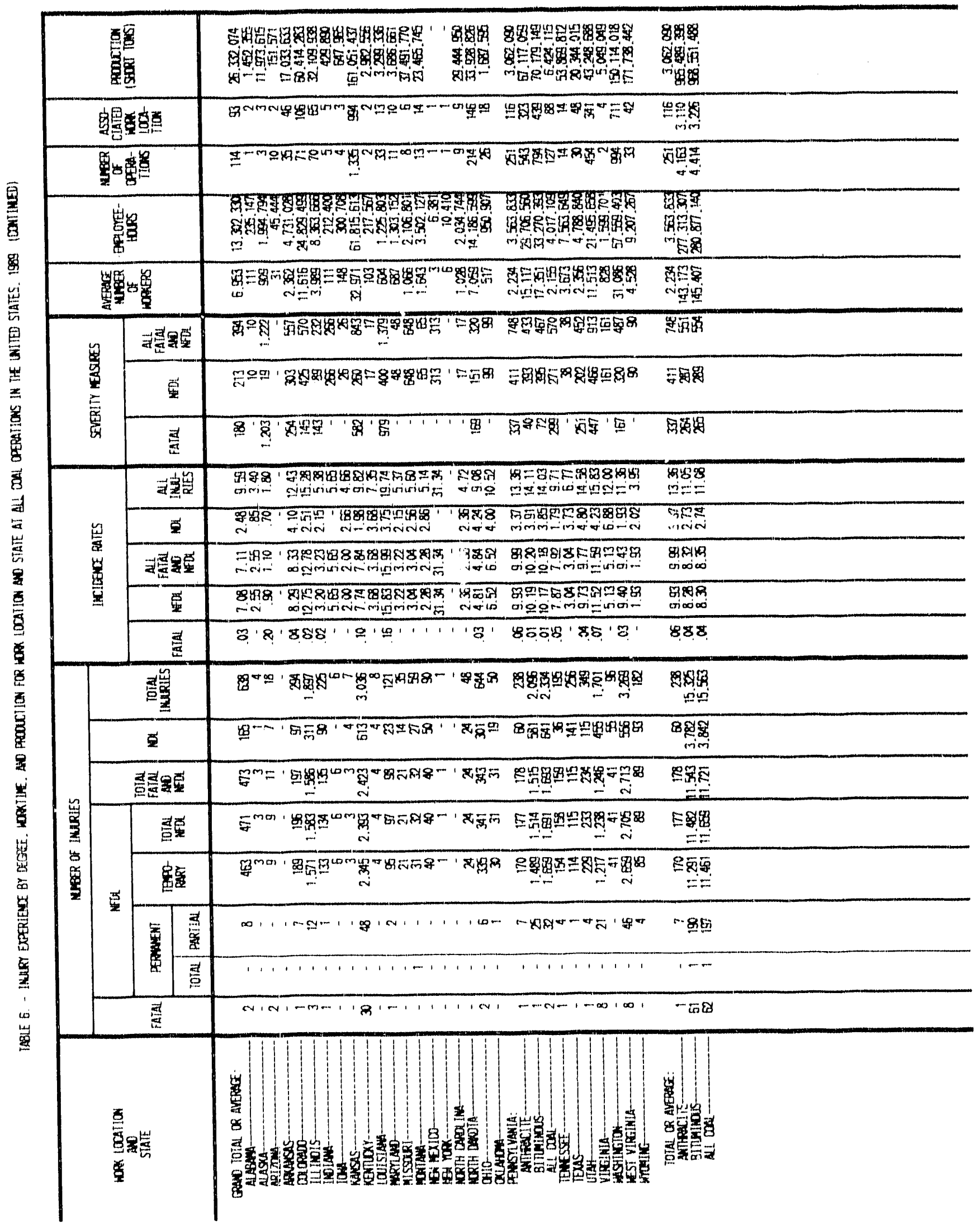




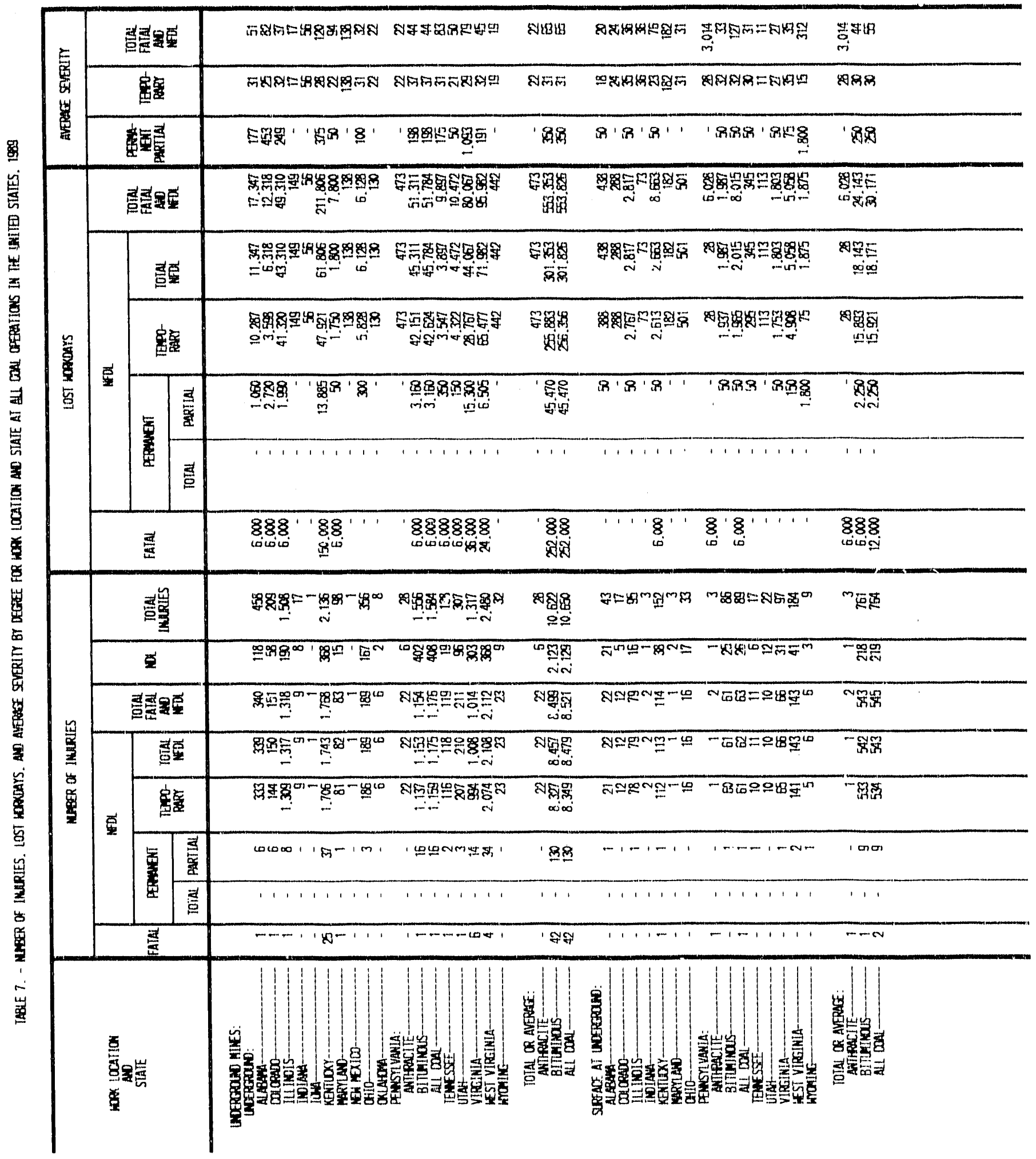




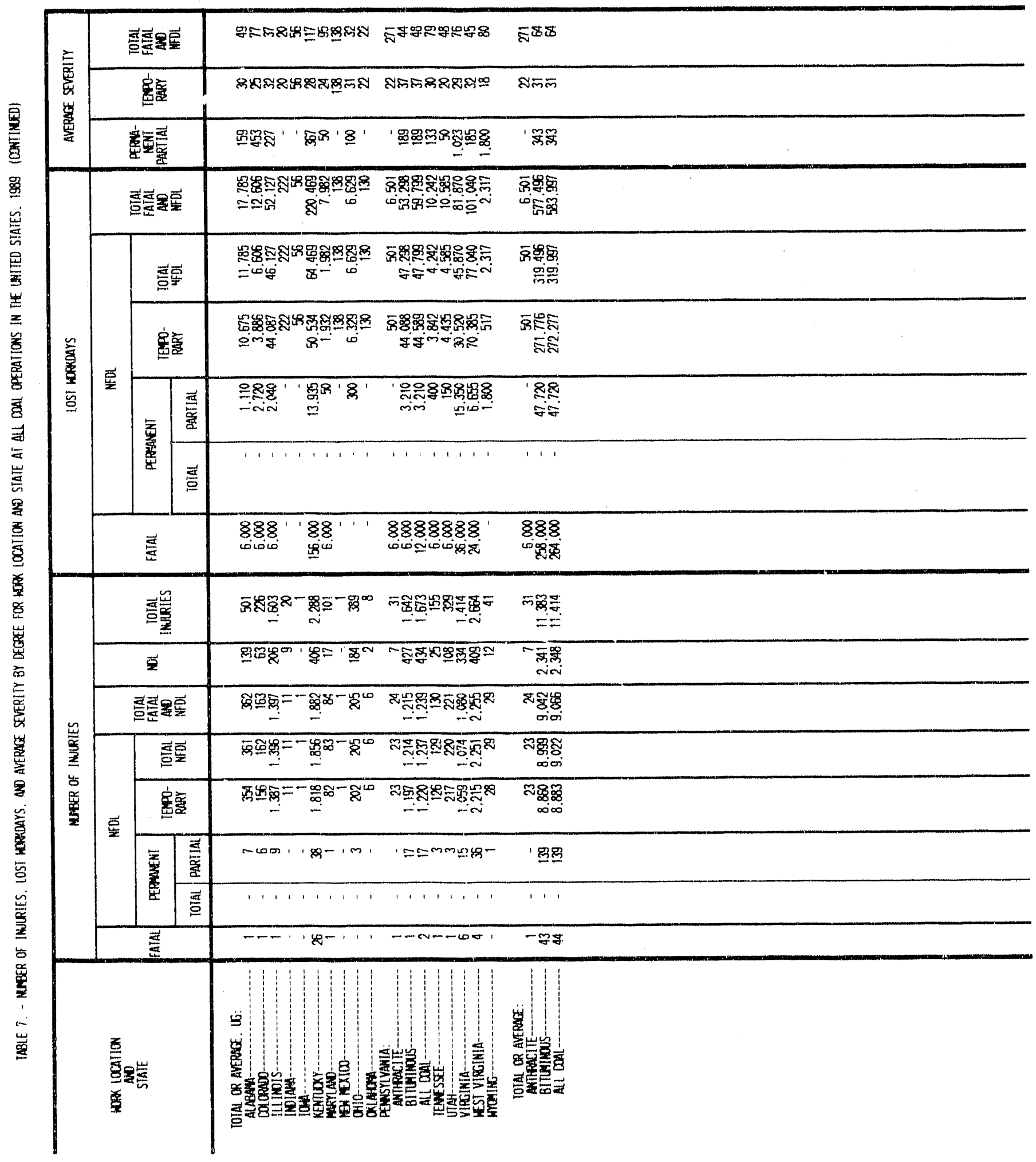




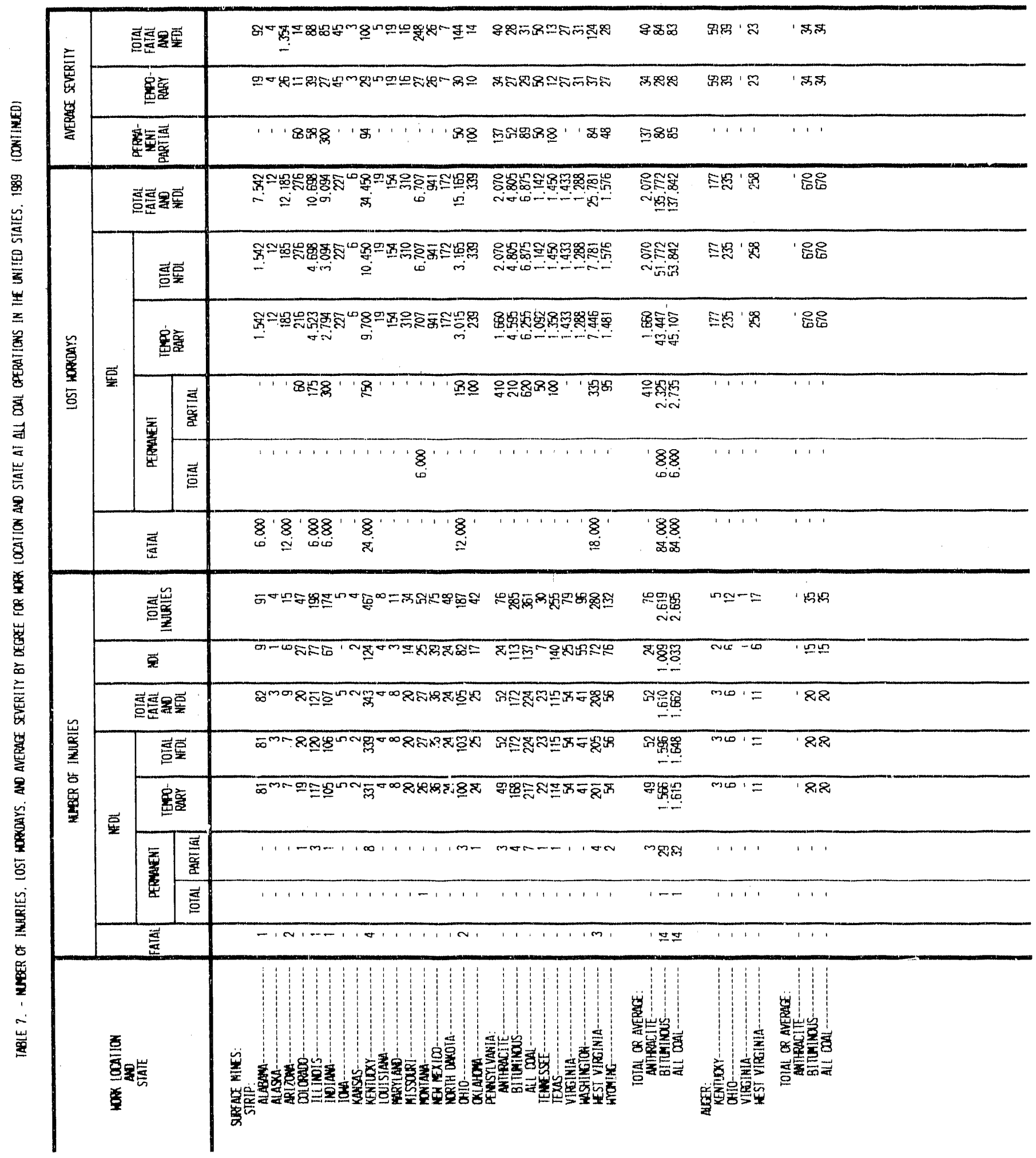




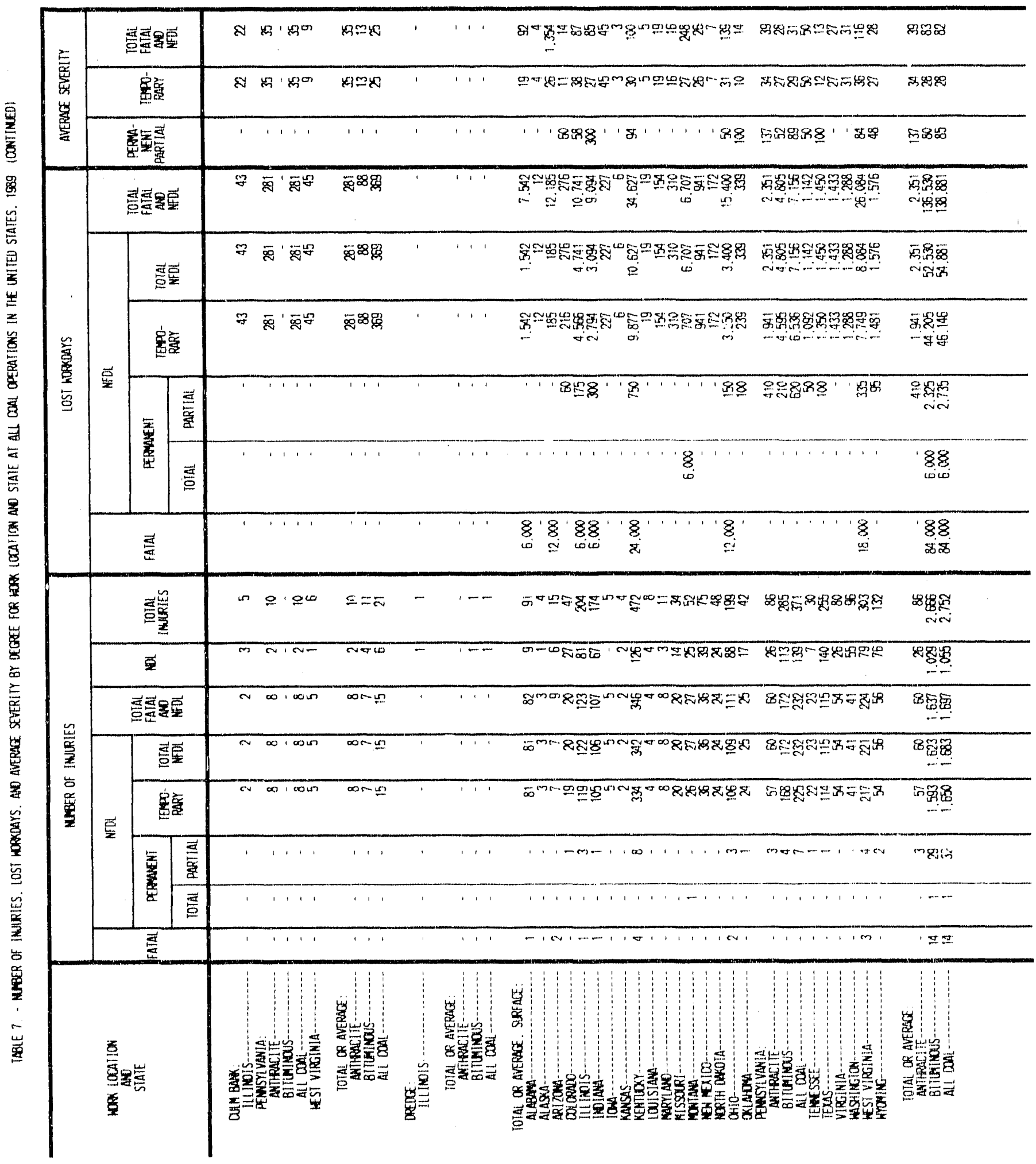




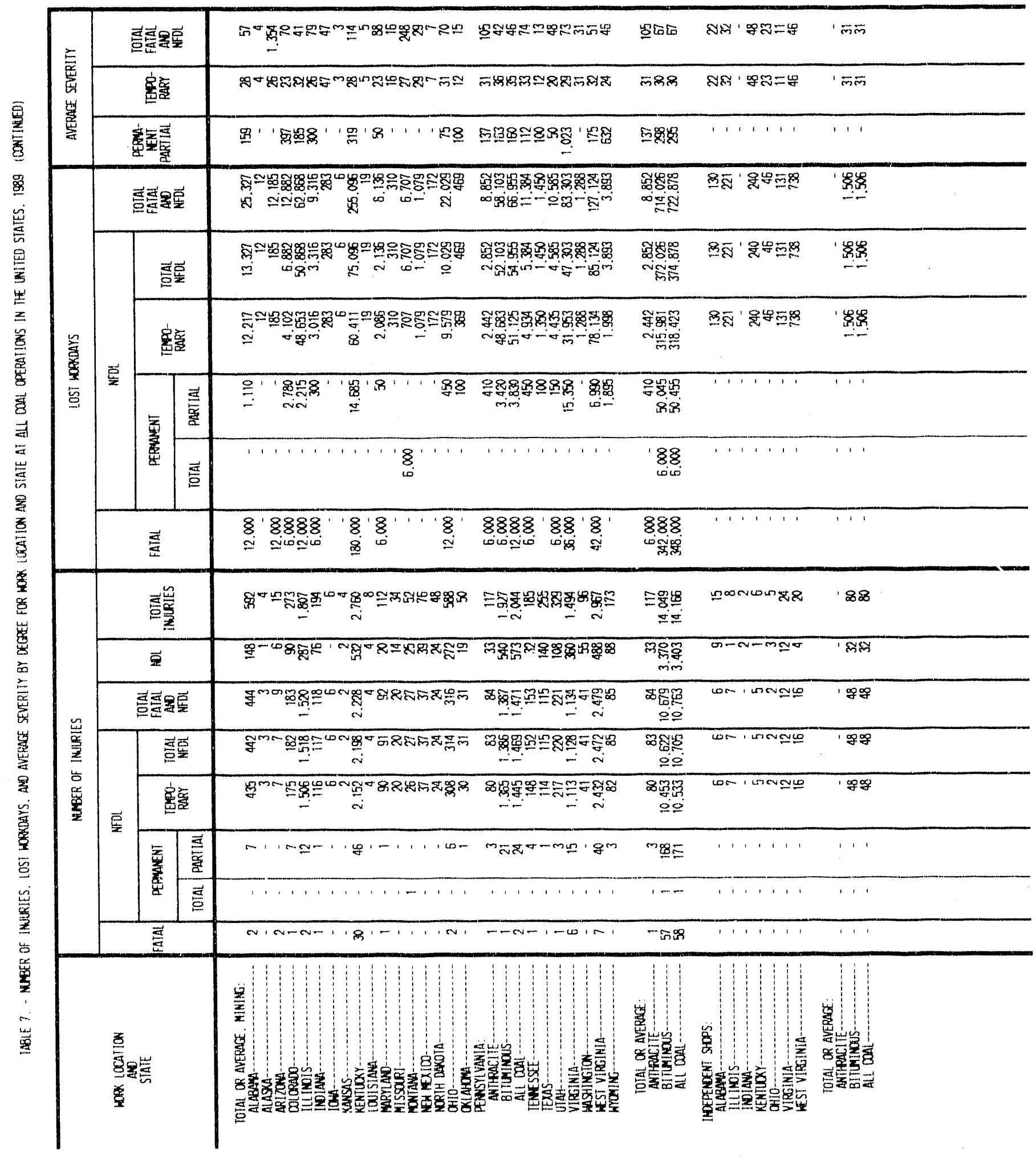




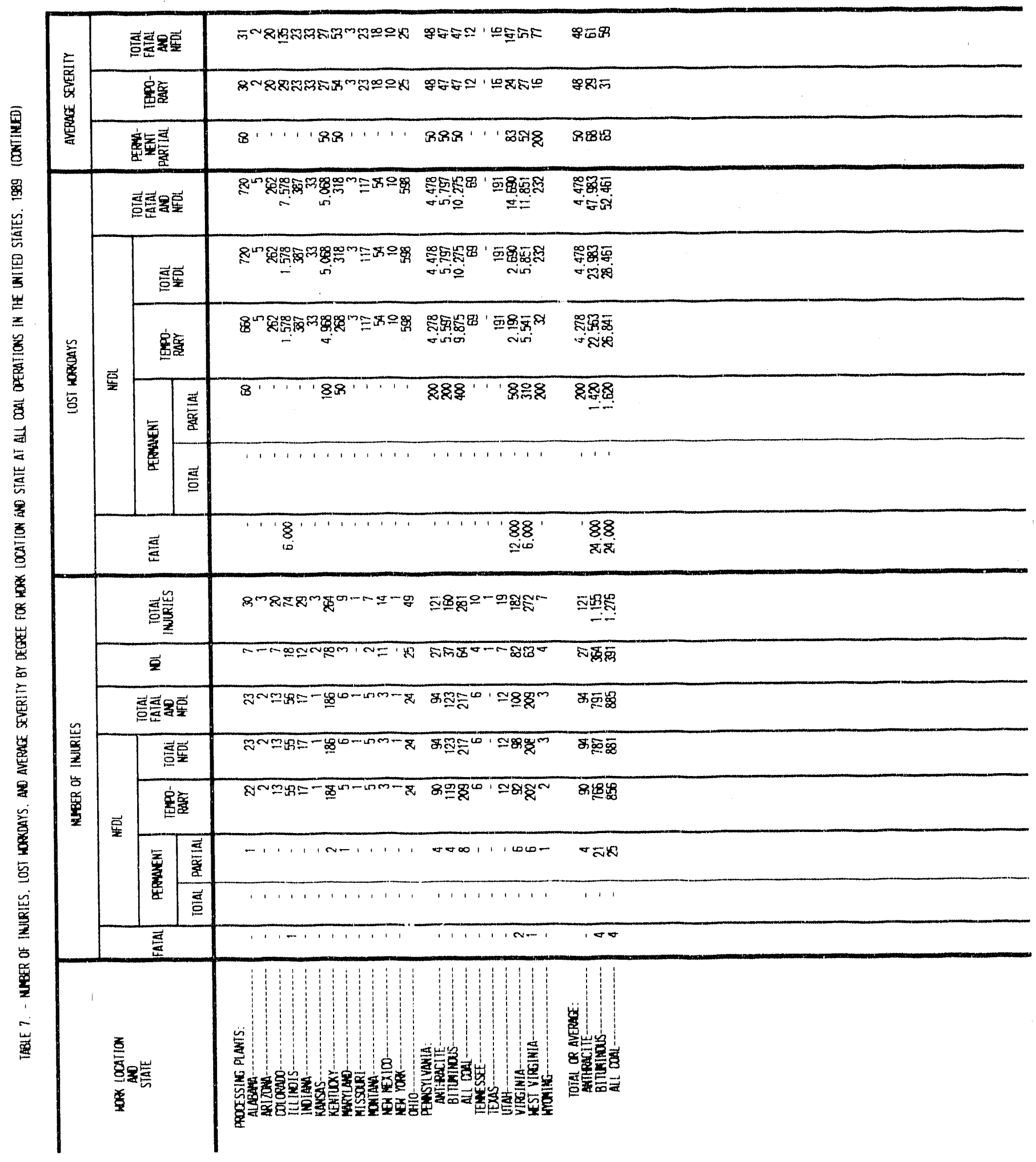




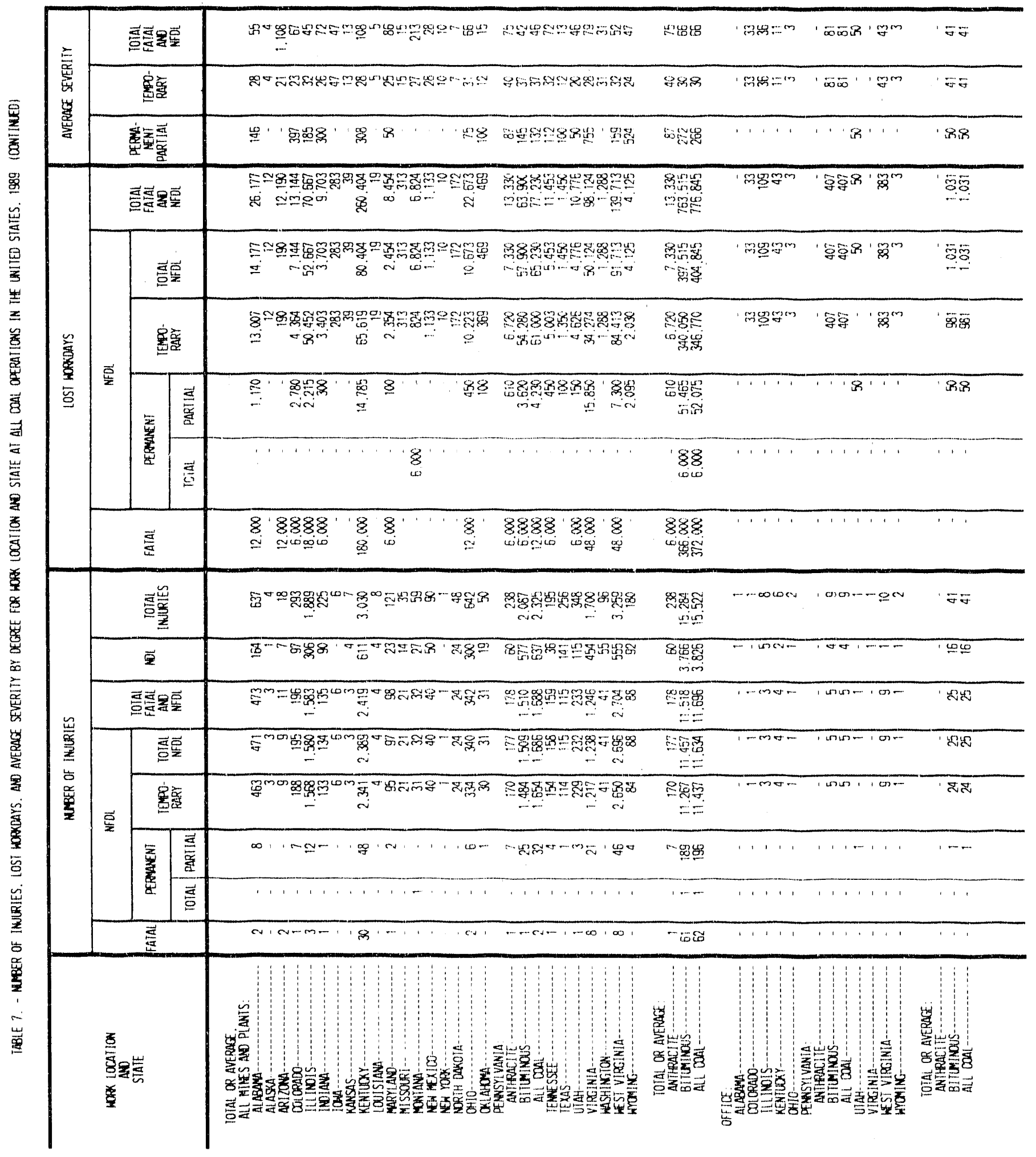




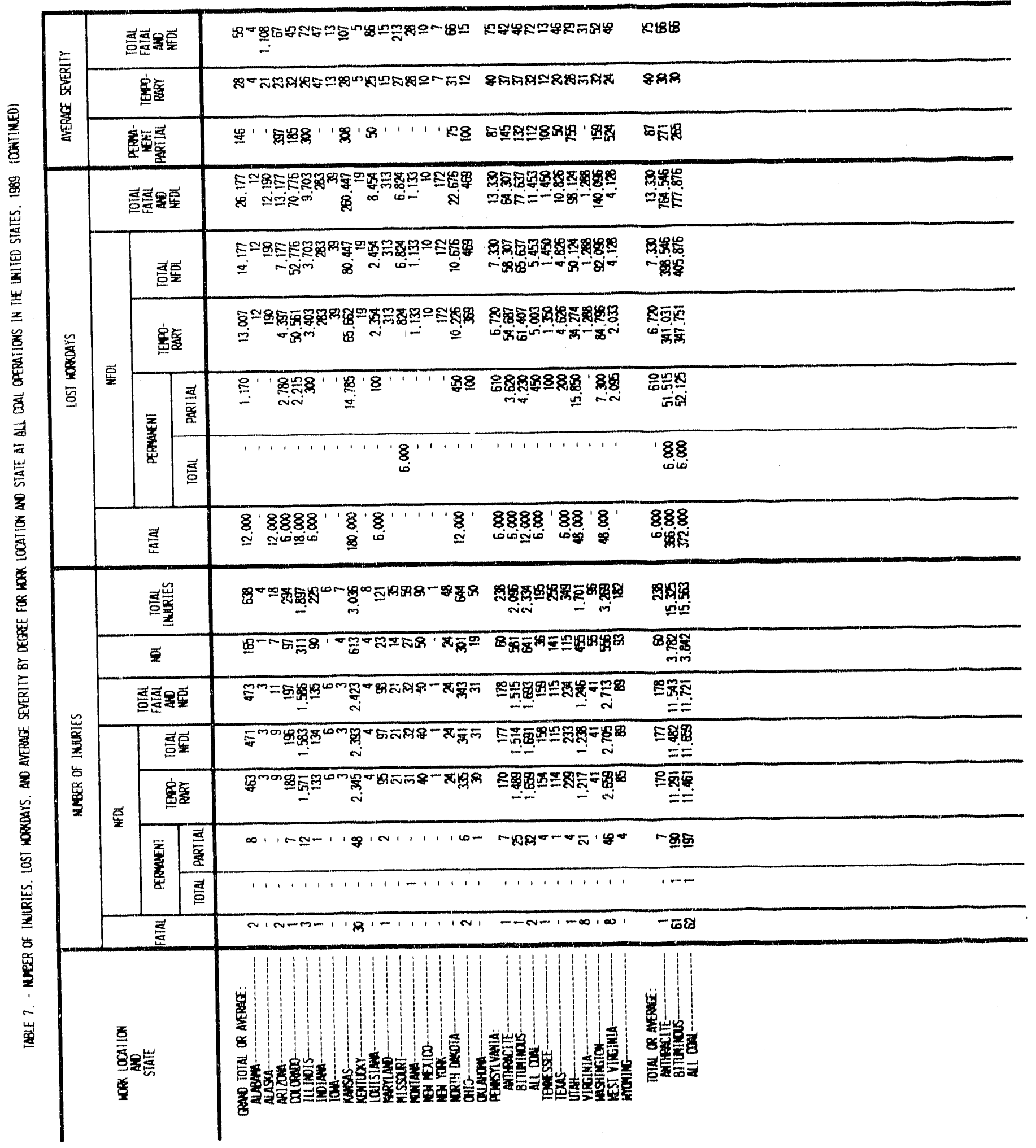




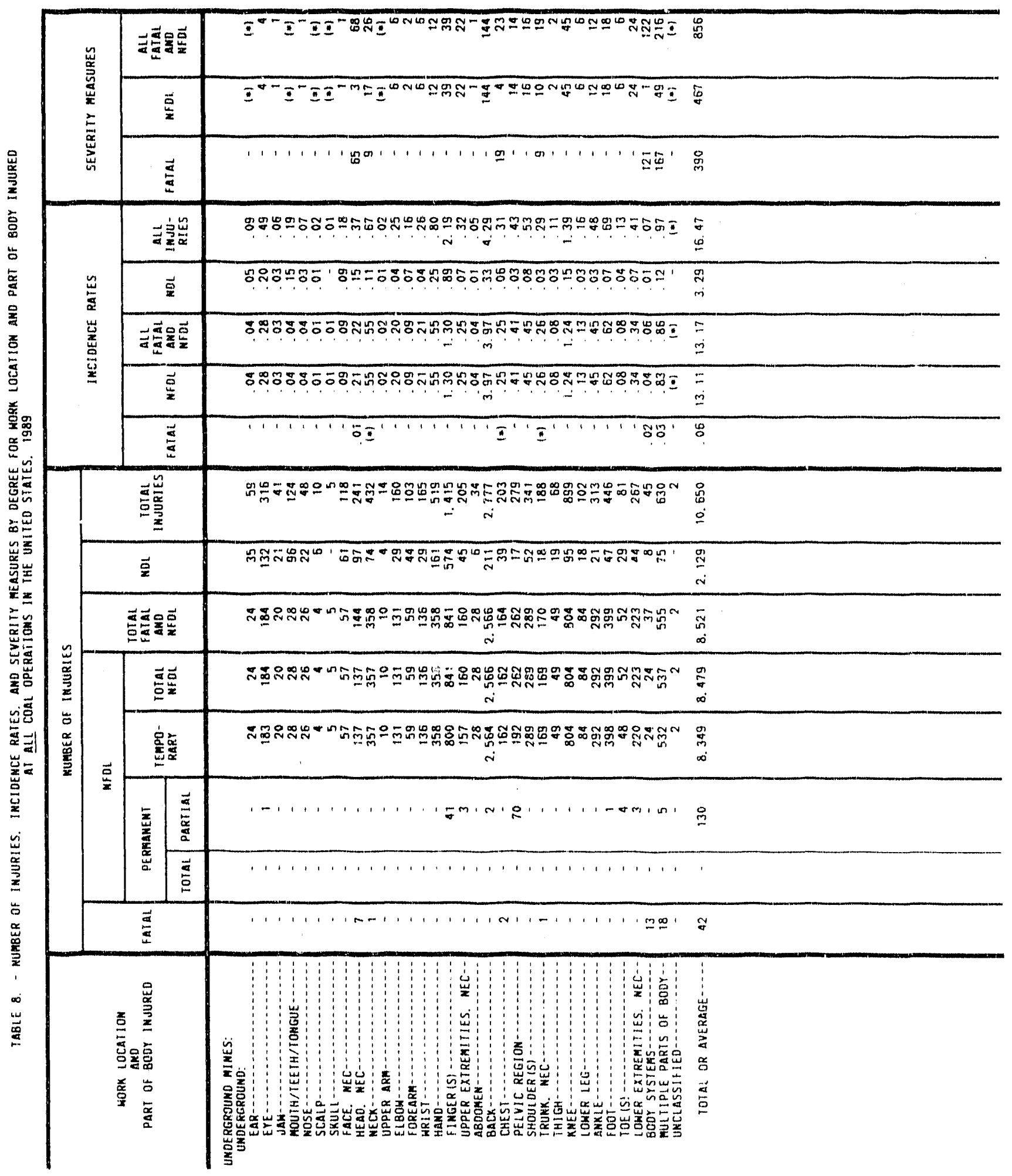




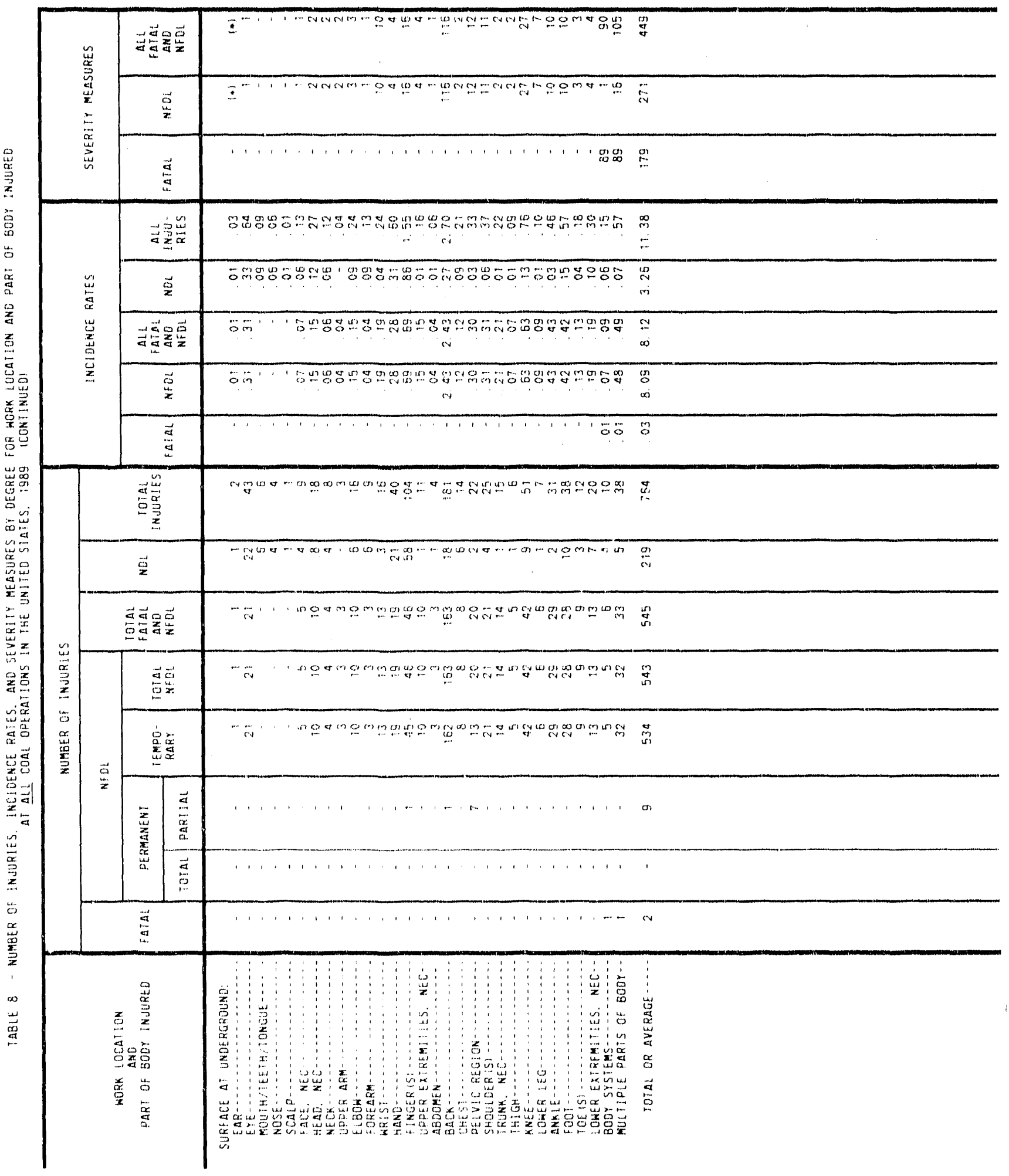




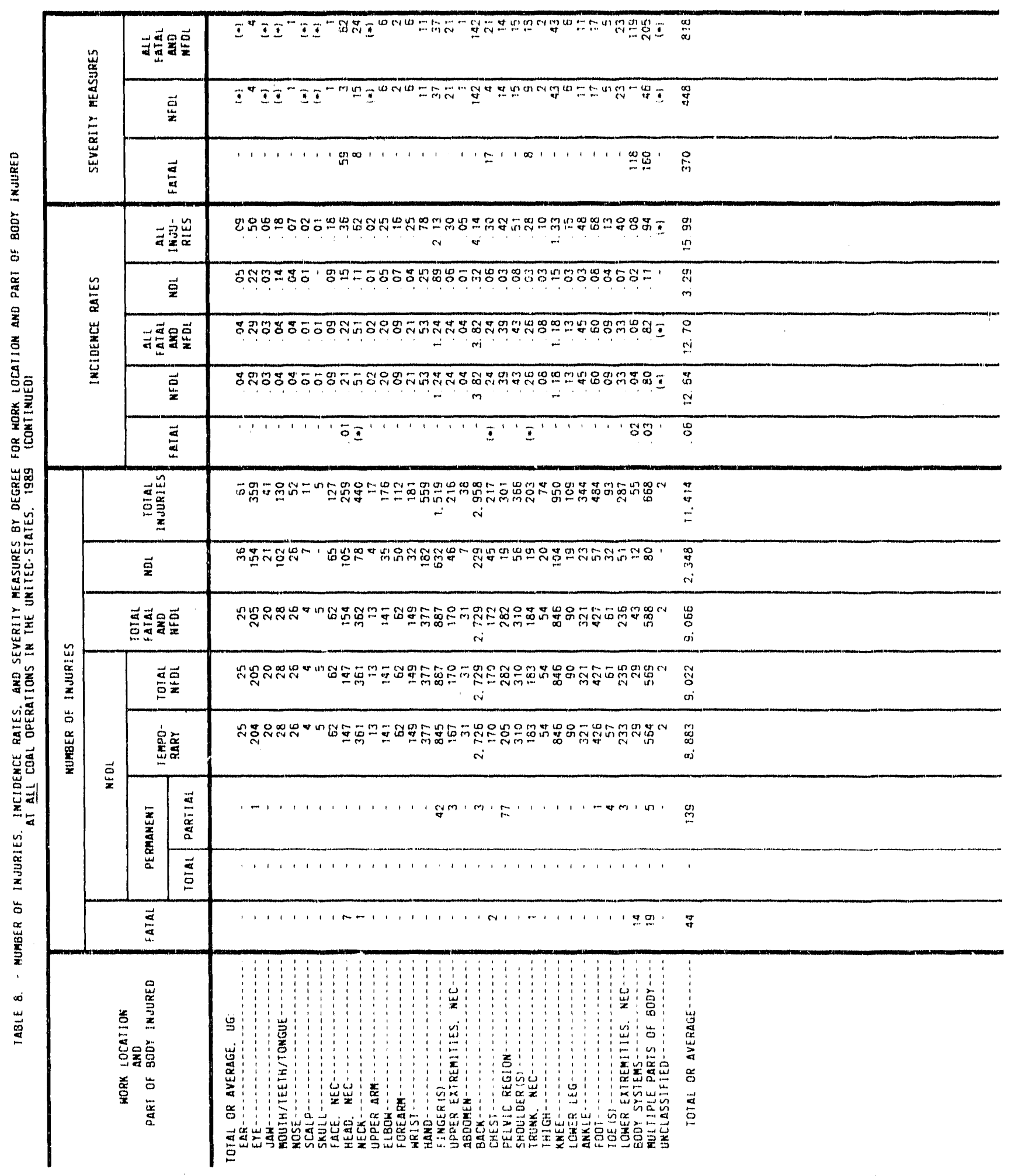




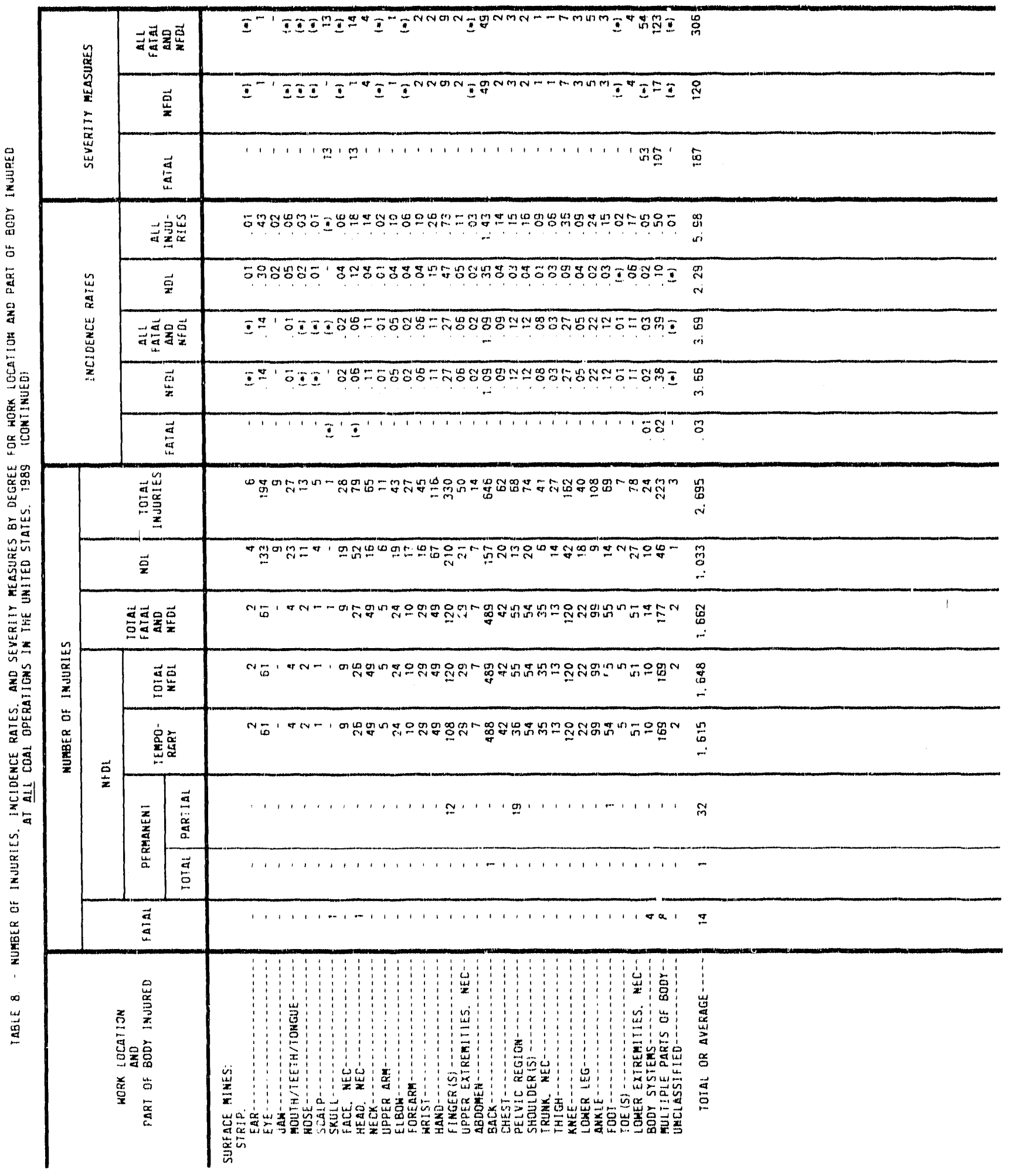




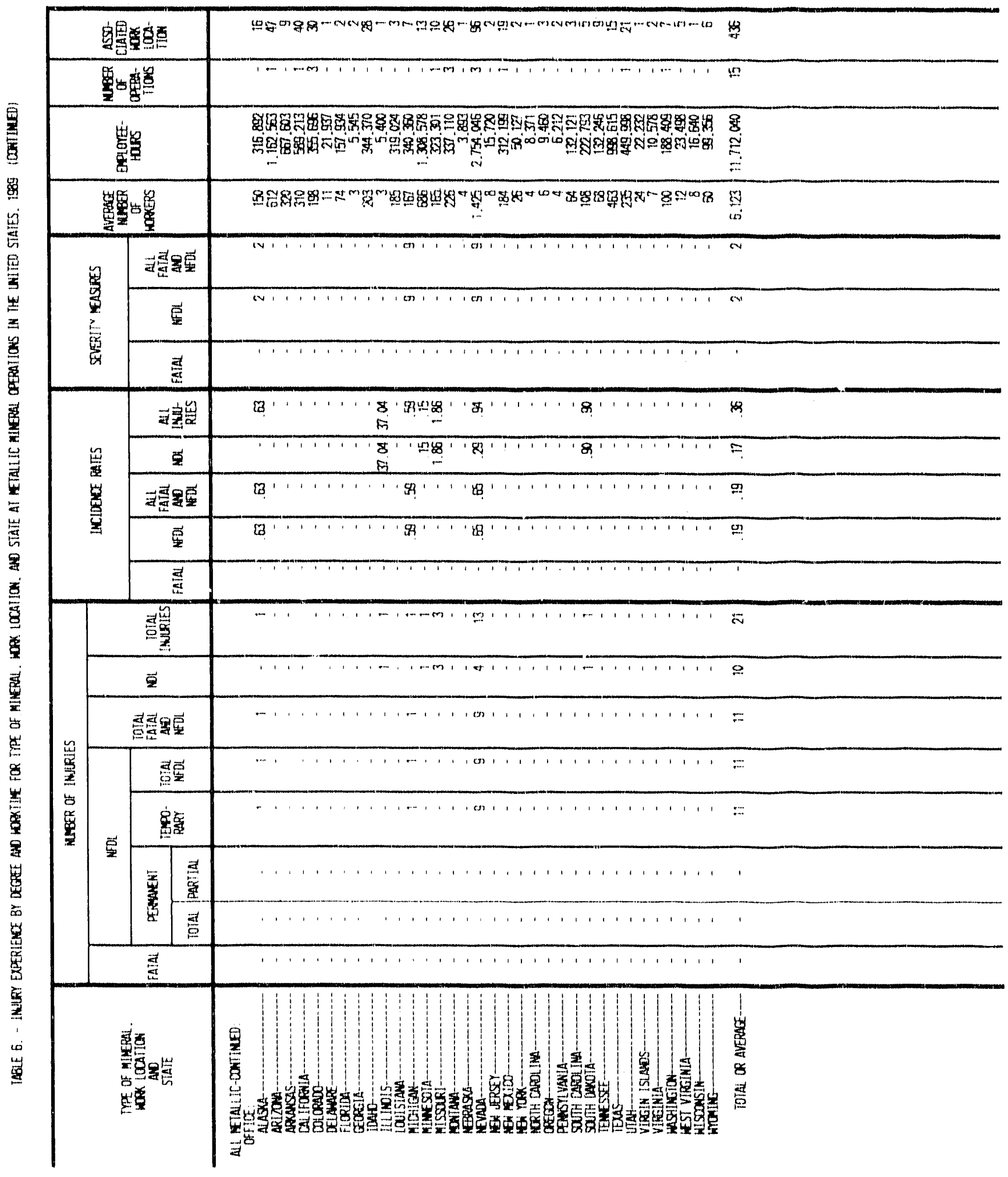




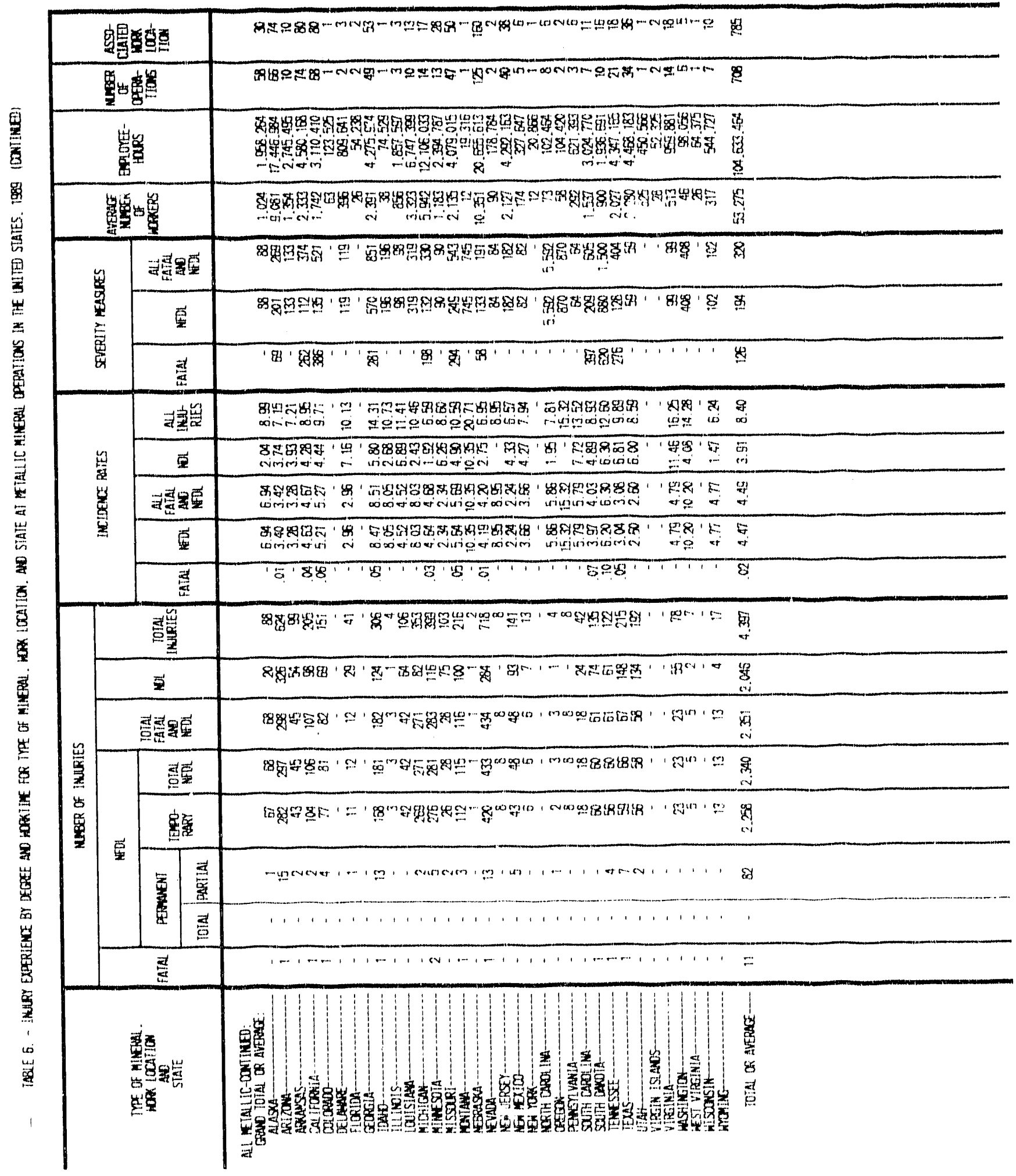




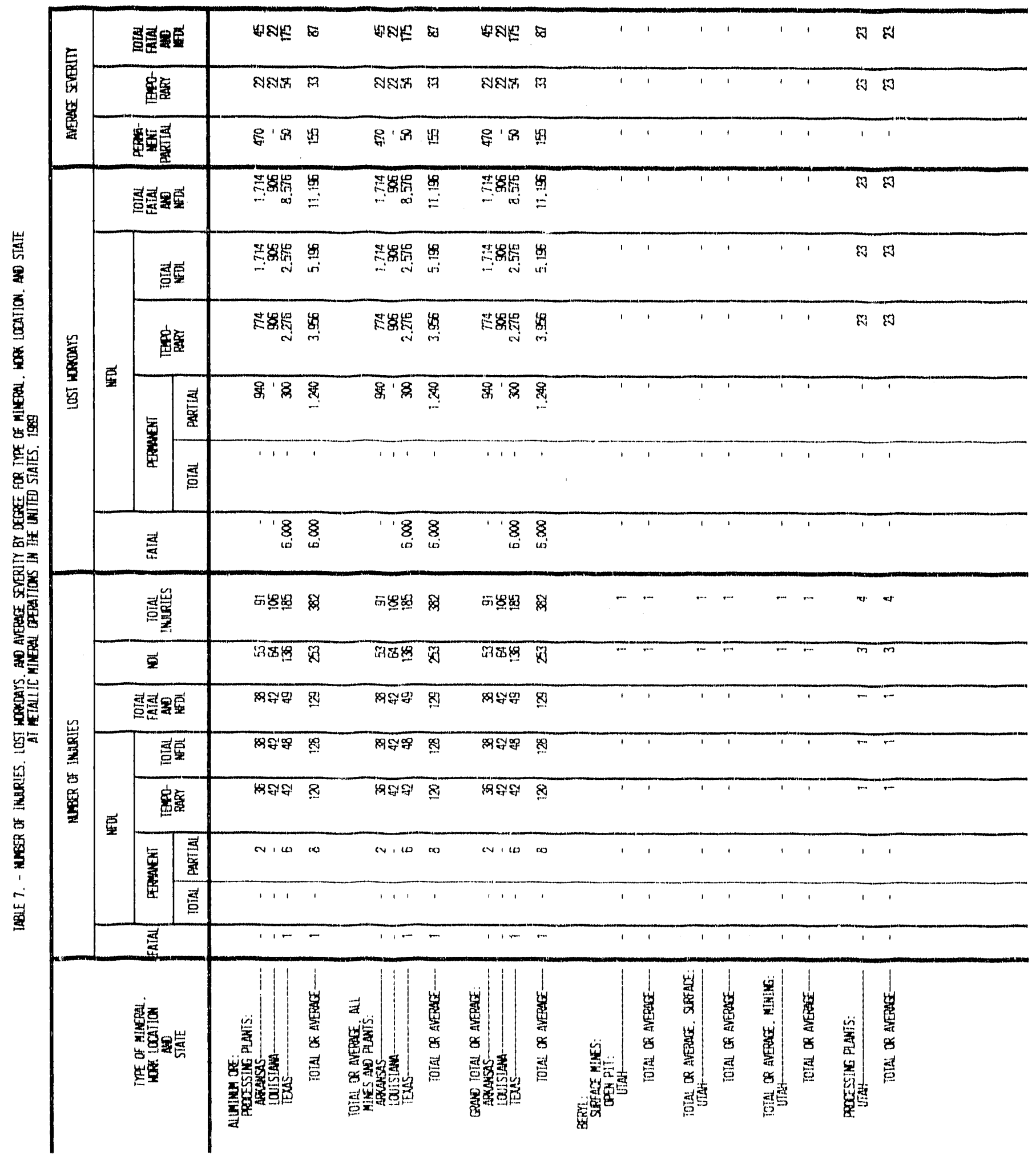




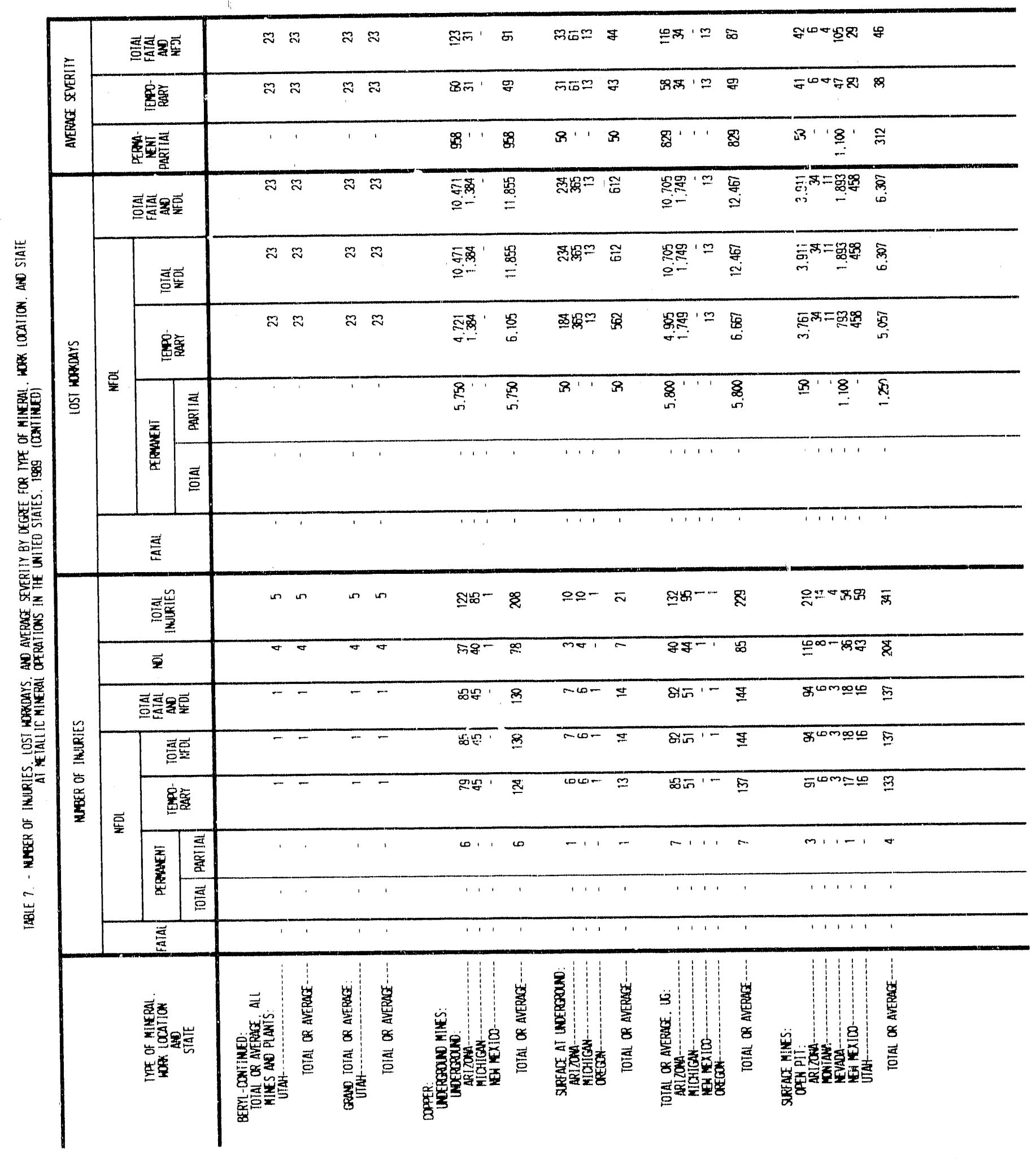




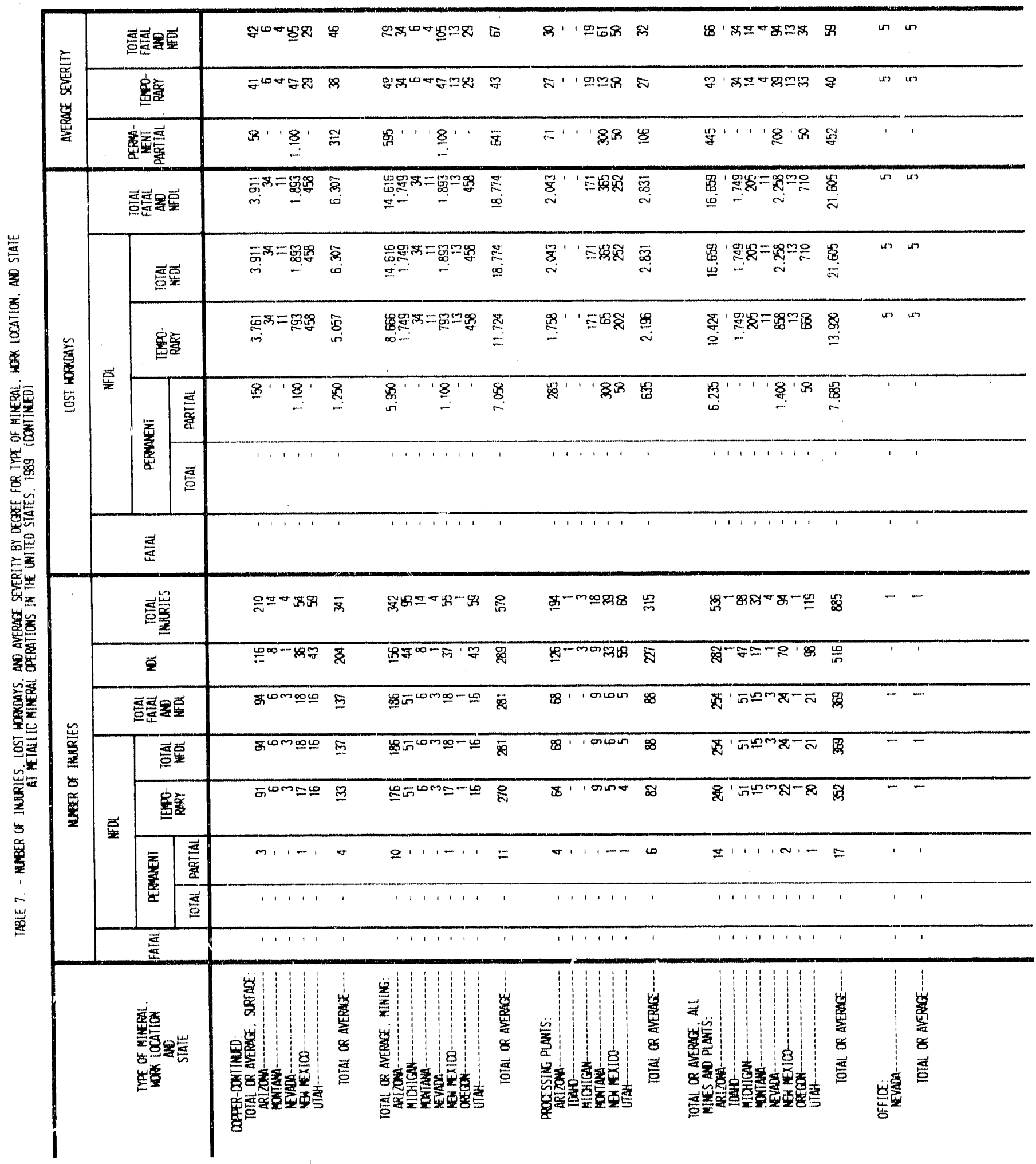




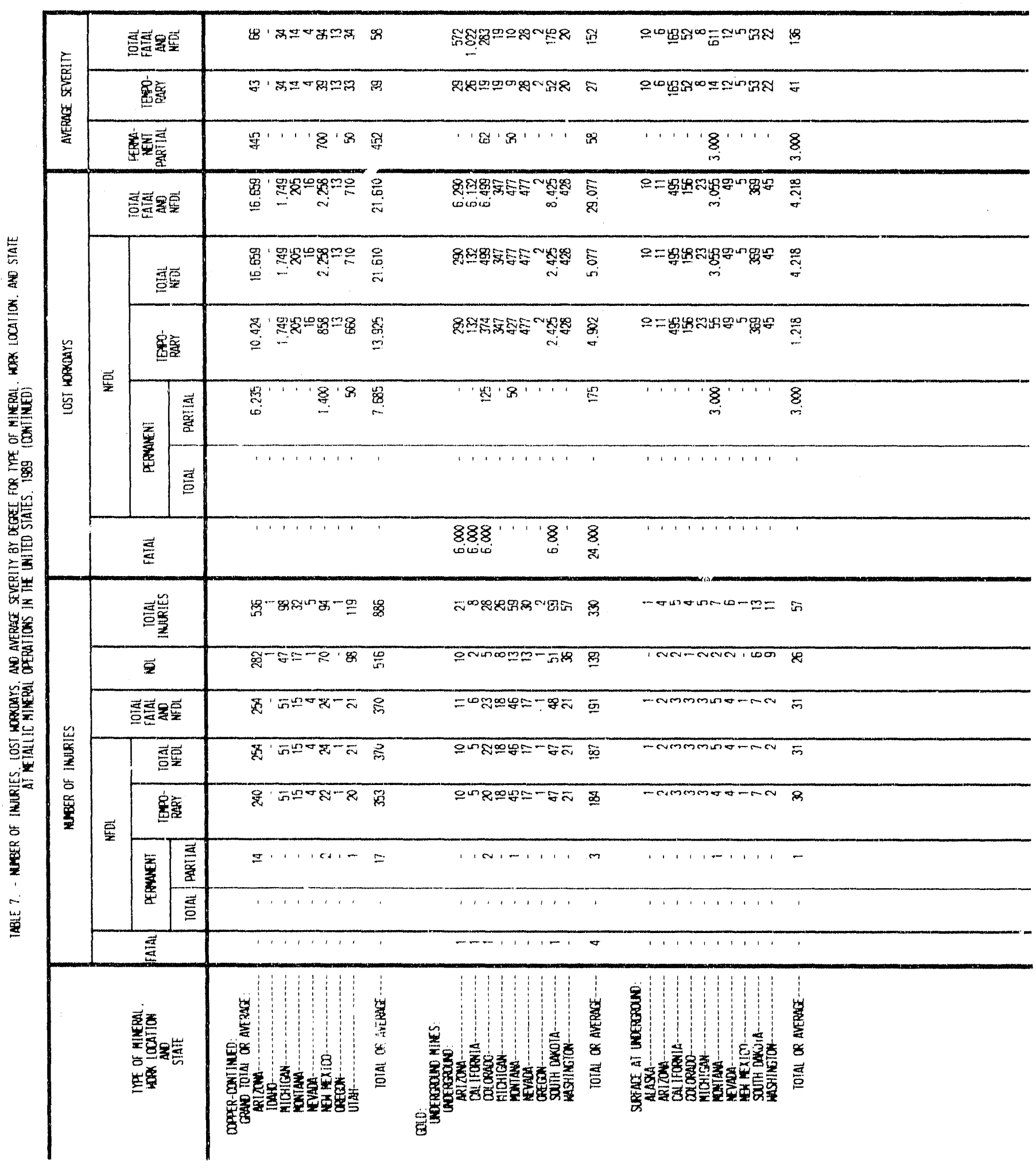




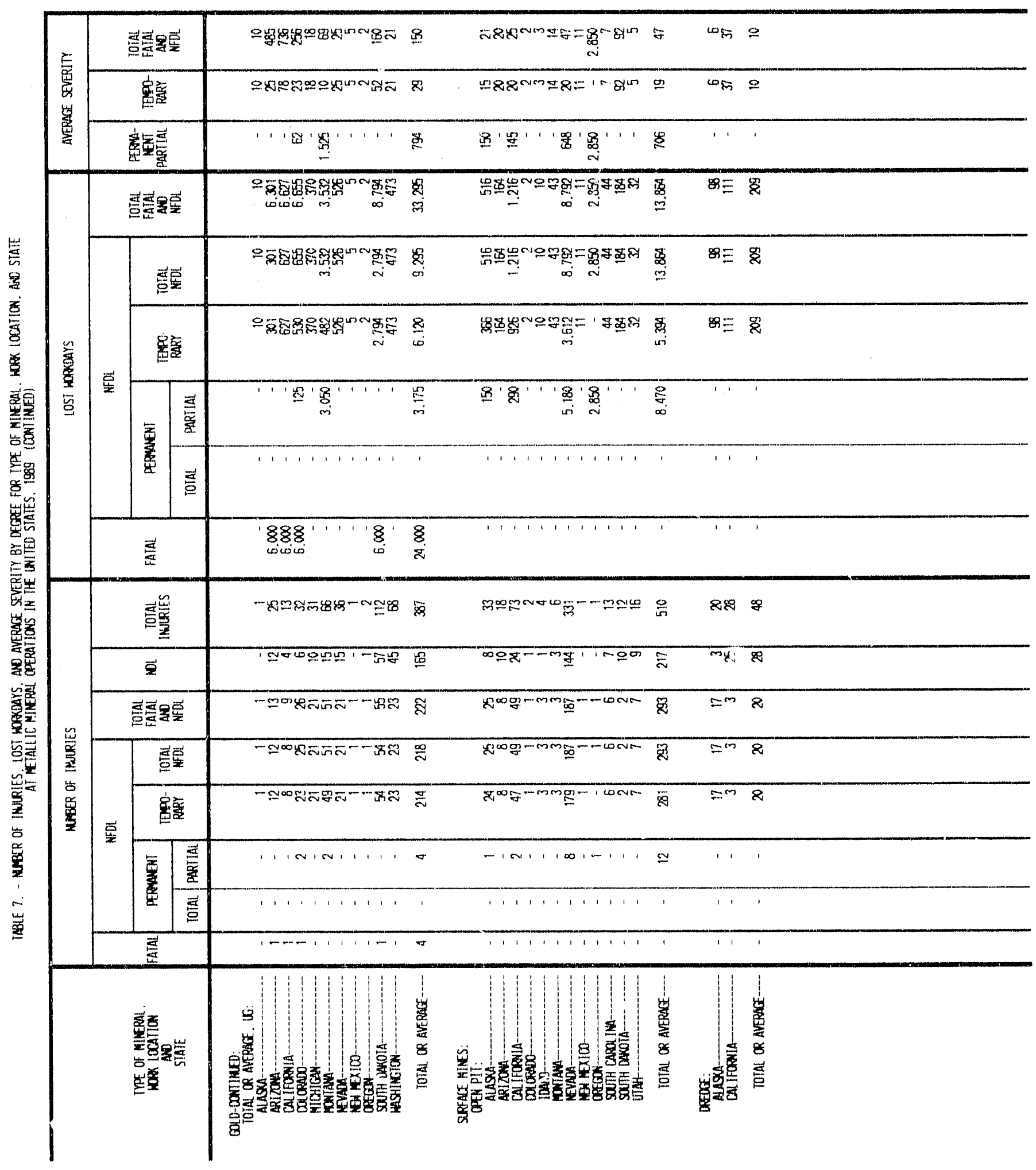




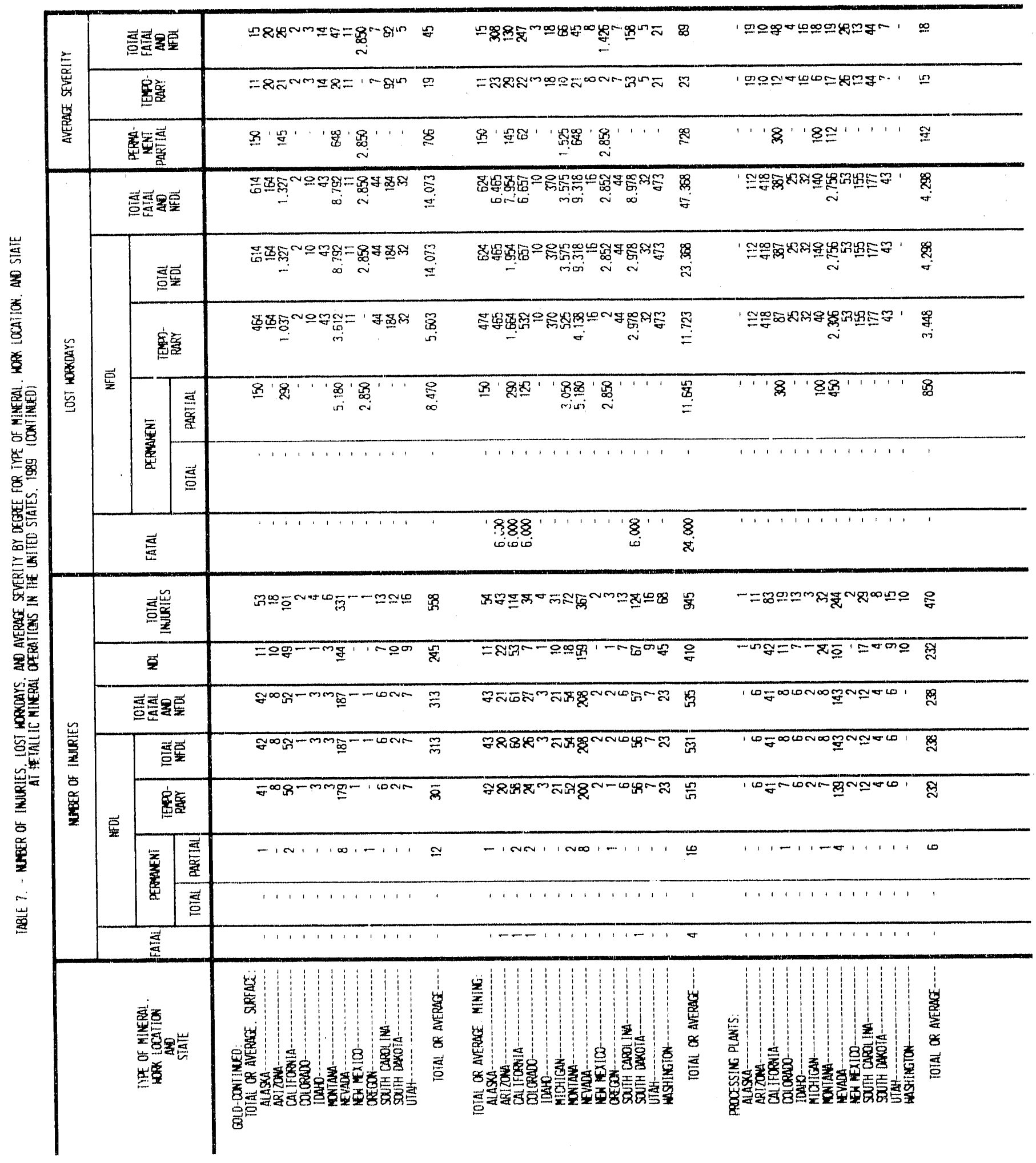




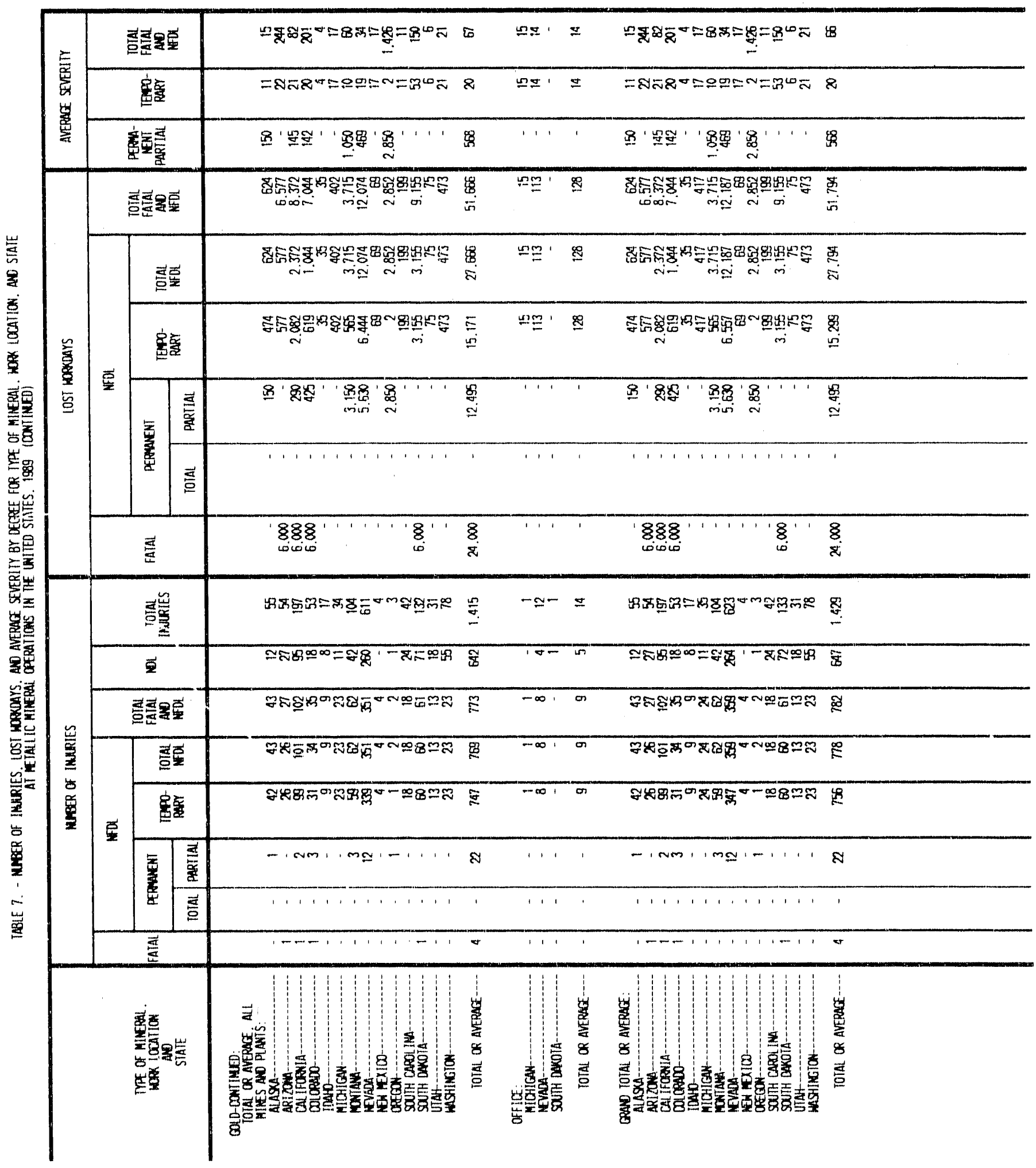




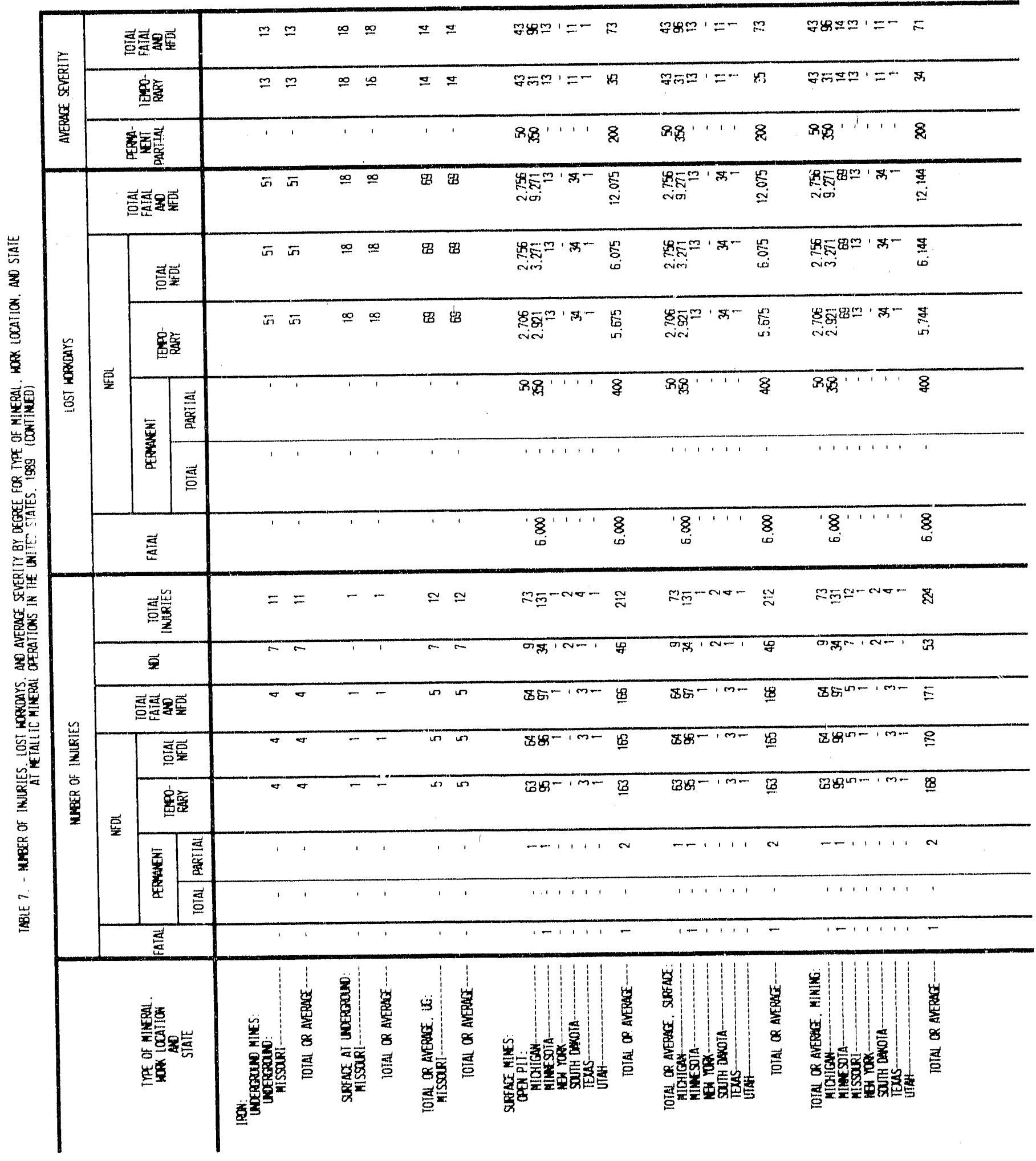




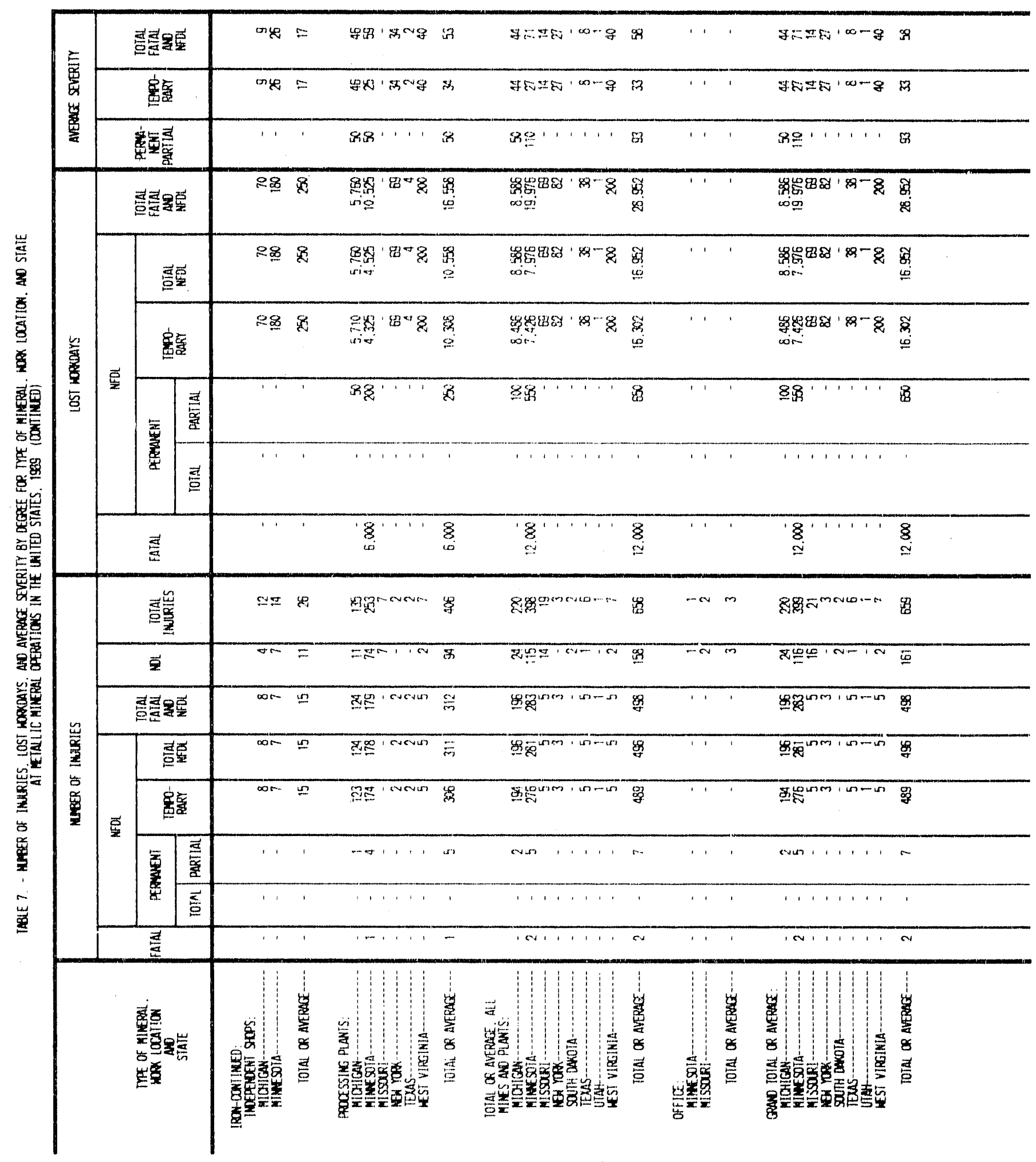




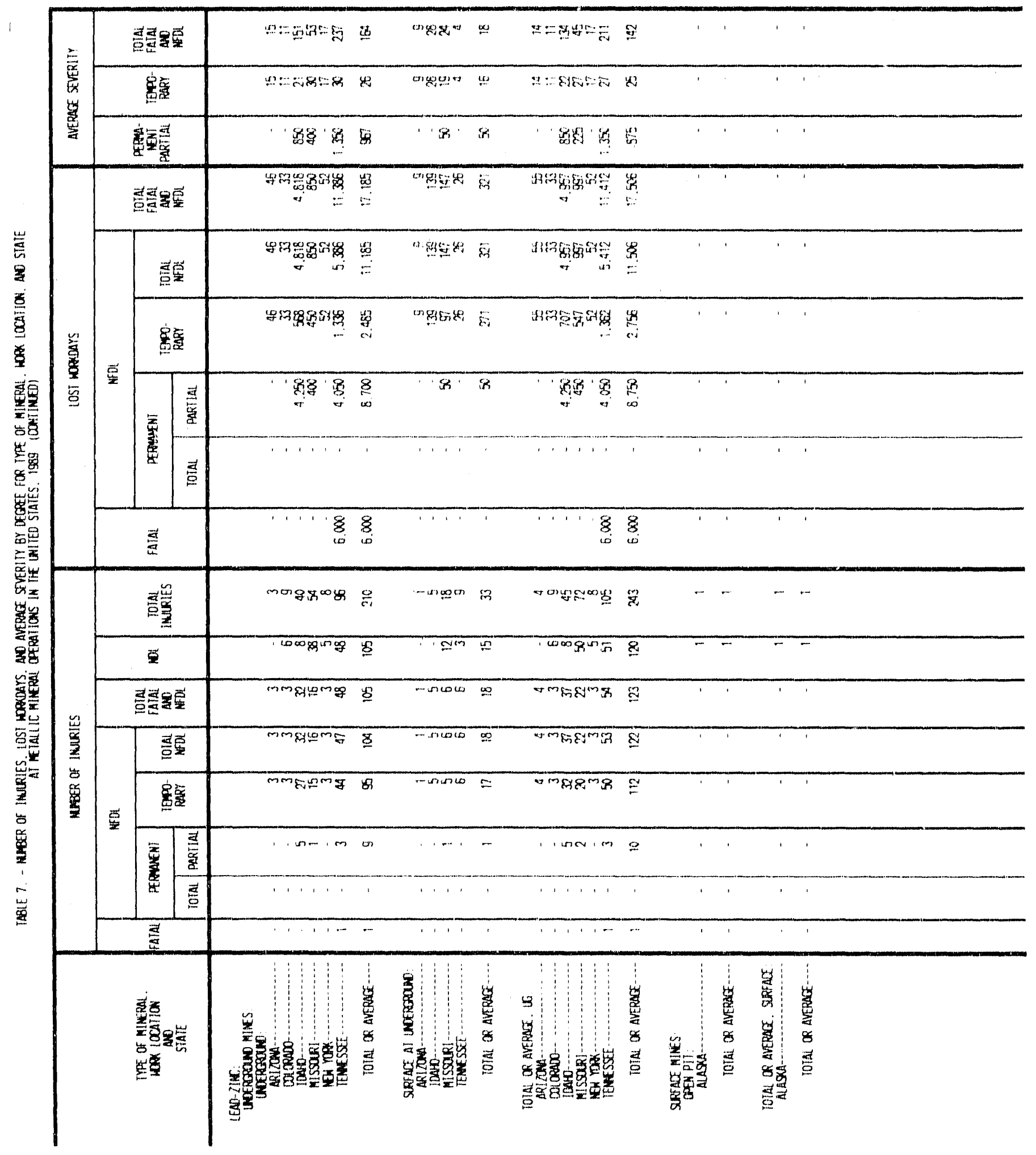




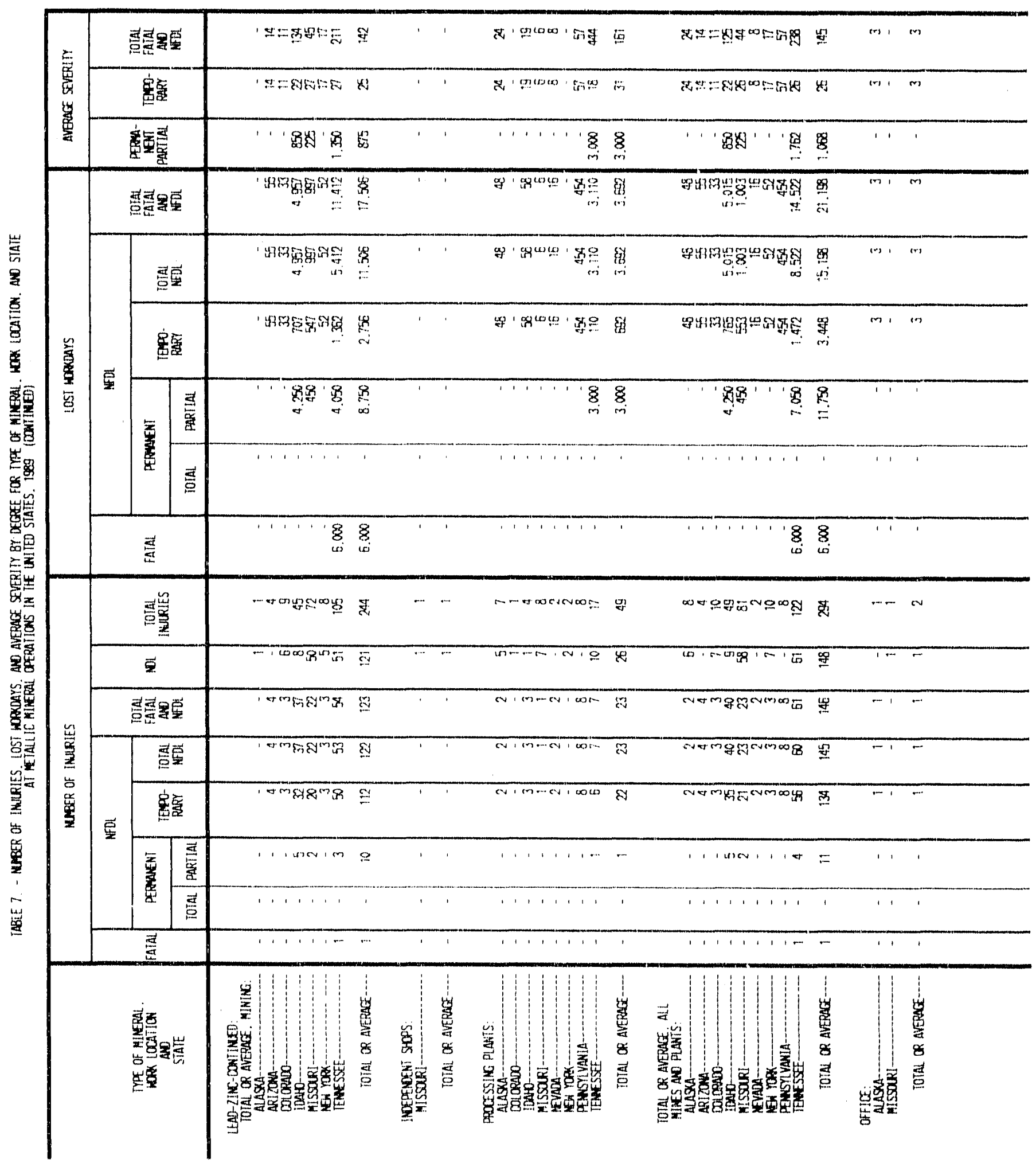




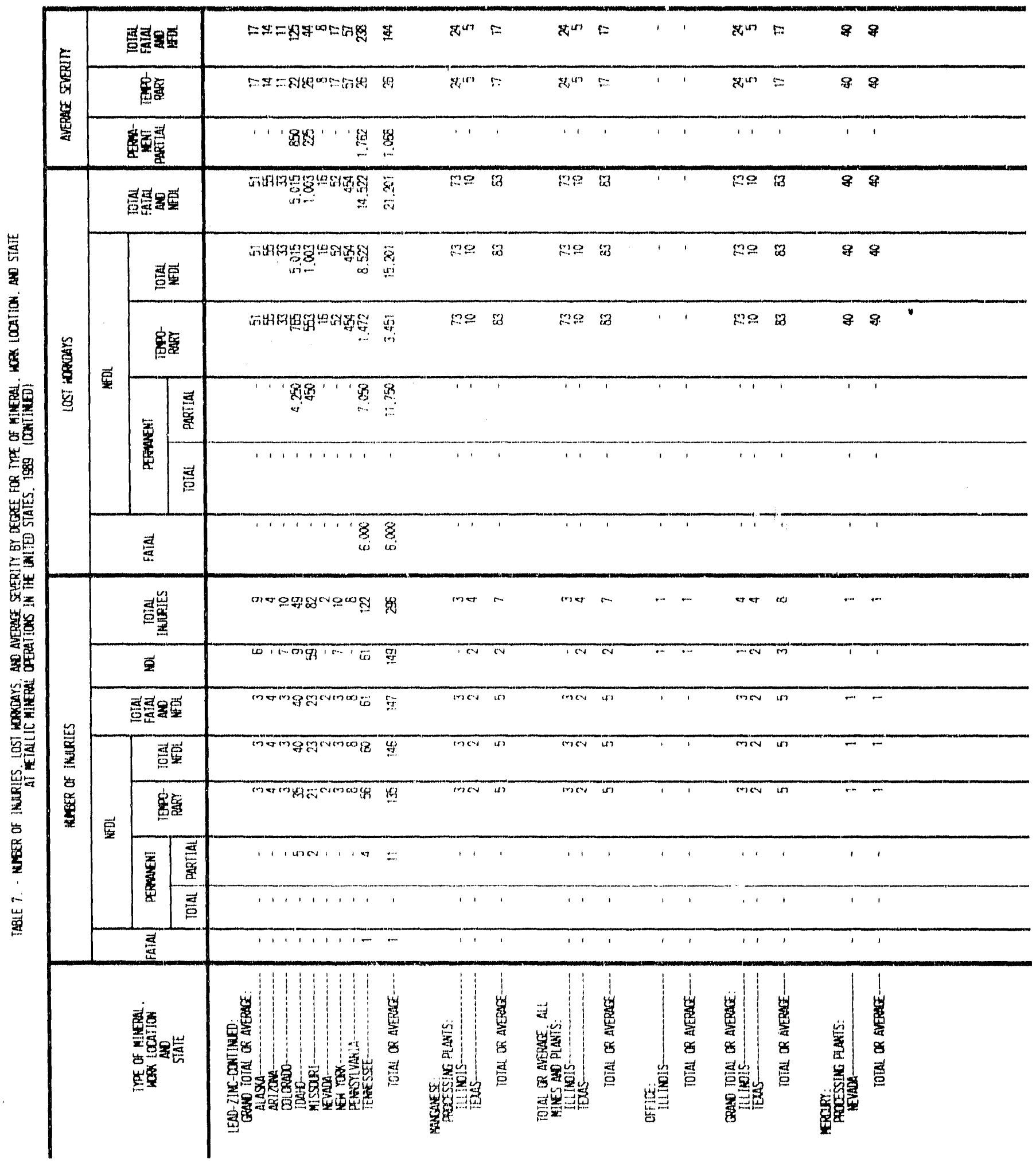




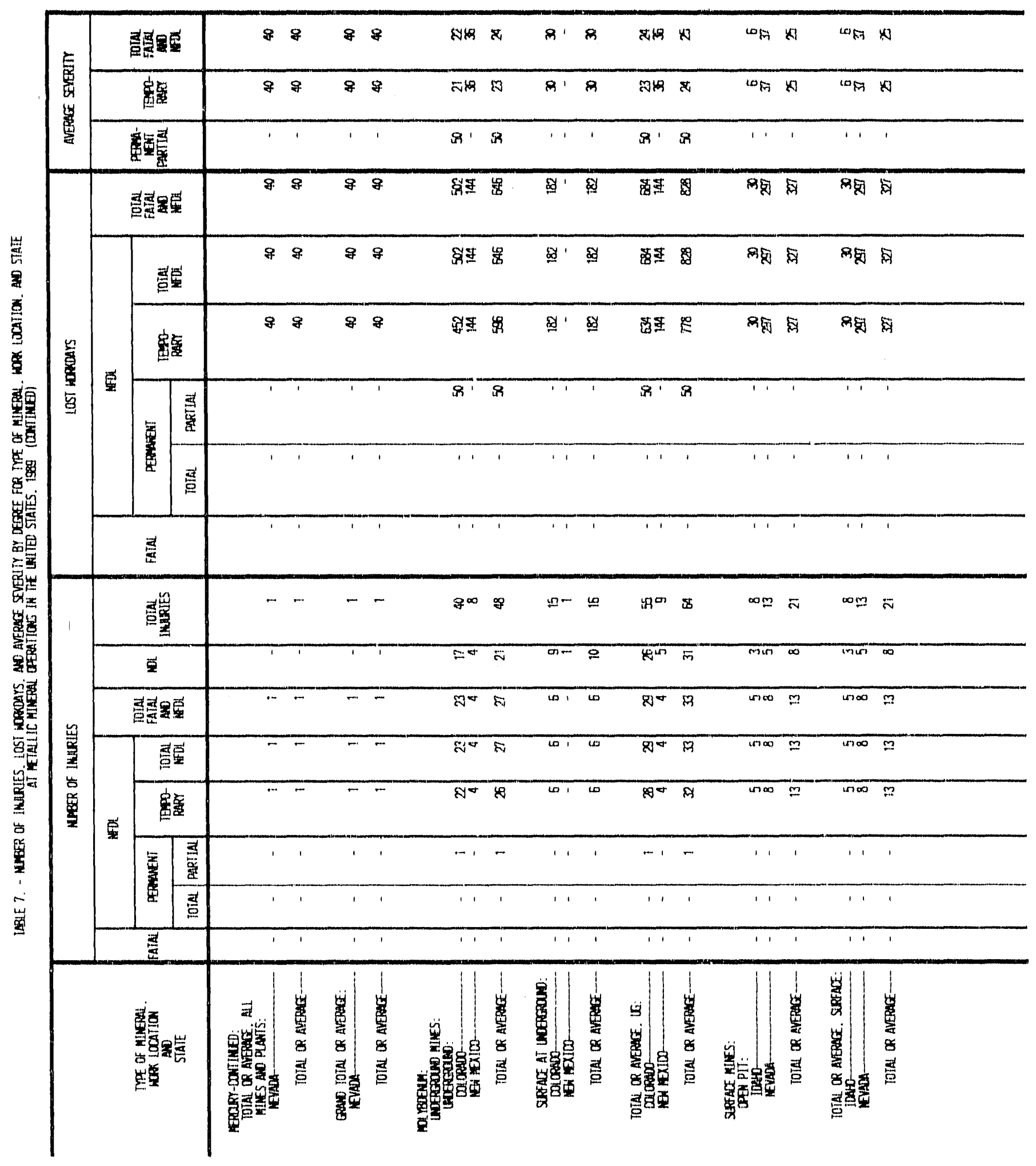




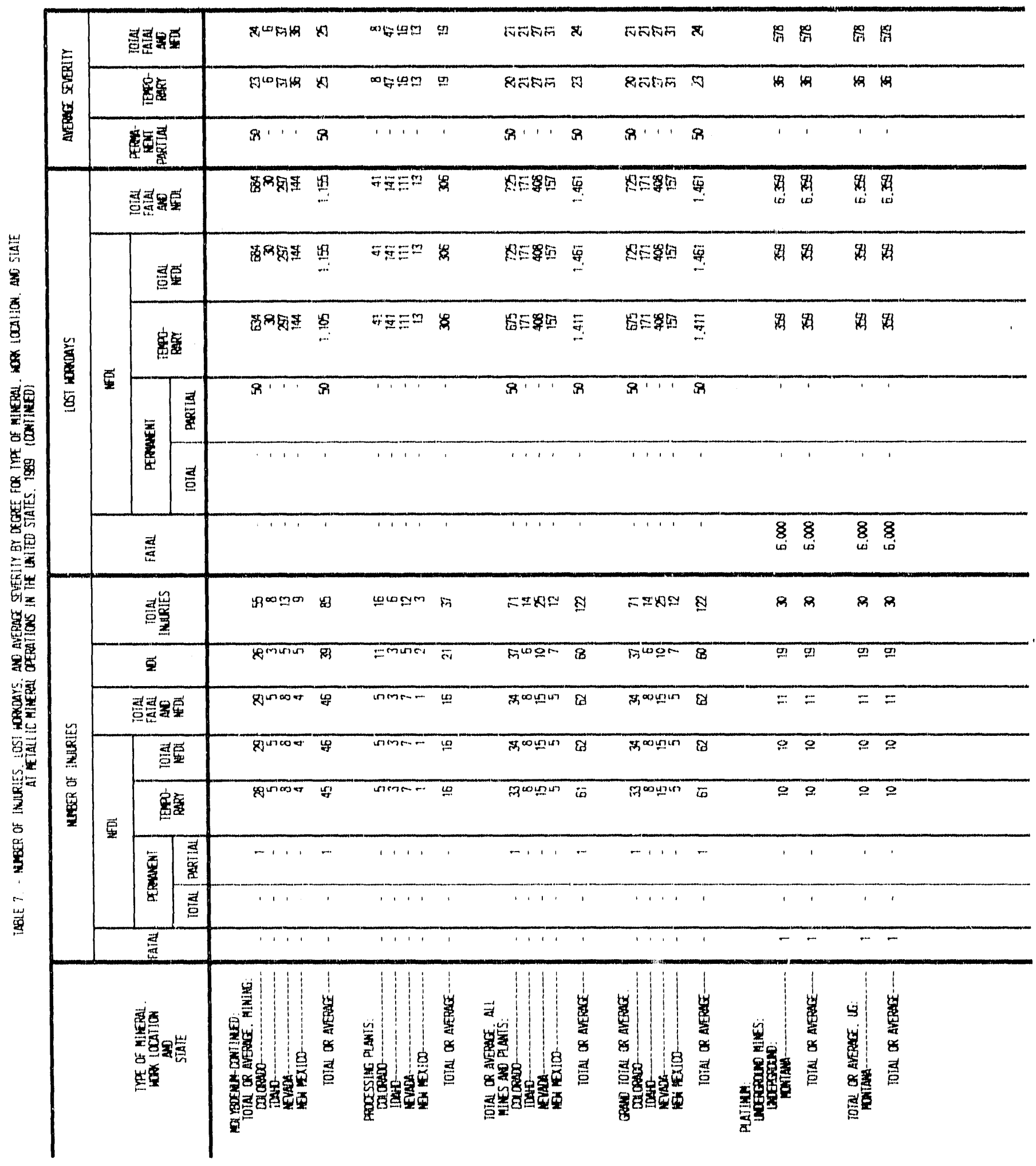




\begin{tabular}{|c|c|c|c|c|c|c|c|c|c|c|c|c|c|c|c|c|}
\hline \multirow{3}{*}{ 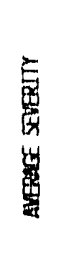 } & \multicolumn{2}{|c|}{ 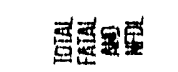 } & \multicolumn{2}{|c|}{ 总搹 } & -1 & \multicolumn{3}{|c|}{ - } & \multicolumn{2}{|c|}{ 一蜜 } & 喿 & 骂 & \multicolumn{2}{|c|}{ 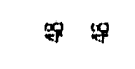 } & \multicolumn{2}{|c|}{ 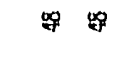 } \\
\hline & \multicolumn{2}{|c|}{ 䗆 } & 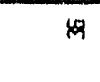 & $\varphi$ & -1 & $\cdots$ & -68 & & -48 & B & $\%$ & 果 & $g$ & 橗 & 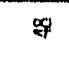 & 羊 \\
\hline & \multicolumn{2}{|c|}{ 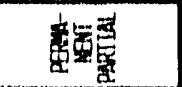 } & ' & ' & 11 & 1 & 11 & 1 & 11 & , & . & . & 1 & ' & ' & ' \\
\hline \multirow{6}{*}{ 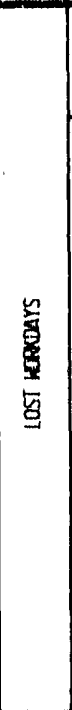 } & \multicolumn{2}{|c|}{ 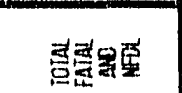 } & 蜔 & 盟 & -1 & - & 一巽 & 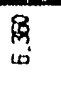 & 留 & 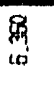 & gi & 器 & 果 & 9 & F & gig \\
\hline & \multirow{4}{*}{ 要 } & 홈롤 & 为 & 妿 & -1 & - & -g & 只 & '-gh & 8 & $\mathscr{s}$ & 吅 & 尔 & $\mathscr{q}$ & 9 & go \\
\hline & & 富害 & 周 & 男 & -1 & - & 一只 & $\mathscr{B}$ & 一蜔 & 品 & 9 & 果 & 骂 & $q$ & 喿 & 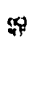 \\
\hline & & 告 & 1 & 1 & 11 & 1 & 11 & & 11 & $T$ & $T$ & 1 & 1 & T & $T$ & T \\
\hline & & $\frac{d t}{E}$ & ' & ' & . & ' & 11 & ' & 11 & ' & ' & ' & ' & \multicolumn{2}{|c|}{$"$} & ' \\
\hline & \multicolumn{2}{|l|}{ 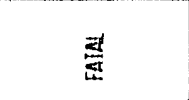 } & $\underset{w i}{8}$ & $\underset{6}{8}$ & 1.1 & 1 & 10 & $\underset{\substack{8 \\
j}}{\text { s. }}$ & $\begin{array}{l}8 \\
10 \\
0\end{array}$ & 悉 & 1 & . & ' & ' & , & 1 \\
\hline \multirow{8}{*}{ 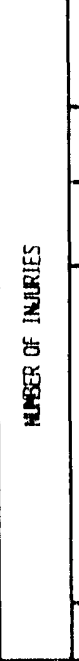 } & \multicolumn{2}{|c|}{ 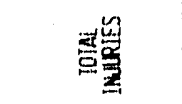 } & in & 8 & $\cdots$ & o & $-\bar{m}$ & ळ્ન & $-\bar{m}$ & $g y$ & N & $\infty$ & N & $N$ & v & a \\
\hline & \multicolumn{2}{|l|}{$\overline{2}$} & T & g & 17 & $=$ & 8 & 8 & 18 & 8 & $=$ & - & $=$ & $=$ & $=$ & $=$ \\
\hline & \multicolumn{2}{|c|}{ 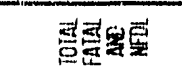 } & $=$ & $=$ & -1 & - & $-=$ & 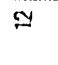 & $-\ldots$ & $\cong$ & $=$ & - & - & $\cdots$ & $=$ & - \\
\hline & \multicolumn{2}{|c|}{ 莡量 } & $?$ & $?$ & -1 & $=$ & 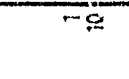 & $=$ & -9 & $\bar{E}$ & $=$ & $=$ & $=$ & $=$ & $\overline{-}$ & $=$ \\
\hline & \multicolumn{2}{|c|}{ 宜 } & ? & ? & -1 & - & -9 & $=$ & $-\Phi$ & $=$ & - & - & - & - & "- & $\cdots$ \\
\hline & 莓 & 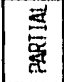 & ' & . & 1. & , & 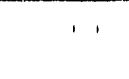 & , & 1, & ! & ' & , & . & , & & \\
\hline & & 善 & ' & . & '. & ' & 1 & ' & 1. & ' & . & ' & ' & ' & , & , \\
\hline & \multicolumn{2}{|l|}{ 䒺 } & - & $\cdots$ & 11 & I & $1-$ & - & $1-$ & - & 7 & $T$ & 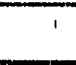 & 1 & 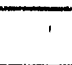 & . \\
\hline & 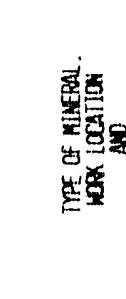 & & 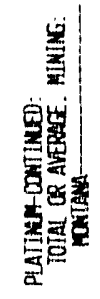 & 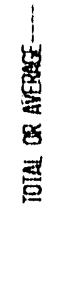 & 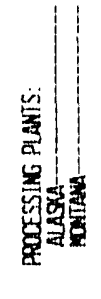 & 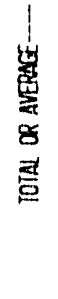 & 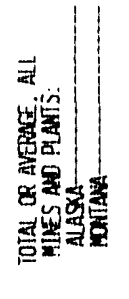 & 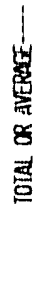 & 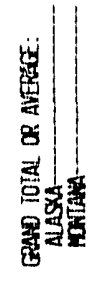 & 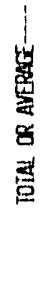 & 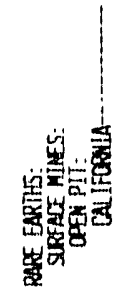 & 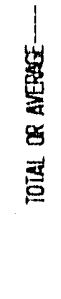 & 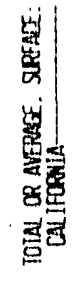 & 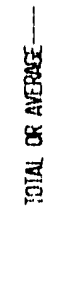 & 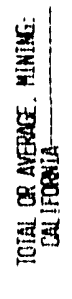 & 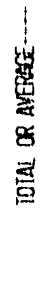 \\
\hline
\end{tabular}




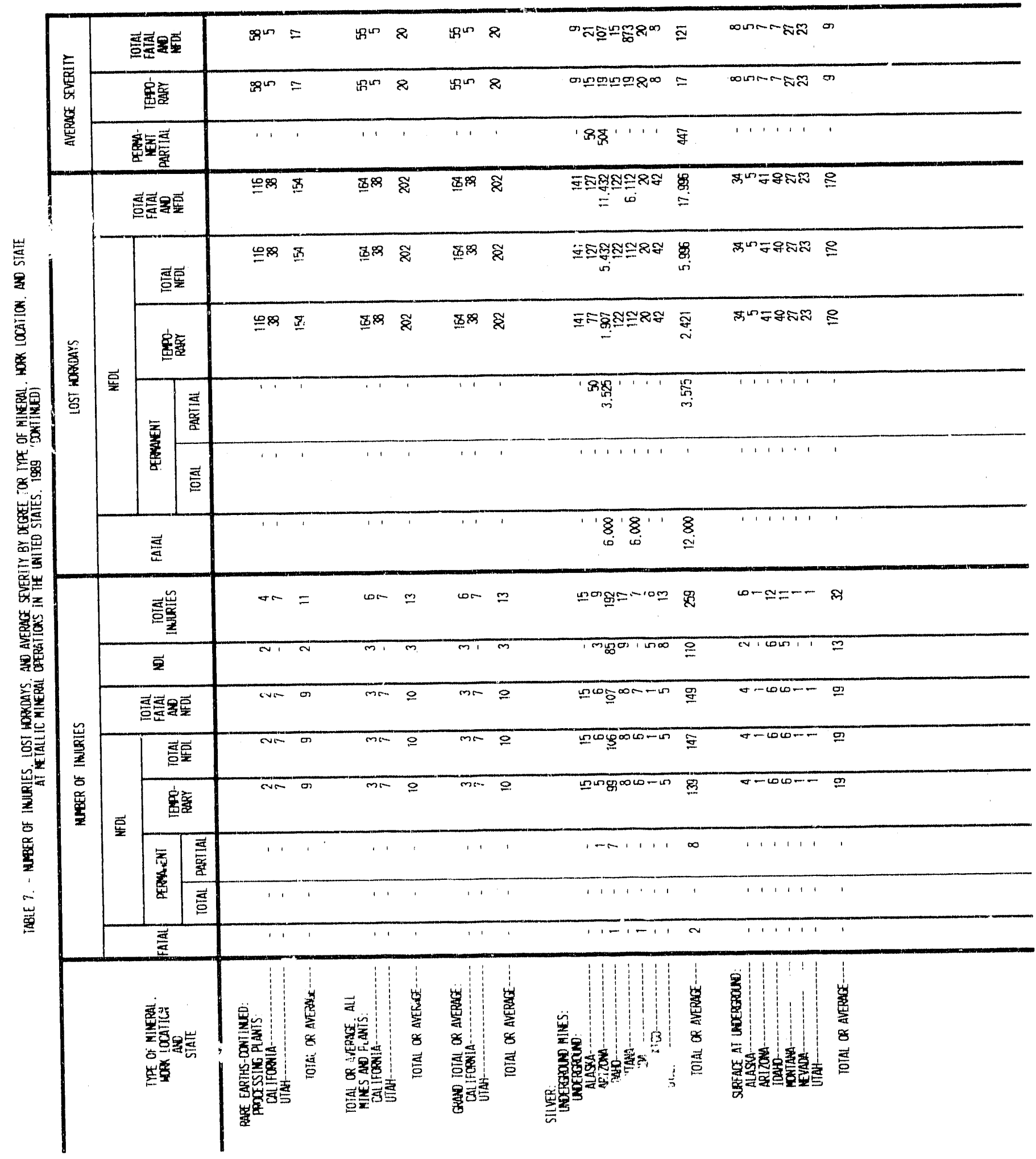




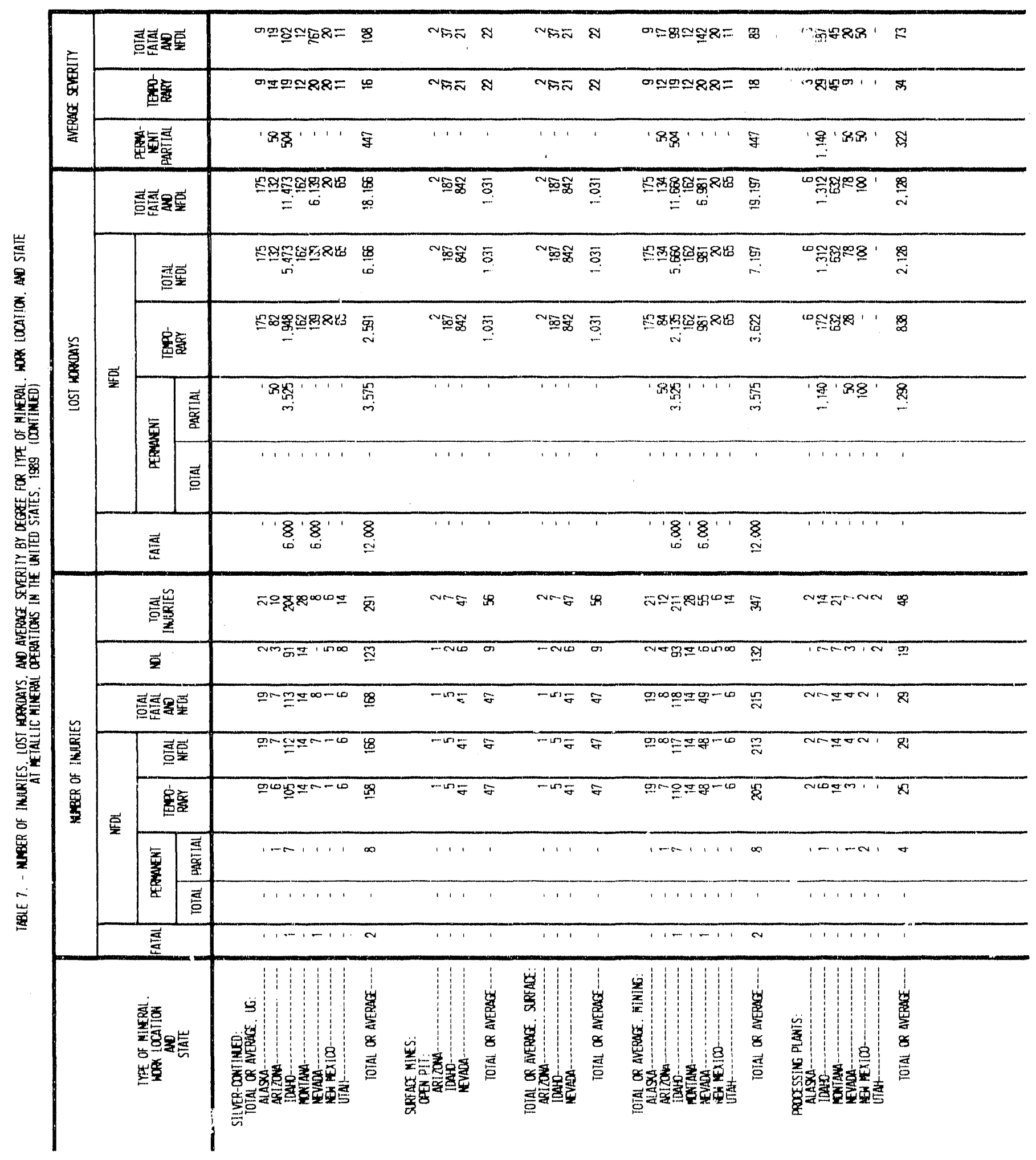




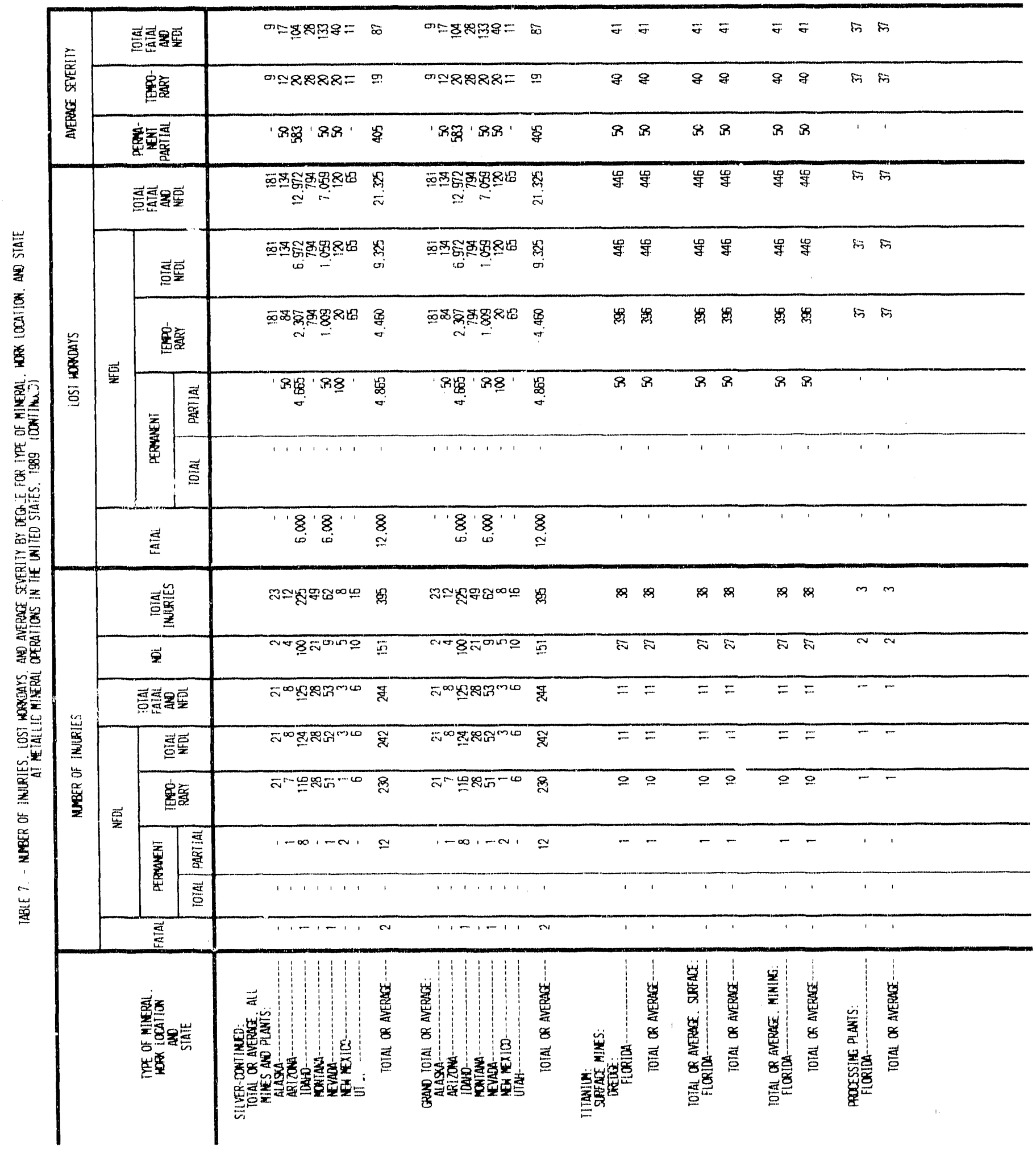




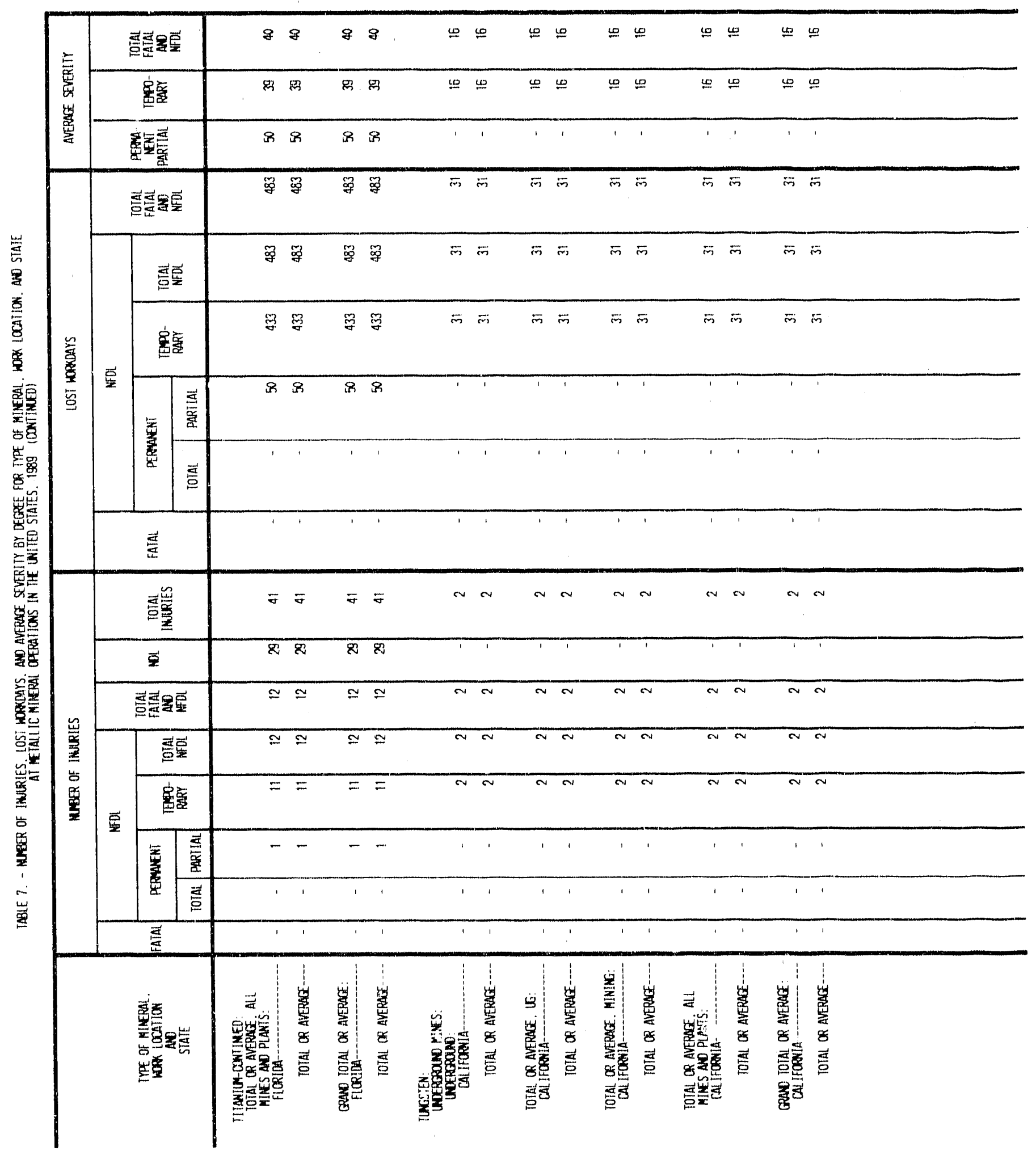




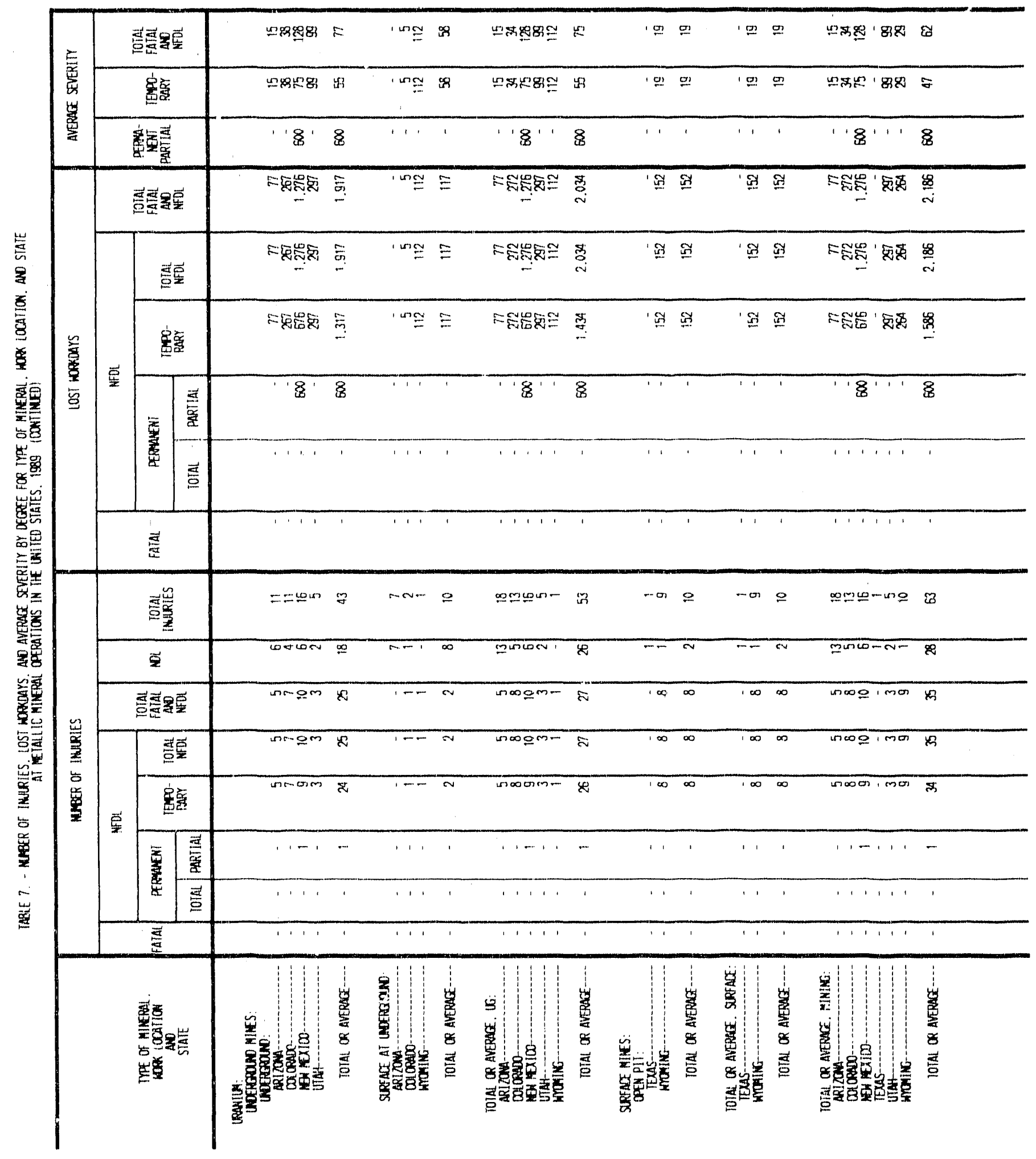




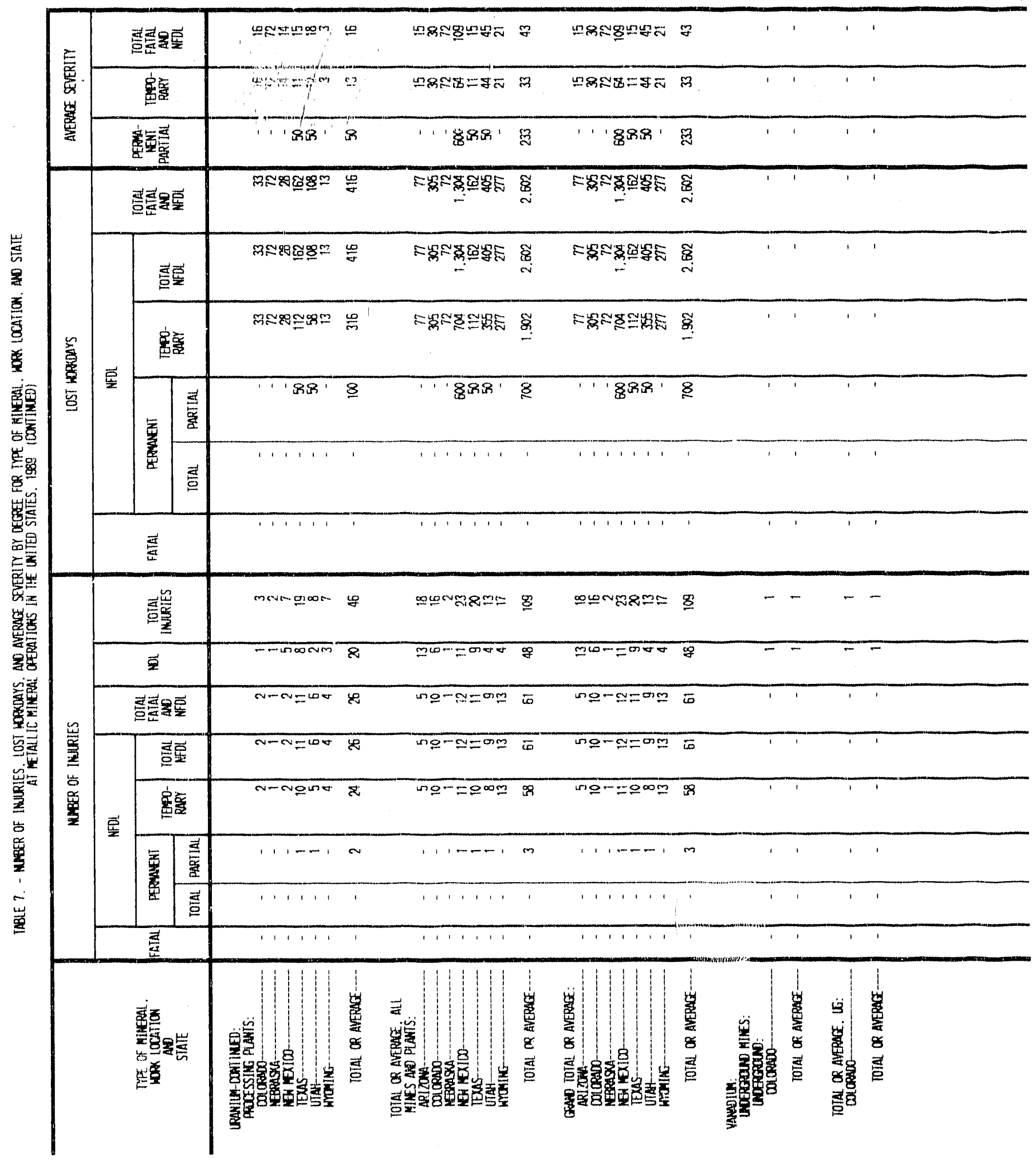




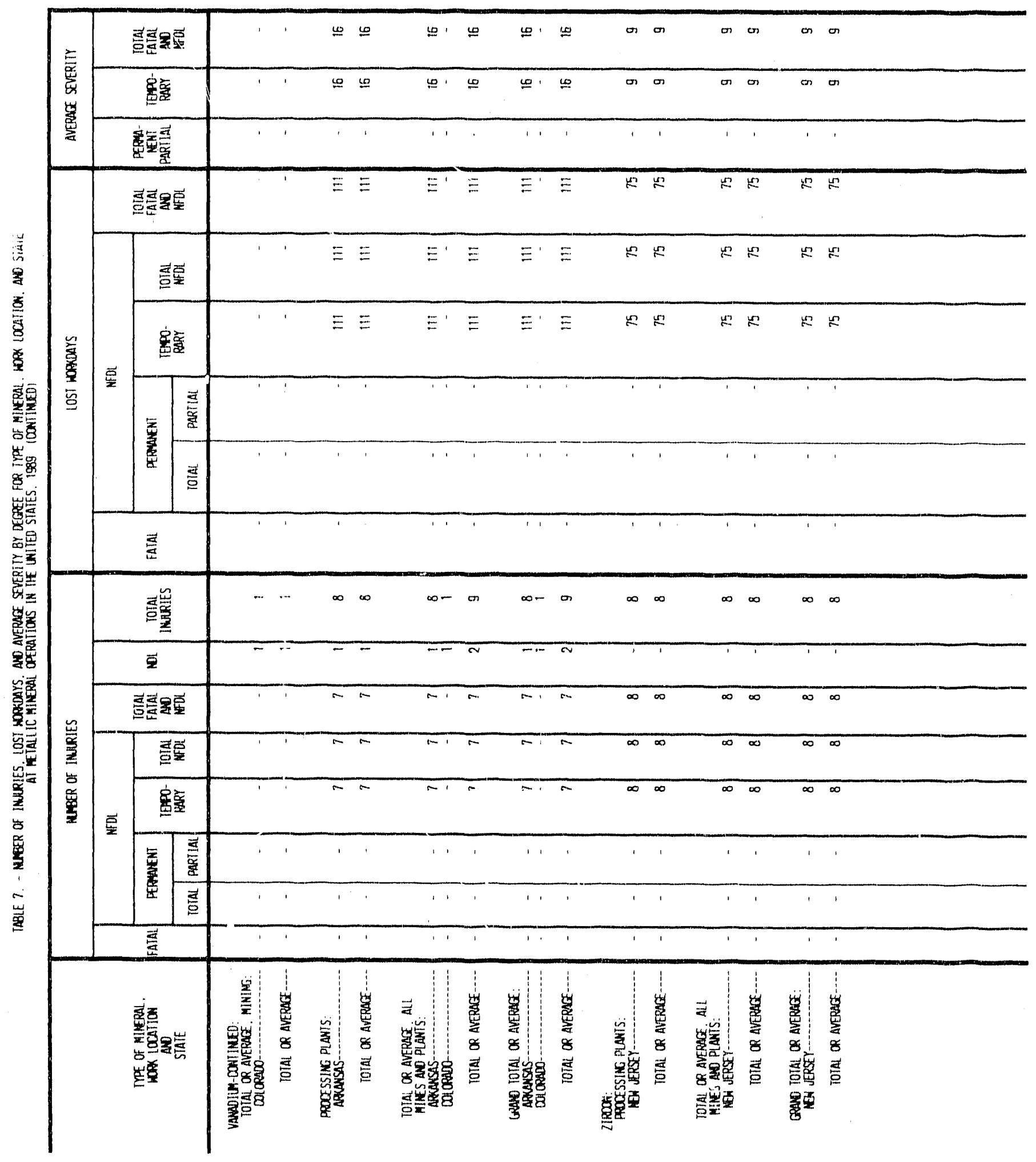




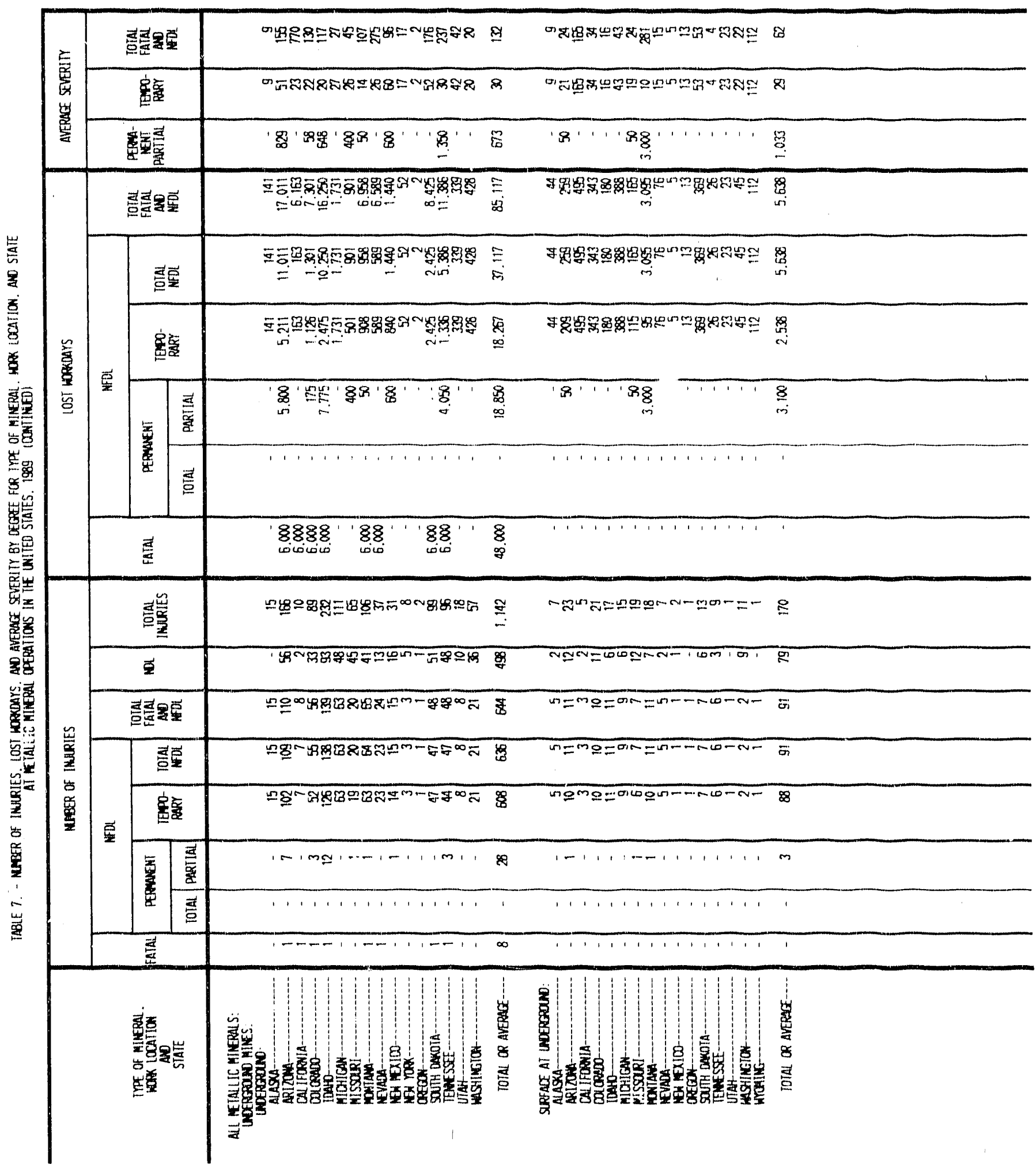




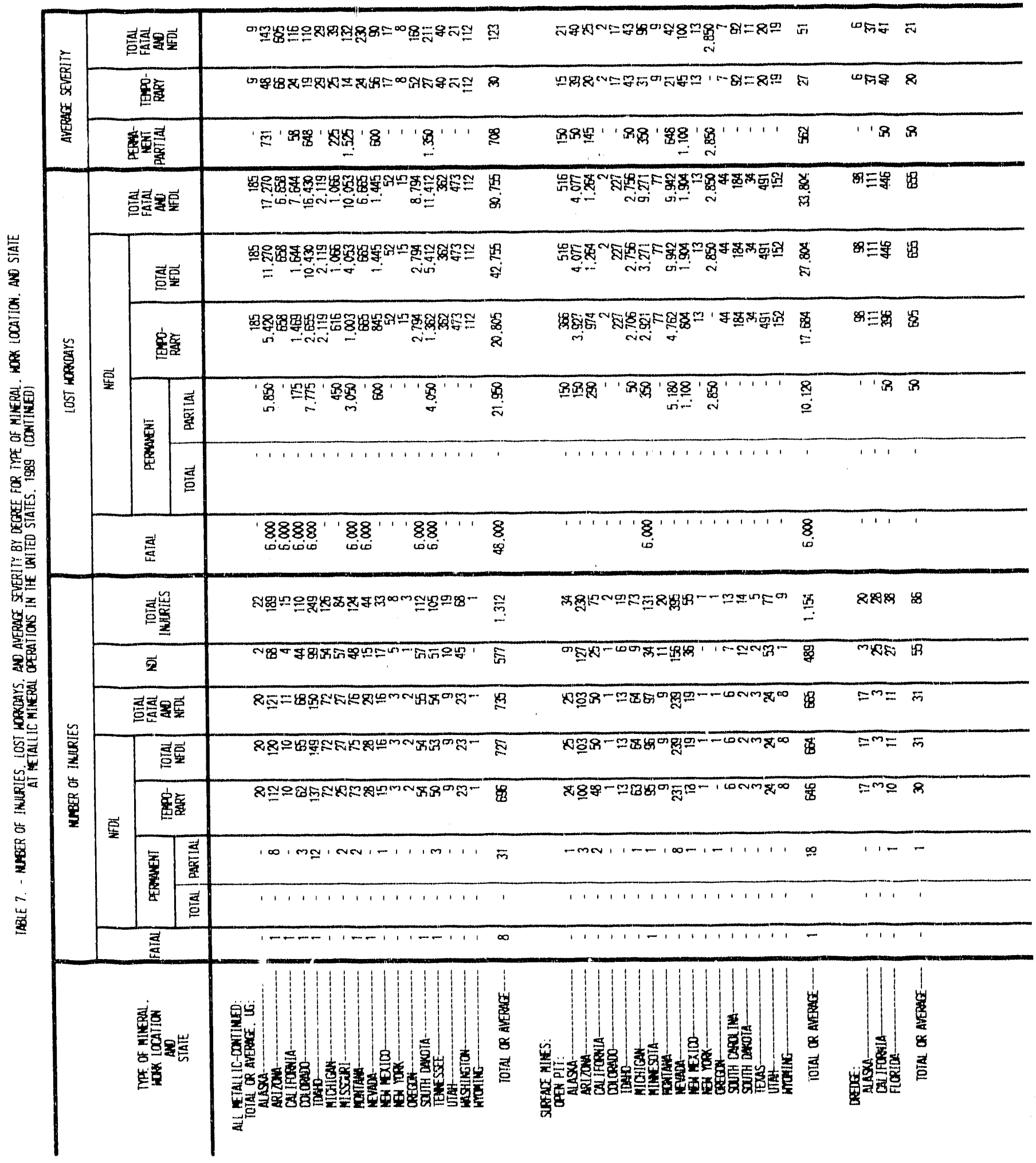




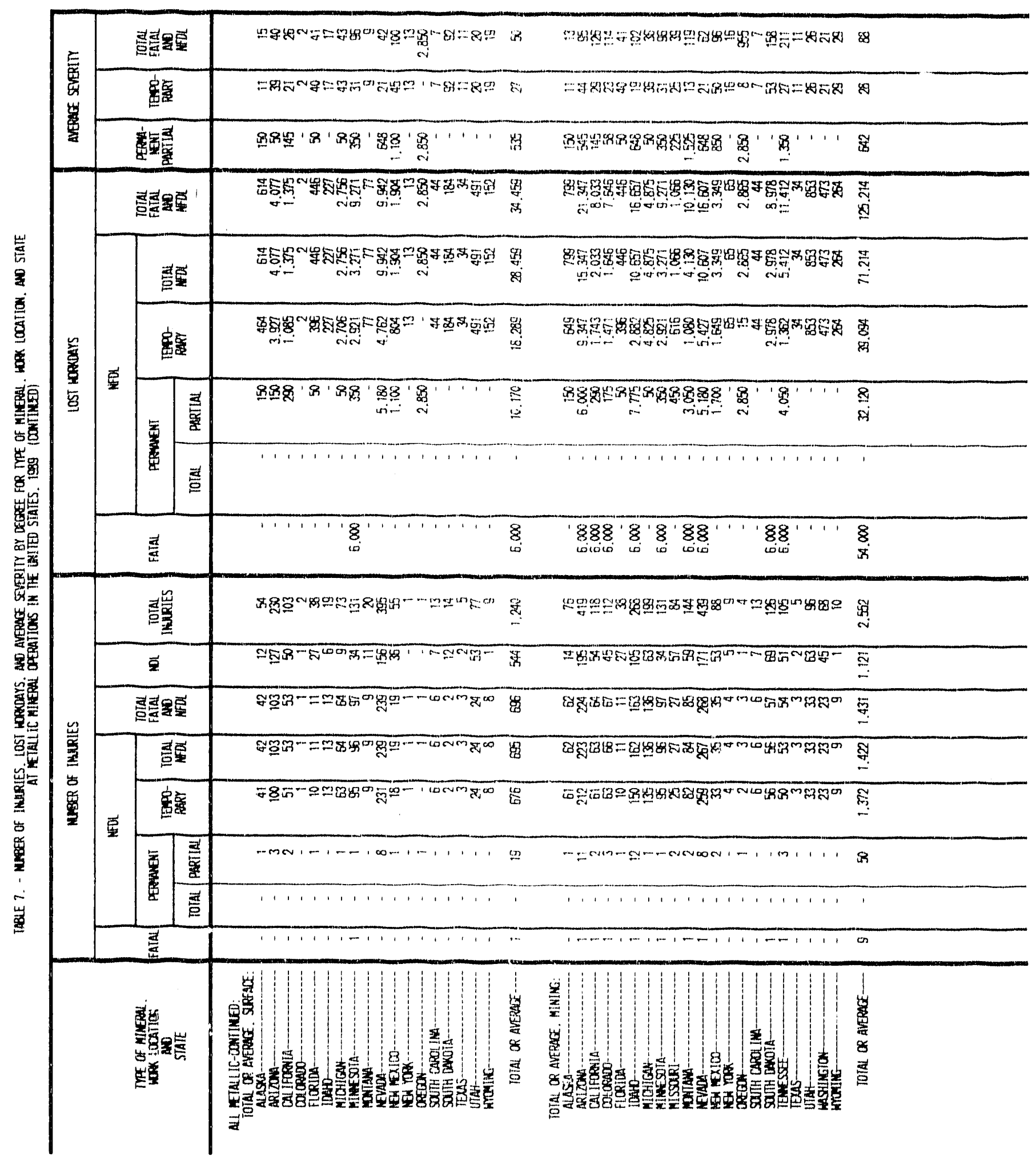




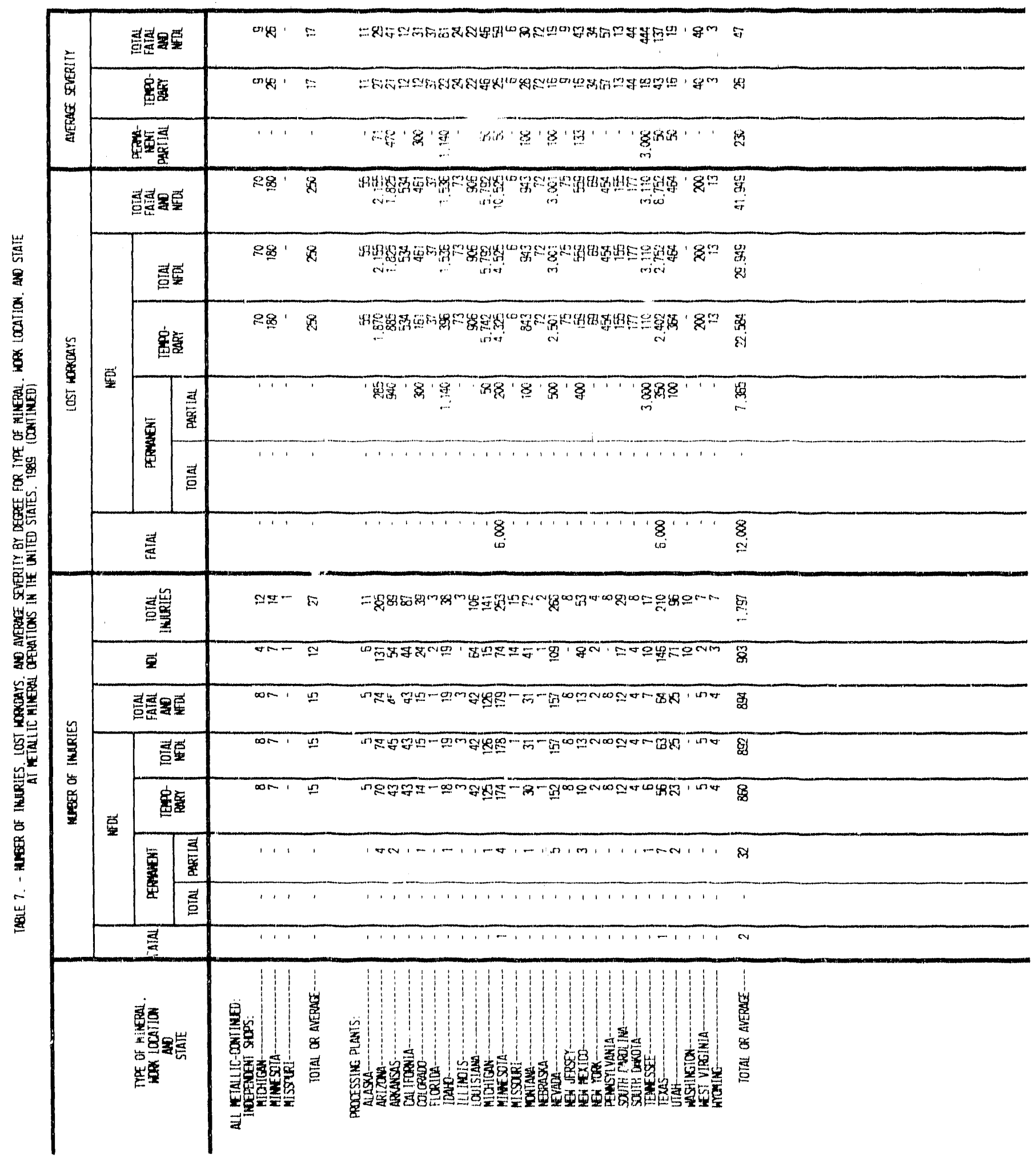




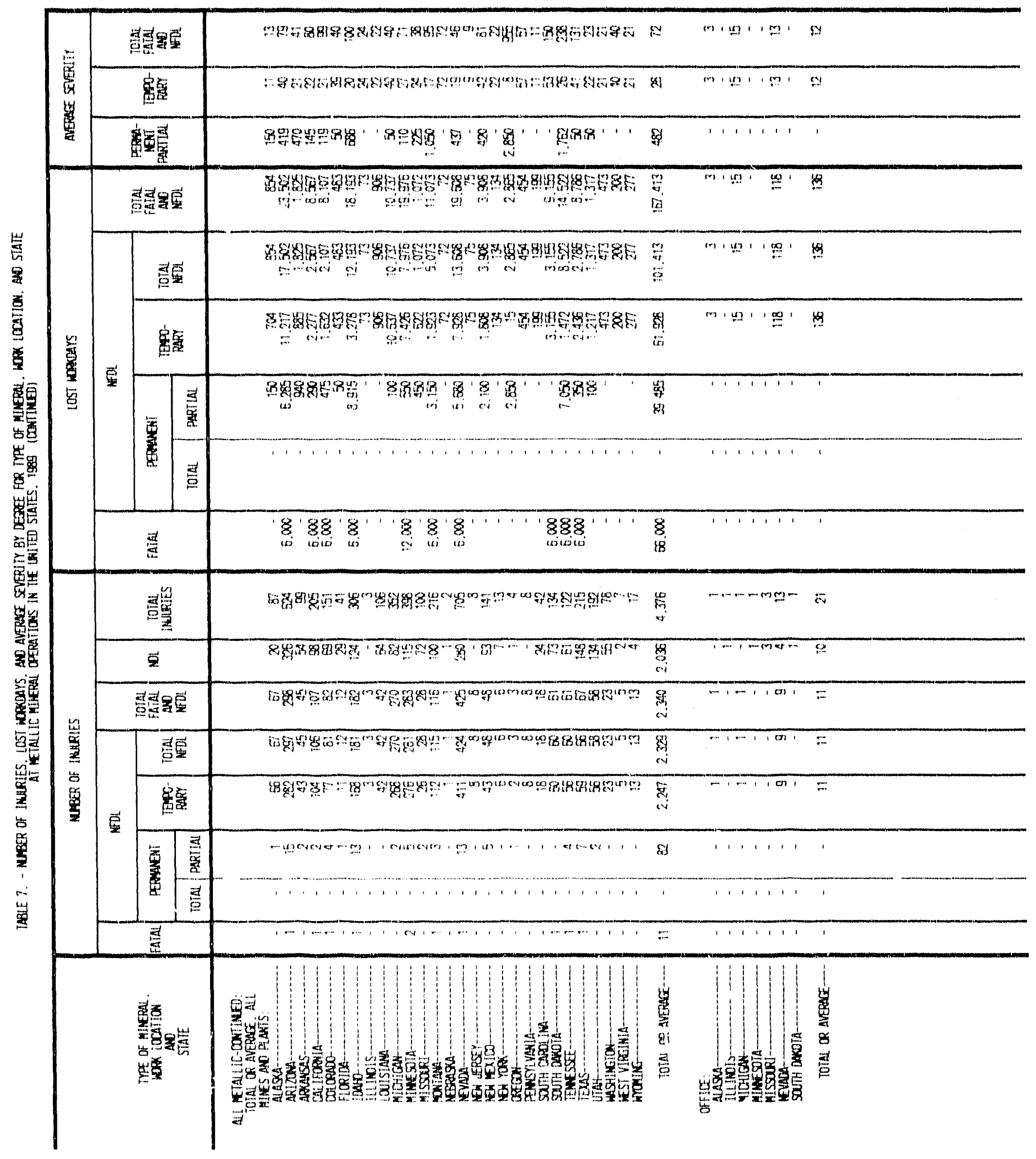




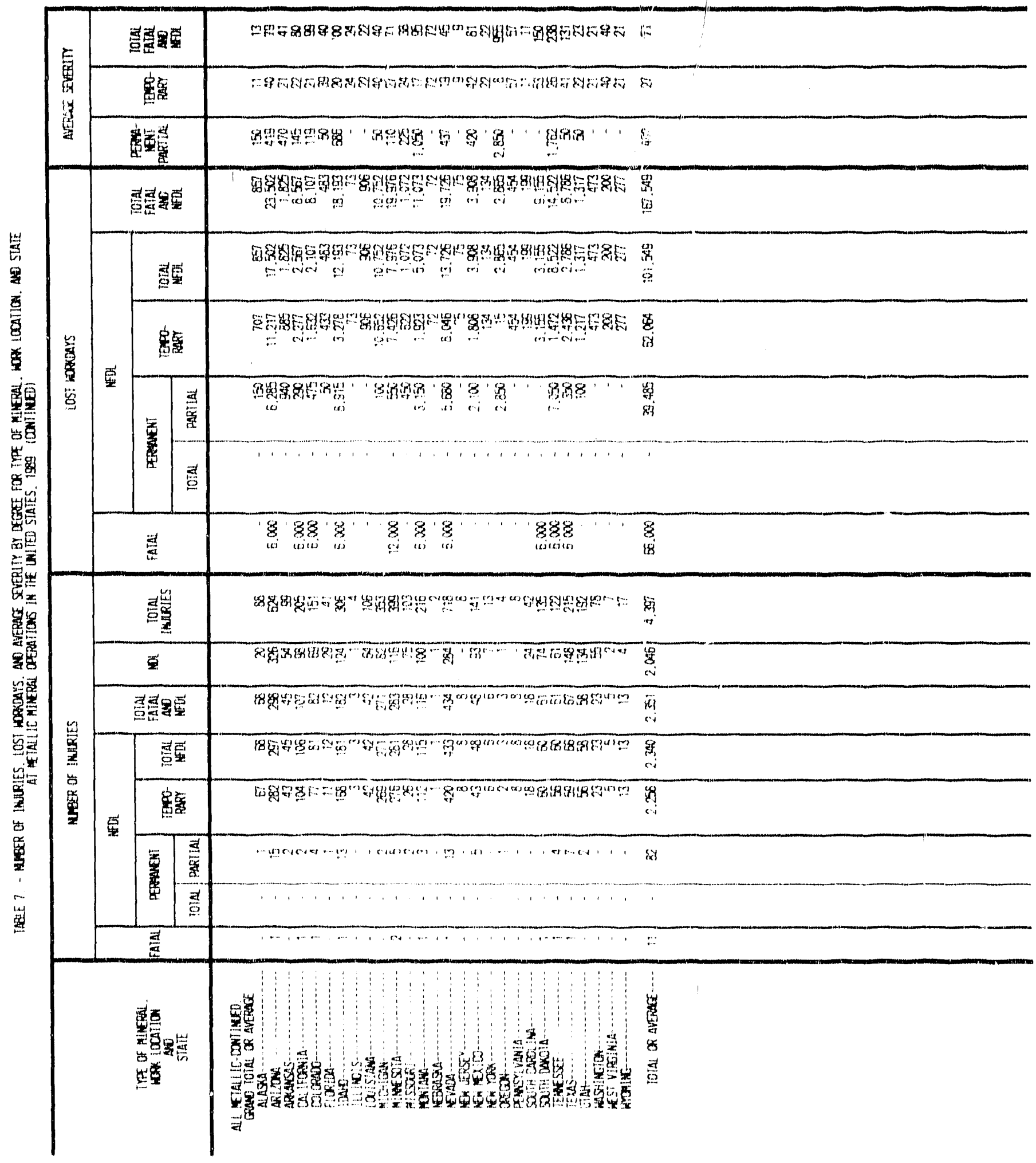




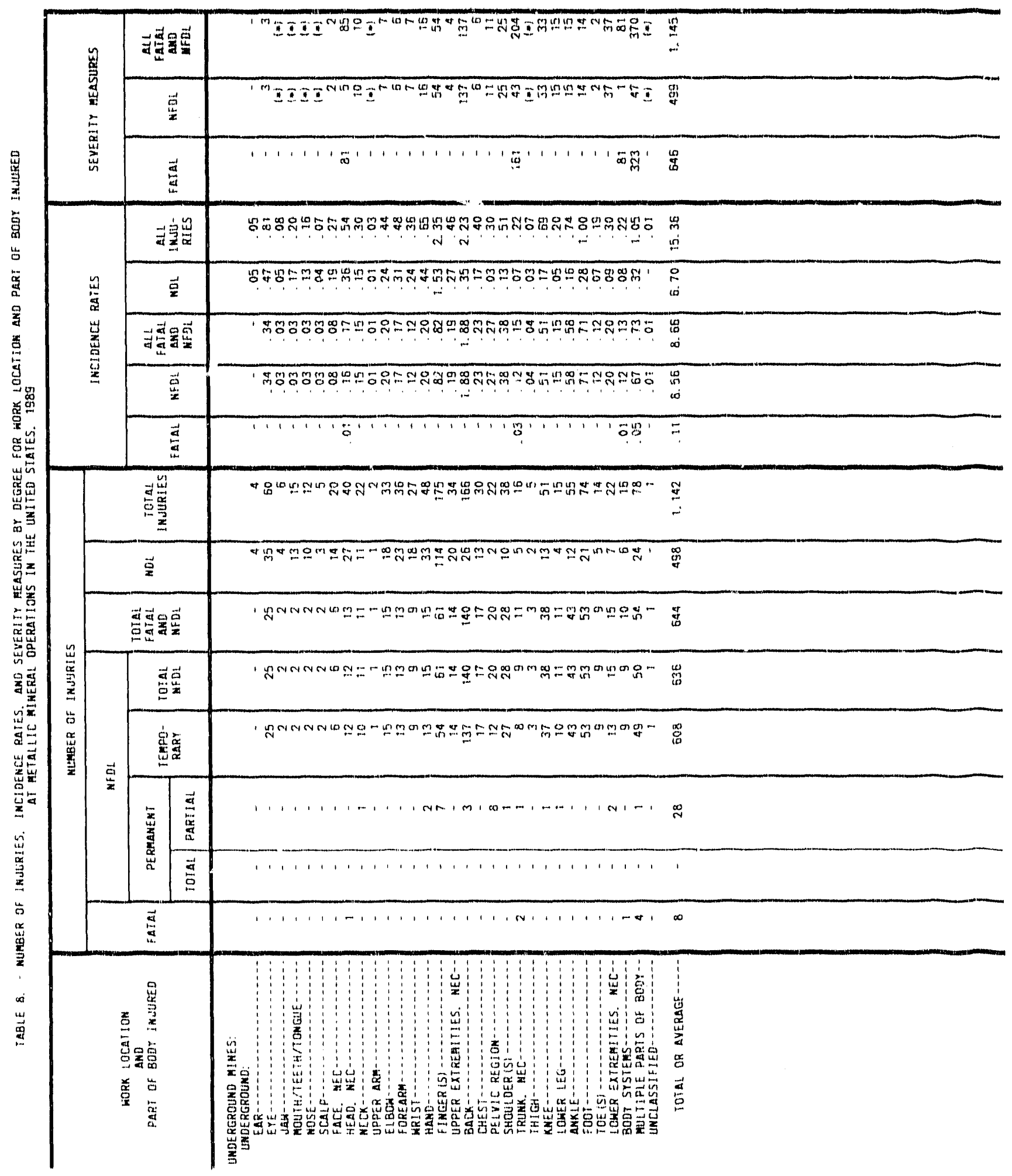




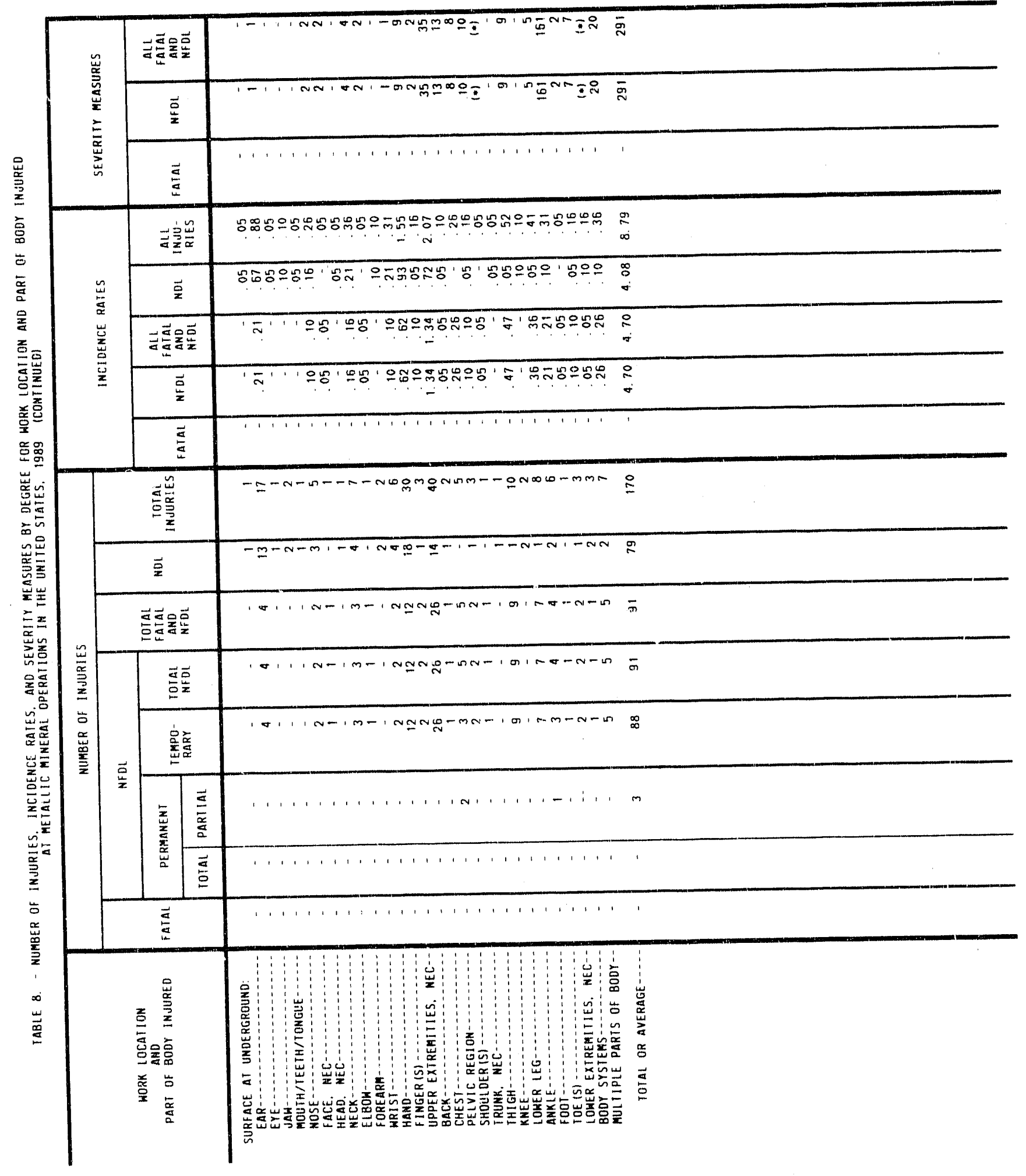




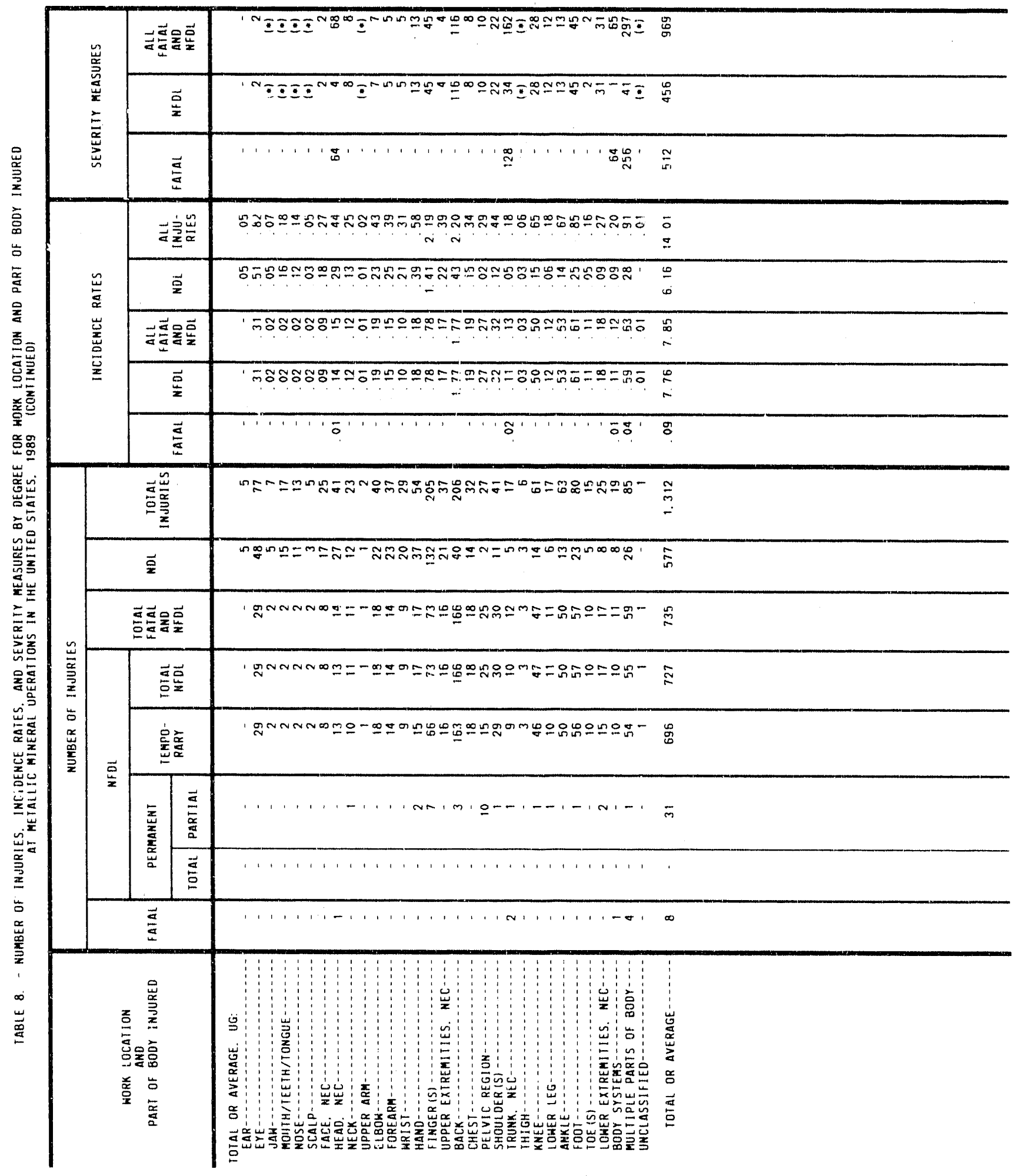




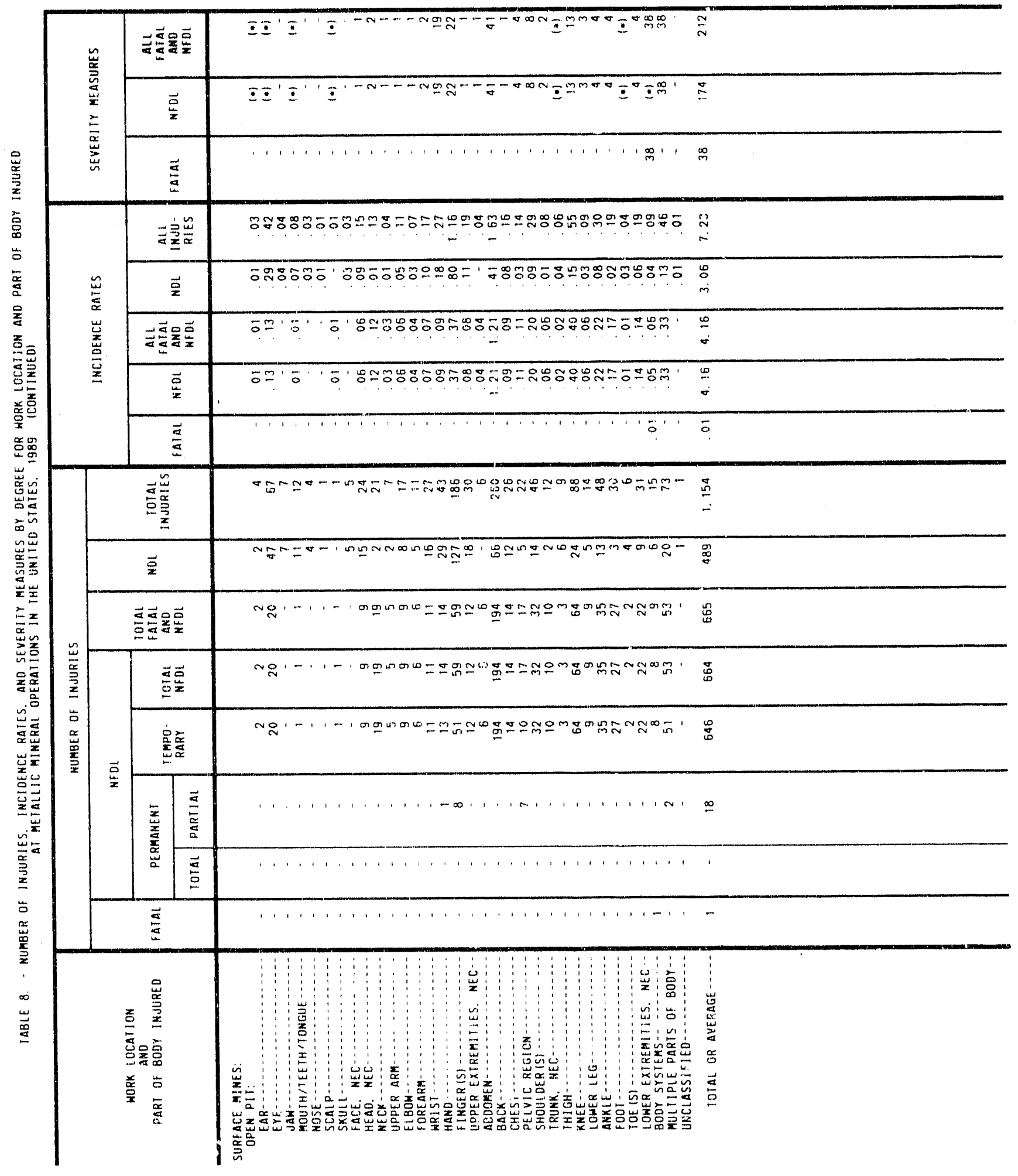




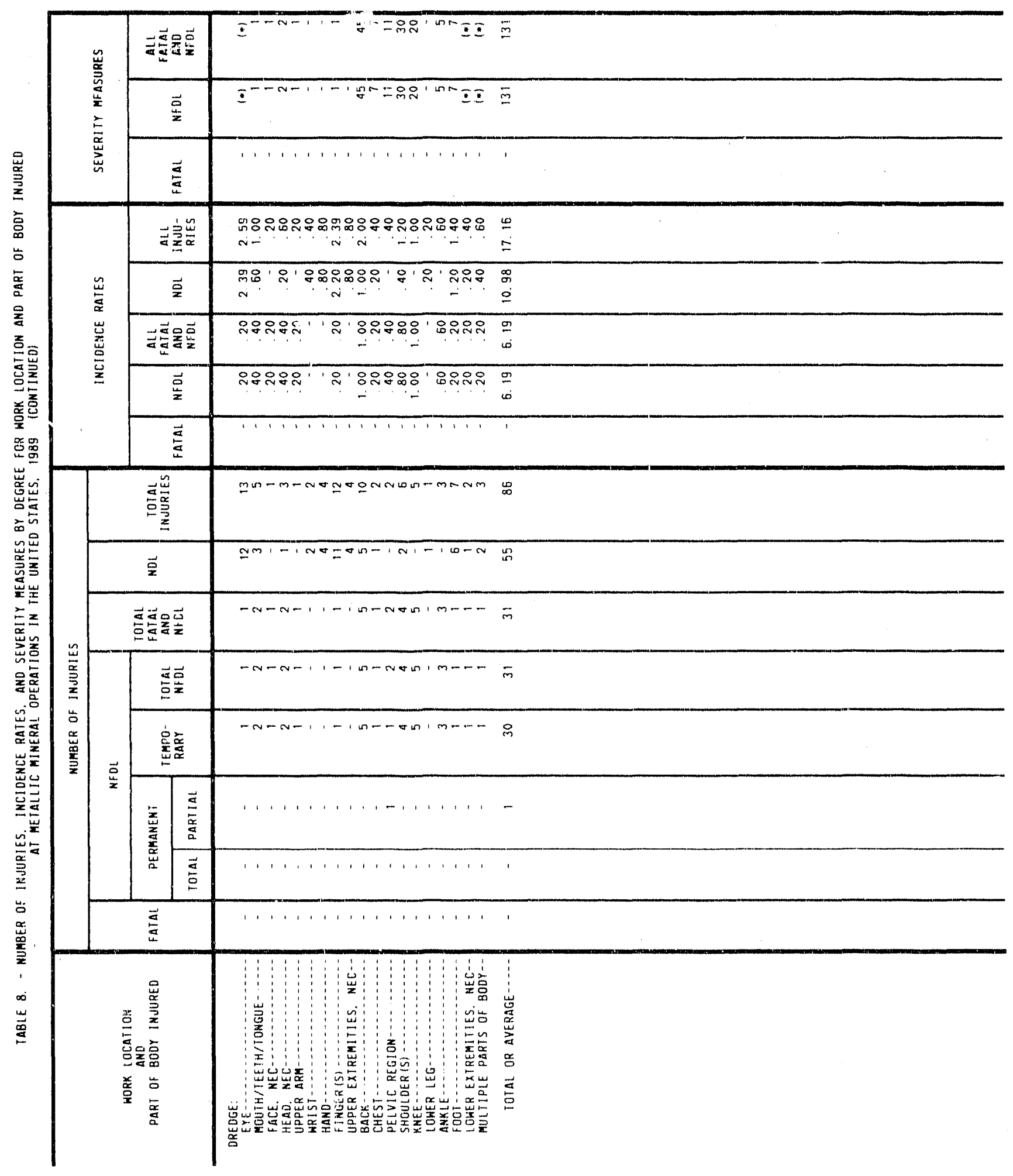




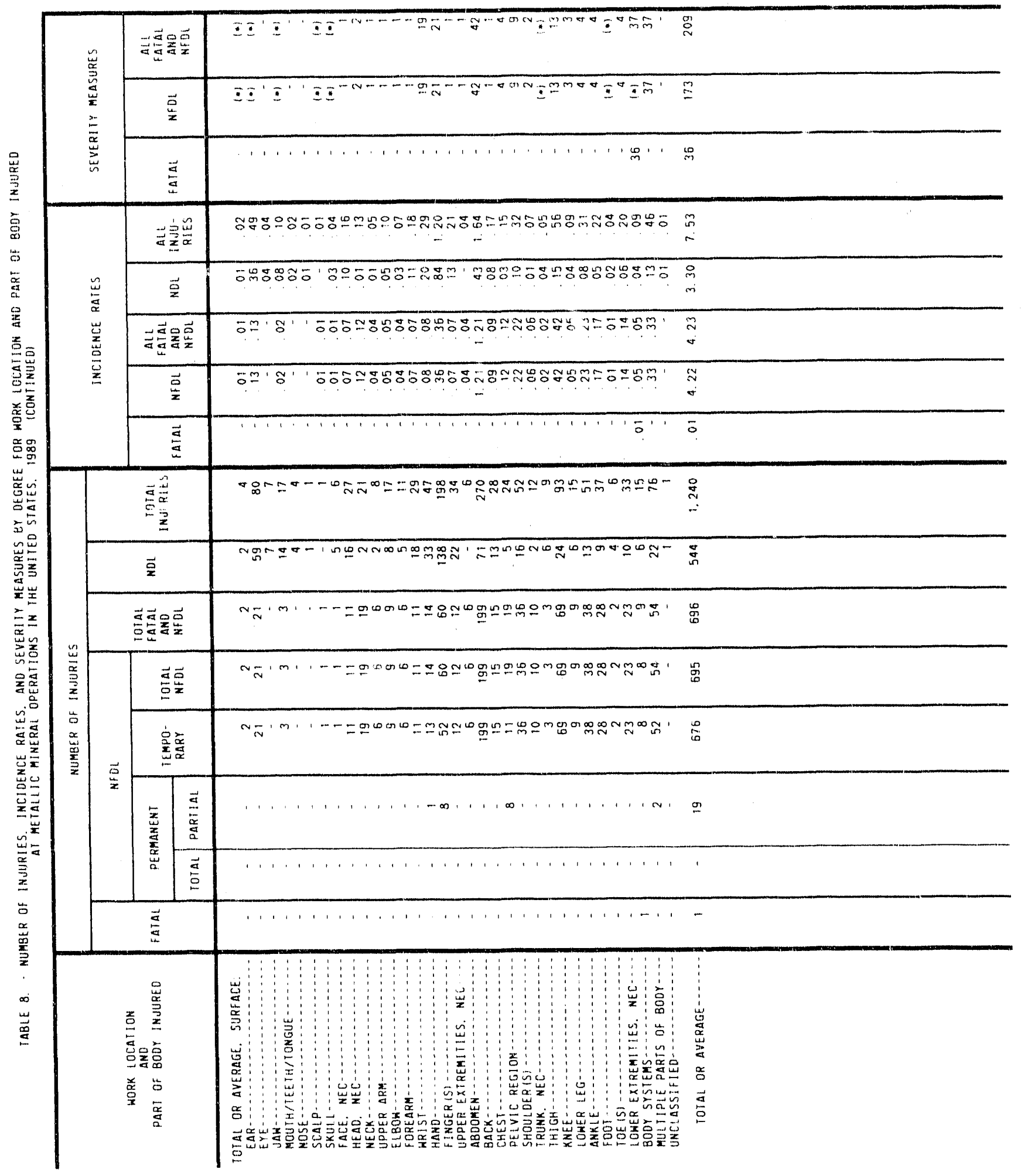




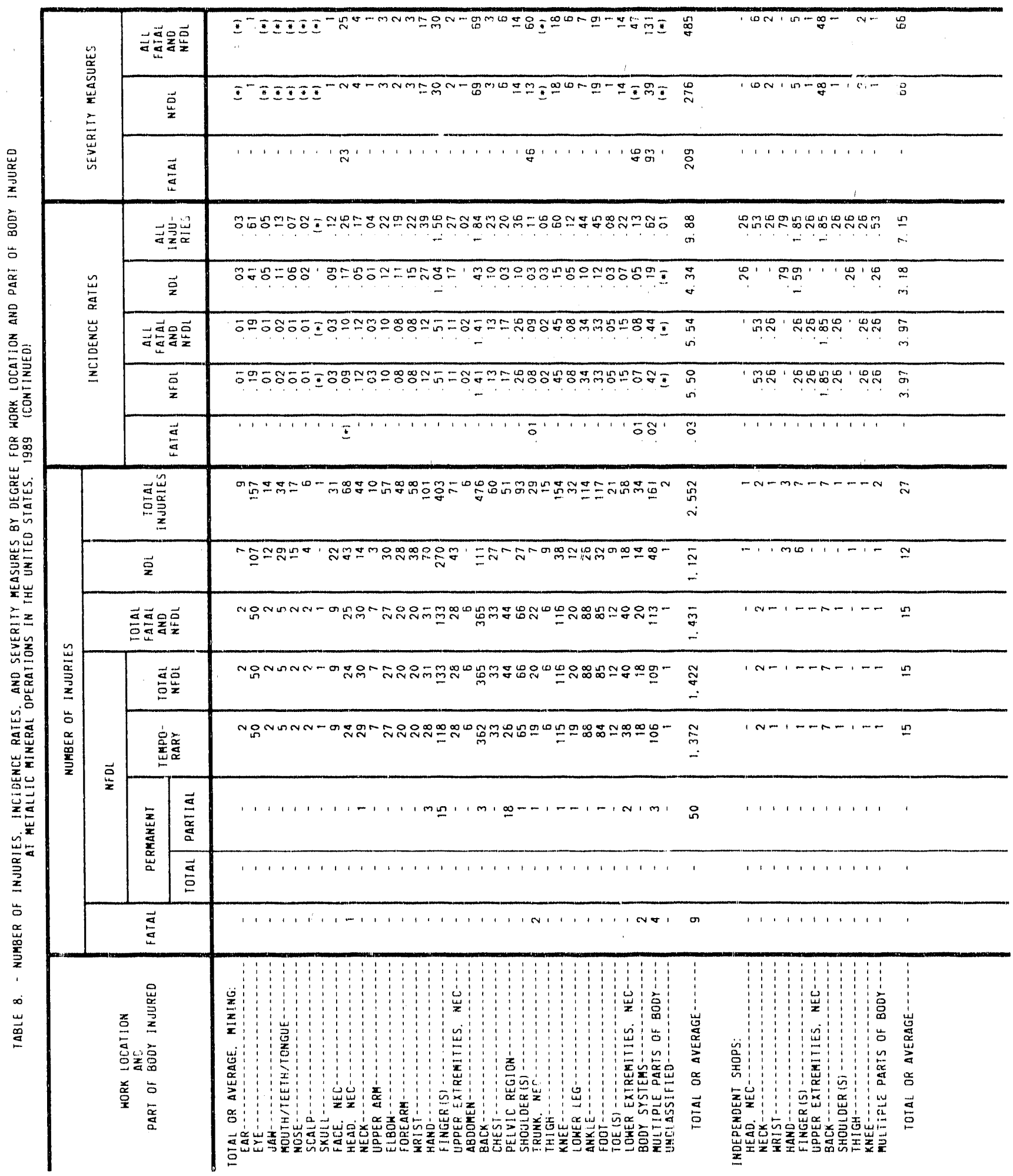




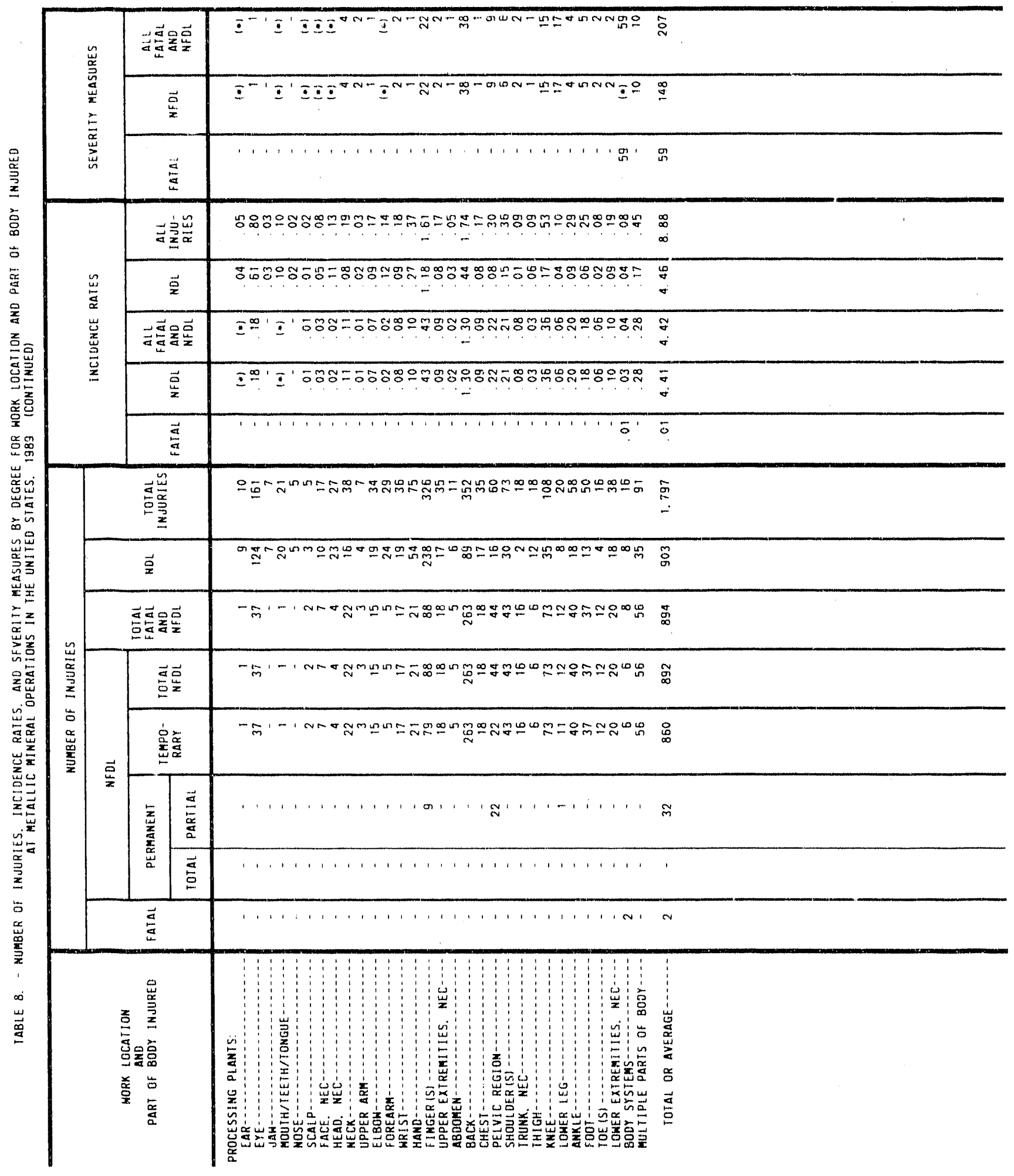




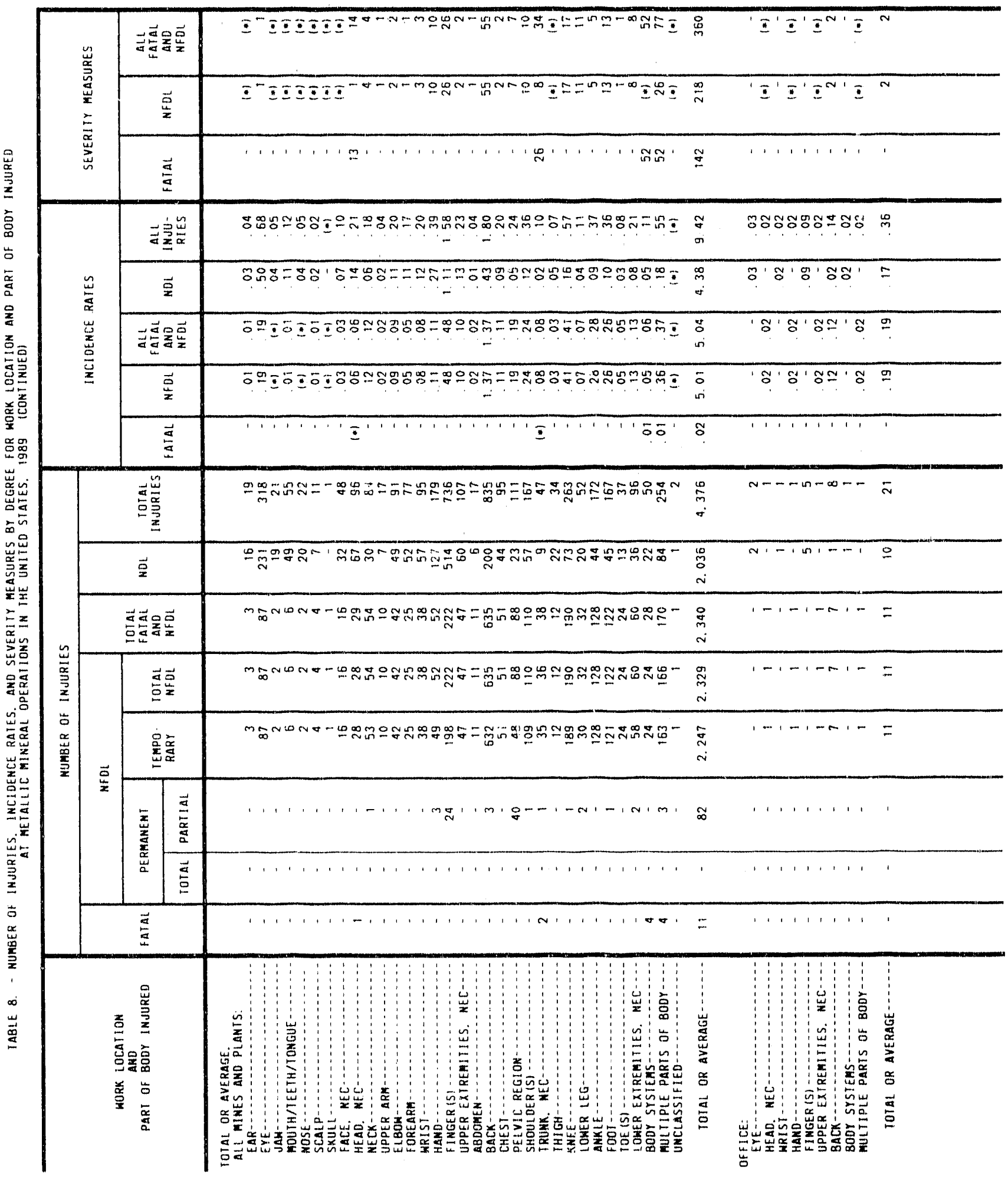




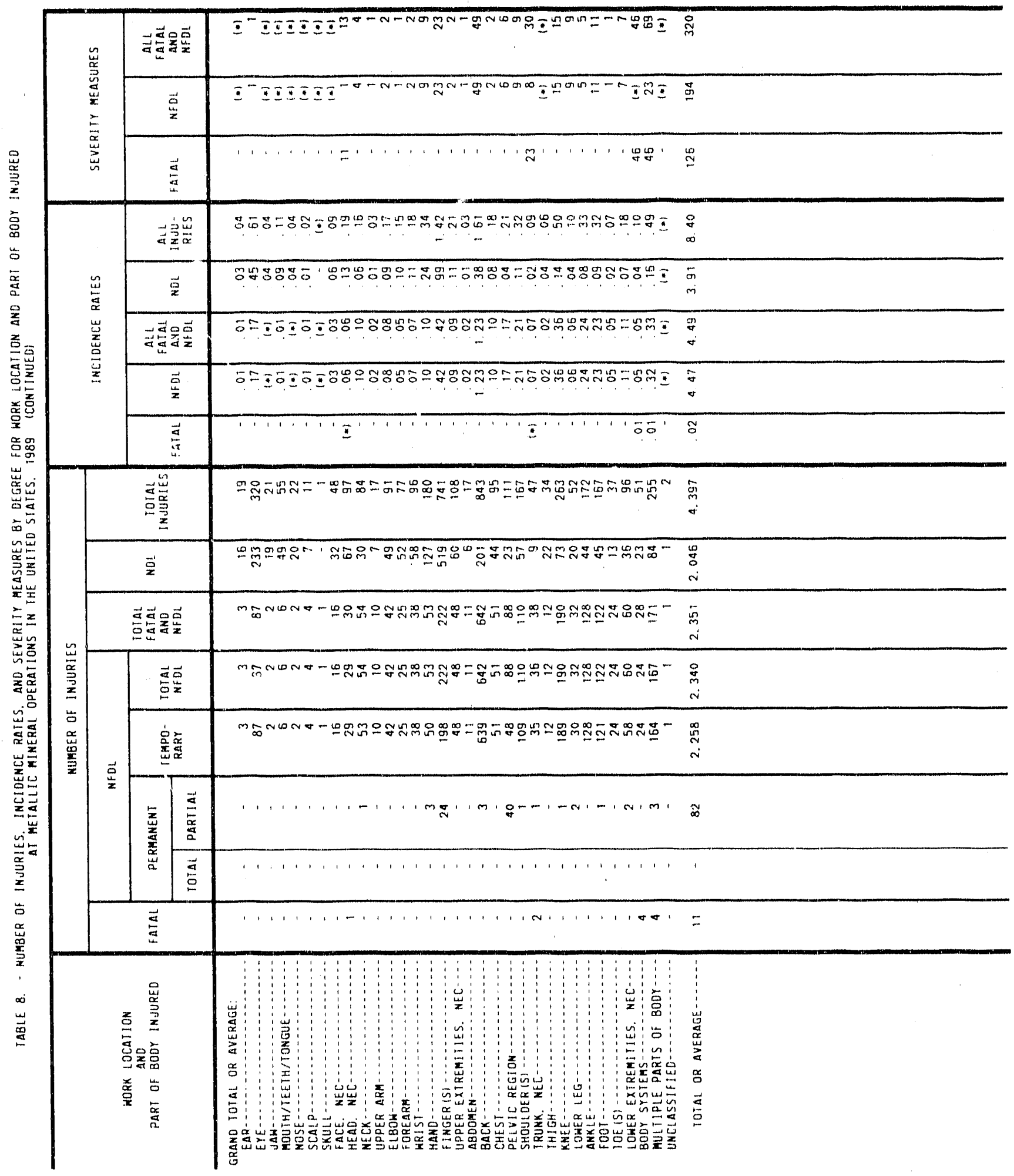




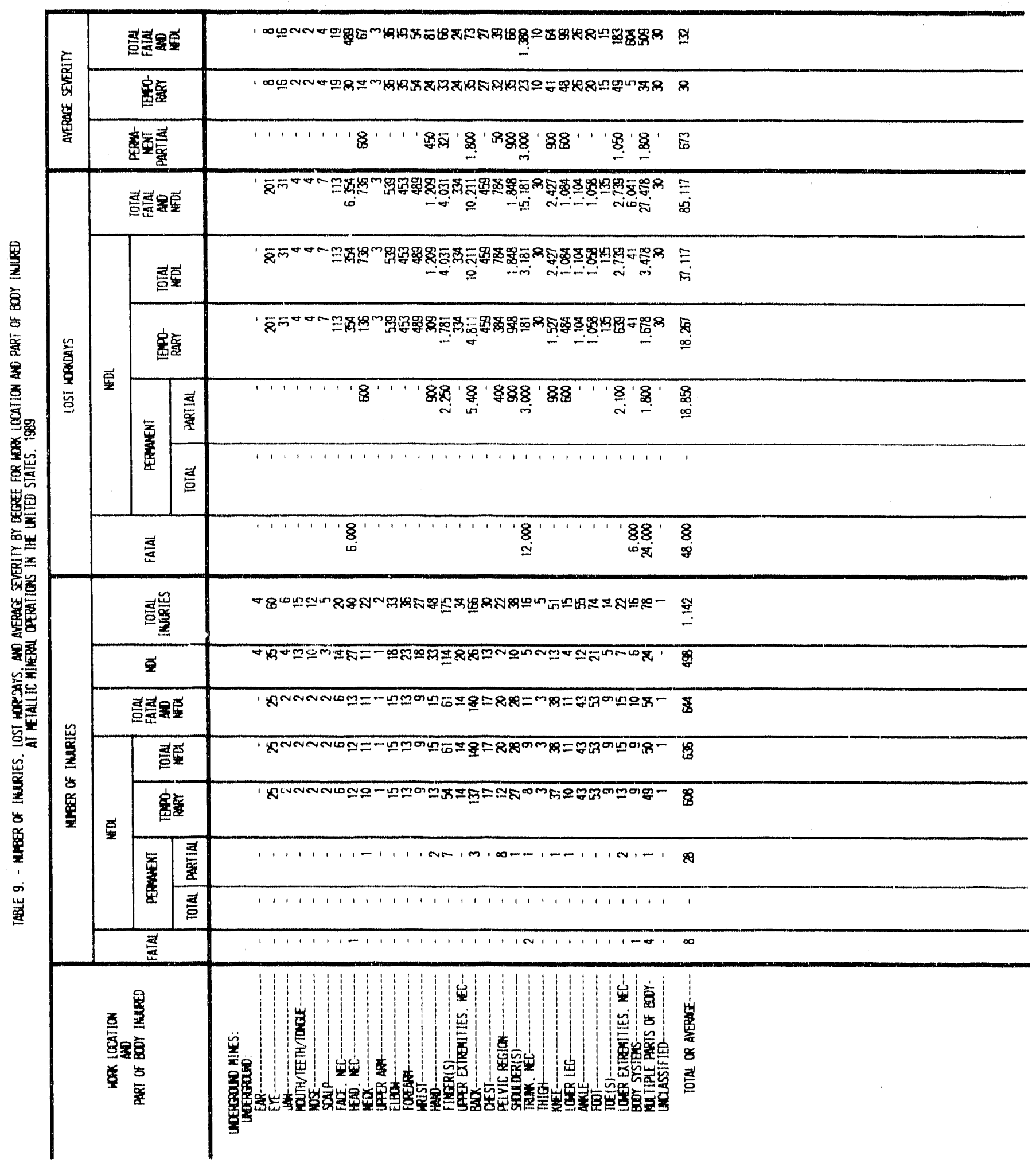




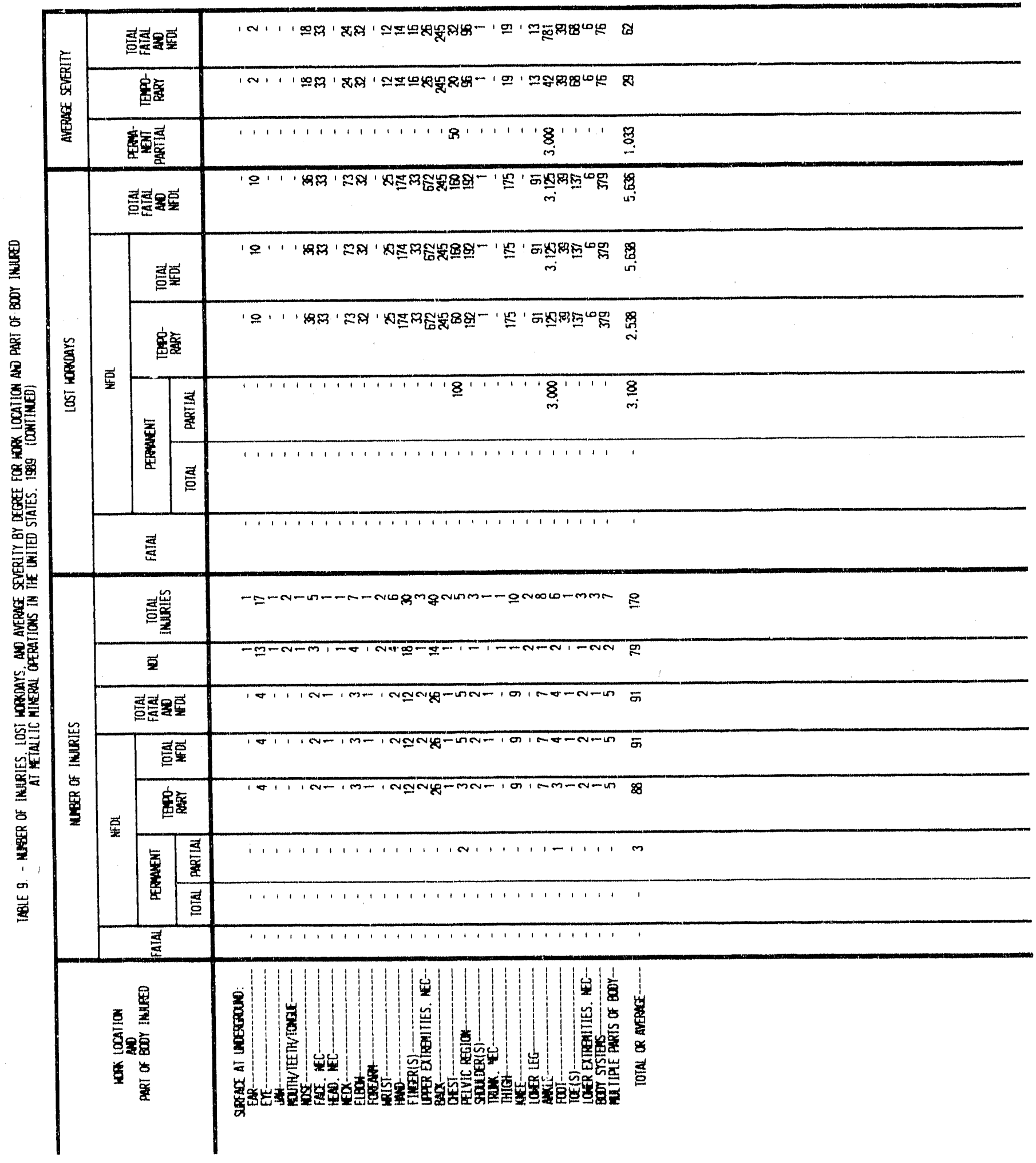




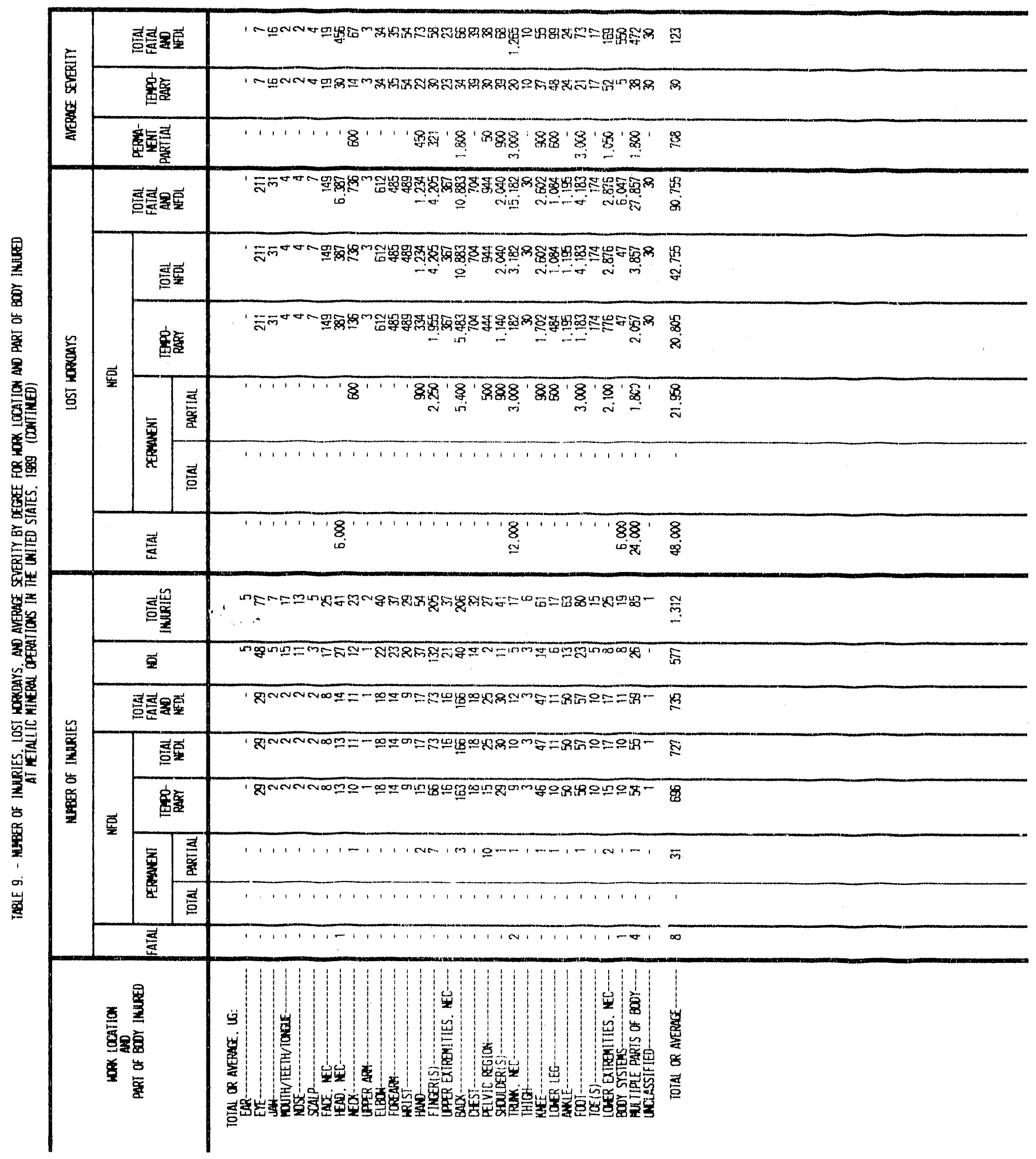




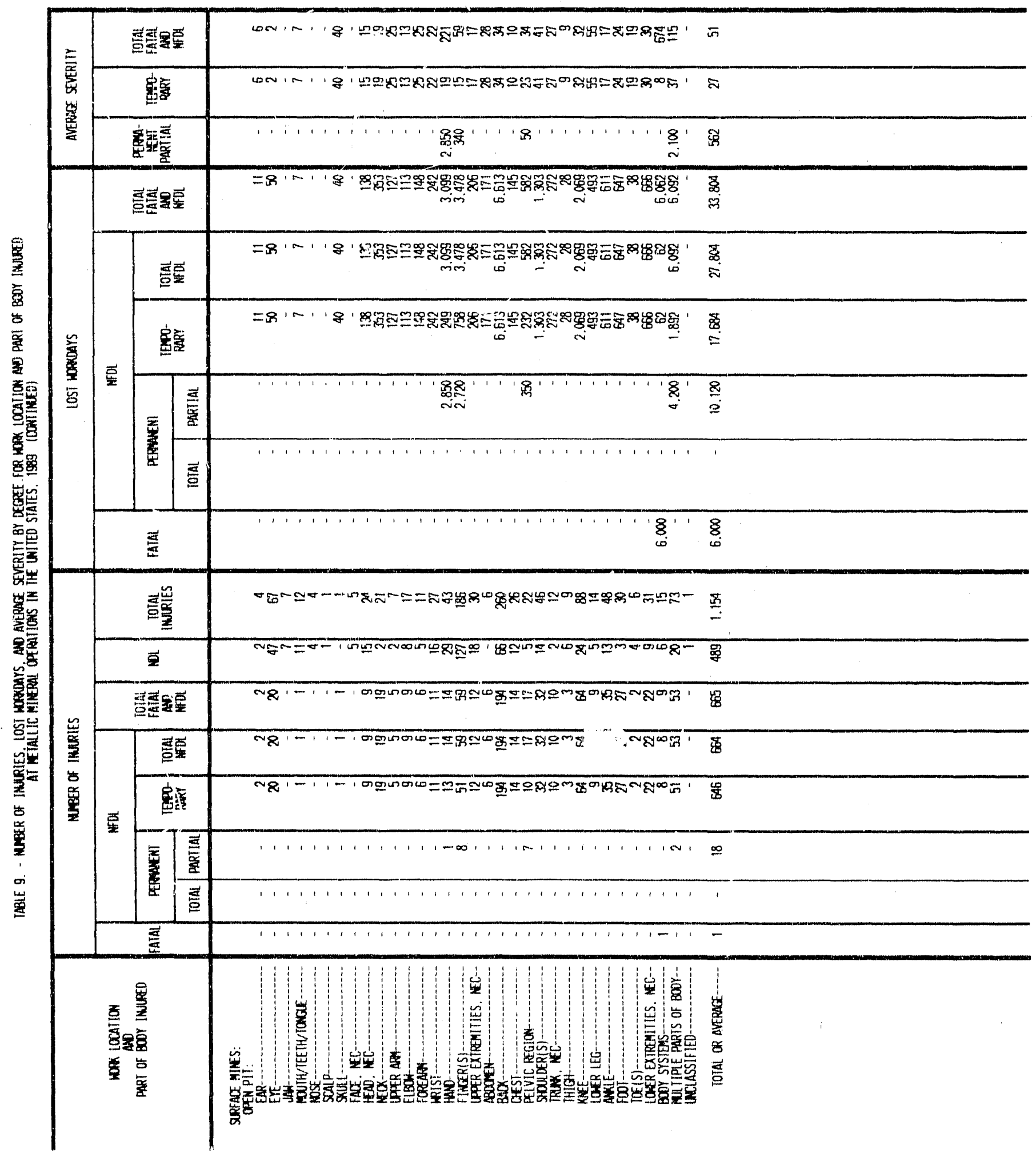




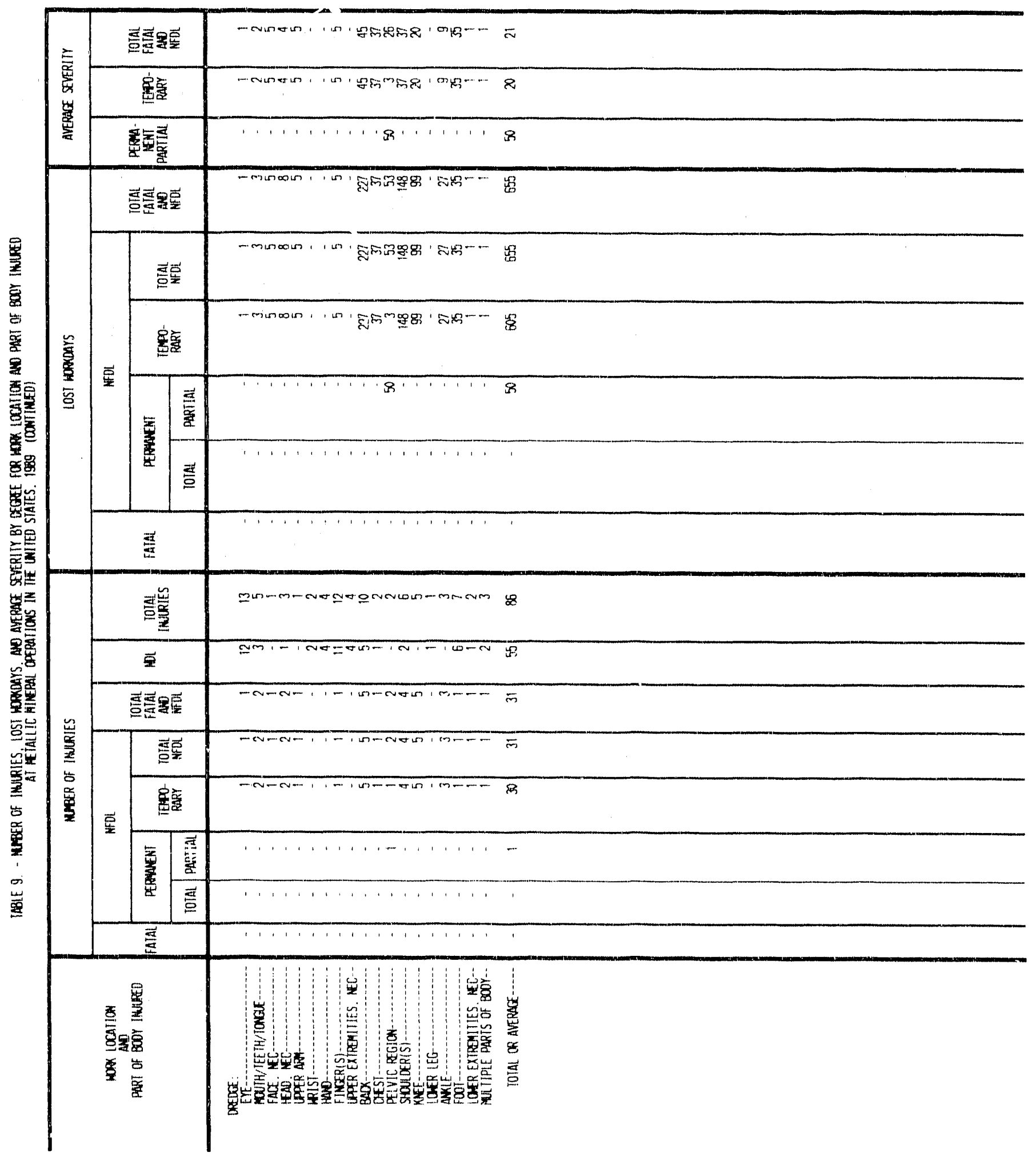




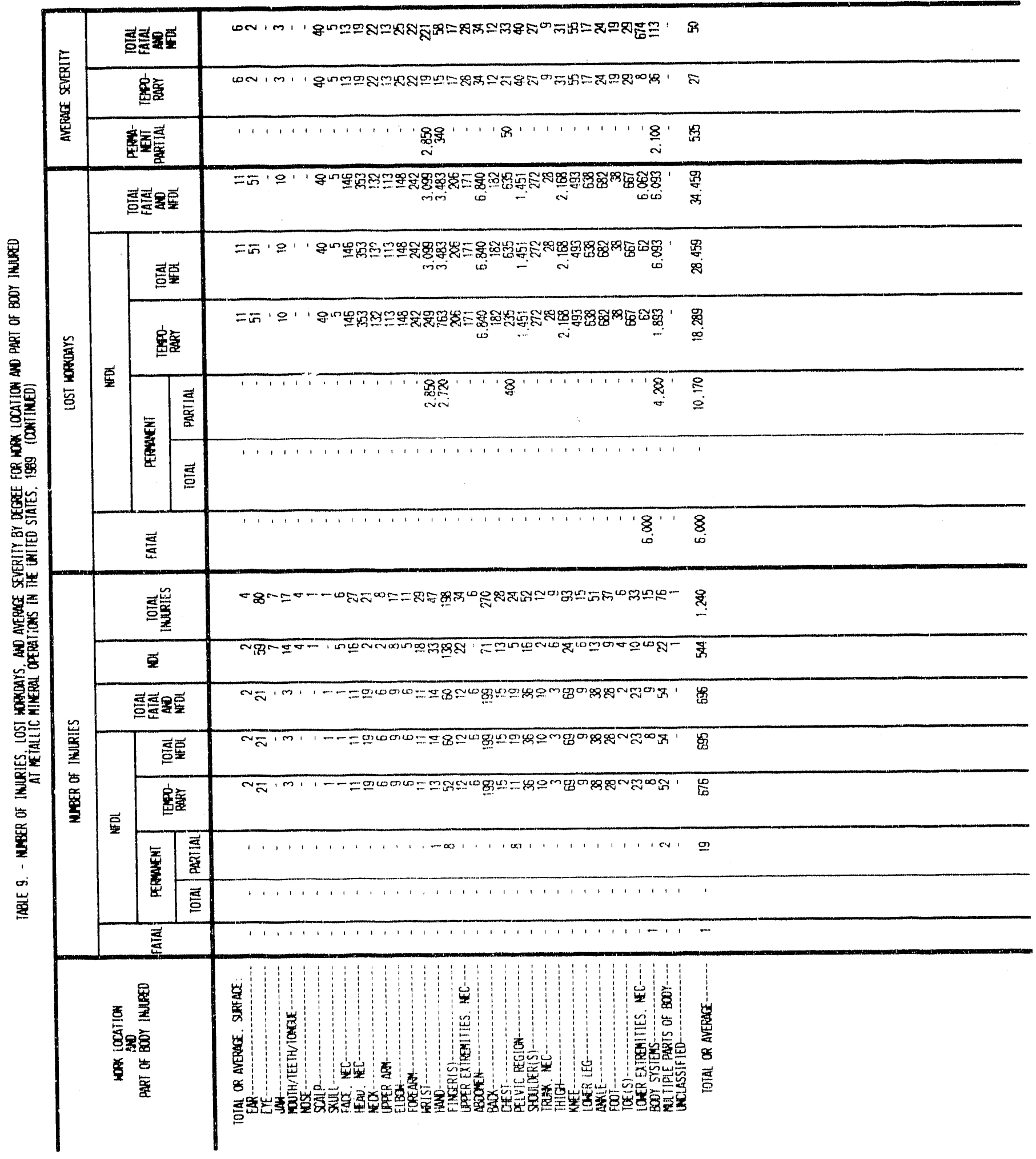




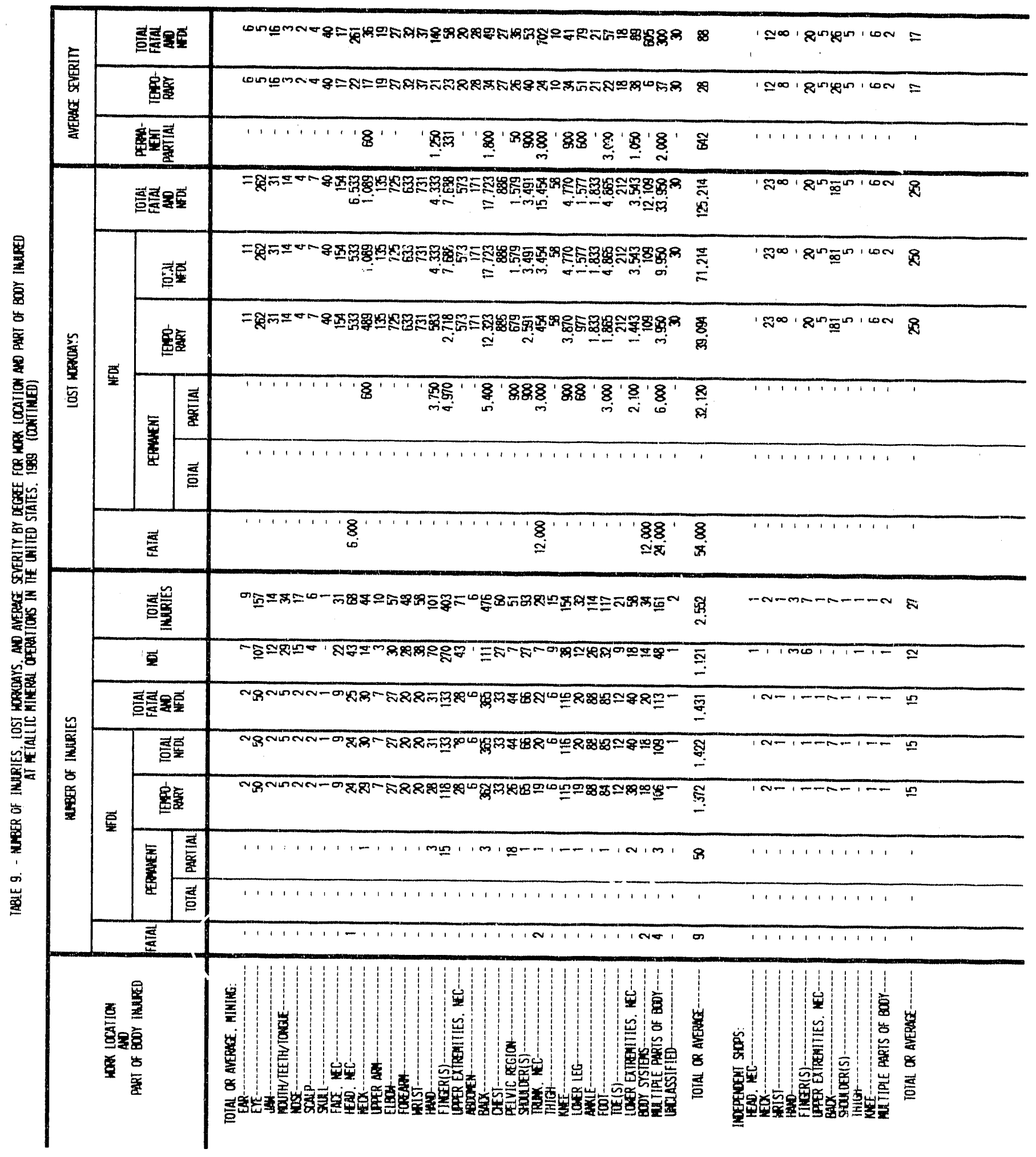




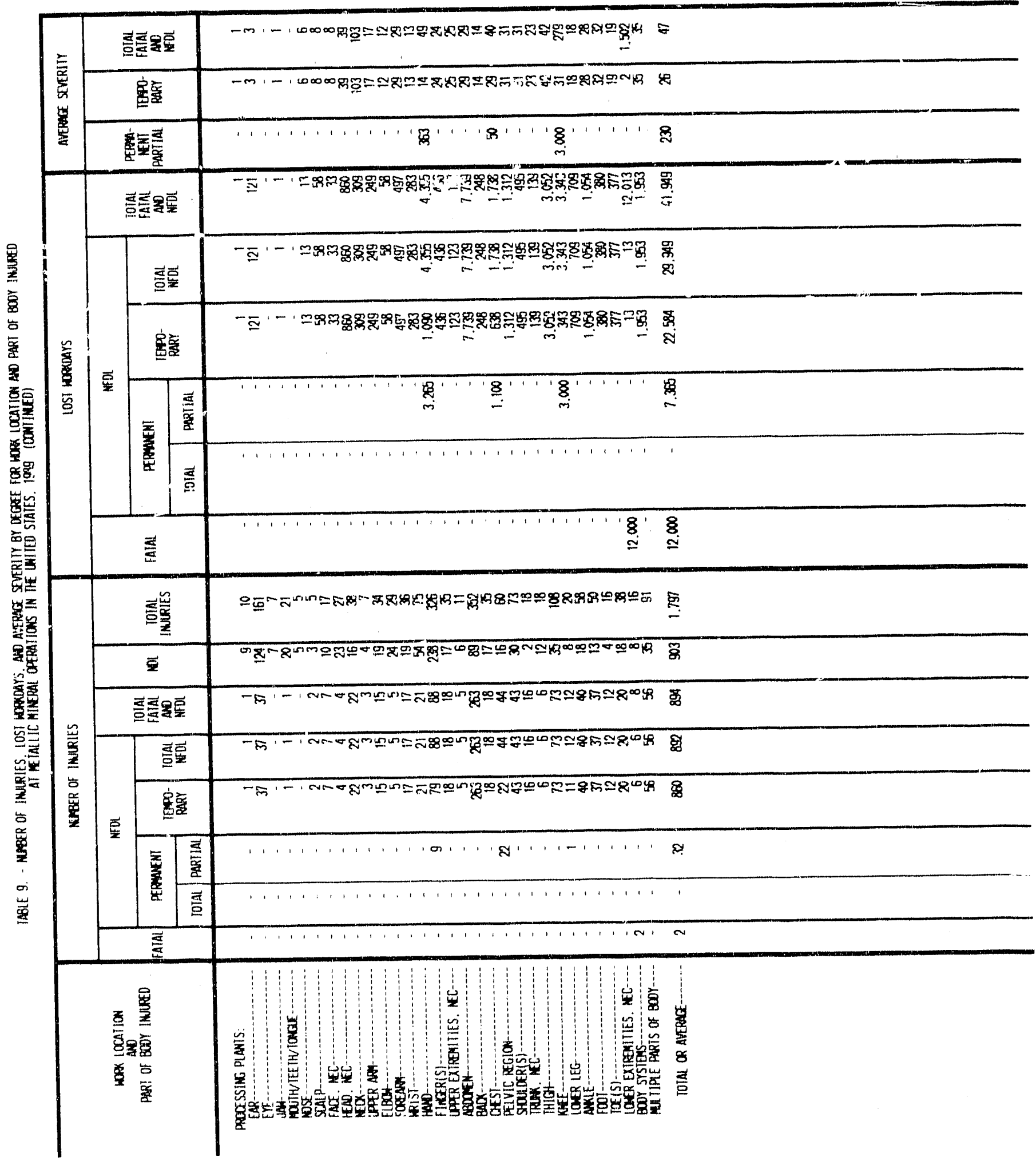




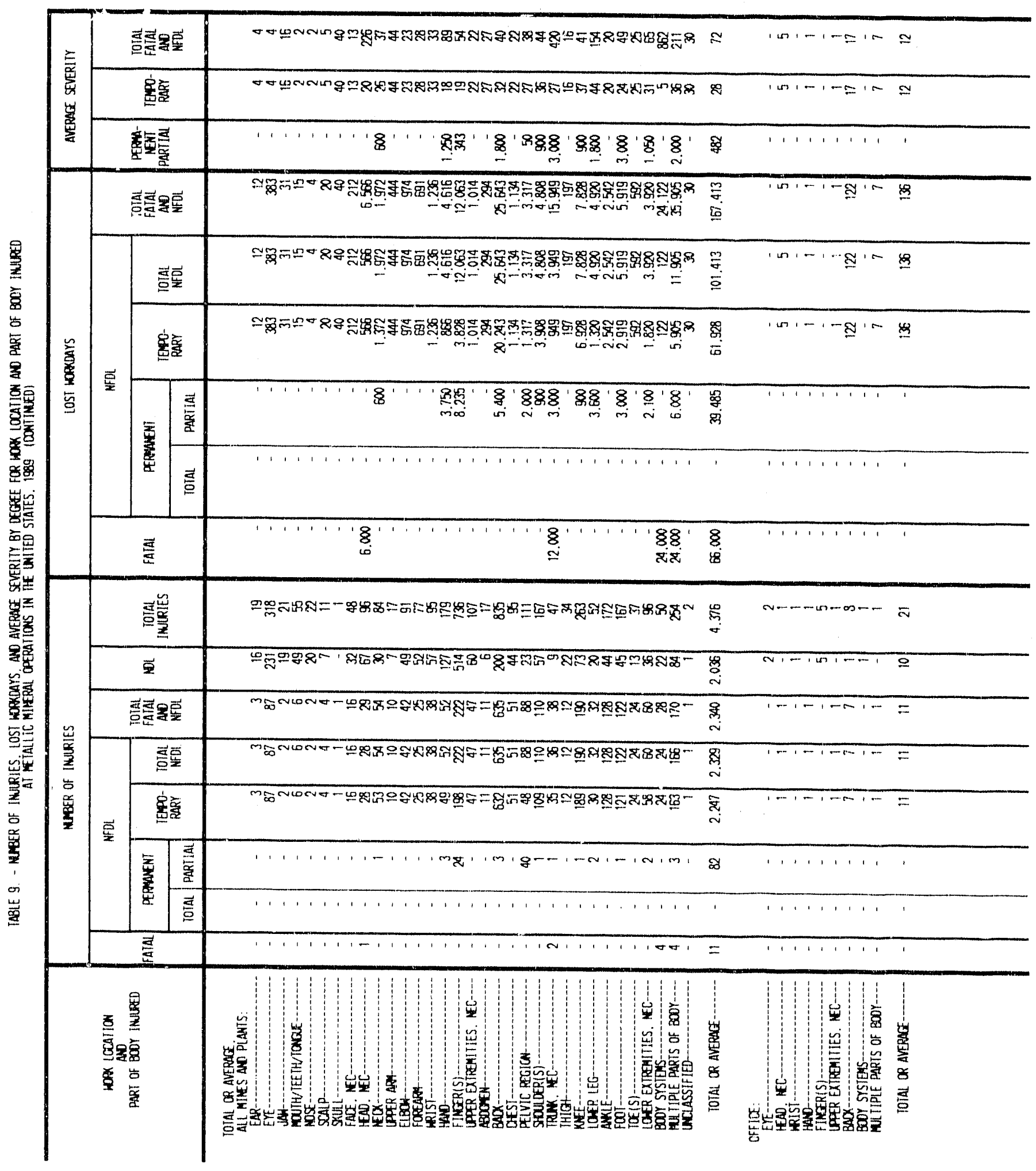




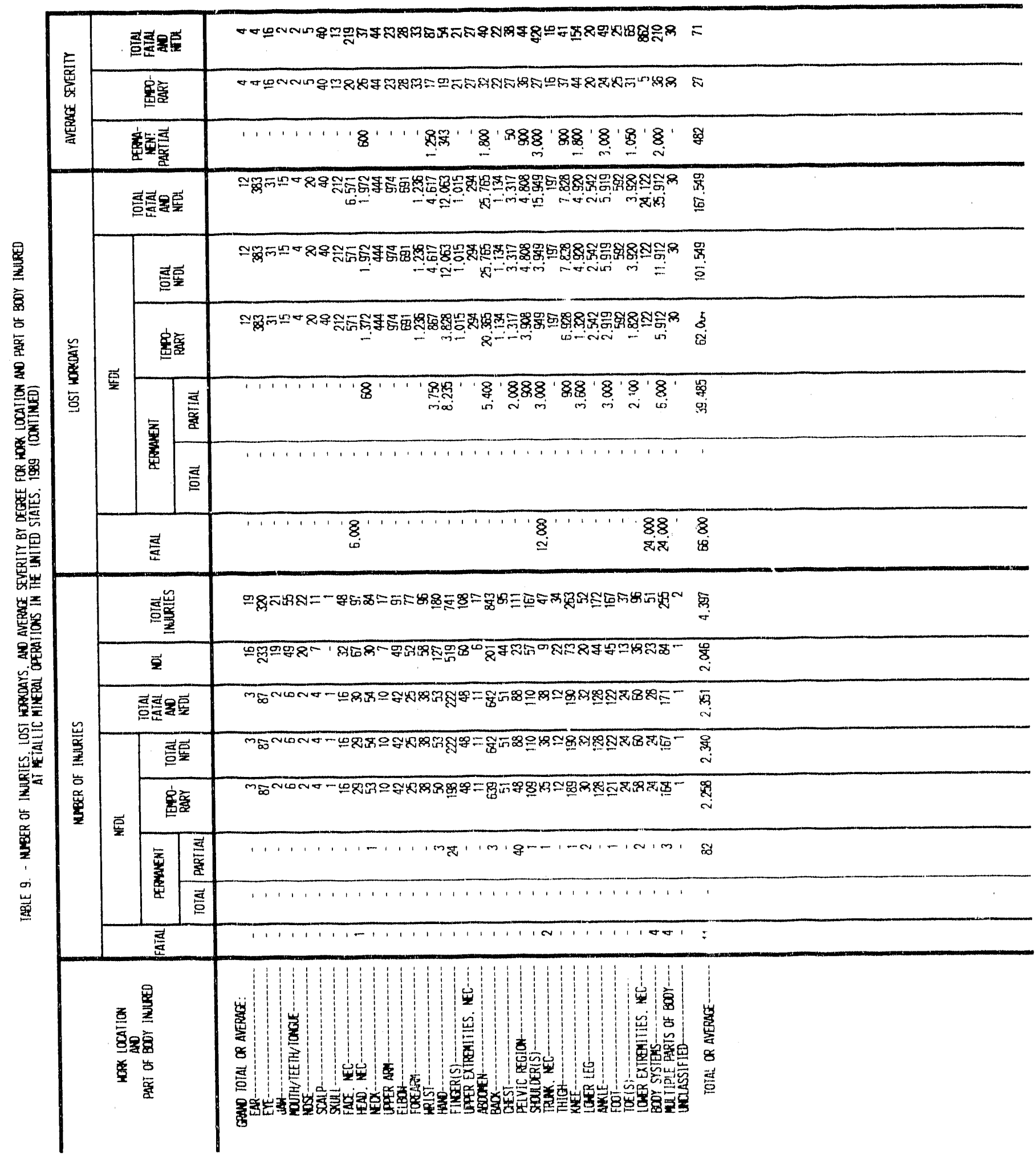




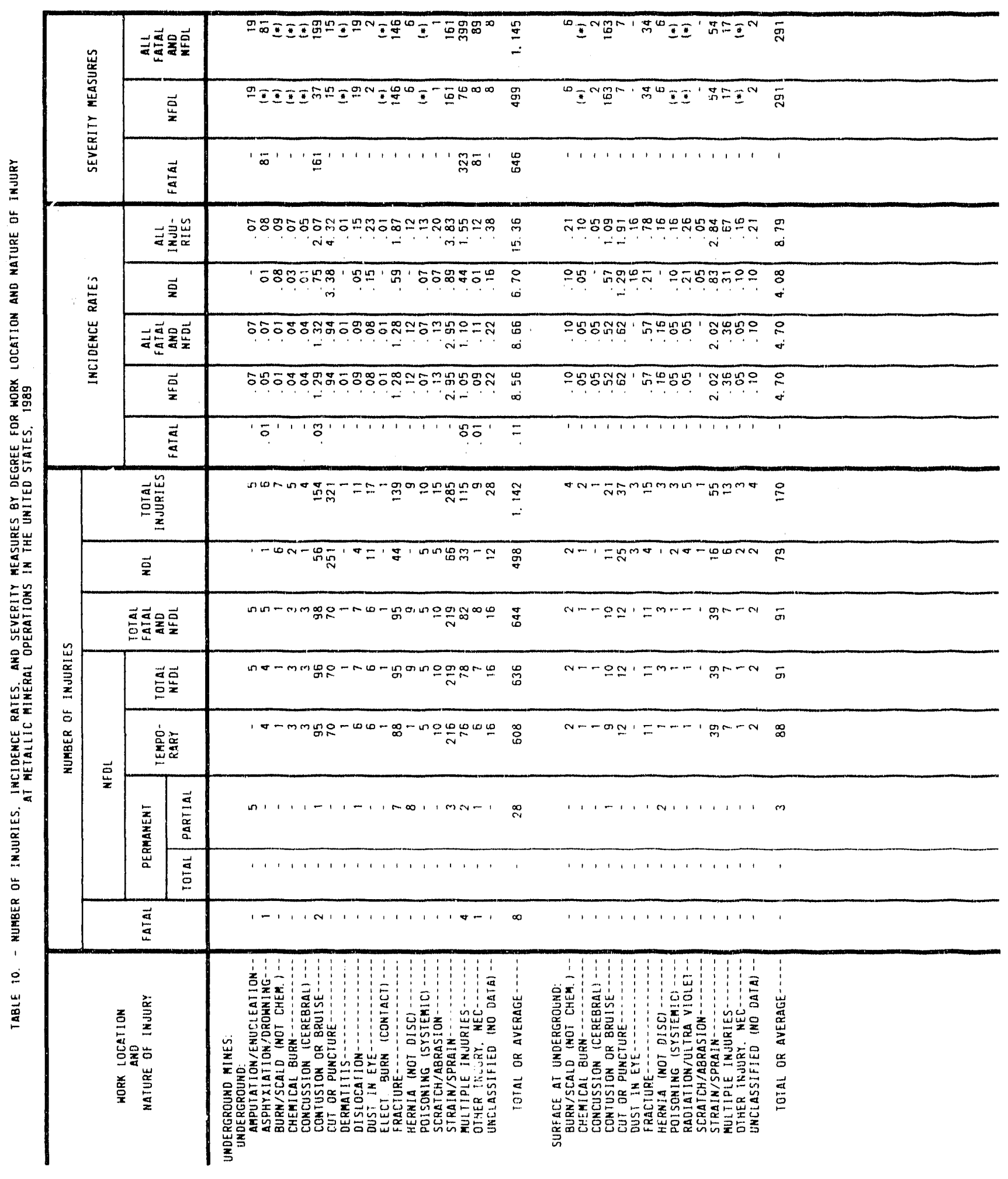




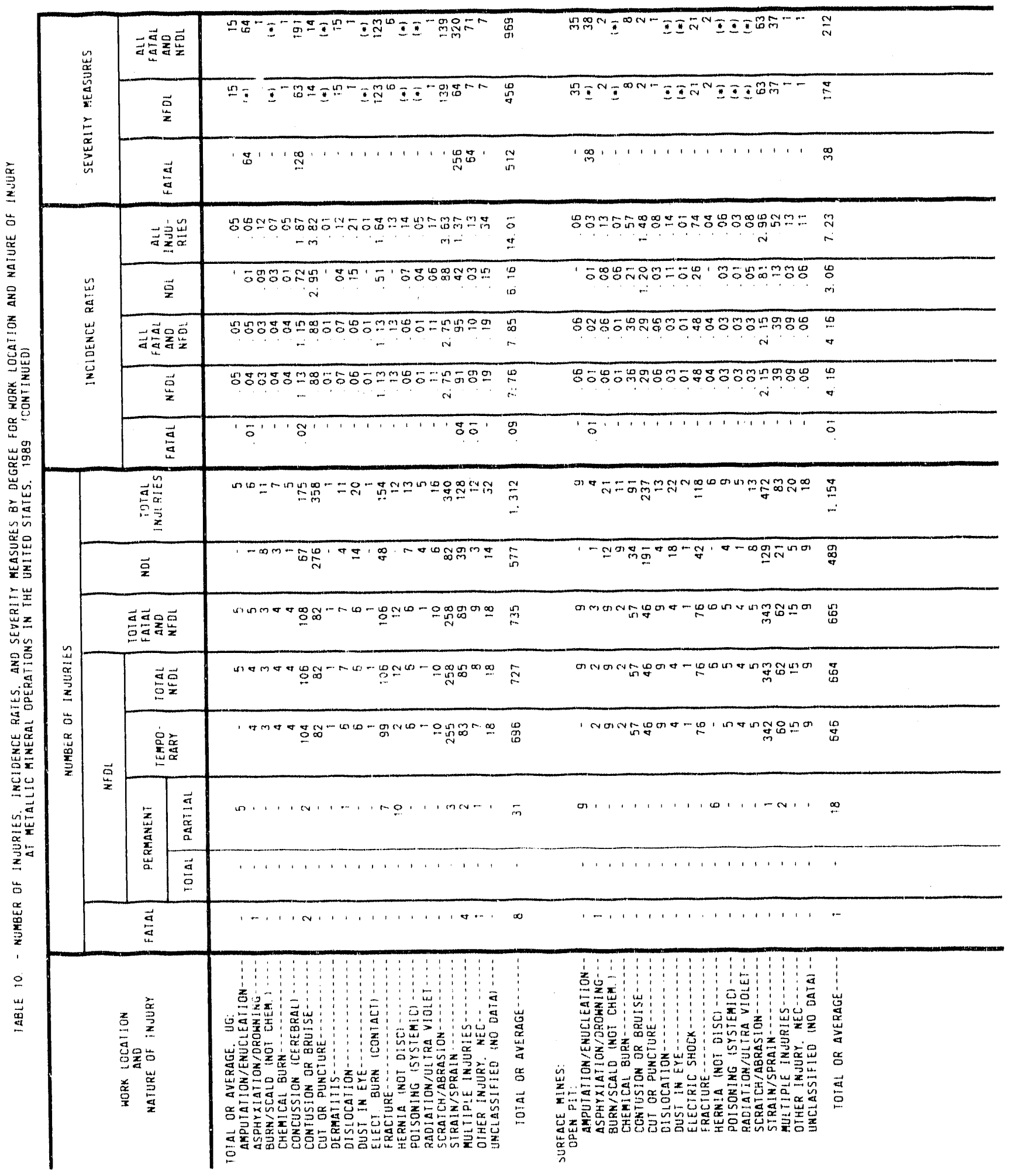




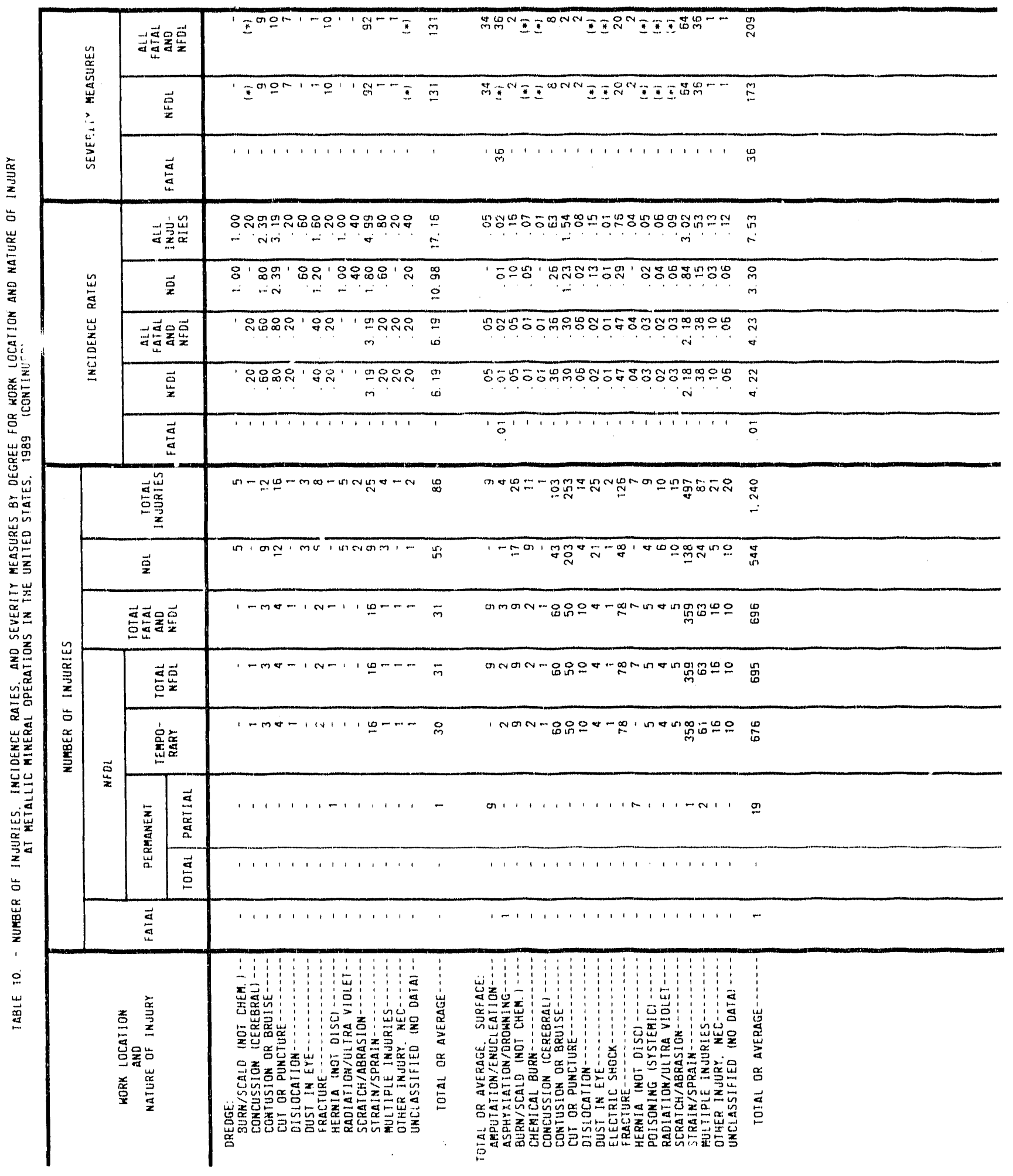




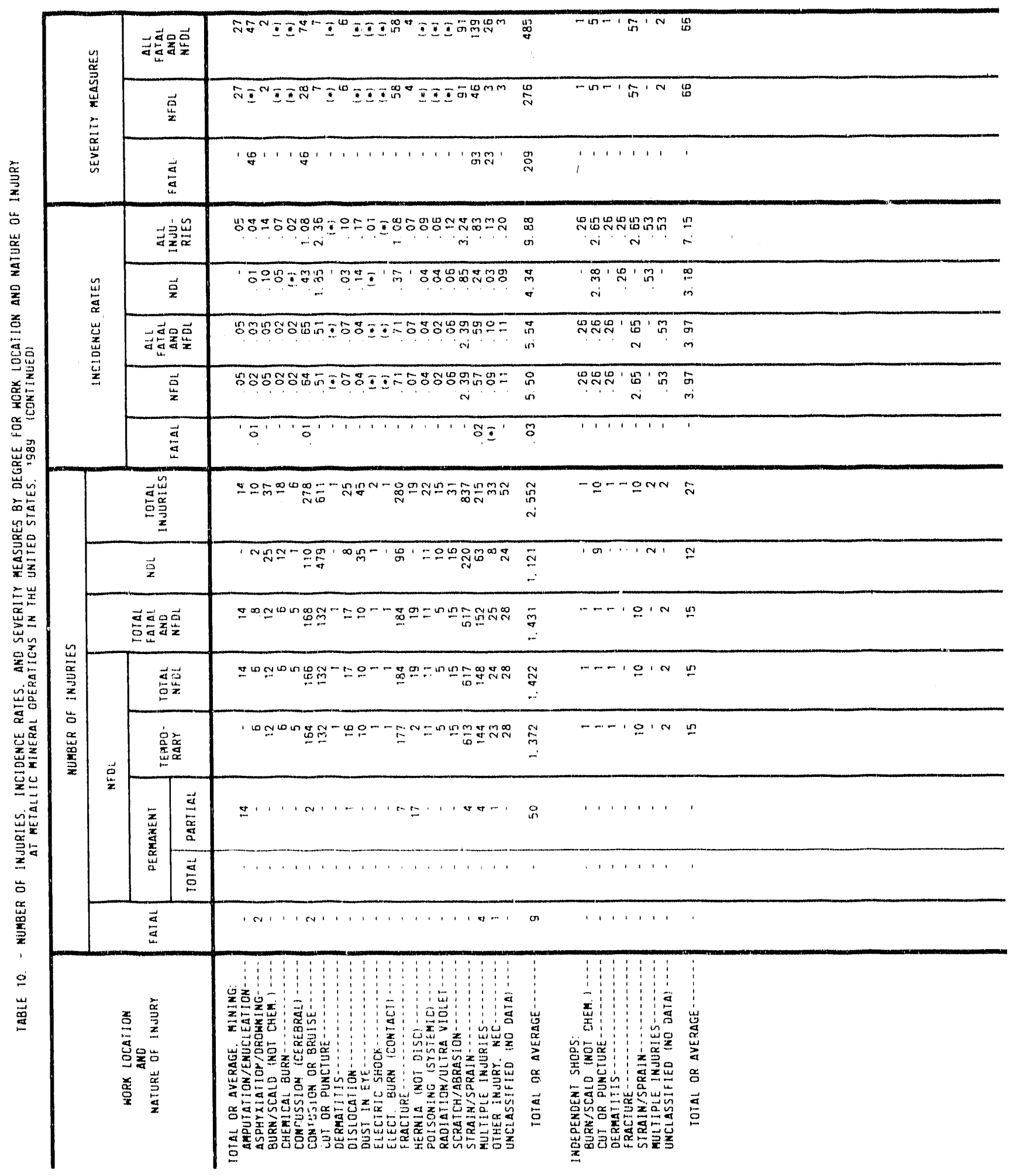




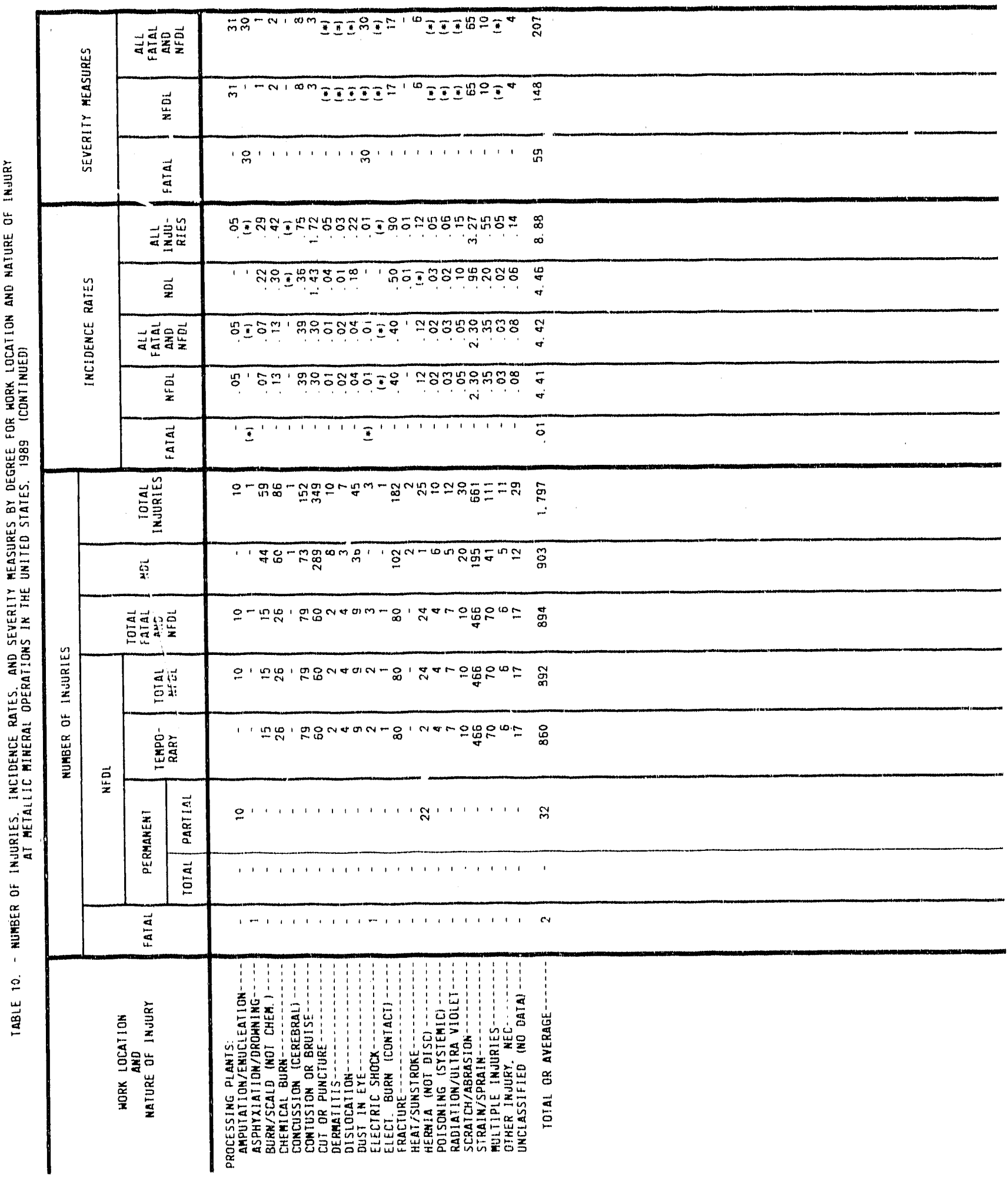




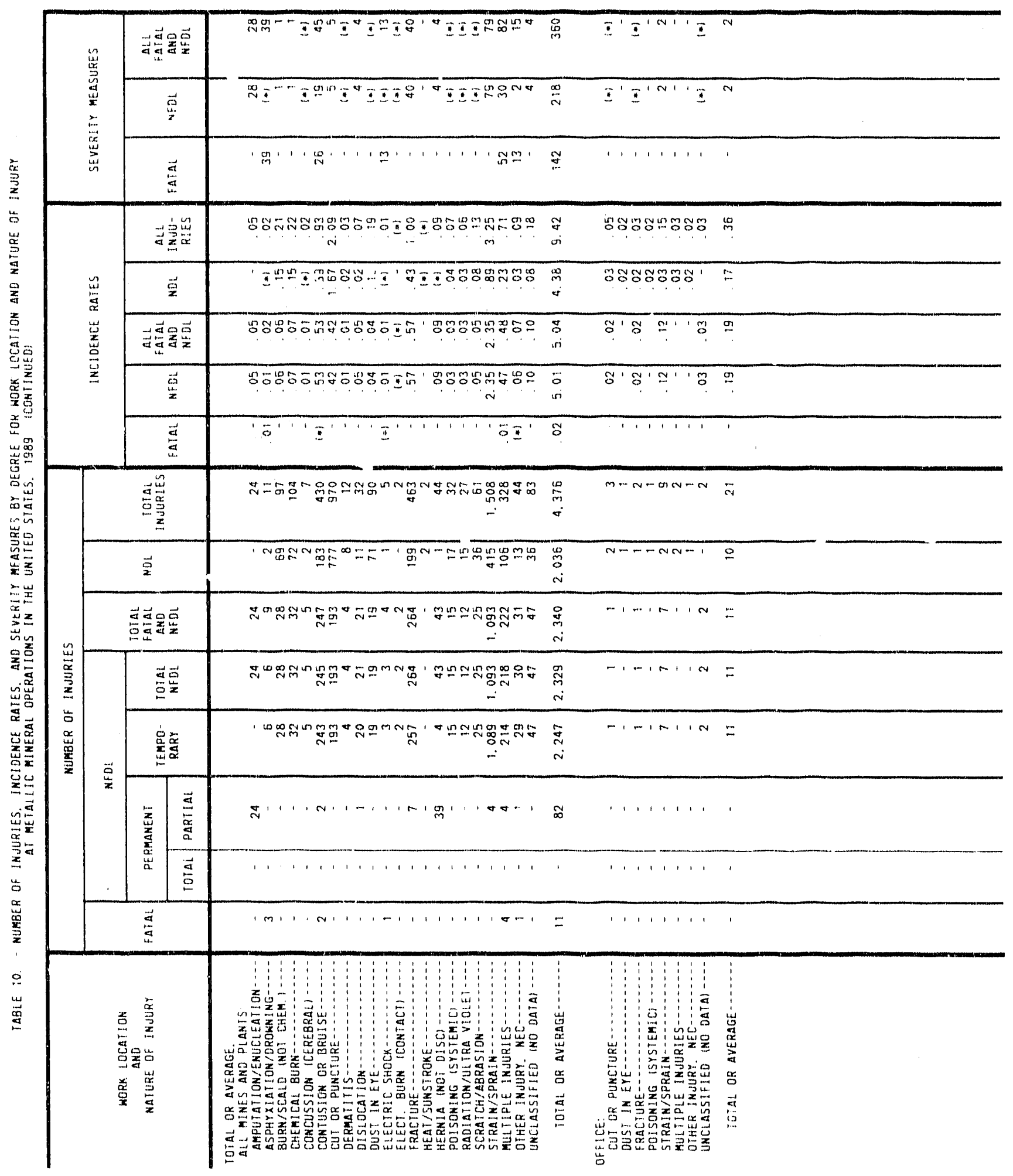




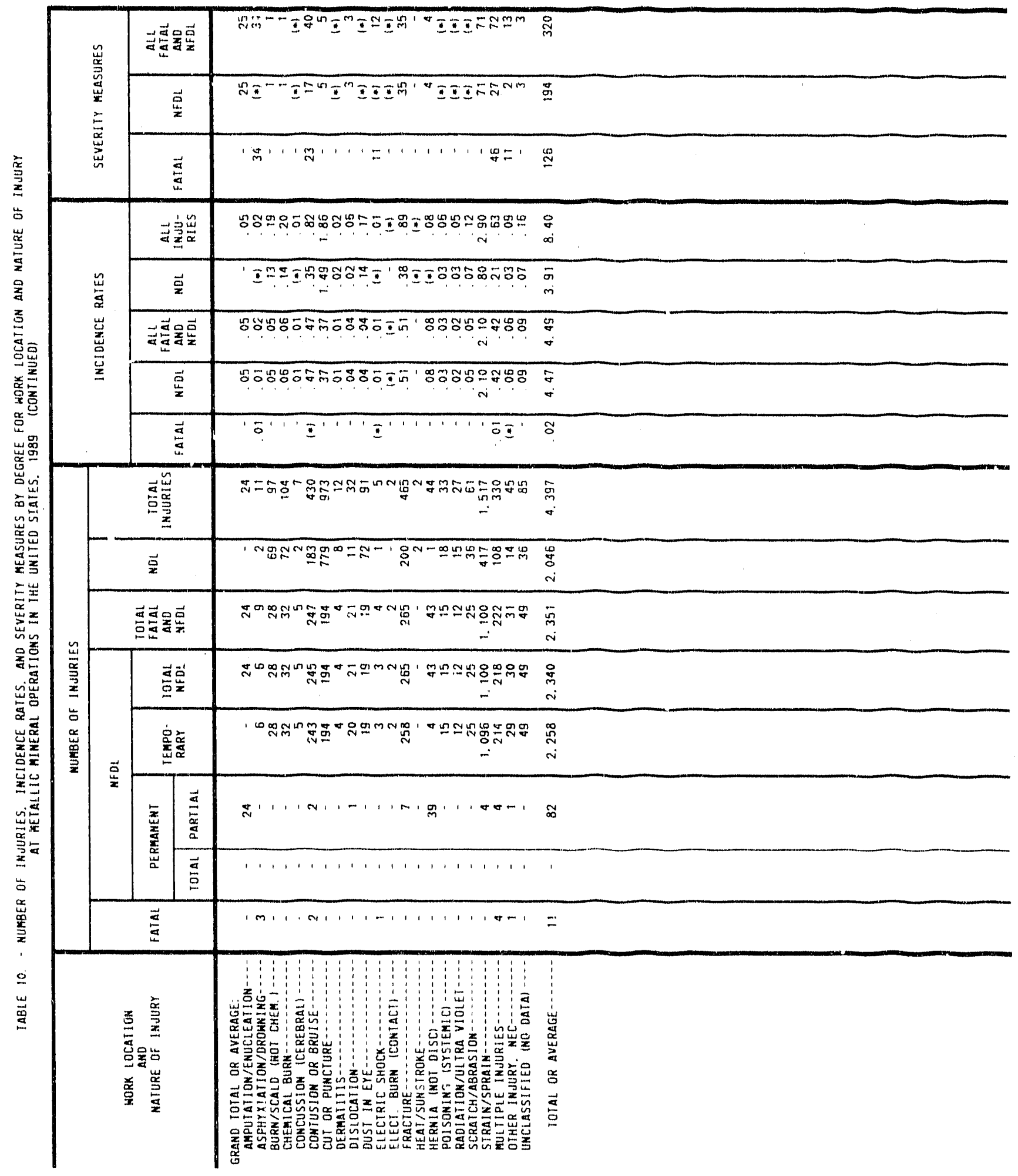




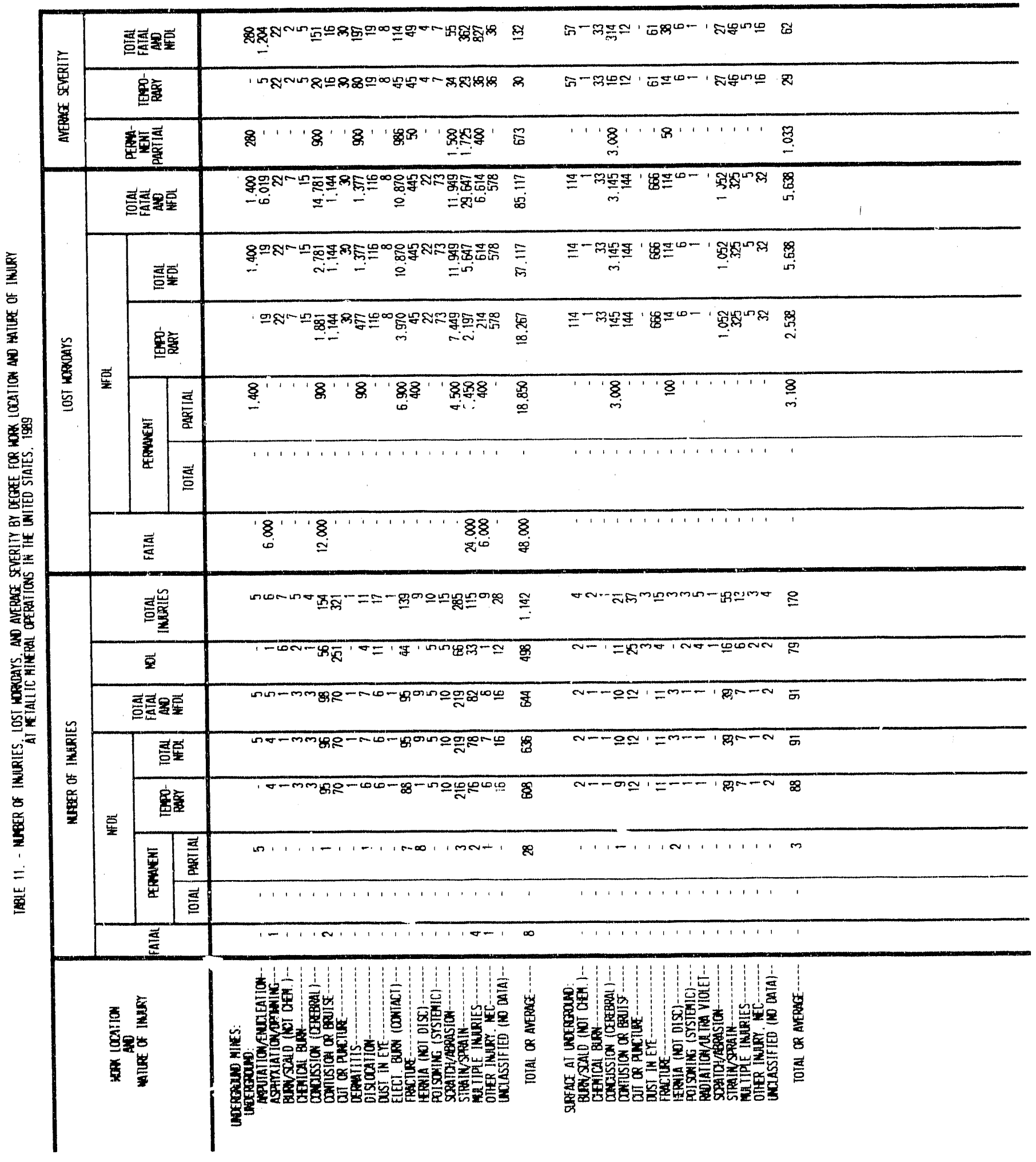




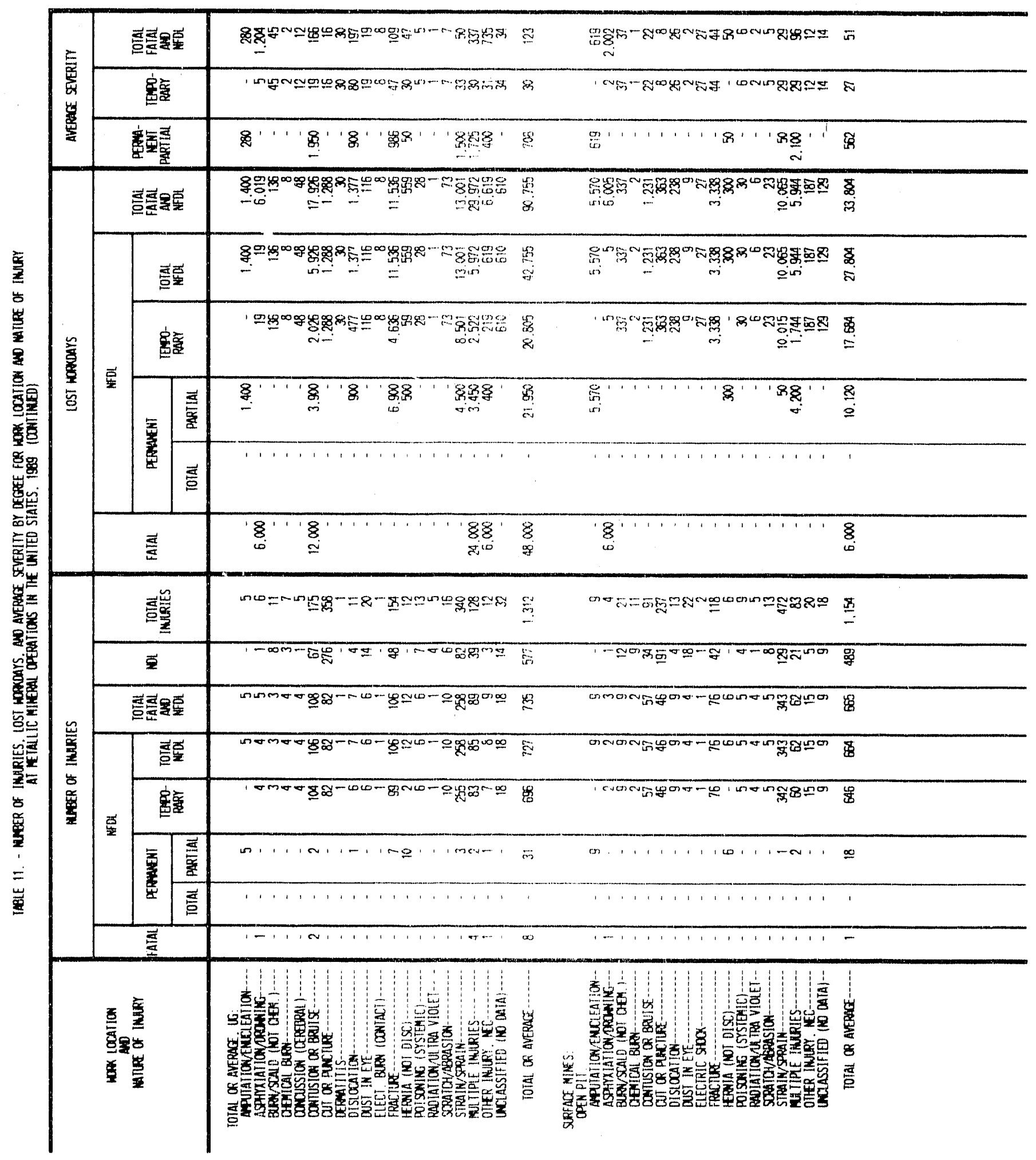




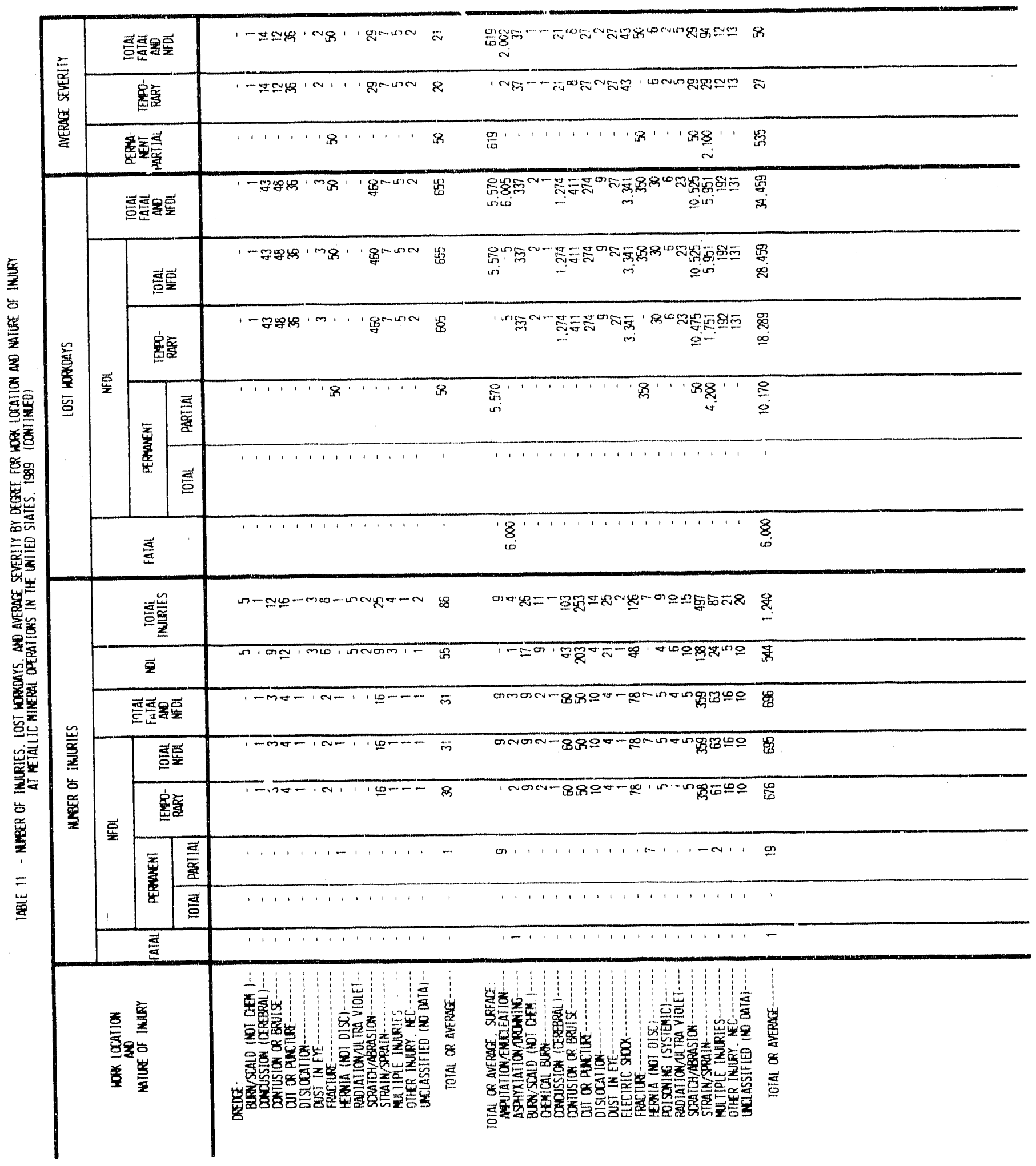




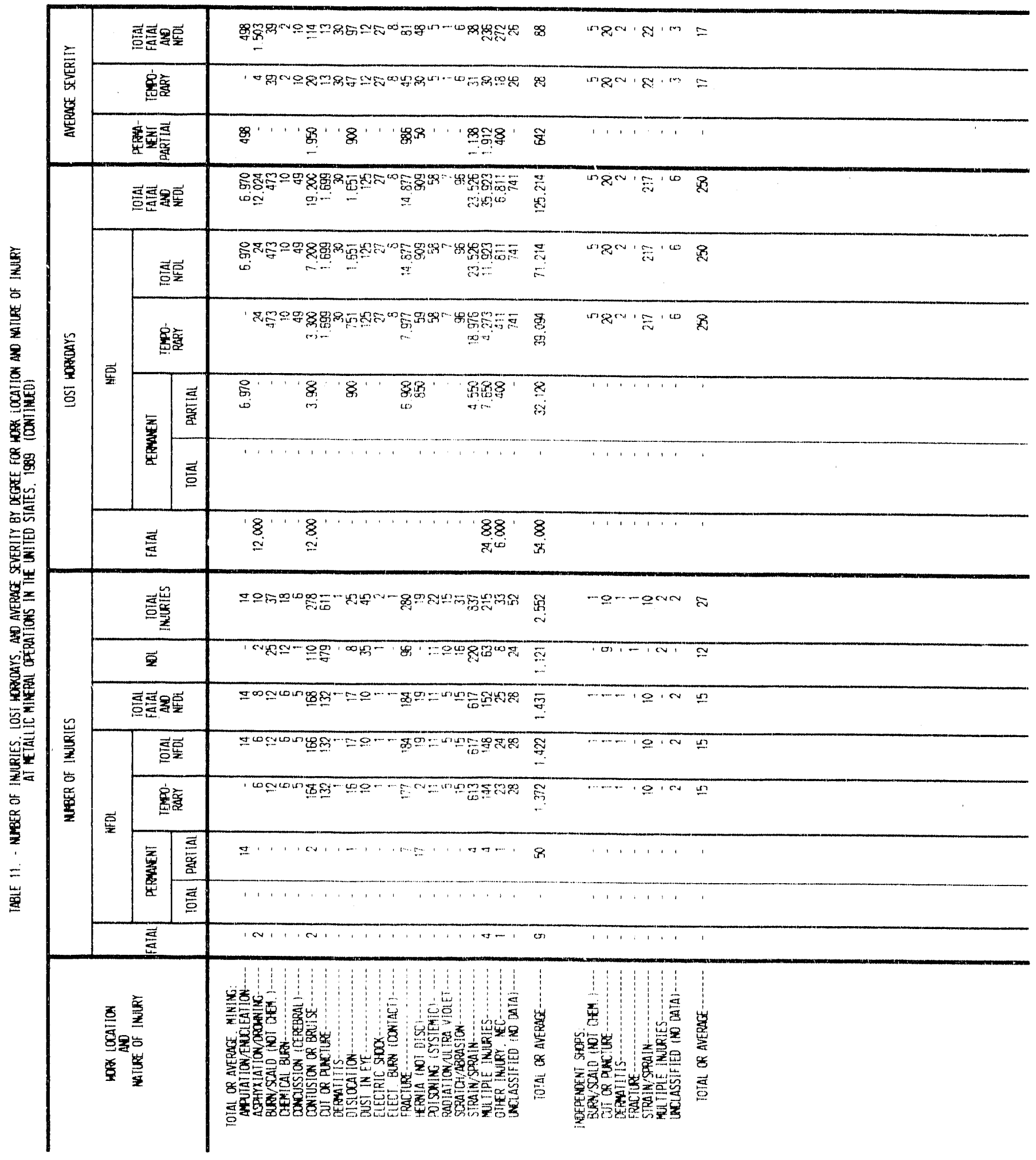




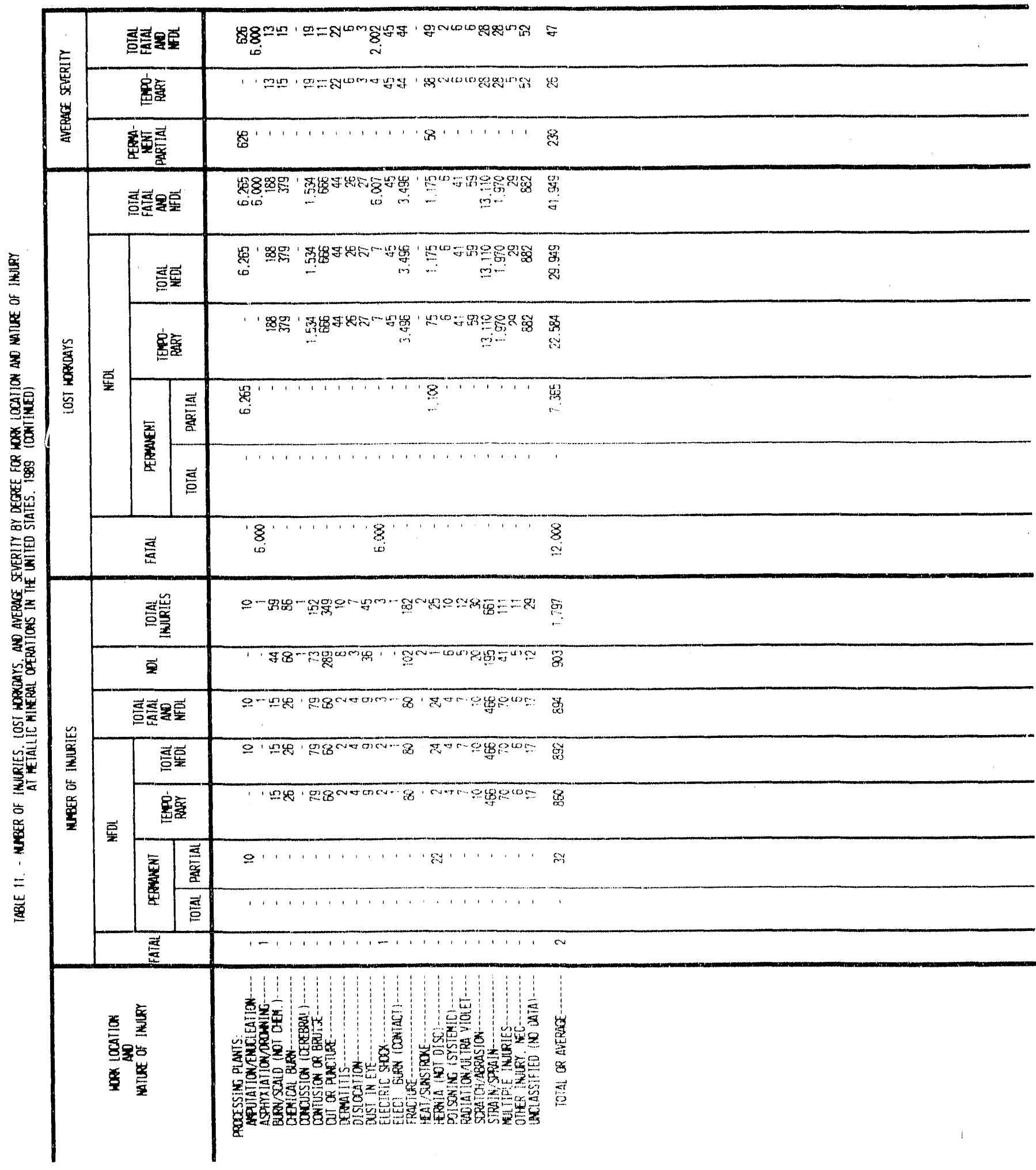




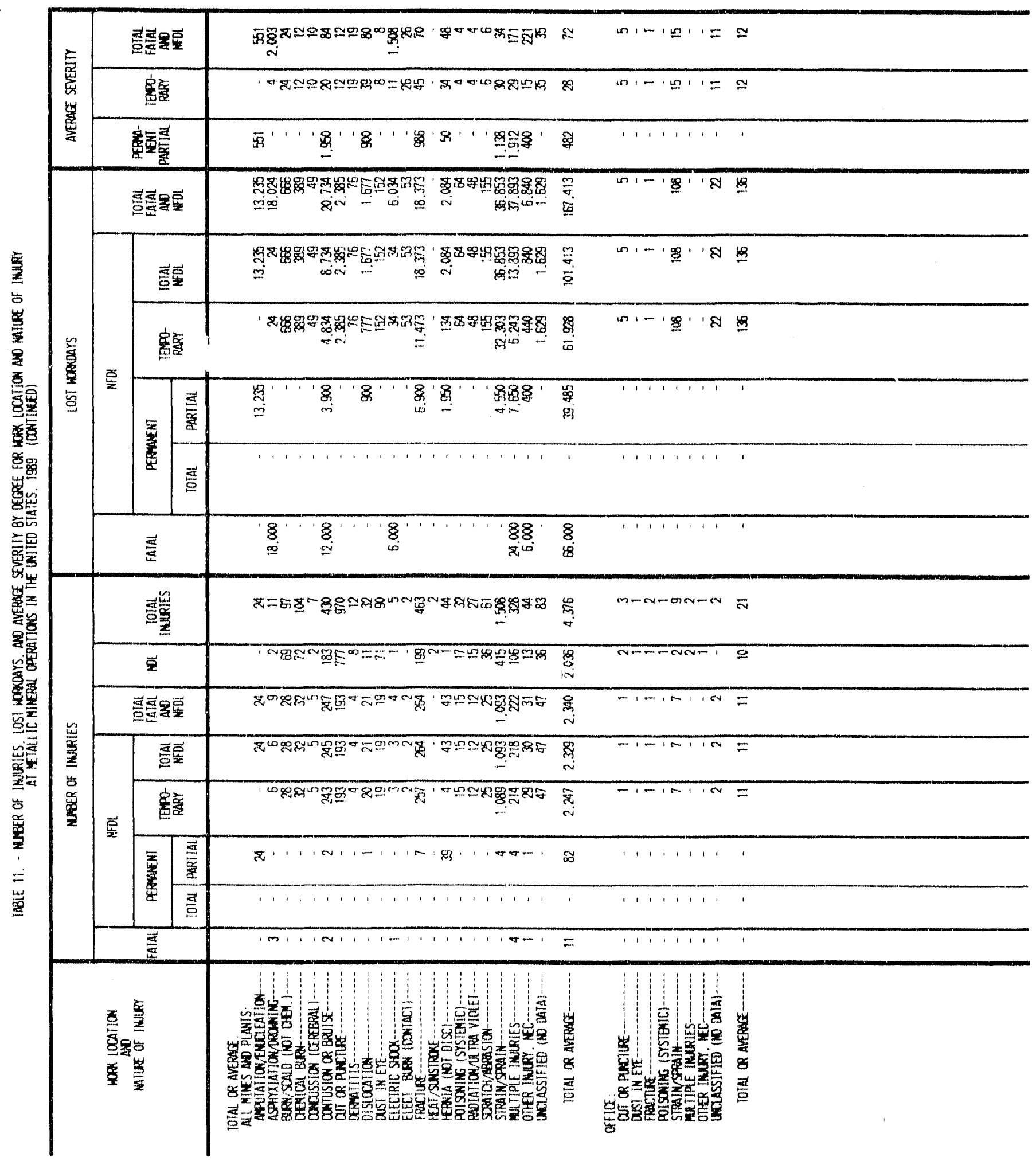




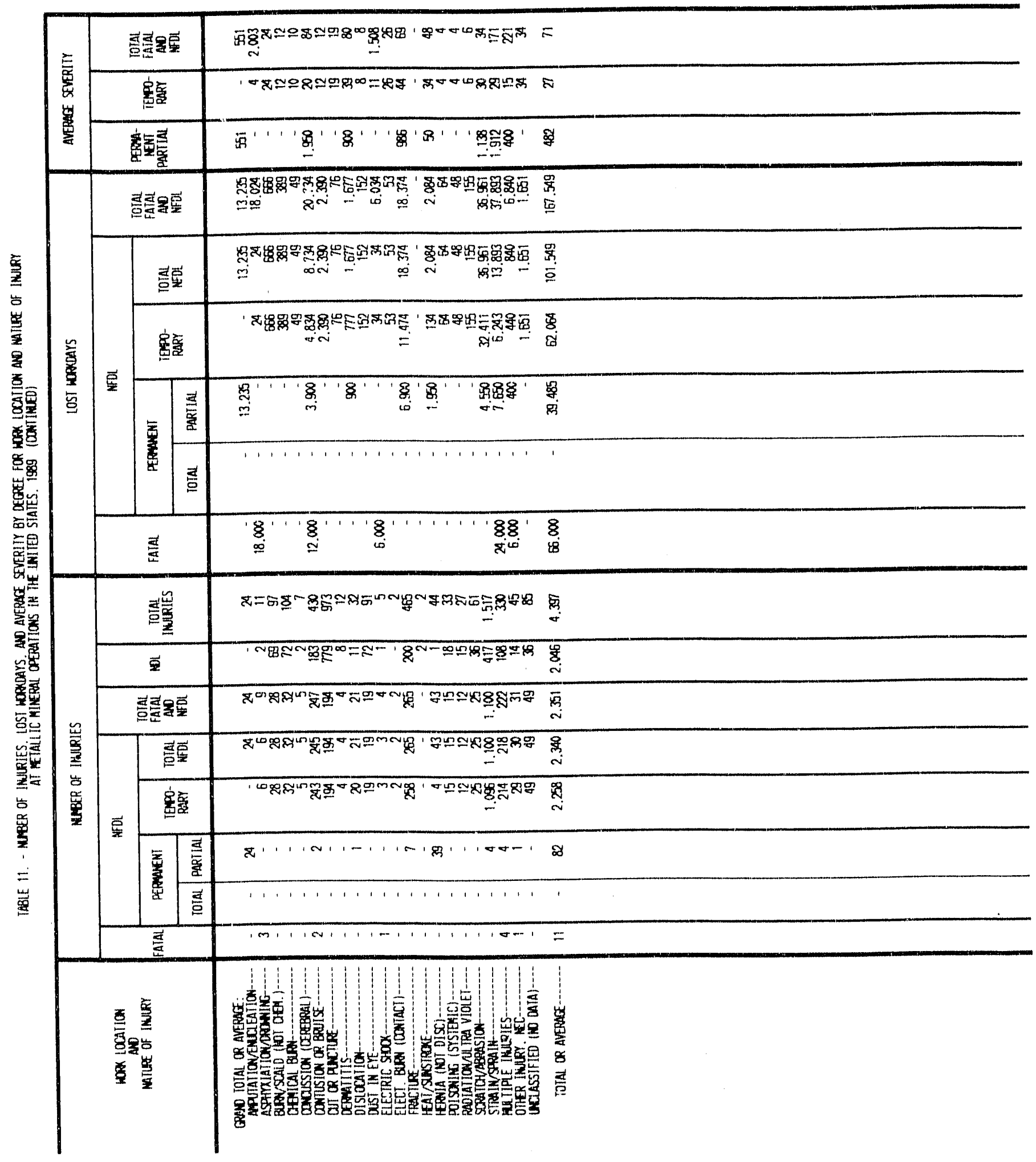




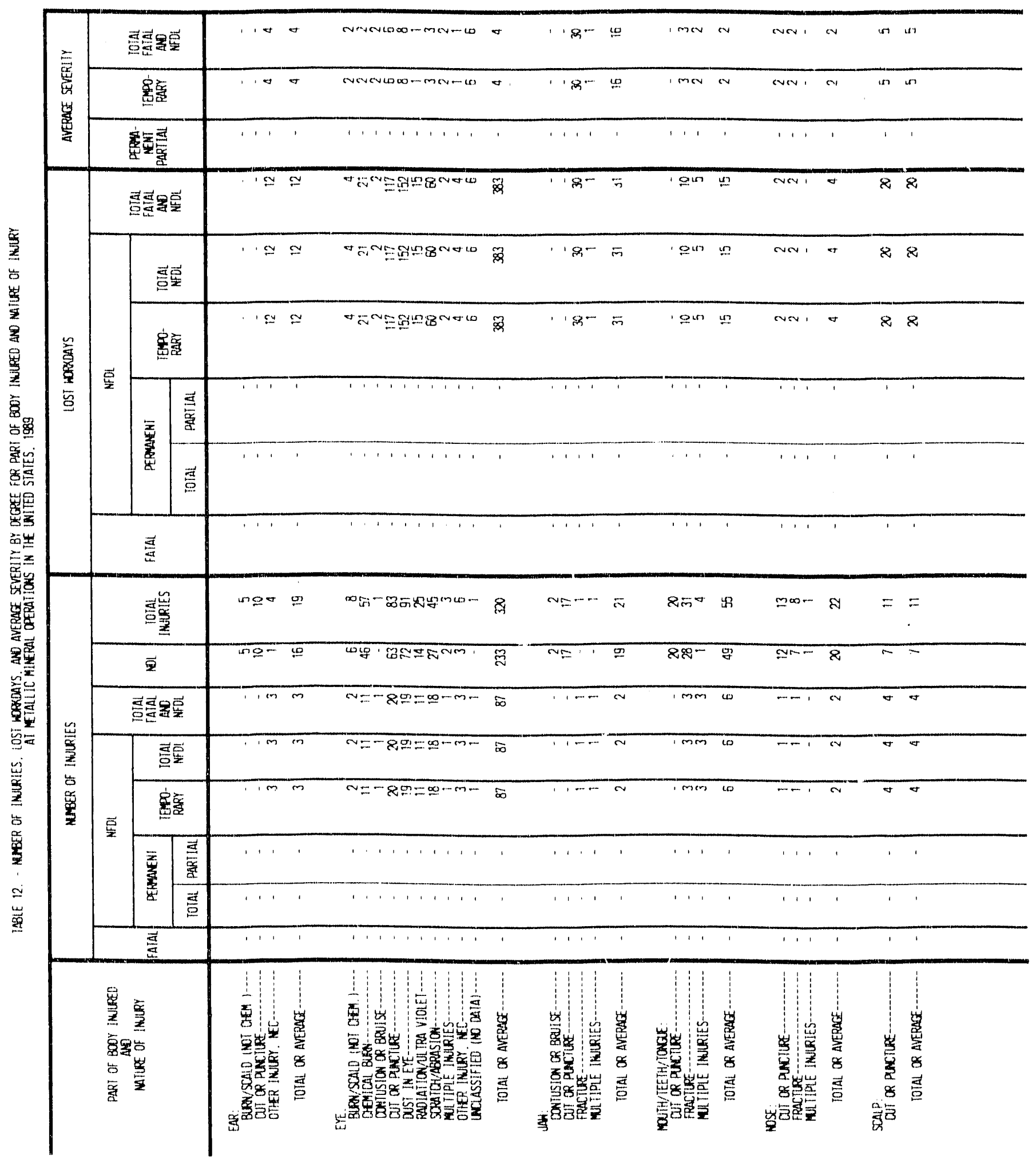




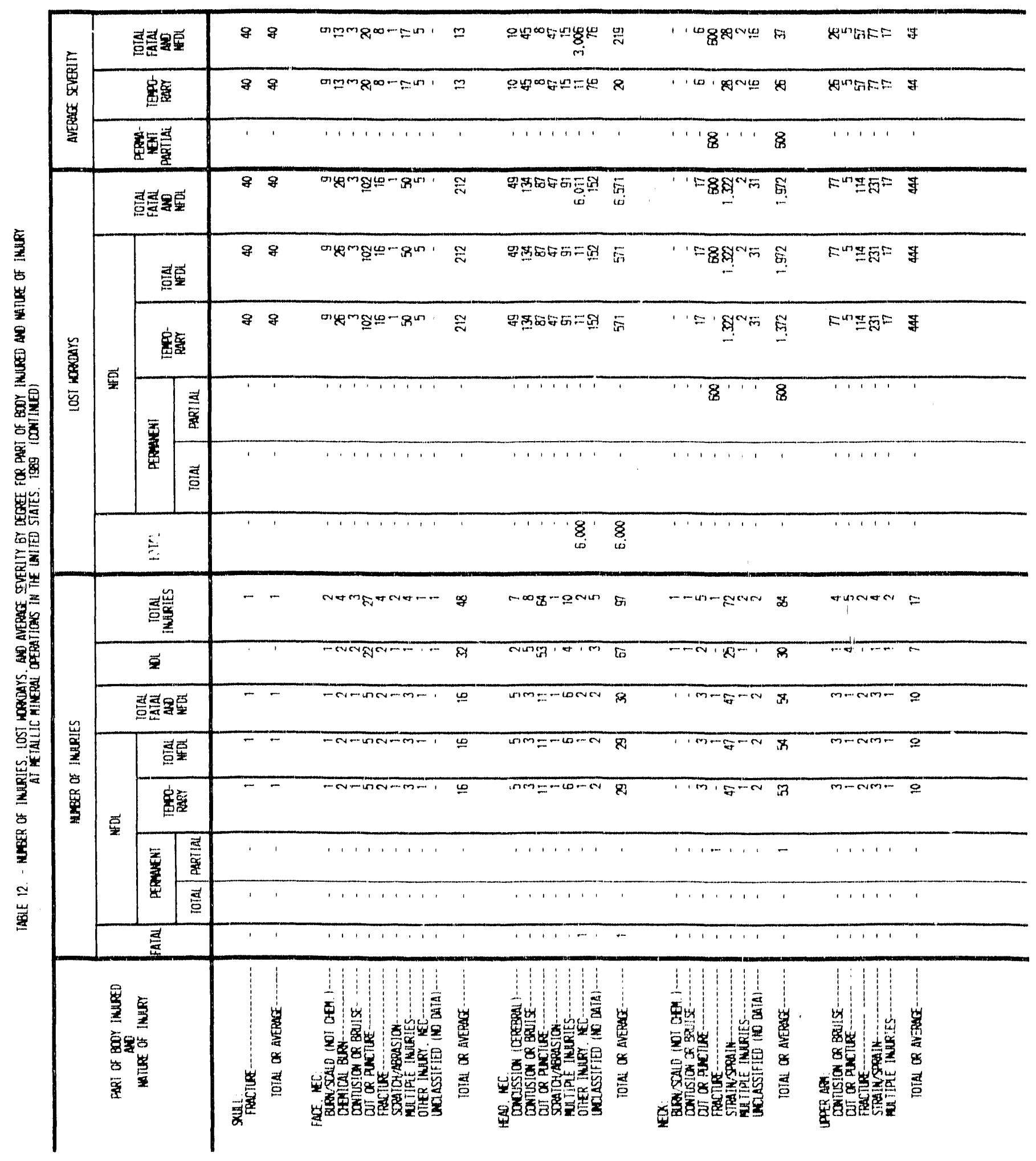




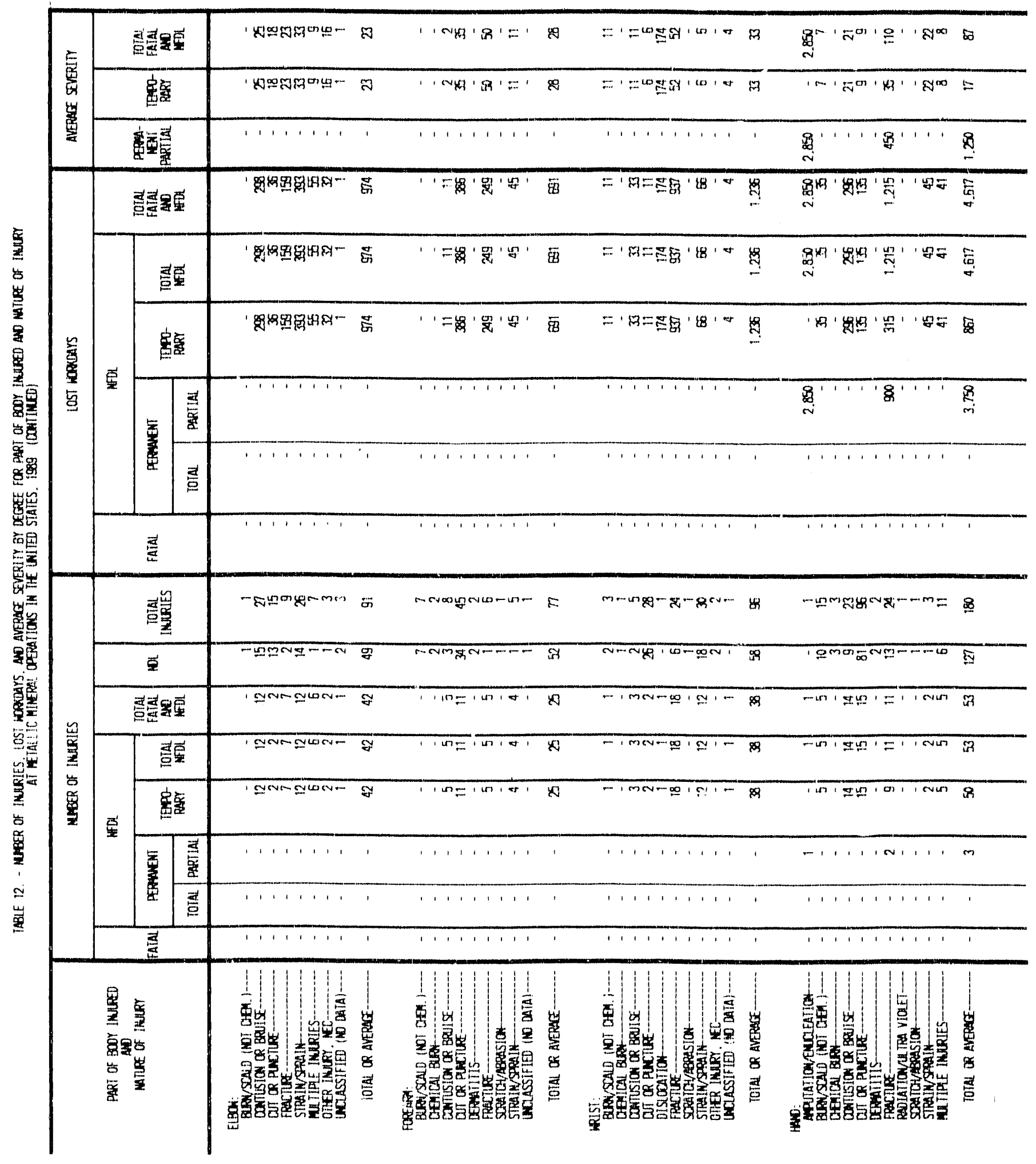




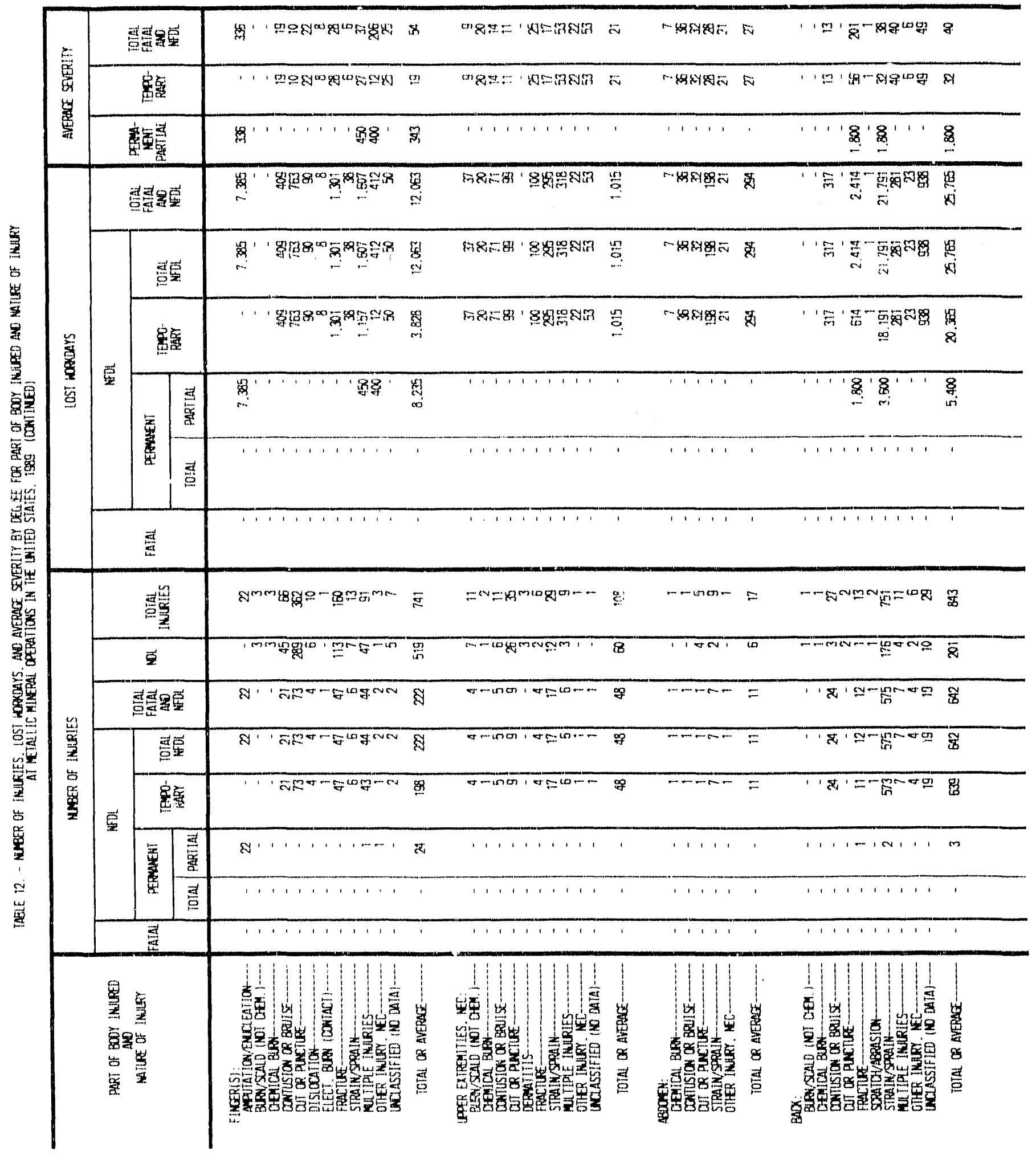




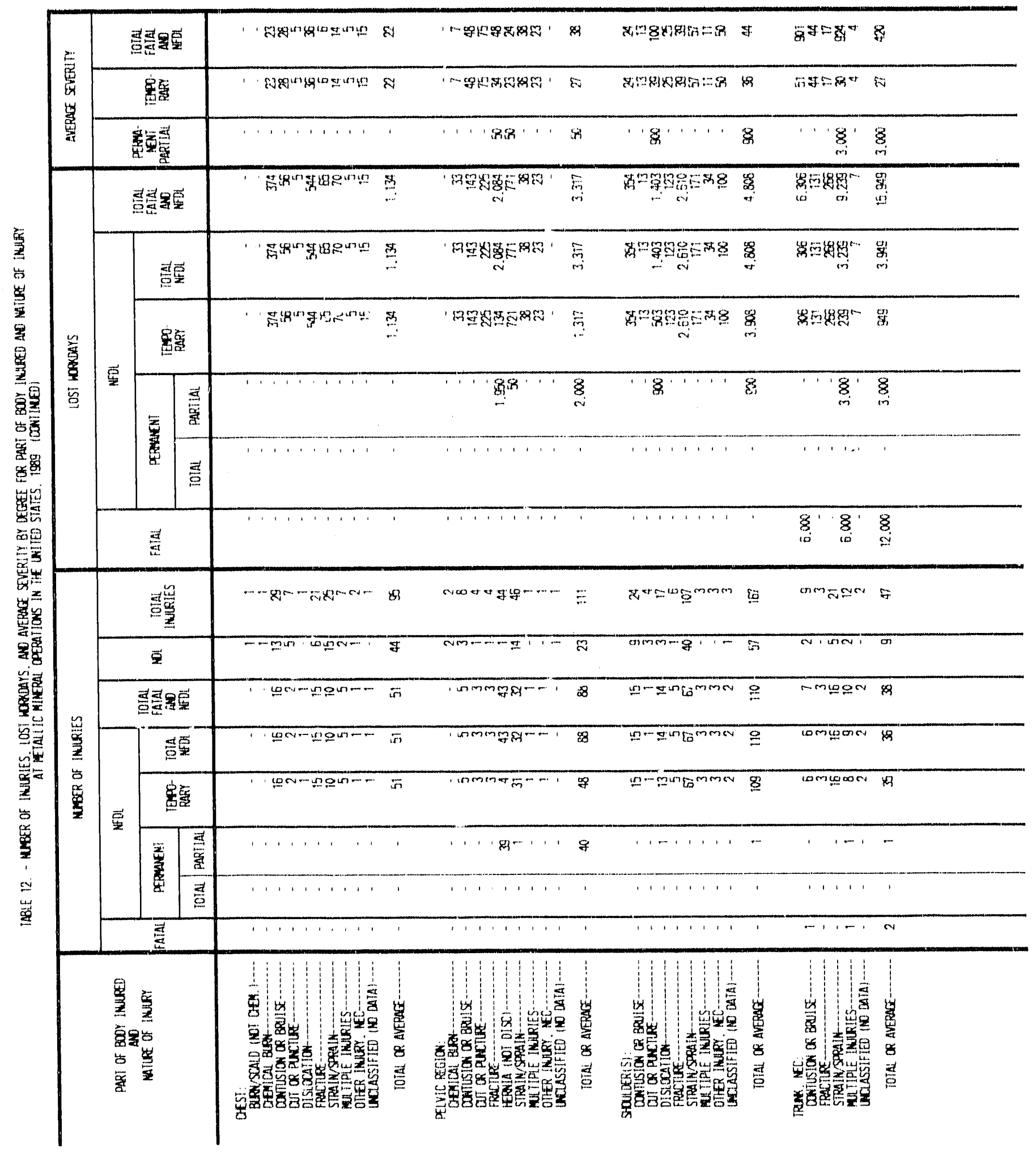




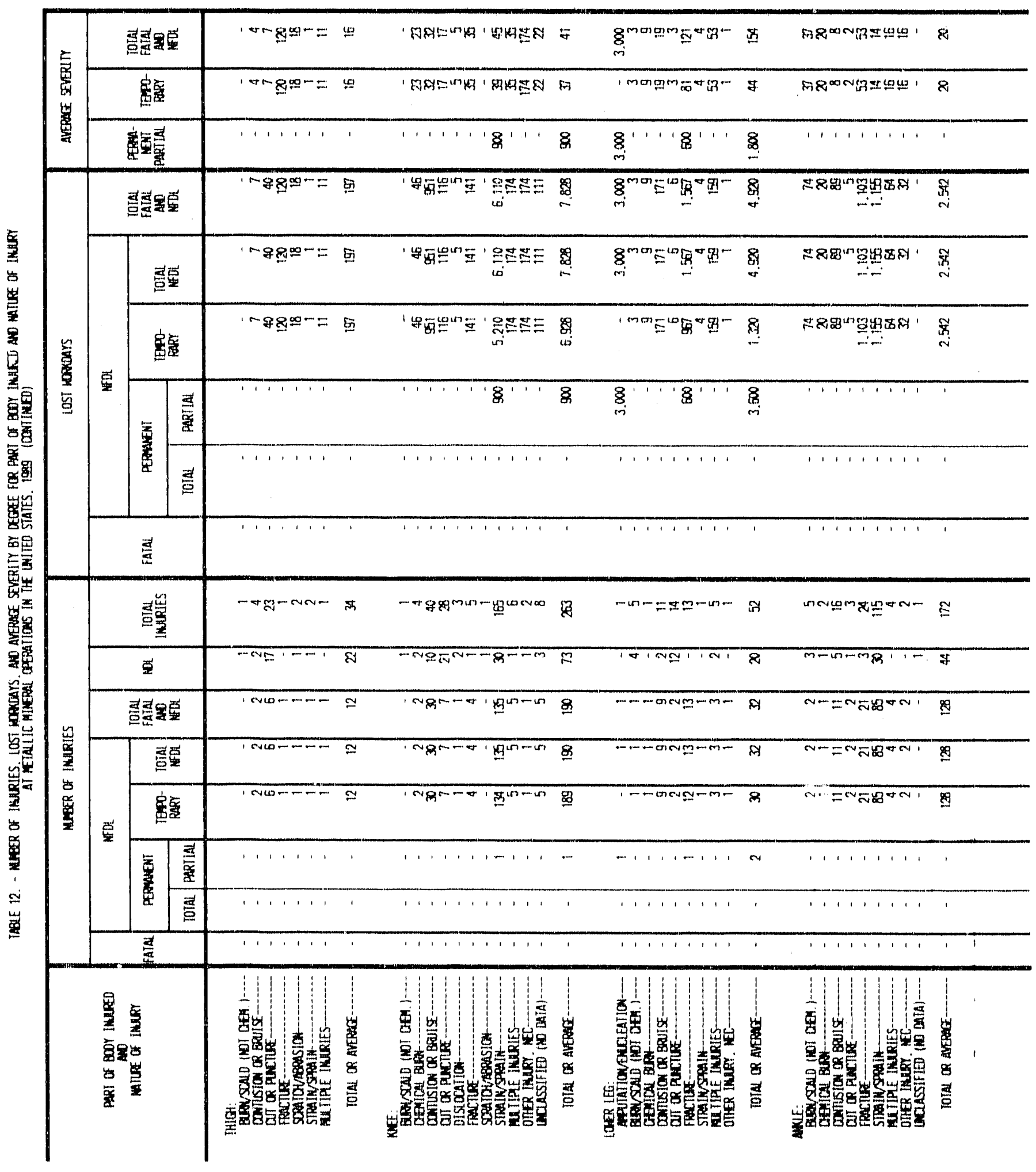




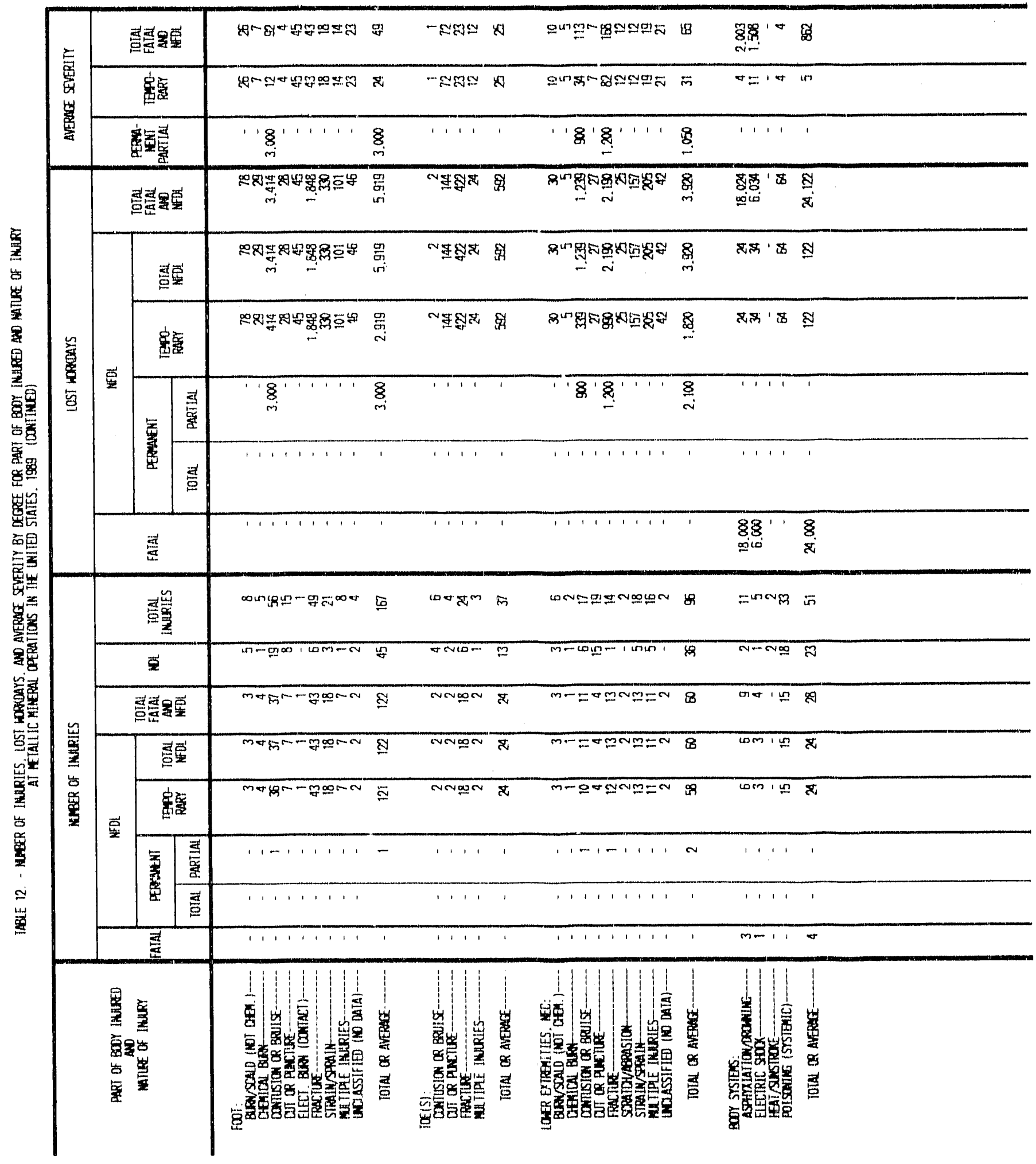




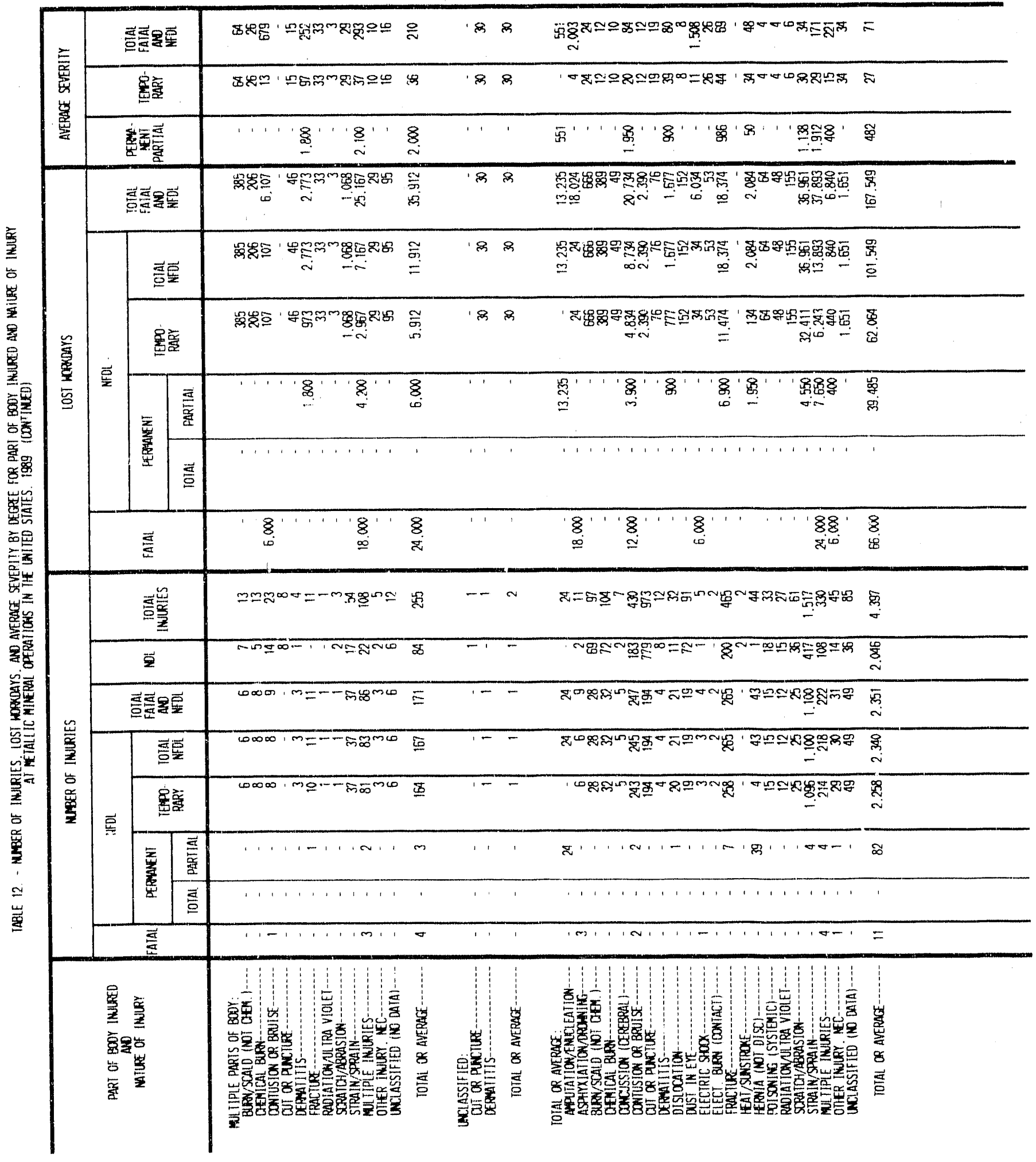




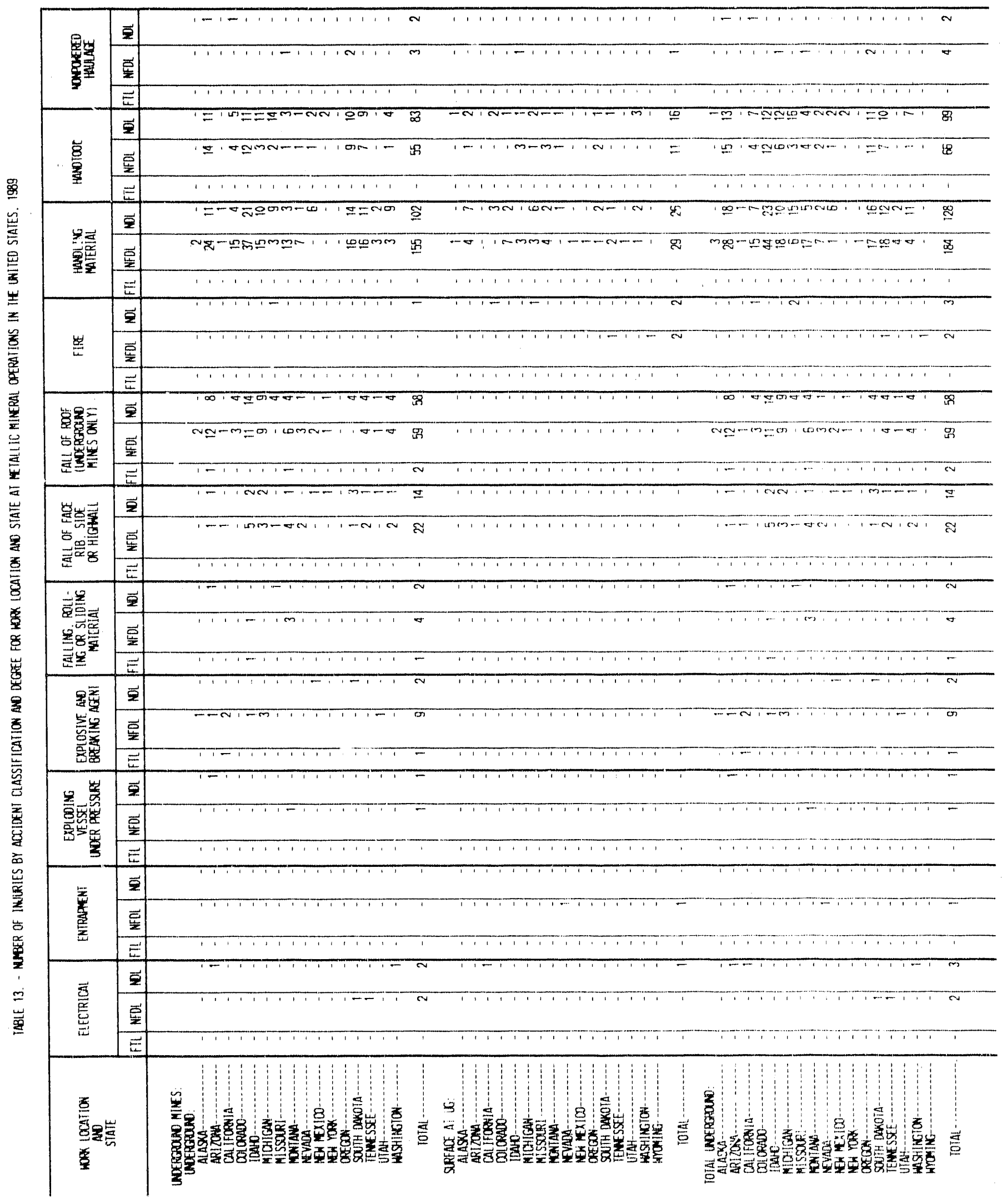




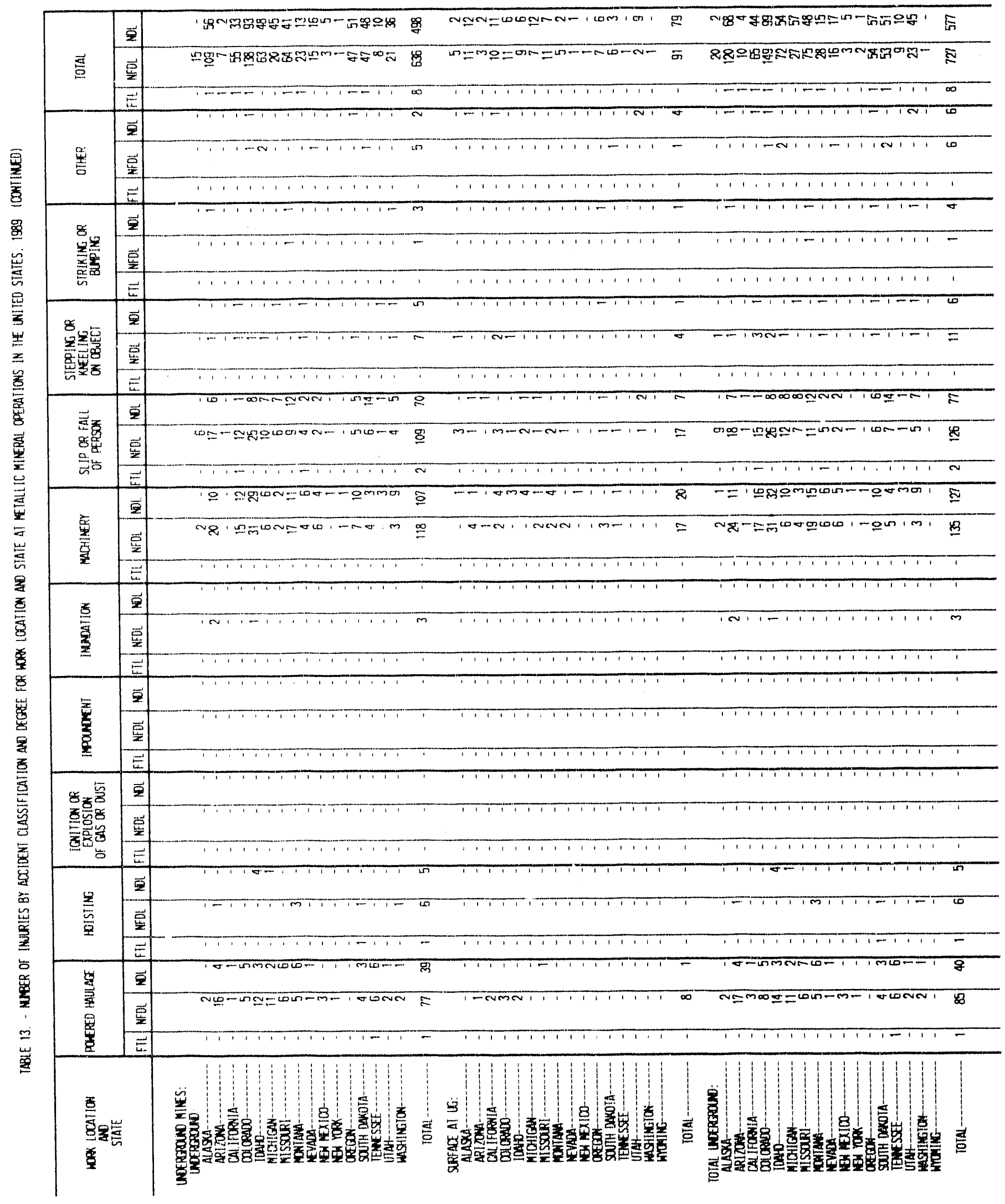




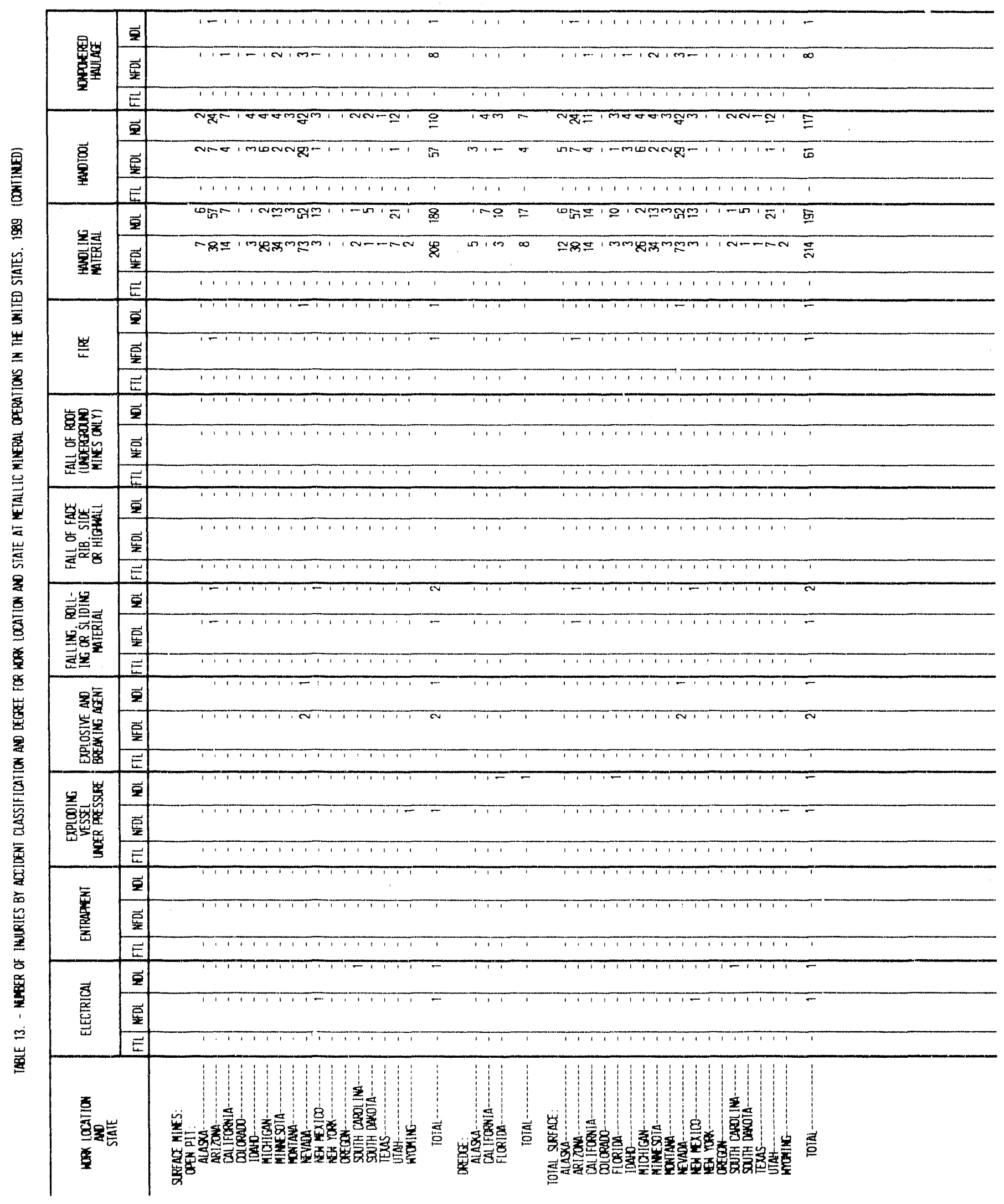




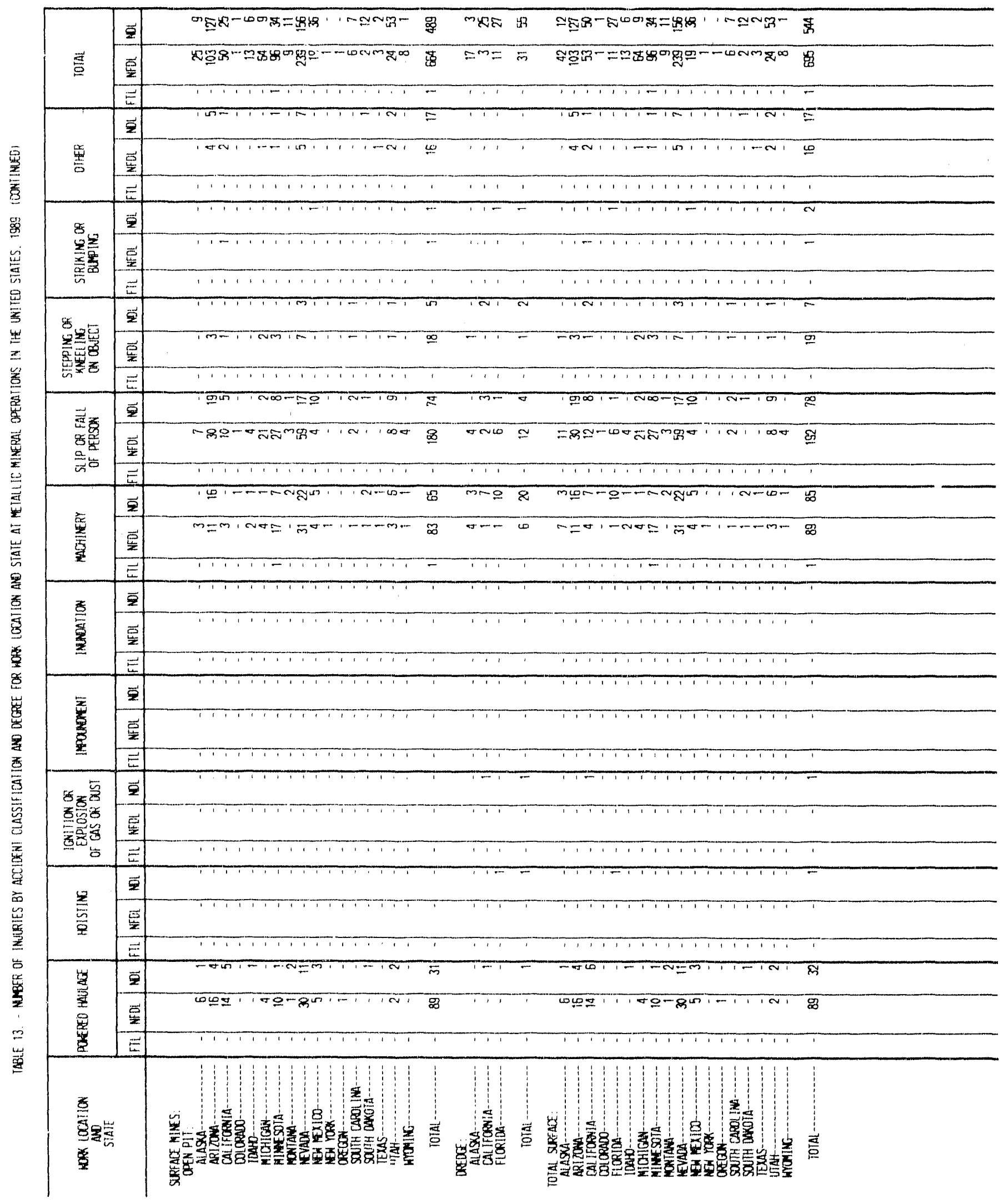




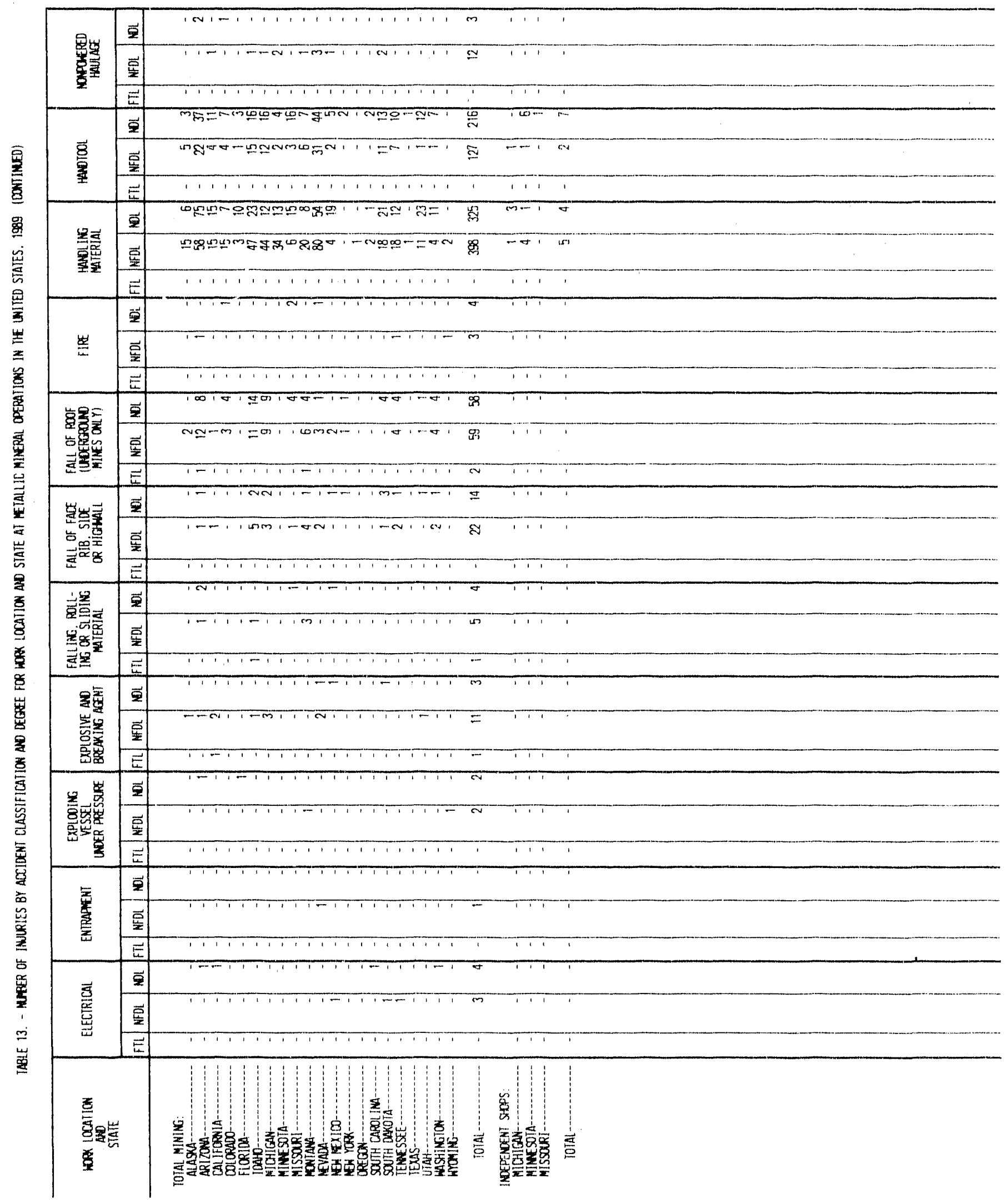




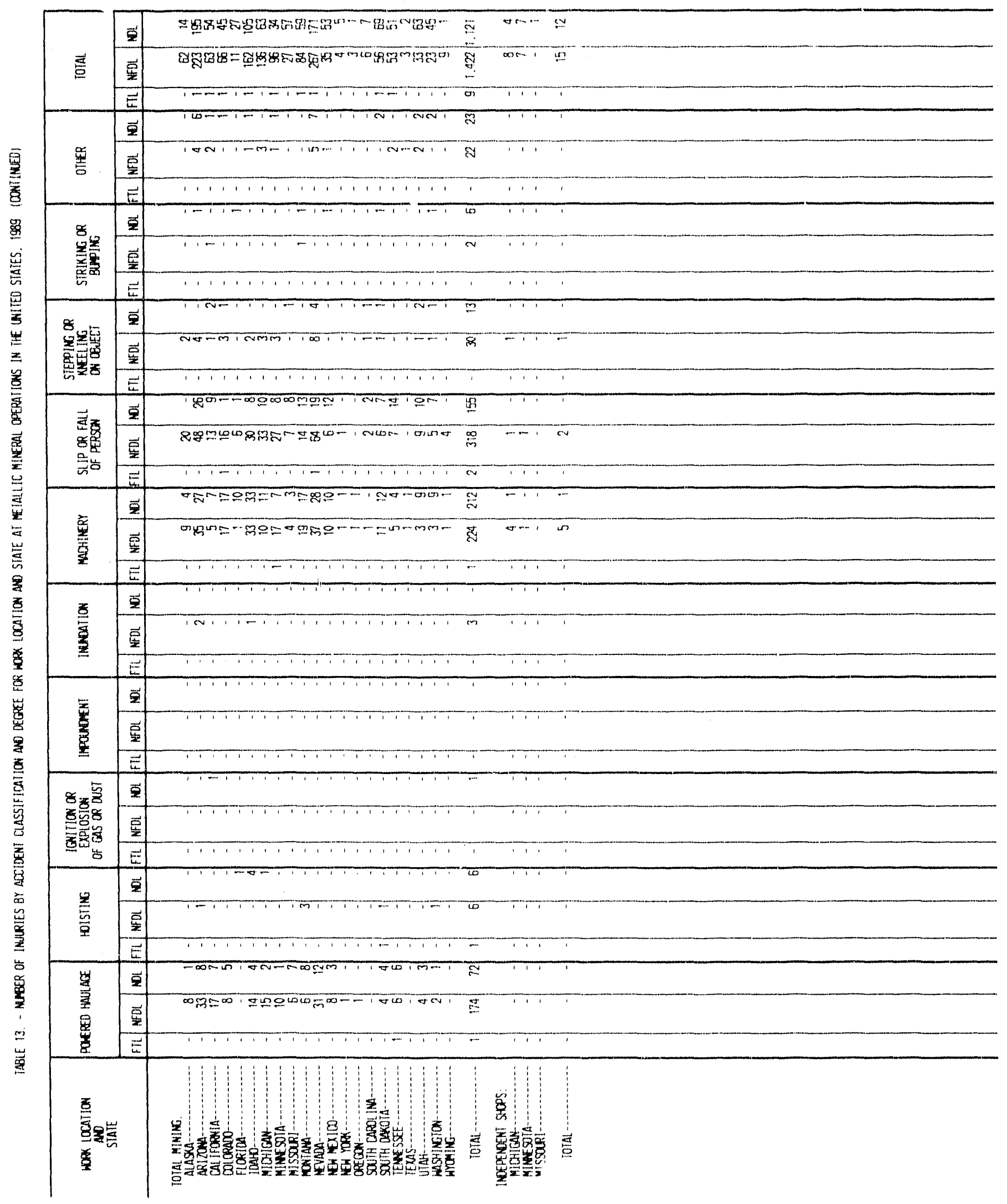




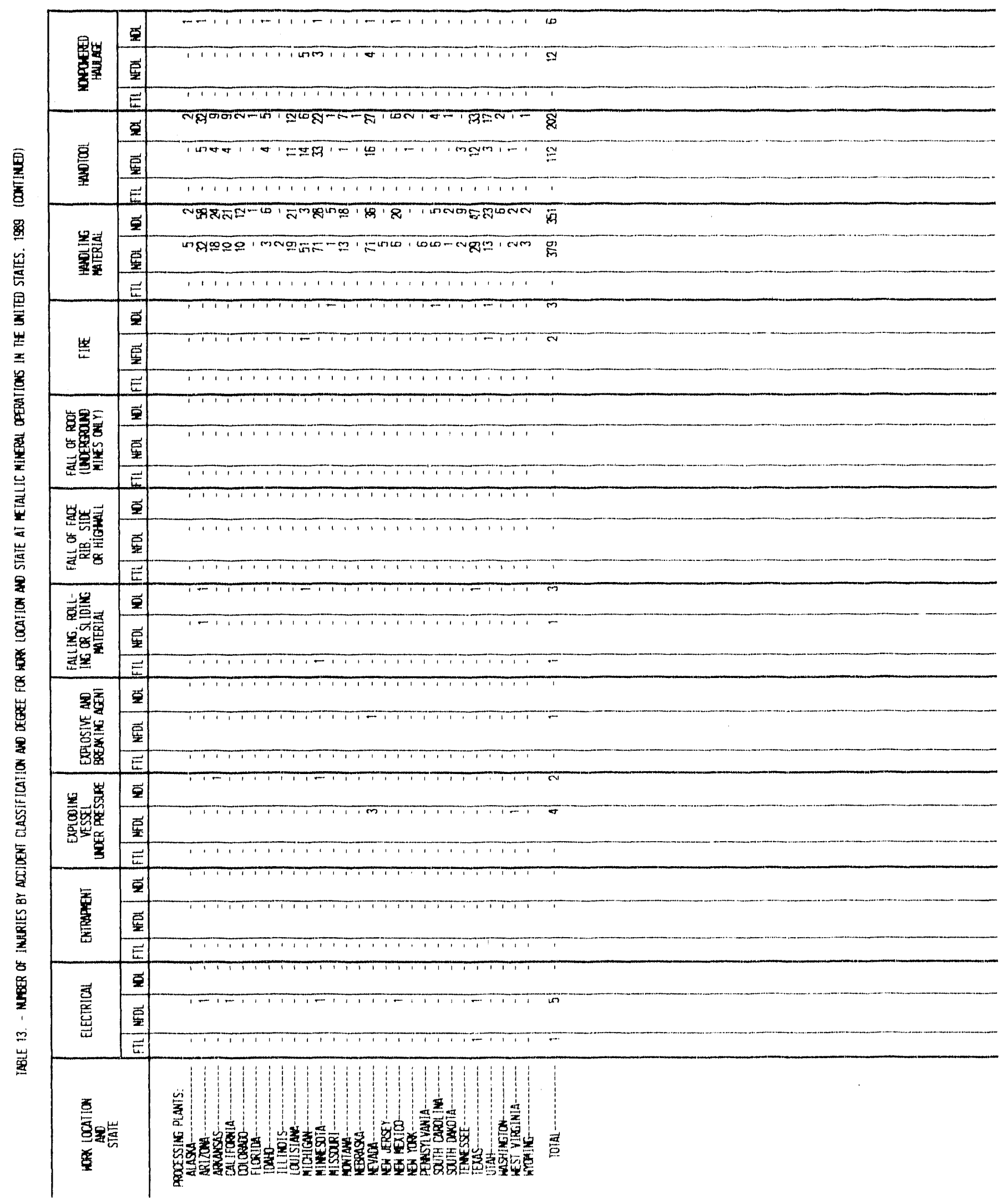




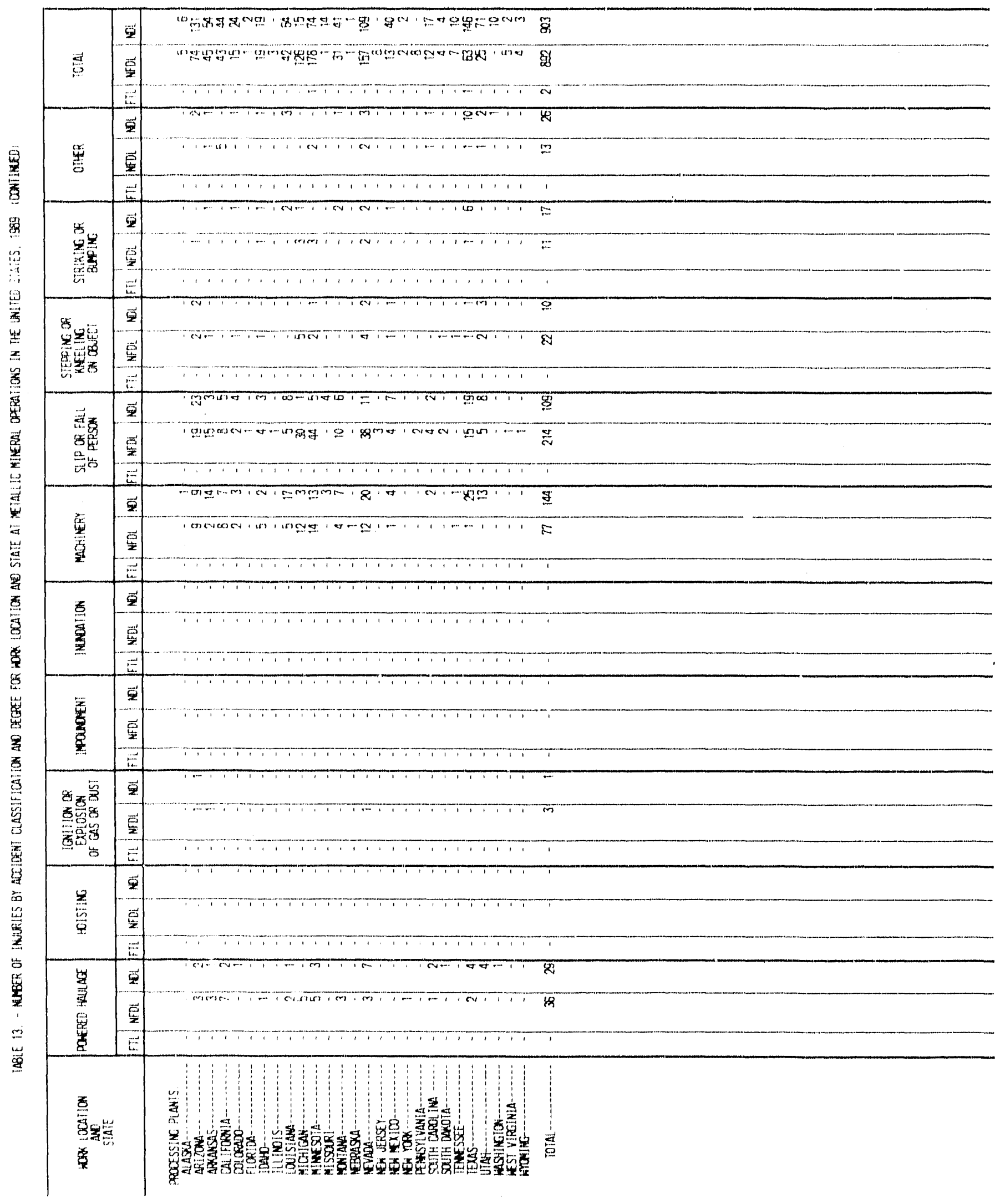




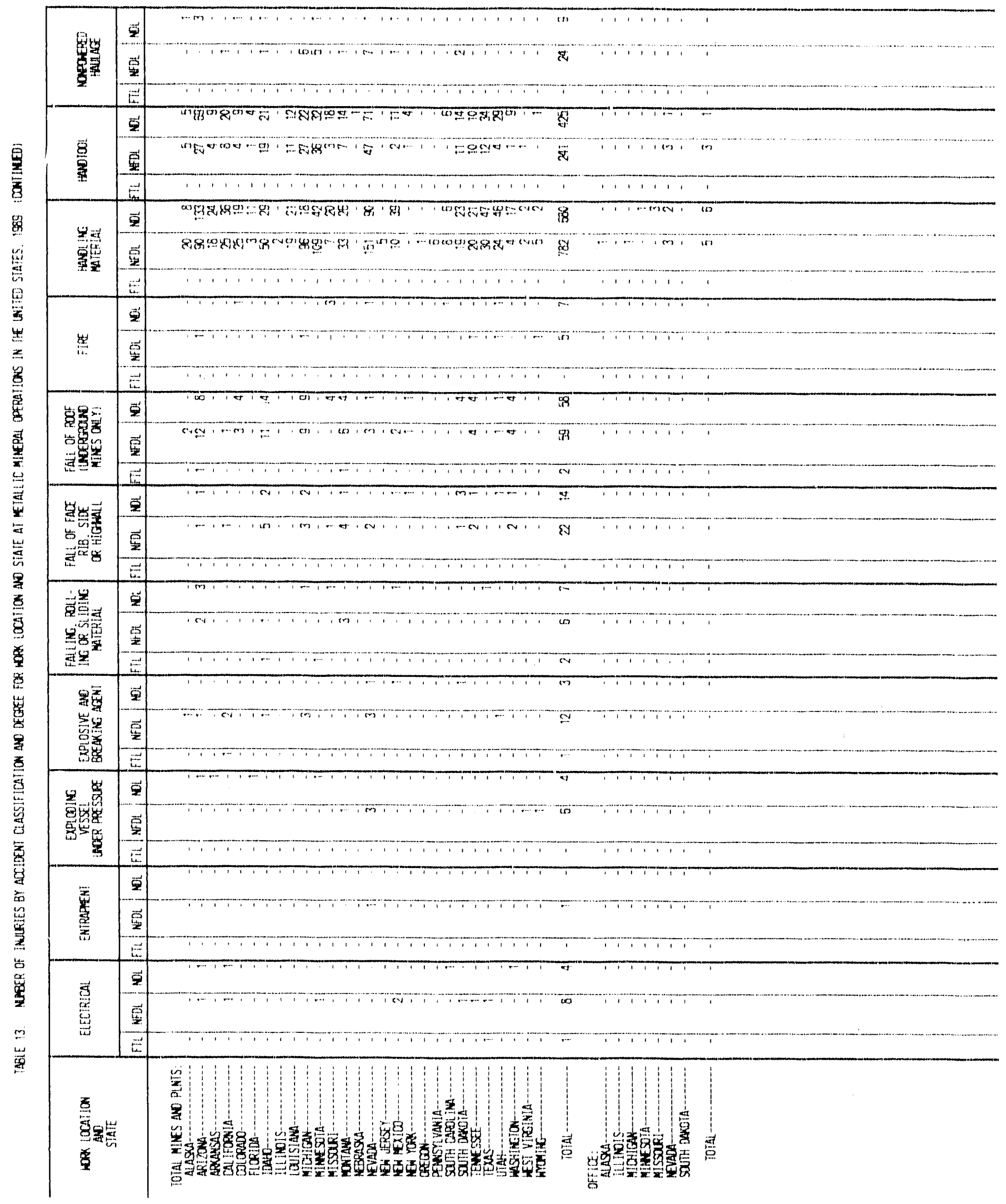




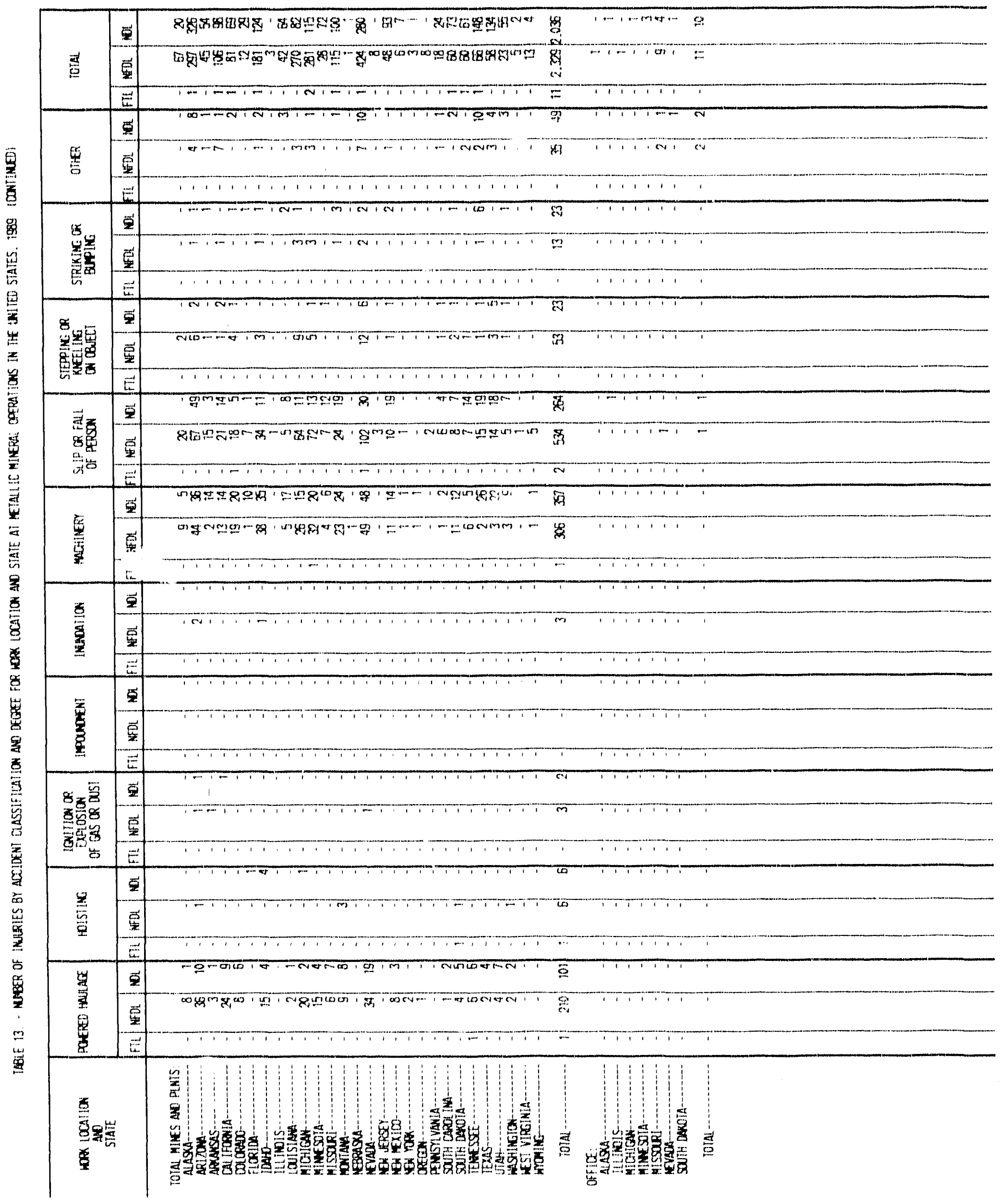




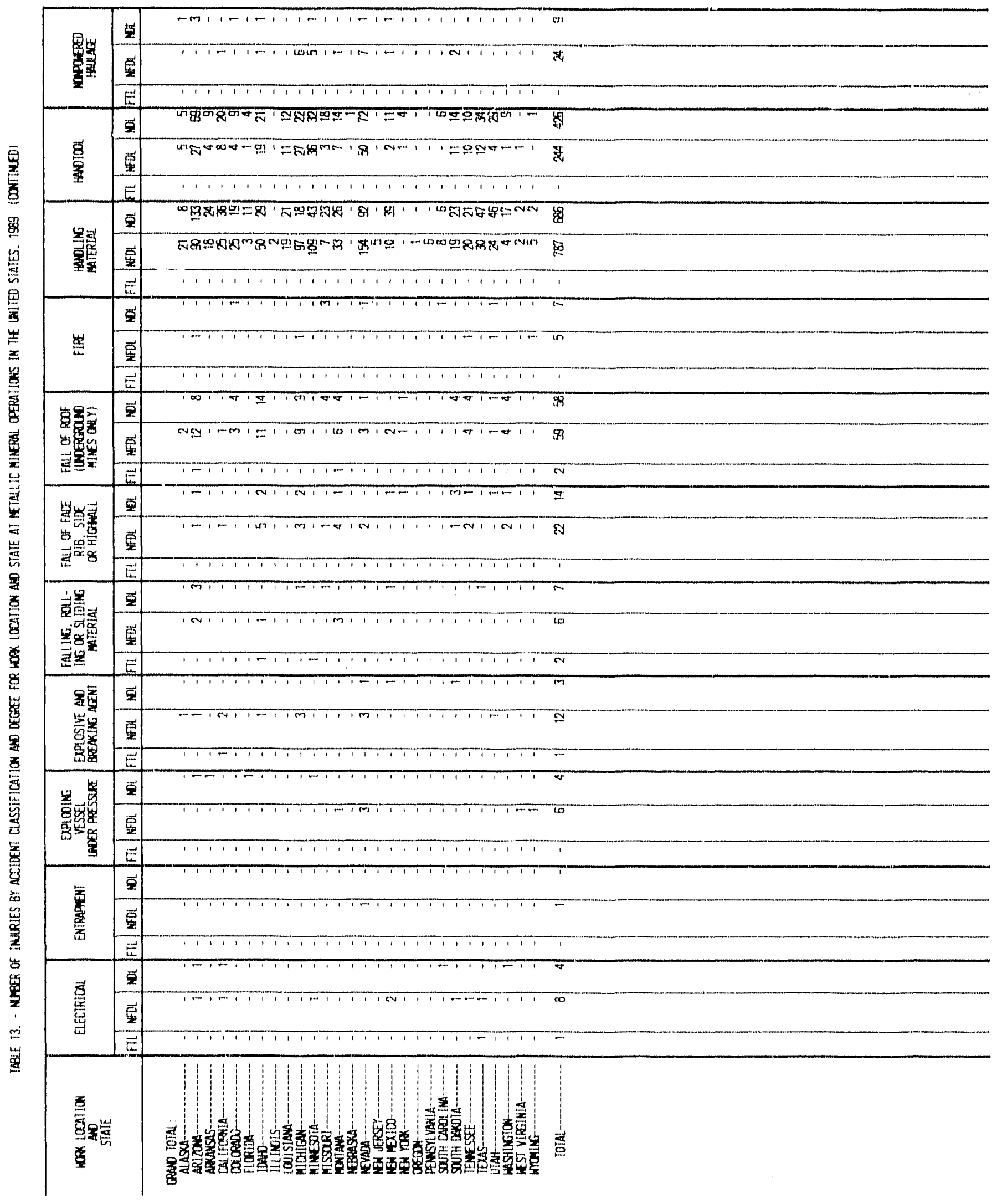




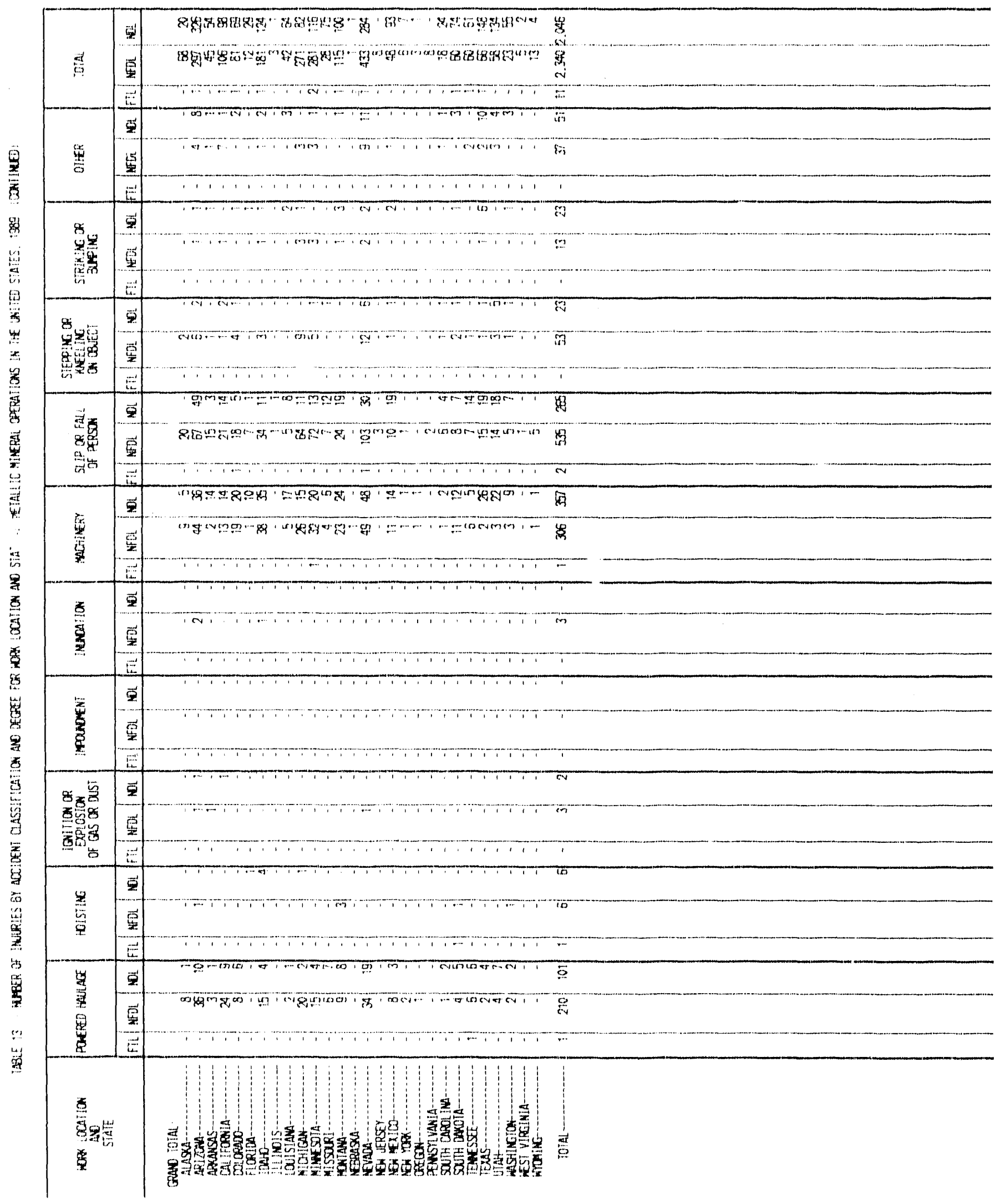




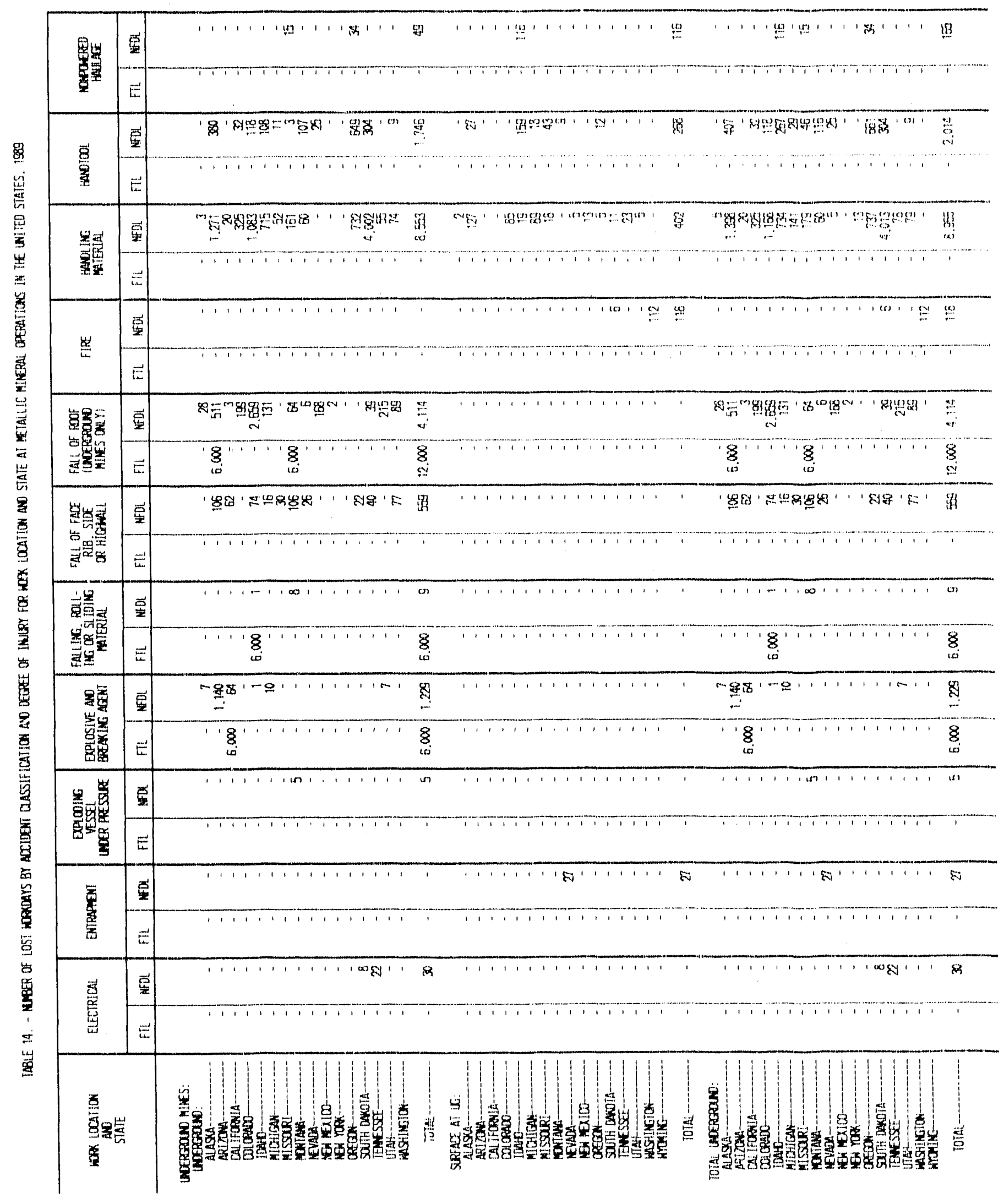




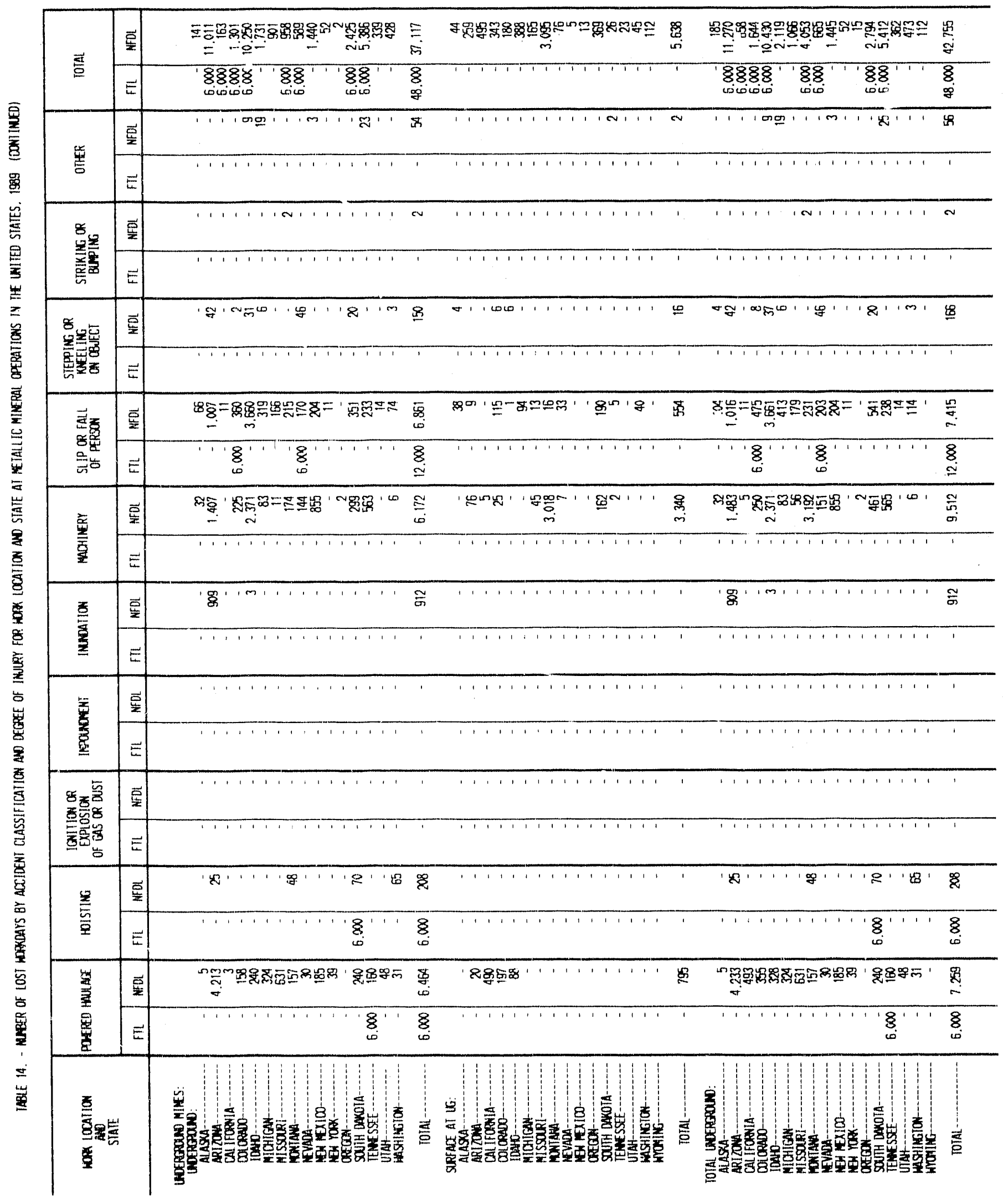




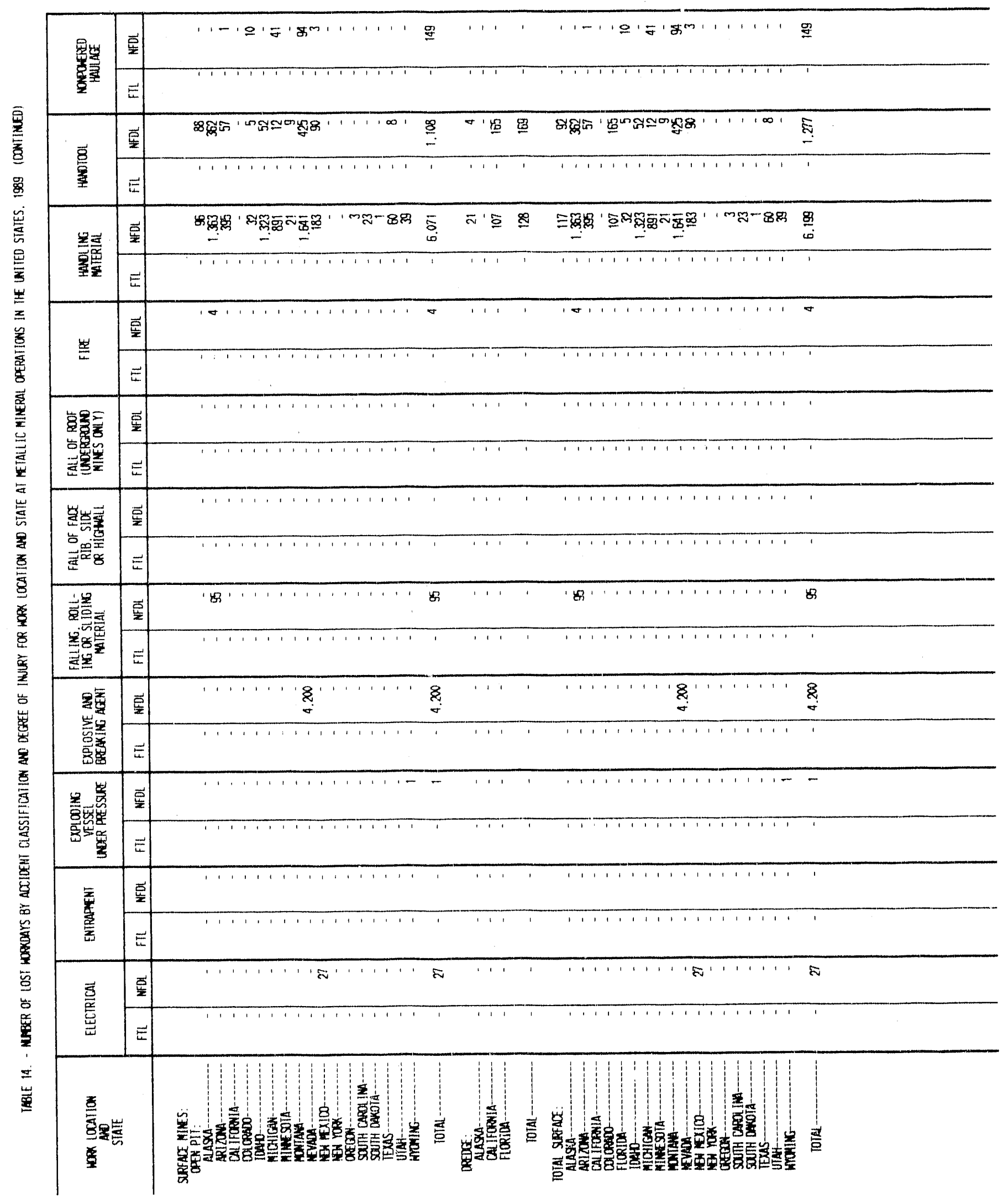




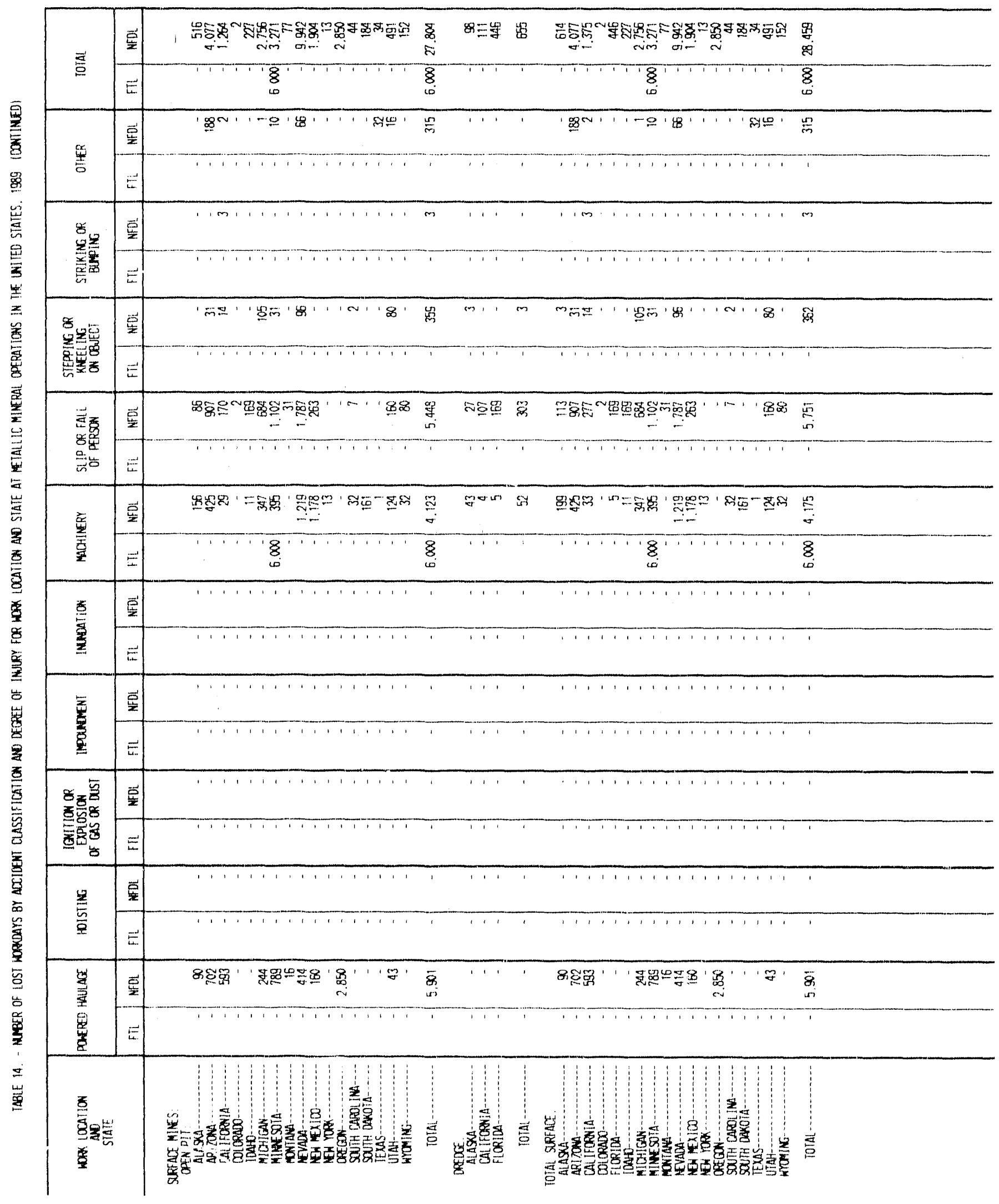




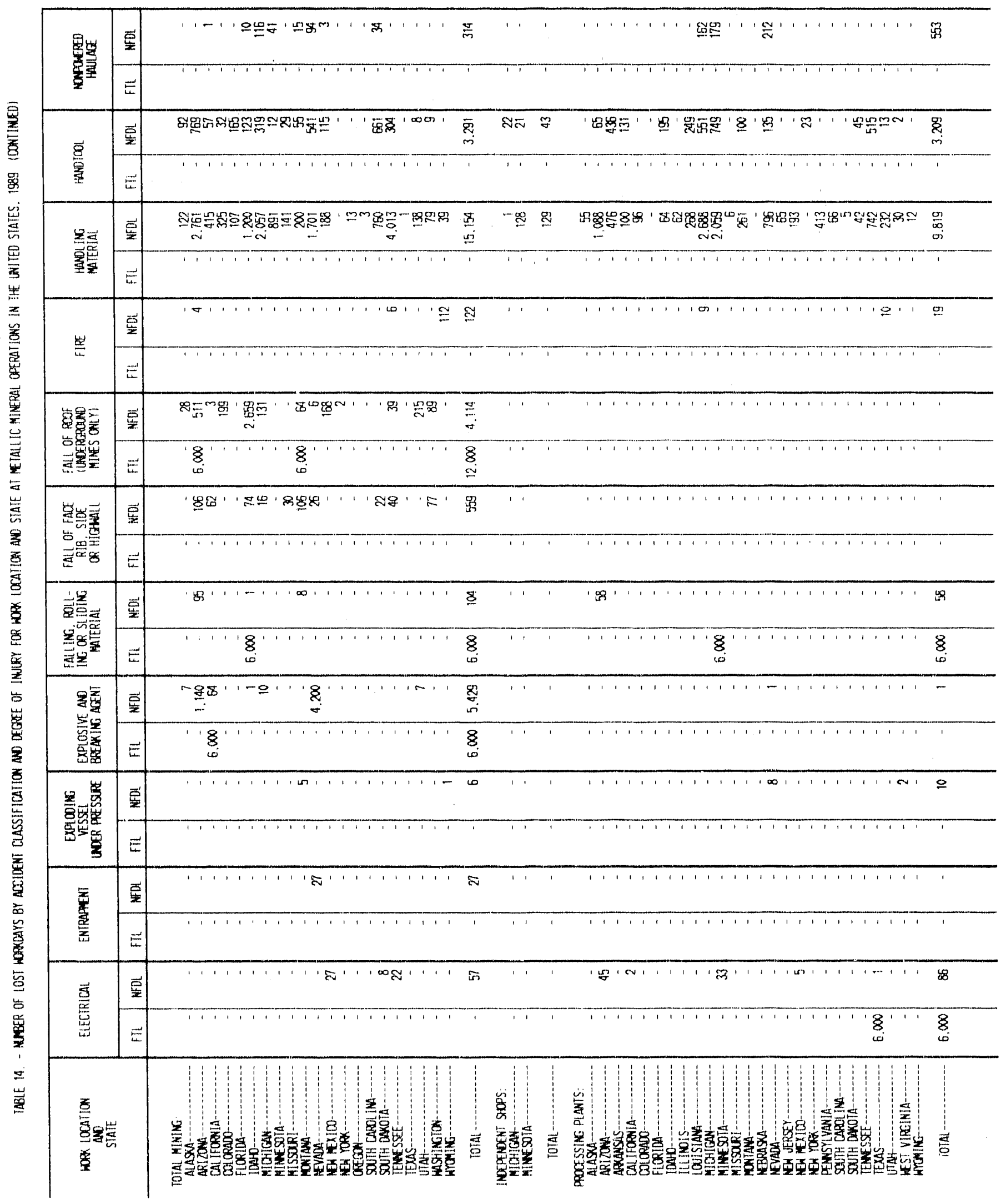




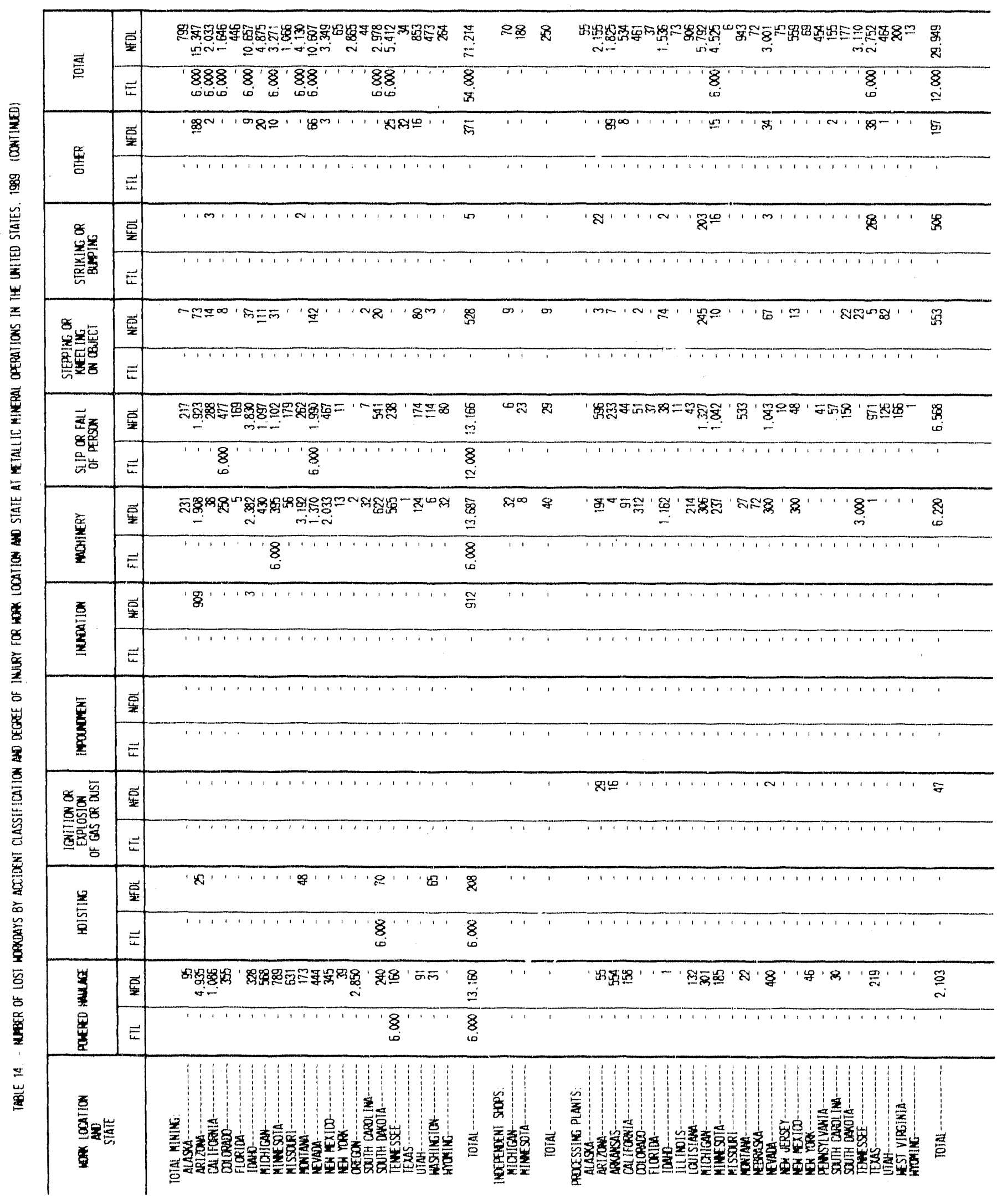




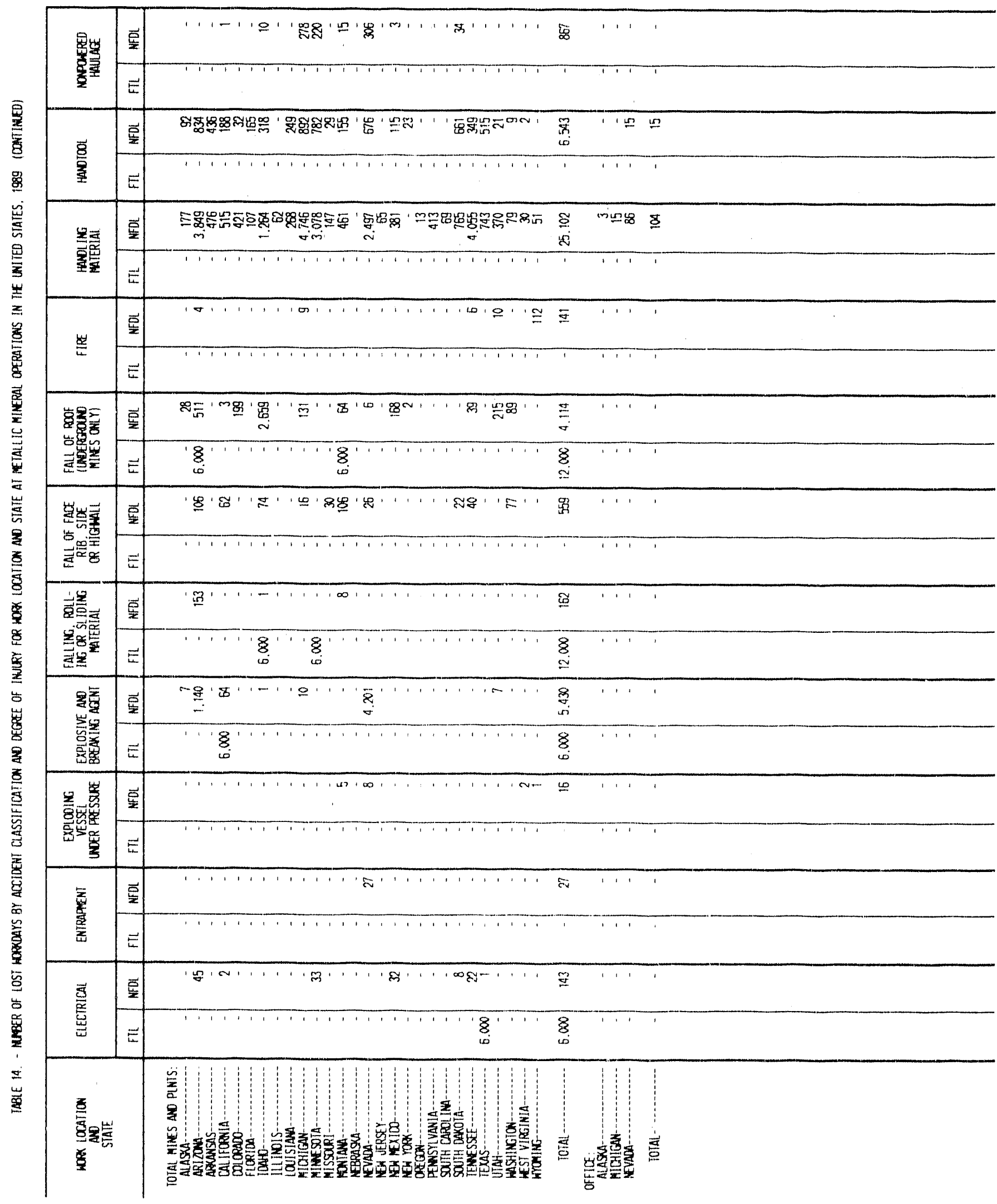




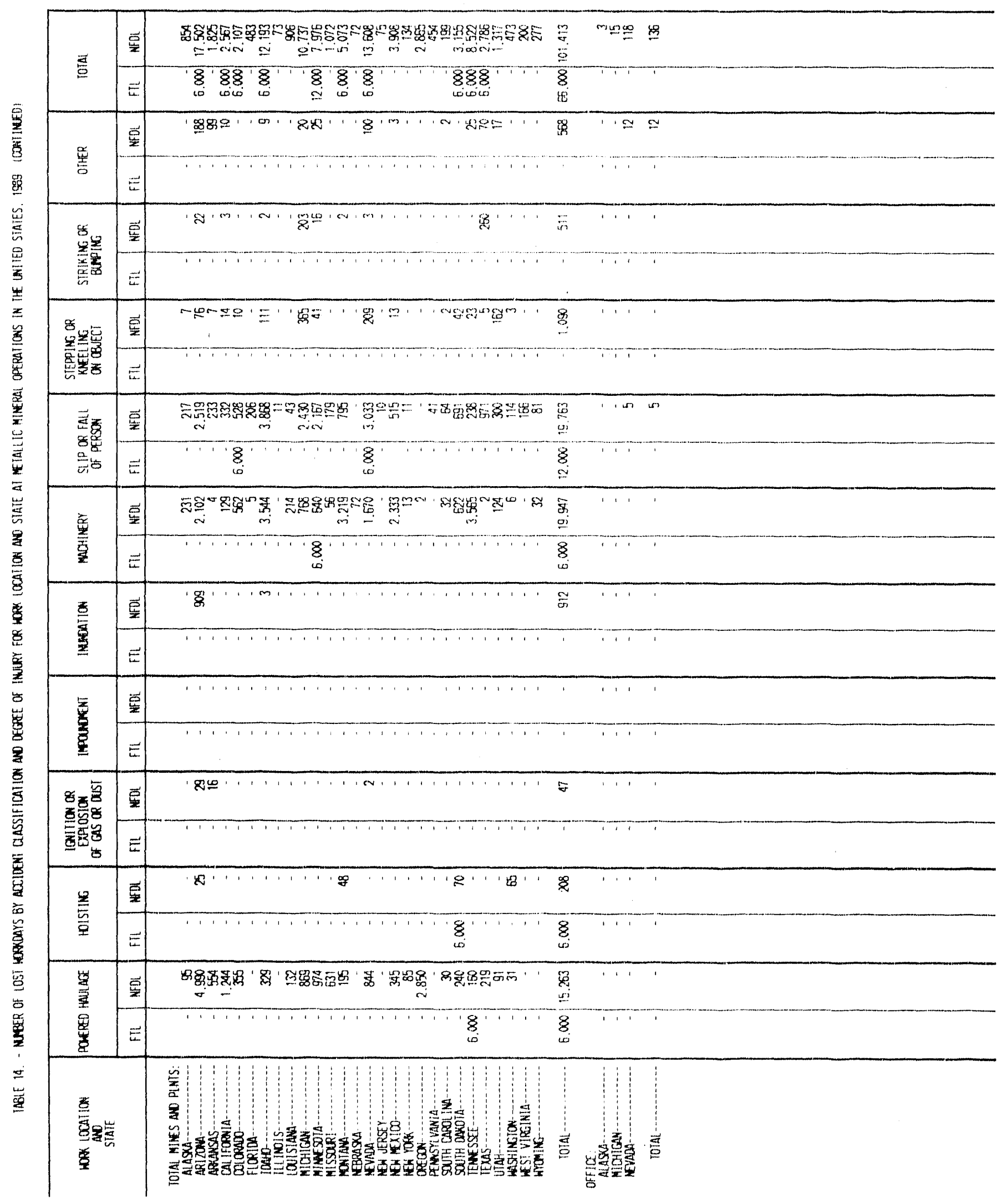




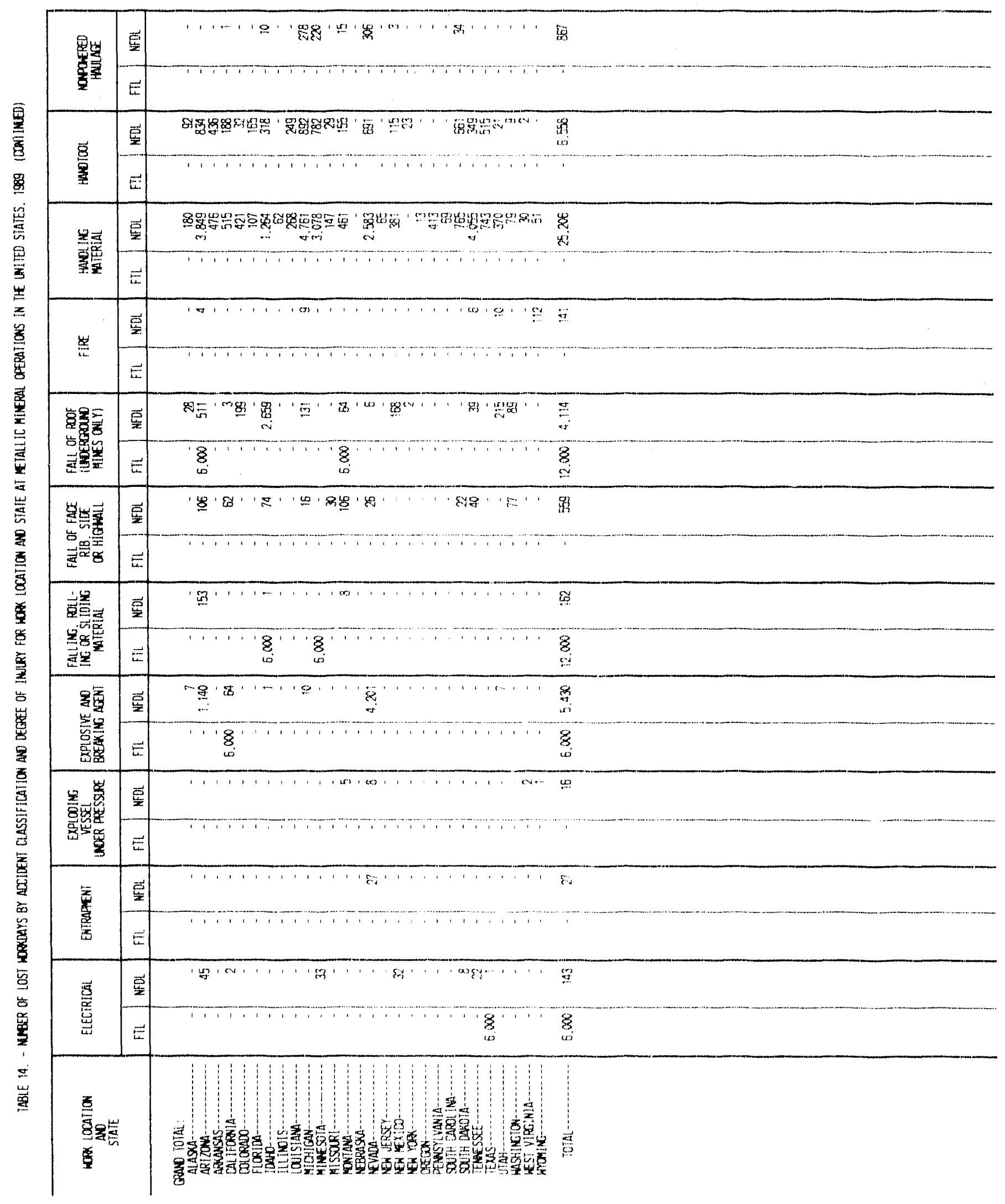




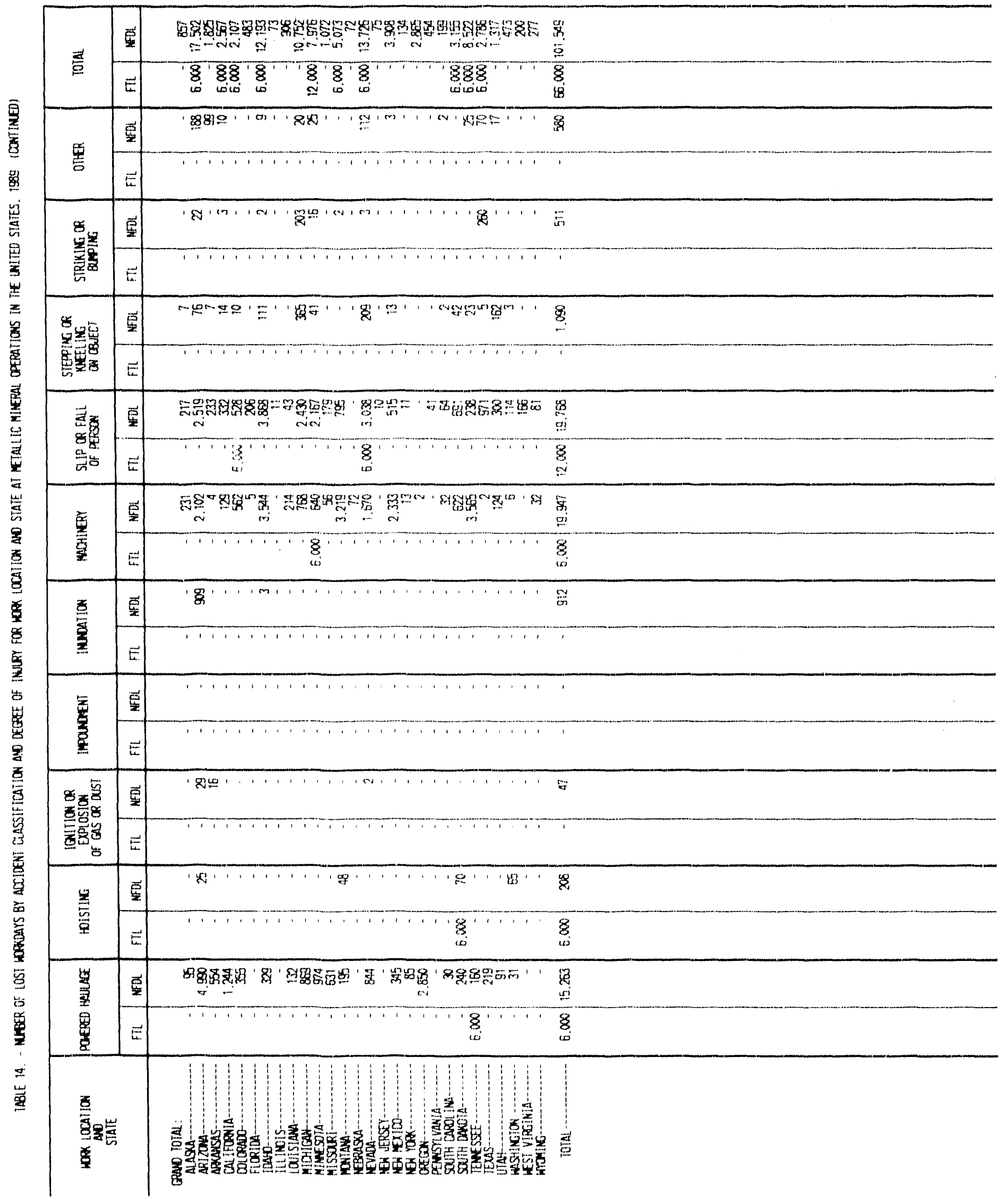




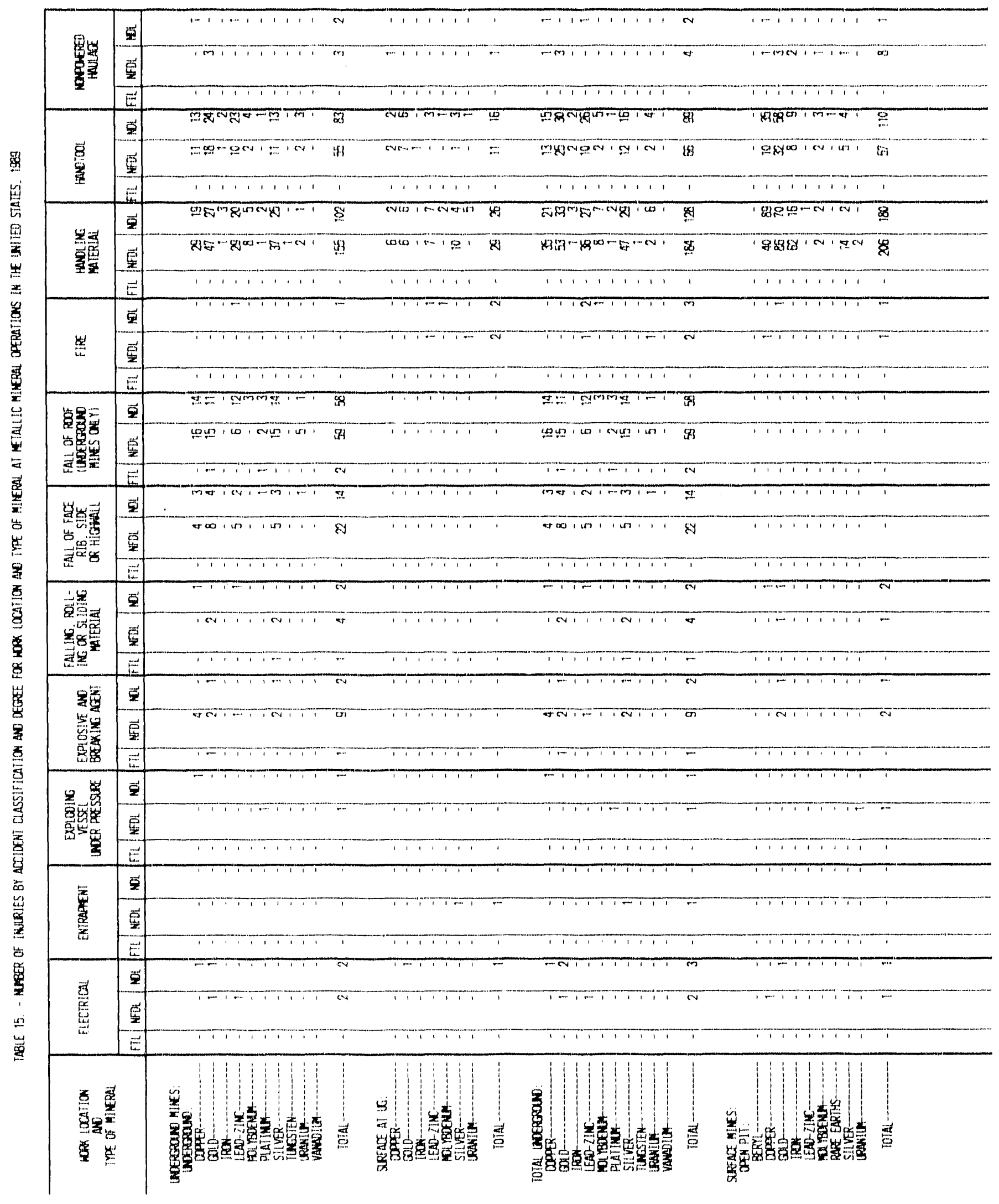




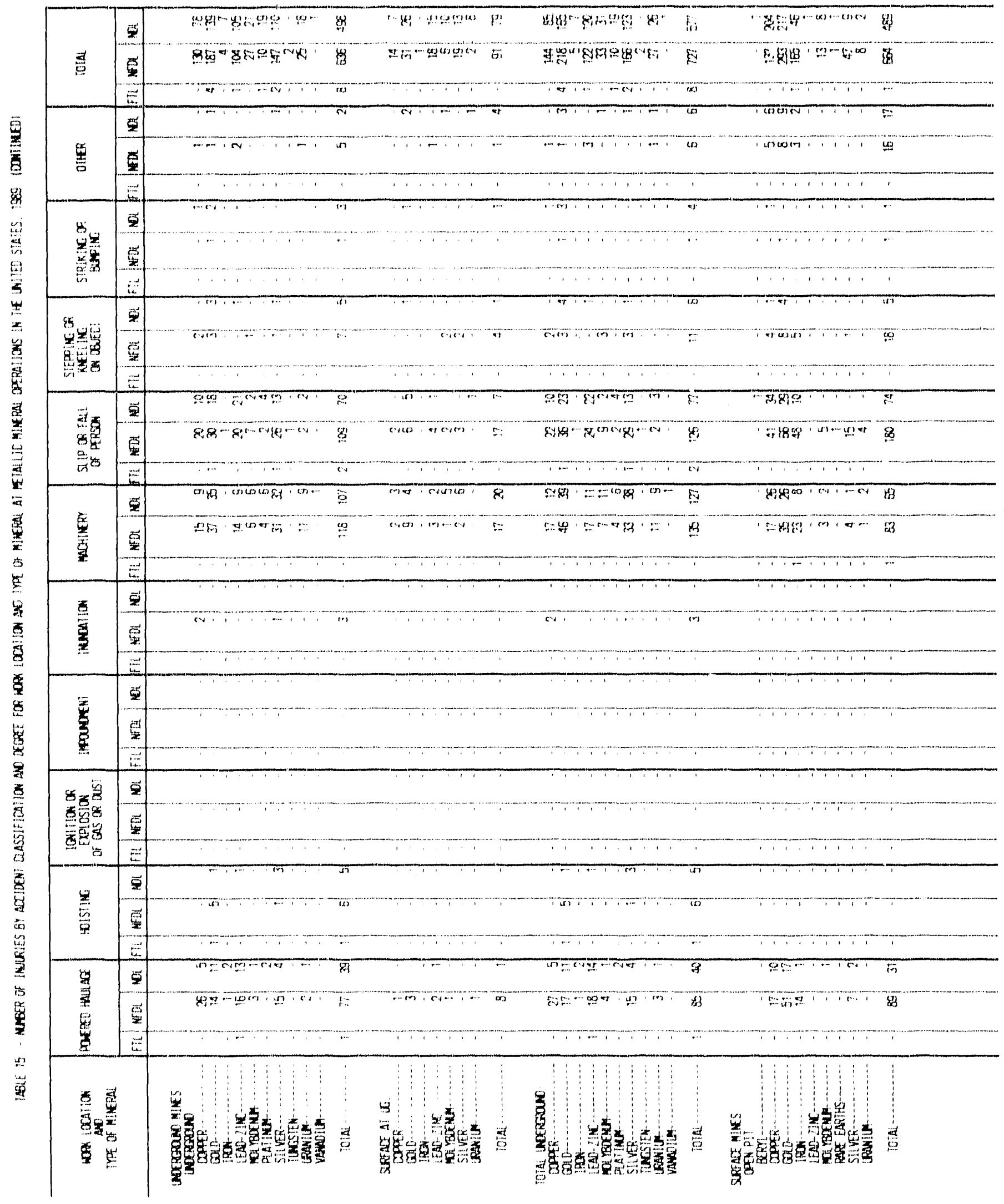




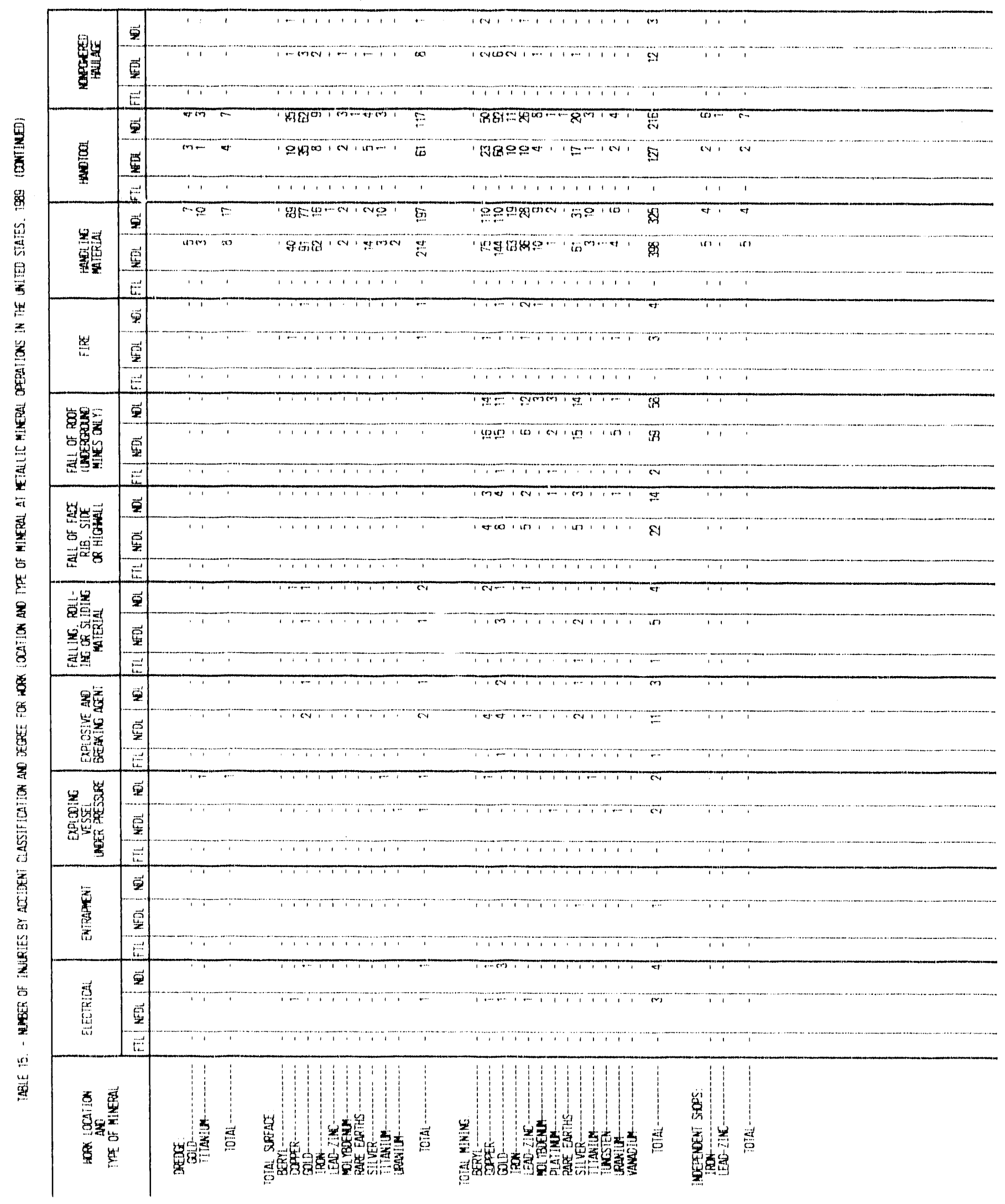




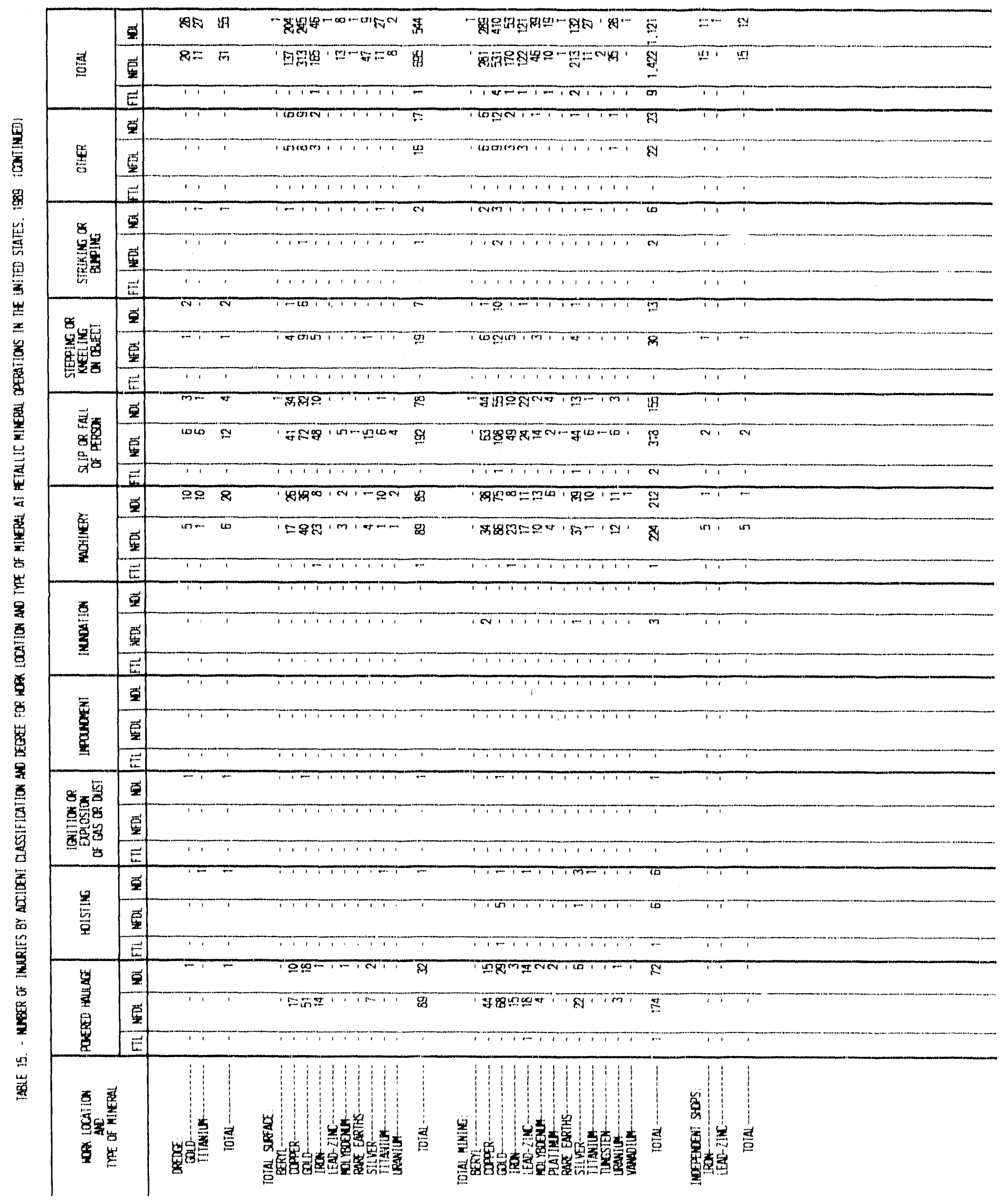




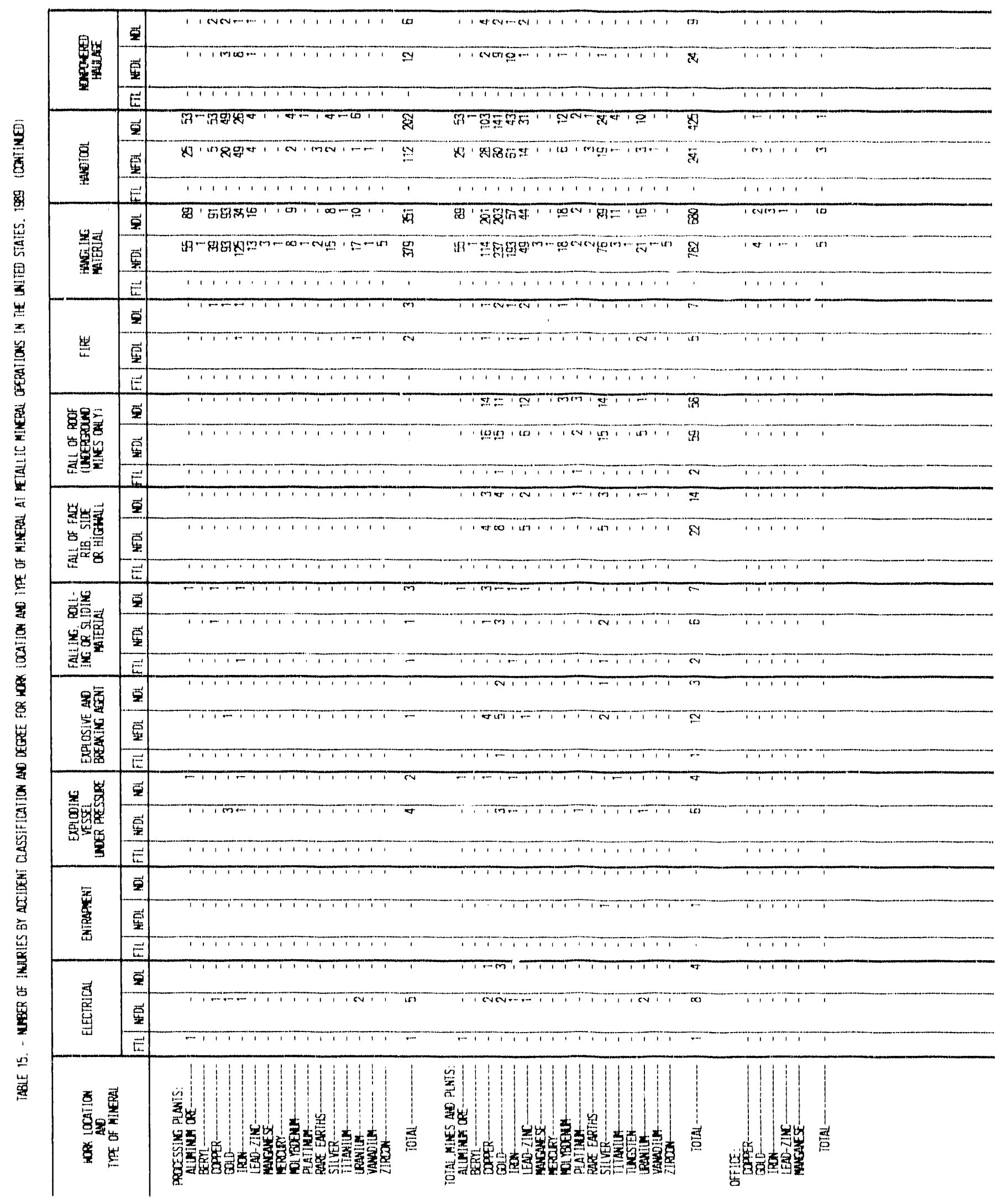




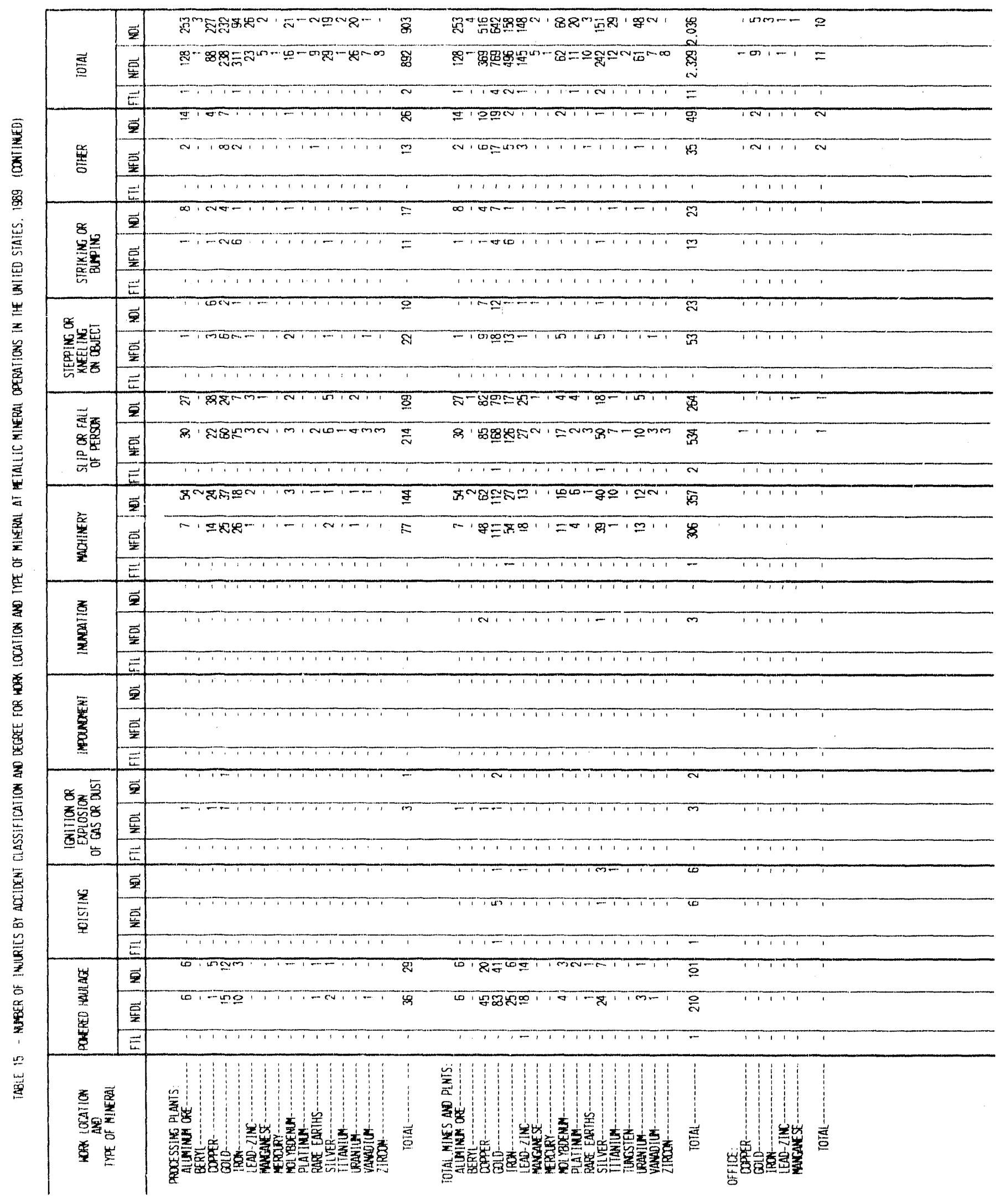




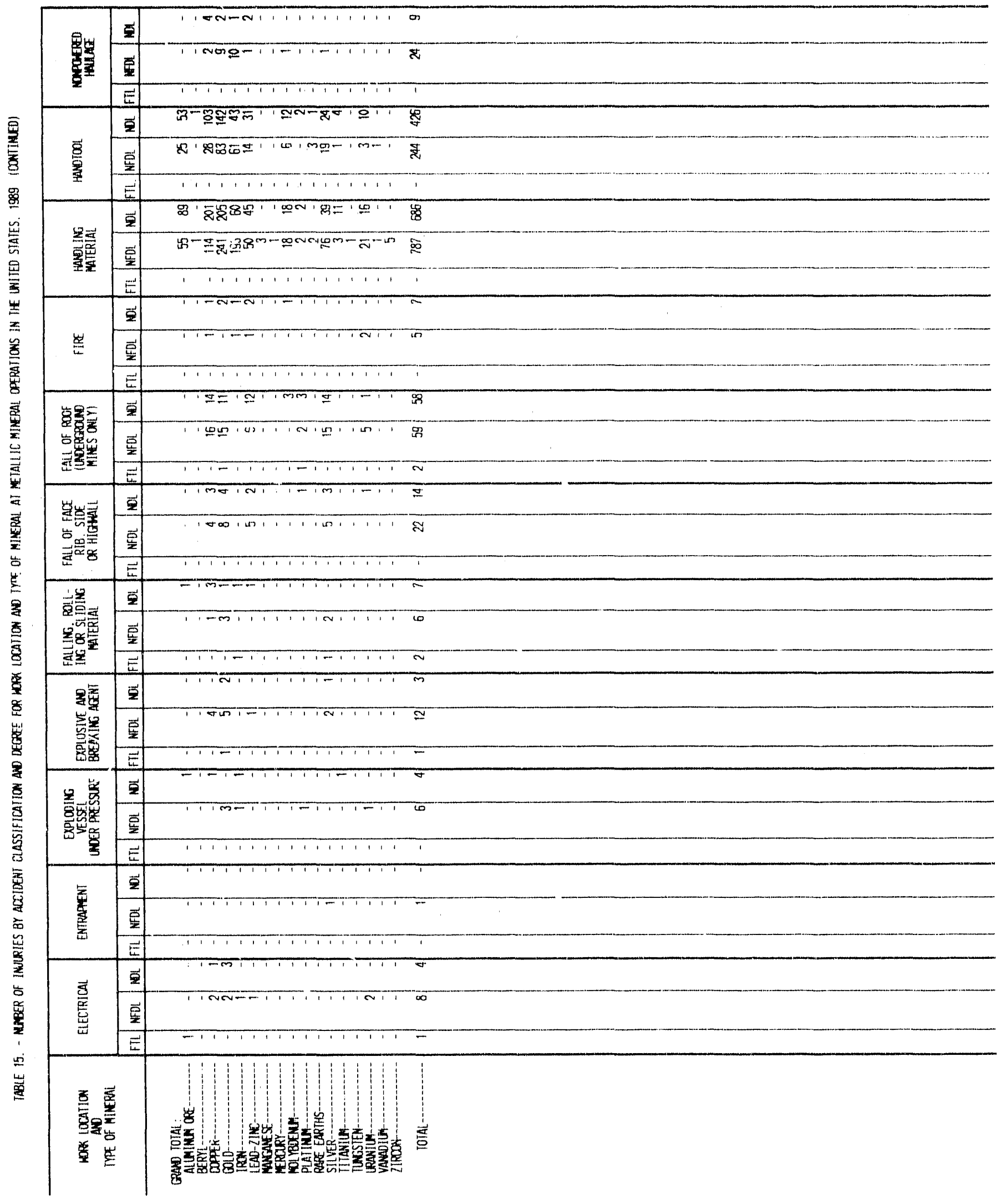




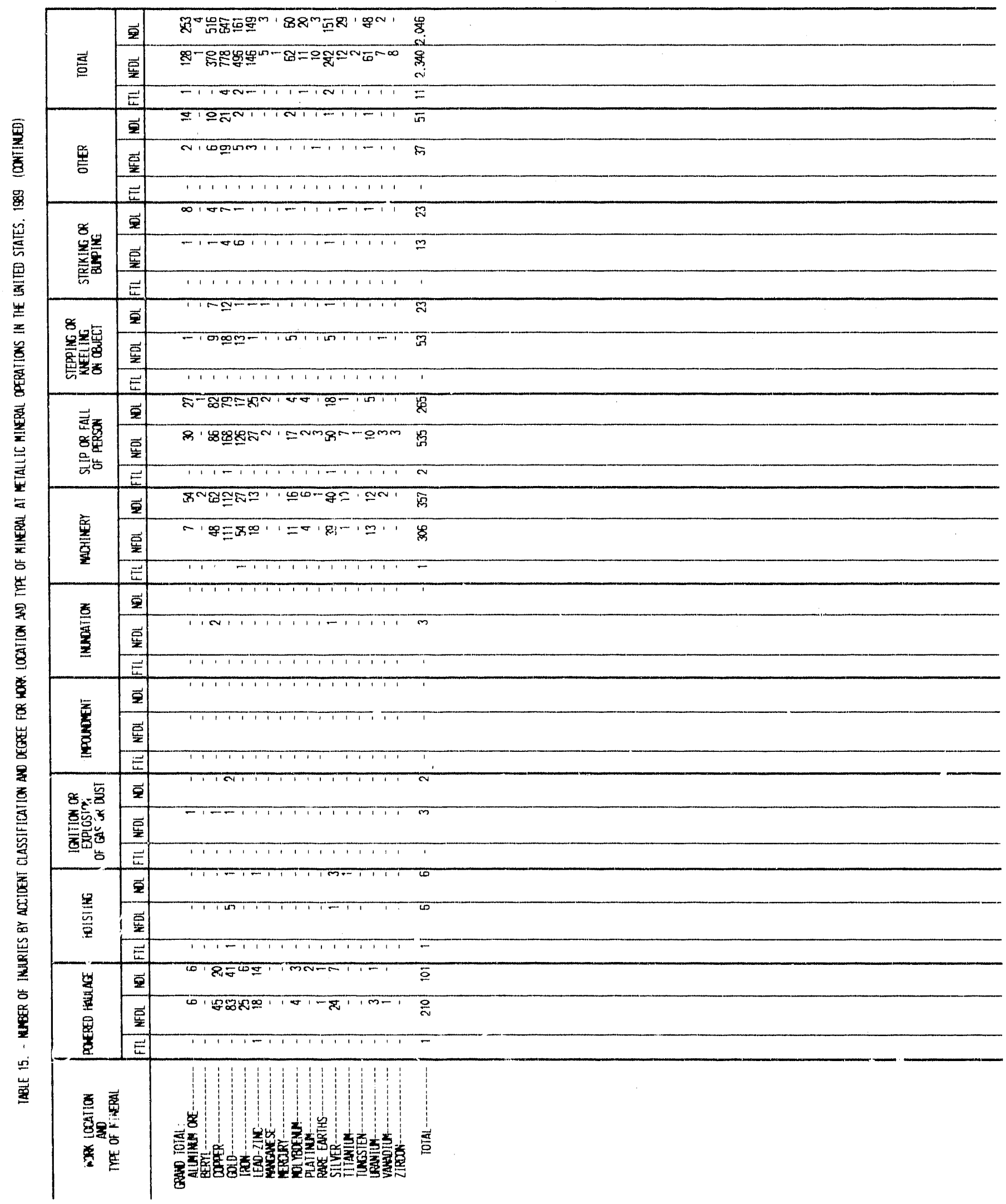




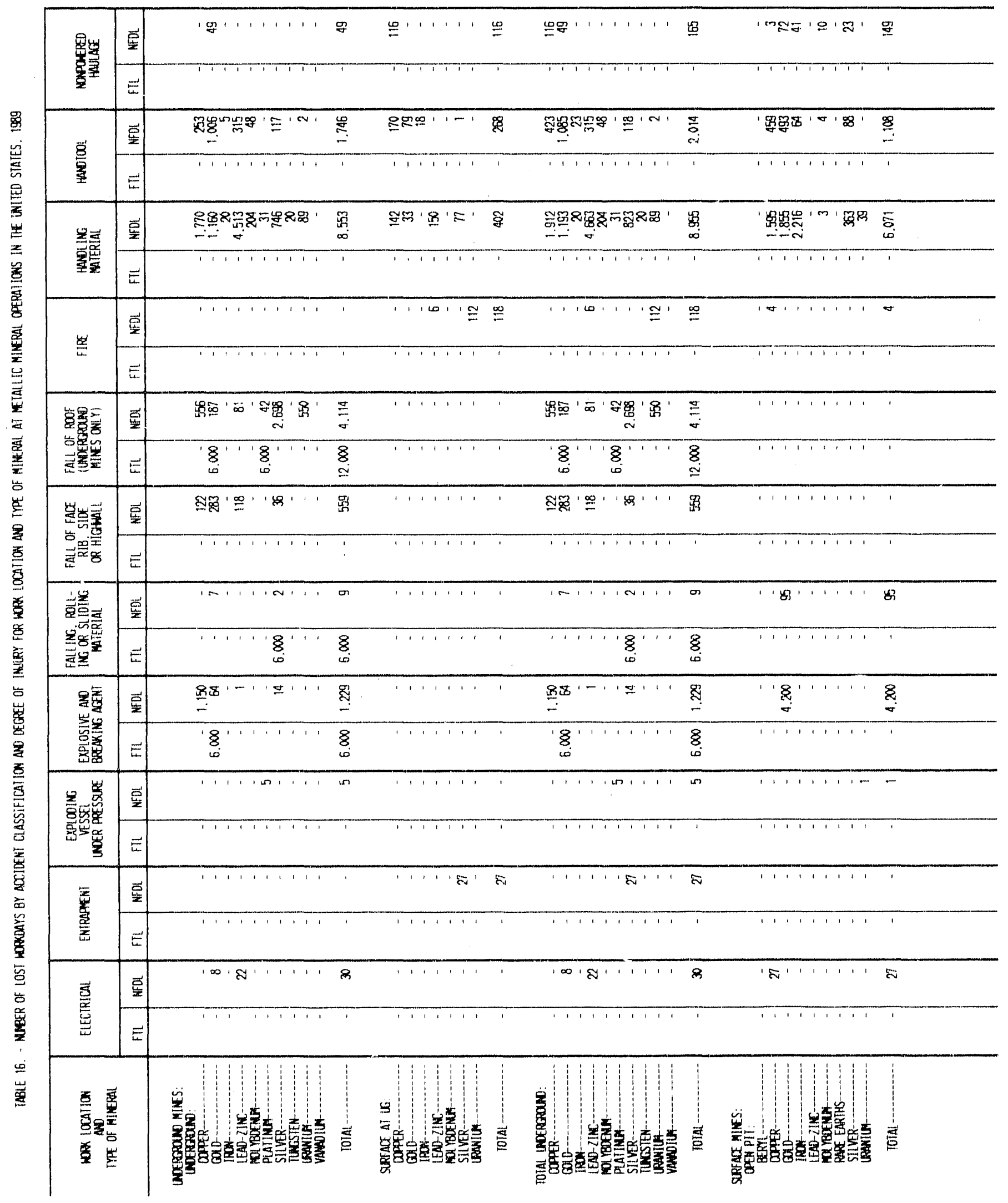




\begin{tabular}{|c|c|c|c|c|c|c|c|c|}
\hline & 㠓 & 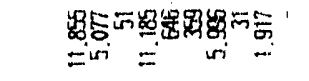 & 品 & 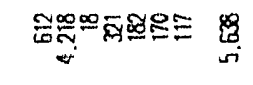 & 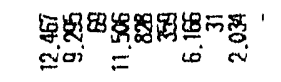 & $\underset{g}{\stackrel{g}{g}}$ & ' & $\underset{\&}{\mathbb{i}}$ \\
\hline 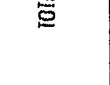 & $E$ & $\begin{array}{l}8 \\
\\
7\end{array}$ & 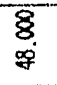 & & $\begin{array}{l}8 \\
8 \\
\text { ¿ }\end{array}$ & $\begin{array}{l}8 \\
\text { gag }\end{array}$ & $1 \underbrace{8}_{6}$ & $\underset{60}{8}$ \\
\hline \multirow{2}{*}{ 哭 } & 总 & $=\infty \cdot \boldsymbol{g}+1, \mathrm{~m}$ & 5 & $1,1,1$. & $=\infty, \Phi, \cdots, m$, & $\mathscr{R}$ & ' \$오임 ' ' ' ' ' ' & $\stackrel{\underline{m}}{m}$ \\
\hline & $F$ & 1, 1. & ' & 1 & & & & \\
\hline \multirow{2}{*}{ 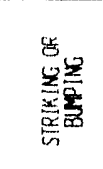 } & 릴 & w & $\sim$ & $\ldots \ldots$ & $n \ldots \ldots$ & $\sim$ & ' 'm....' & m \\
\hline & $=$ & '. & & ' & ' ' ' & & 11 & ' \\
\hline \multirow{2}{*}{ 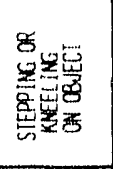 } & 产 & 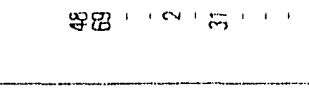 & $\$$ & ' ' ', & 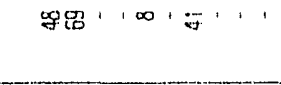 & 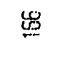 & 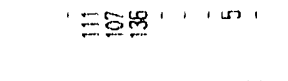 & 塄 \\
\hline & 光 & (1.1. & ' & ' & ' ' & ' & 1. & ' \\
\hline \multirow{2}{*}{ 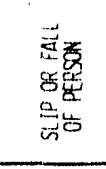 } & $\overrightarrow{\vec{z}}$ & 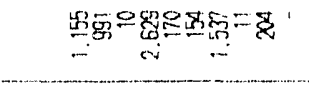 & $\begin{array}{l}\ddot{3} \\
10\end{array}$ & 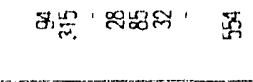 & 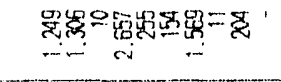 & $\stackrel{\frac{5}{5}}{=}$ & 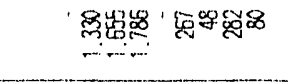 & 粱 \\
\hline & $\vec{a}$ & 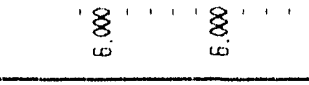 & 8 & ' ' ' & 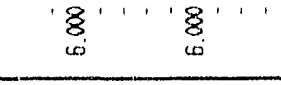 & $\stackrel{8}{8}$ & 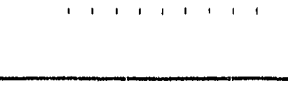 & \\
\hline \multirow{2}{*}{$\begin{array}{l}\text { 焉 } \\
\text { 营 }\end{array}$} & 产 & 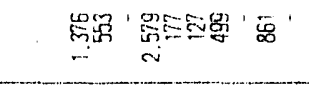 & $\stackrel{c}{c}$ & 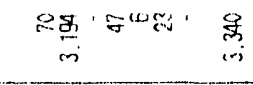 & 紫 ' & 总 & 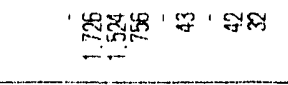 & $\underset{\sim}{\stackrel{\sim}{+}}$ \\
\hline & 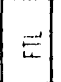 & $\ldots \ldots \ldots$ & & $\cdots$ & , & & 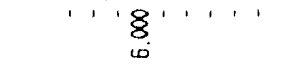 & $\begin{array}{c}8 \\
0 \\
0\end{array}$ \\
\hline \multirow{2}{*}{ 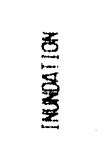 } & 竞 & 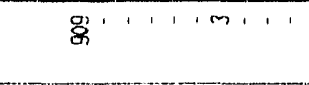 & $\check{\sigma}$ & & 罯' ' & $\cong$ & & 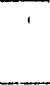 \\
\hline & $\equiv$ & & & & & & & \\
\hline \multirow{2}{*}{ 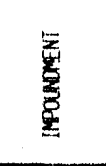 } & 总 & 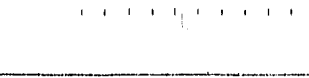 & ' & 1,1 & '. '. & ' & $11+1+1+1+1$ & ' \\
\hline & $E$ & & & & & & & \\
\hline \multirow{2}{*}{ 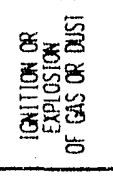 } & 帝 & 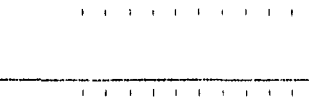 & & & & ' & , 1 & \\
\hline & 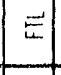 & & & & & & & \\
\hline \multirow{2}{*}{$\frac{2}{5}$} & 总 & 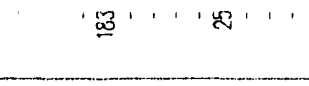 & 嵒 & 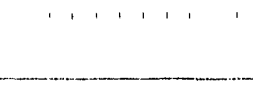 & 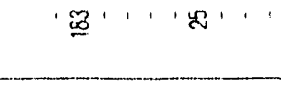 & 蛋 & ' ' ' & ' \\
\hline & $E$ & '冬' & $\underset{6}{8}$ & $1 \cdots$ & $\begin{array}{l}8 \\
0 \\
0\end{array}$ & $\underset{6}{8}$ & 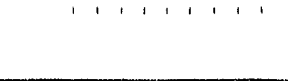 & ' \\
\hline \multirow{2}{*}{ 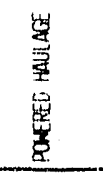 } & 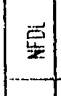 & 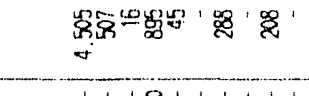 & 亦 & 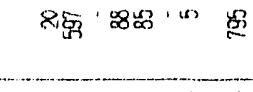 & 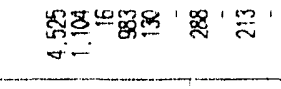 & $\stackrel{9}{g}$ & 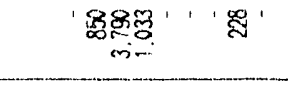 & in \\
\hline & $E$ & 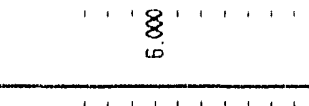 & $\underset{6}{8}$ & $1,1,1$ & ' ' ' & $\underbrace{8}_{6}$ & $1,1,1$, & ' \\
\hline \multicolumn{2}{|c|}{ 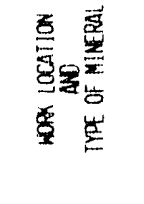 } & 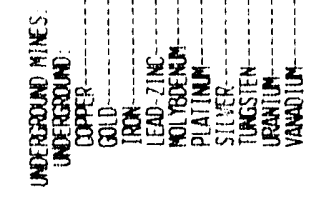 & 咅 & 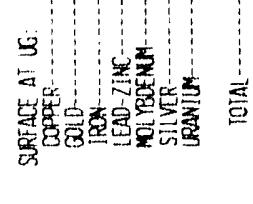 & 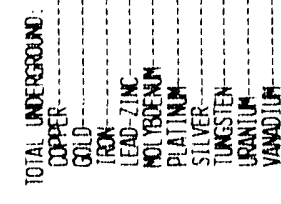 & 运 & 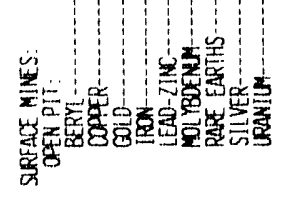 & s. \\
\hline
\end{tabular}




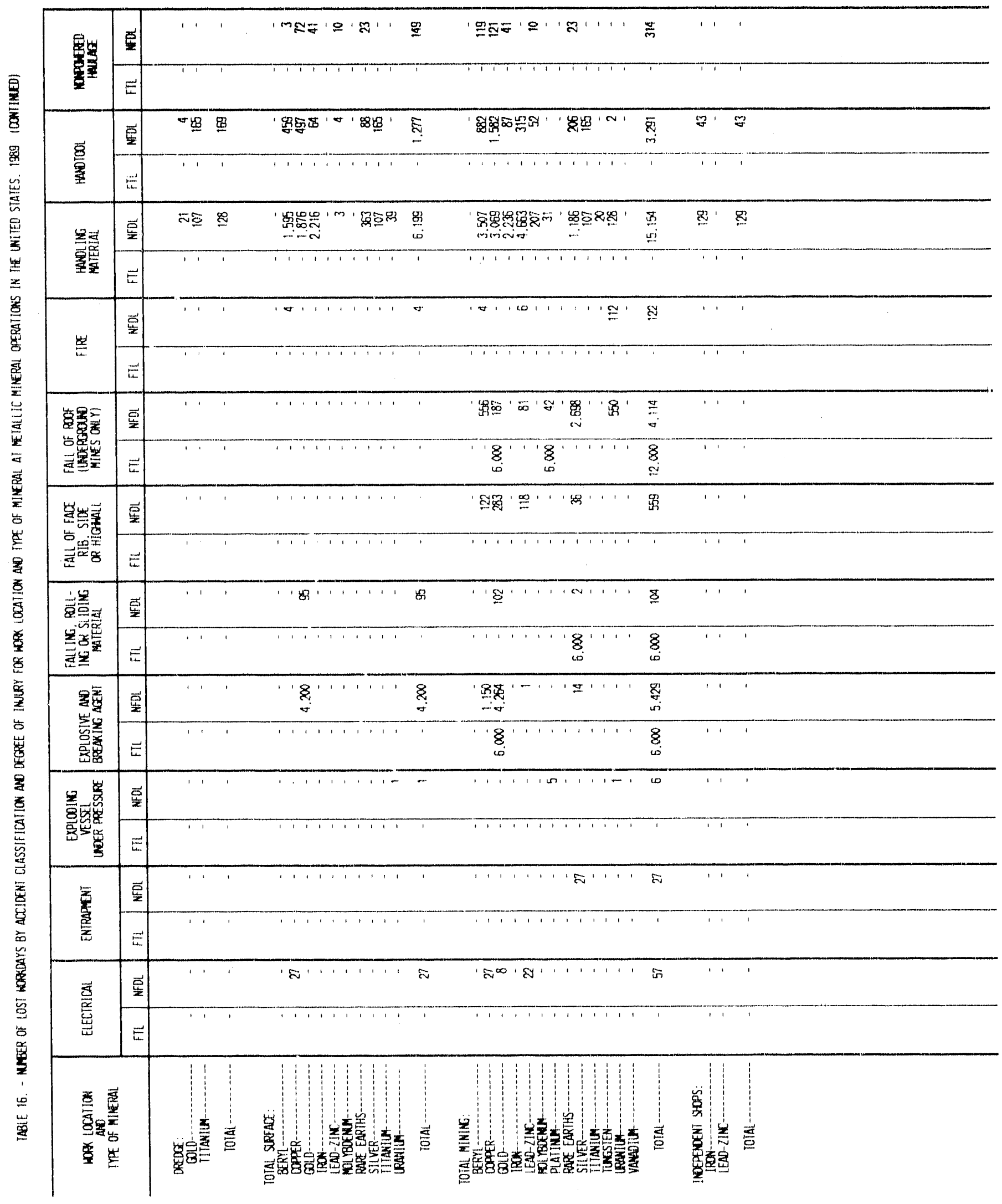




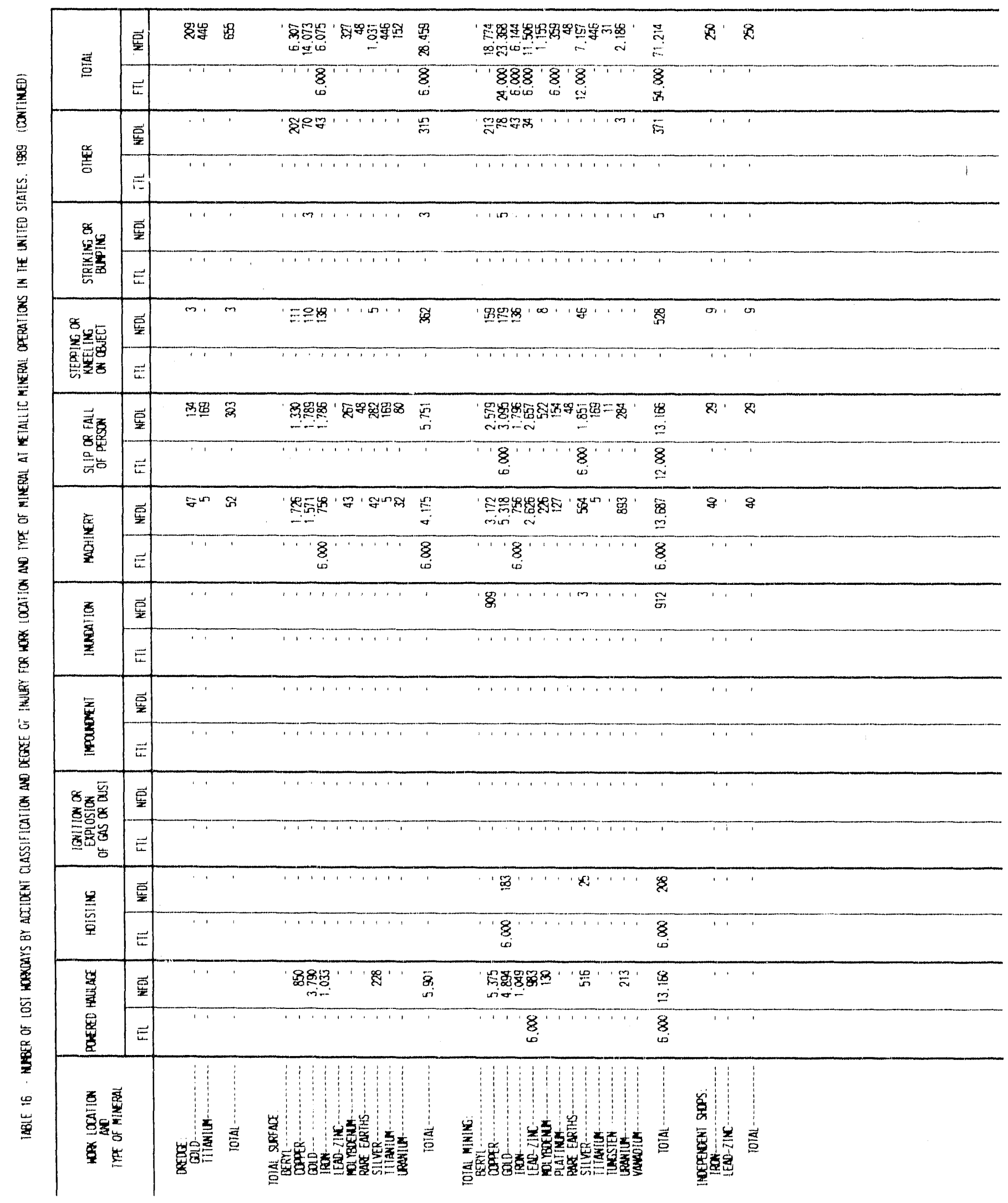




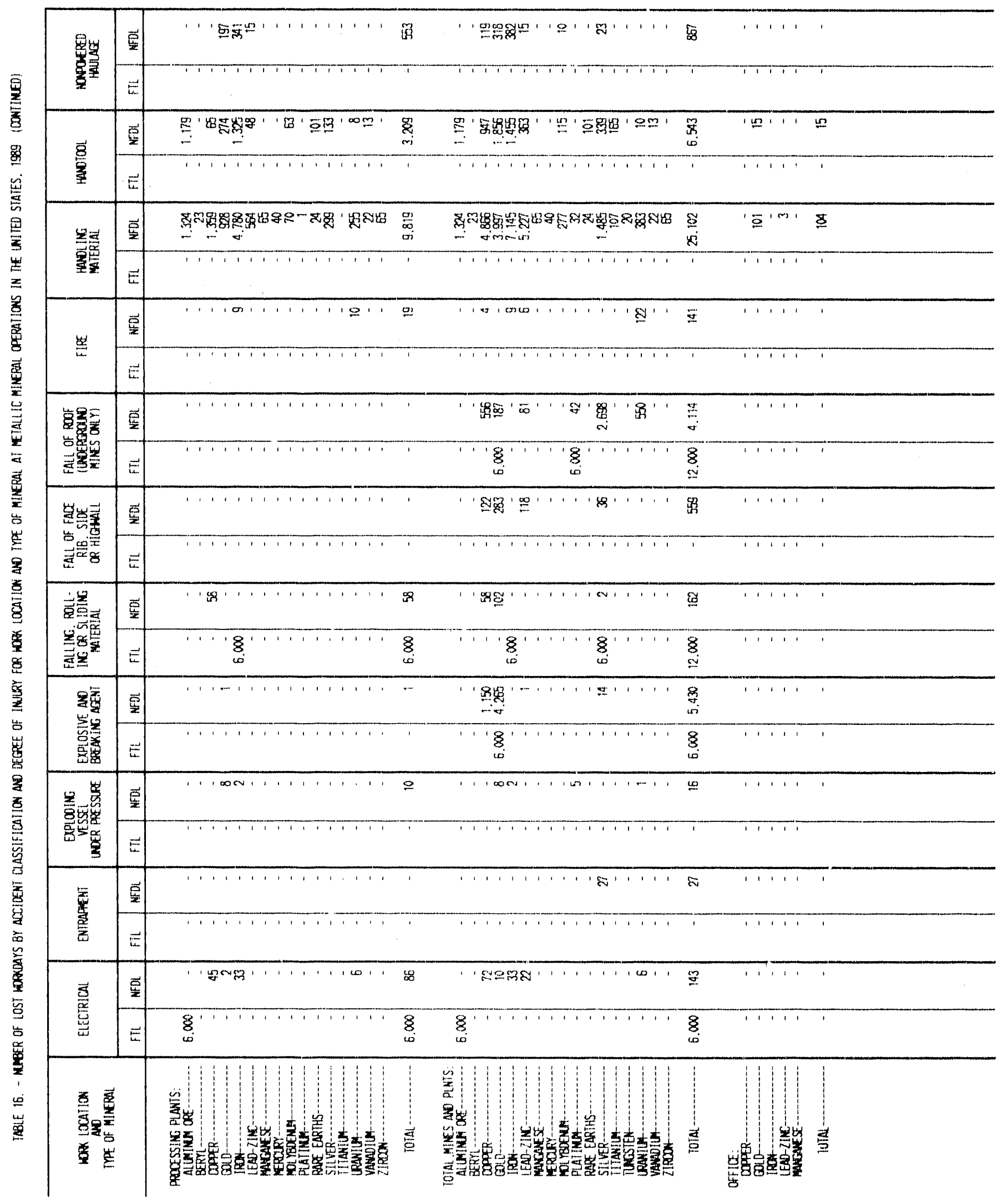




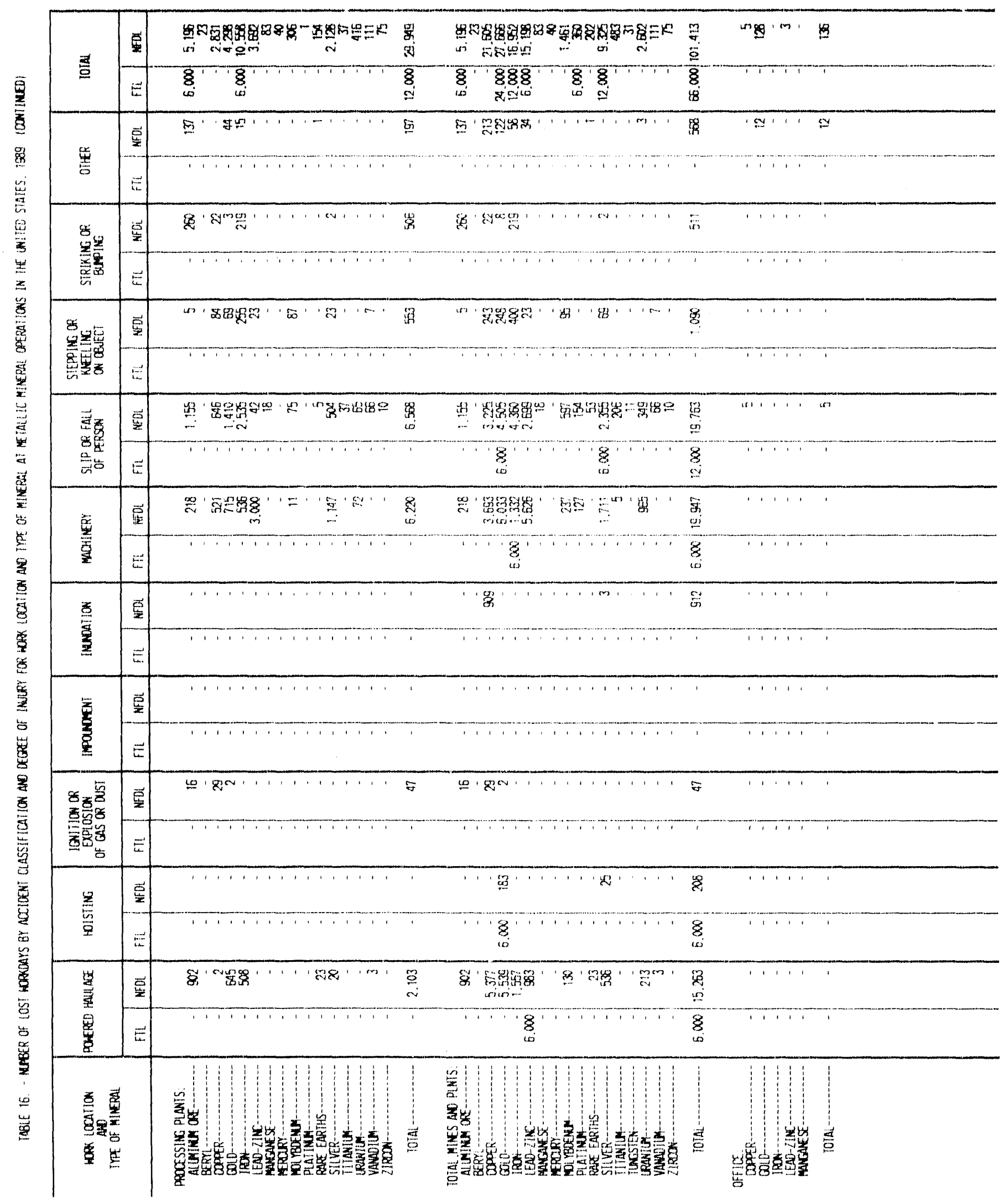




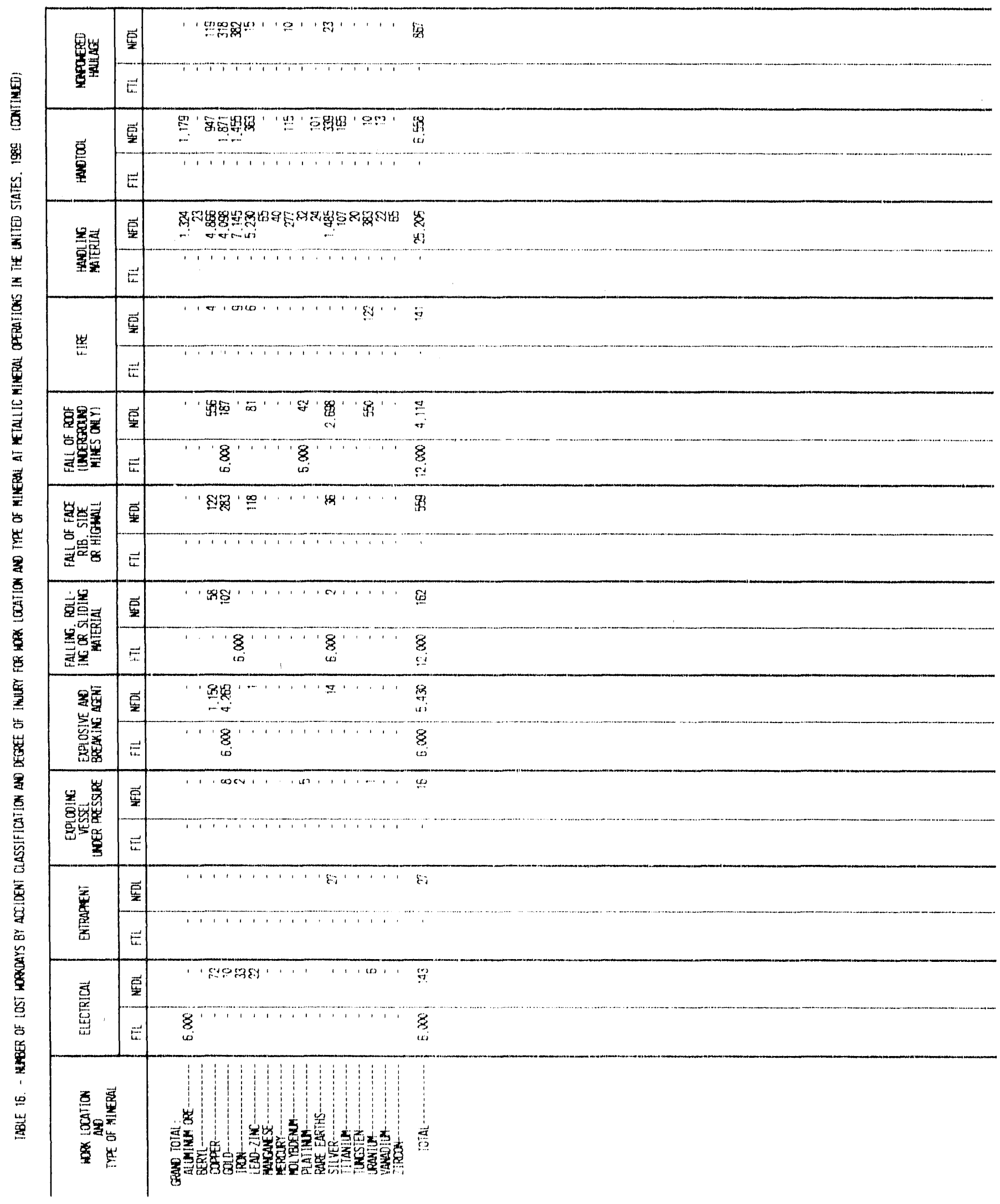




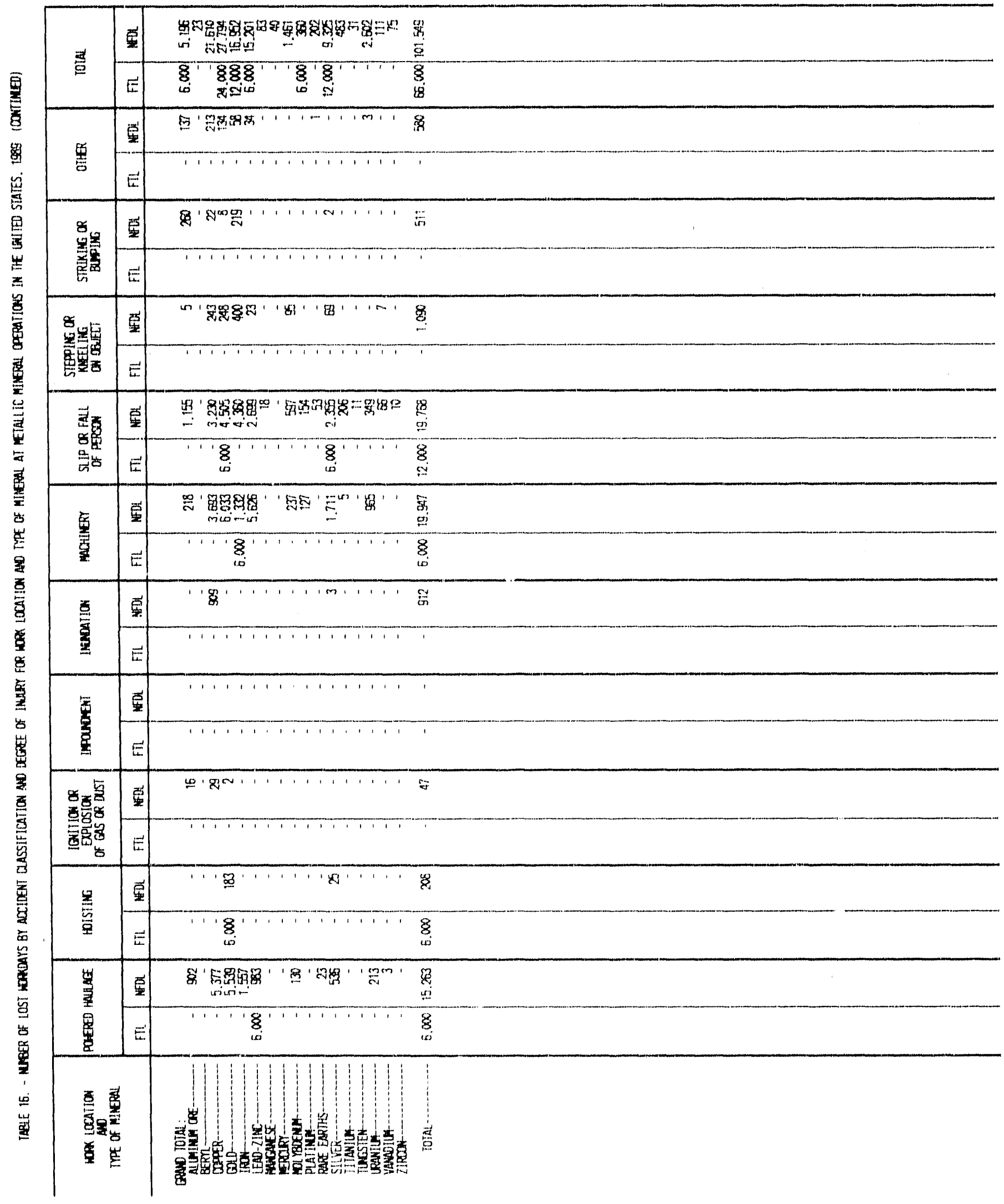




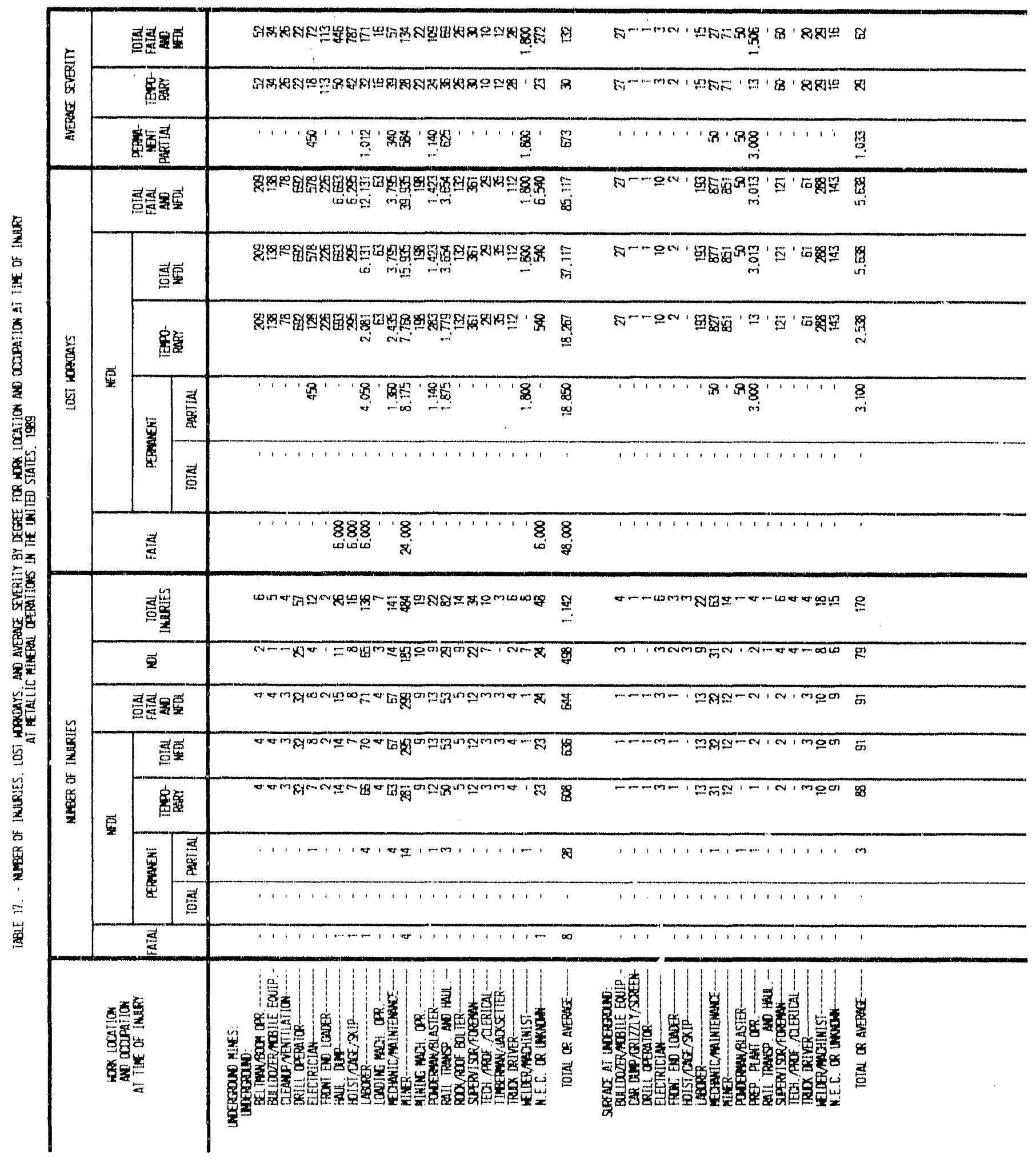




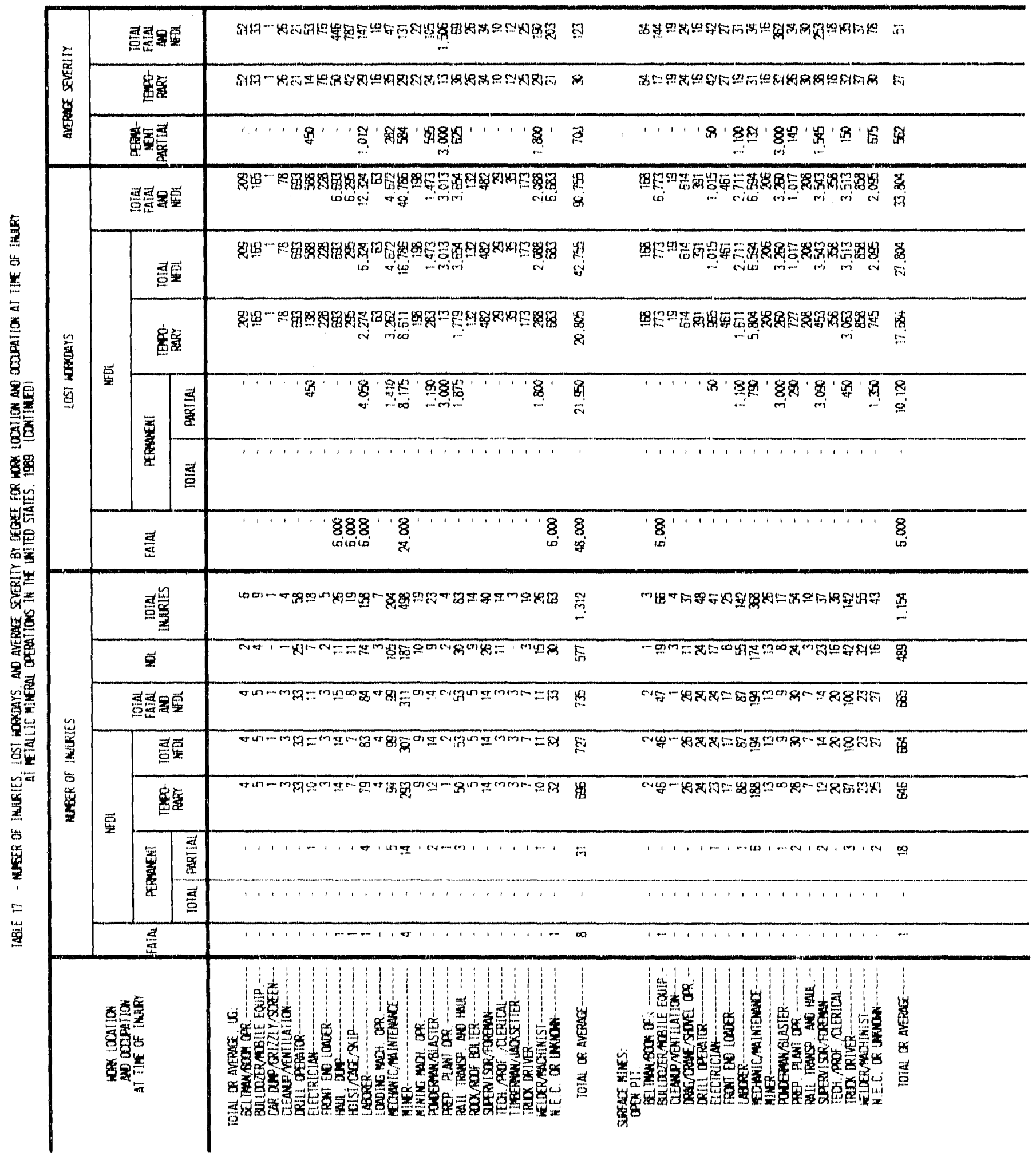




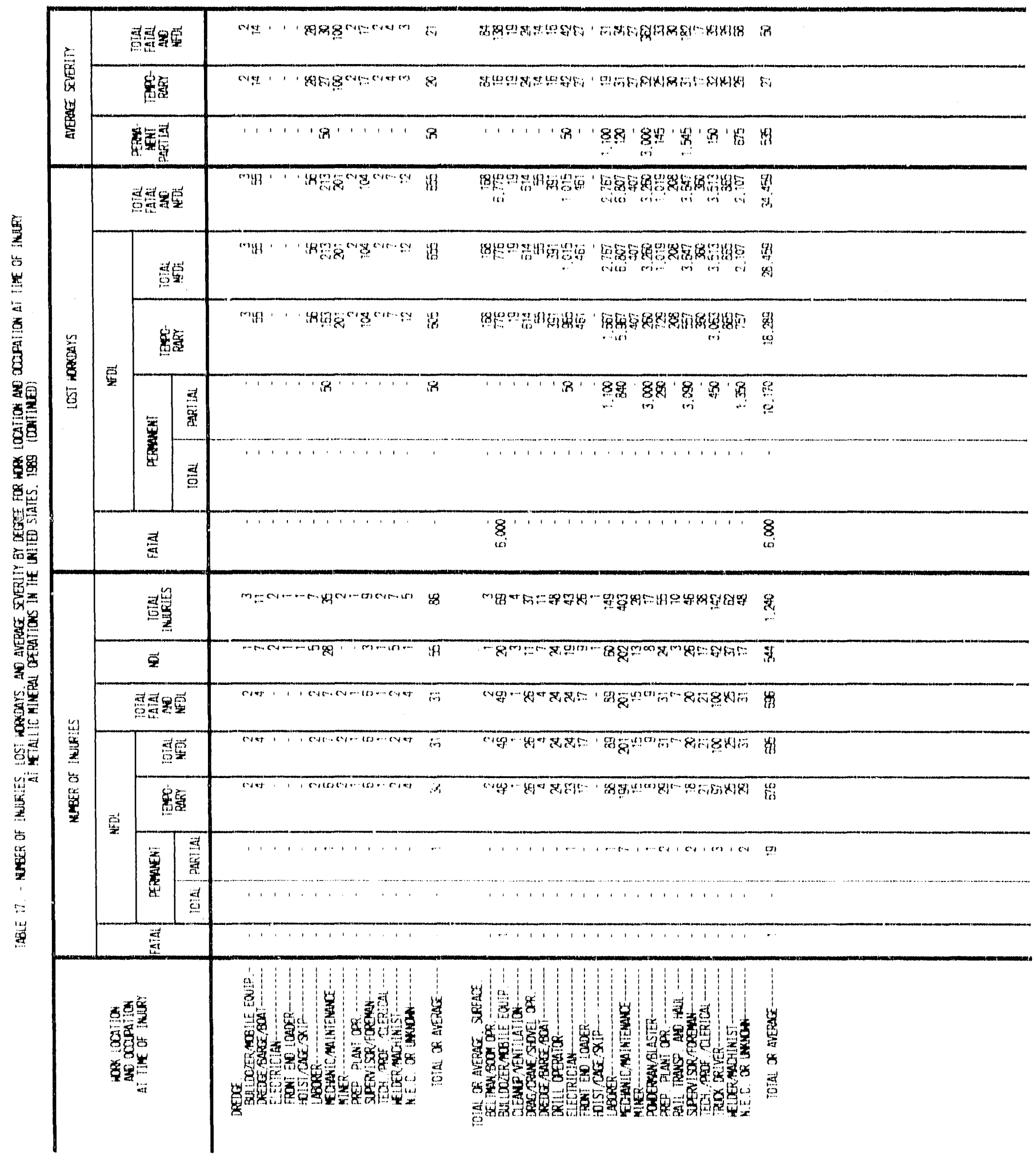




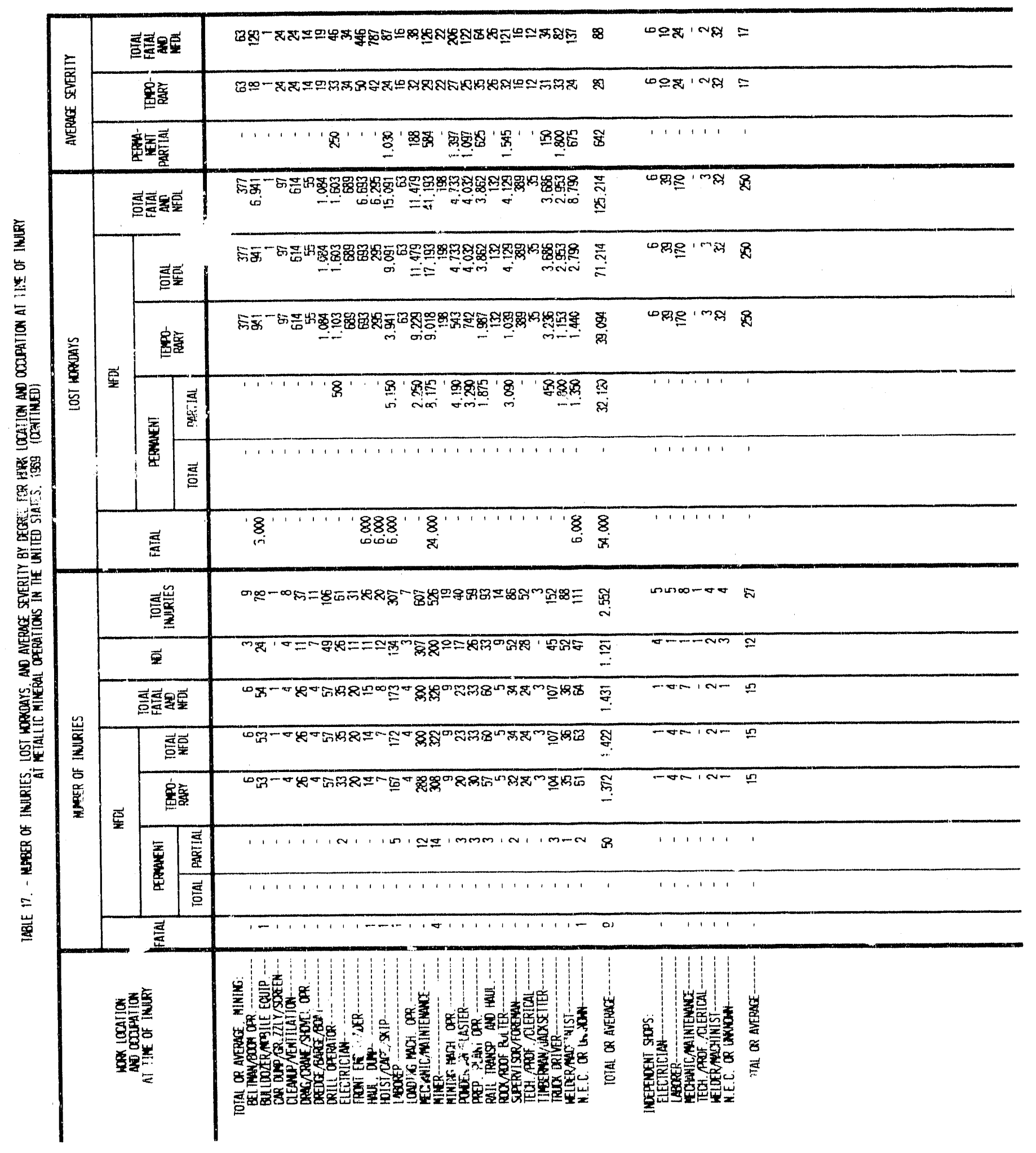

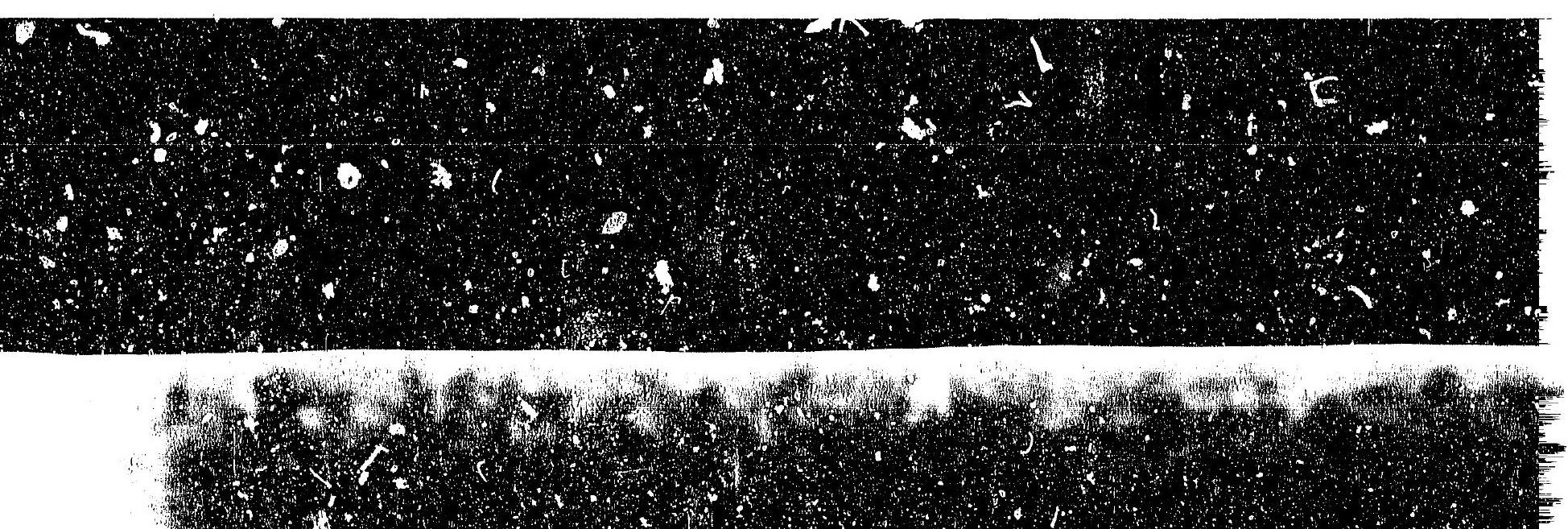




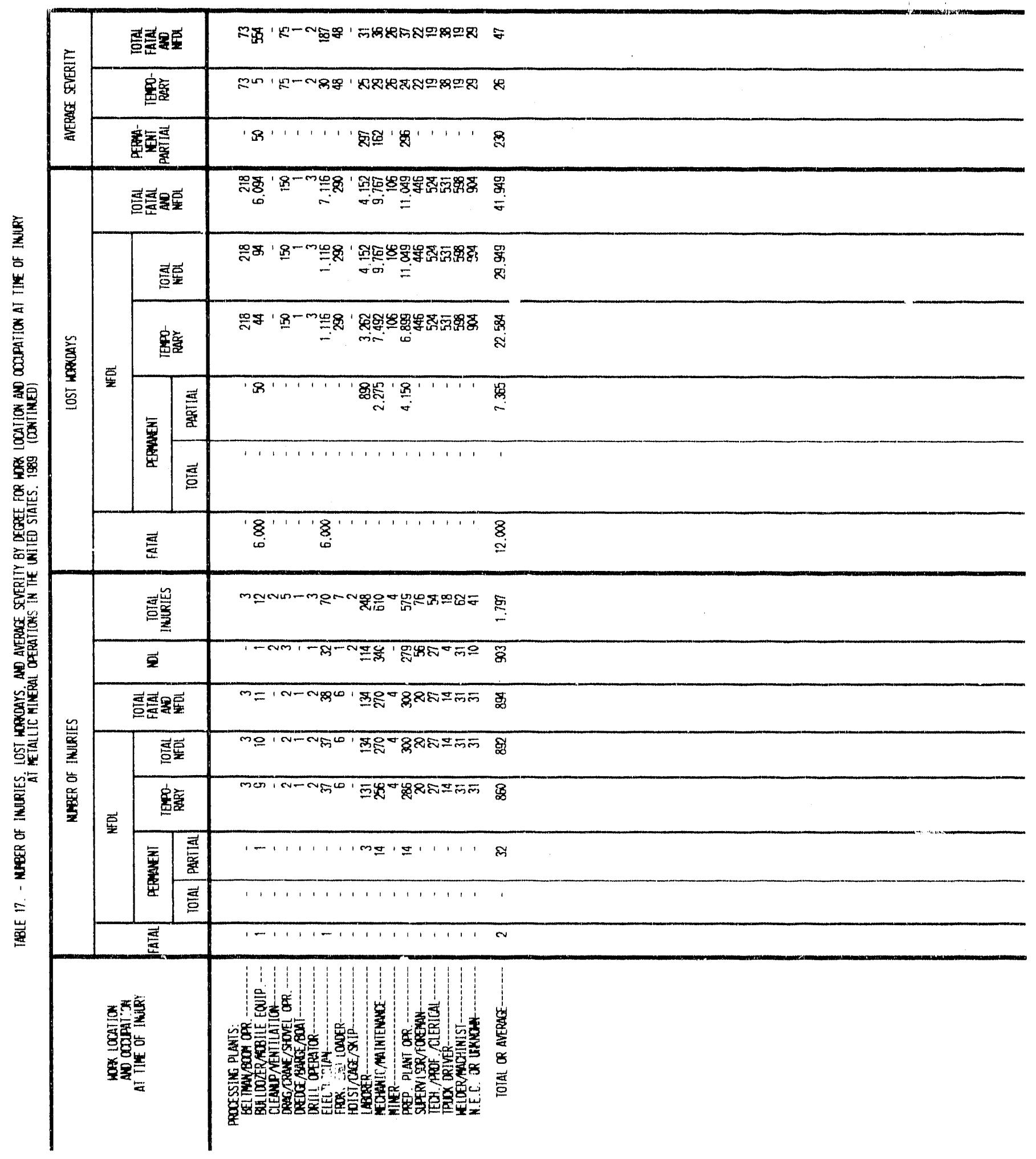




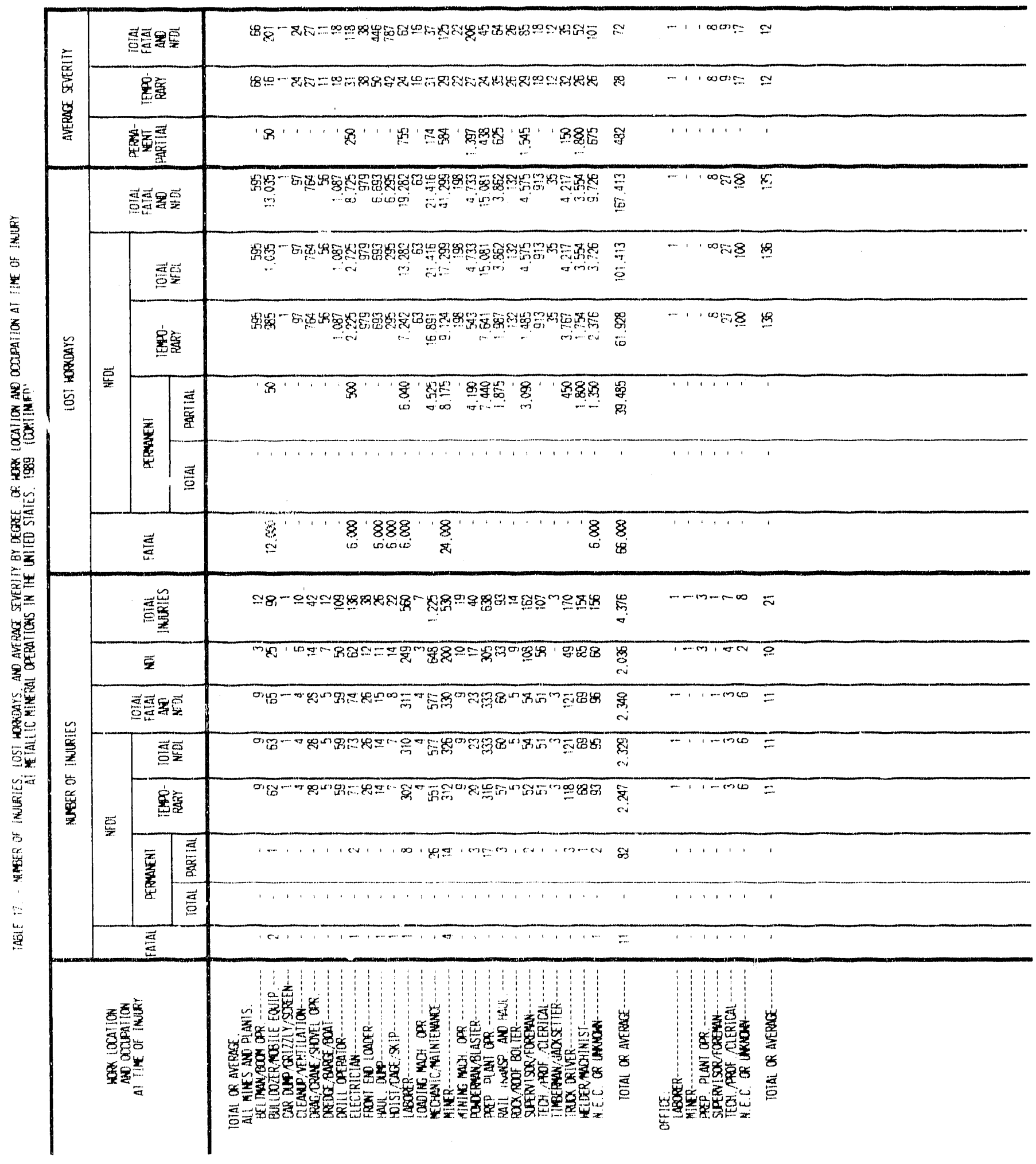




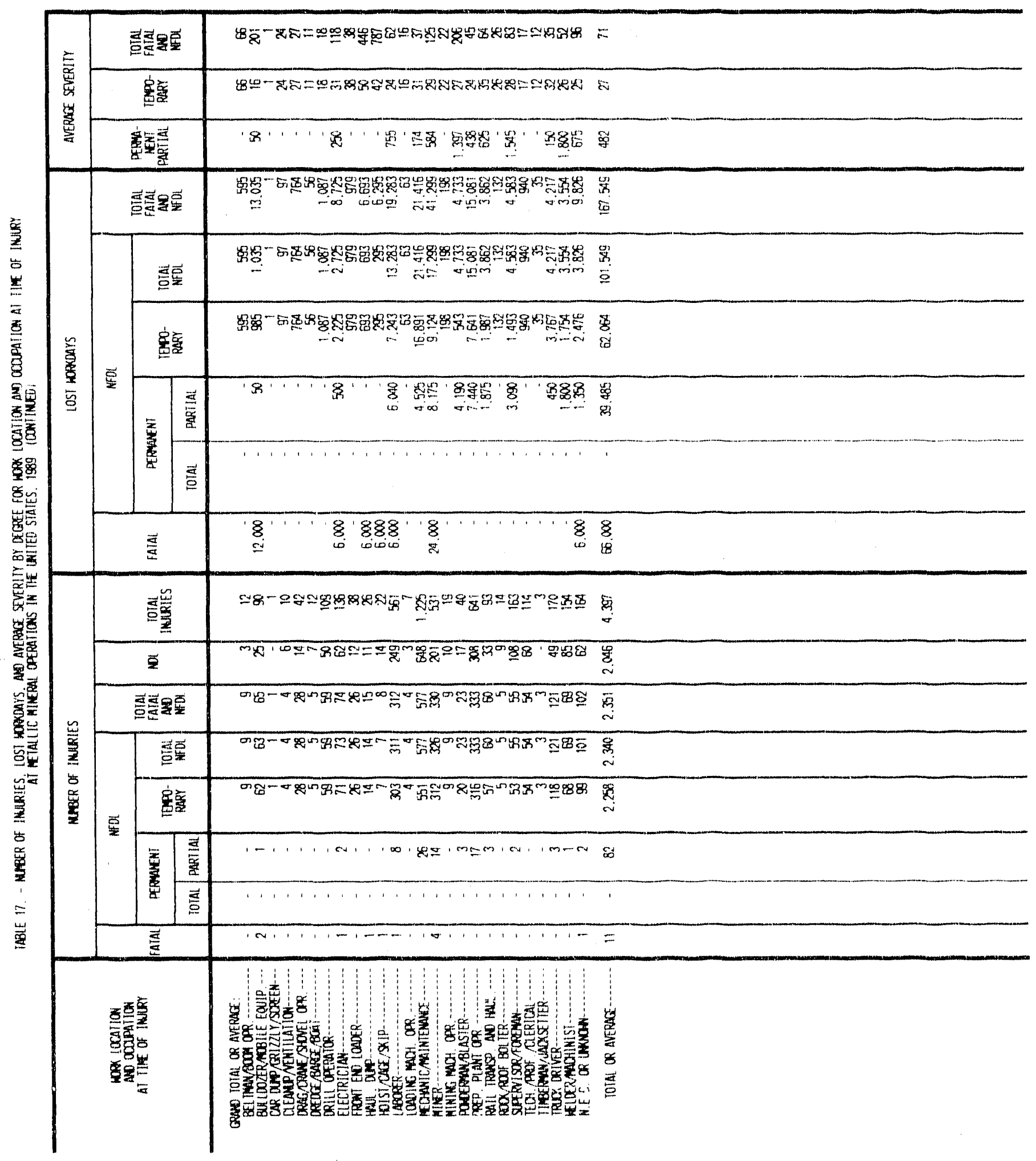




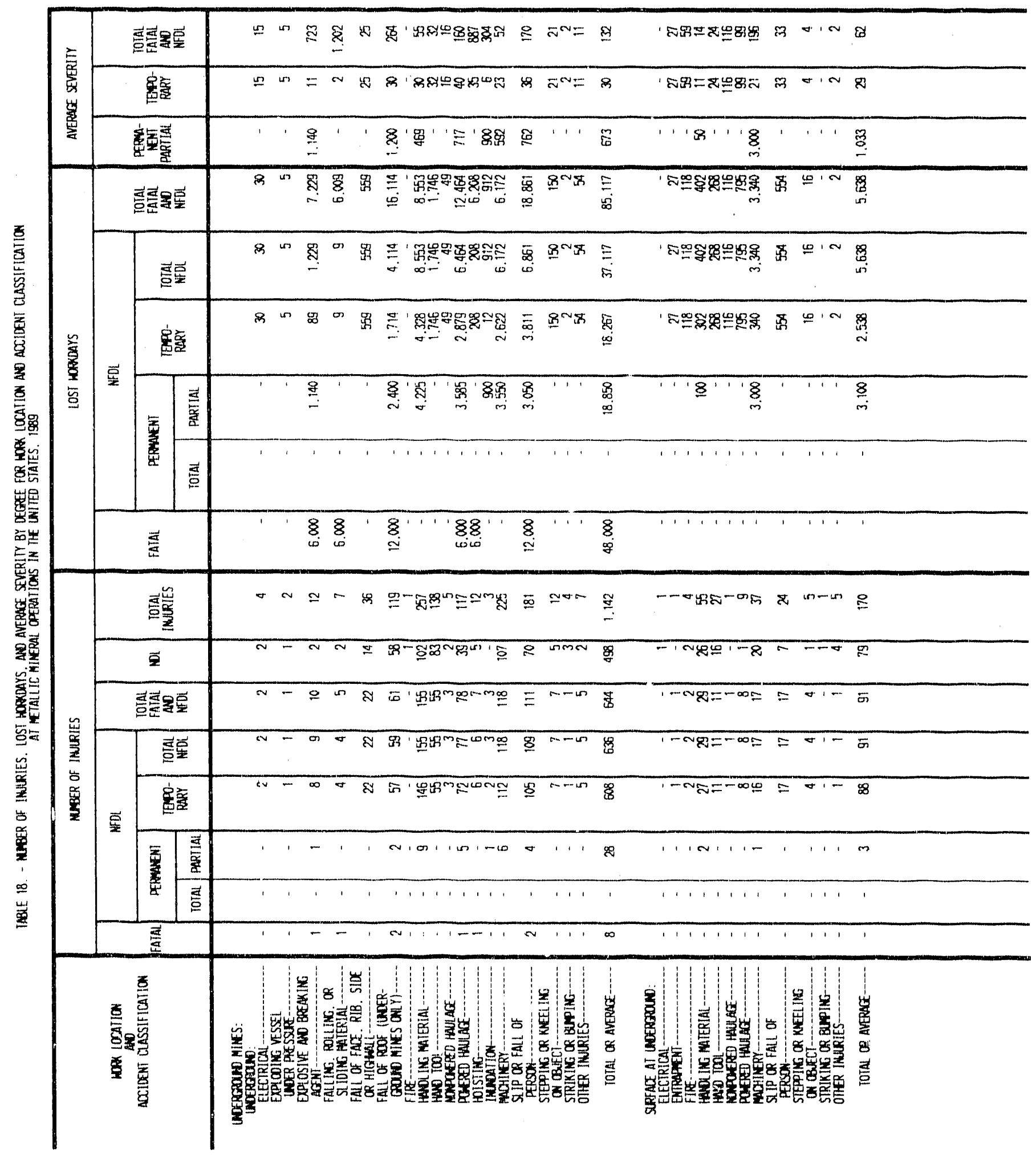




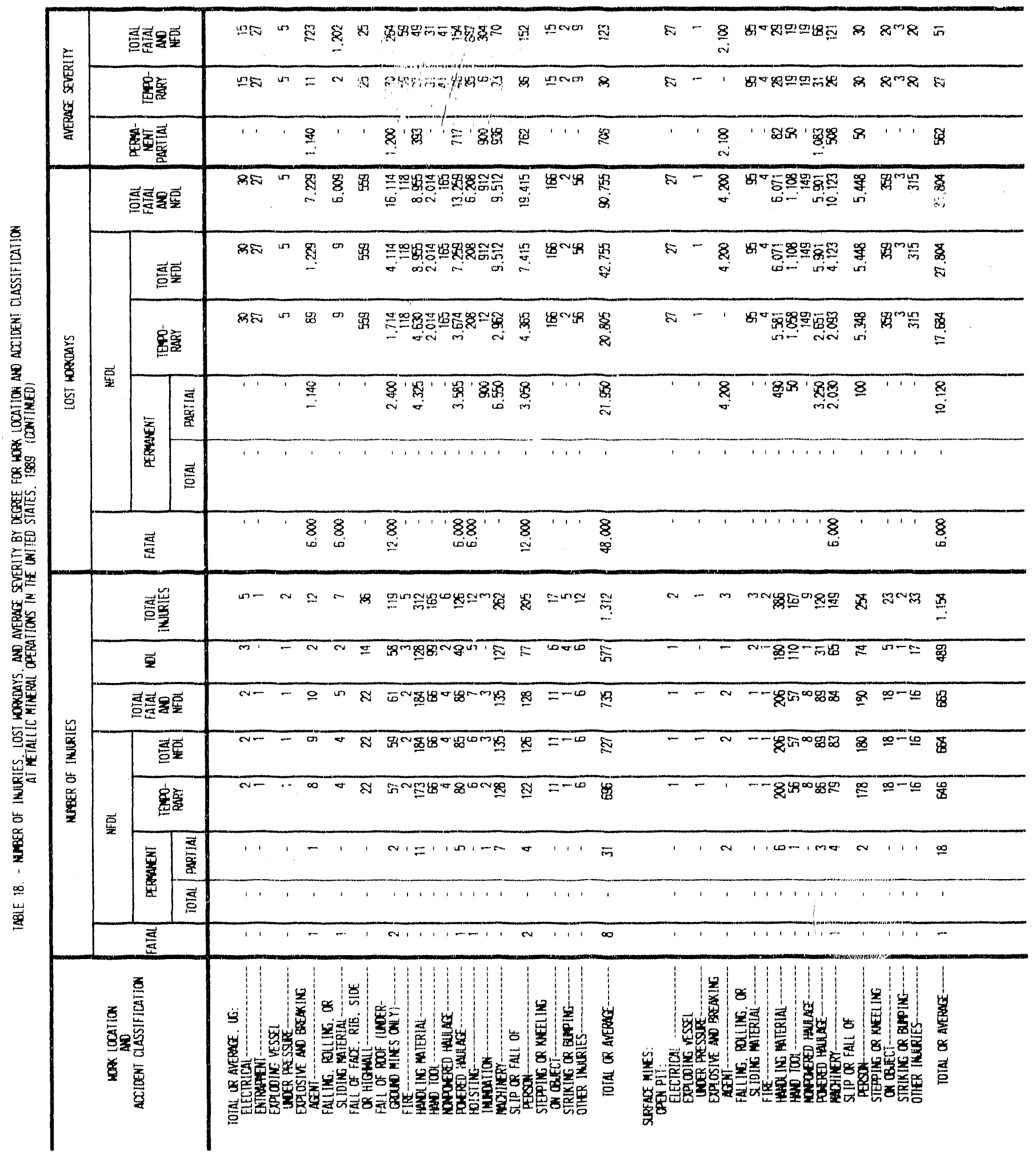




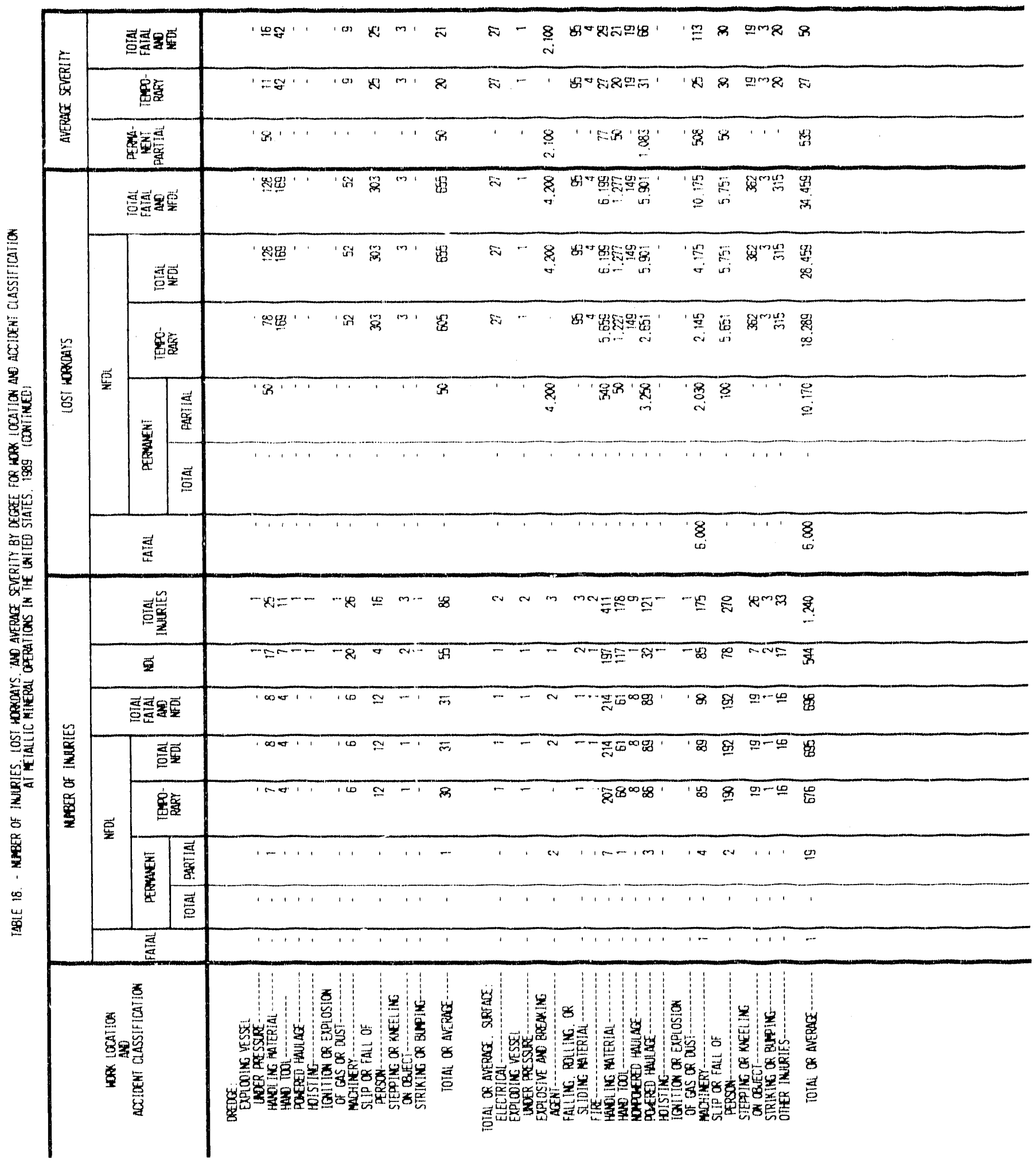




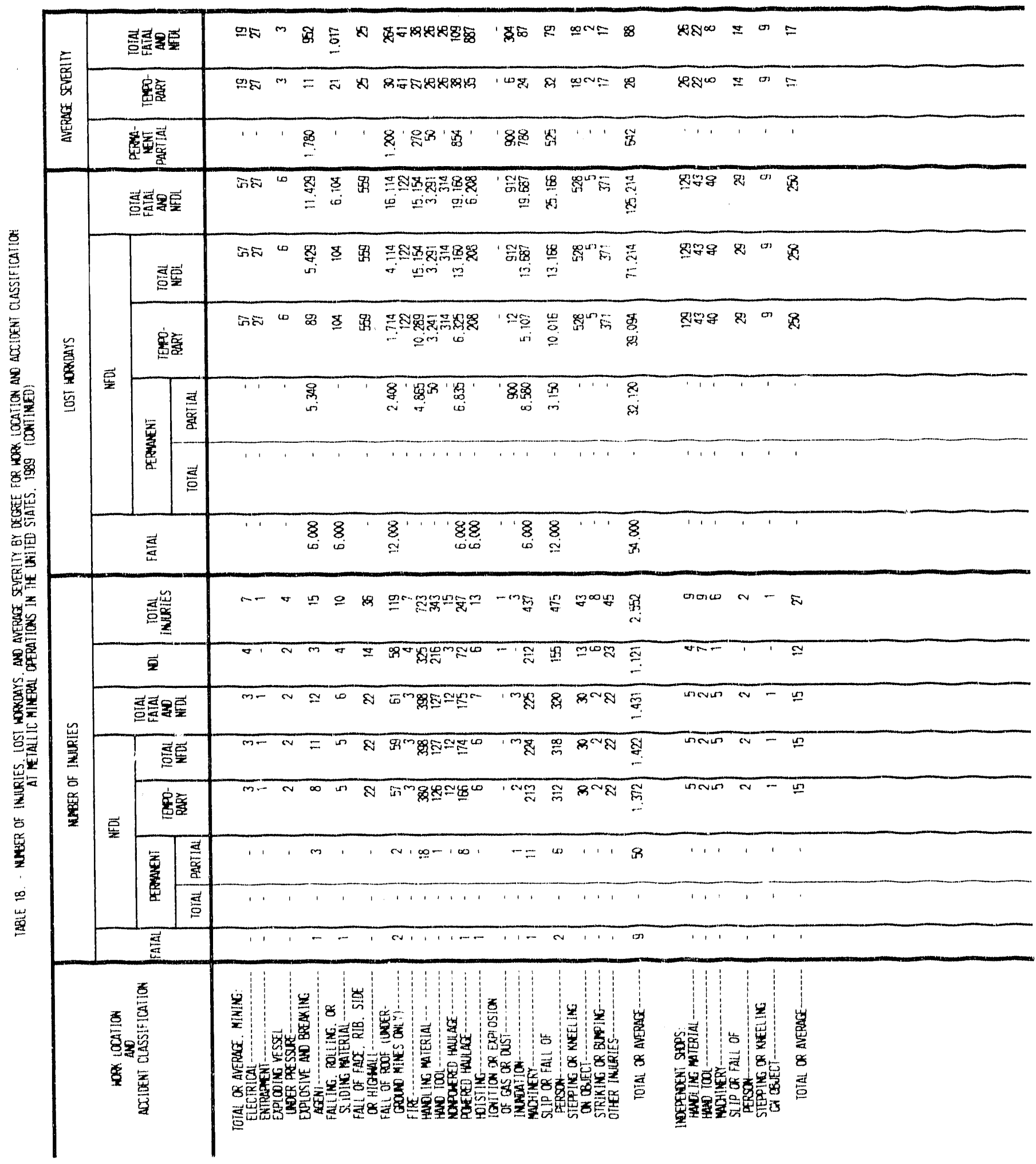




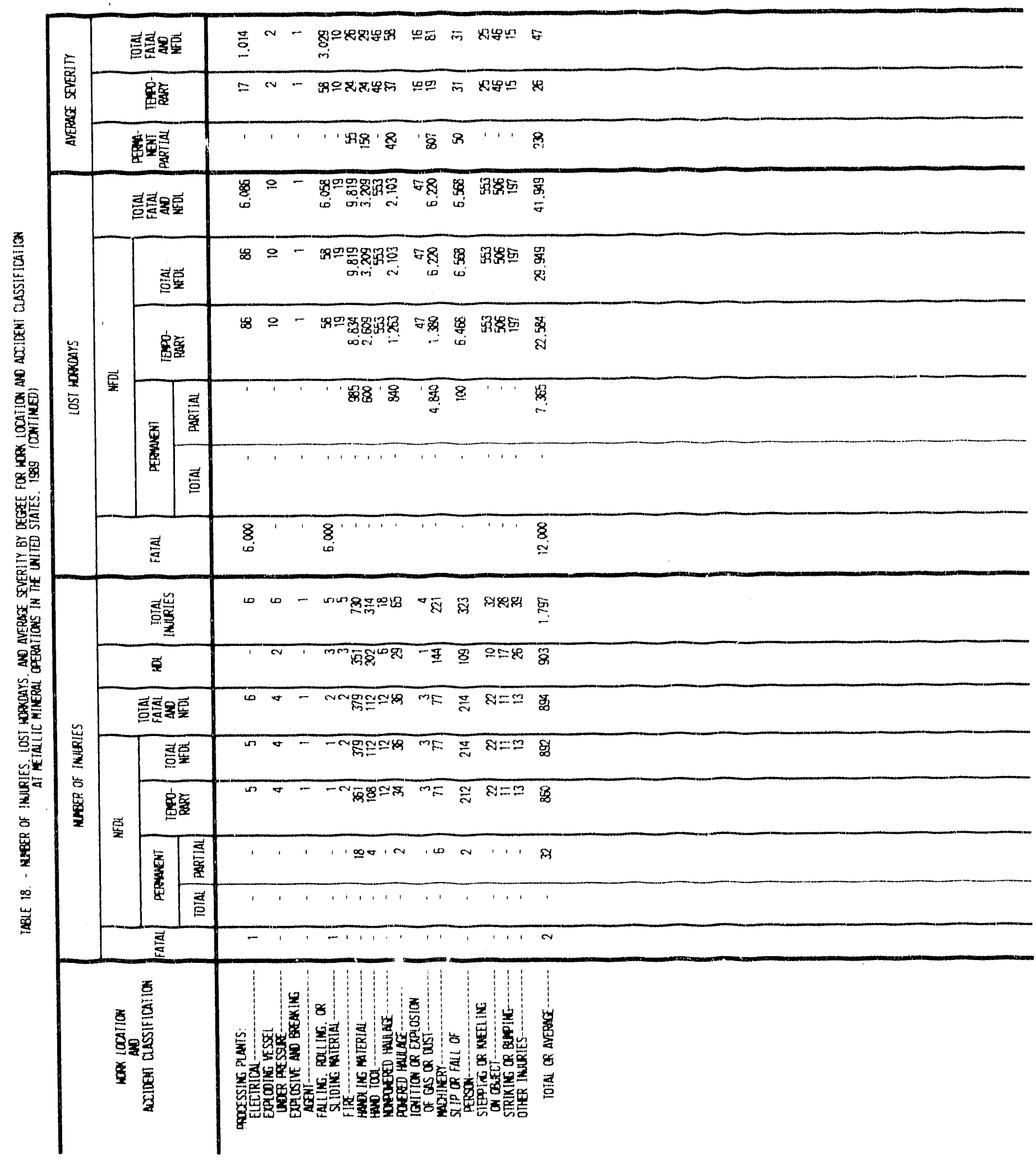




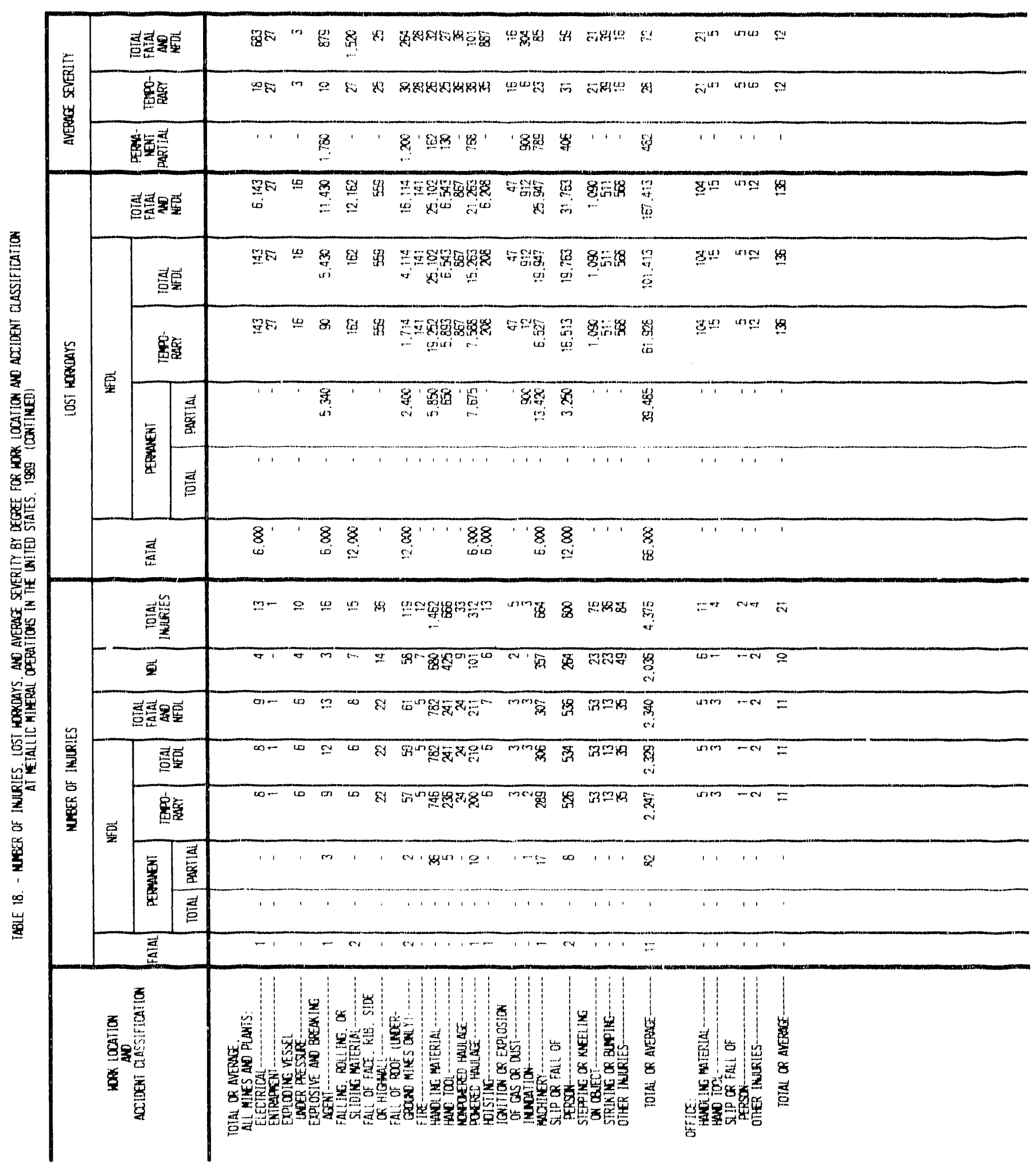




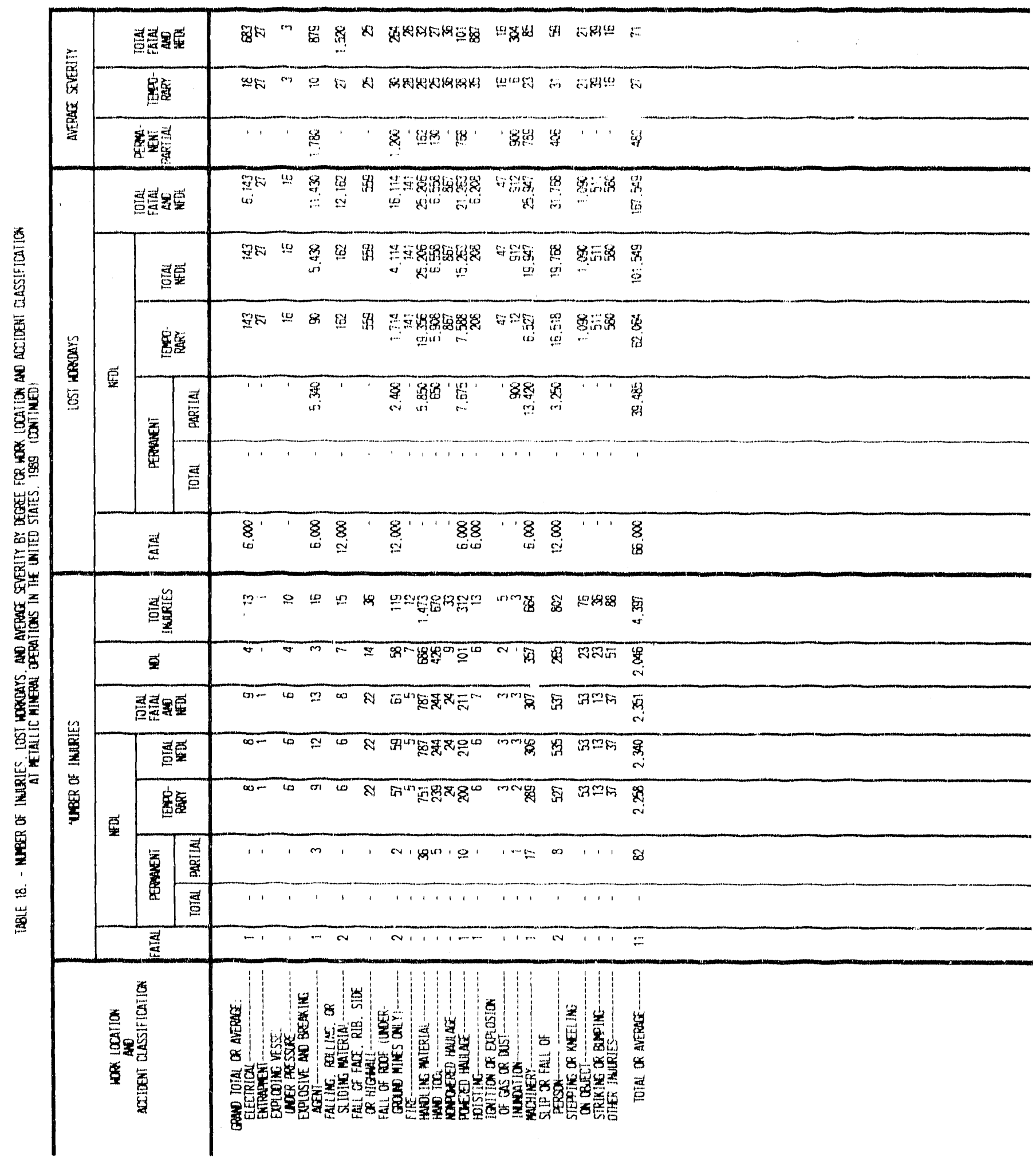




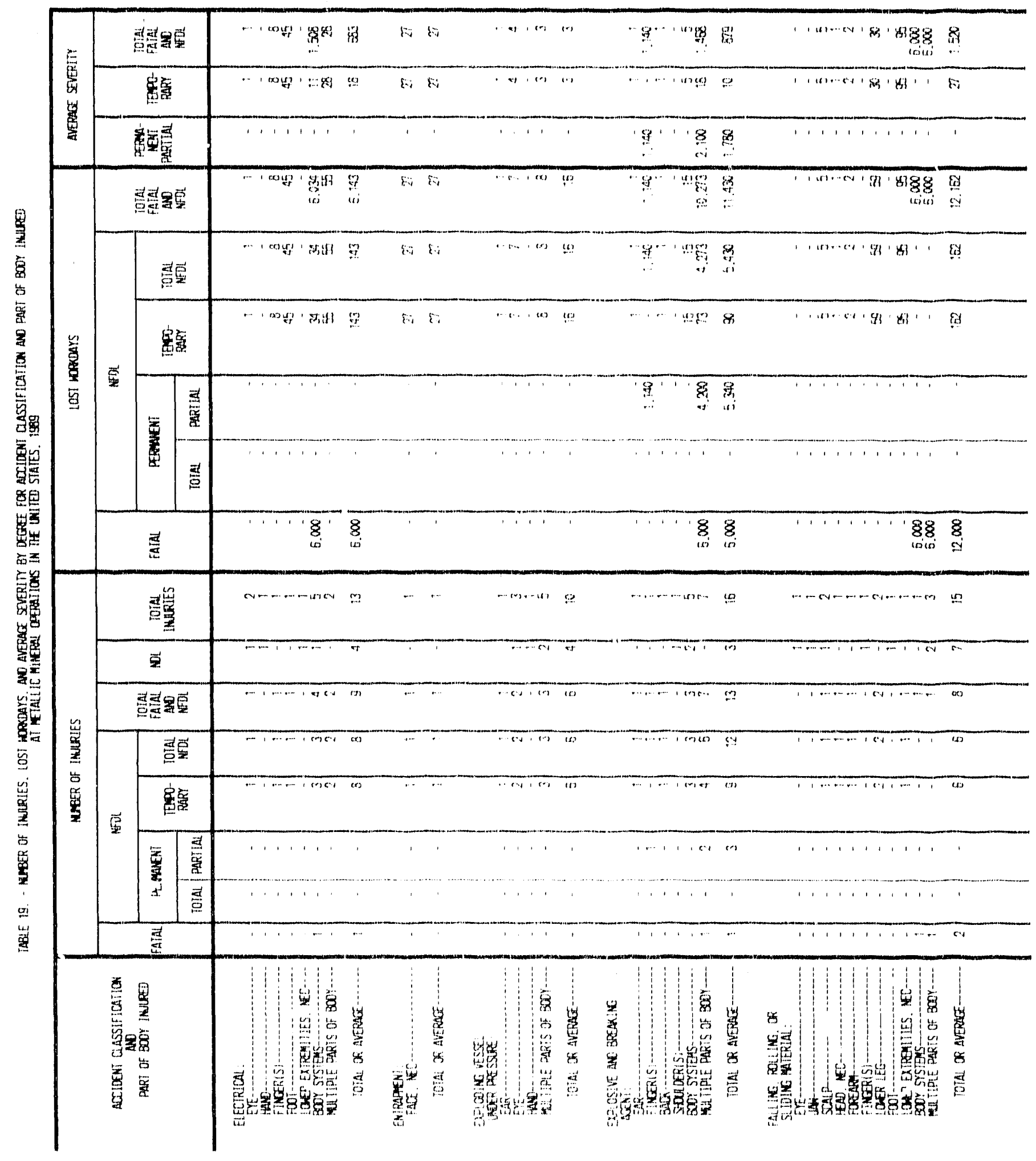




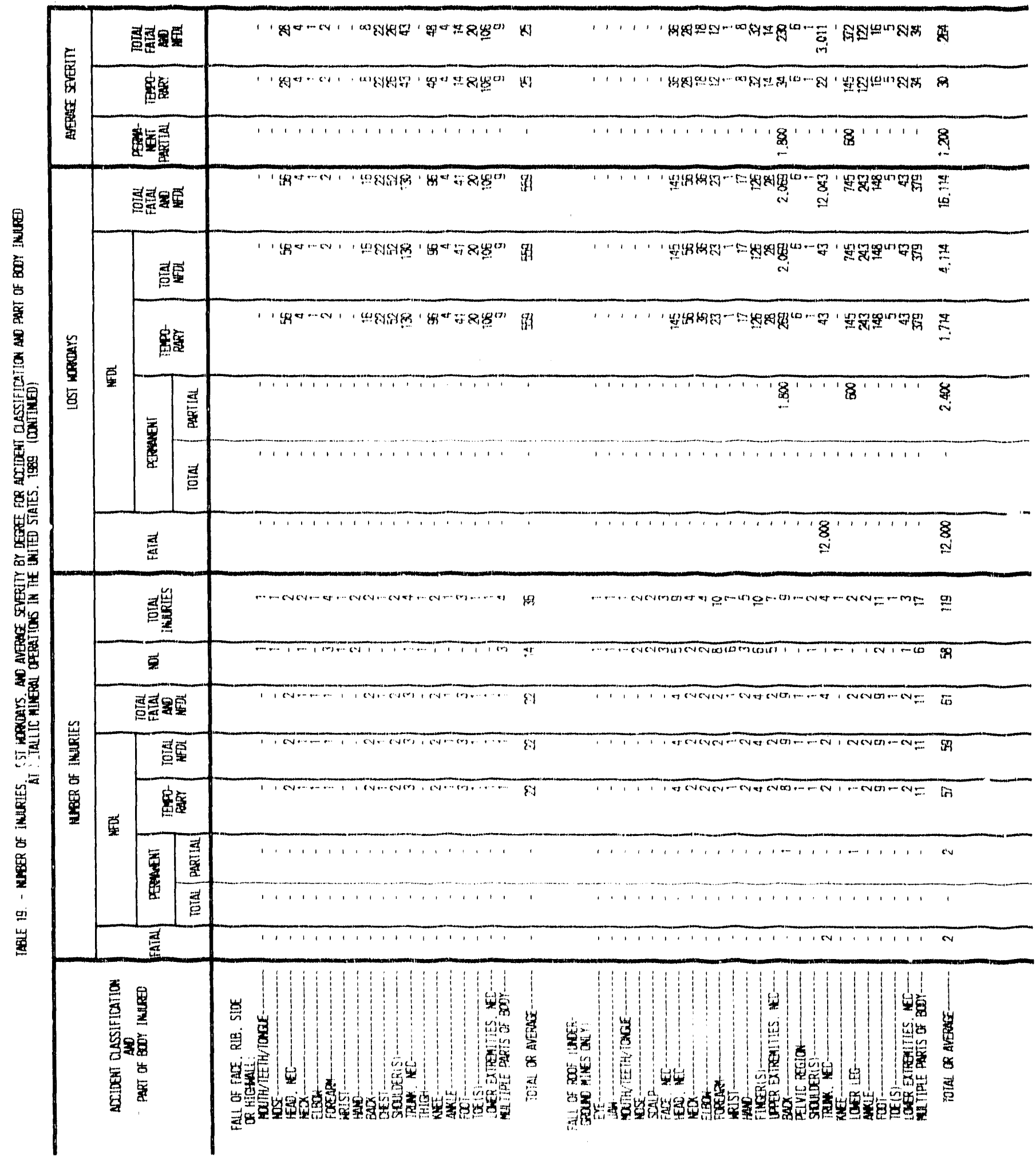




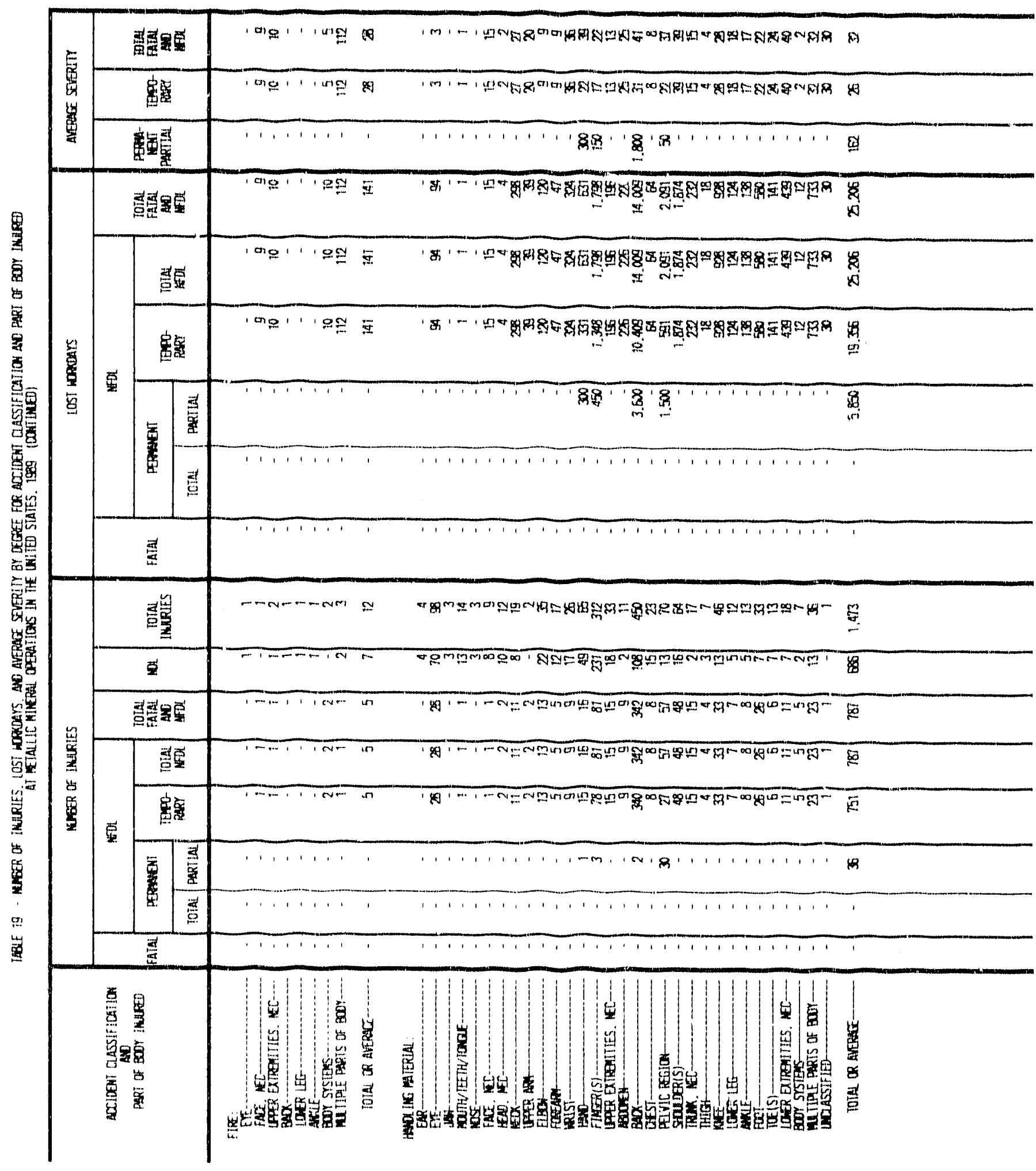




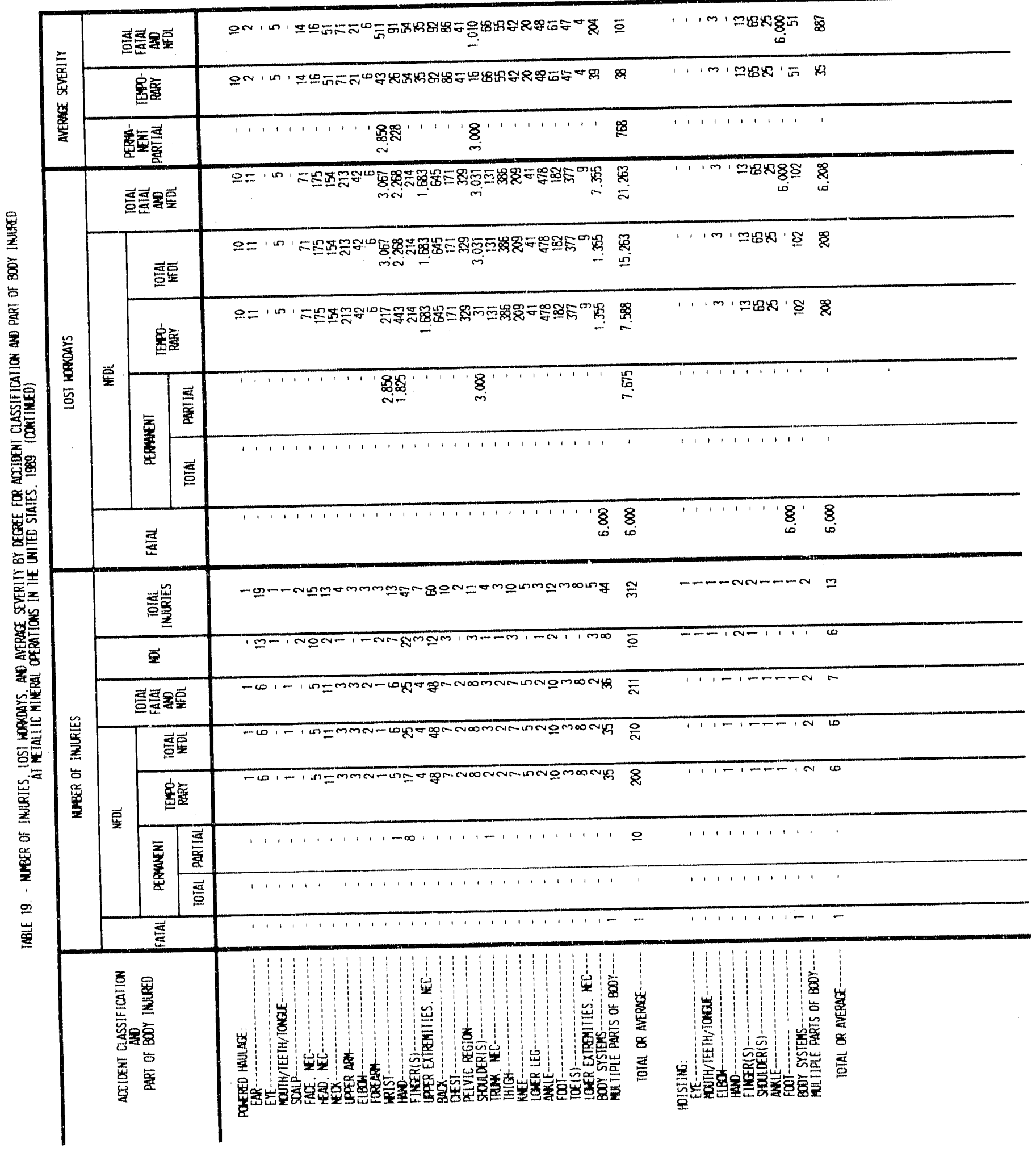




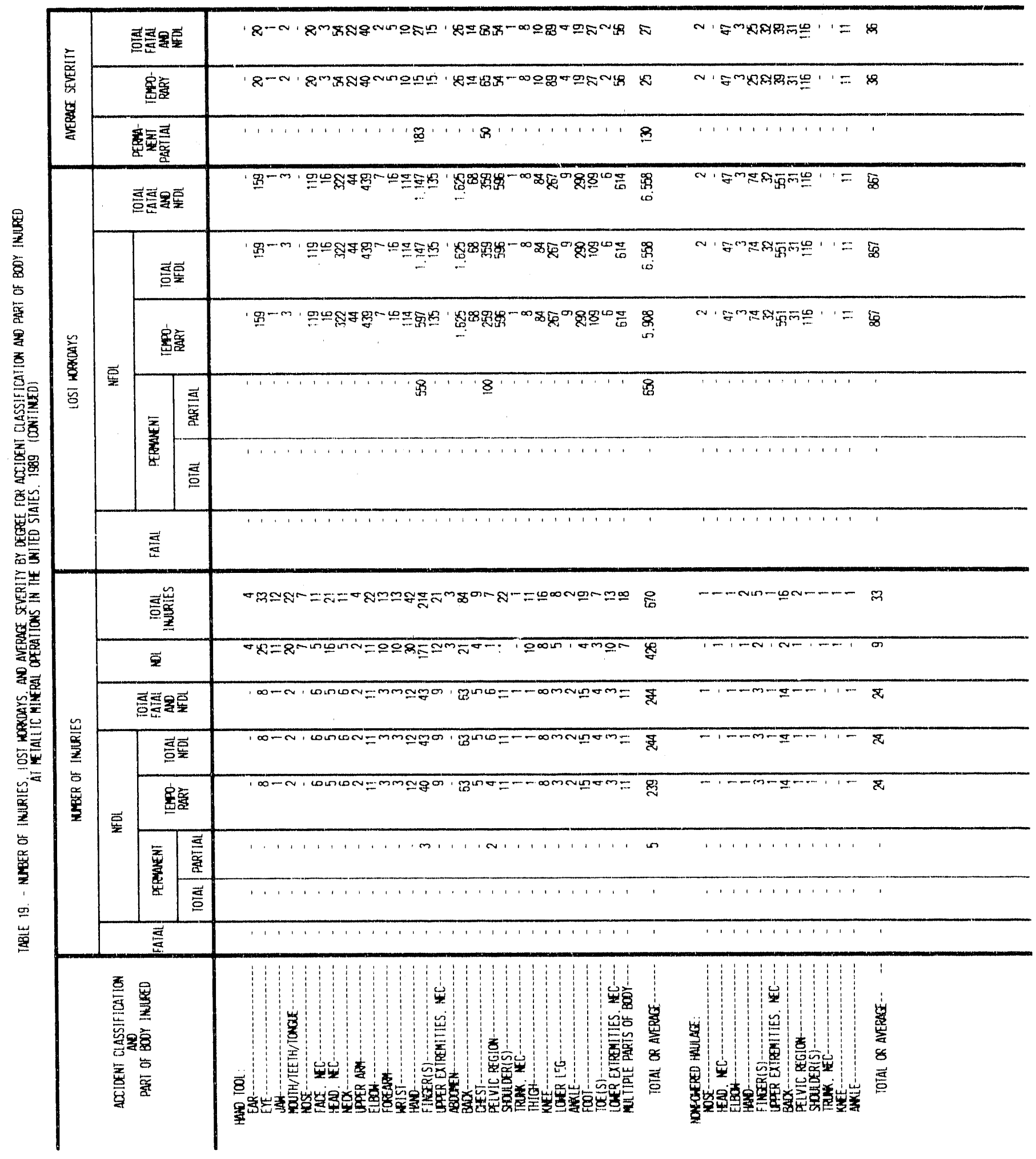




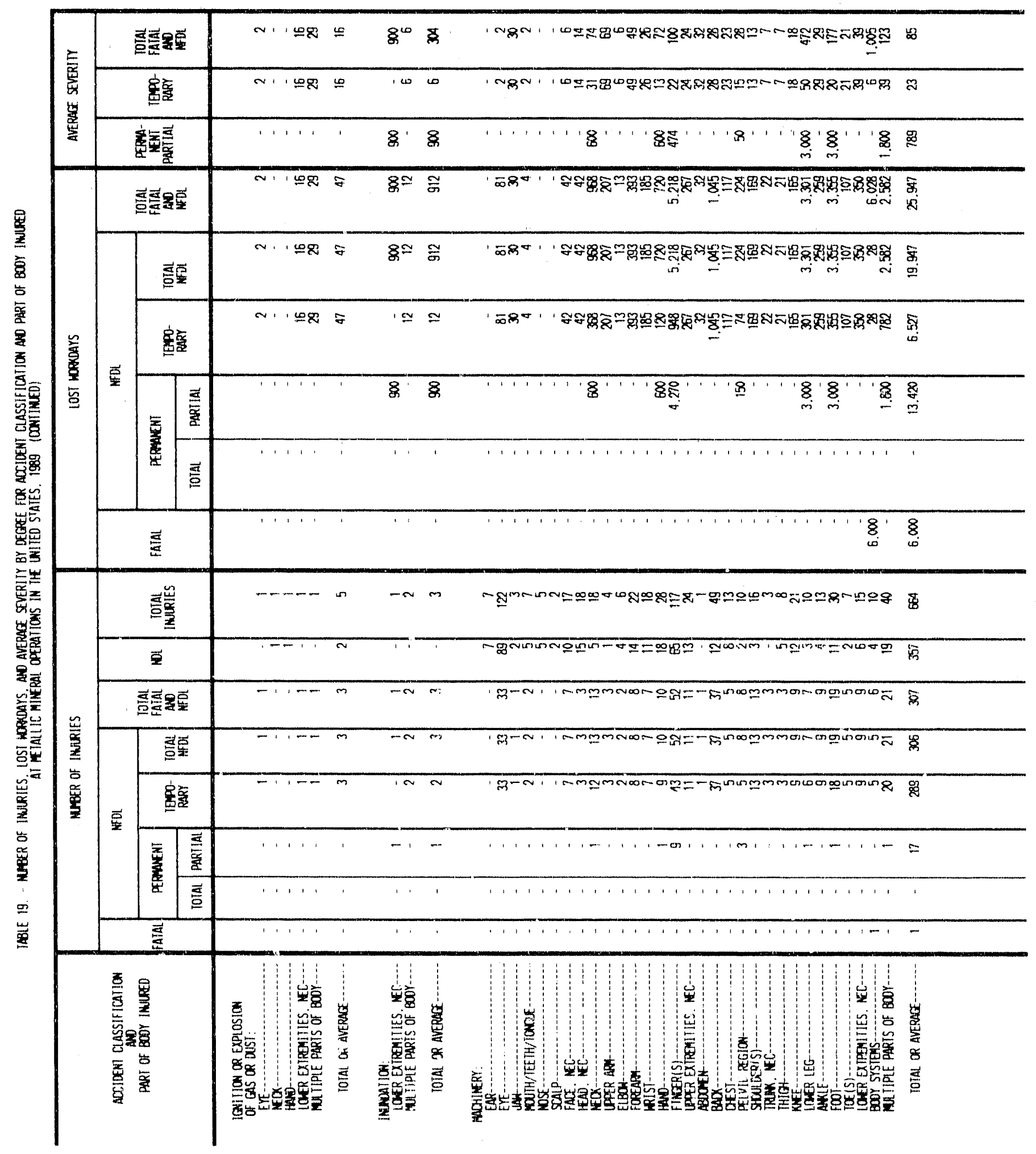




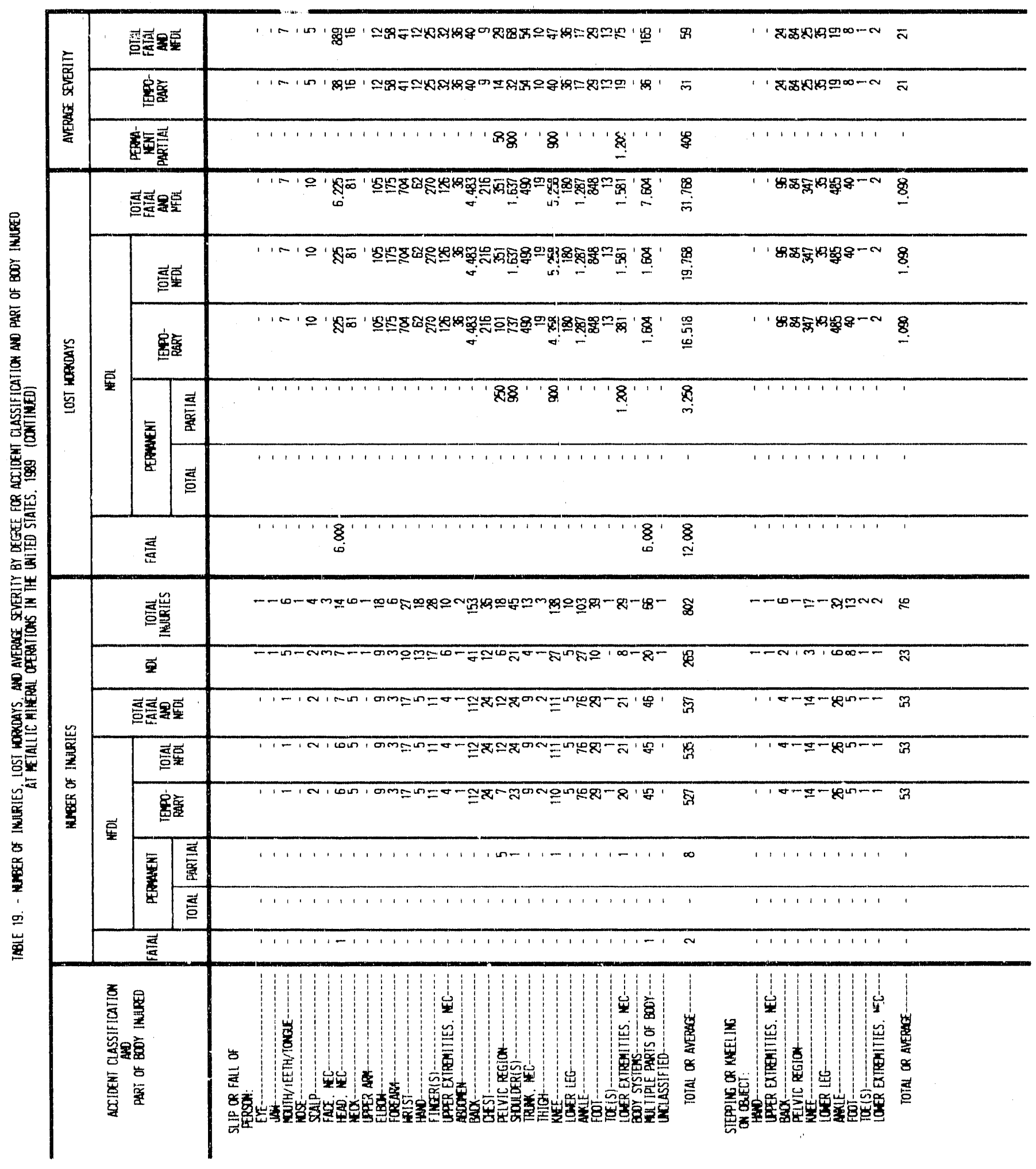




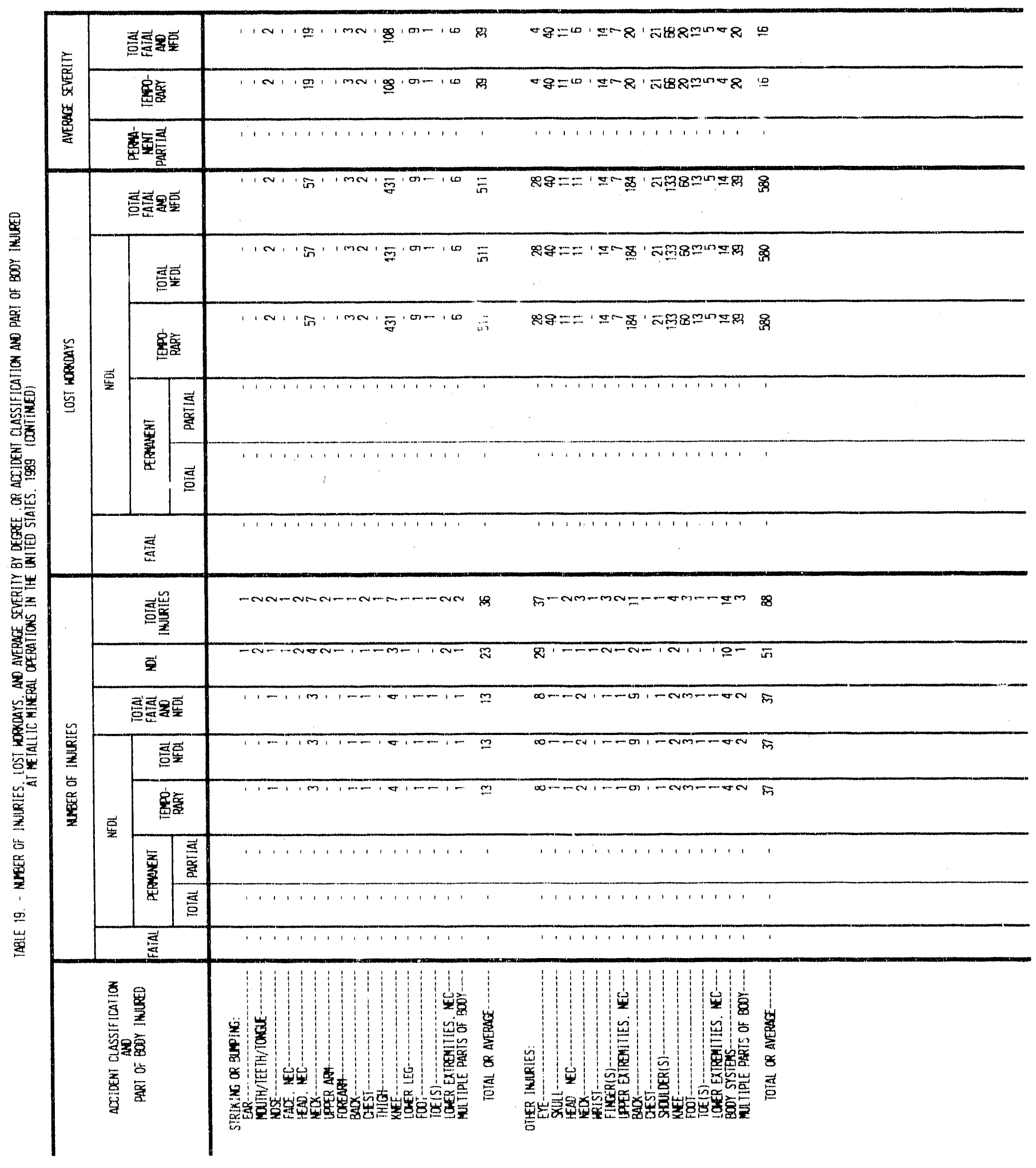




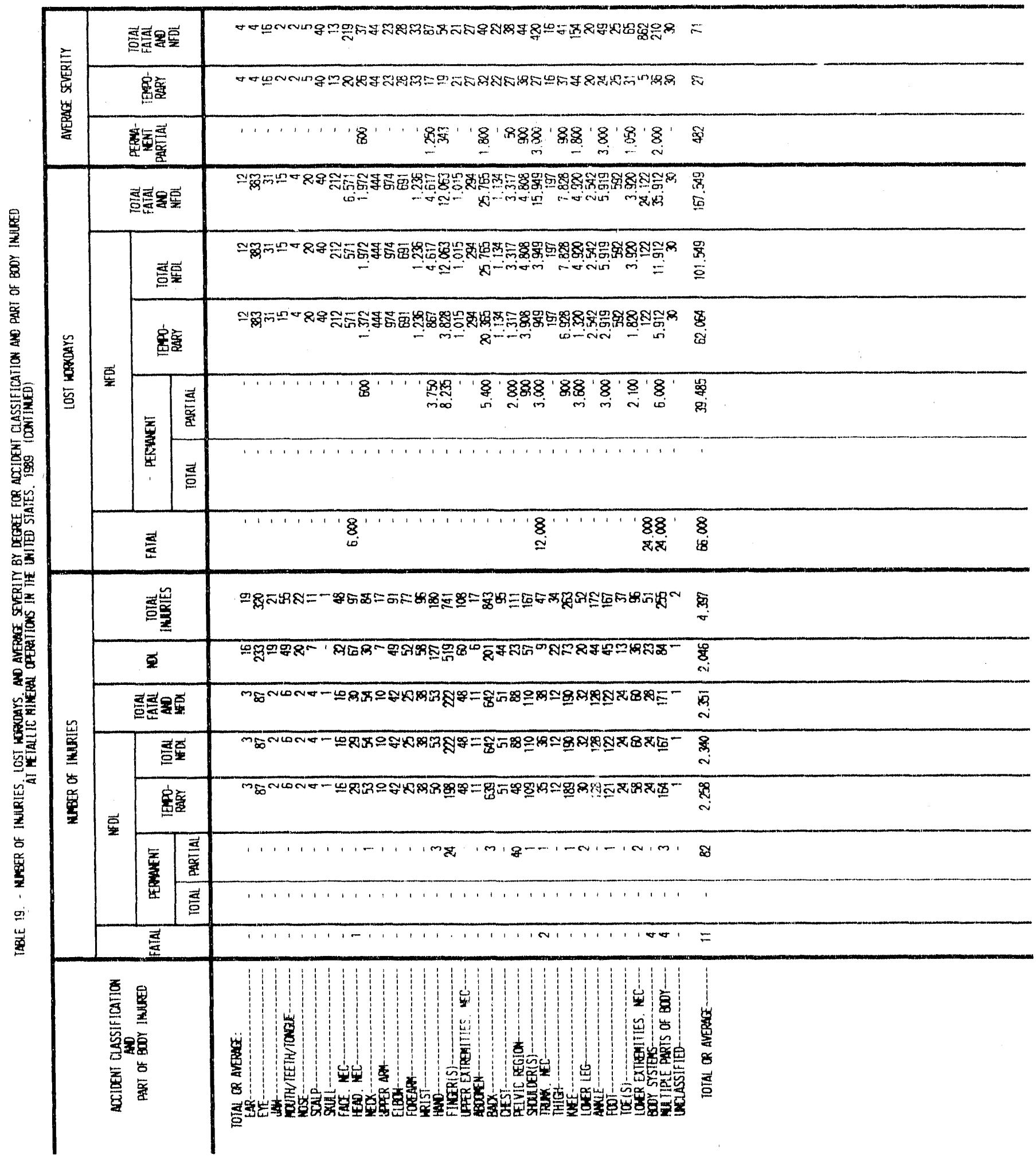




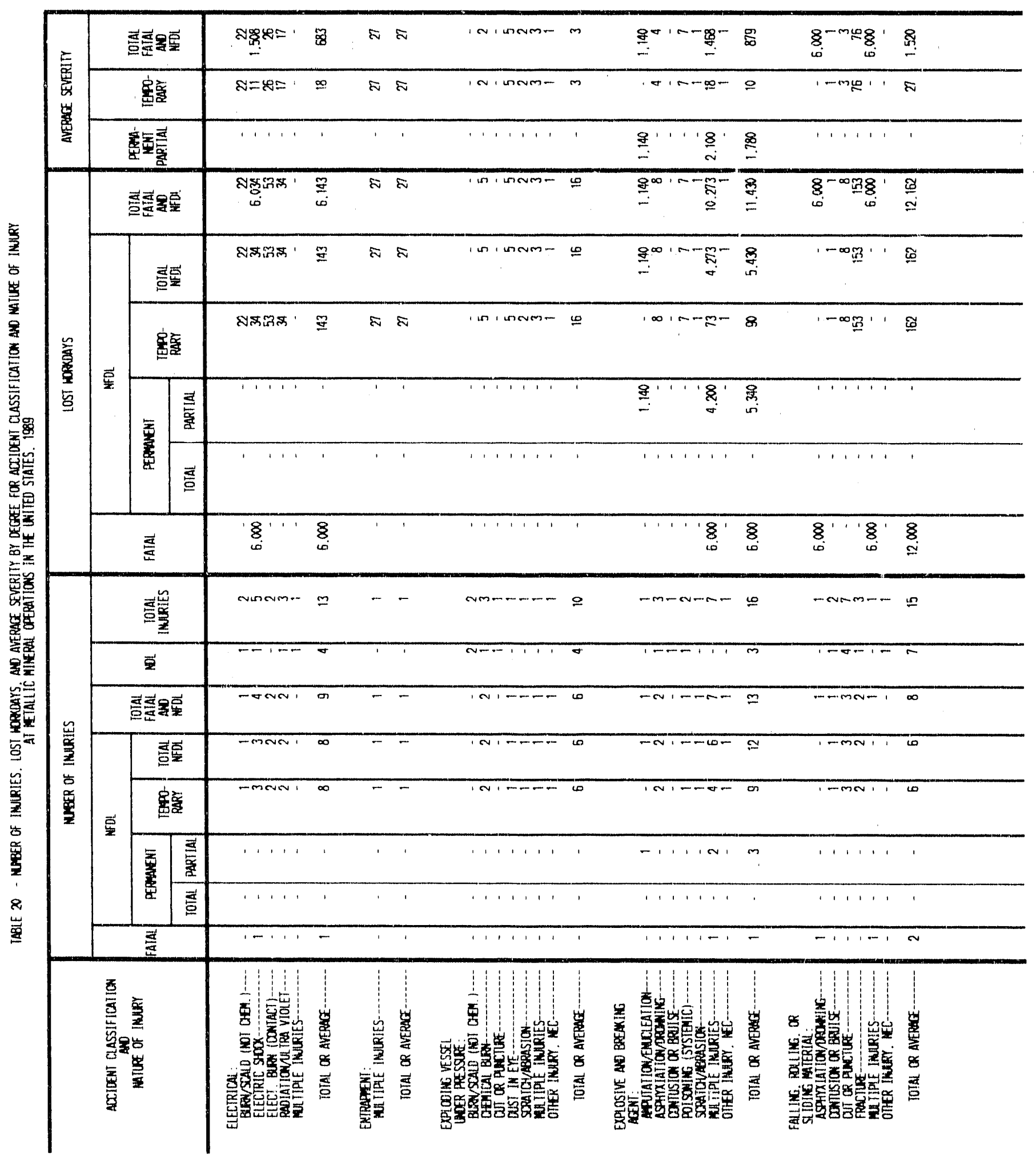




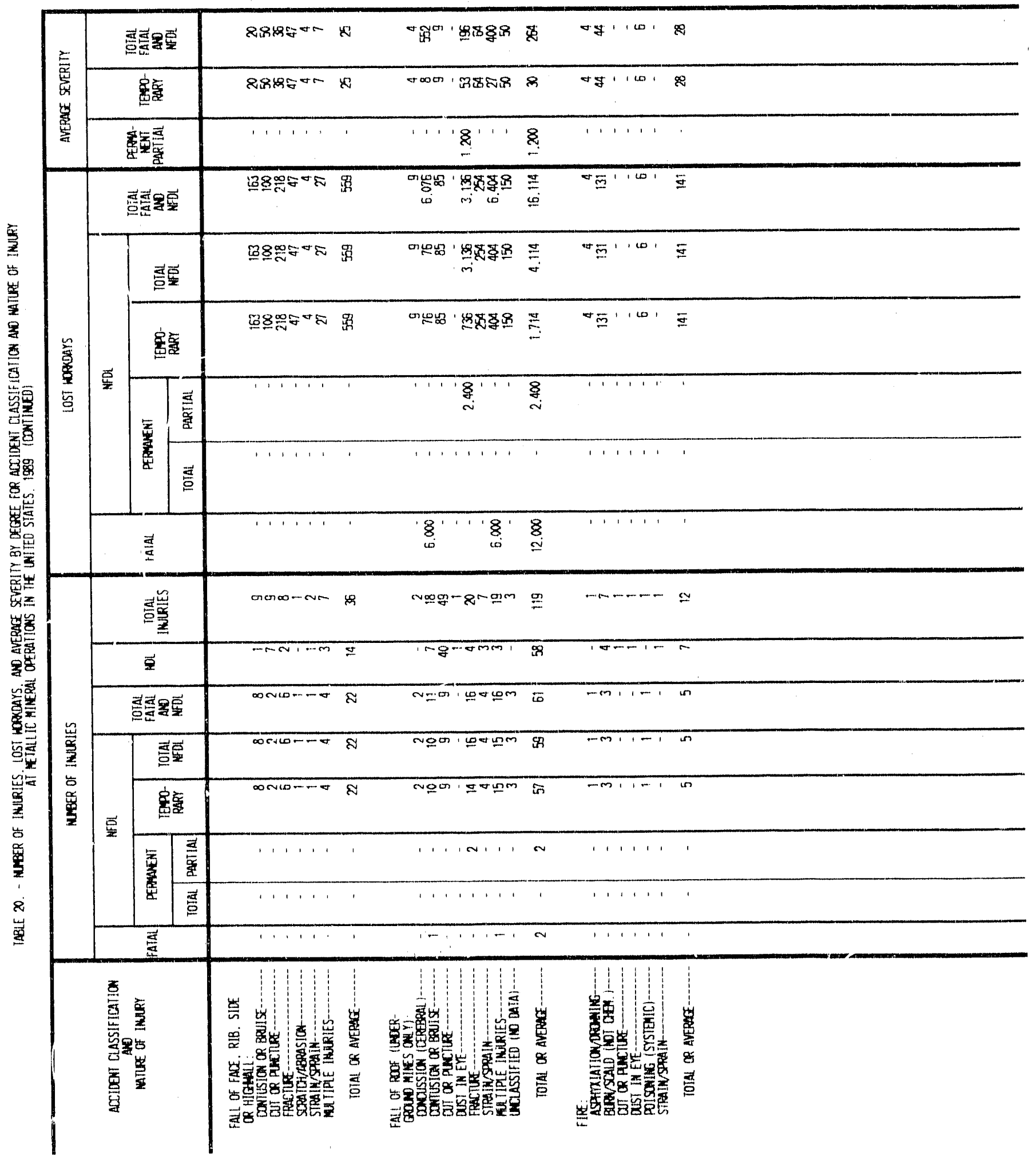




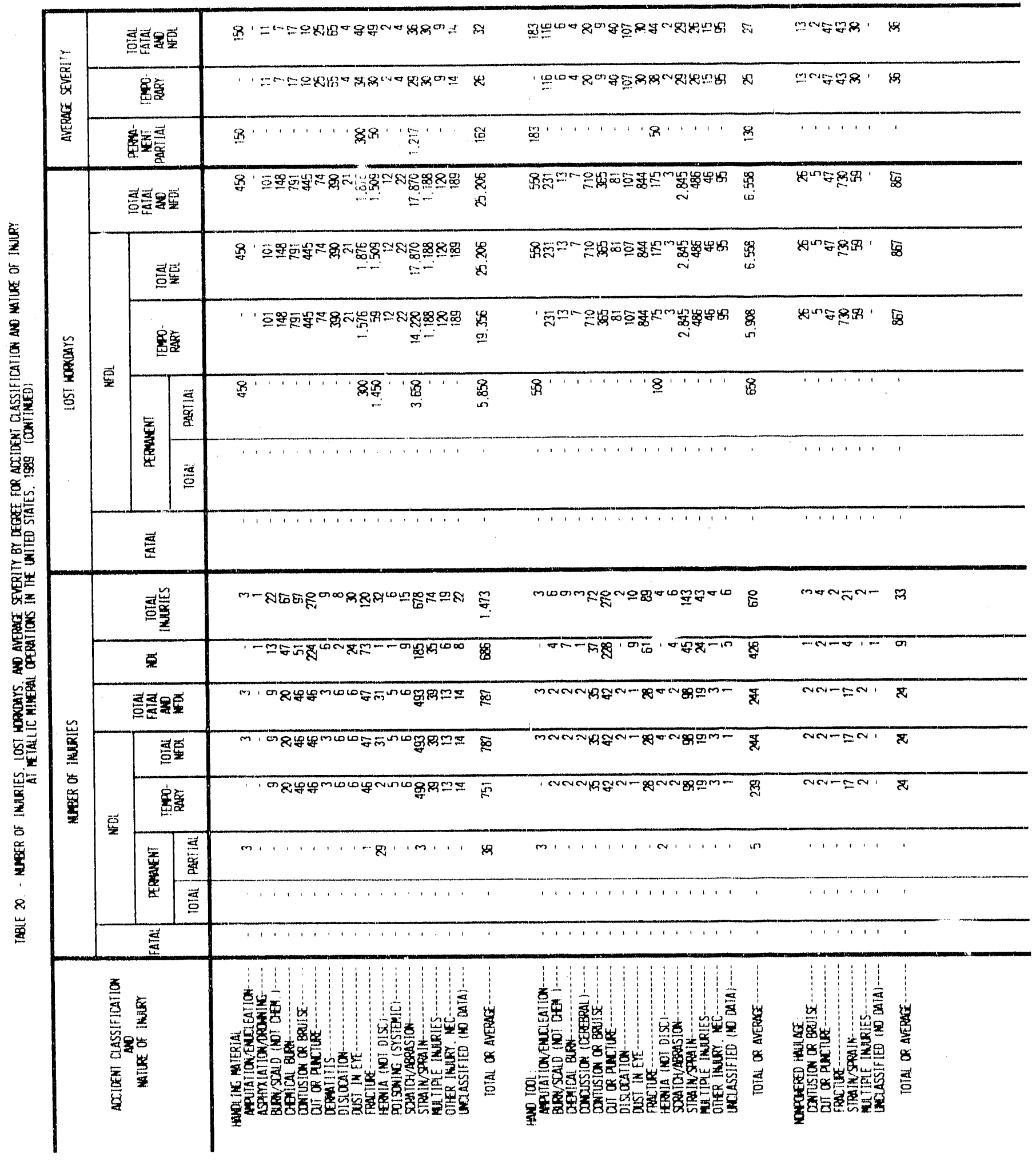




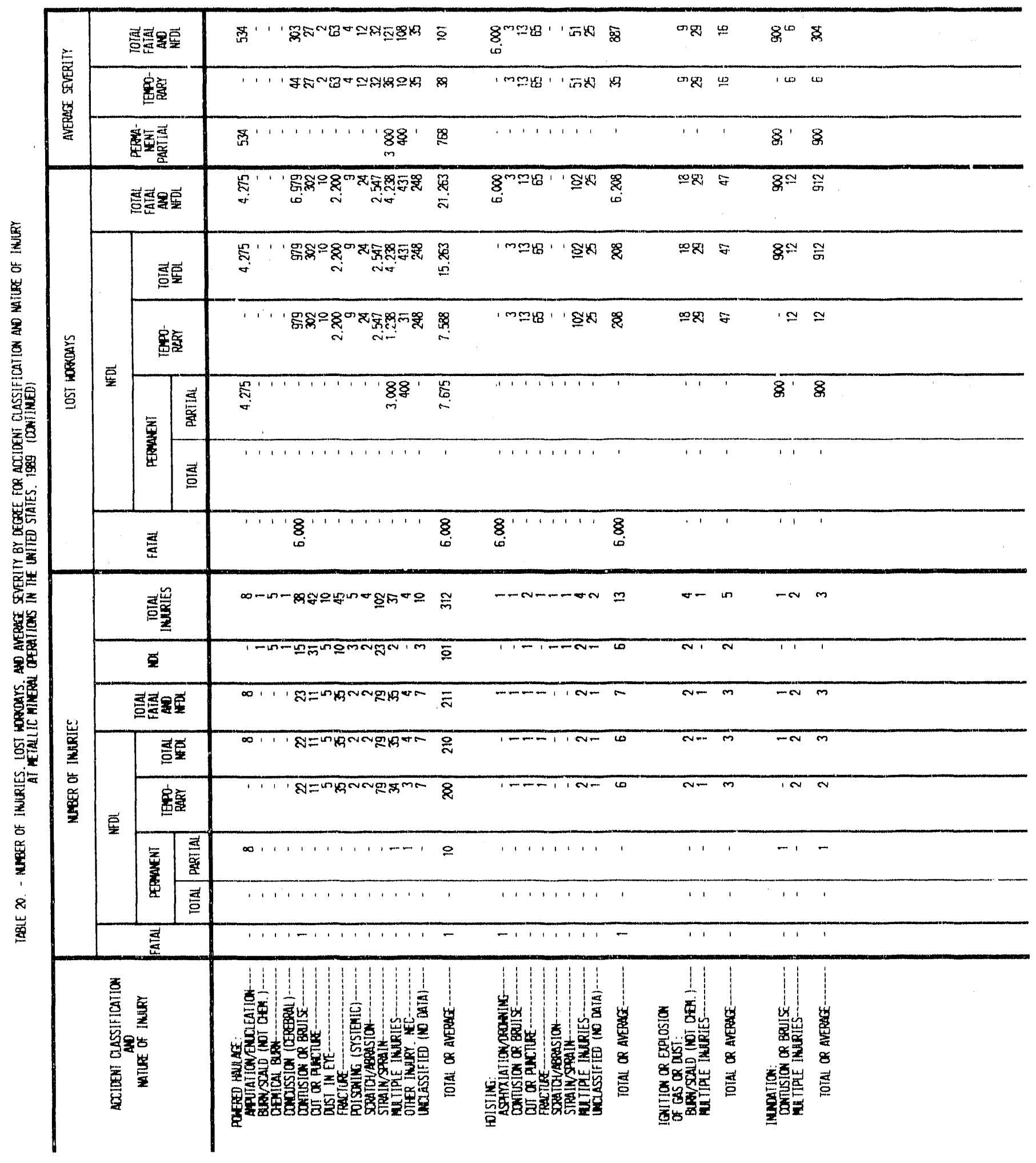




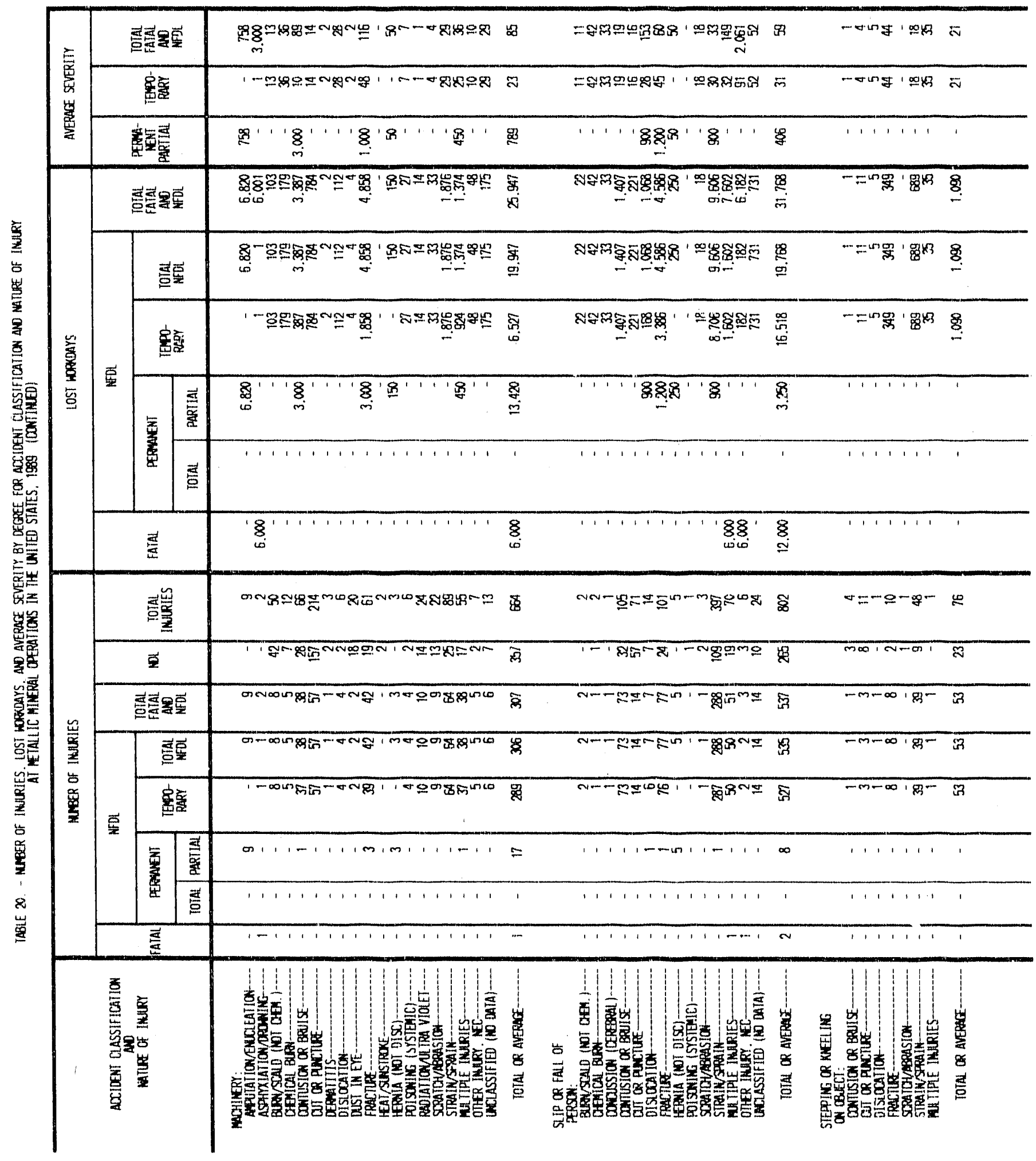




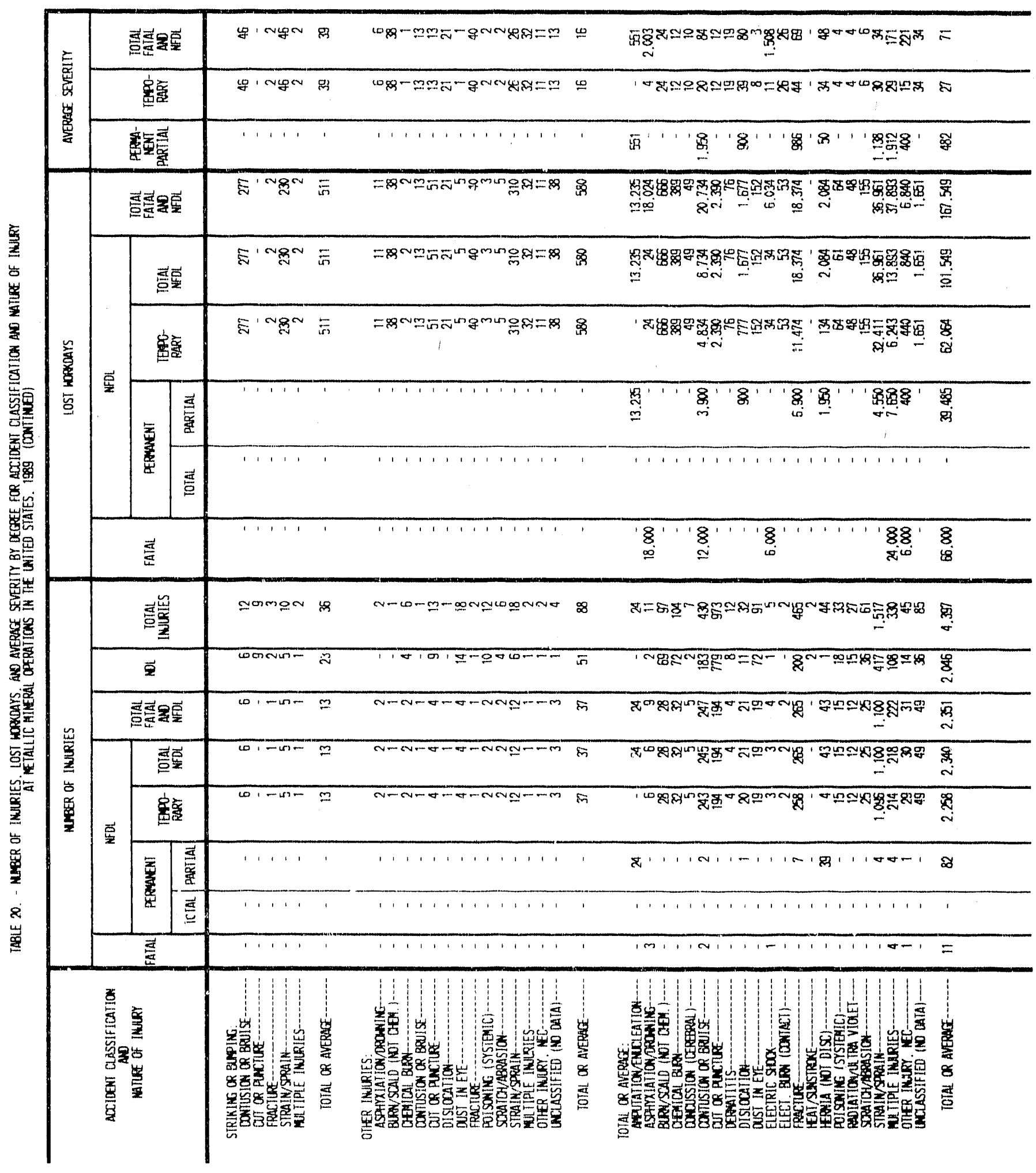




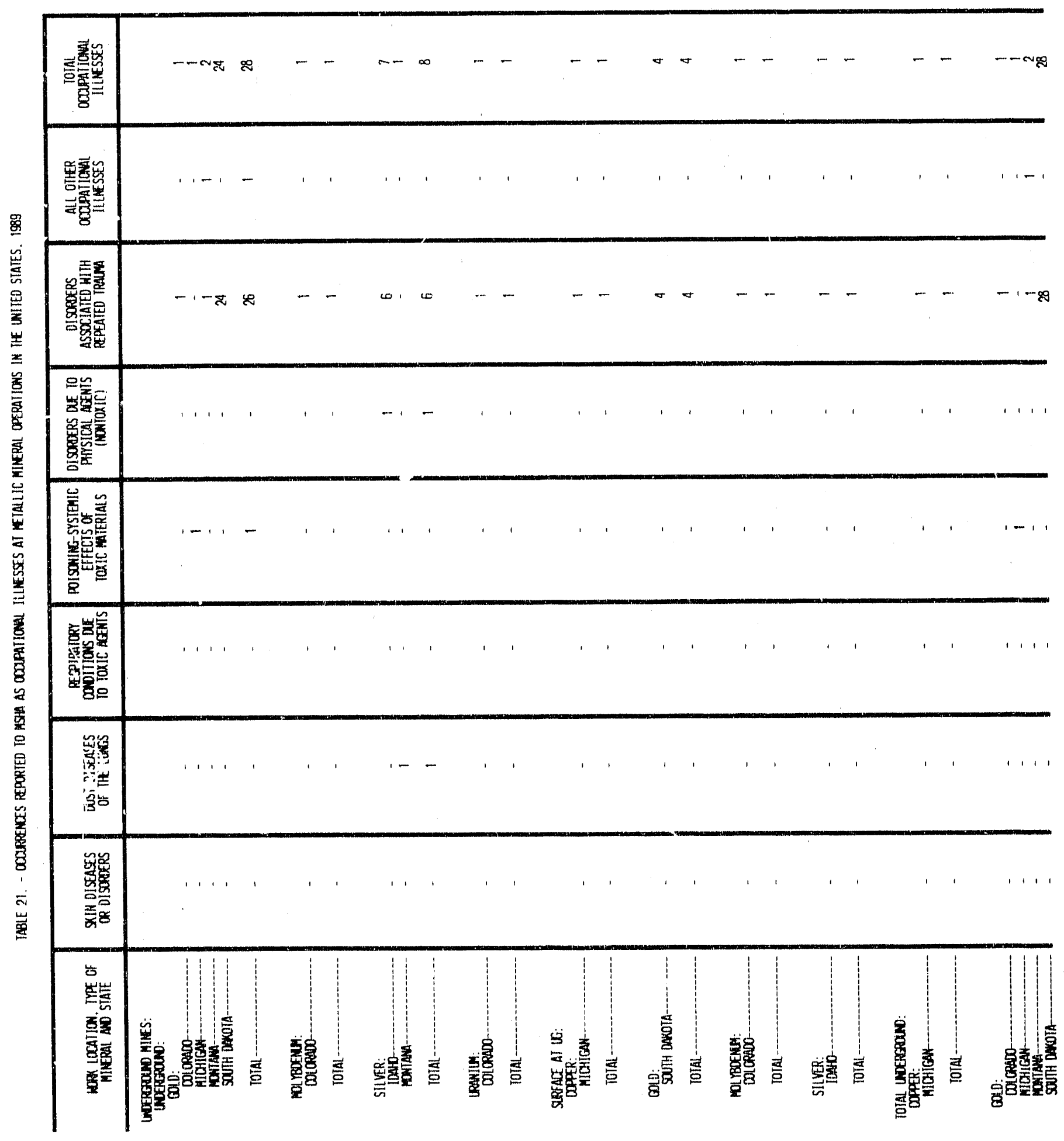




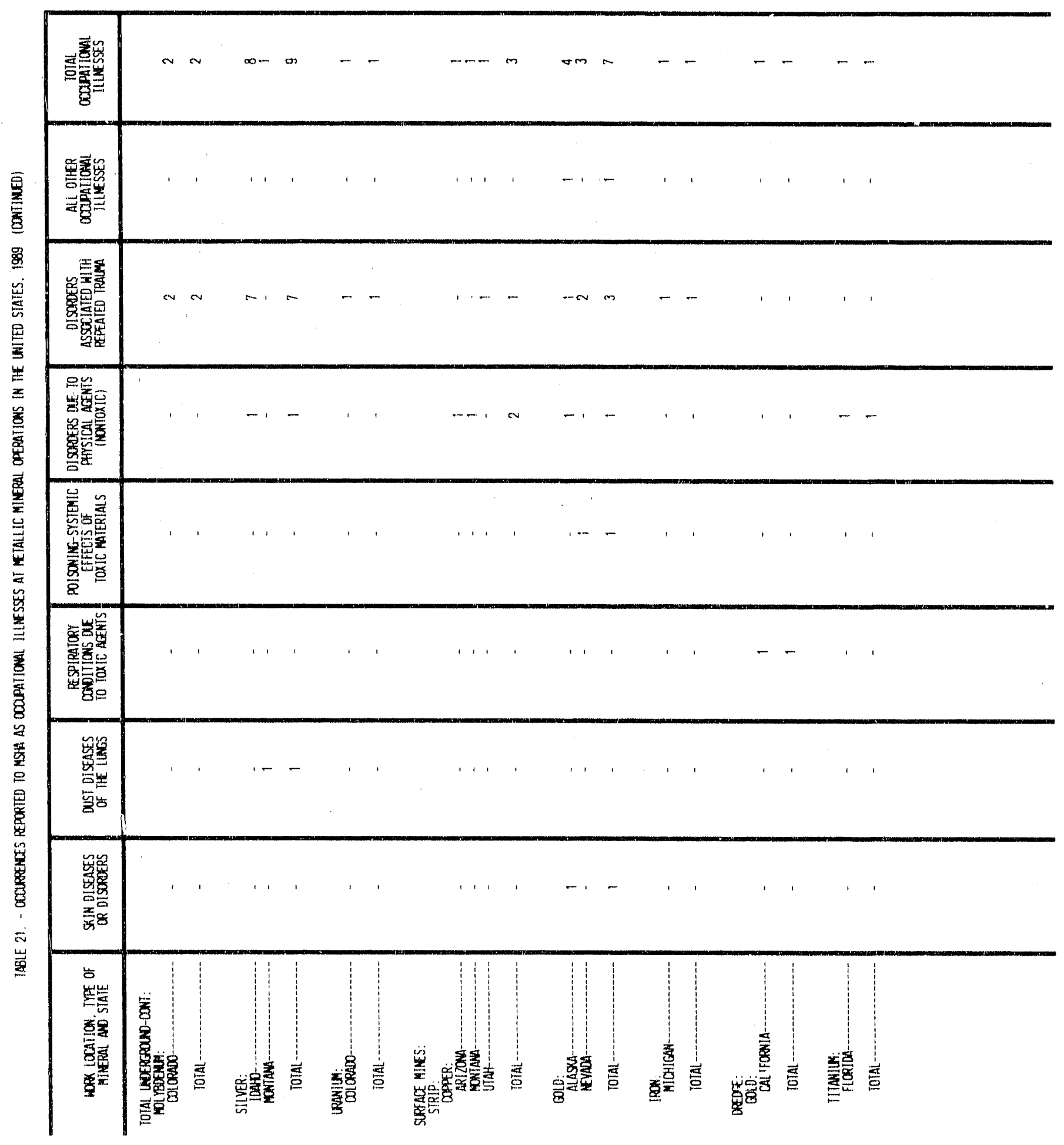




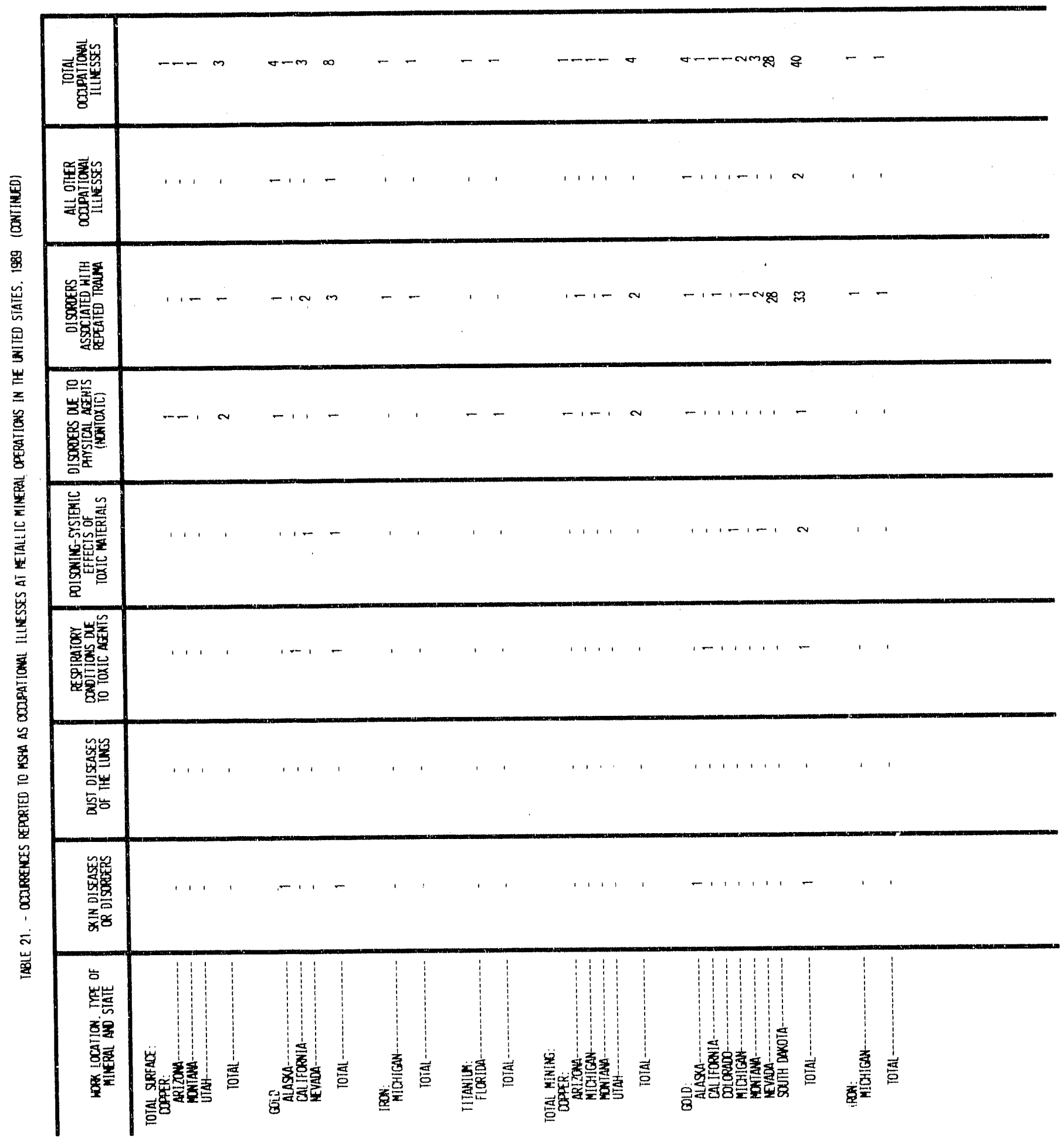




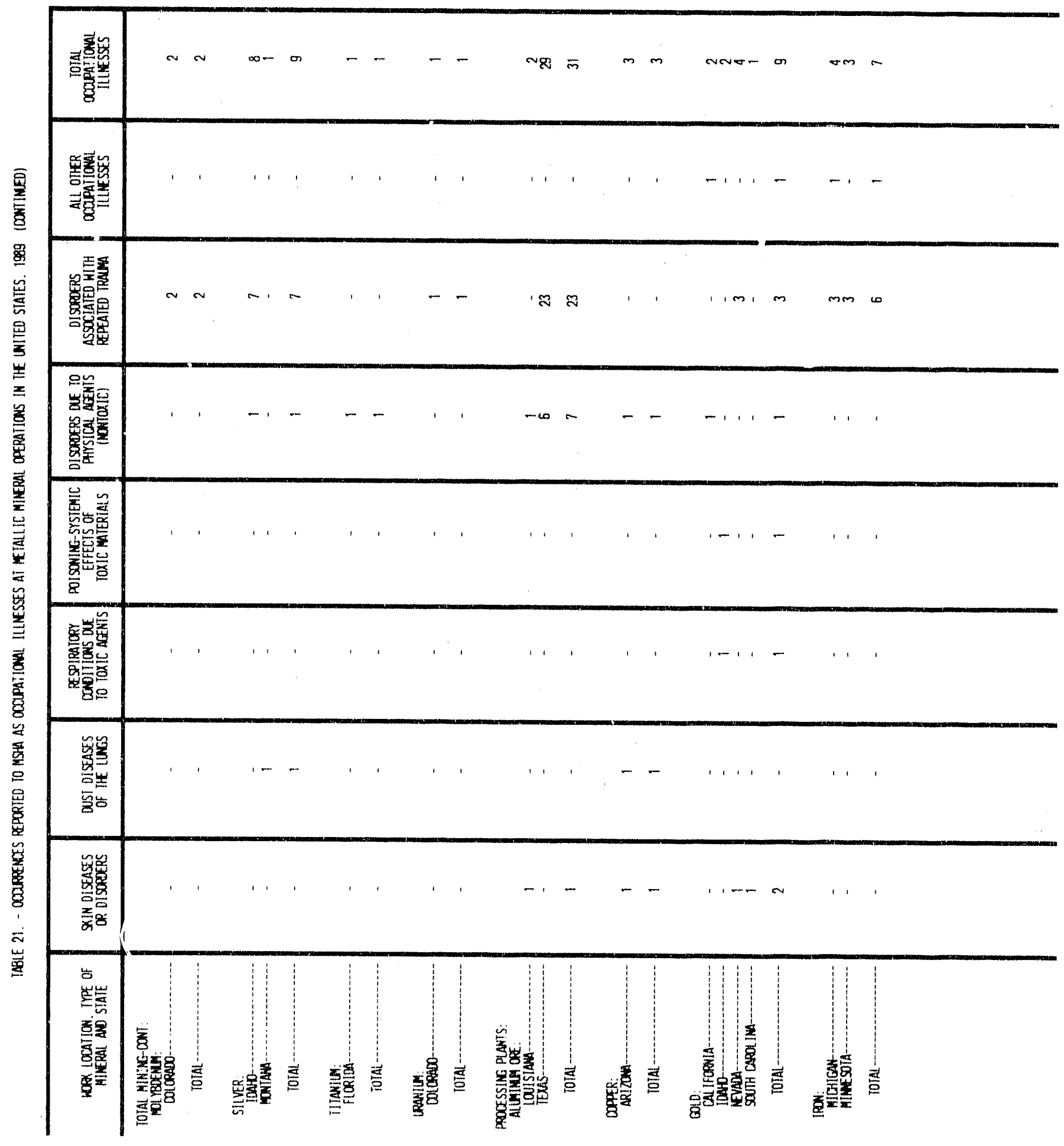




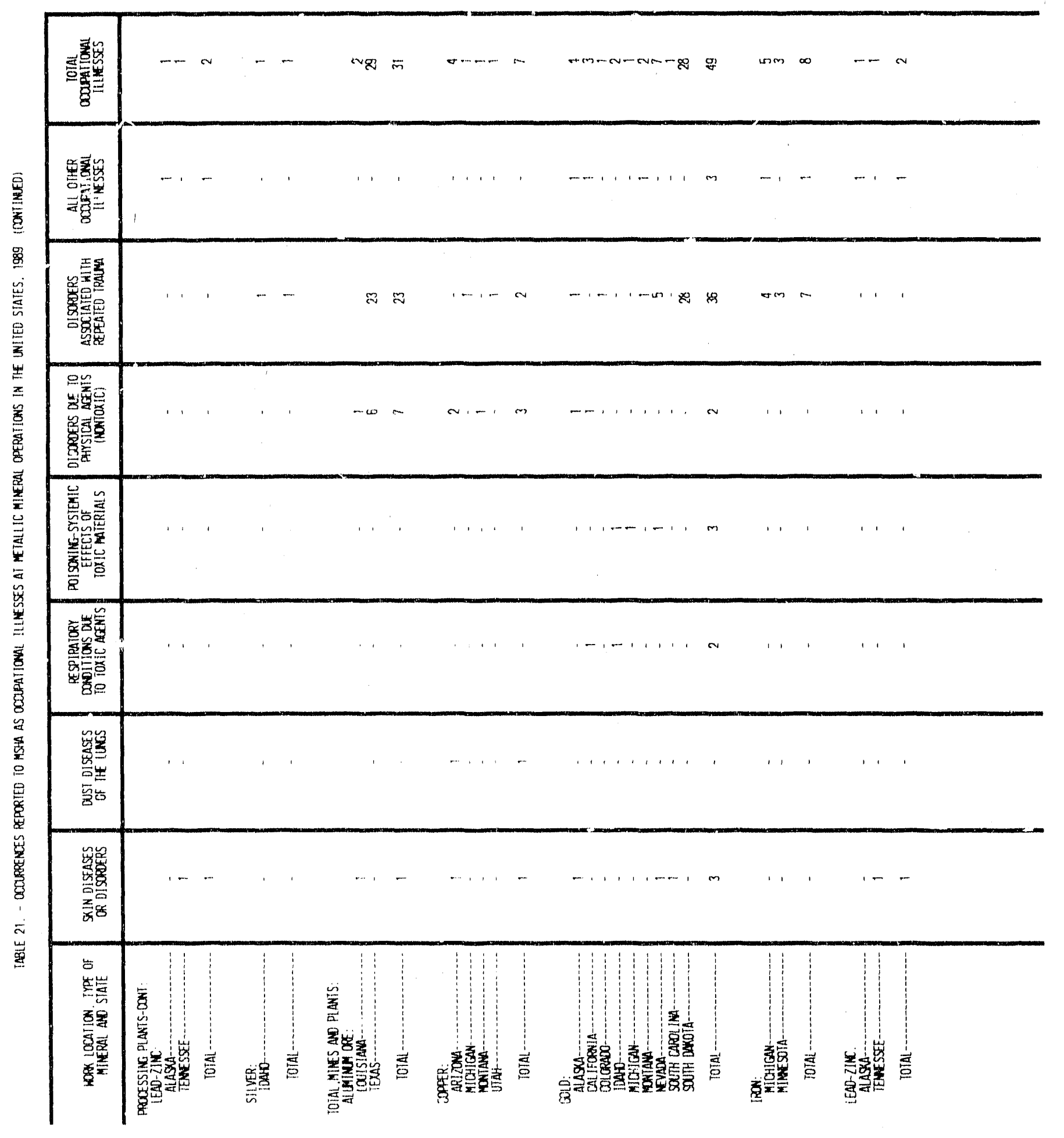




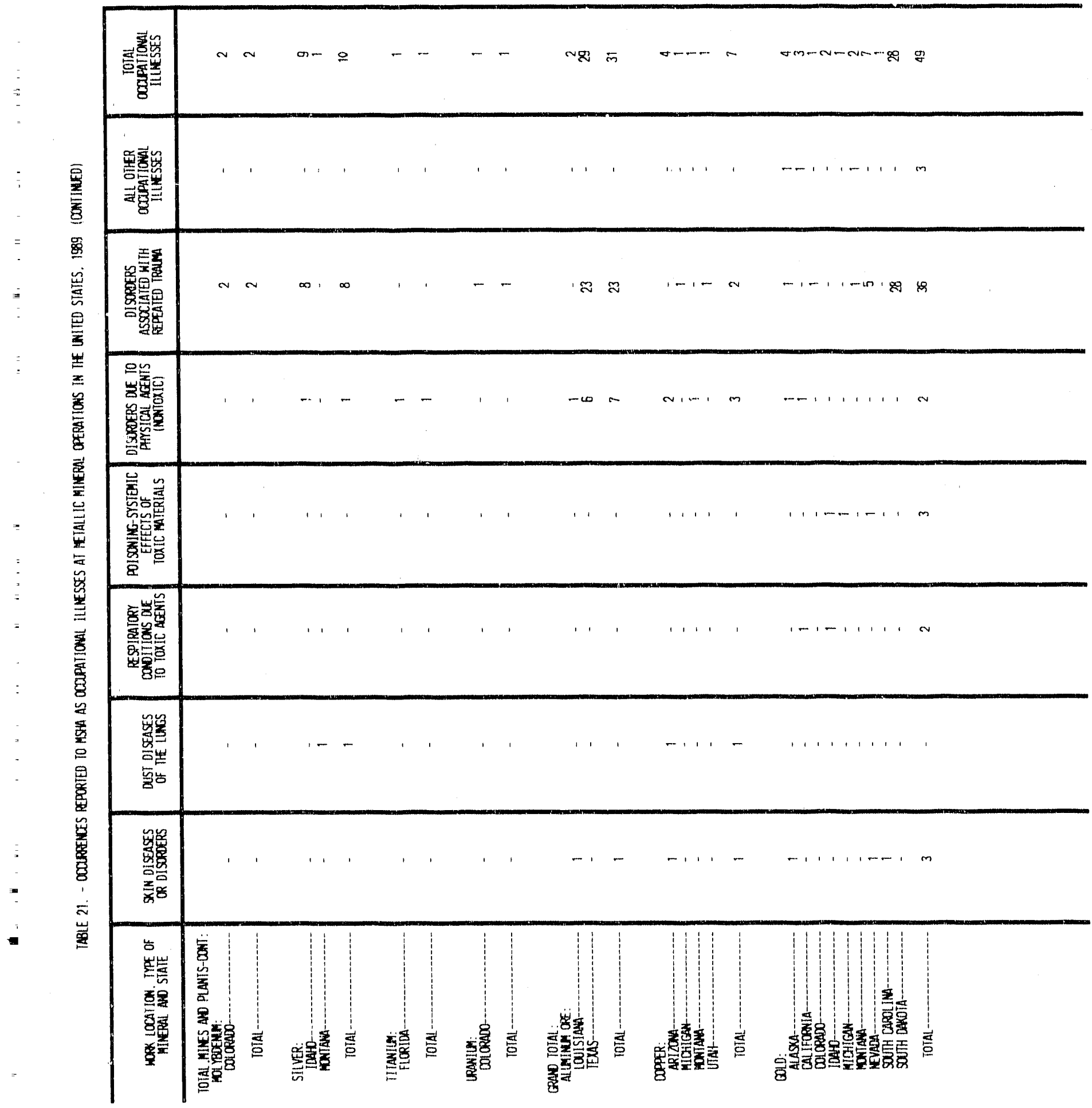




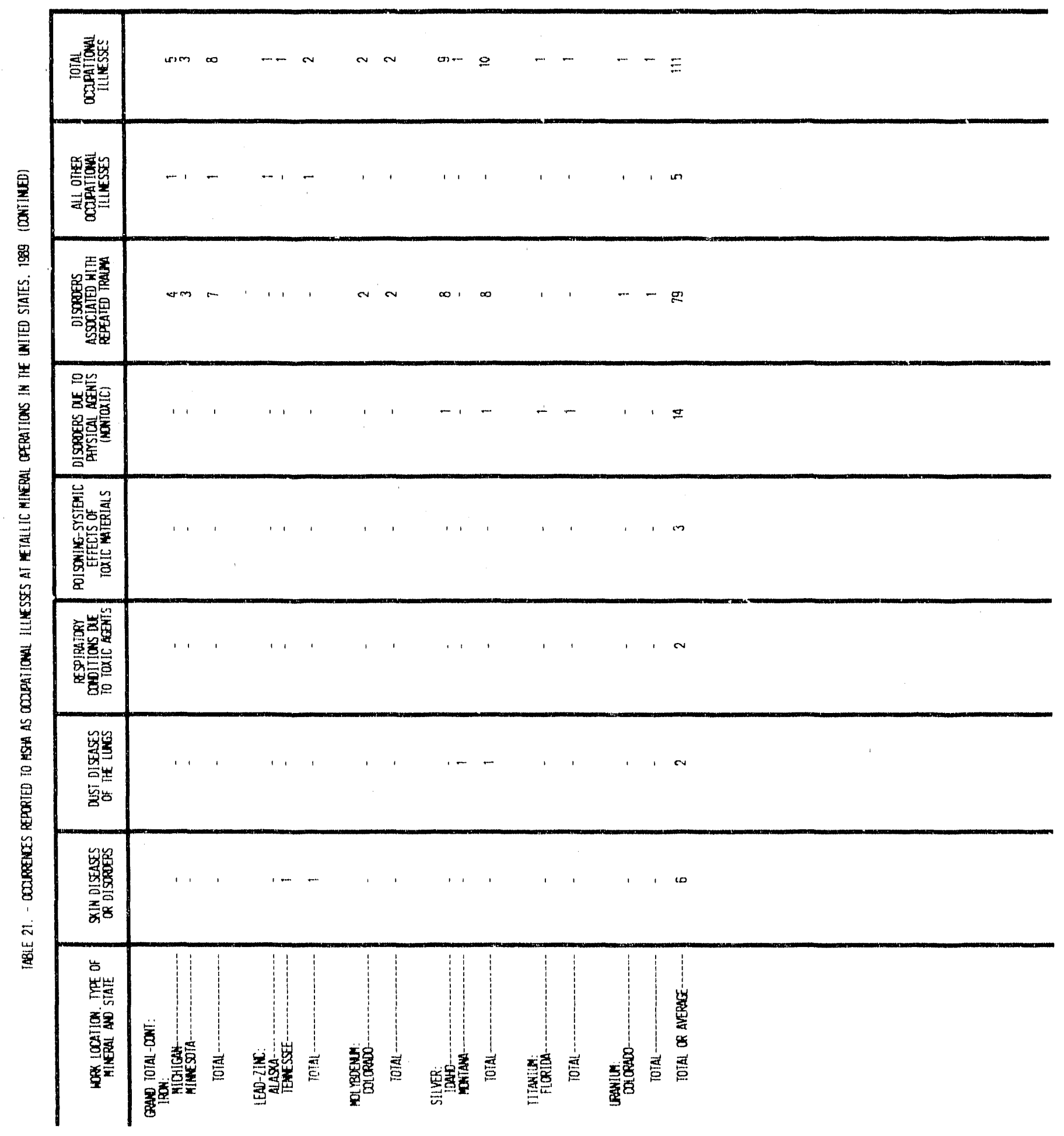




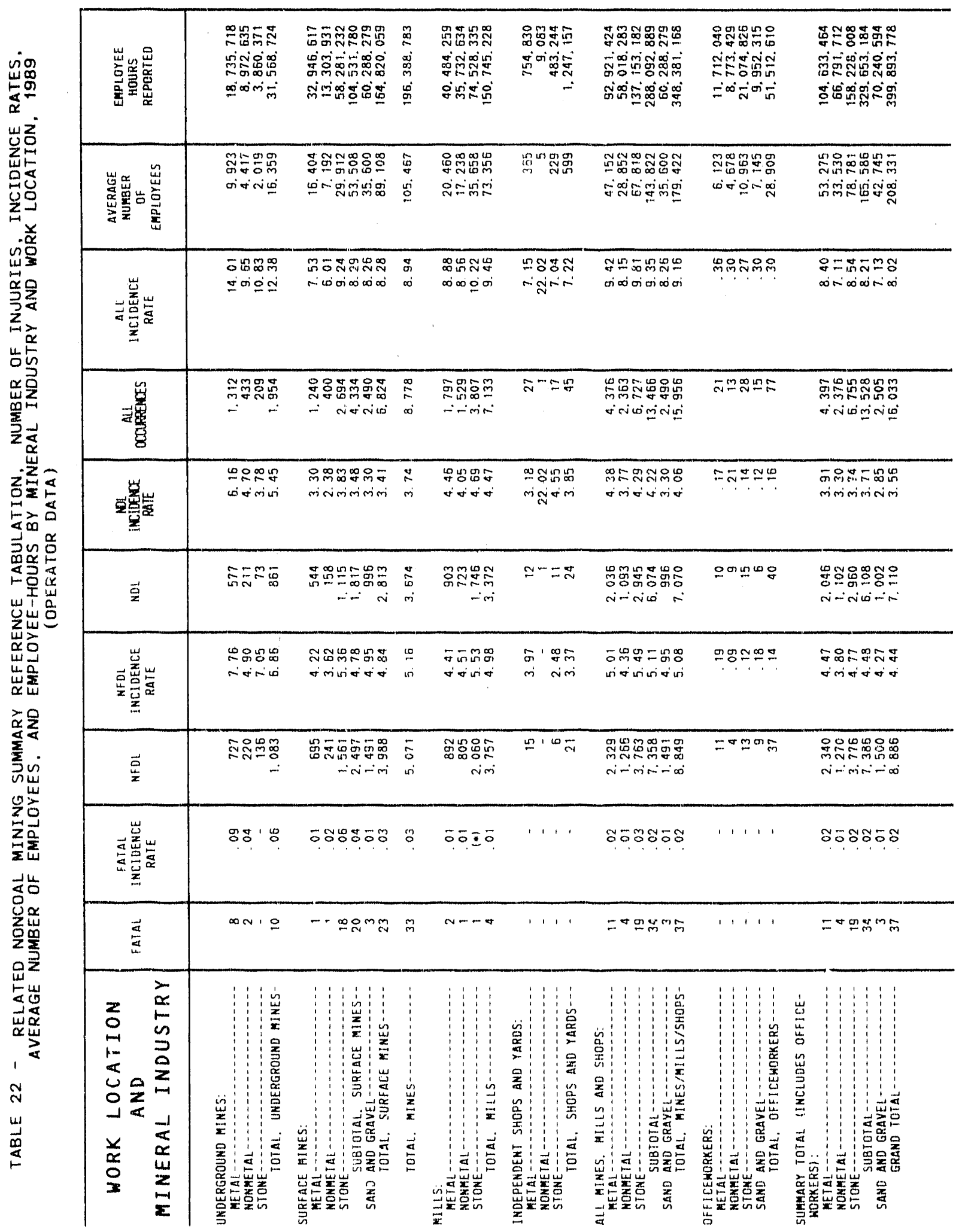




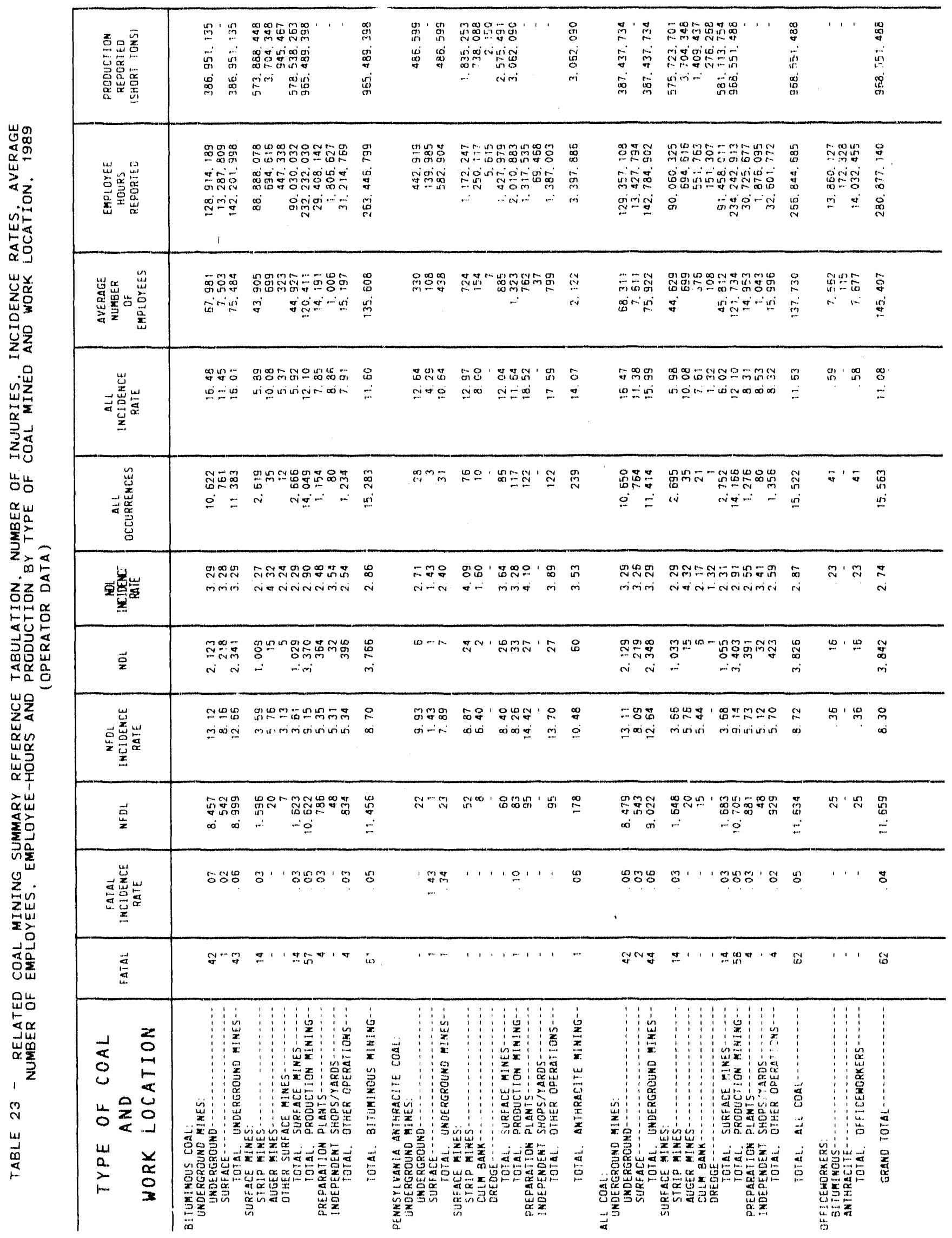


CONTRACTOR DATA 
The following reference guide facilitates locating particular data elenents contained in the annual statiatical tables for contractors. The number appearing in each cell indicates the table number in which that particular element may be found. All elements 1 isted under the stub headings appear as vertical headings in the tables. Elements 1 isted under column headings appear as horizontal headings. The only exception to this is table 1, in which the headings have been reversed.

To use this guide, read down or ucross the elements 1 isted in both the stub and column headings to determine which combinations are of interest. For example, 10st workdays by work location appear in eight different. tables, numbers $3,7,9,11,14,16,17$, and 18. However, only one of these, table 11, provides a further division by nature of injury as well. The occupation of the injured is found in table 17 and, as seen in the column headings, provides the number of injuries, 10st workdays, and average severity.

Although injuries and illnesses occurring to contractor employees are identifiable as to state in which they oncurred or commodity mined, natching exposure hours are not available. Incidence rates for such employees are possible only at the national level for the various subunits and only for the coal industry as a whole and the uoncoal industry as a whole. Consequently, certain tables have been entirely eliminated from the contractor portion of this report. The remaining tables are numbered to correspond with the comparable table in the operator portion of this report. 
ERTERENCE GUIDE TO GETALLIC ETATISTICAS TASLES (CONTRACTOR DATA)

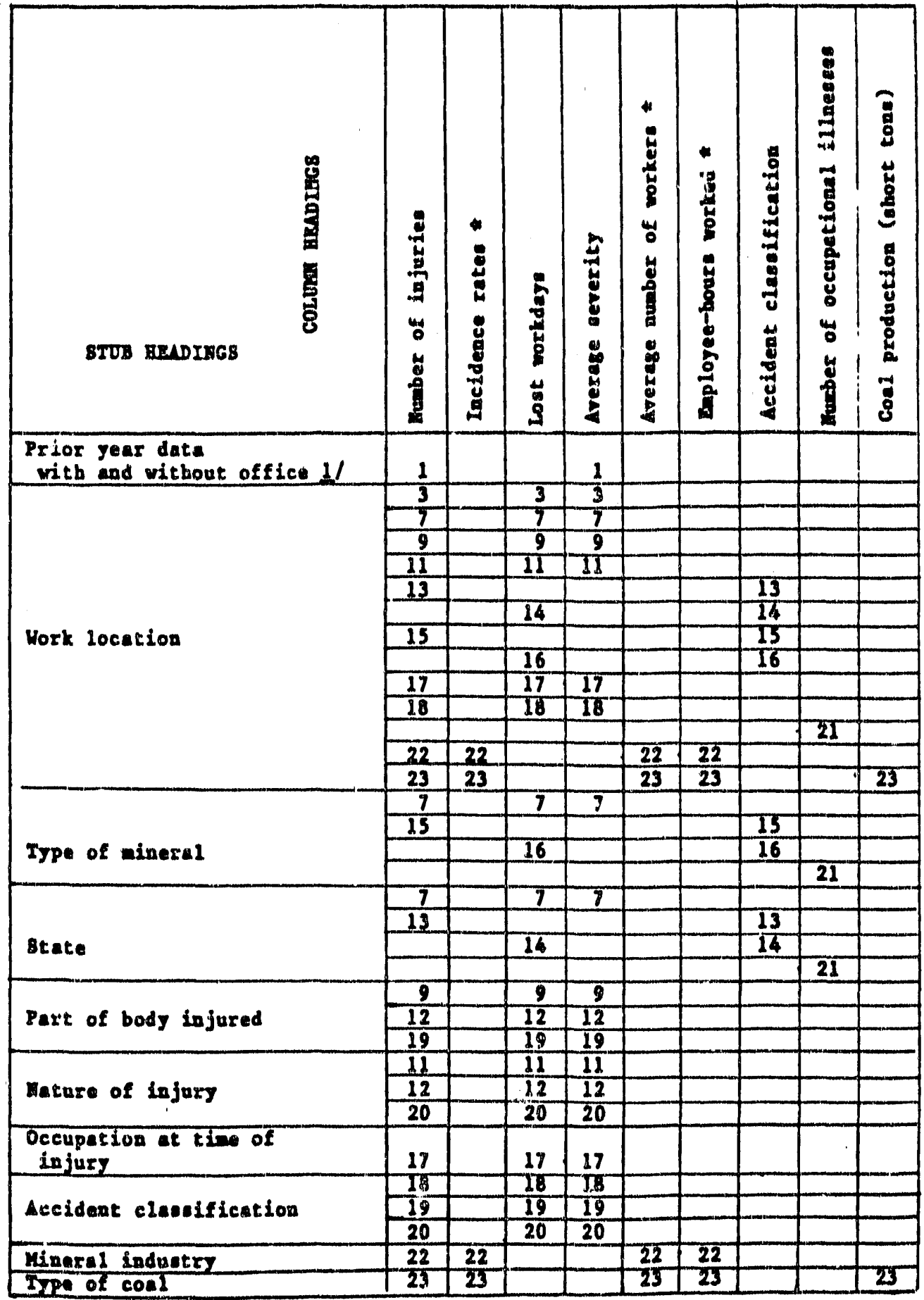

I/ Yor table 1 only, the inading have been revareed to oimplify this cuide; that 10, the date elemante indicated in the colum beadinge for this table actualis appear an verticall headingo.

- Contractor dats appearing in Table 22 for tha colum headingu denoted with an soteriak (*) in thio guide are obrainable oniy for the noacoal induetry at wole and do not rapranent a particular alberal inductry. 


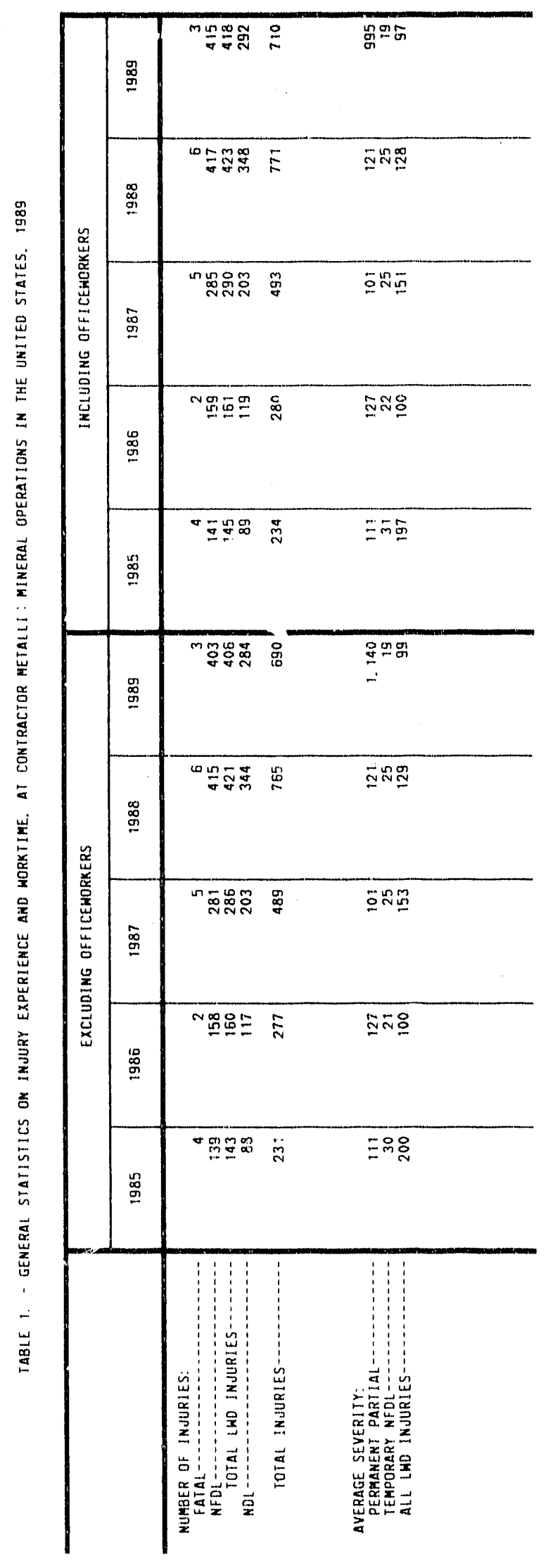




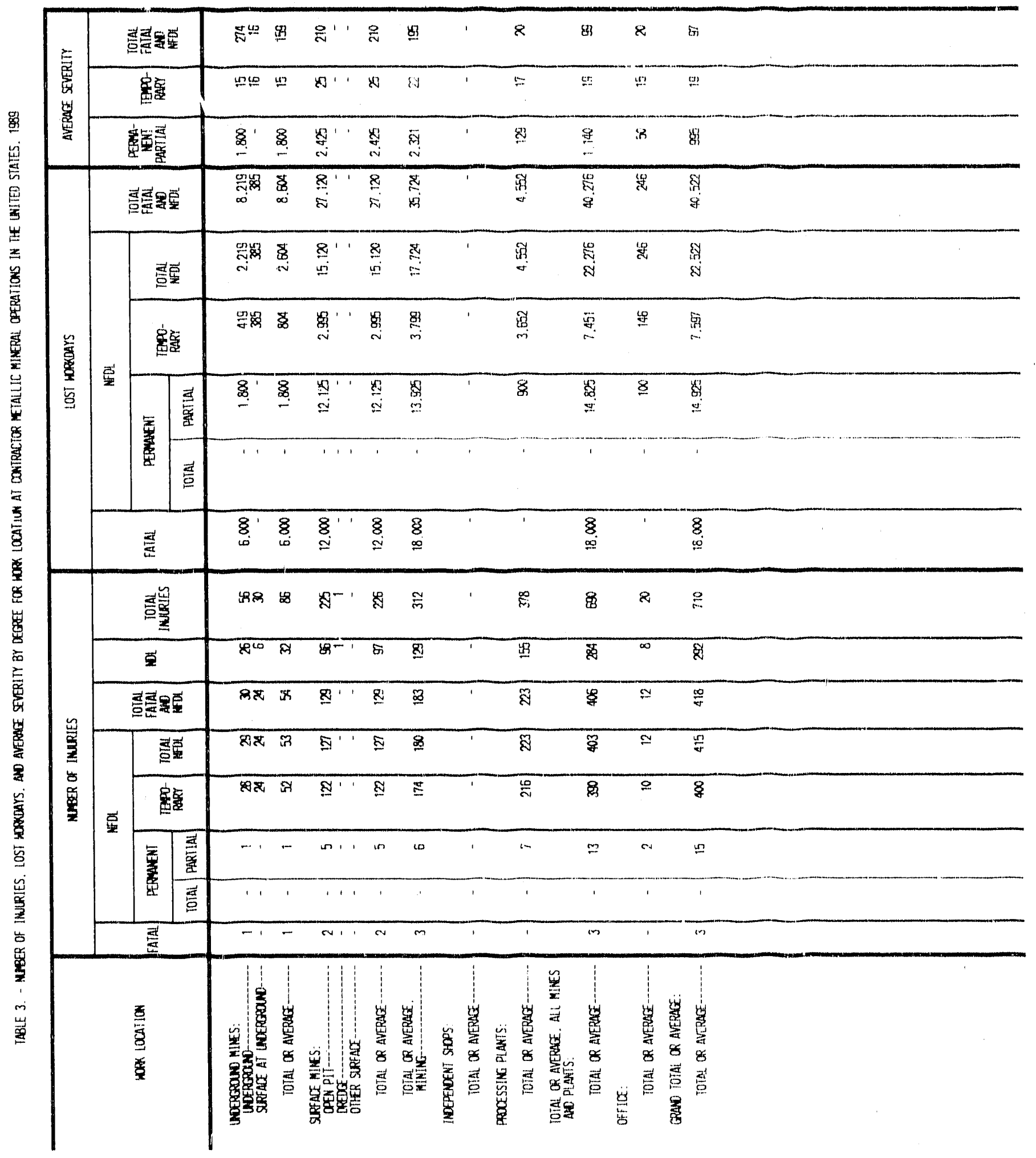




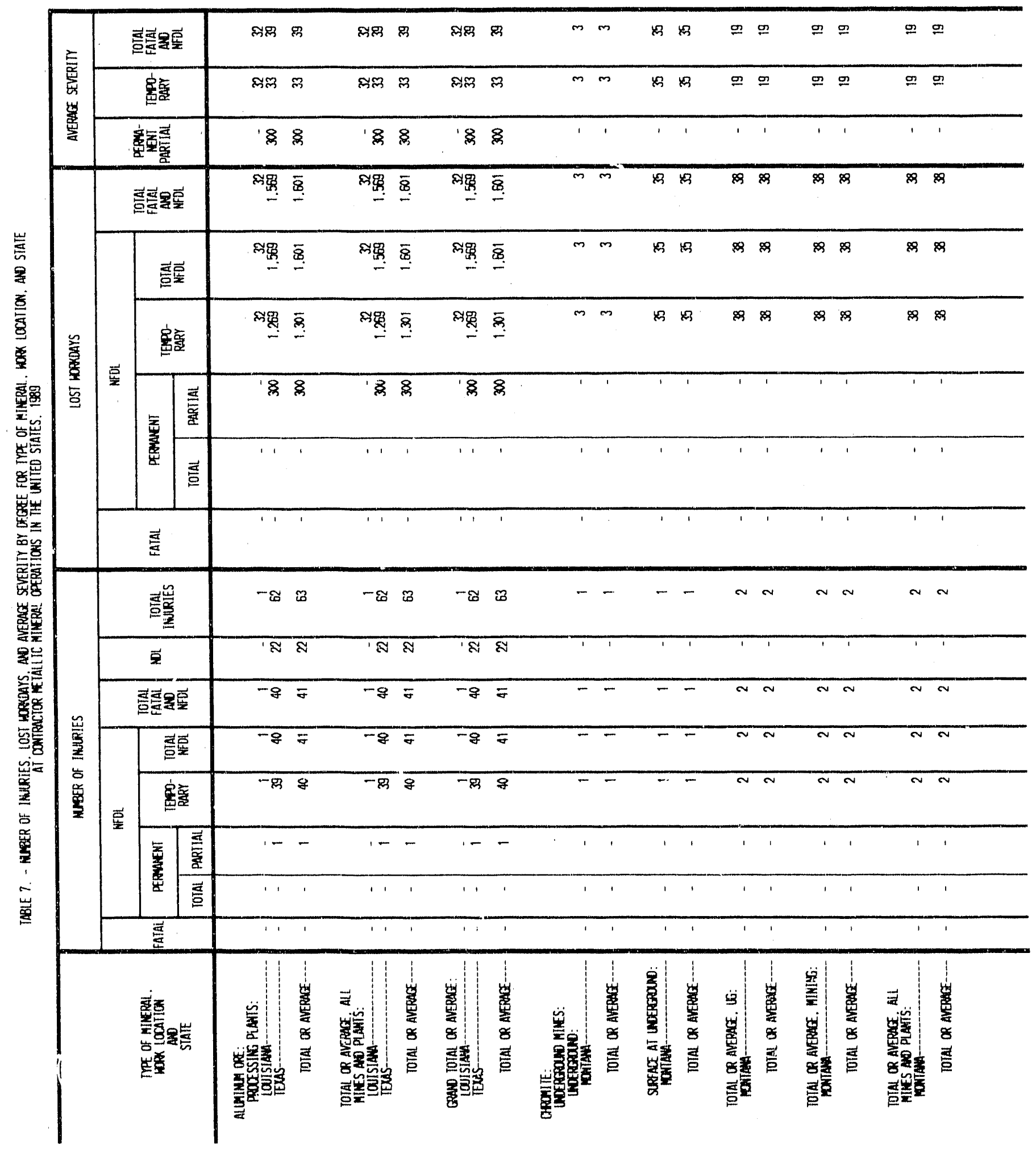




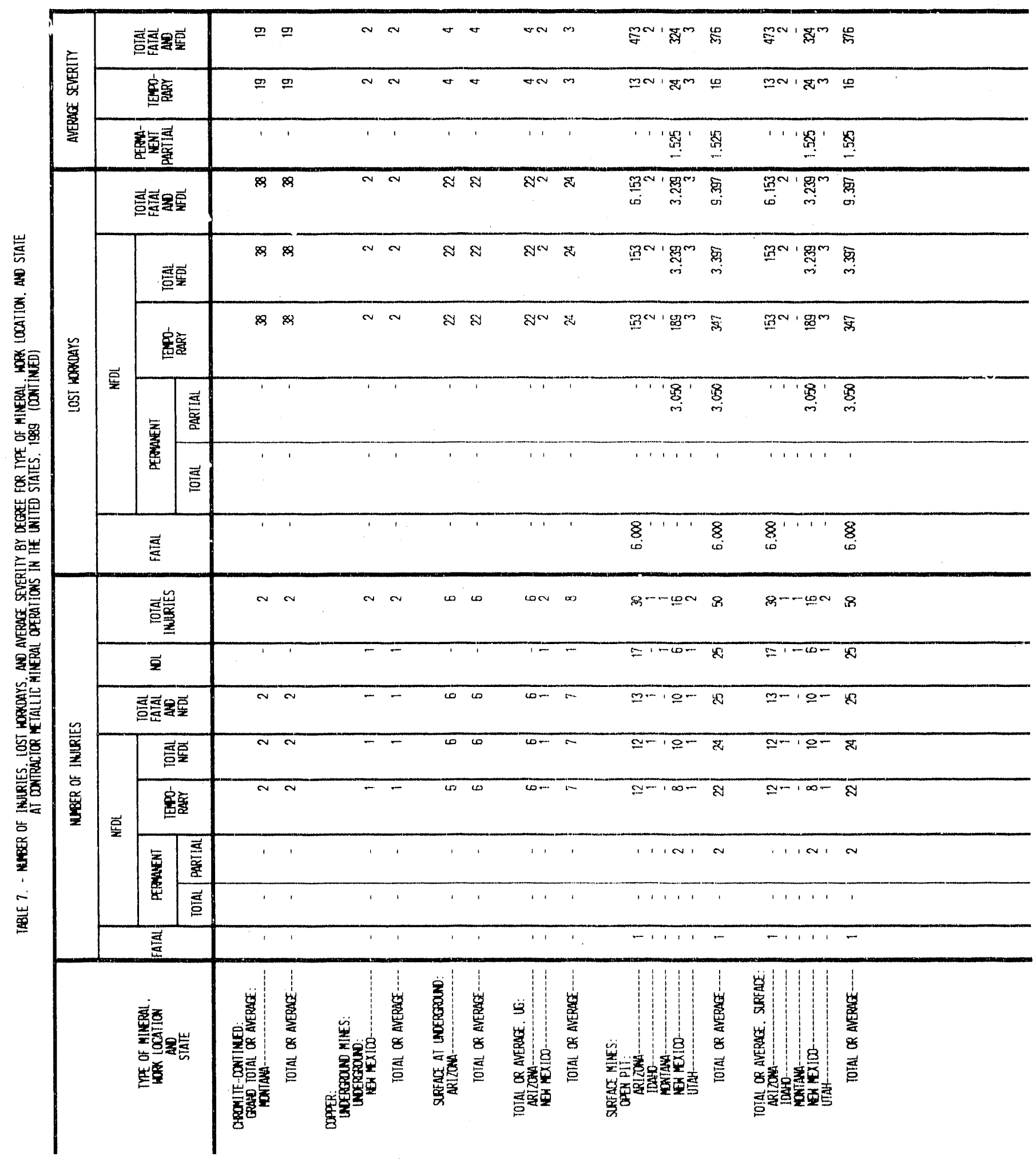




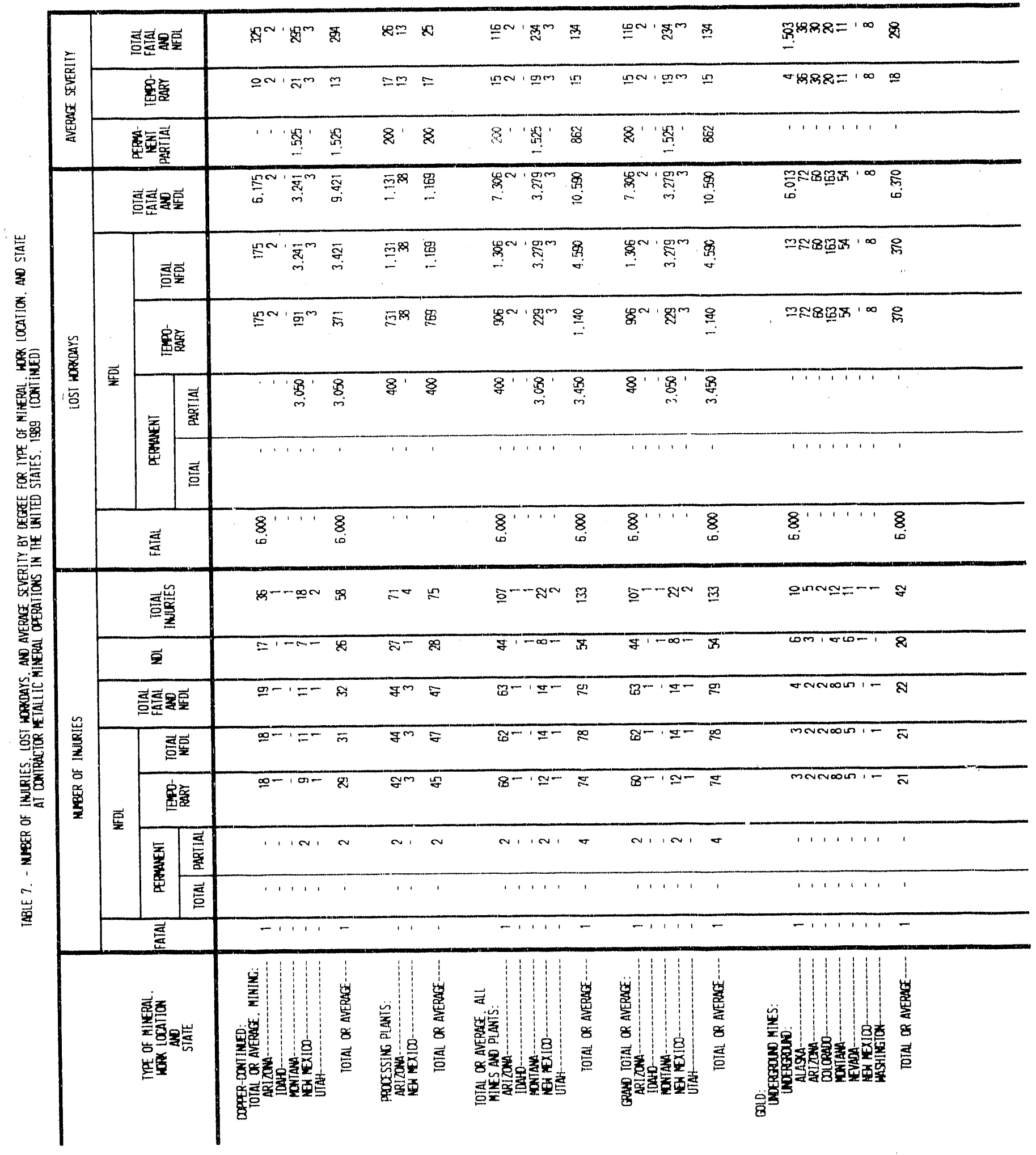




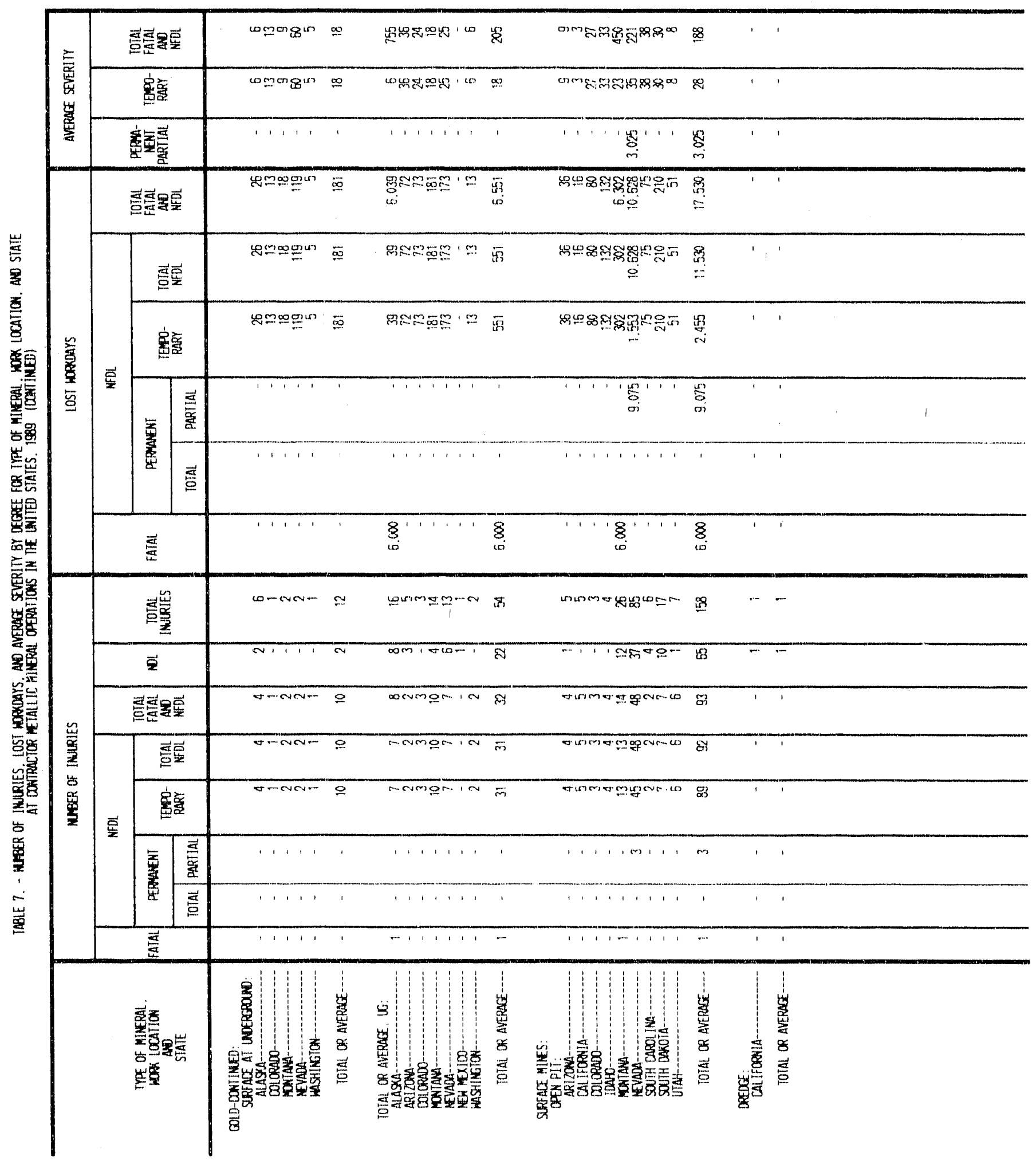




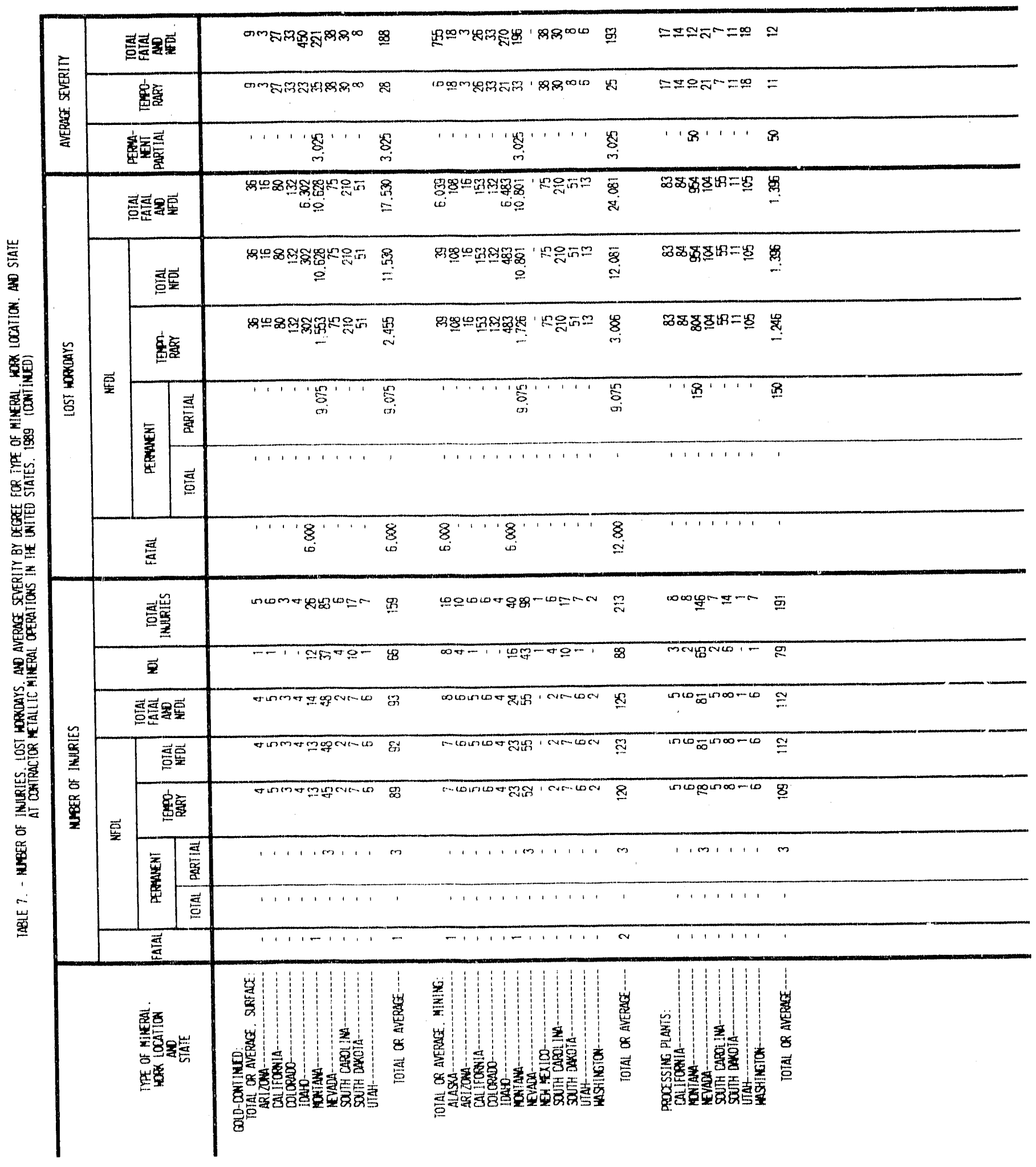




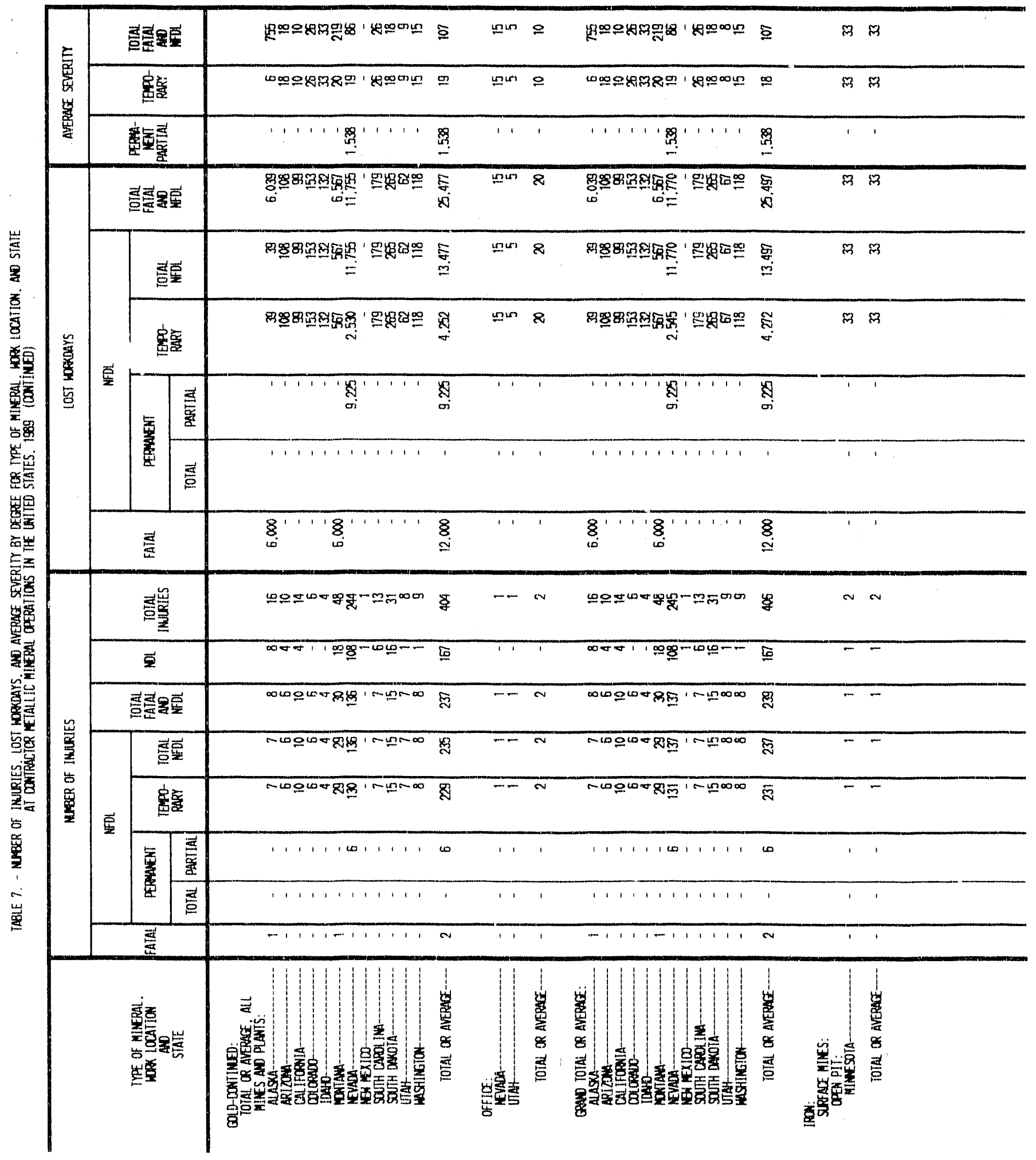




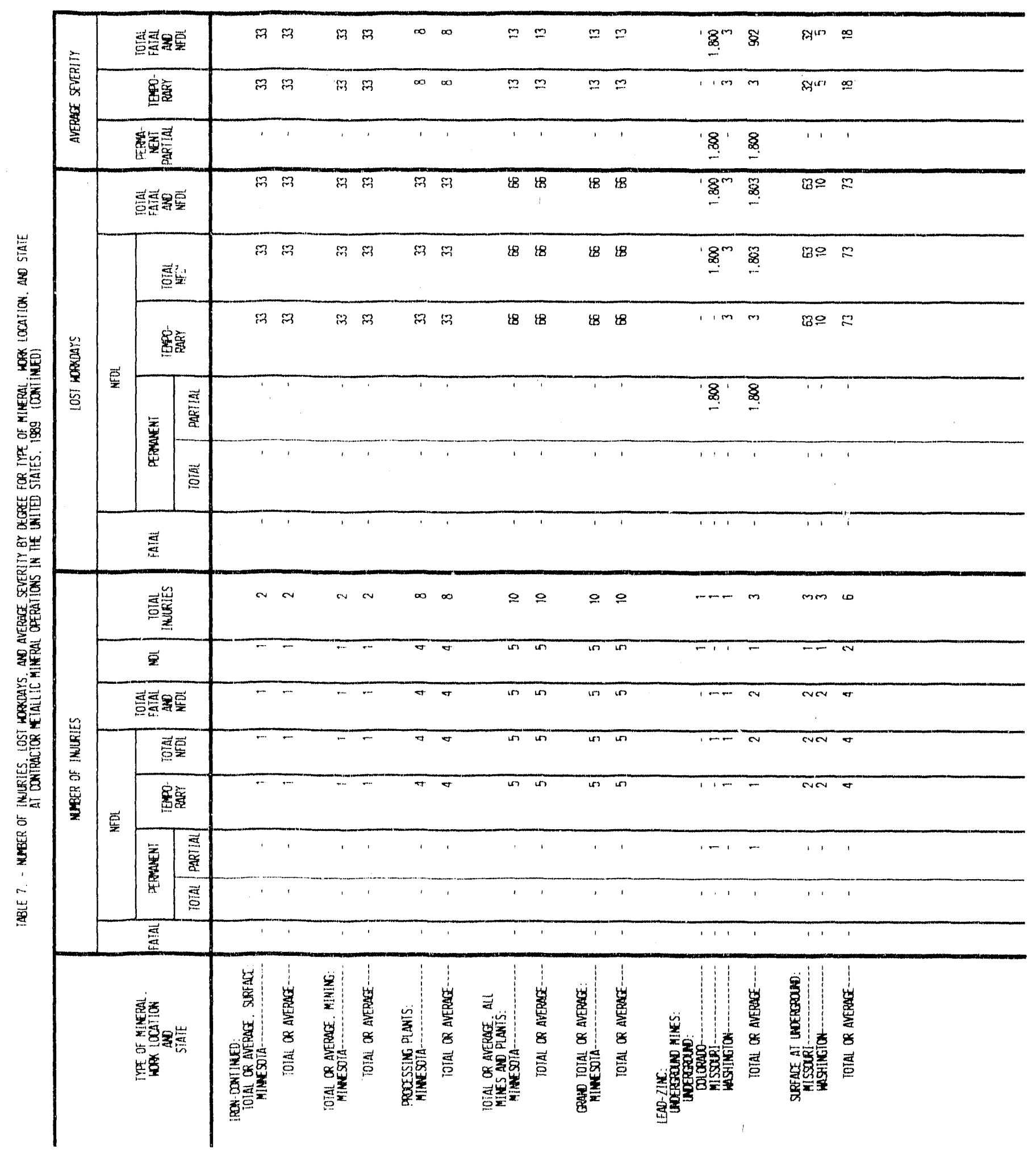




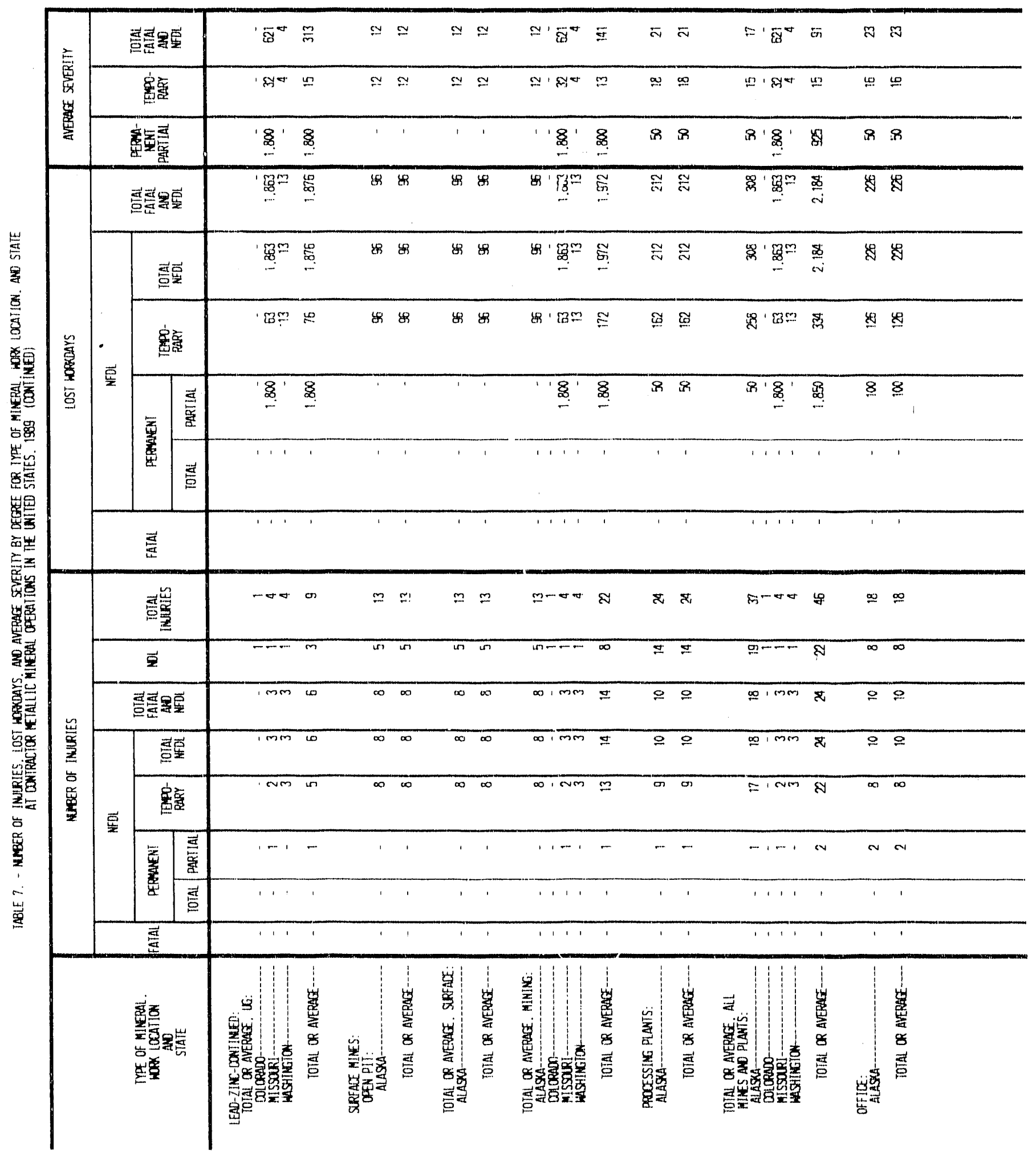




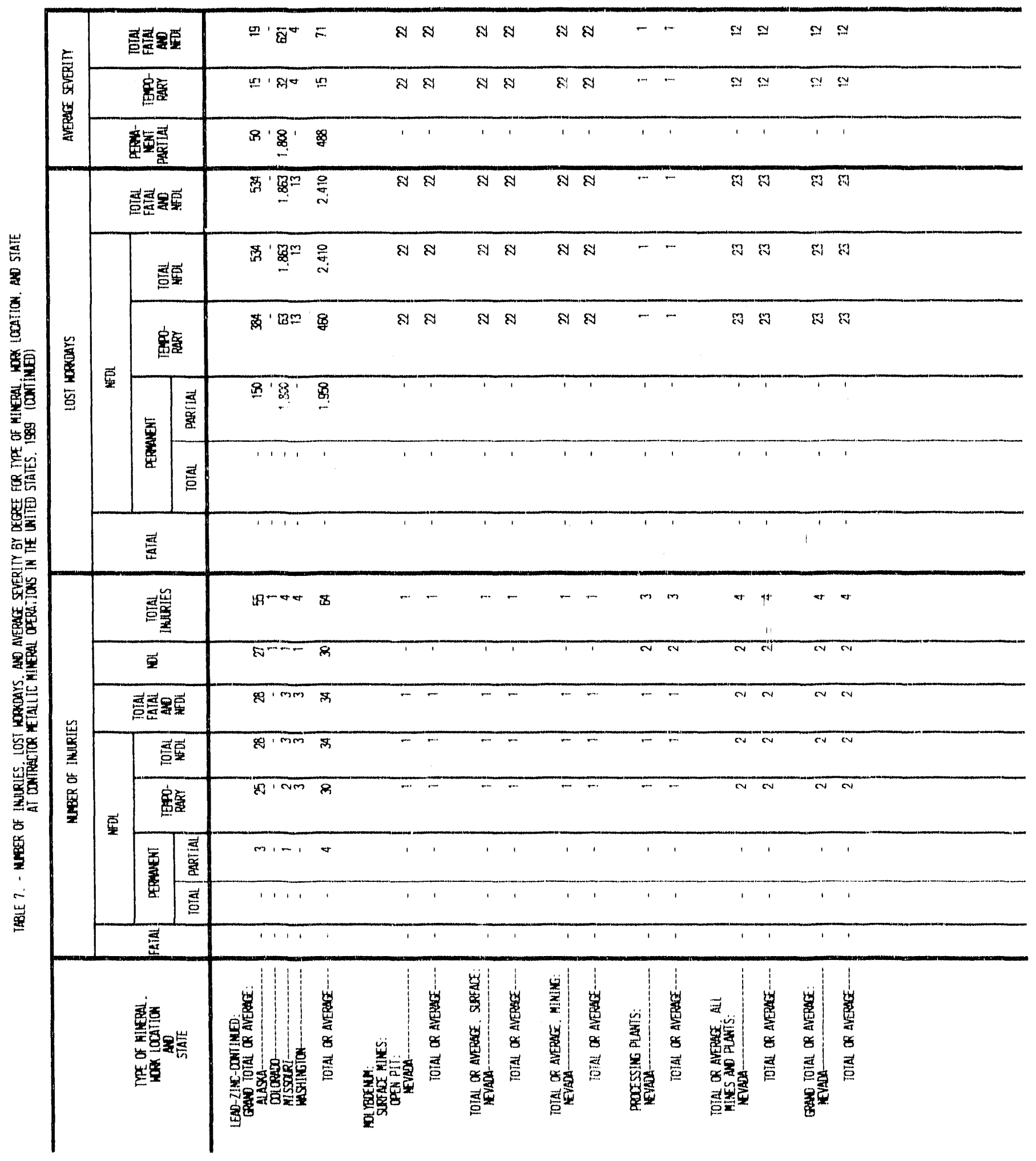




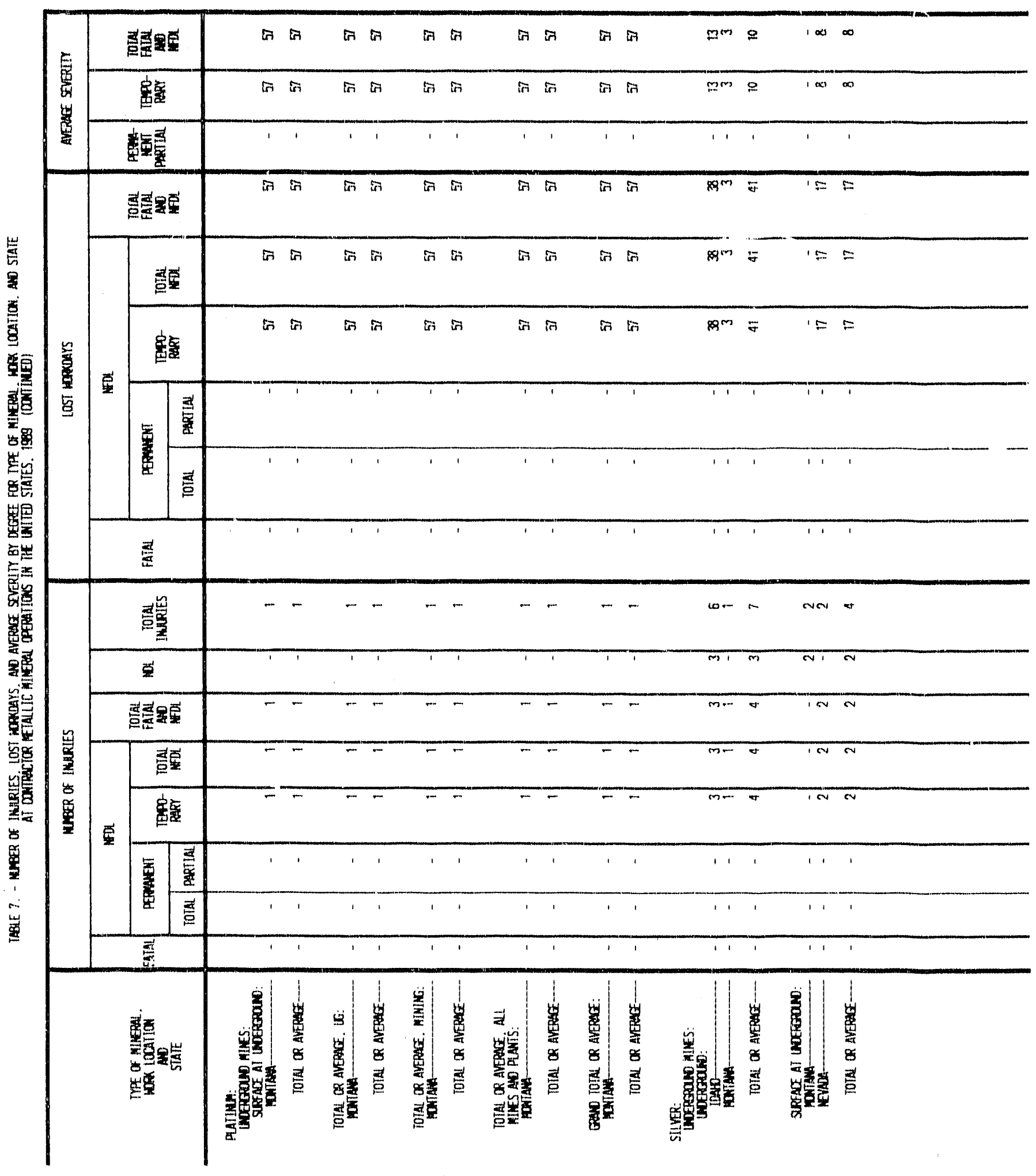




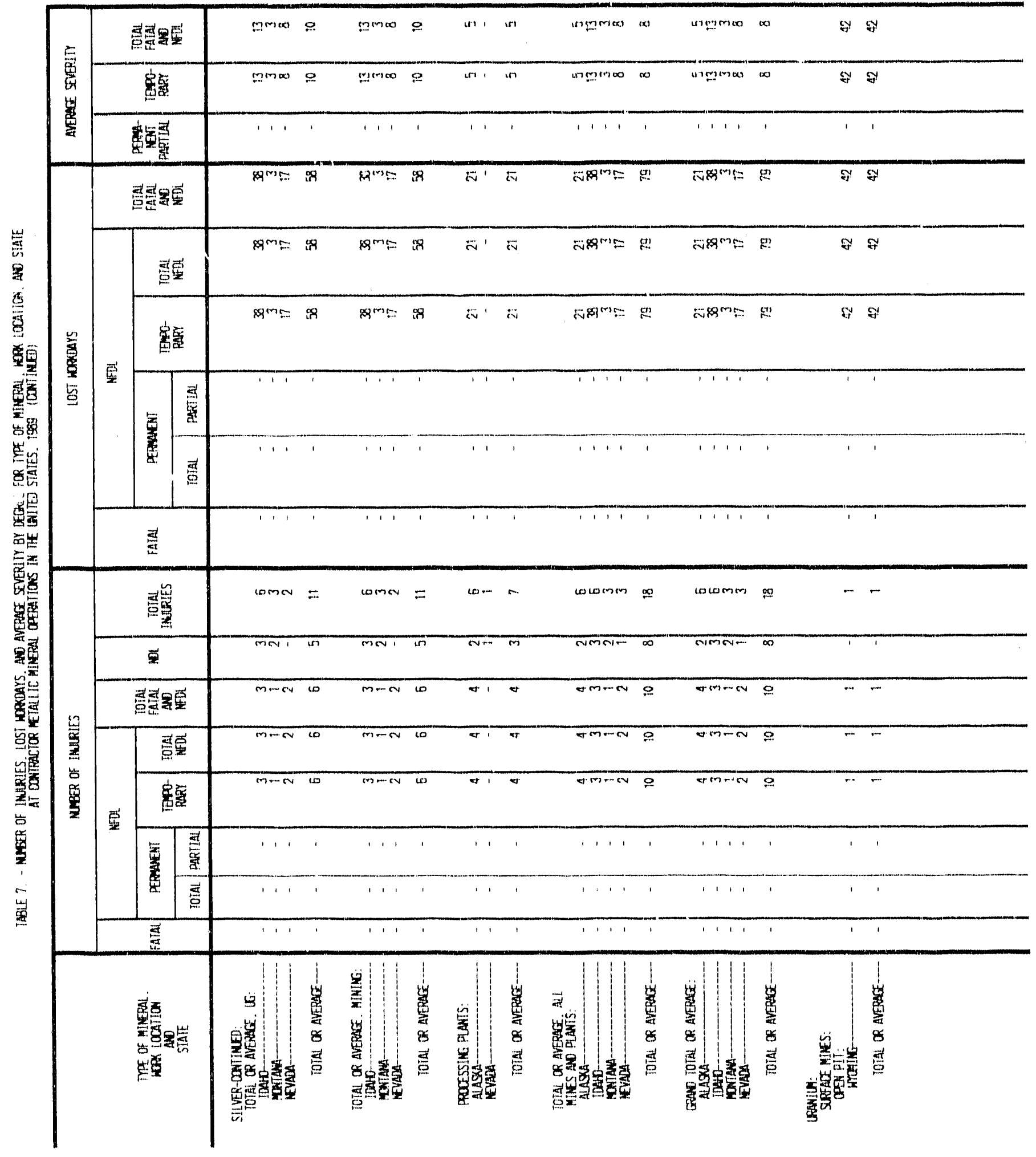




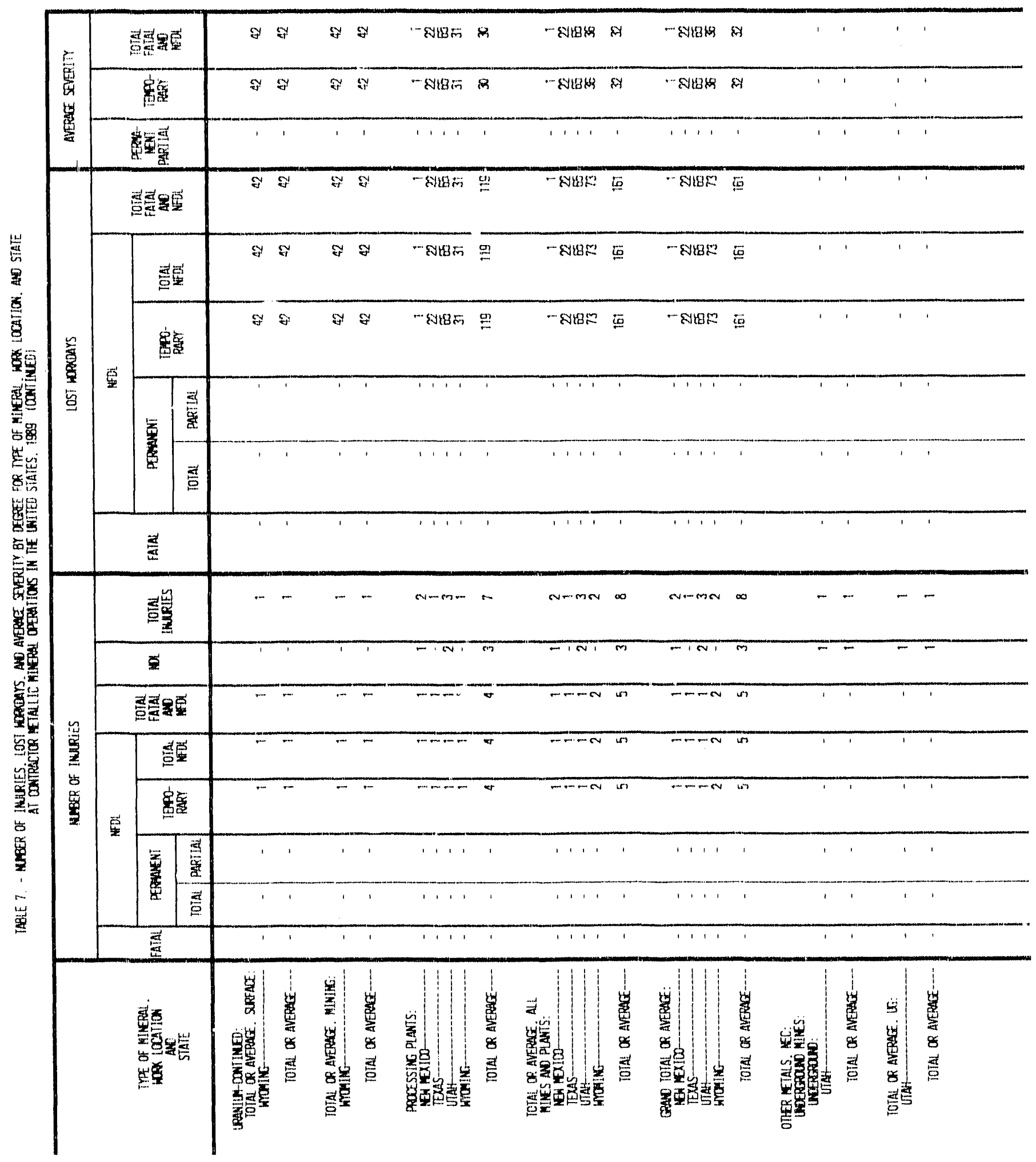




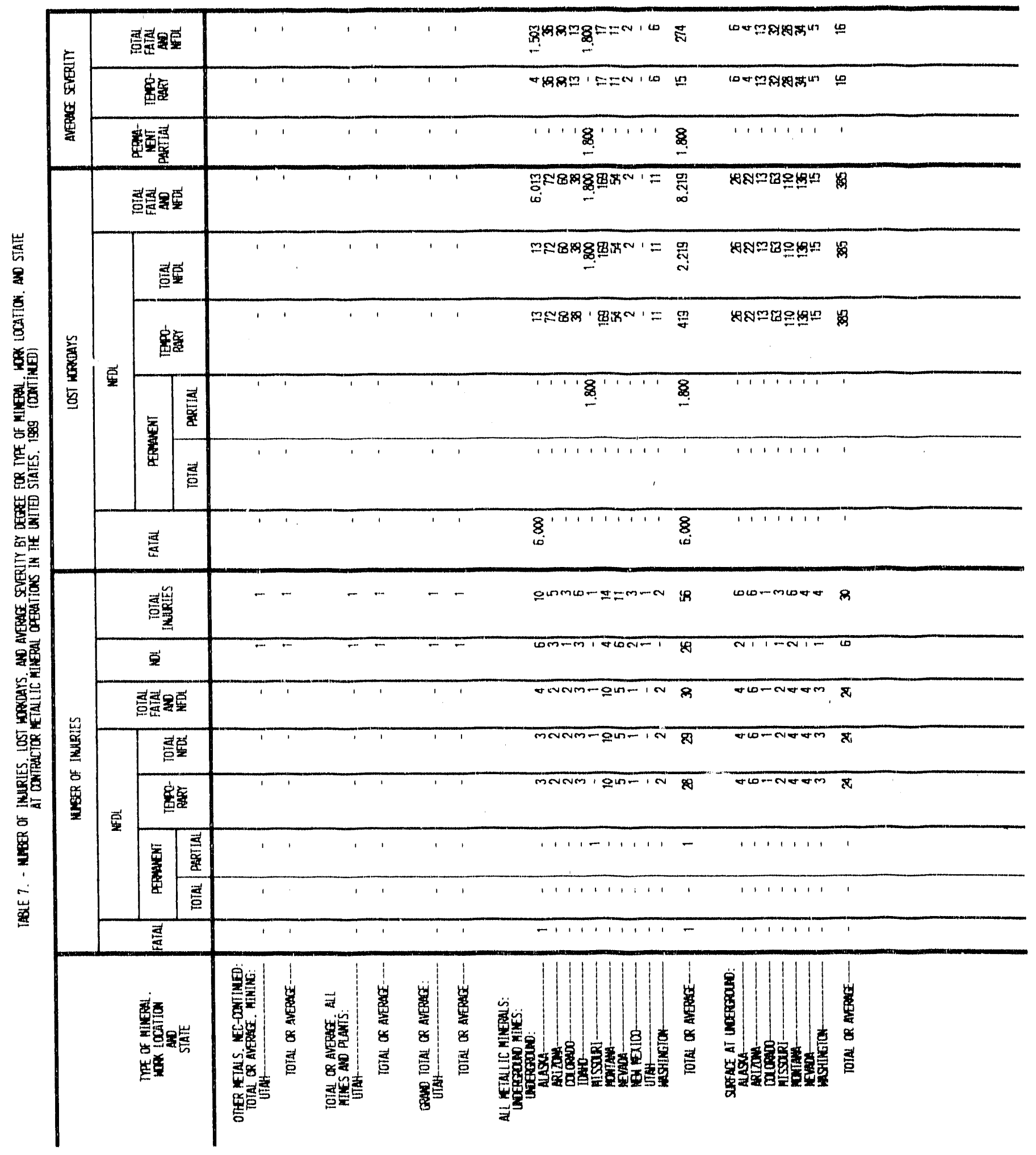




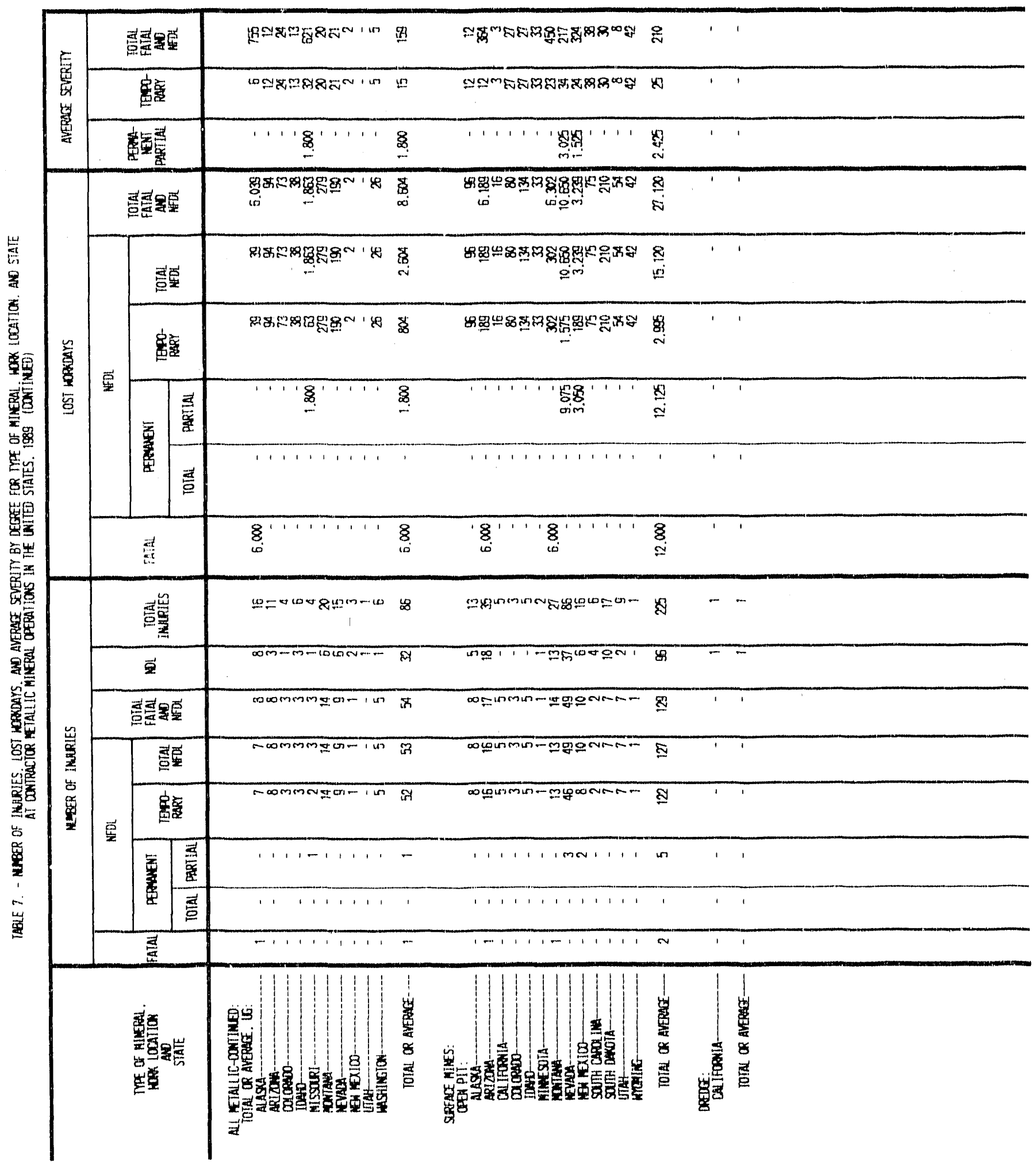




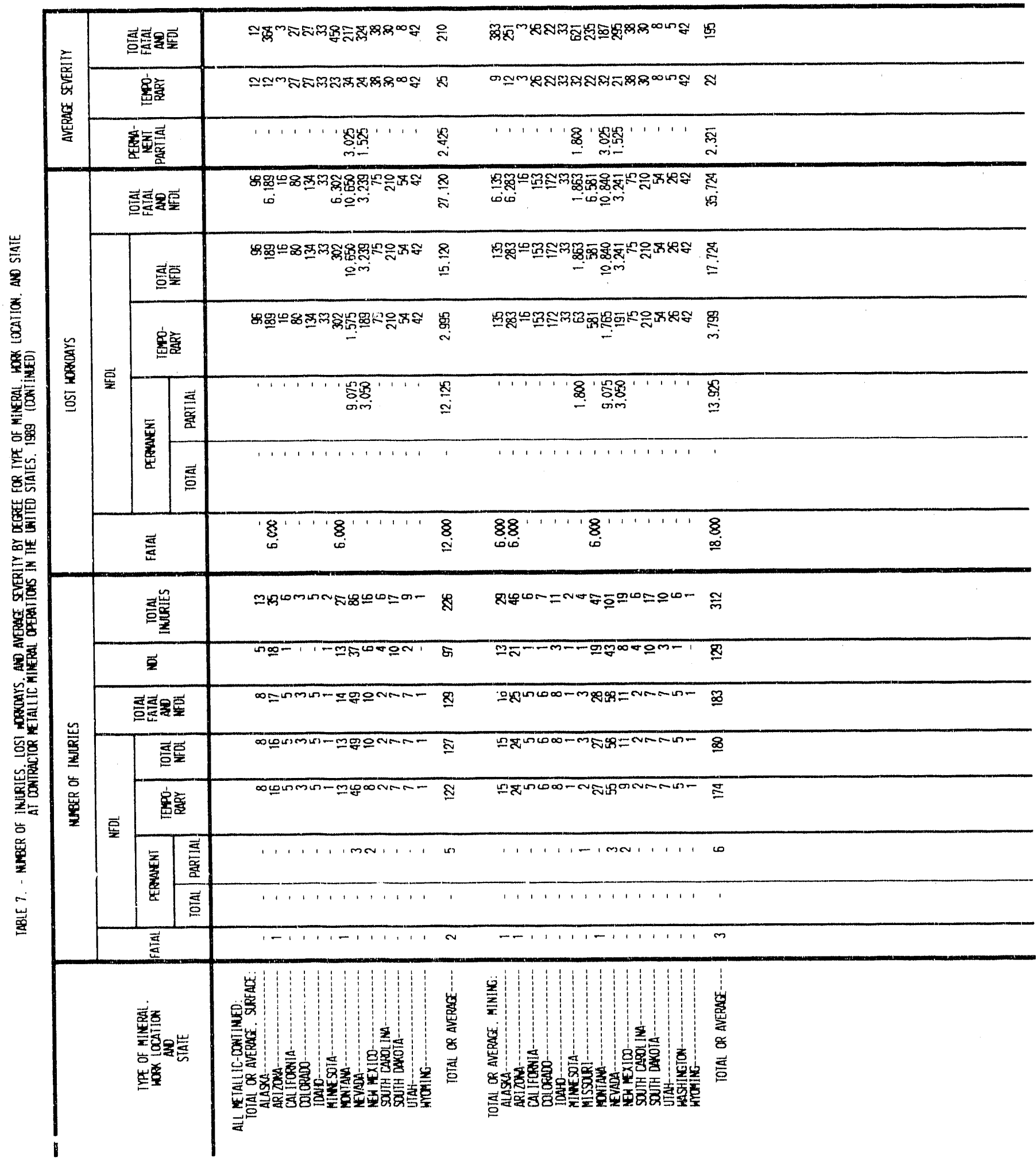




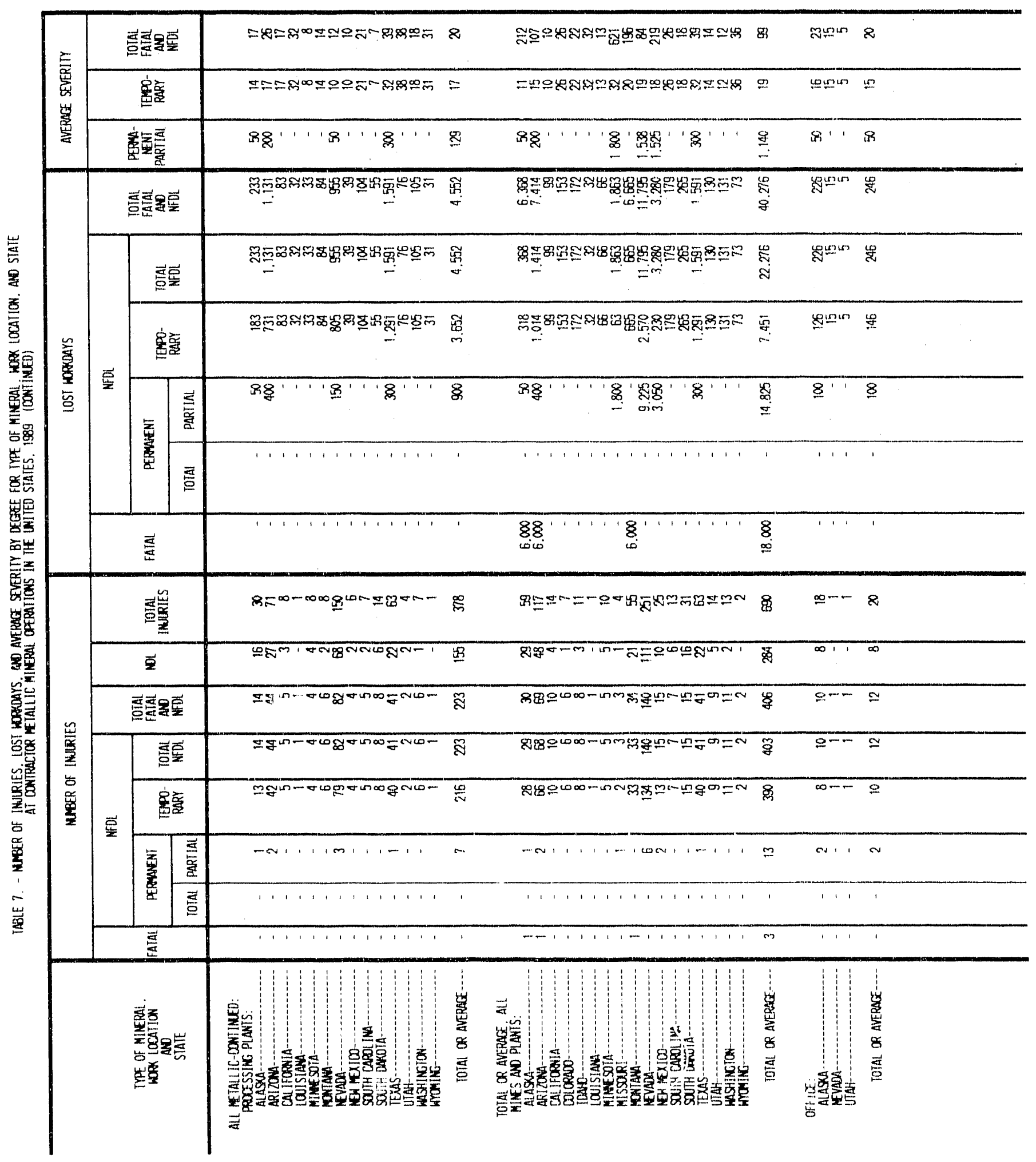




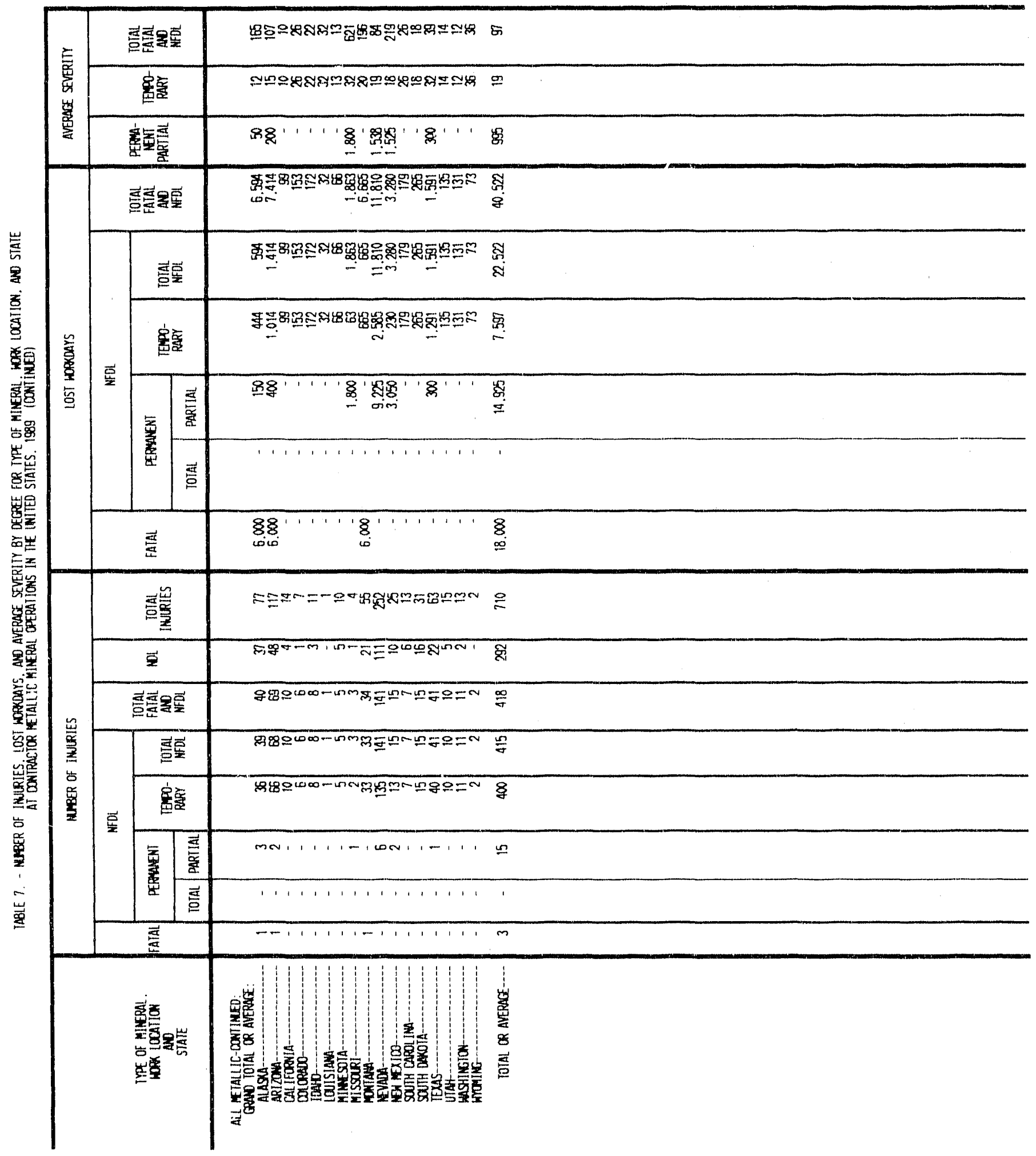




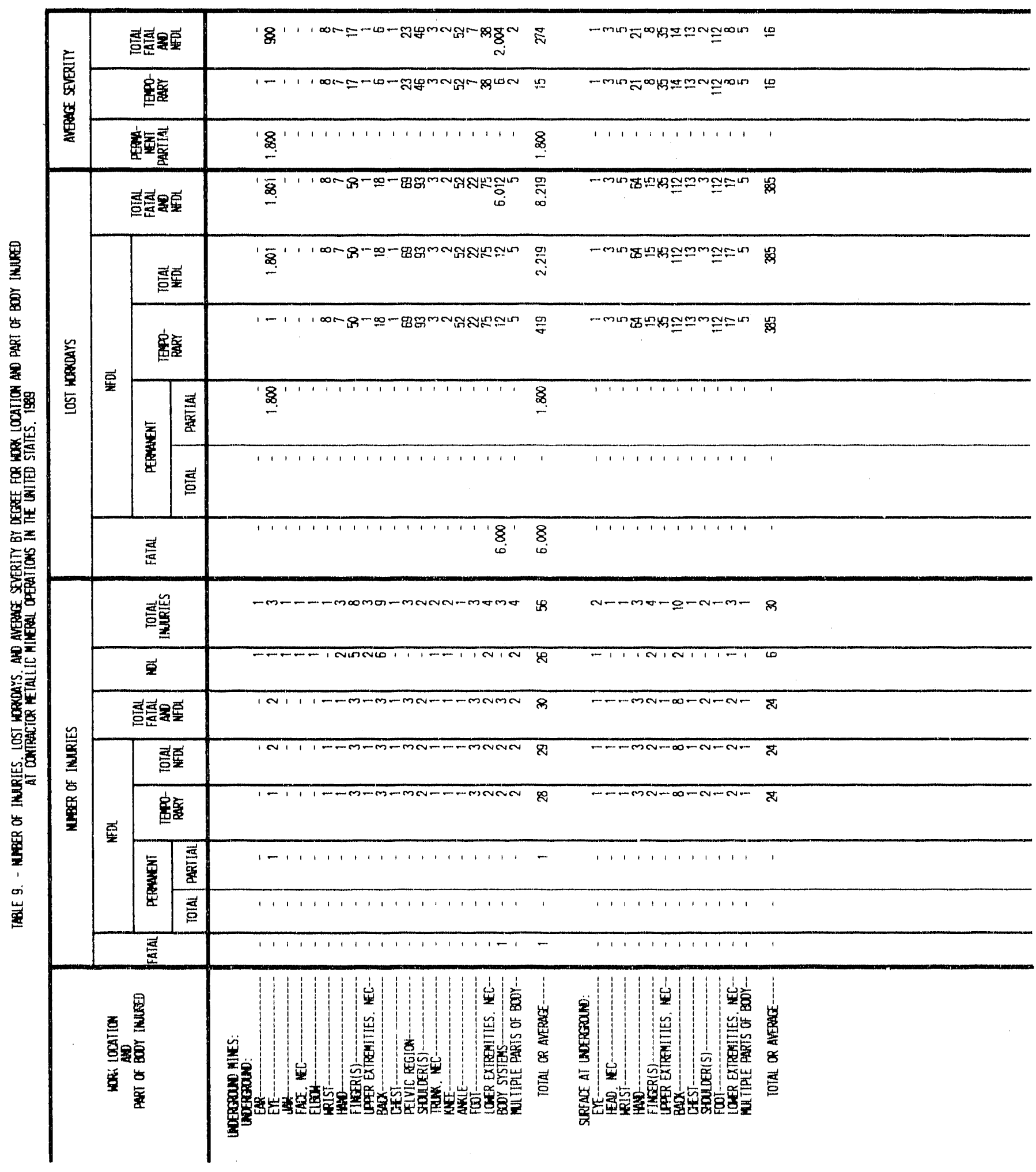




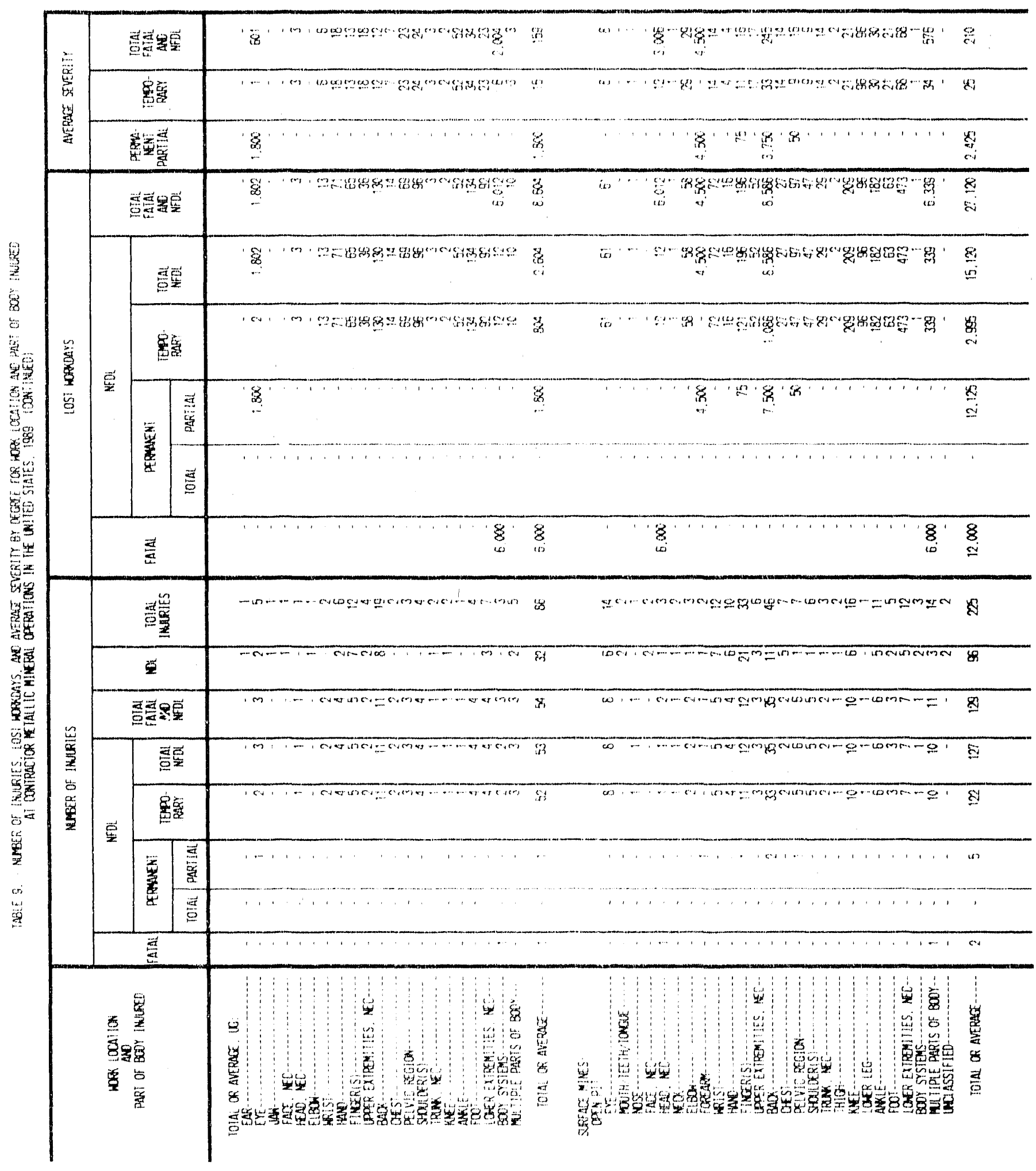




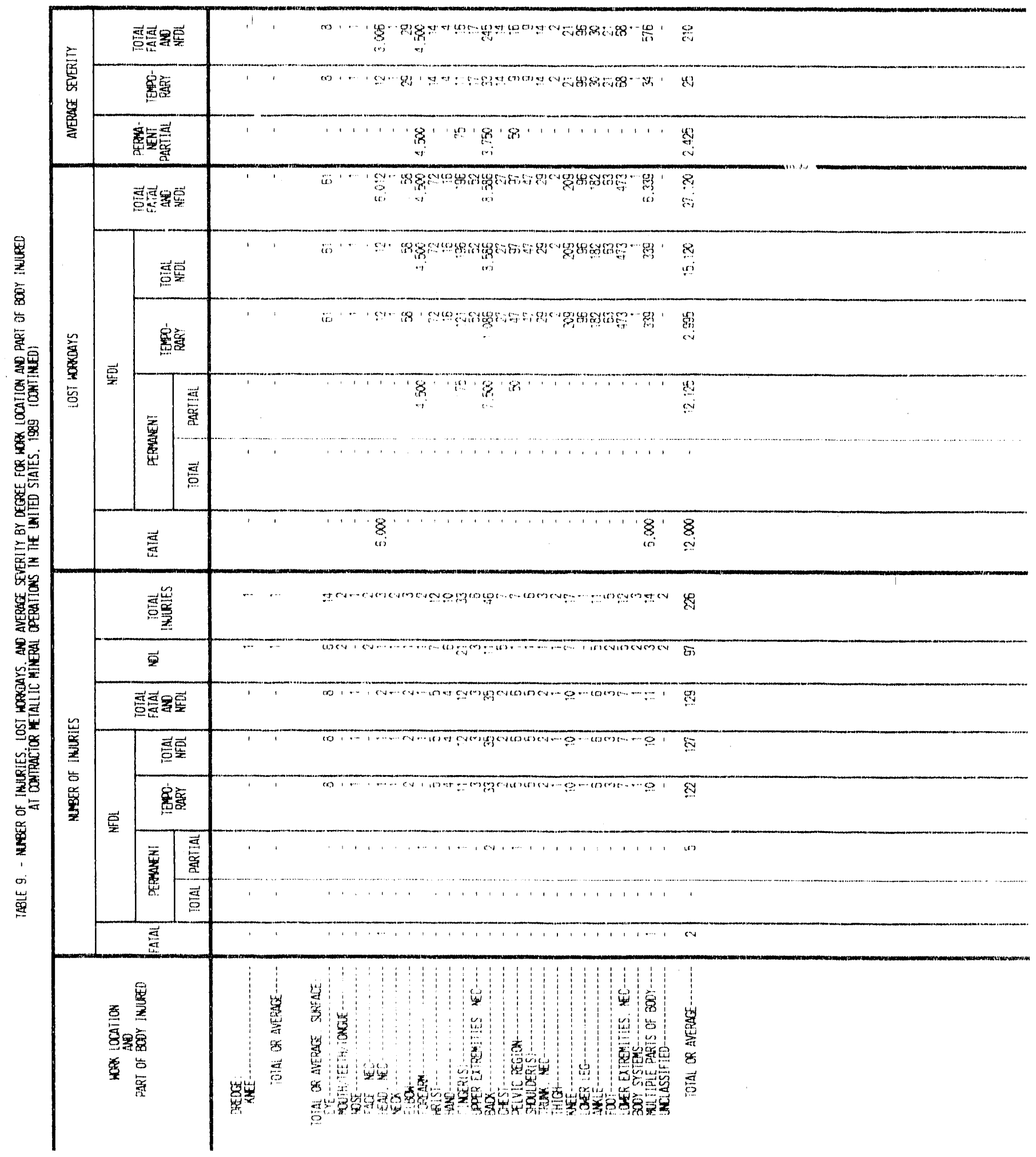




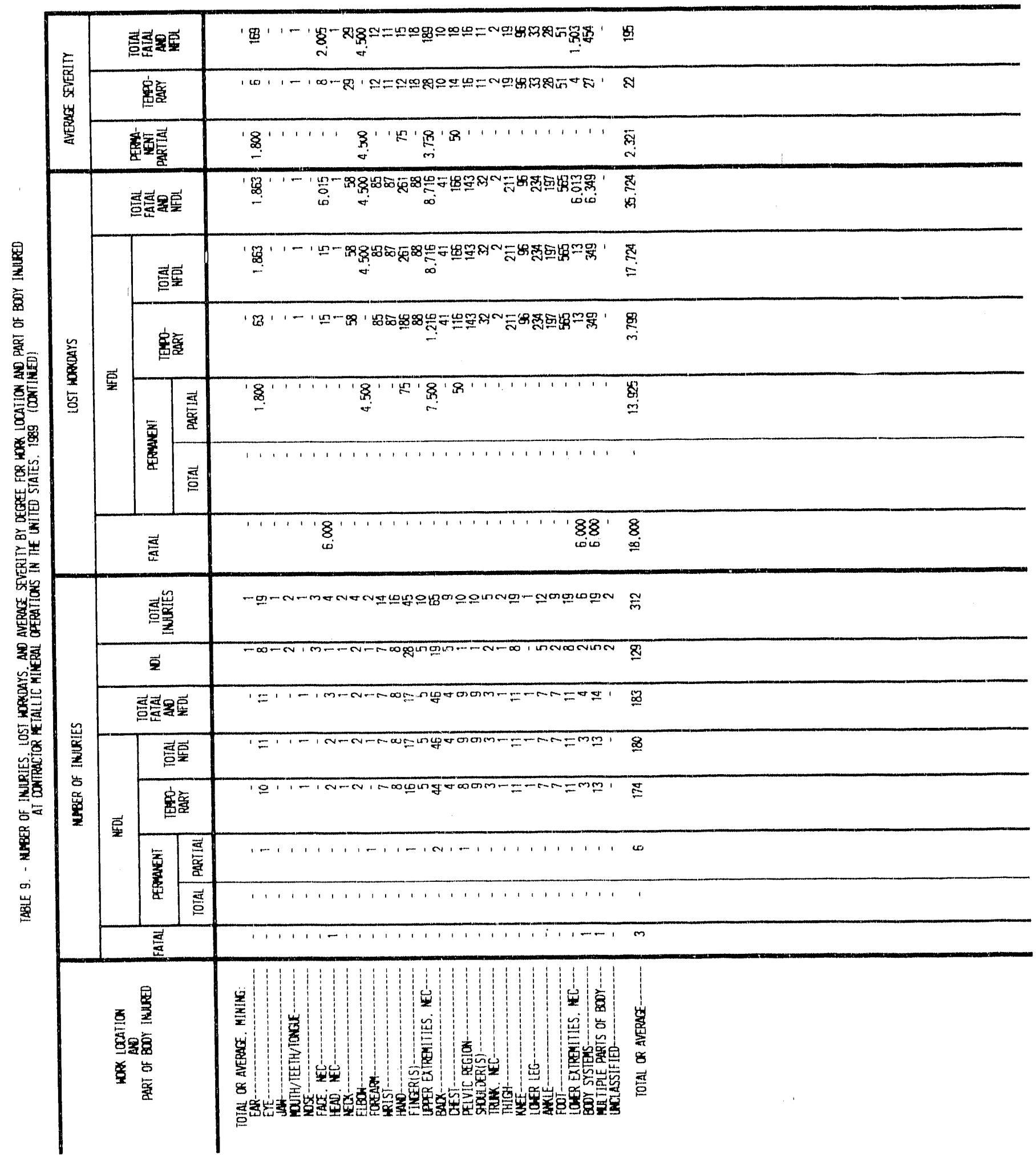




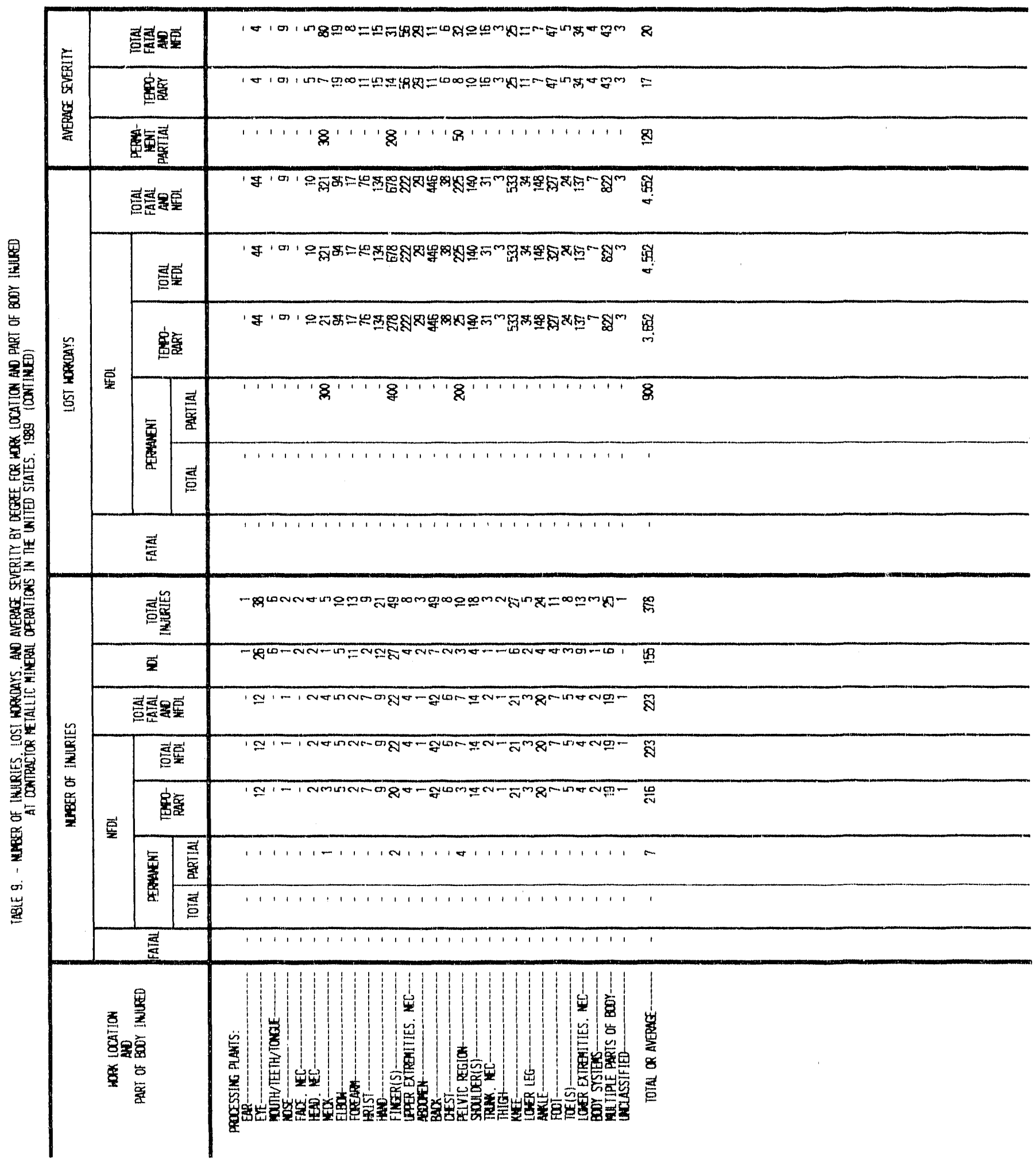




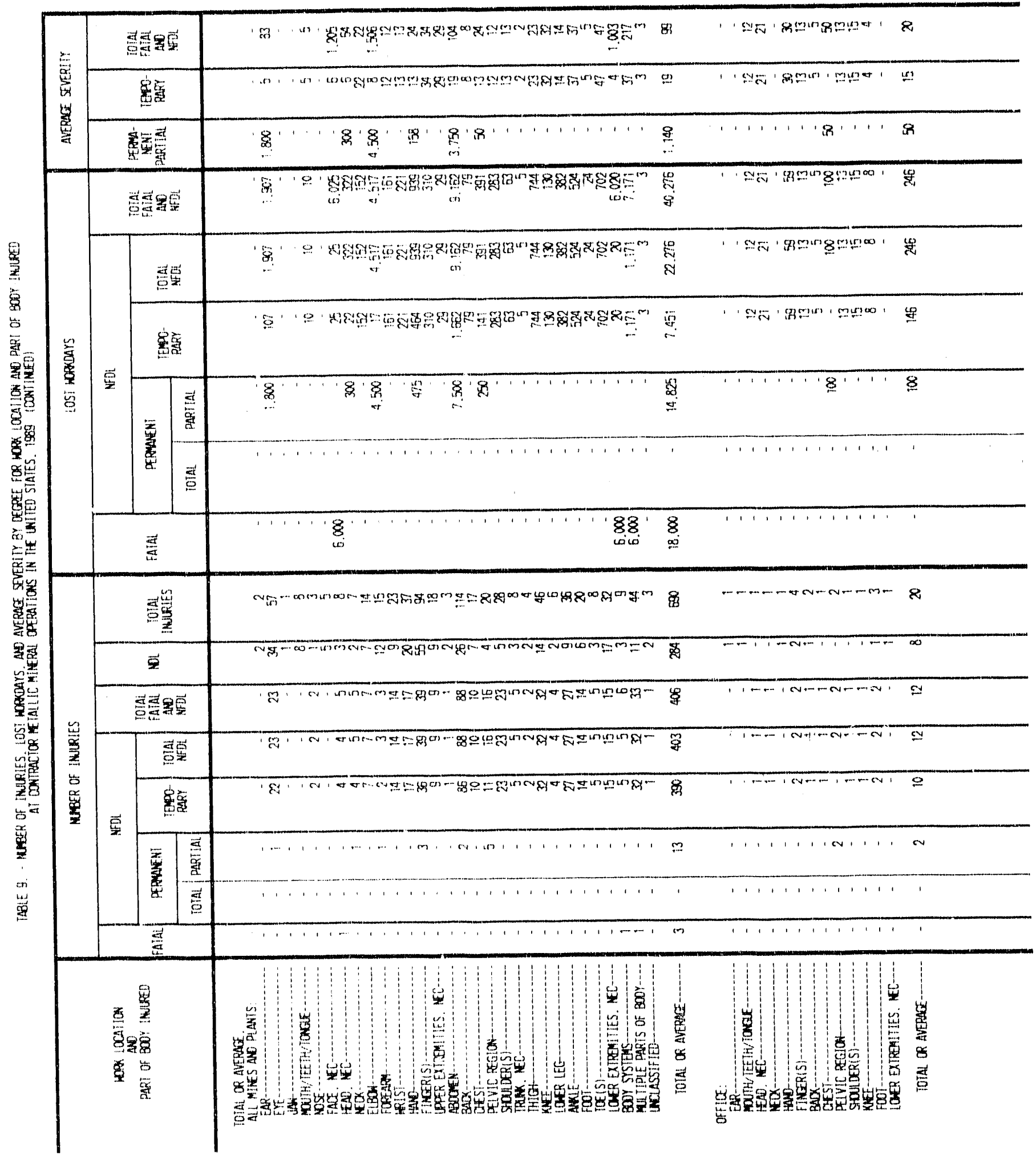




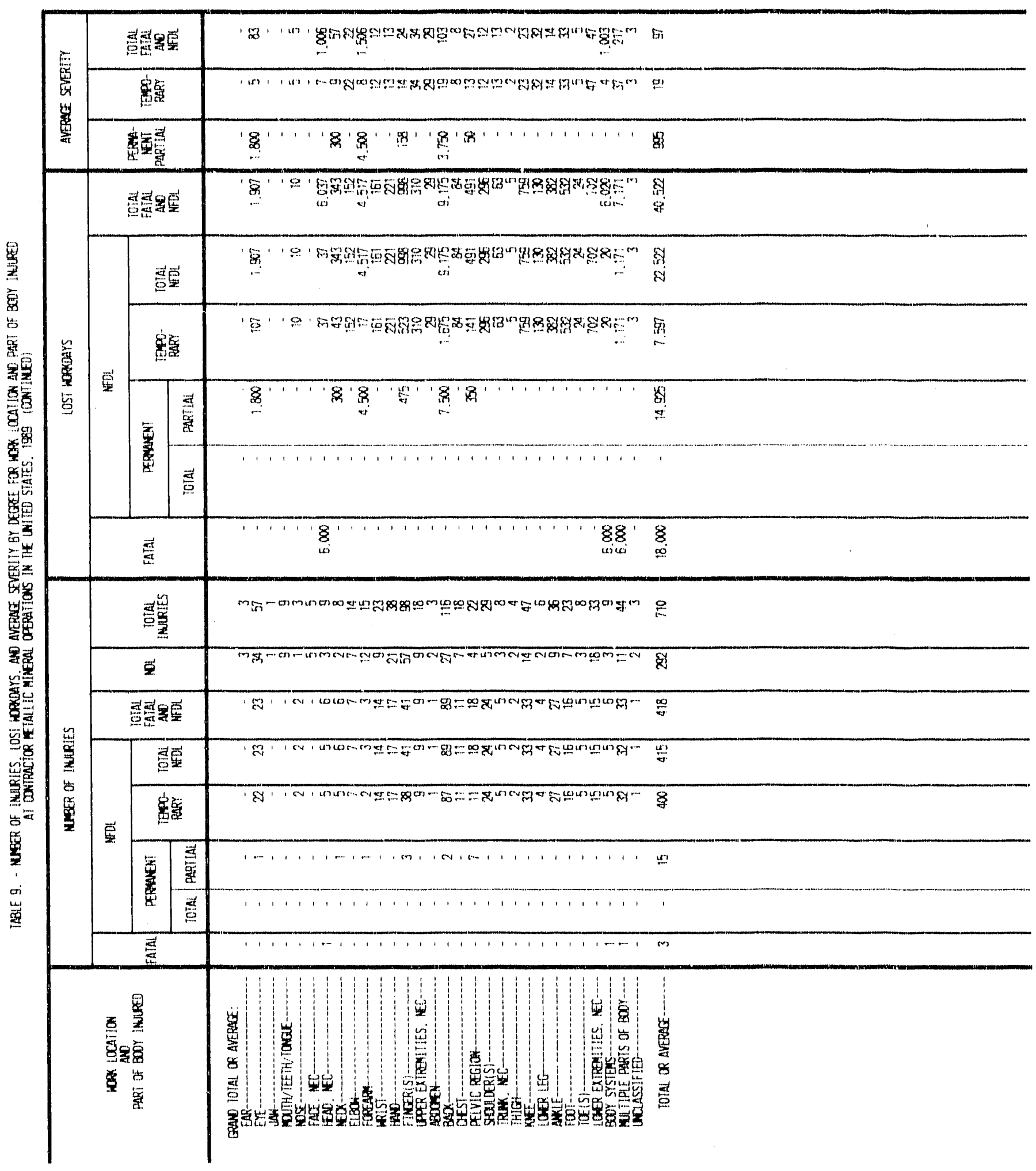




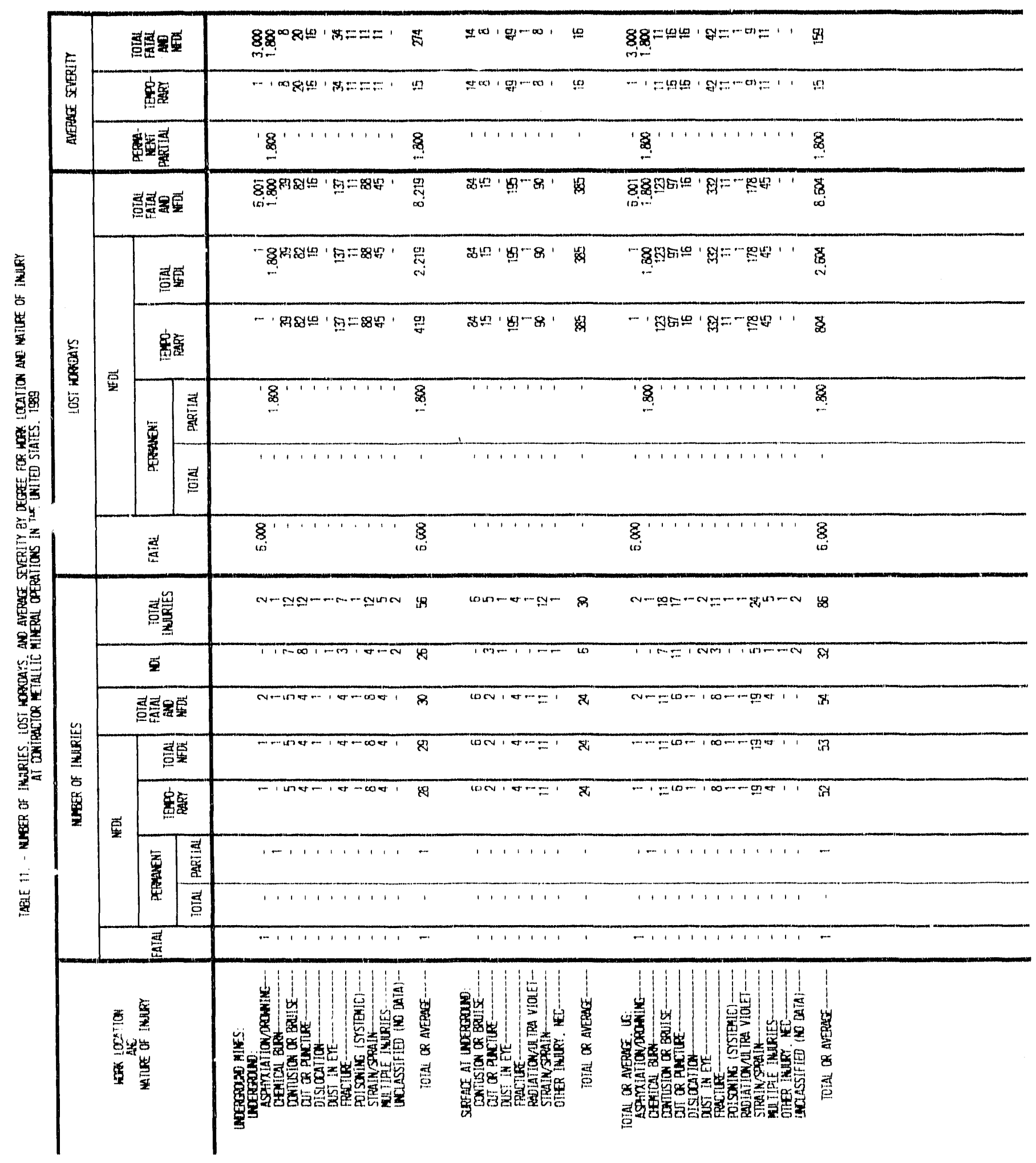




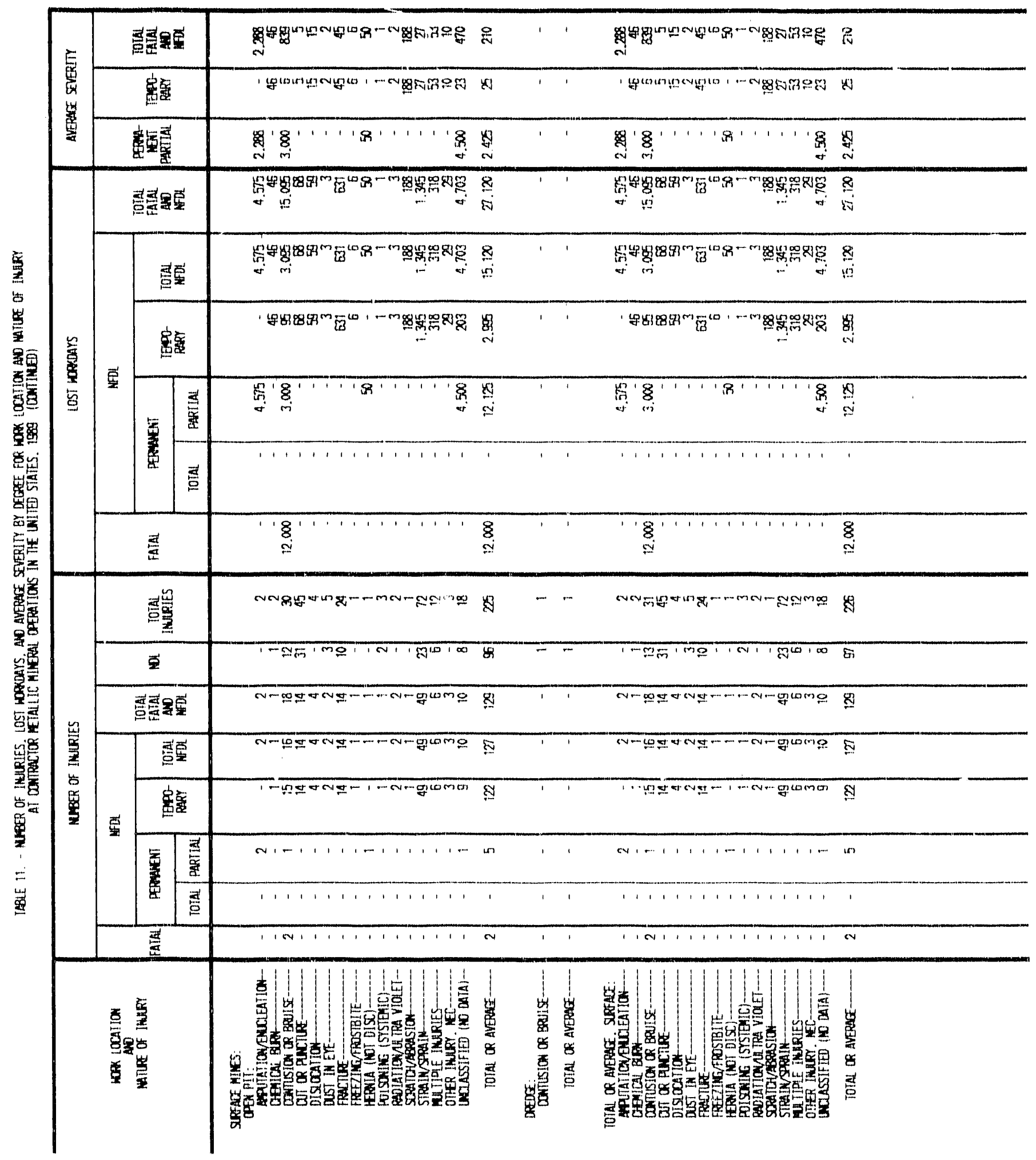




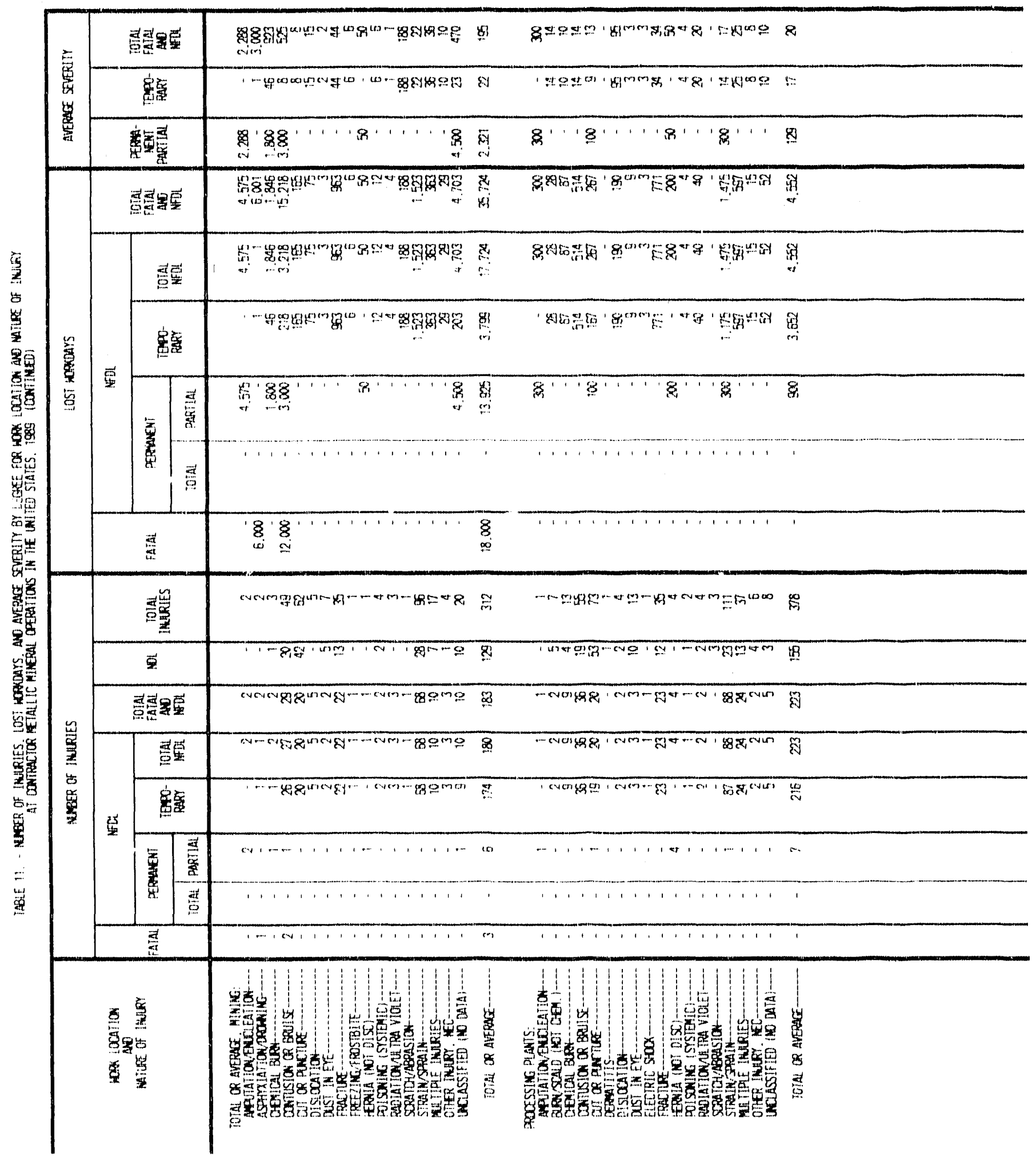




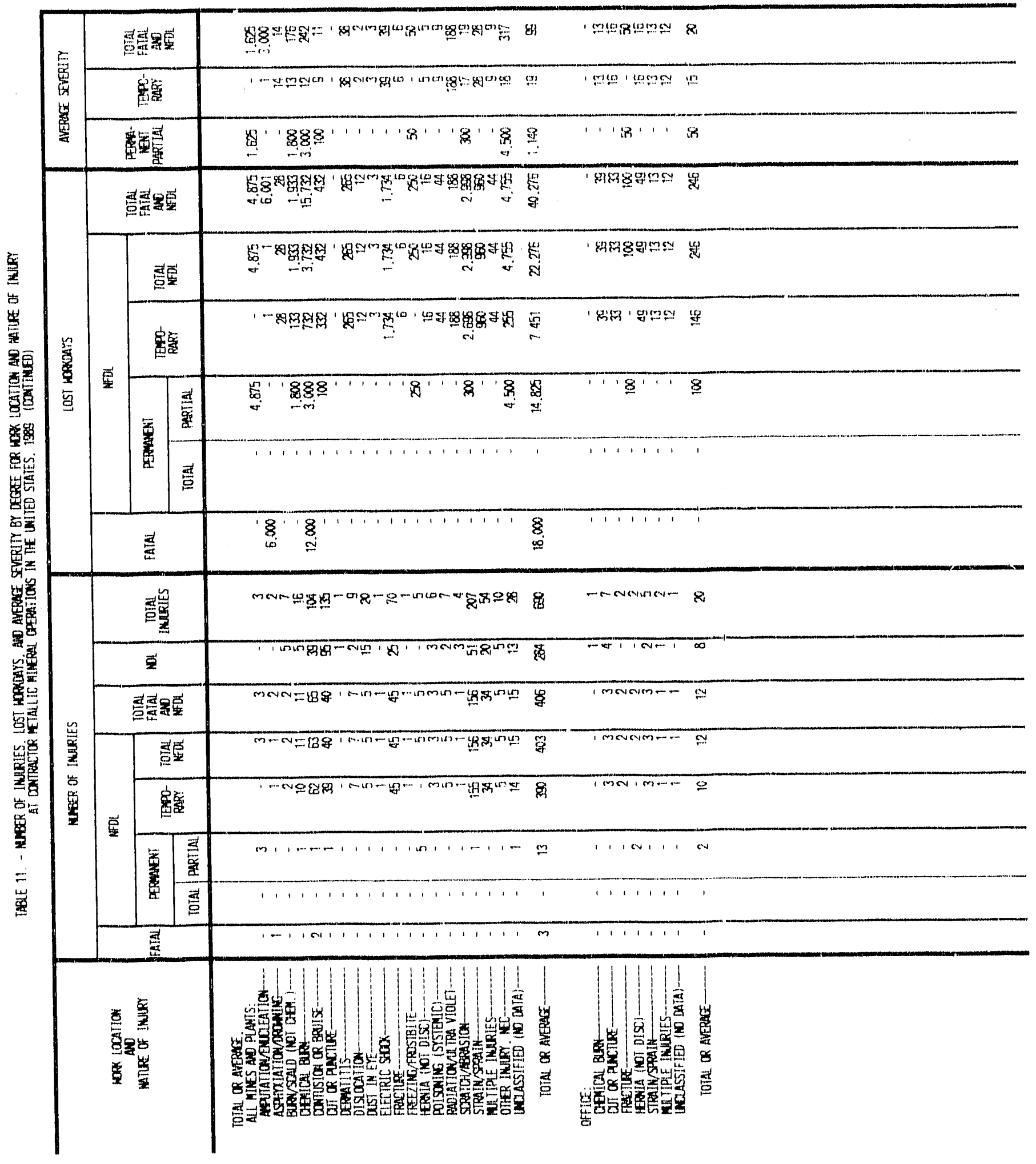




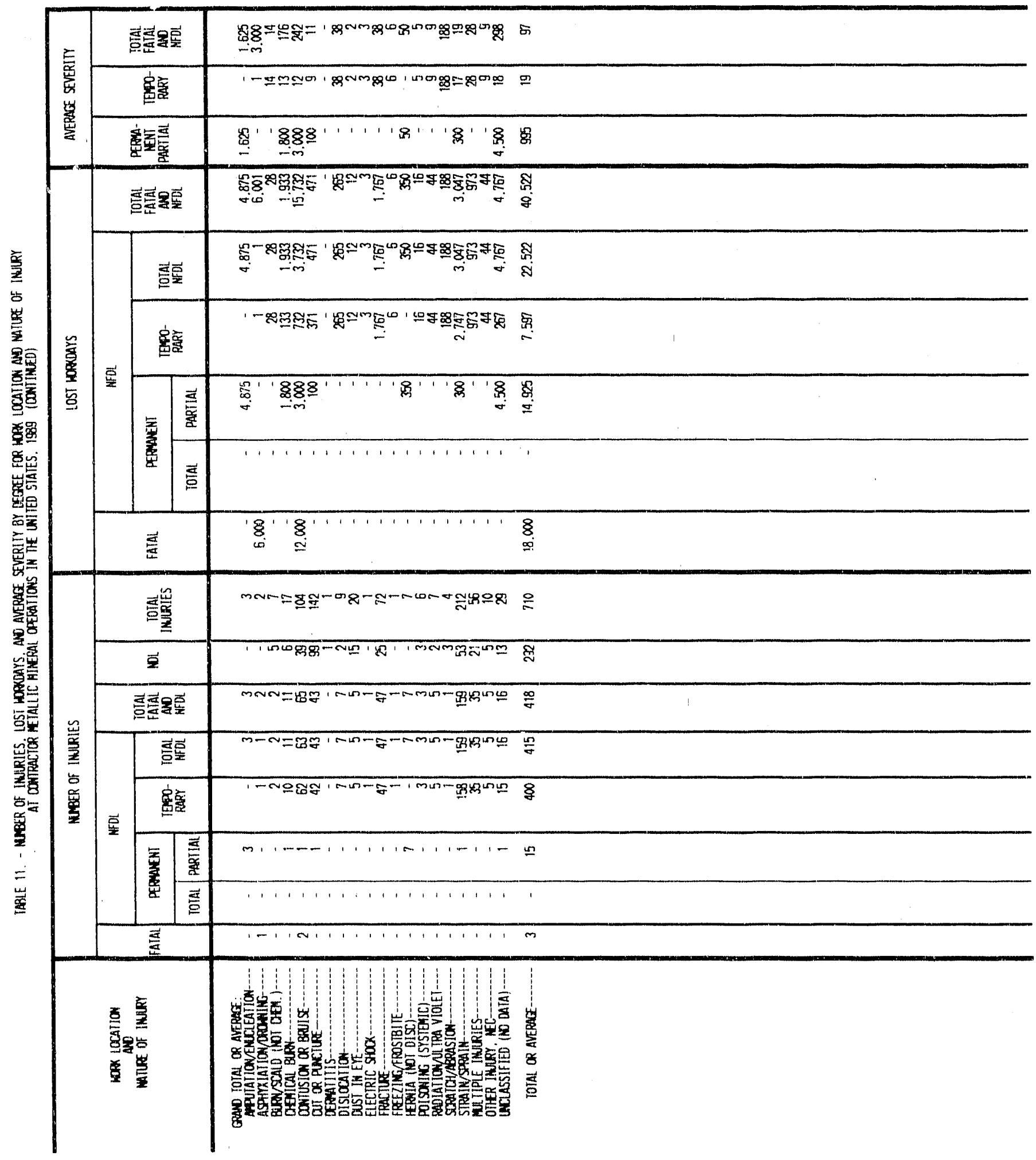




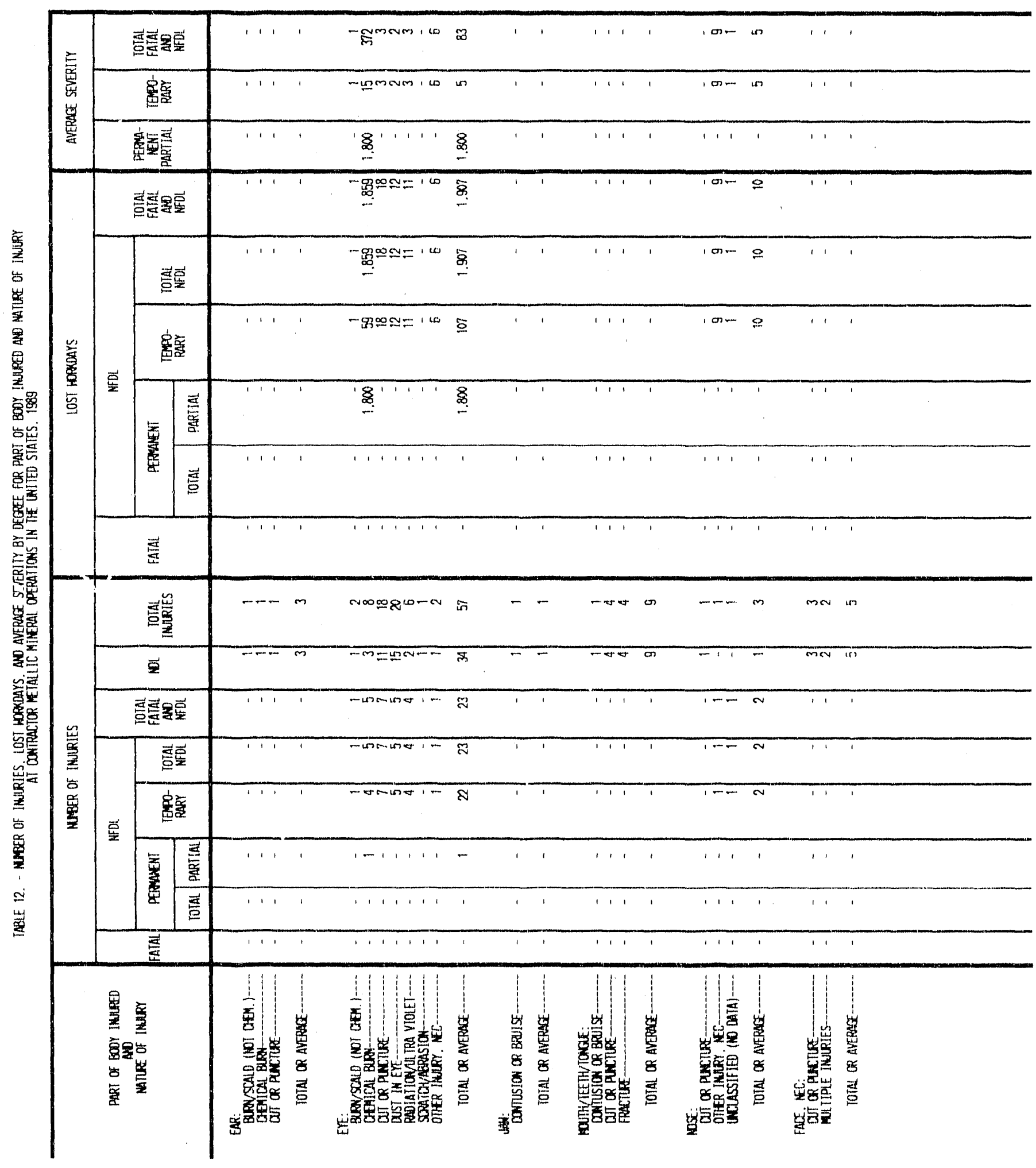




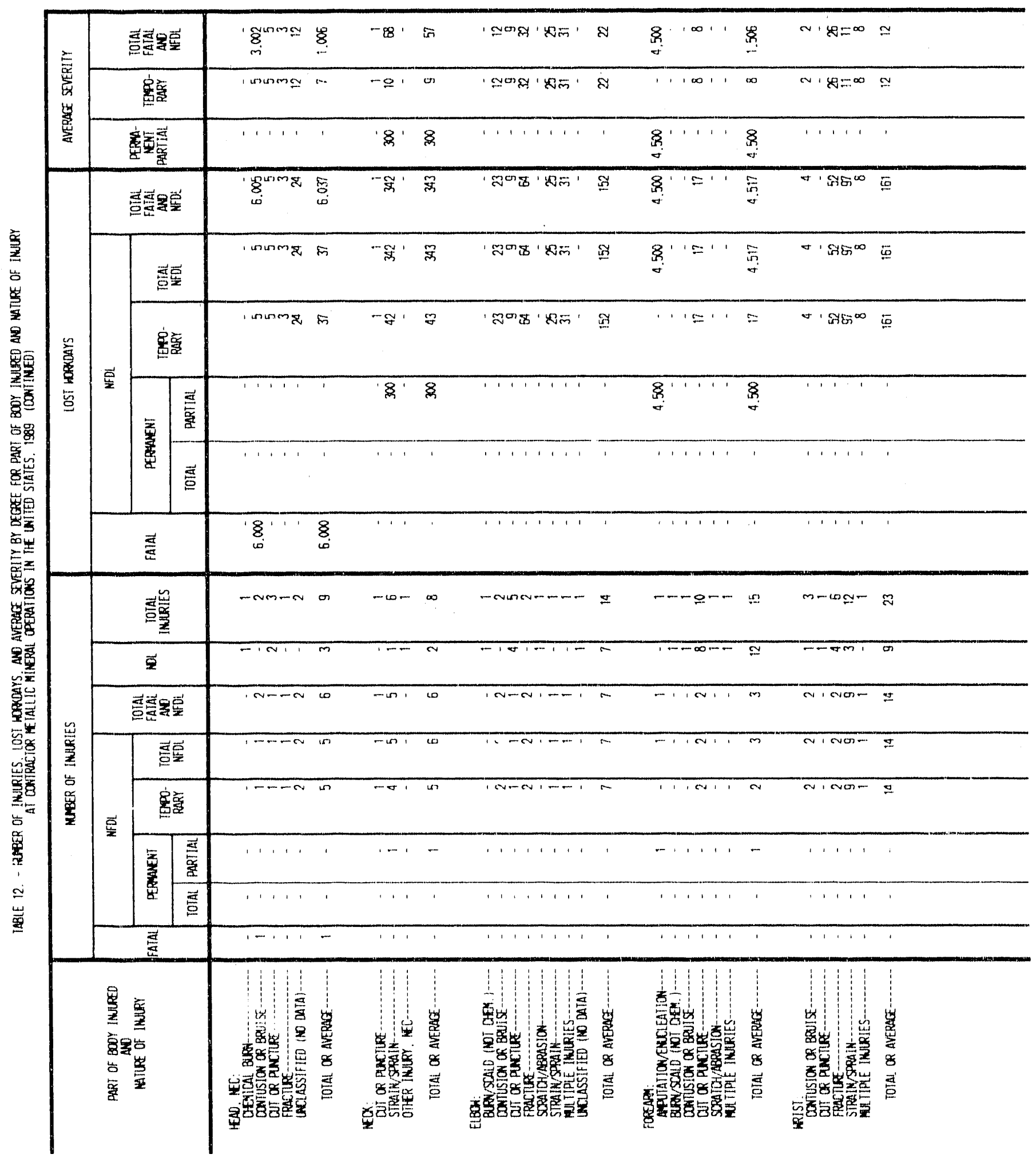




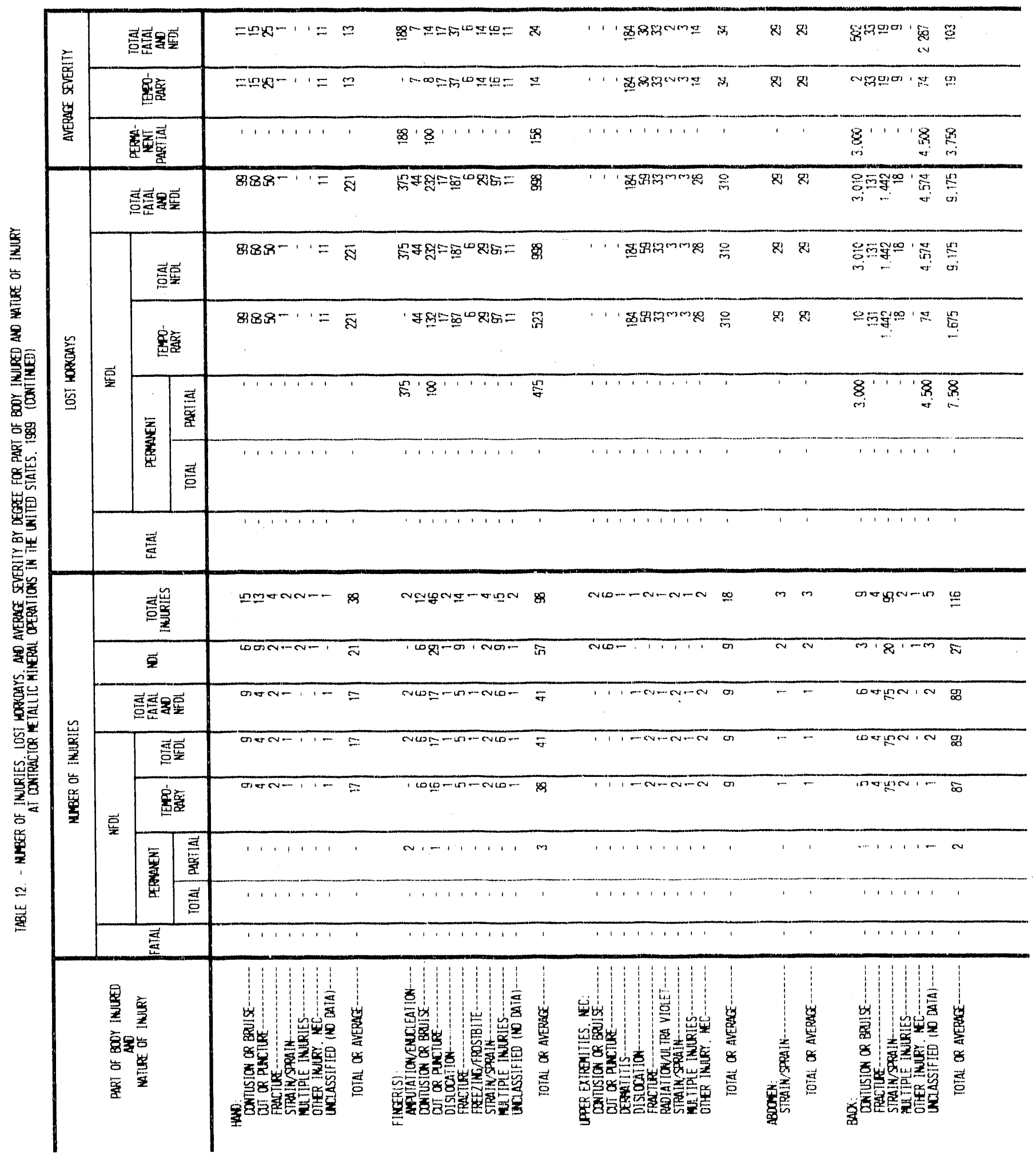




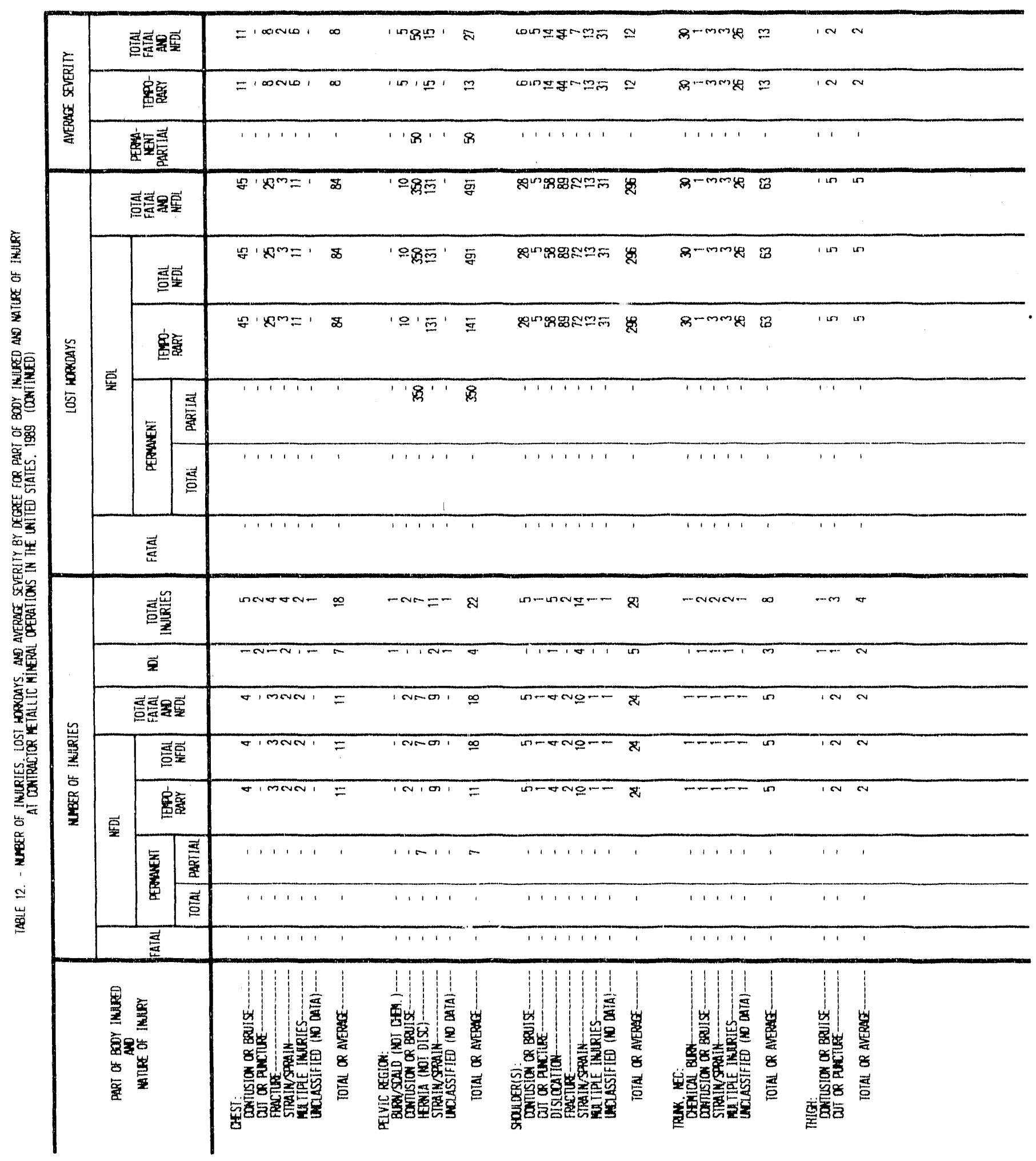




\begin{tabular}{|c|c|c|c|c|c|c|c|c|c|c|c|c|}
\hline \multirow{3}{*}{ 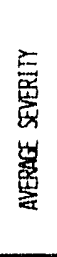 } & \multicolumn{2}{|c|}{ 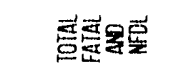 } & - Syl \& & $\approx$ & $E \cdot \operatorname{gg}(1)$ & $\mathscr{F}$ & ong्र & \pm & $\cos \sin (\infty$ & 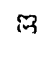 & Neo I & in \\
\hline & \multicolumn{2}{|c|}{ 起童 } & - & 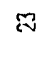 & sige & s & ज鸟ㄴ. & \pm & $\cos 48 \cdot \infty$ & $\mathscr{m}$ & Neol & in \\
\hline & \multicolumn{2}{|c|}{ 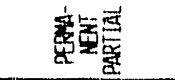 } & $1+1,11$ & ' & 1,1 & ' & $1 \quad 1 \quad 1$ & ' & 11111 & ' & 11 & ' \\
\hline \multirow{6}{*}{ 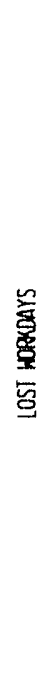 } & \multicolumn{2}{|c|}{ 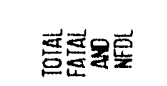 } & $\bar{m}^{\circ} \mathrm{N} g=N$ & 品 & $\tilde{n} \cdot \xi^{\infty}$ & 8 & 므몰ㄹㄹ & $\mathscr{s}$ & 写브의 & జ్ల & NN 1 & $\bar{x}$ \\
\hline & \multirow{4}{*}{ 要 } & 홈물 & $\bar{n} \operatorname{nin}^{\infty} \mathrm{N} g=N$ & 吕 & $\Sigma 5^{10}$ & 픈 & 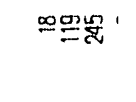 & \&్థ & 孚正骂问 & $\mathscr{N}$ & $\sim_{\text {\&ू. }}$ & 齐 \\
\hline & & 䔡 & $\pi \sin ^{\infty} 28=0$ & 吕 & हi $5^{\circ}$ & 8 & D哭哭 & \%్ & 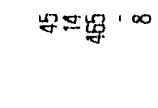 & $\underset{\pi}{2}$ & "va ! & $\mathbb{X}$ \\
\hline & & 颚 & 11111 & ' & 111 & T & 111 & ' & 11111 & ' & 11 & $T$ \\
\hline & & 홍 & 1,1111111 & ' & 1,1 & ' & 111 & ' & 1111 & ' & 111 & ' \\
\hline & \multicolumn{2}{|c|}{ 苑 } & 1111 & ' & 1,1 & I & 1,1 & ' & 11,1 & ' & 11 & ' \\
\hline \multirow{8}{*}{ 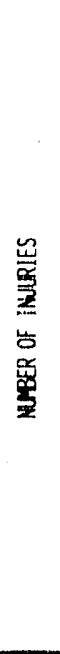 } & \multicolumn{2}{|c|}{ 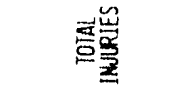 } & nm-monar & $\Xi$ & -arer & 10 & NCOE - & 4 & winion- & $\approx$ & Nun- & $\infty$ \\
\hline & \multicolumn{2}{|l|}{$\Phi$} & 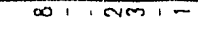 & \pm & 101 & $\infty$ & $\cdots+$ & का & $-a v-m 1$ & $m$ & -5 & $m$ \\
\hline & \multicolumn{2}{|c|}{ 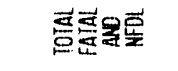 } & nin-mळor & $m$ & -10 & $\sigma$ & cvirse' & 8 & these 10 & $\omega$ & $-\sigma$ & 157 \\
\hline & \multicolumn{2}{|c|}{ 롱물 } & rm-m!on- & $m$ & $\cdots+\mathrm{cor}$ & $\sigma$ & Ning & $\overline{\mathrm{N}}$ & lnme & $\dddot{0}$ & -81 & in \\
\hline & \multicolumn{2}{|c|}{ 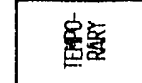 } & Nm-mছNm & $m$ & -10. & $\sigma$ & $\sin 8$ & $E$ & whmo 1. & $\Phi$ & $-\infty$ & in \\
\hline & \multirow{2}{*}{$\begin{array}{l}\text { 峿 } \\
\text { 悹 }\end{array}$} & 亲 & $\cdots 1, \ldots$ & ' & , ' ' & . & . 1 1 & ' & 11111 & ' & 111 & ' \\
\hline & & $\underline{\underline{E}}$ & ' & ' & $\cdot$ & ' & , & ' & . ' & ' & 1,1 & ' \\
\hline & \multicolumn{2}{|l|}{$\overrightarrow{\underline{E}}$} & $\ldots \ldots$ & ' & ' & , & ' & . & 1,1 & ' & 11 & ' \\
\hline & 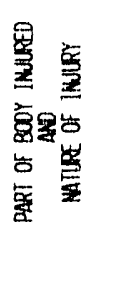 & & 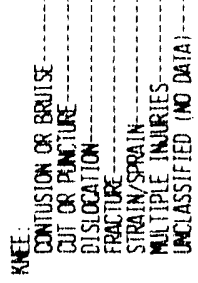 & 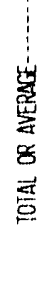 & 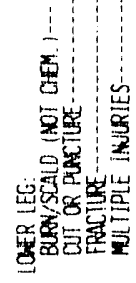 & 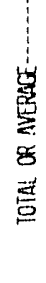 & 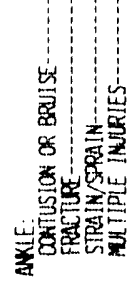 & 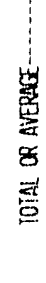 & 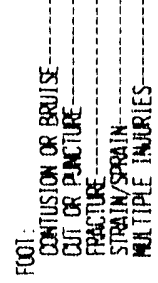 & 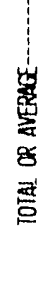 & 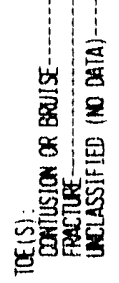 & 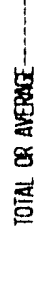 \\
\hline
\end{tabular}




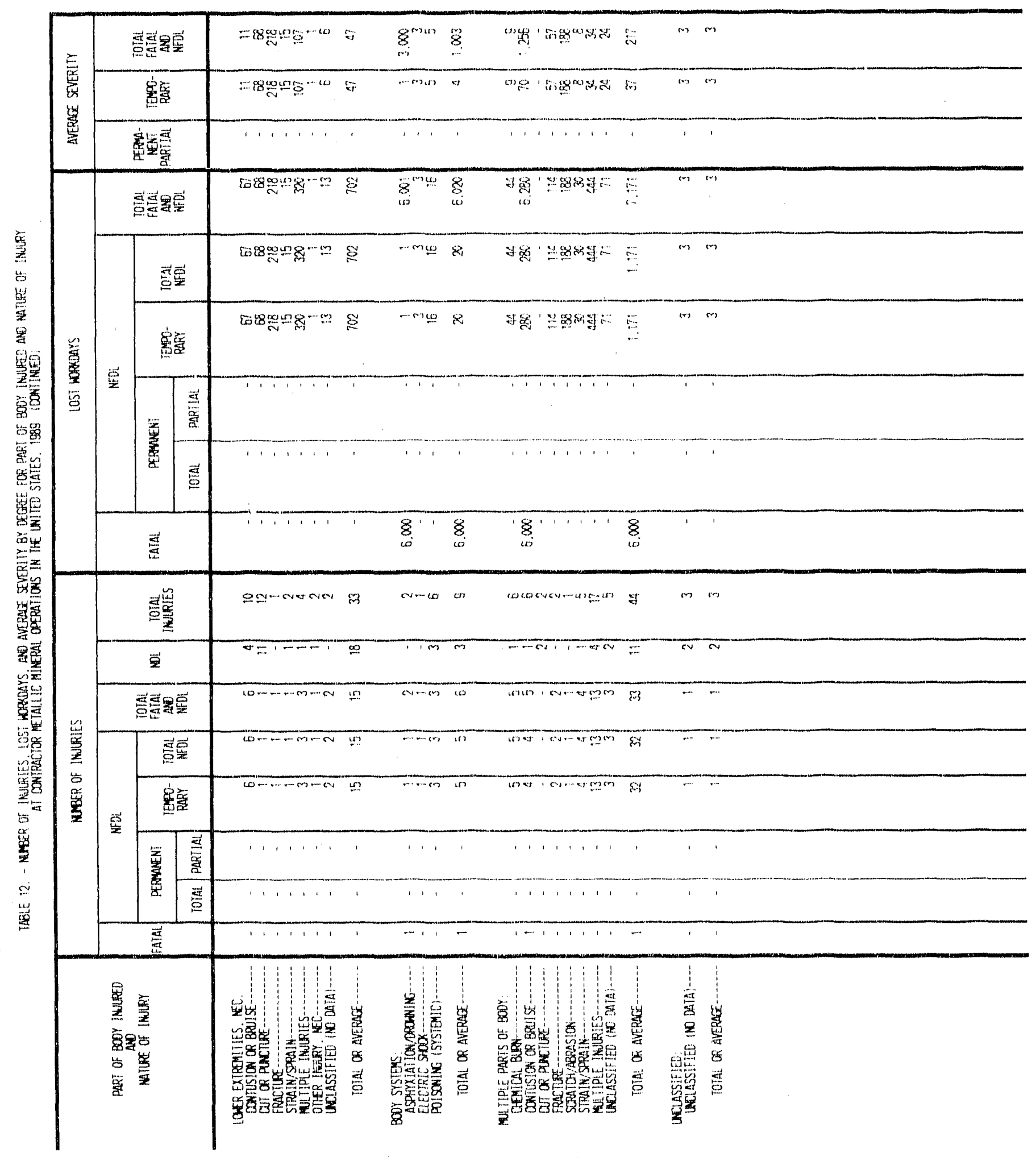




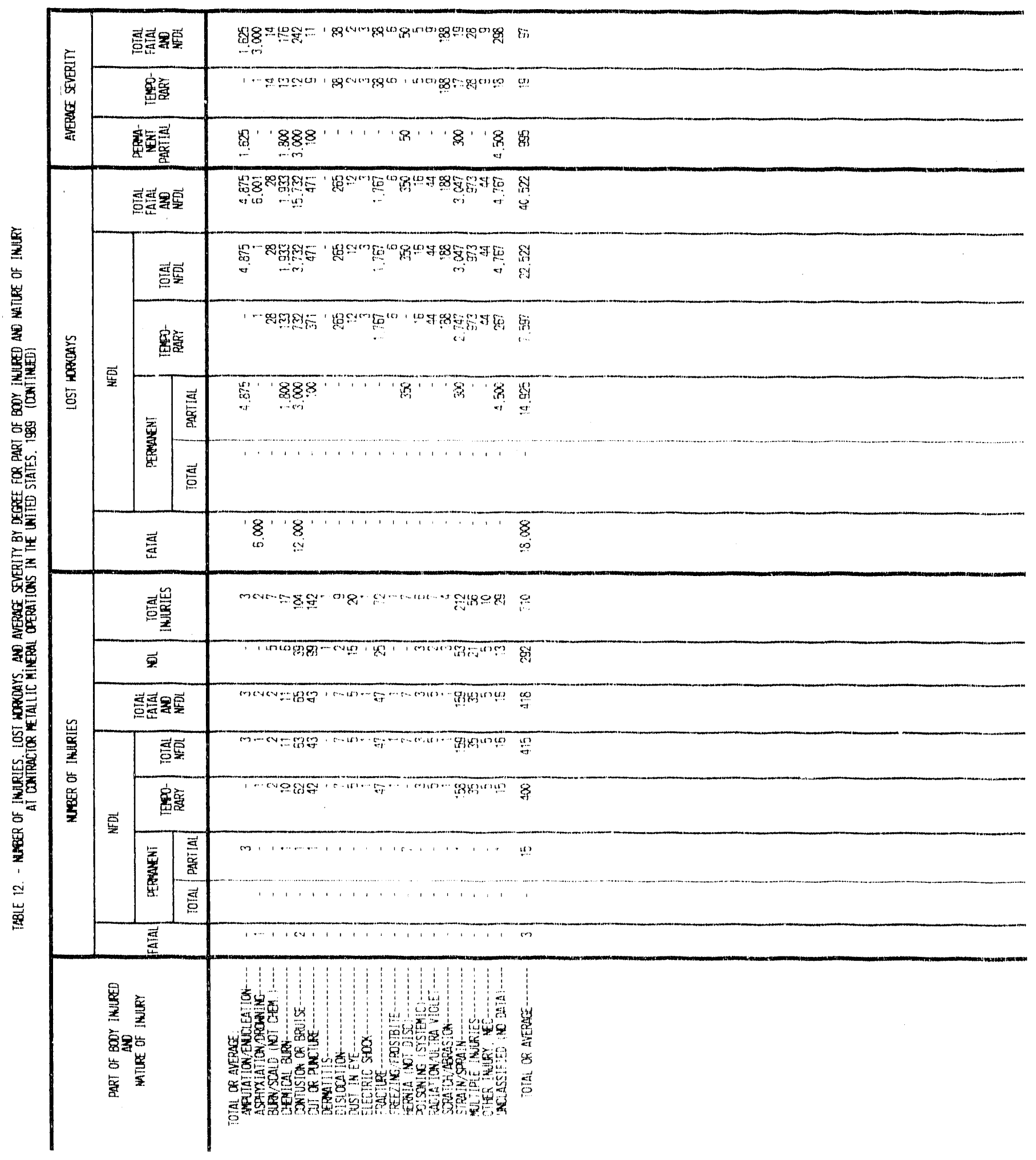




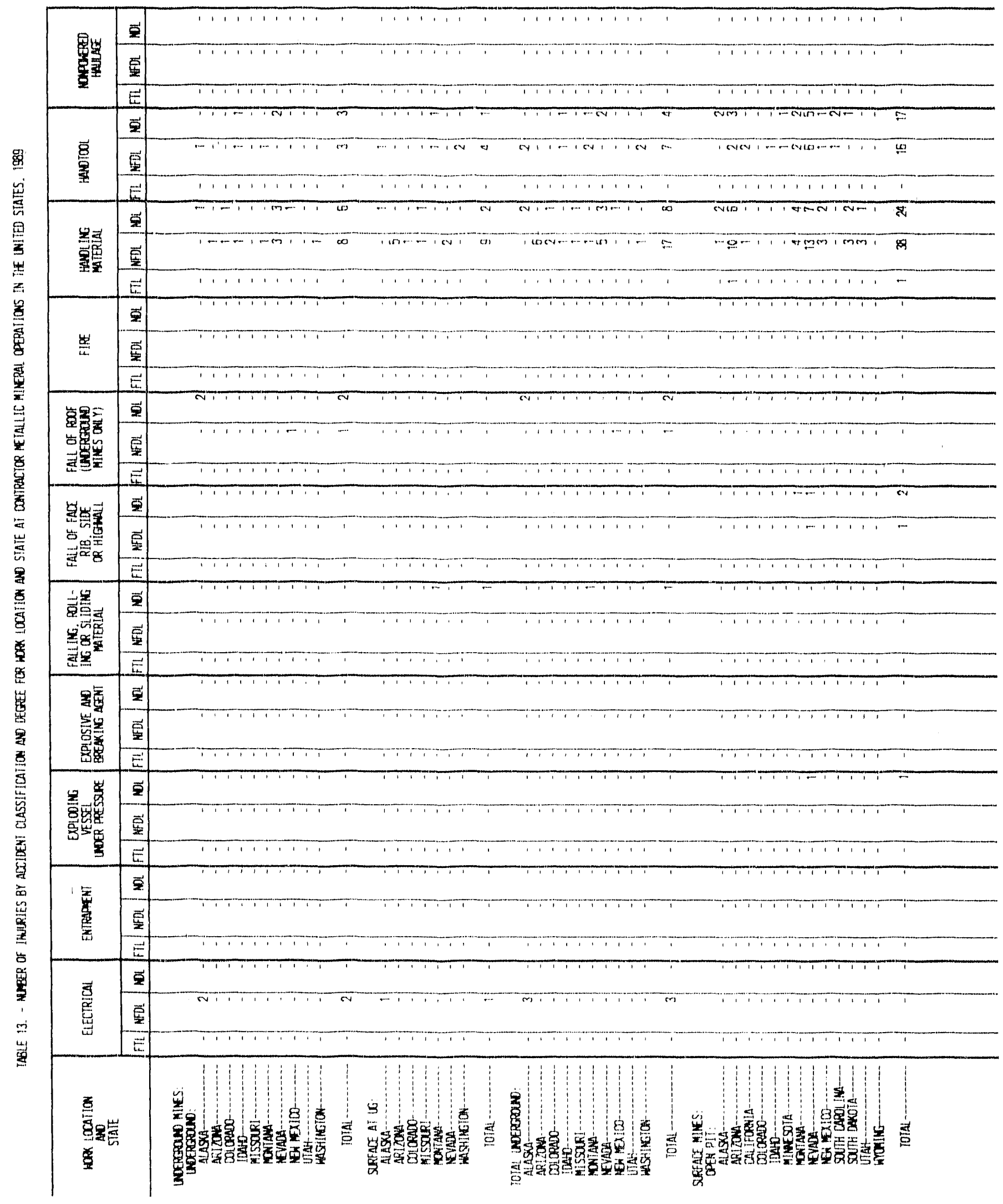




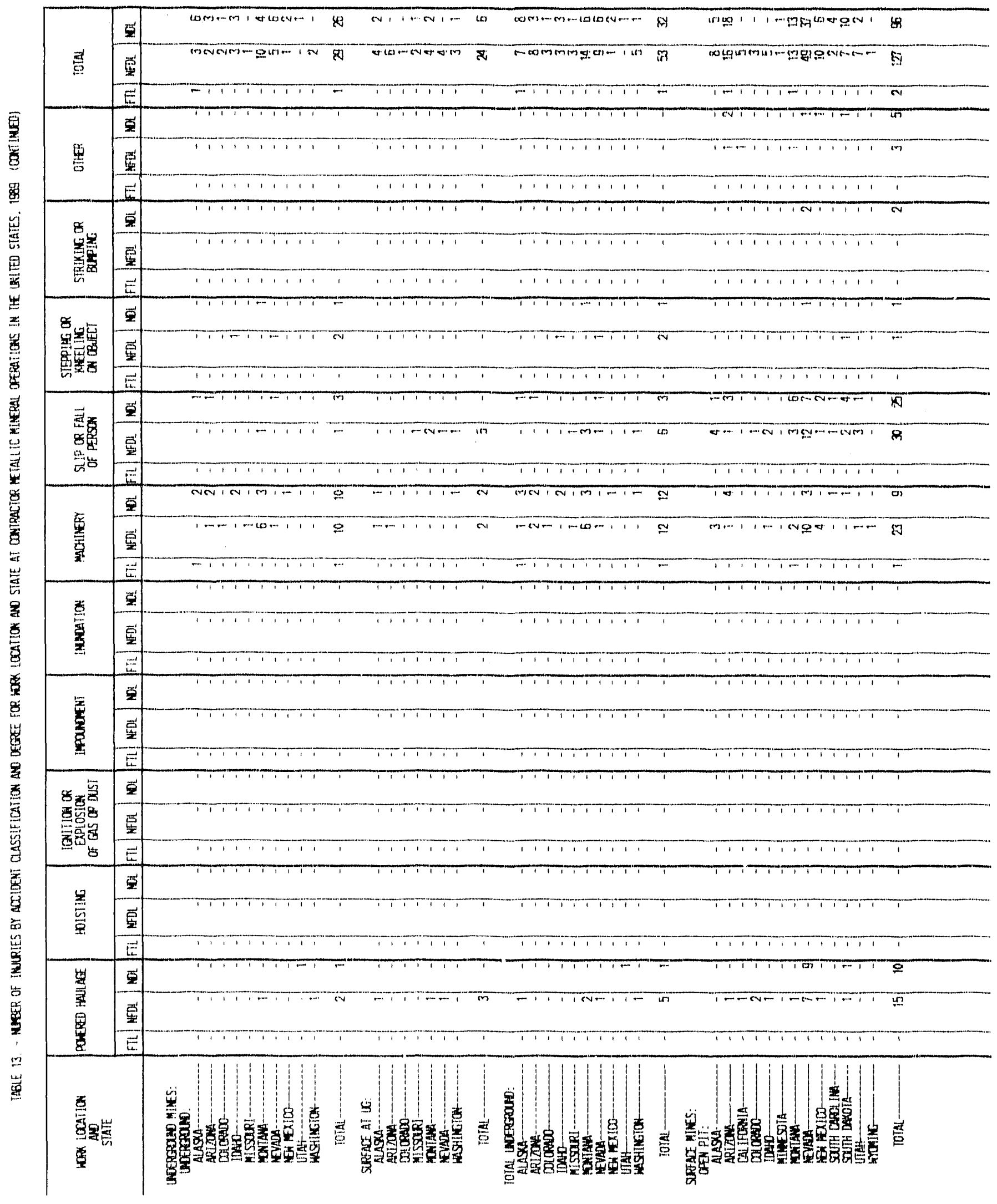




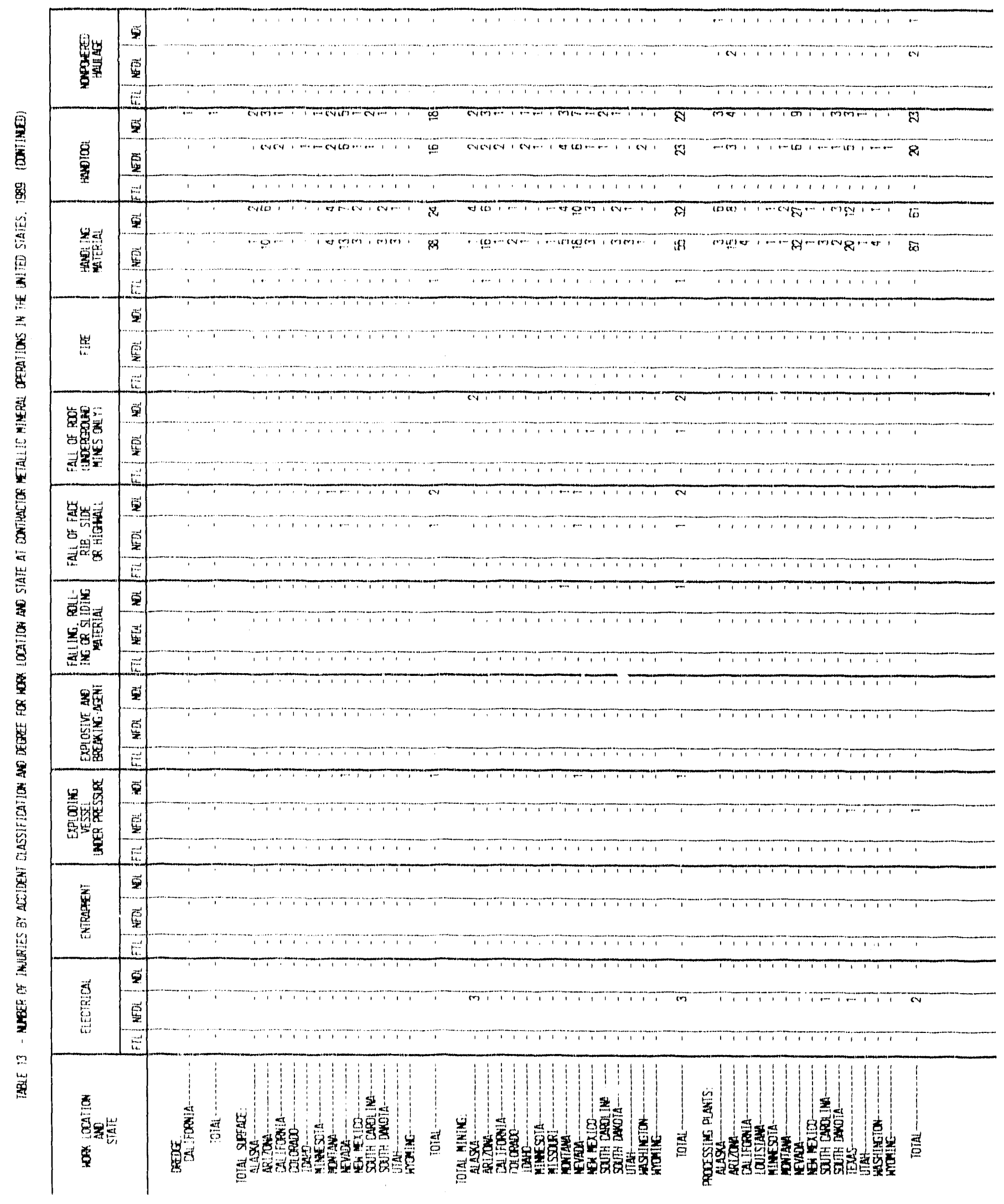




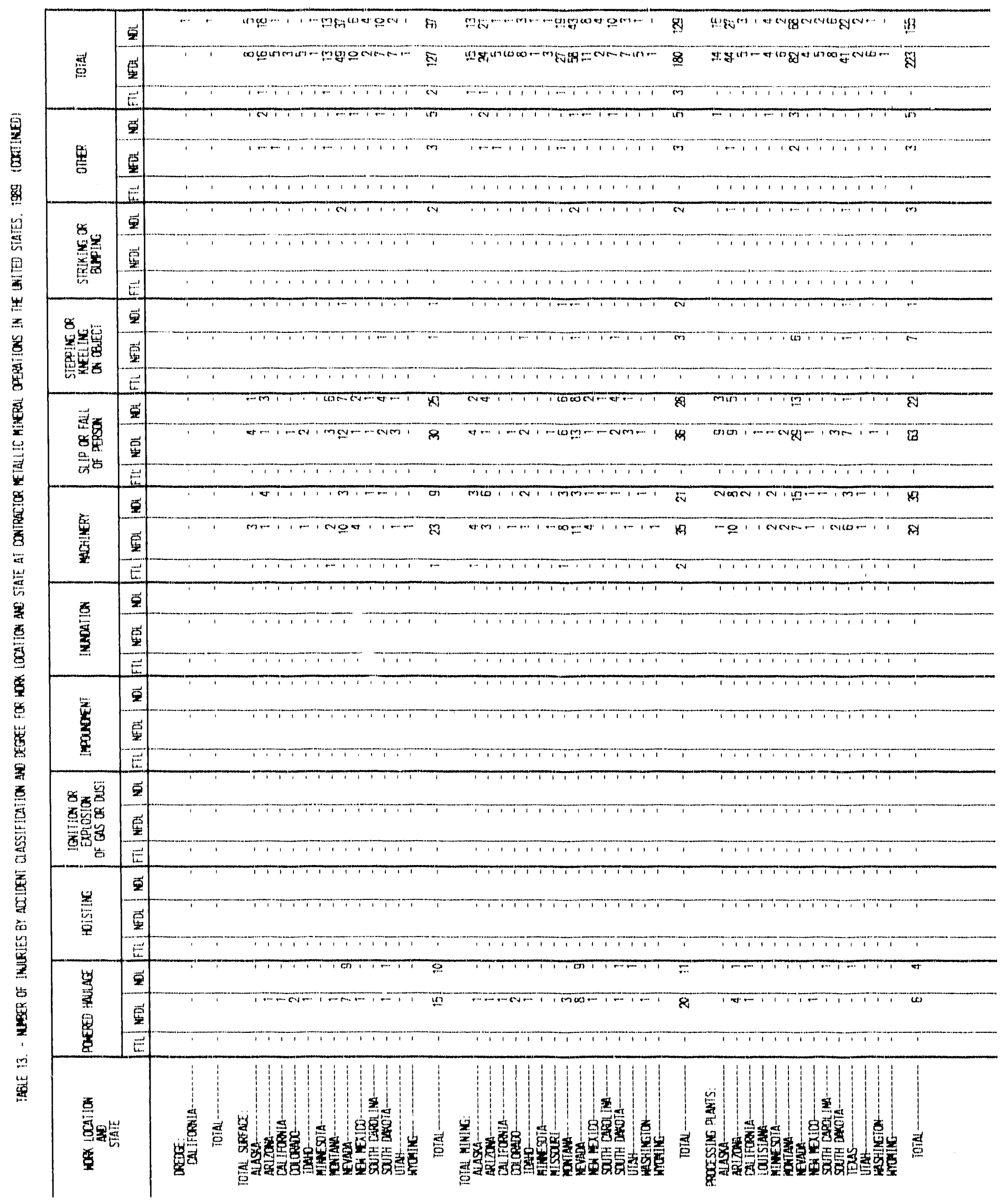




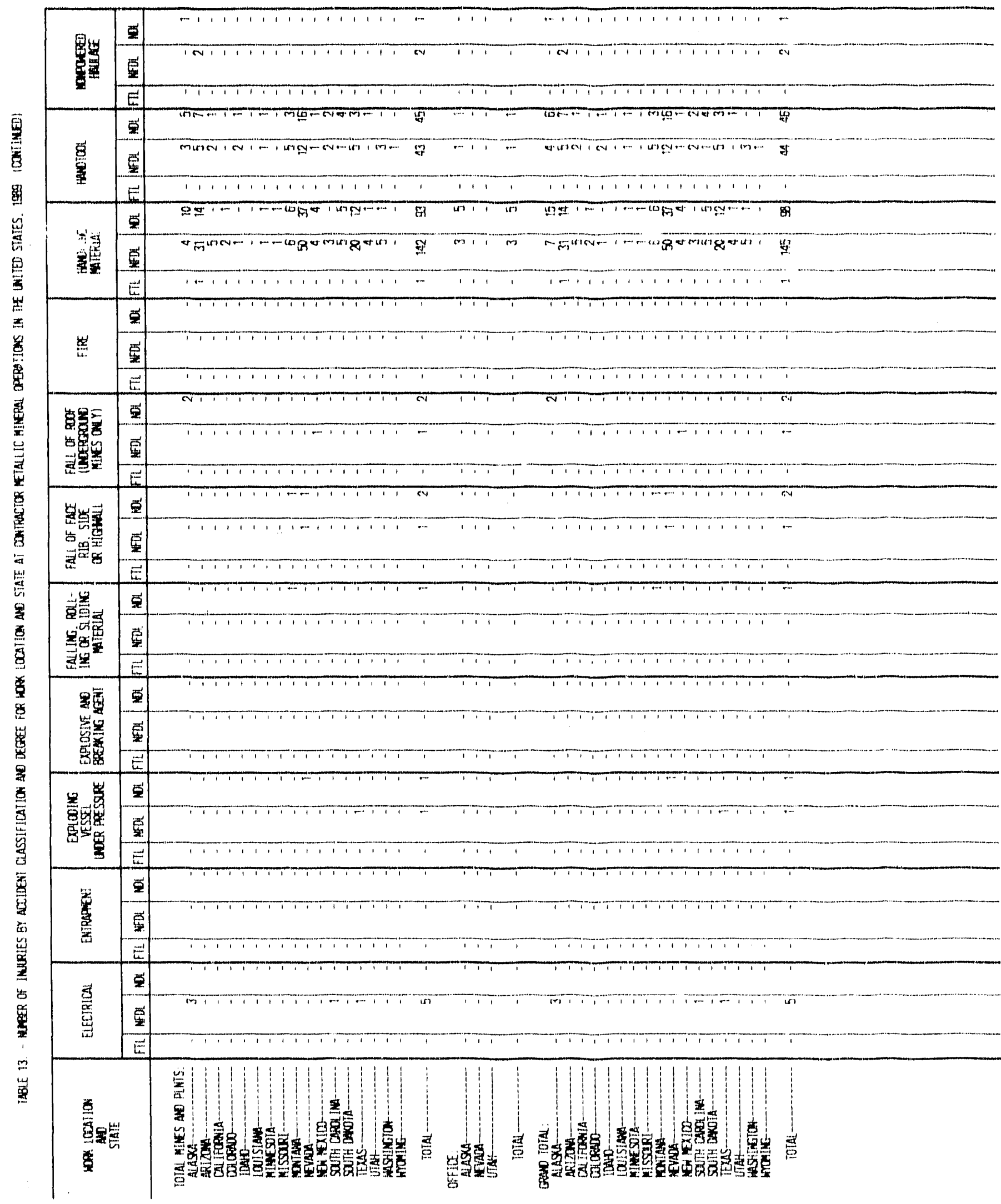




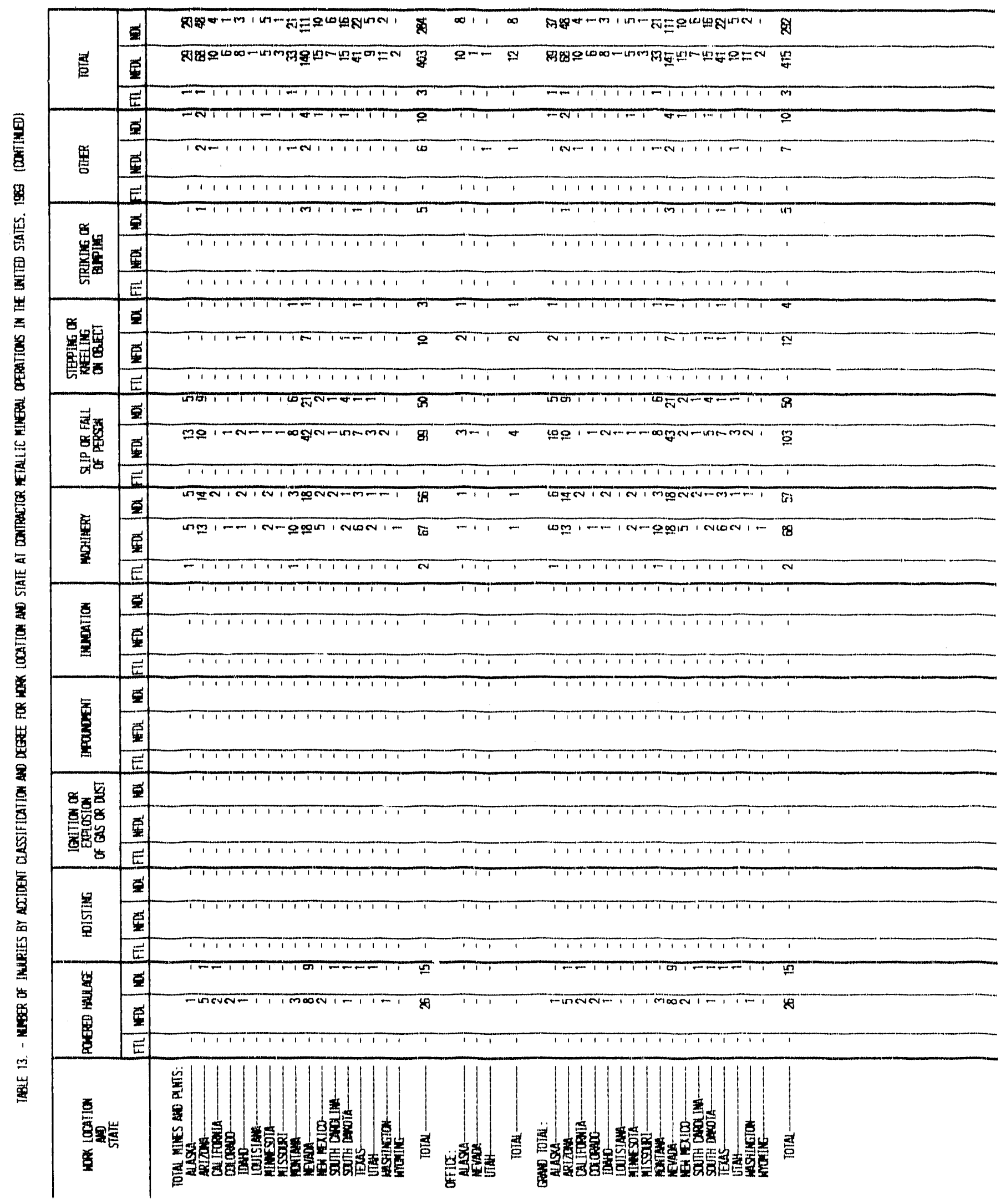




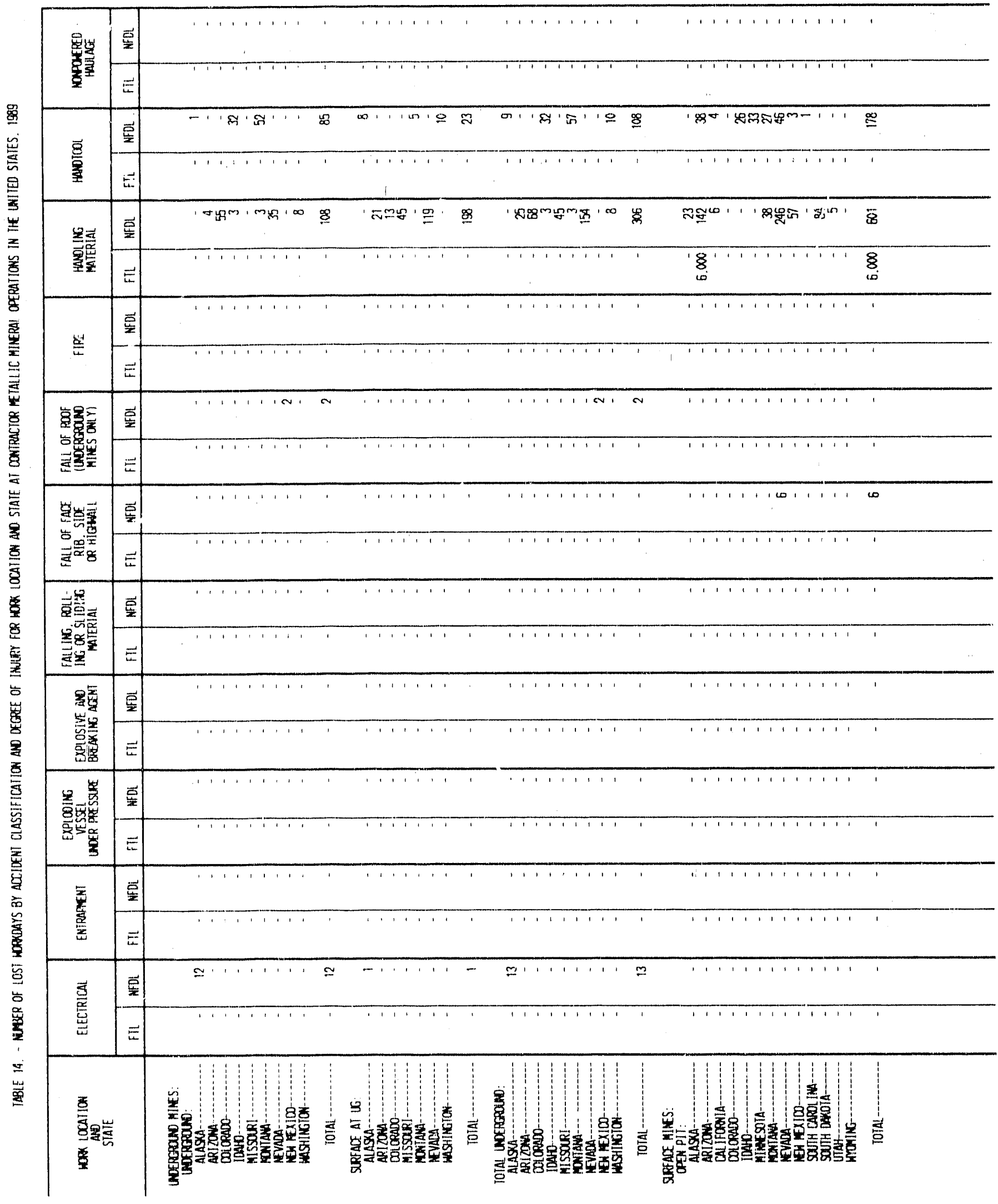




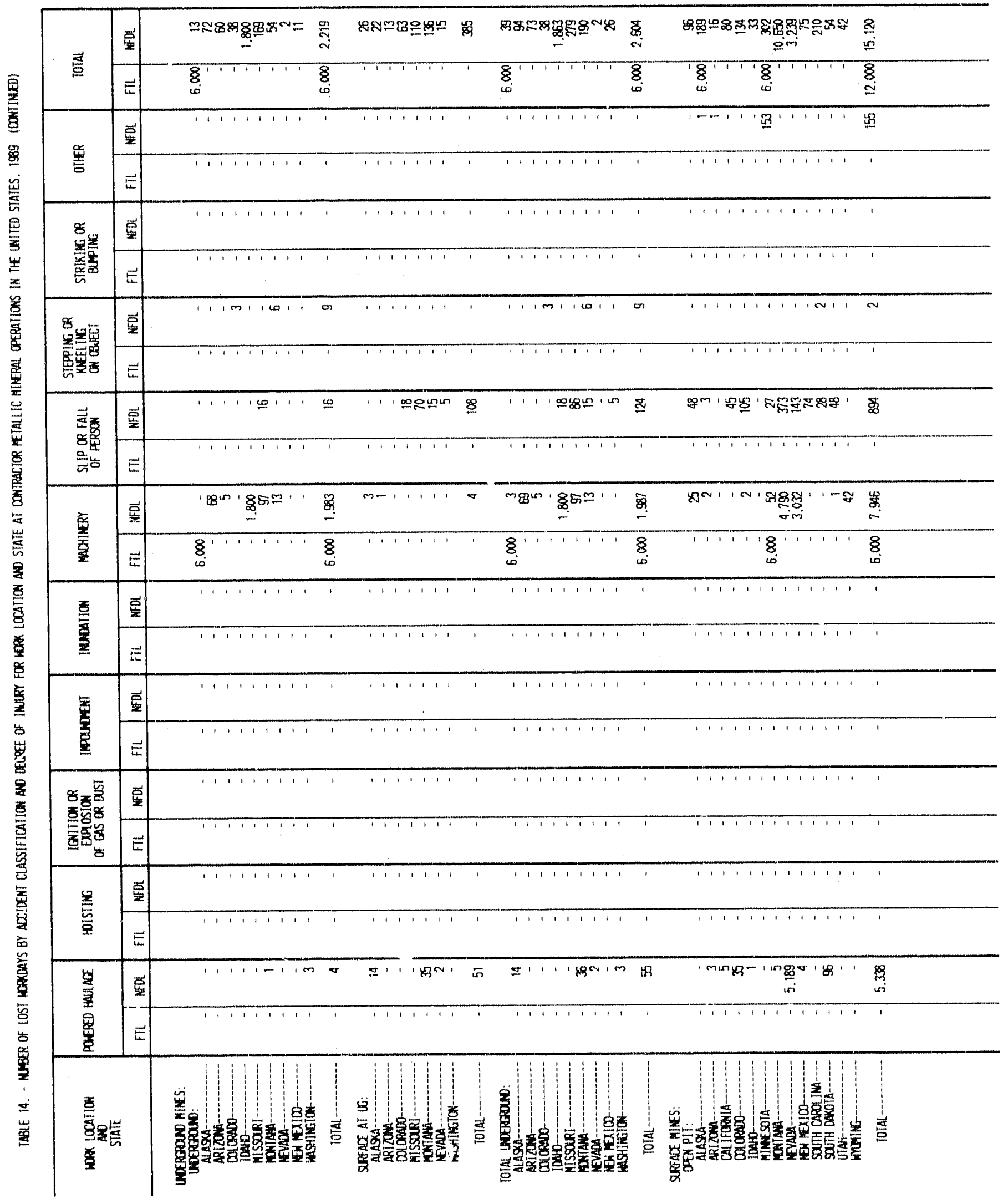




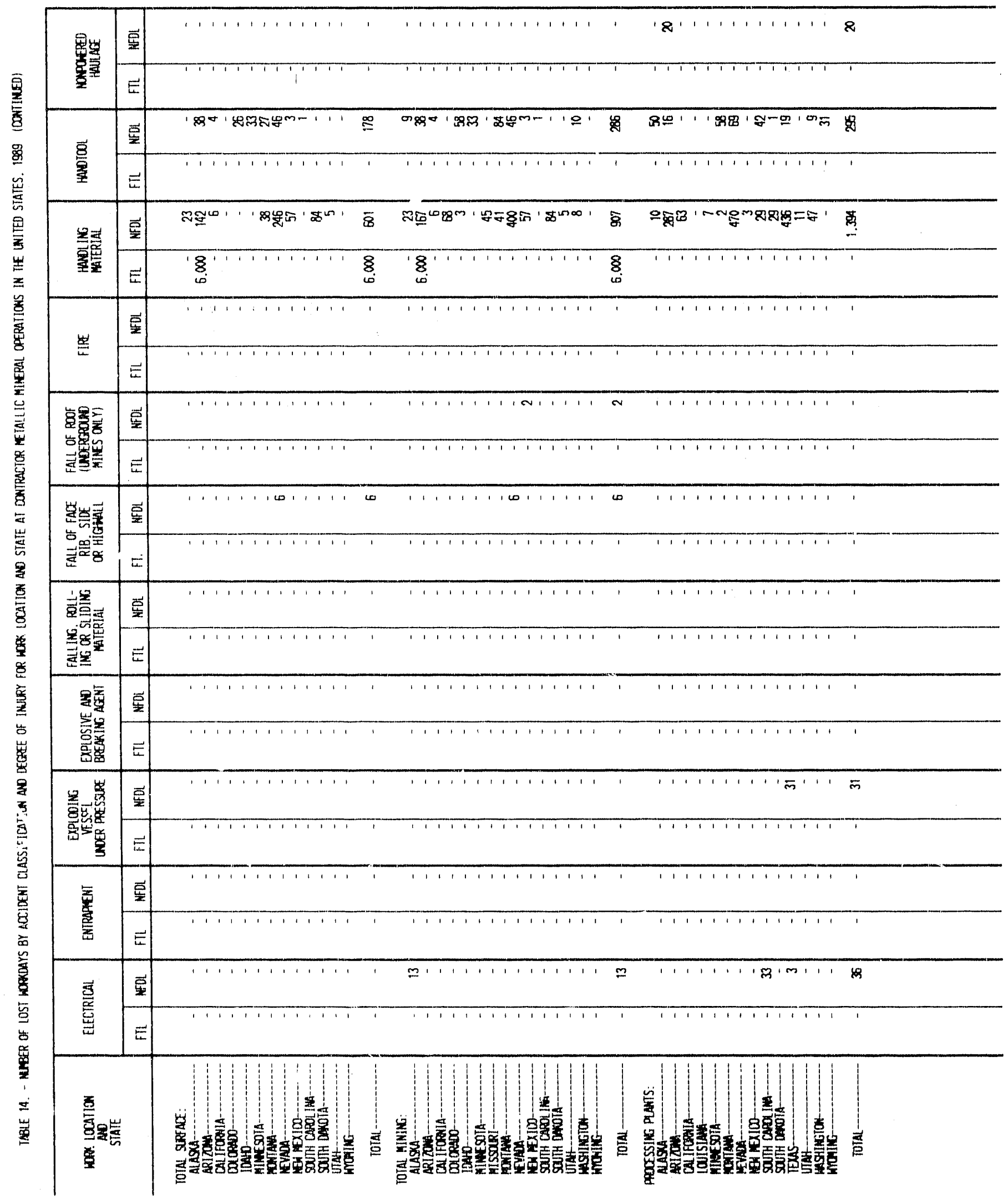




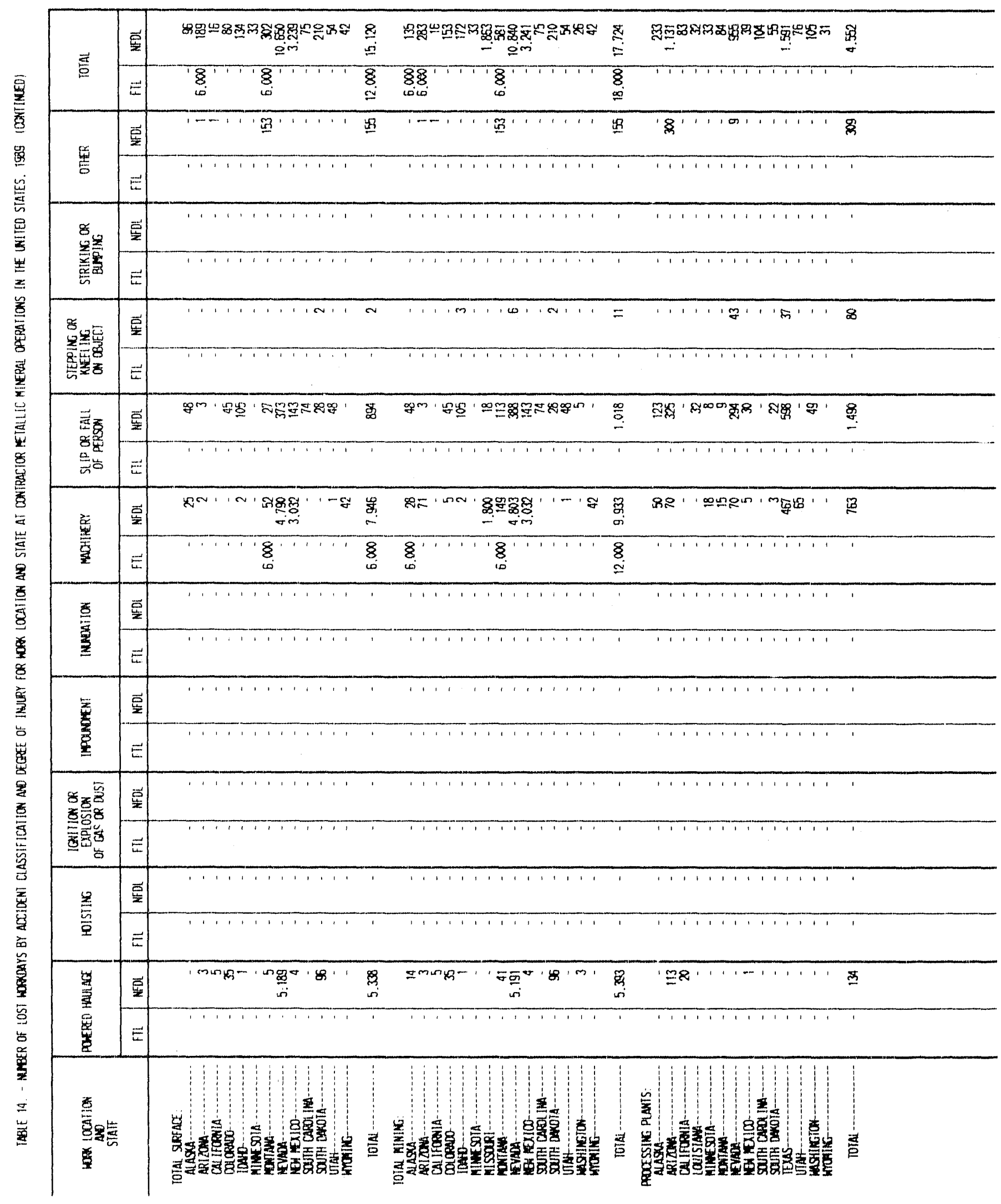




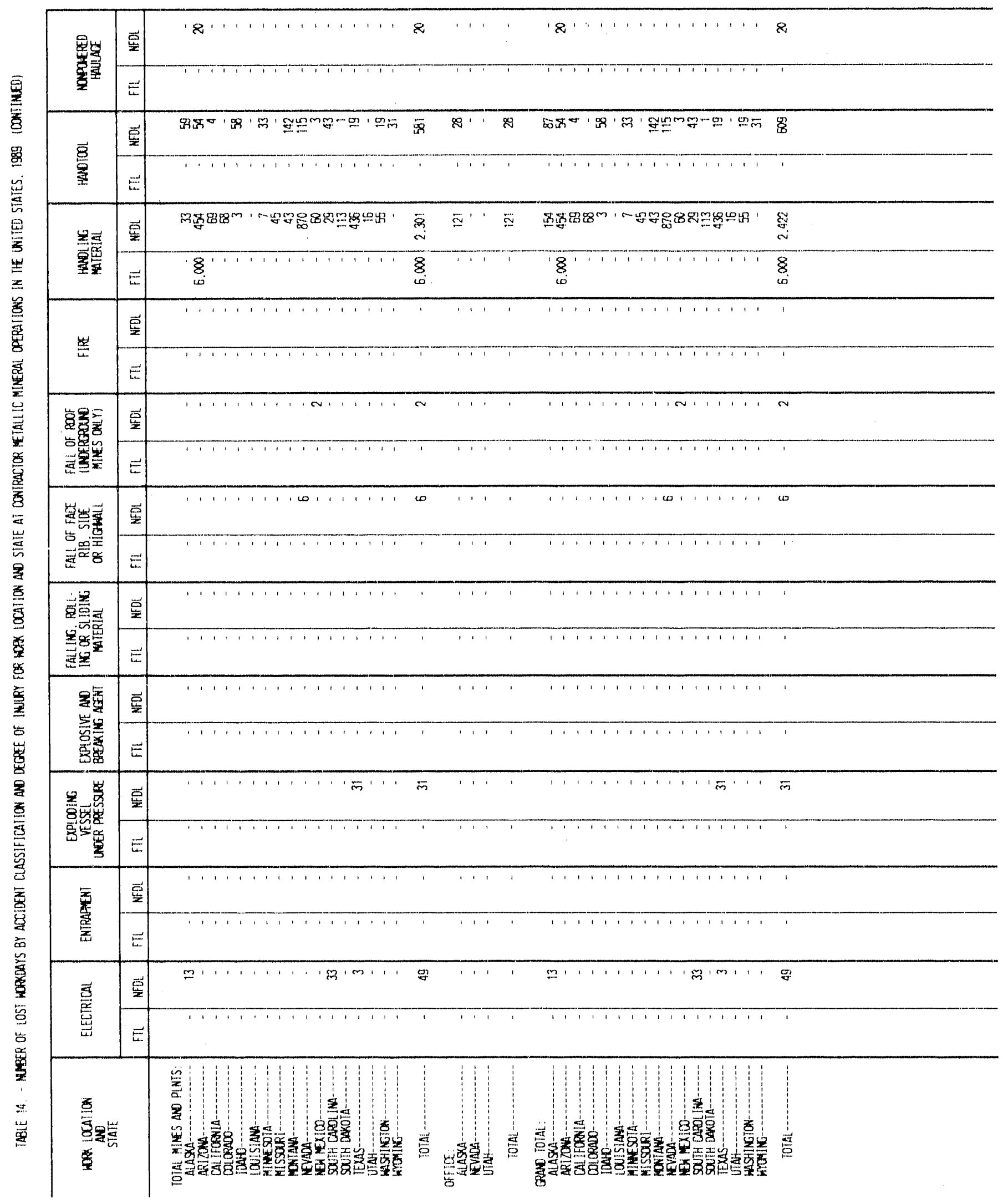




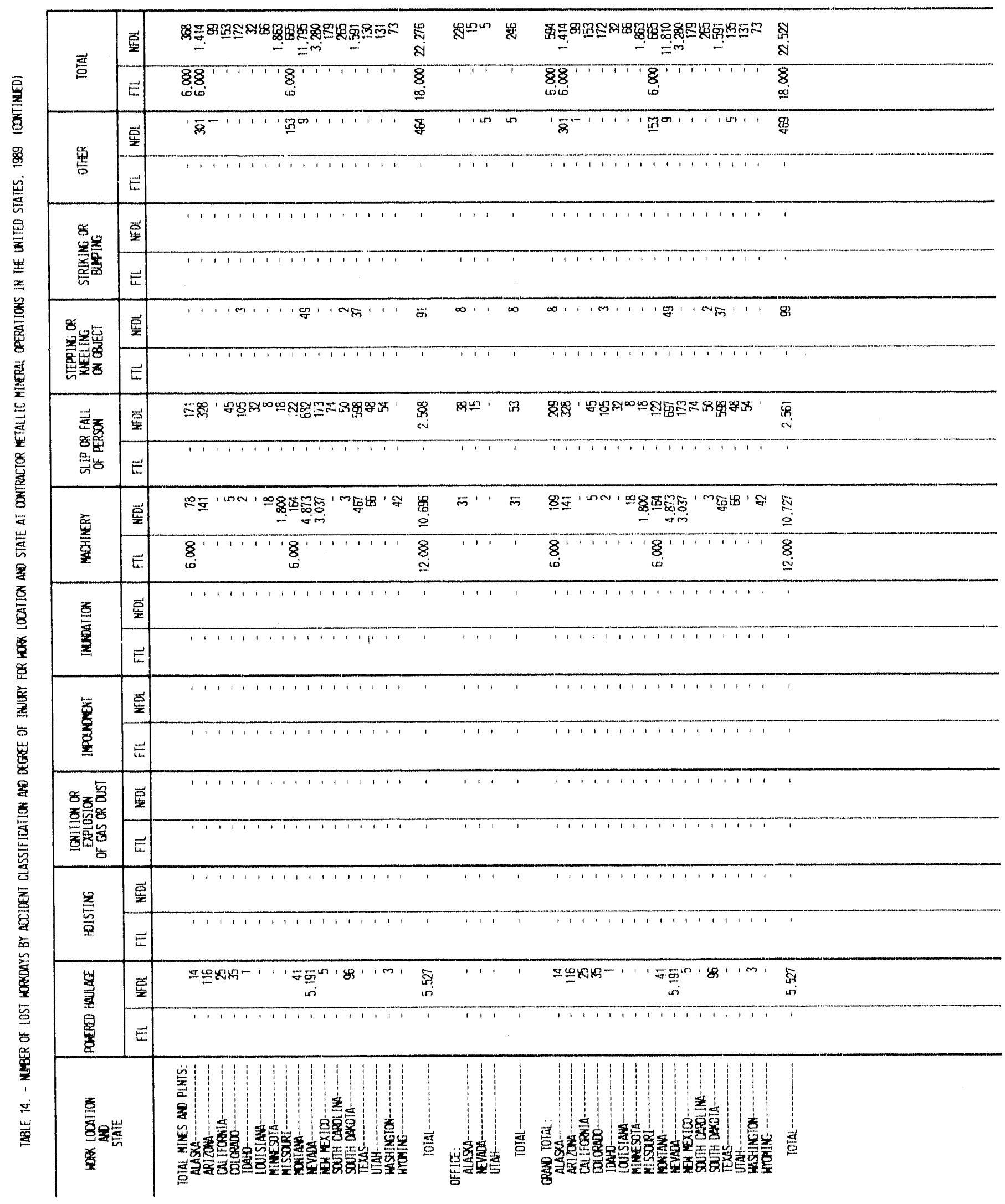




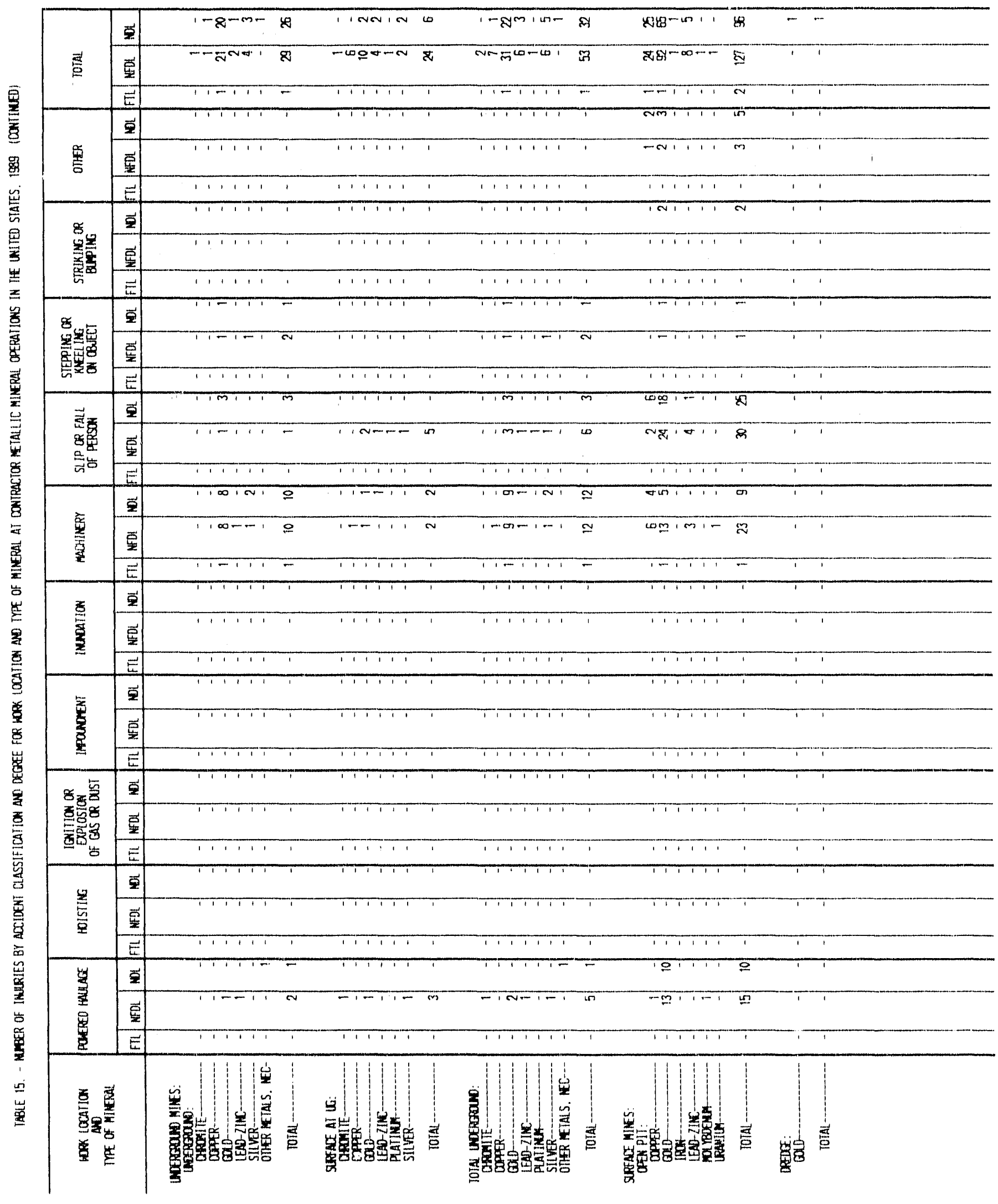




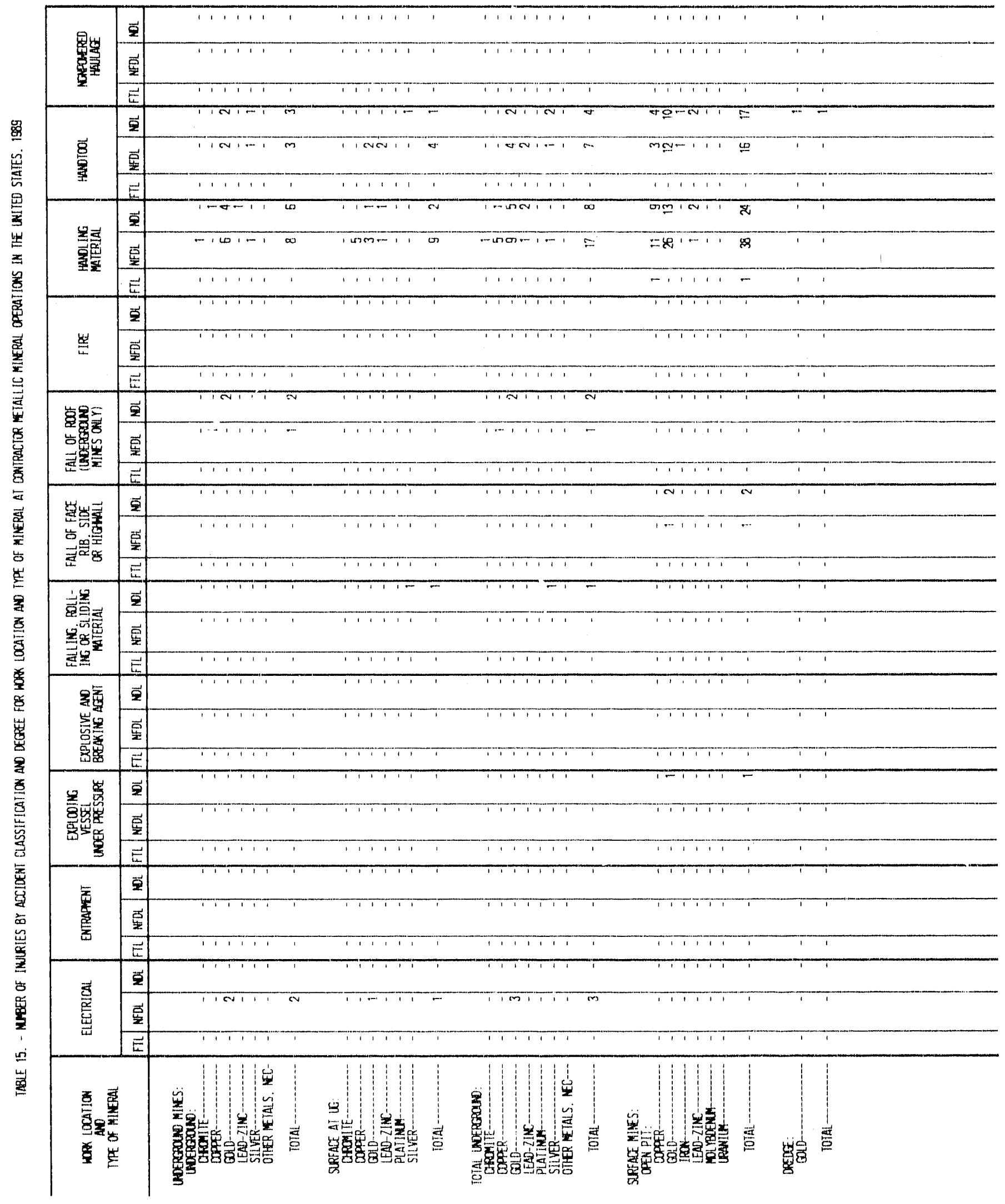




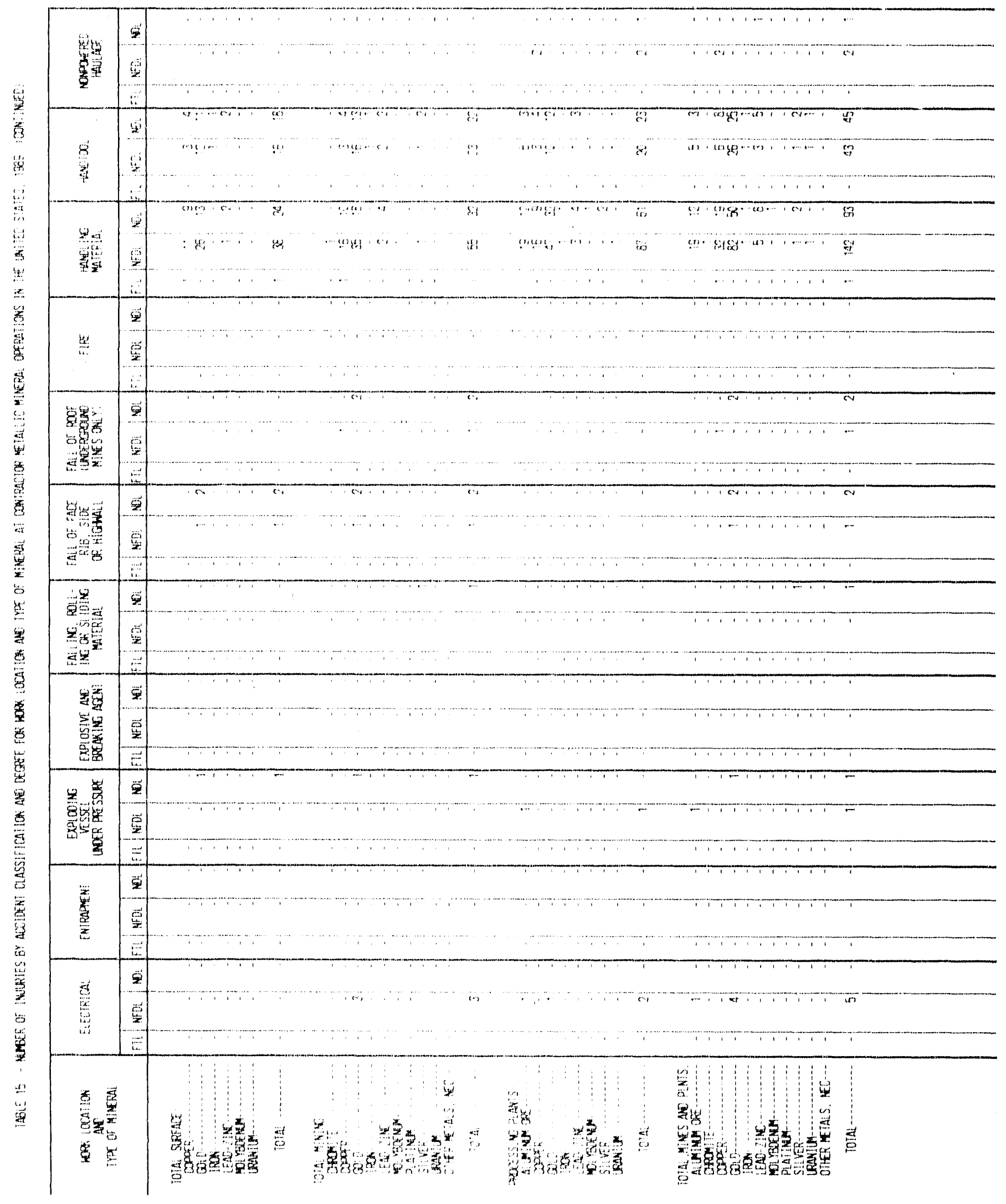




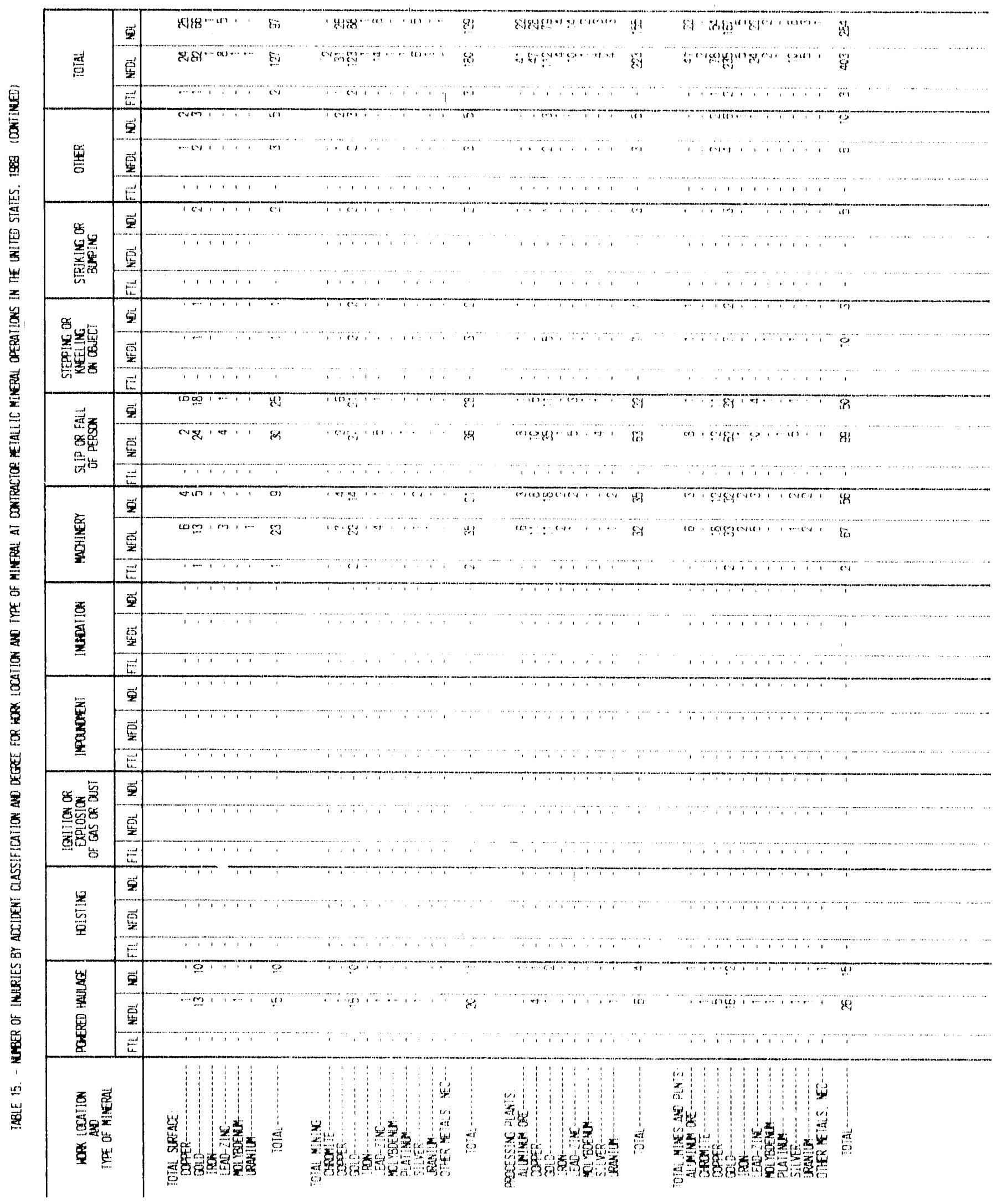




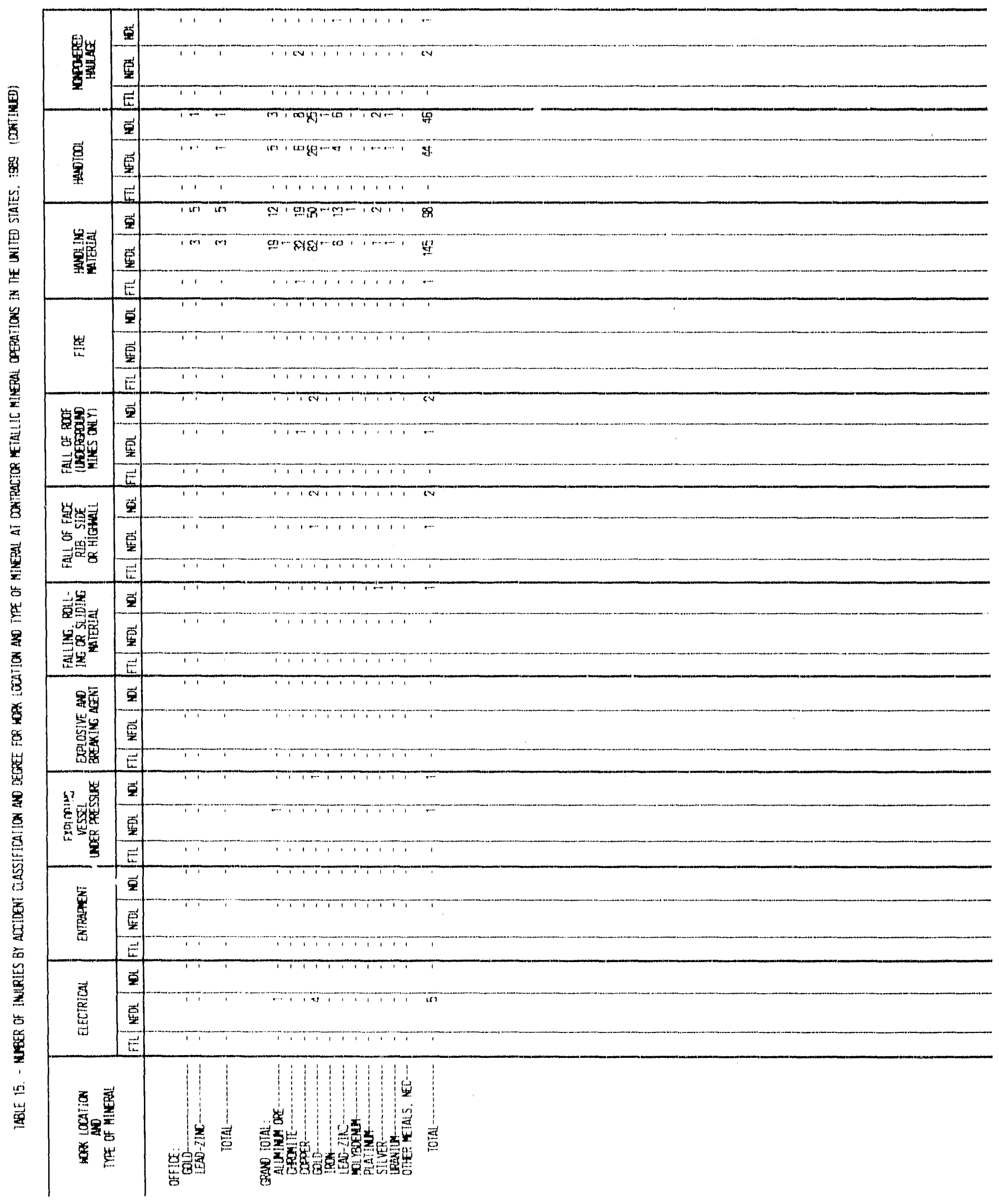




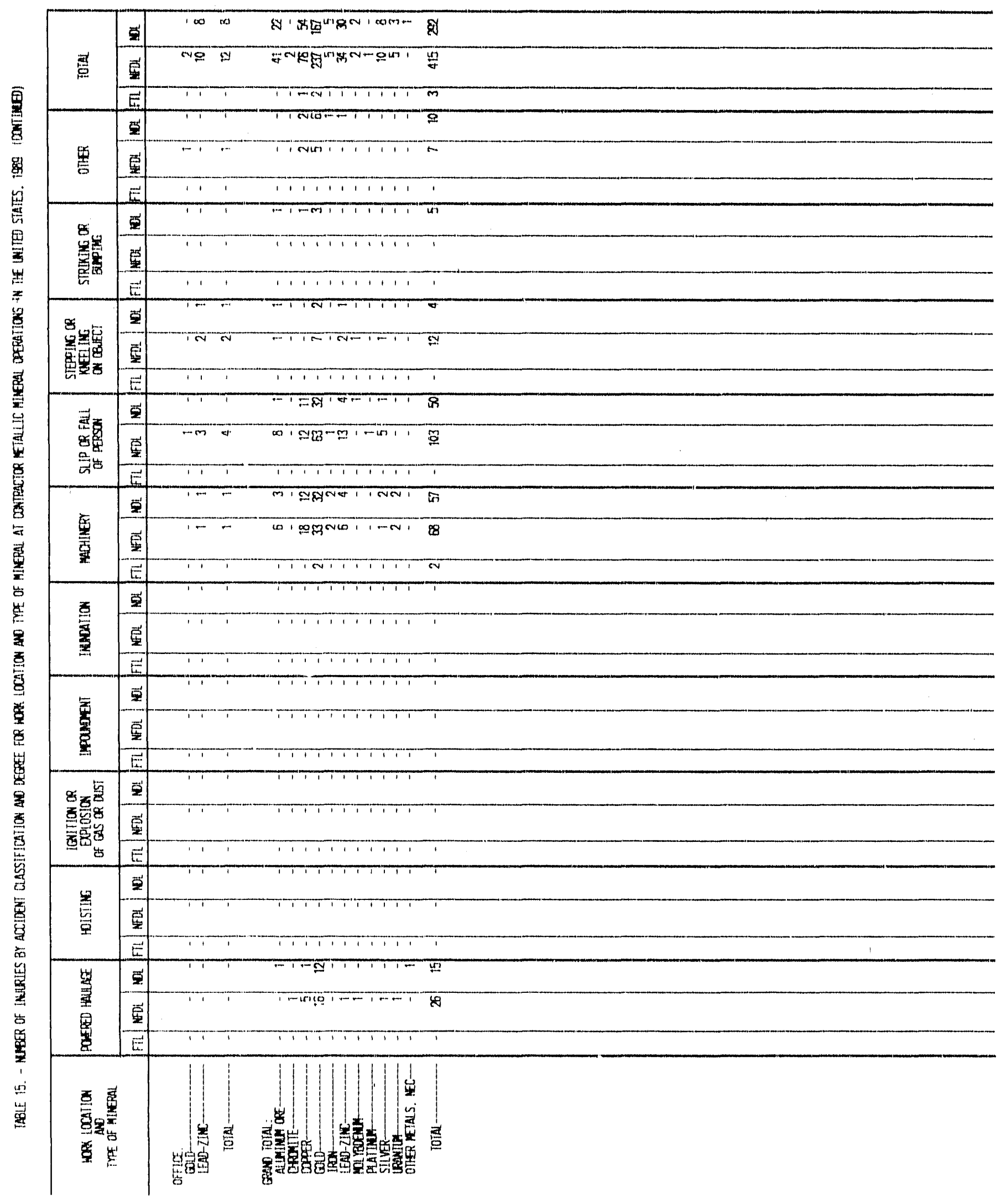




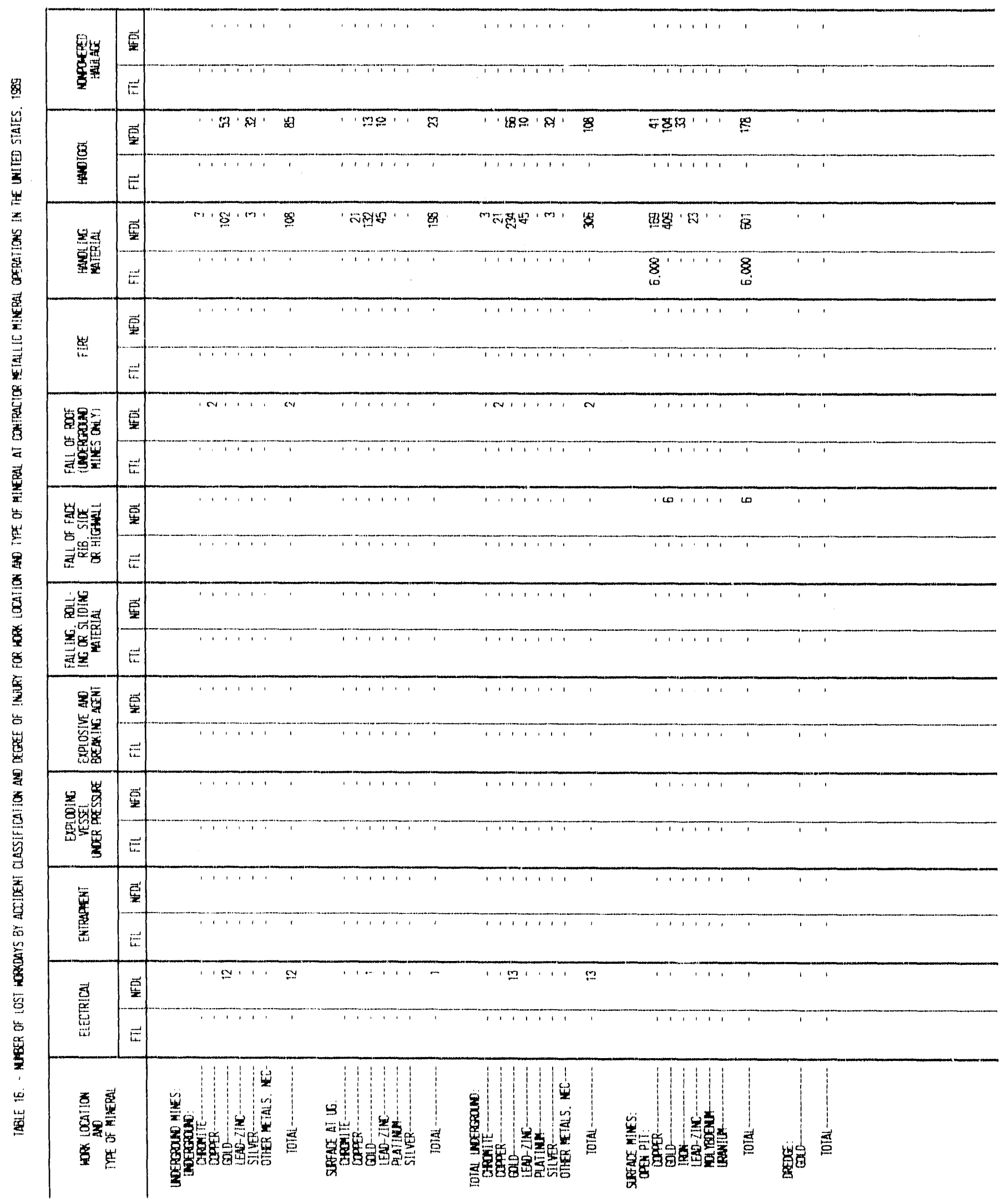




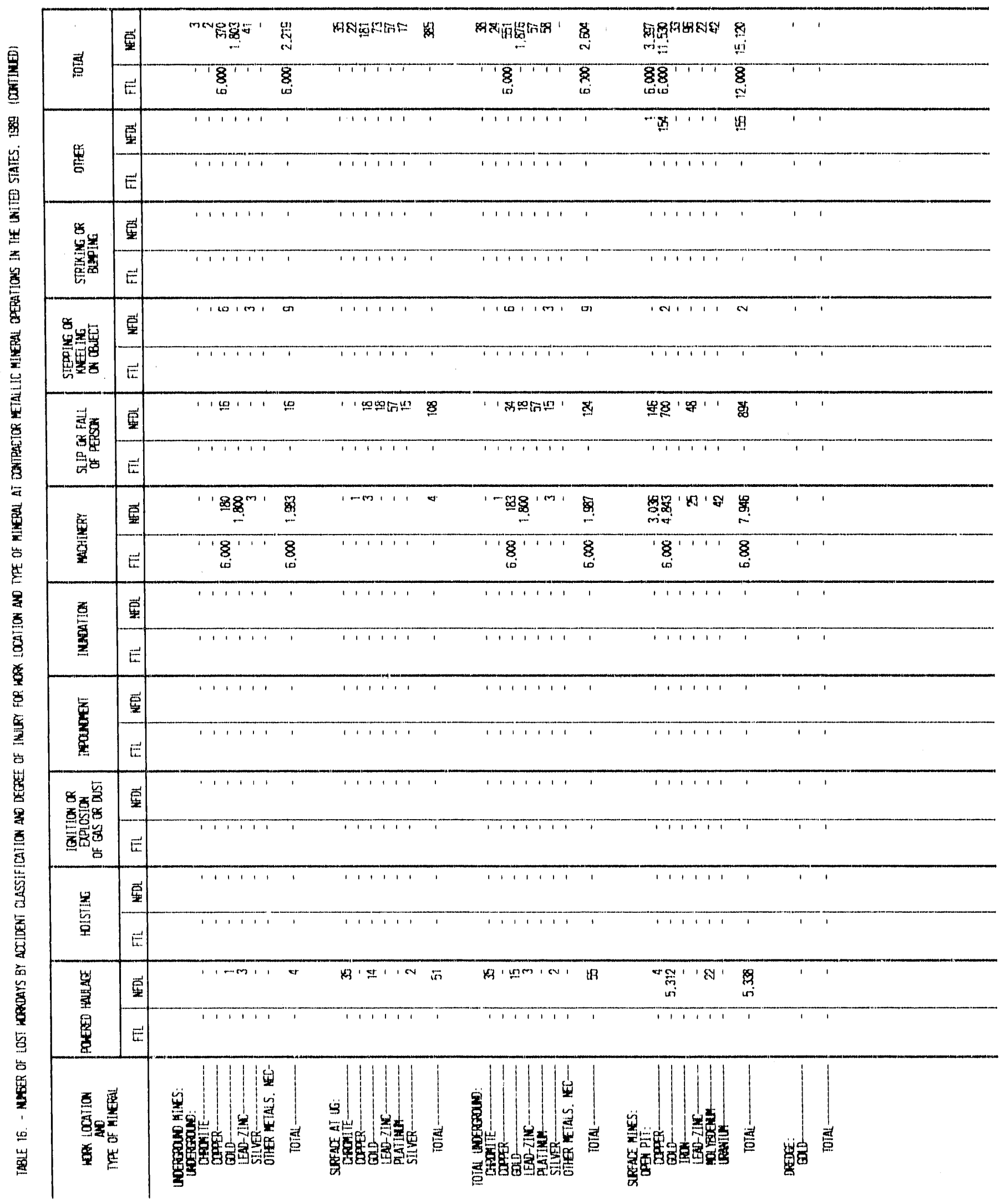



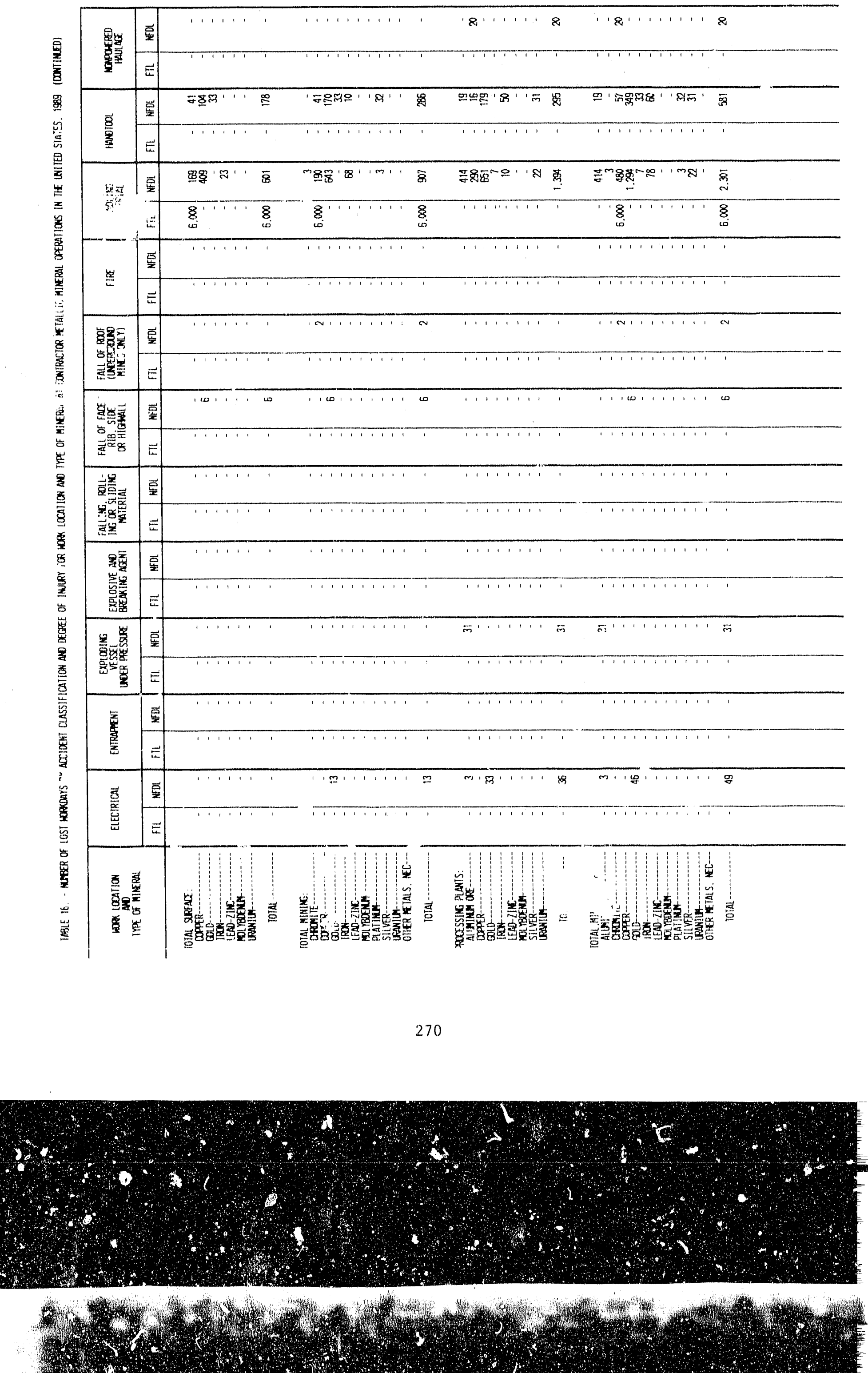


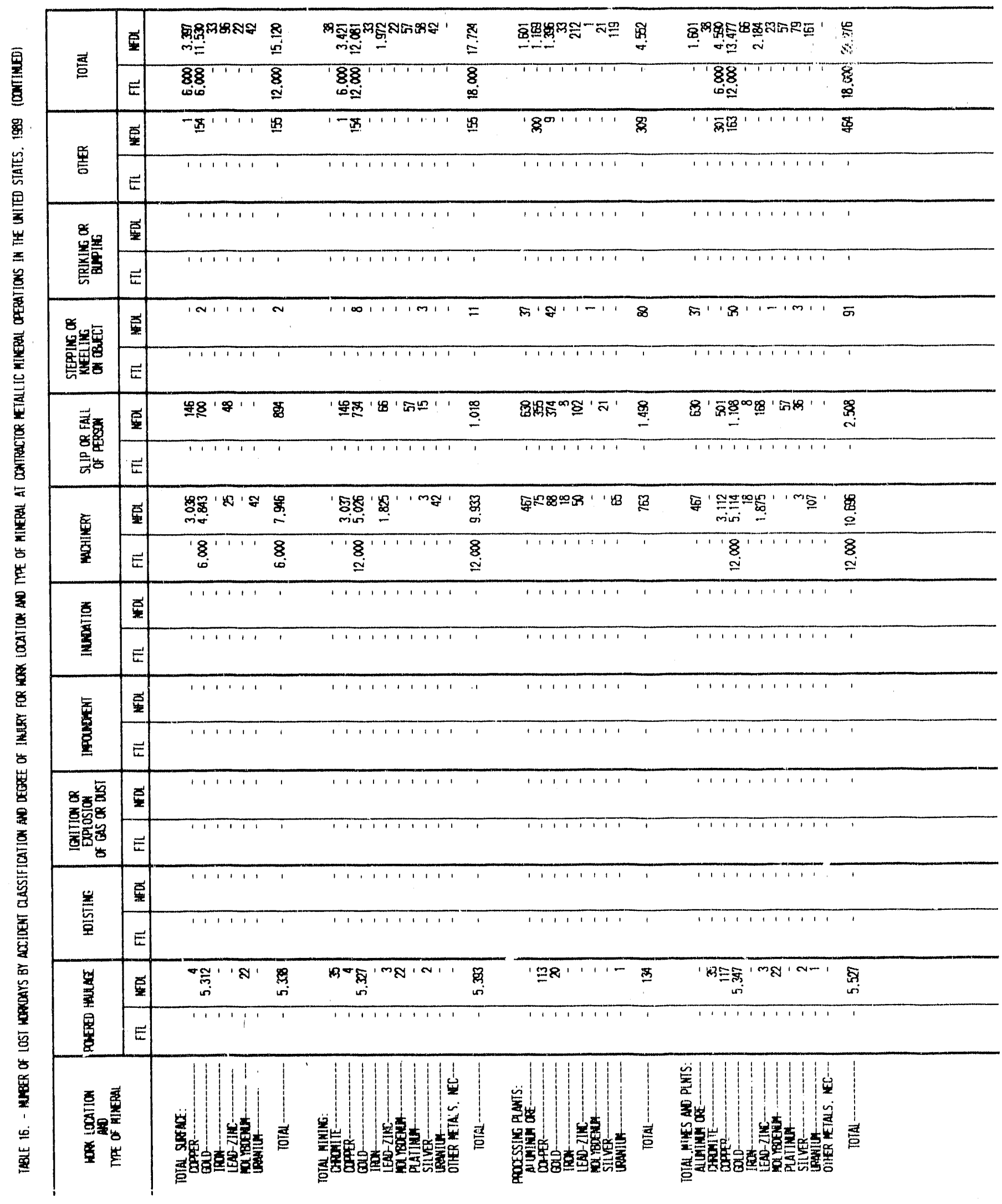




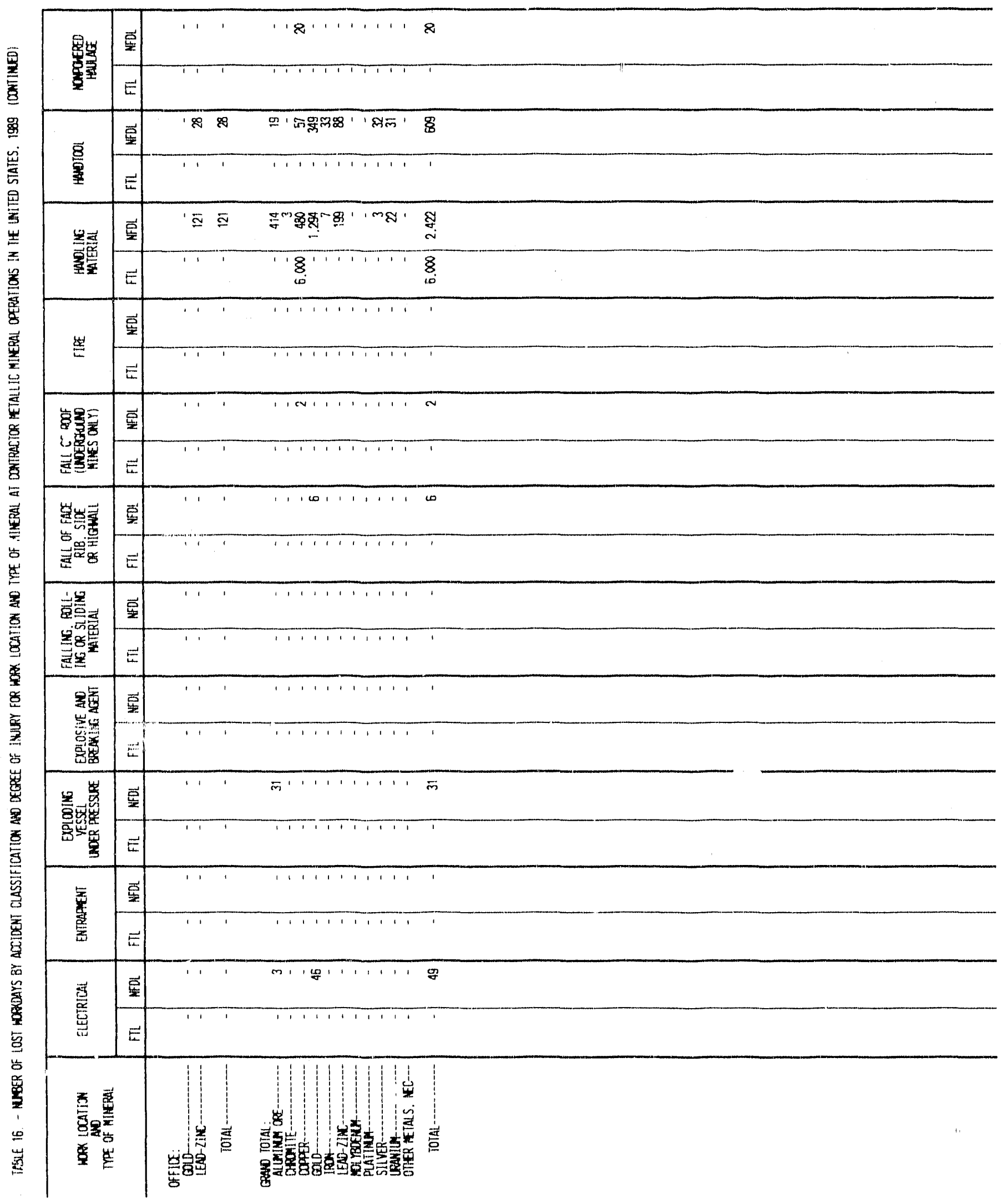




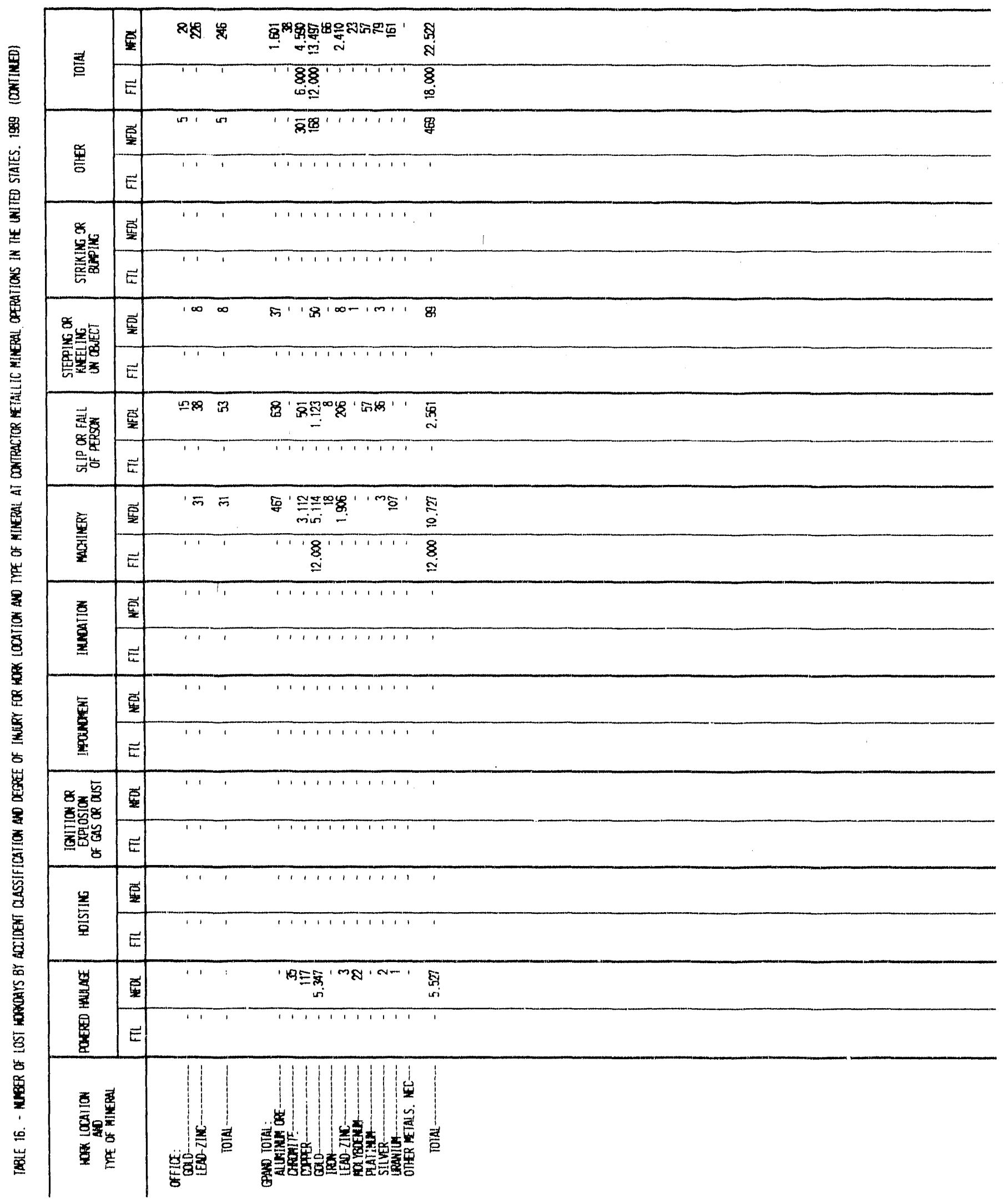




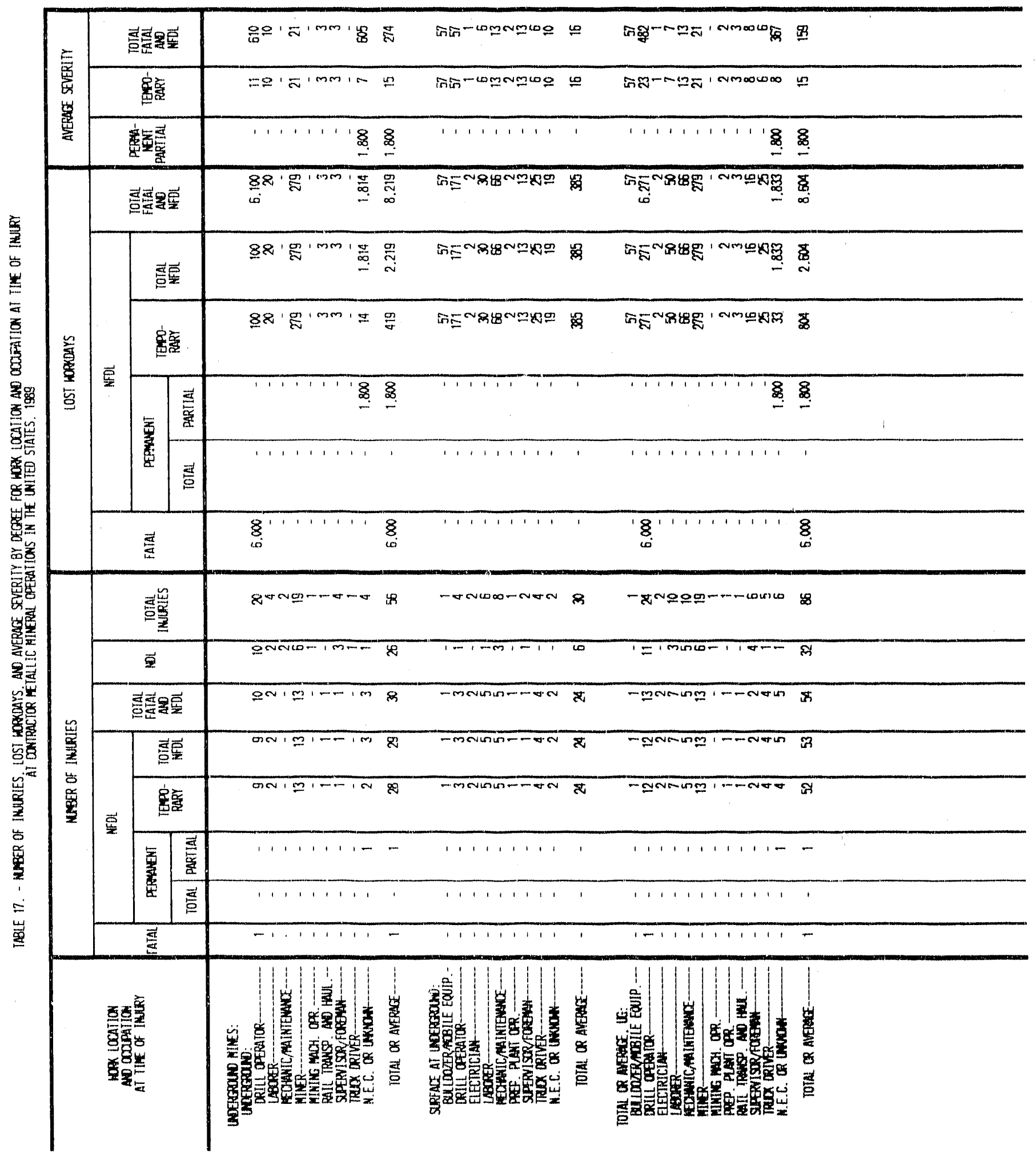




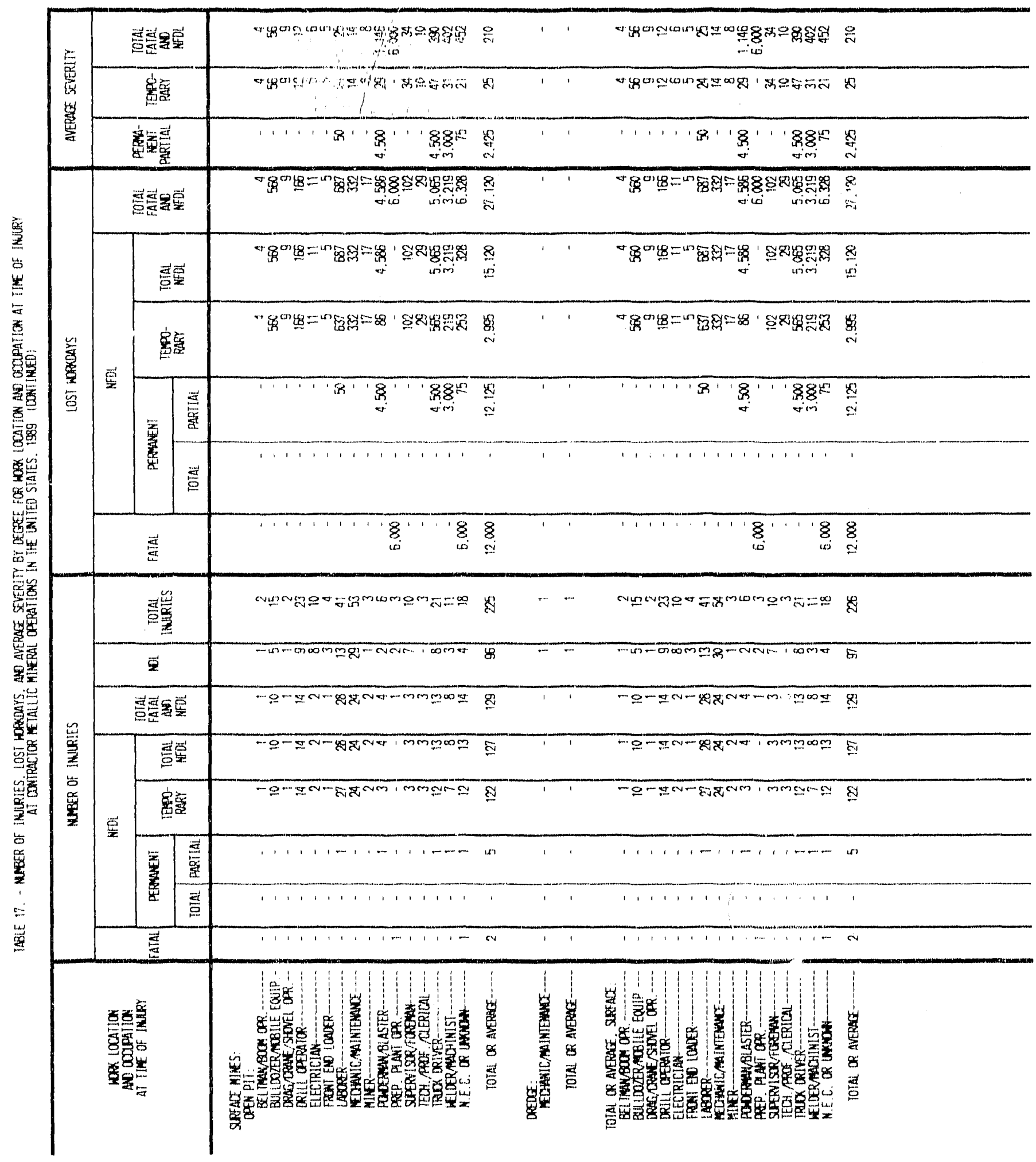




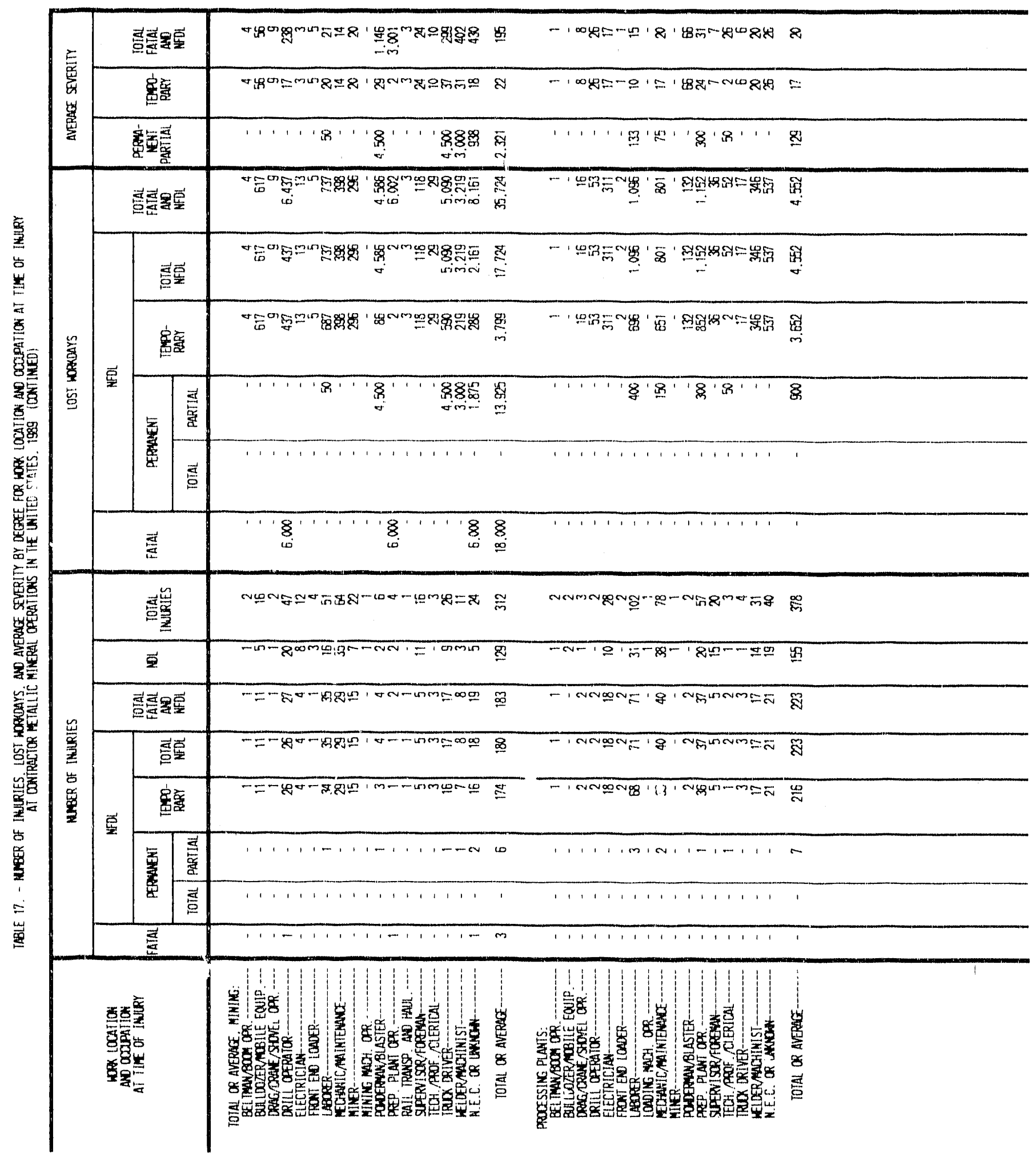




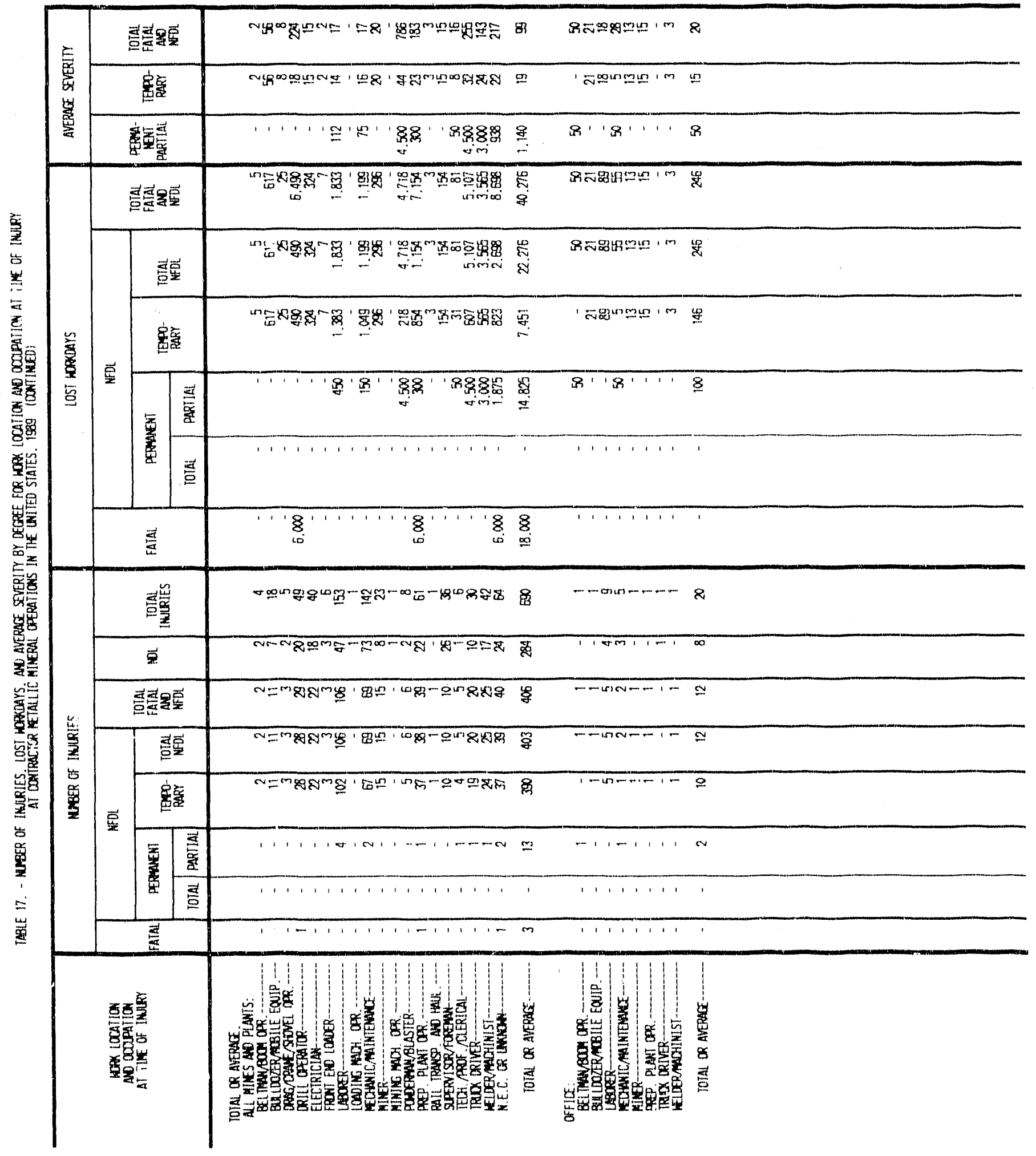




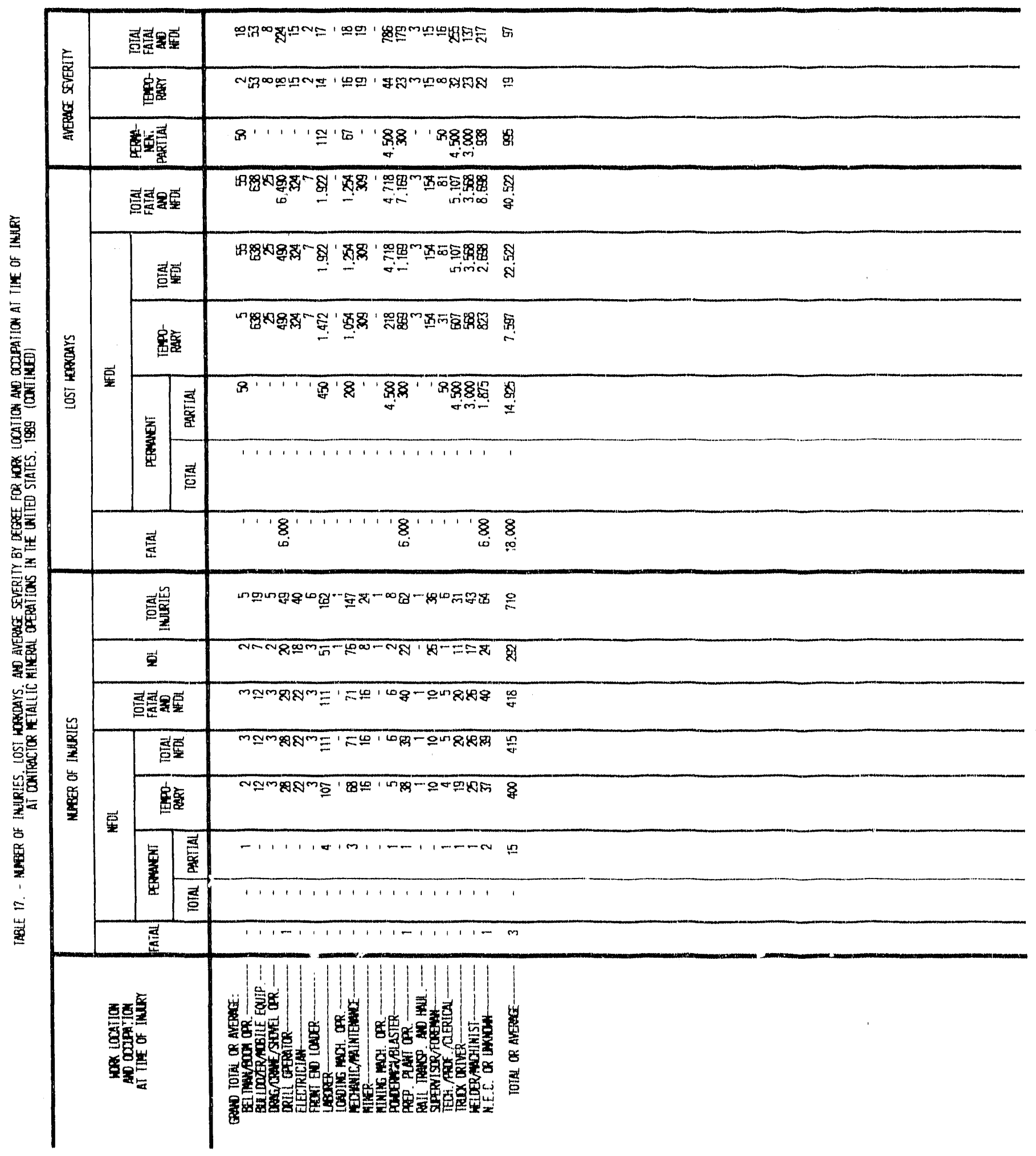




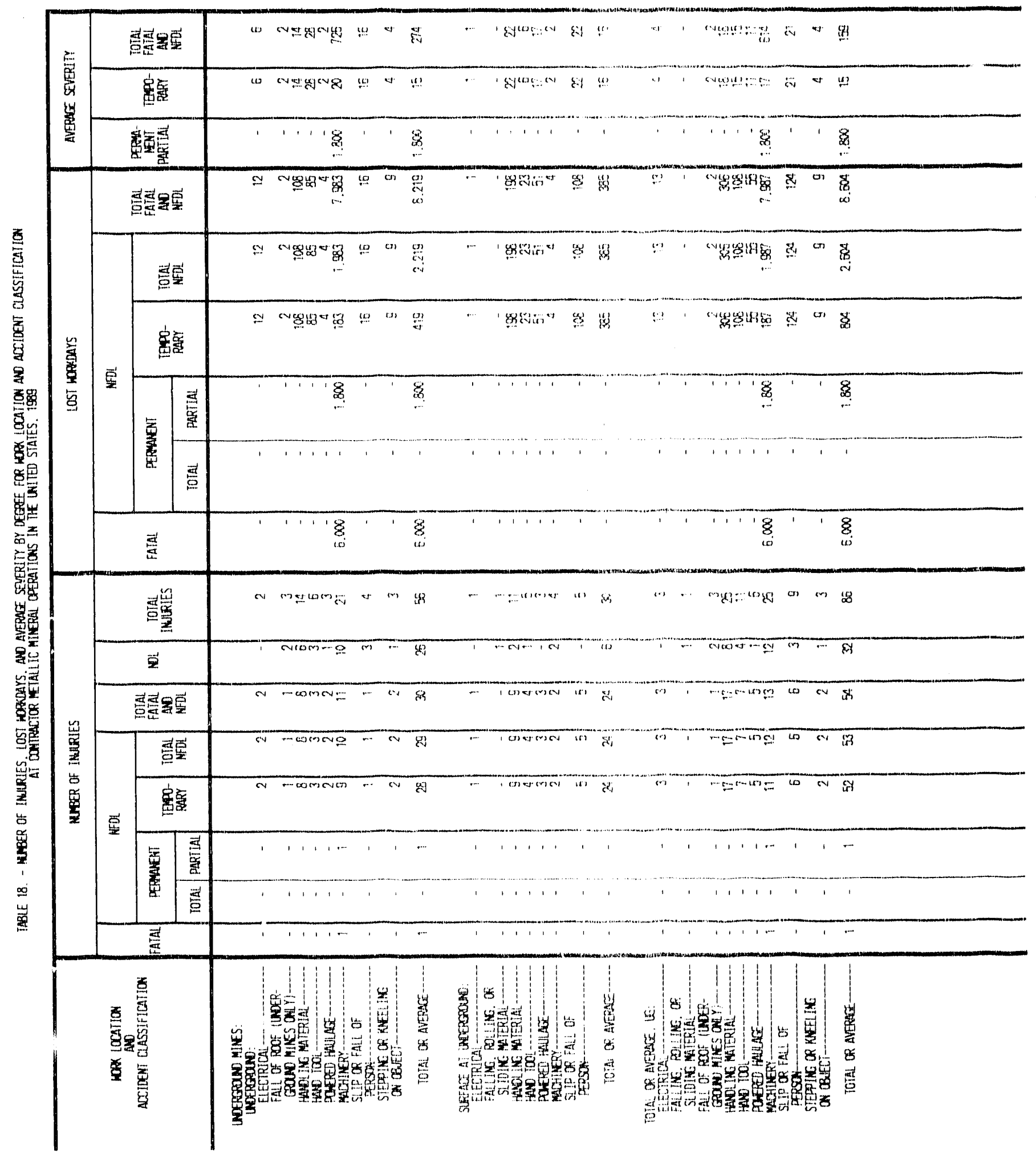




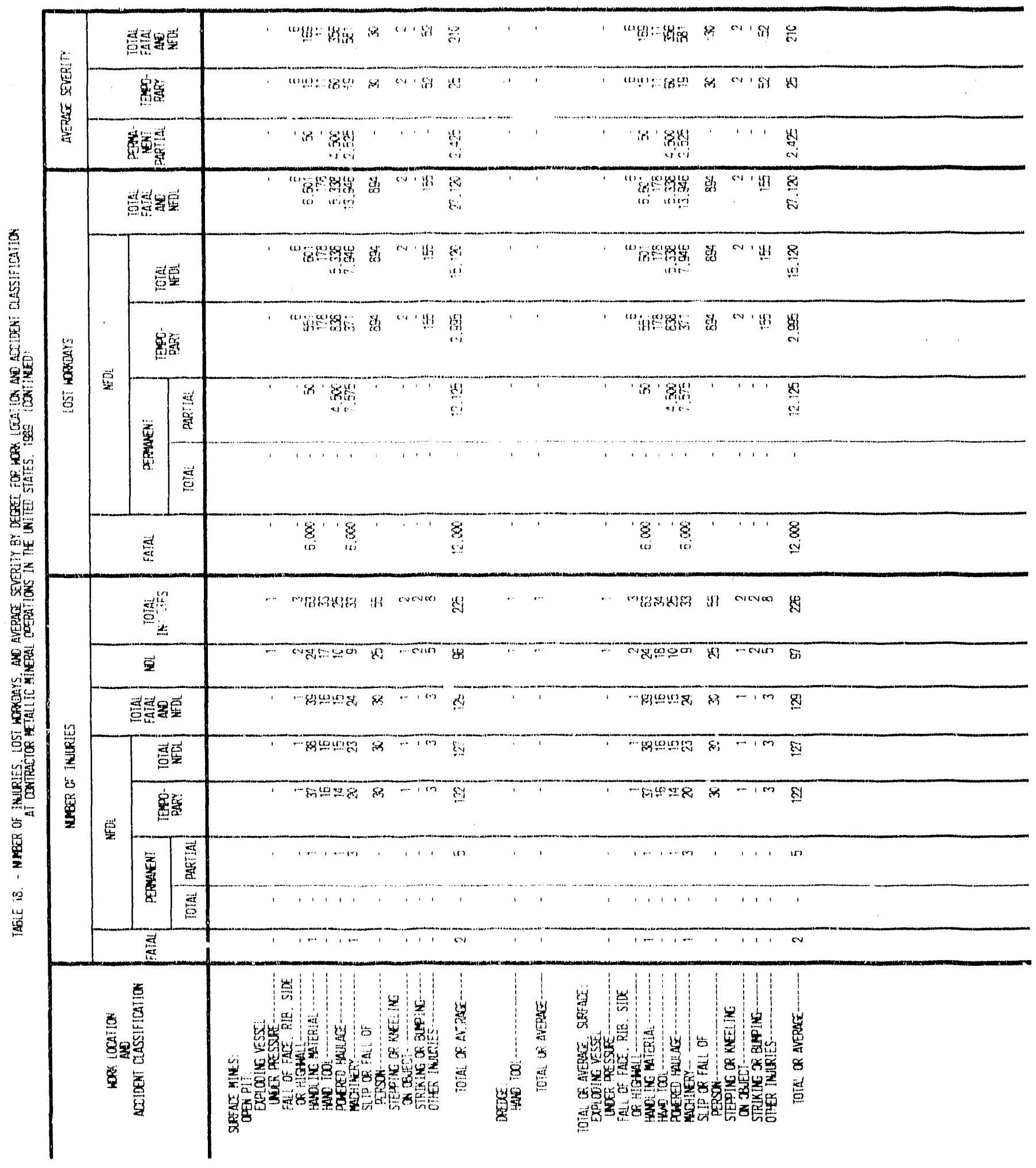




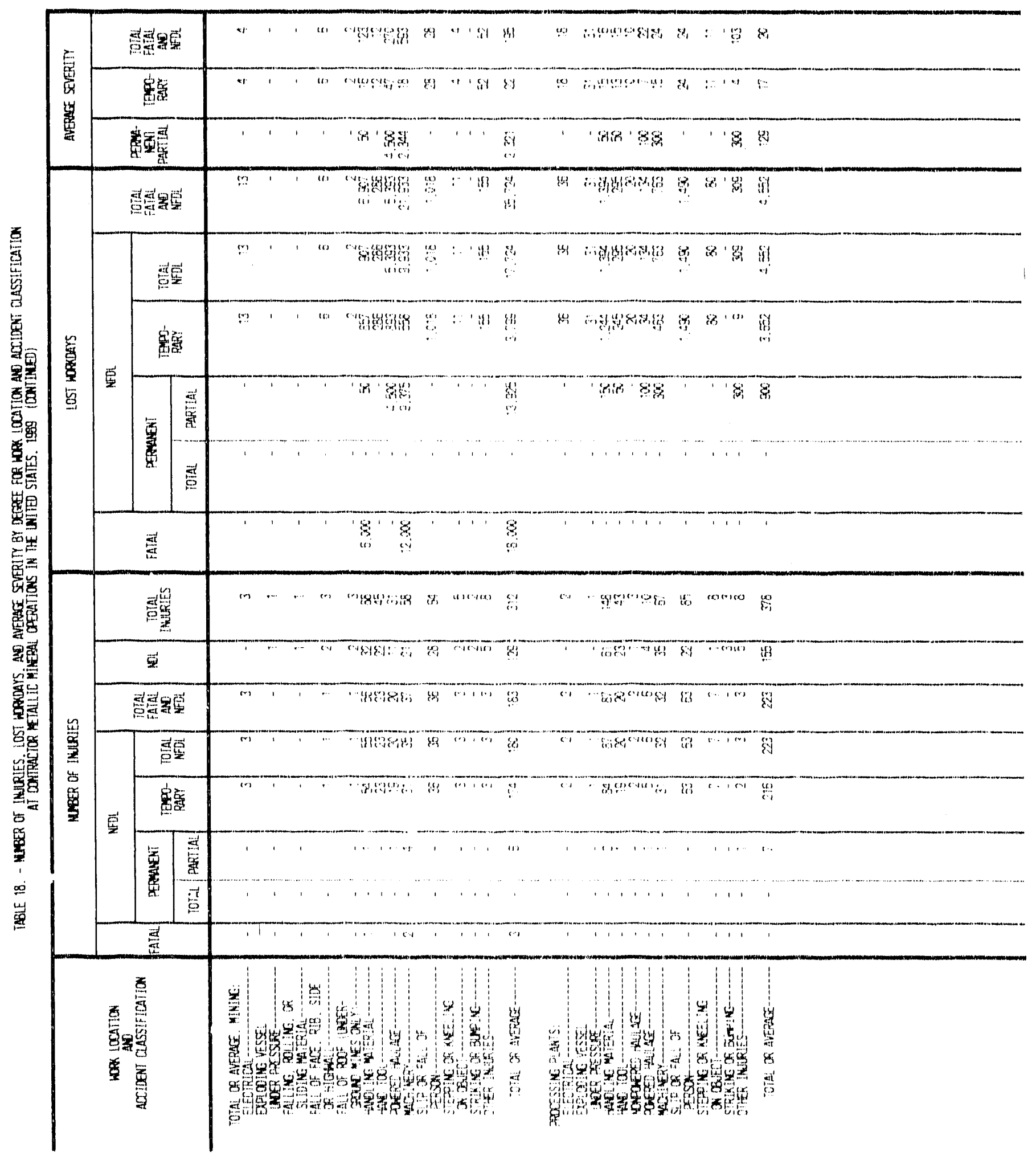




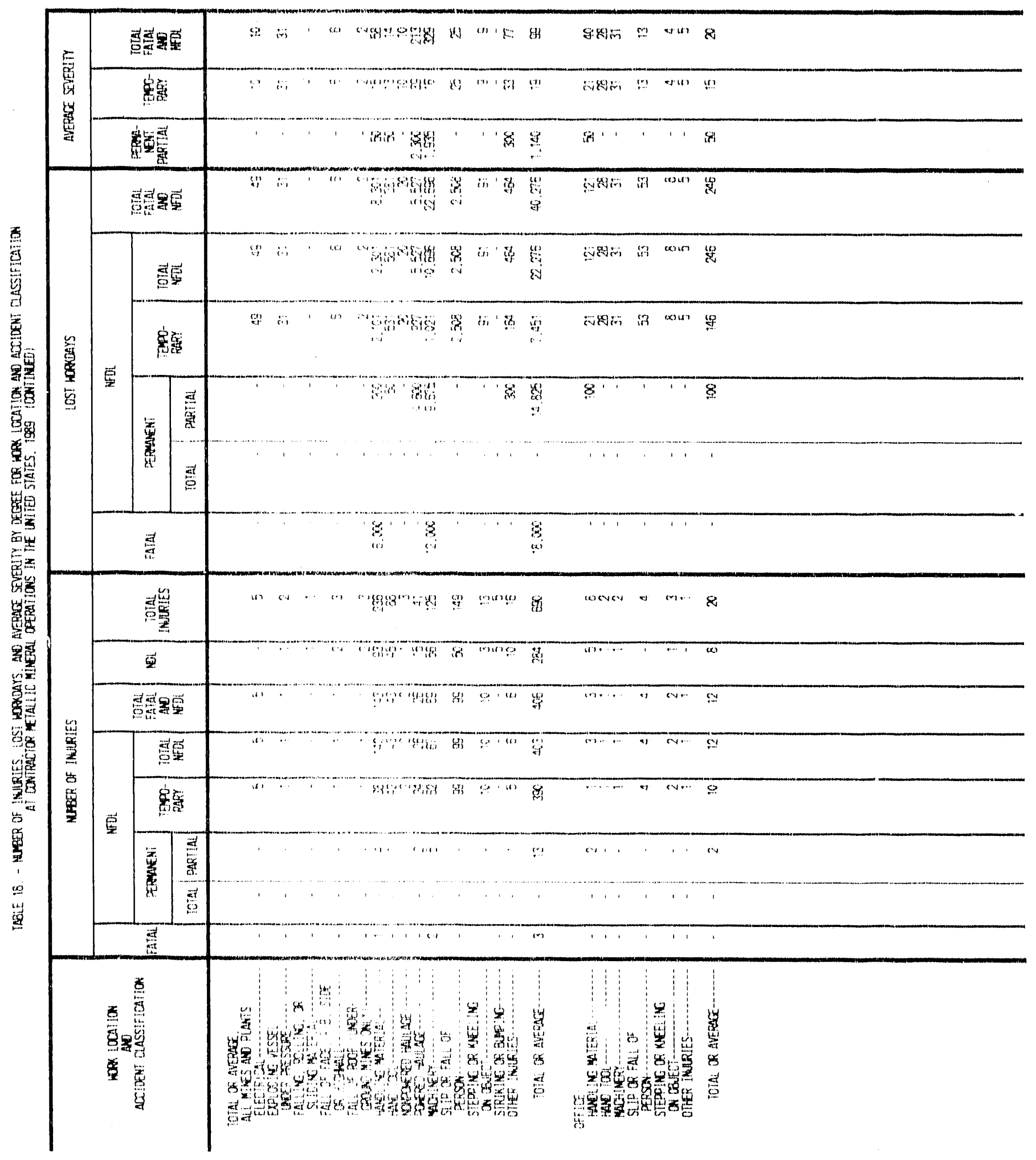




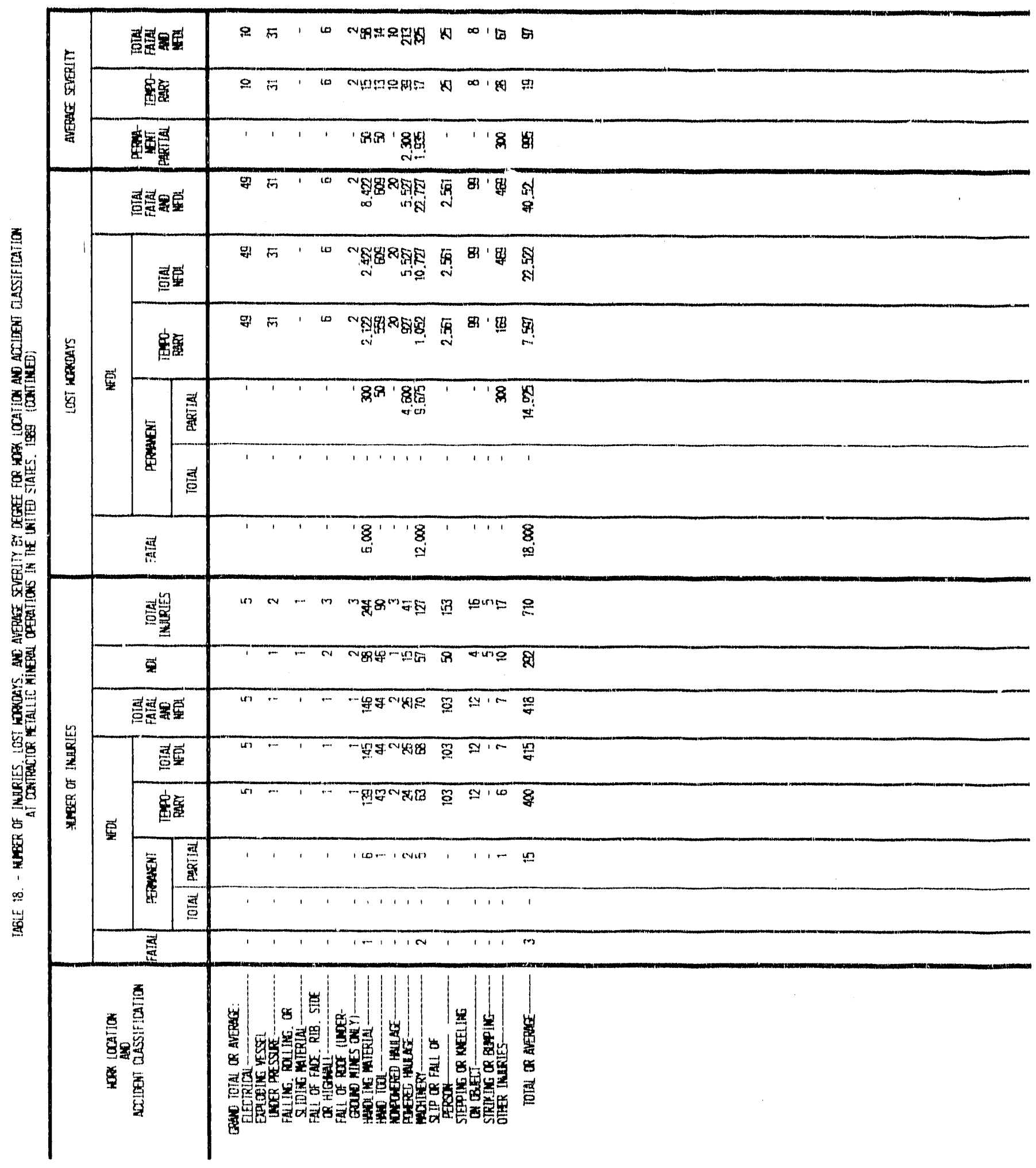




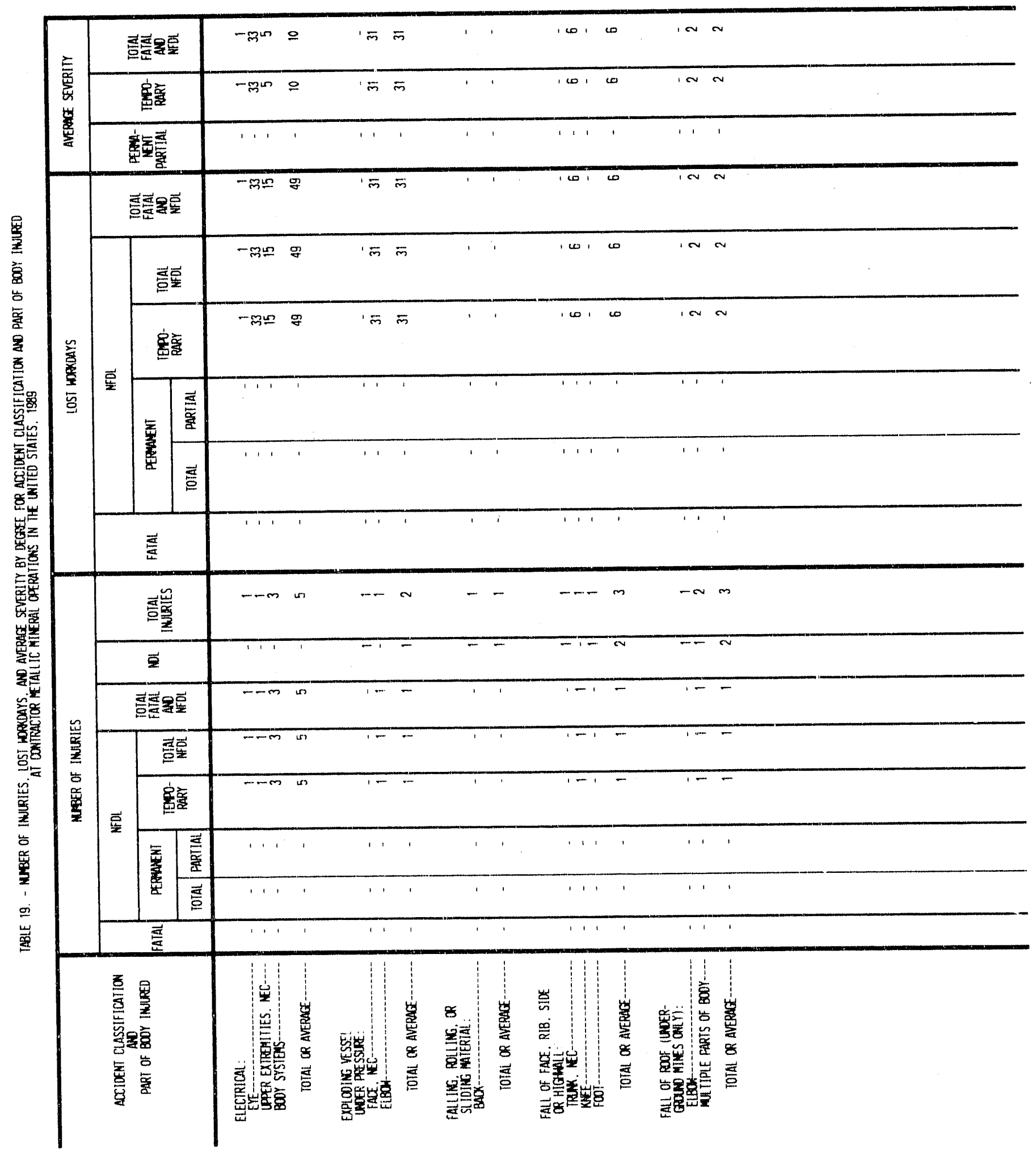




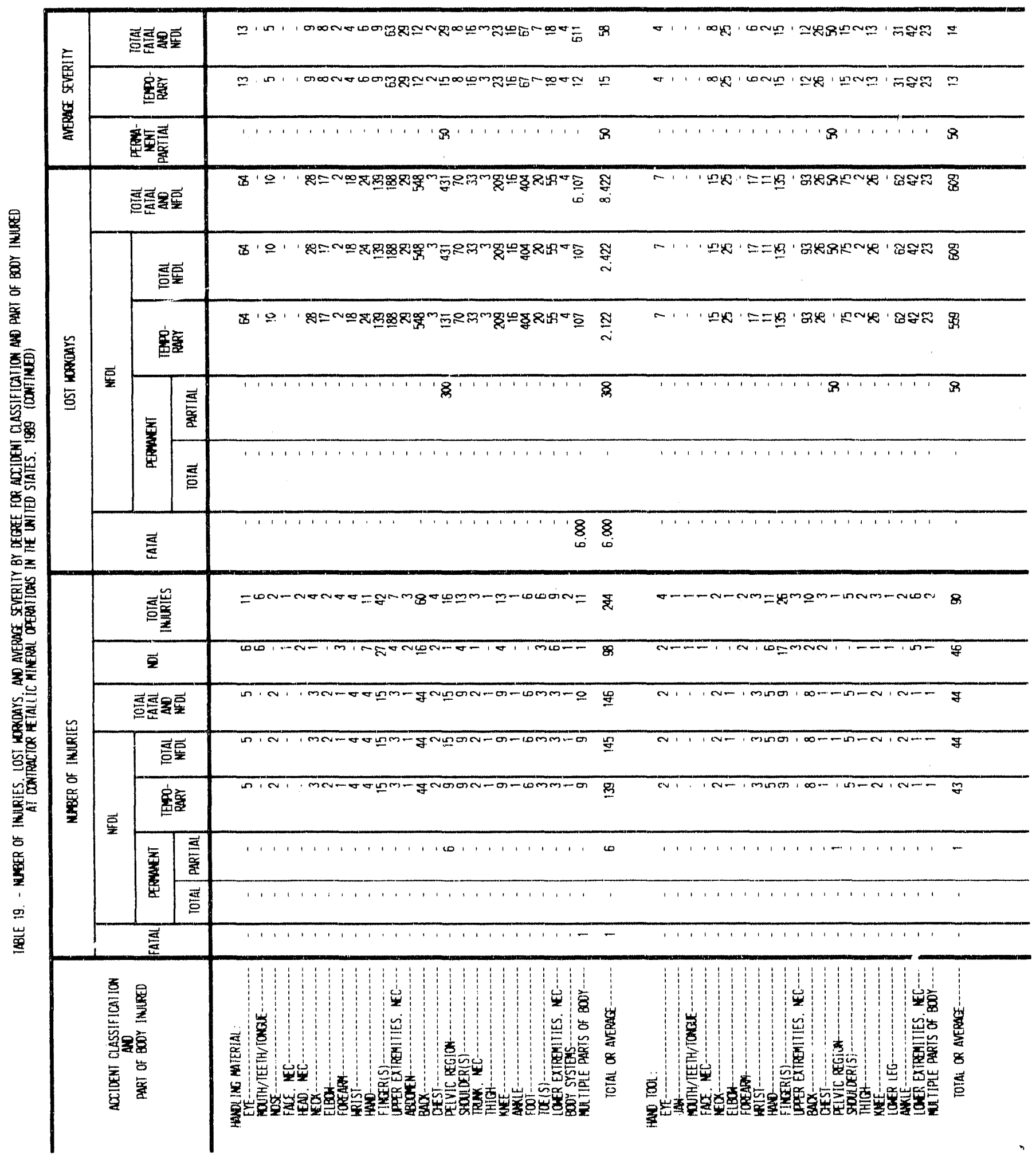




\begin{tabular}{|c|c|c|c|c|c|c|c|c|}
\hline \multirow{3}{*}{ 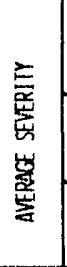 } & \multicolumn{2}{|c|}{ 형편요롤 } & $n$ & & 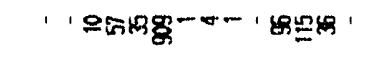 & $\stackrel{m}{\sim}$ & 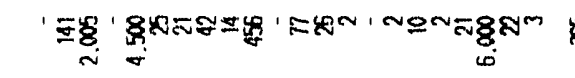 & $\$$ \\
\hline & \multicolumn{2}{|c|}{ 鲁签 } & $r=$ & 우 & 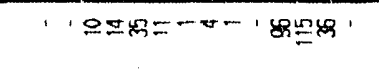 & $g$ & 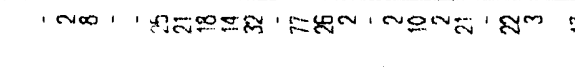 & $=$ \\
\hline & \multicolumn{2}{|c|}{ 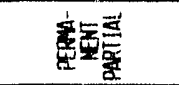 } & ' ' & ' & '多 $\underset{8}{\prime}$ & sis & 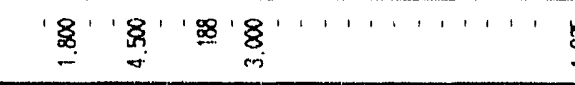 & $\stackrel{9}{-}$ \\
\hline \multirow{6}{*}{ 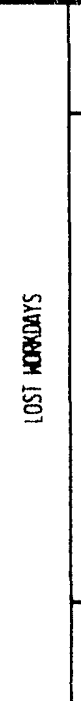 } & \multicolumn{2}{|c|}{ 홍편원룰 } & -9 & 8 & 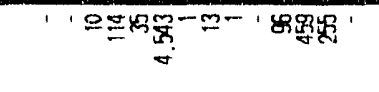 & స్్ㄱ & ' & 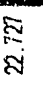 \\
\hline & \multirow{4}{*}{ 亘 } & 害垔 & $r m$ & $\&$ & 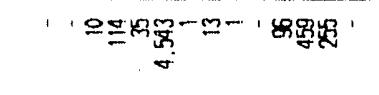 & స్్ㄱ & ' & 焉 \\
\hline & & 容悹 & $m$ & \& & 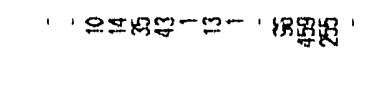 & 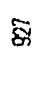 & 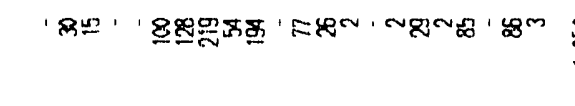 & 范 \\
\hline & & 亲 & & & $8 \underset{8}{8} \sin ^{1}+1111$ & $\underset{8}{8}$ & 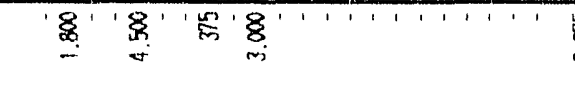 & 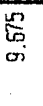 \\
\hline & & 홈 & ' ' & . & , $\cdots$ & ' & $\cdots \cdots \cdots 1$, & ' \\
\hline & \multicolumn{2}{|l|}{ 咅 } & .1 & ' & 11 & I & $\underbrace{\prime}_{\omega}+\cdots, \ldots$ & $\stackrel{8}{8}$ \\
\hline \multirow{8}{*}{ 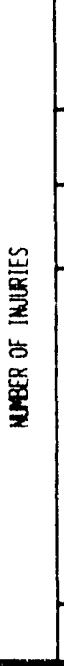 } & \multicolumn{2}{|c|}{ 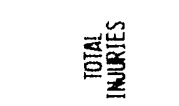 } & $-\infty$ & $m$ & m-evm-in-mam-inar & $\bar{\alpha}$ & 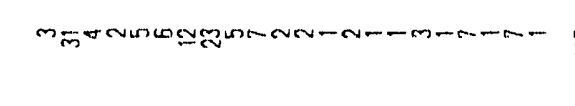 & $\Phi$ \\
\hline & \multicolumn{2}{|l|}{$\overrightarrow{\underline{2}}$} & $1-$ & - & 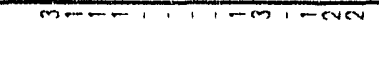 & 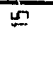 & mimi & $\overline{\mathrm{m}}$ \\
\hline & \multicolumn{2}{|c|}{ 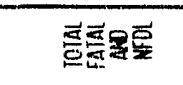 } & - & $\sim$ & $a n-m-1-2 m$ & $\bar{t}$ & 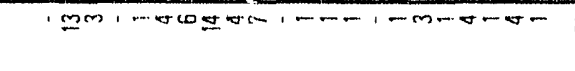 & 2 \\
\hline & \multicolumn{2}{|c|}{ 咅要 } & $=$ & $\sim$ & fan & $\bar{L}$ & 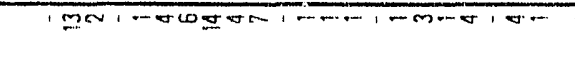 & 8 \\
\hline & \multicolumn{2}{|c|}{ 实䇾 } & $=$ & $\sim$ & 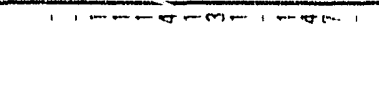 & $\overline{2}$ & 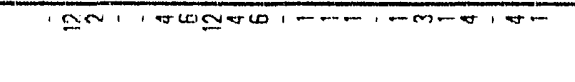 & $\check{\Xi}$ \\
\hline & & 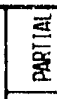 & ' ' & , & $\cdots$ & $\sim$ & -1 ering & $m$ \\
\hline & 案 & $\underline{\underline{\Delta}}$ & $\cdots$ & ' & , , , & , & $\ldots \ldots$ & . \\
\hline & \multicolumn{2}{|l|}{ 迹 } & '. & ' & $11, \ldots$ & ' & $1,1,1,1,1$ & $\sim$ \\
\hline & \multicolumn{2}{|l|}{ 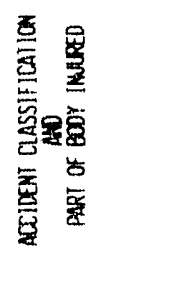 } & 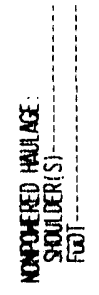 & 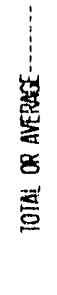 & 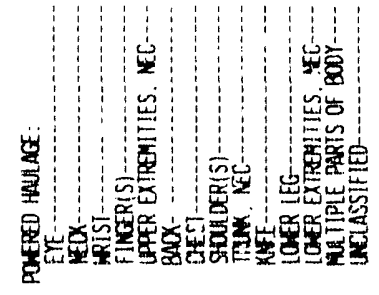 & 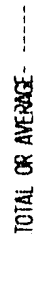 & 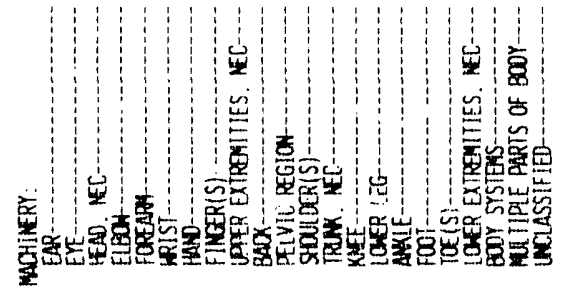 & 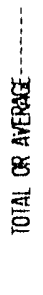 \\
\hline
\end{tabular}




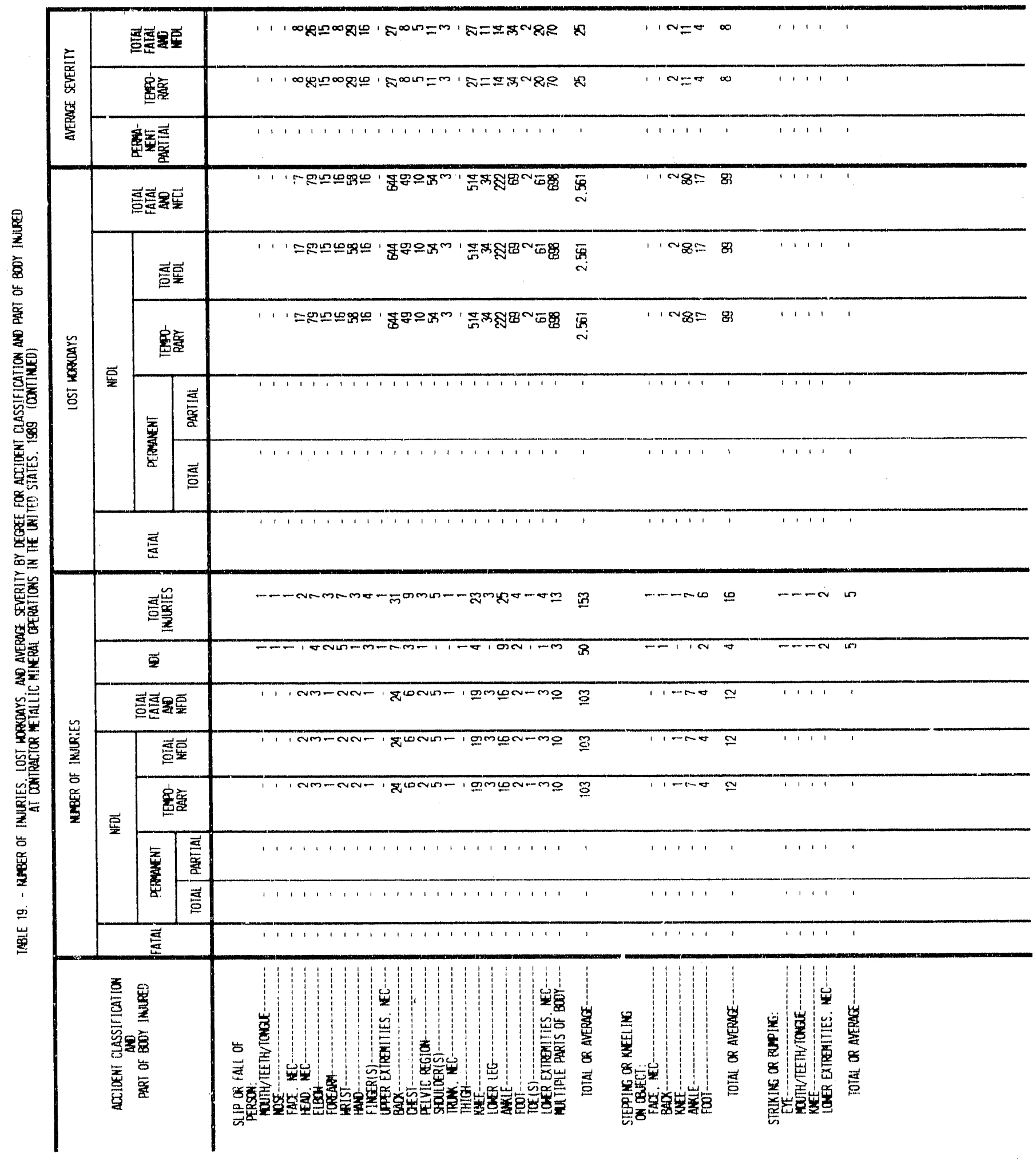




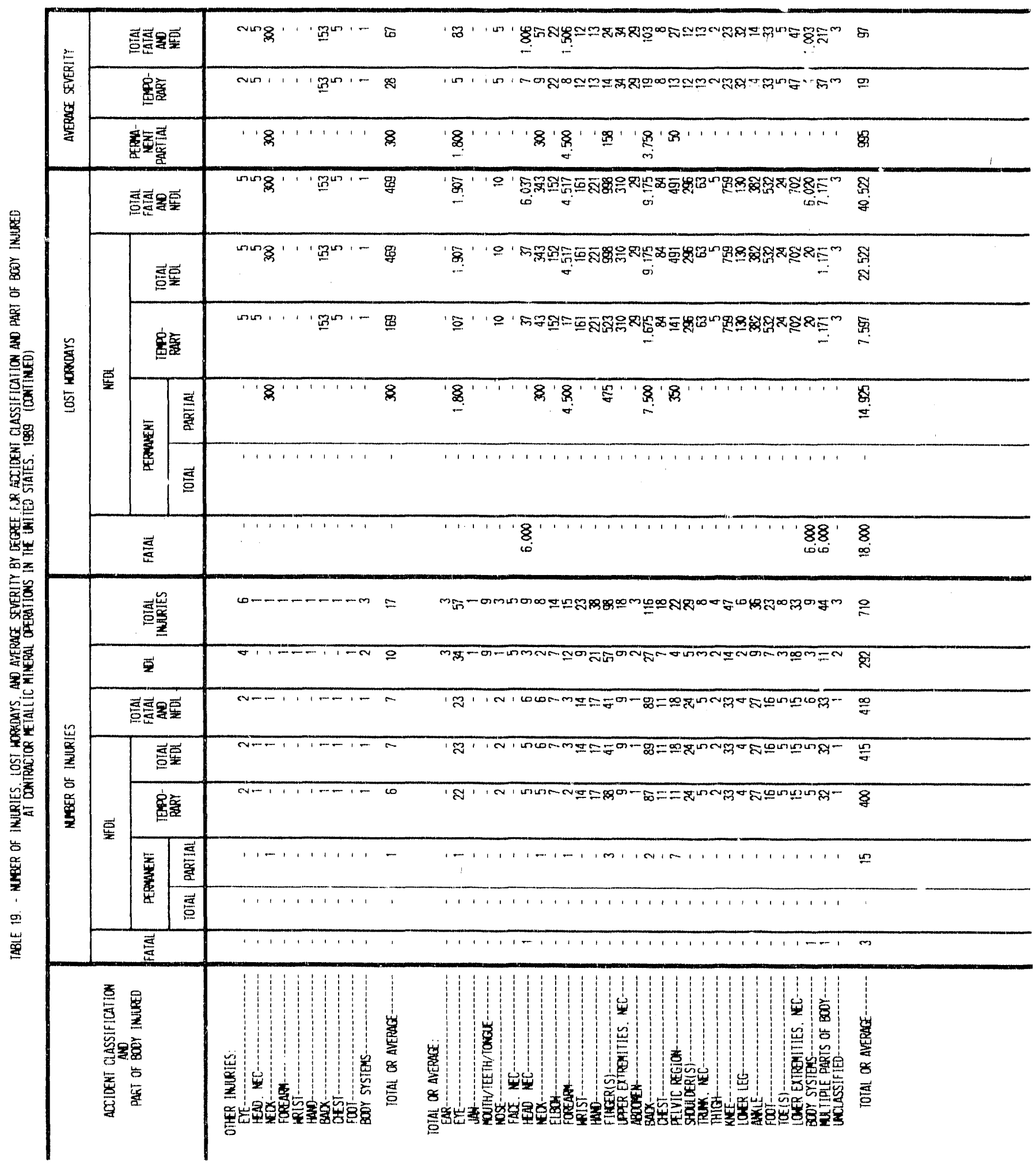




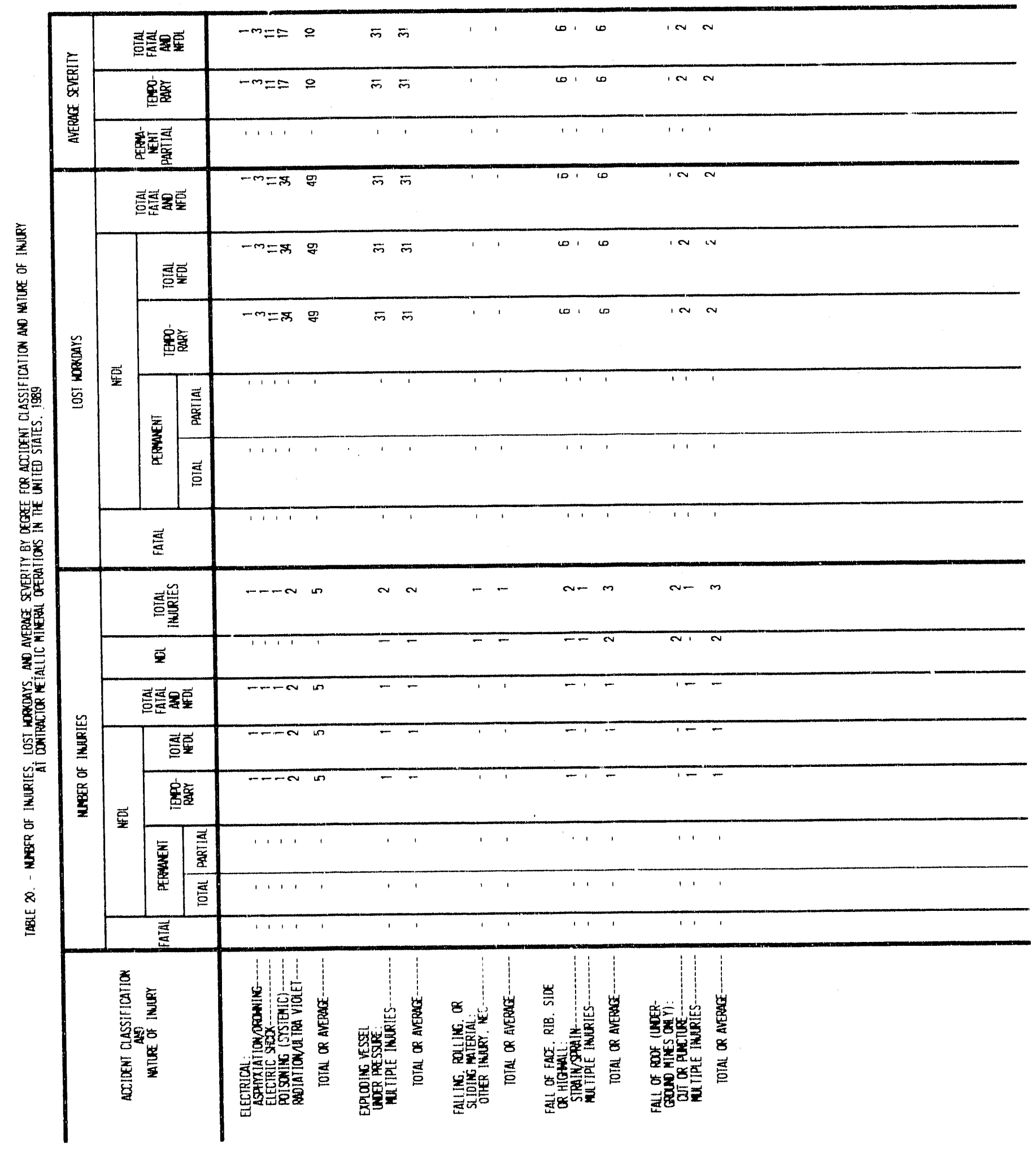




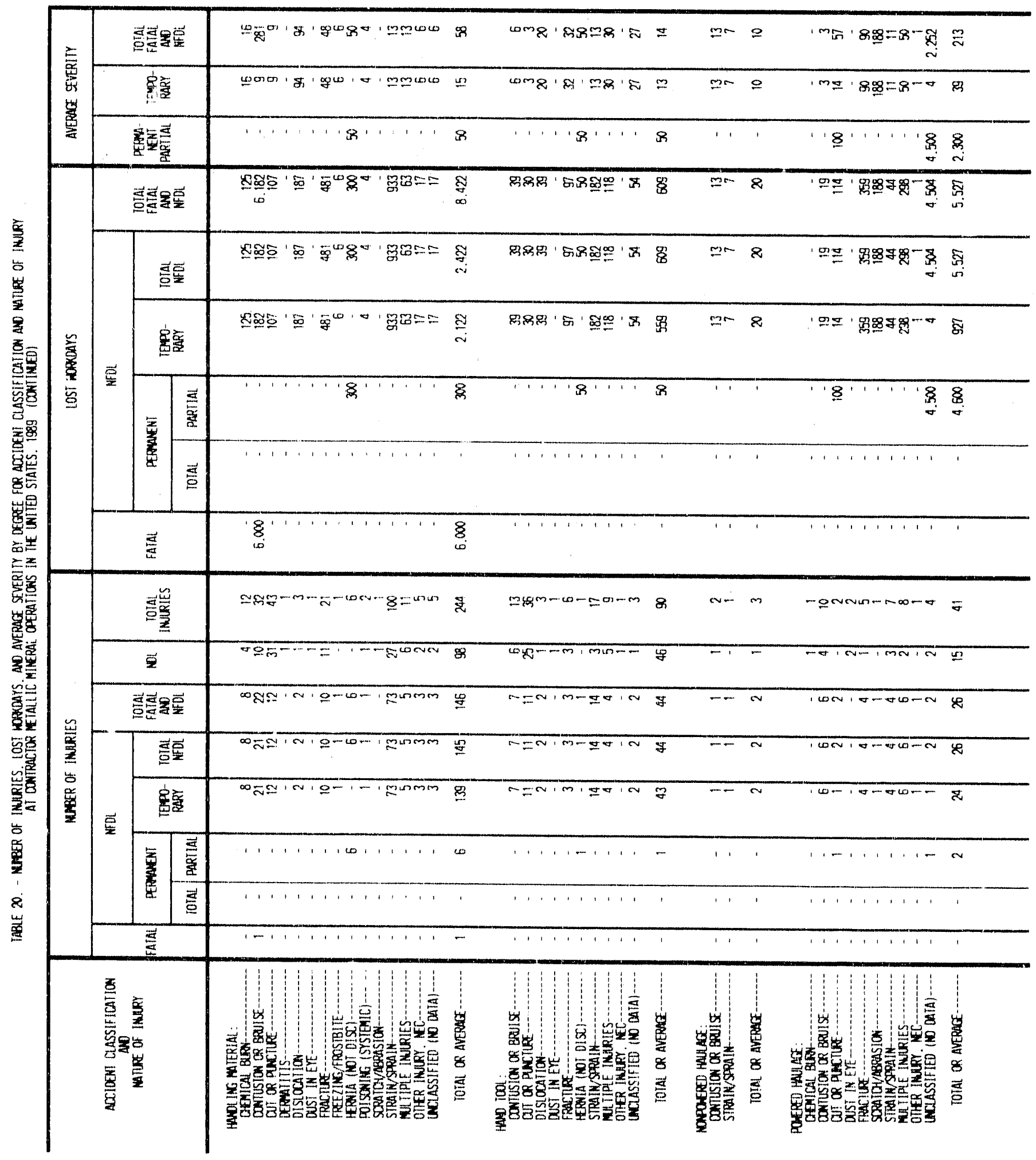




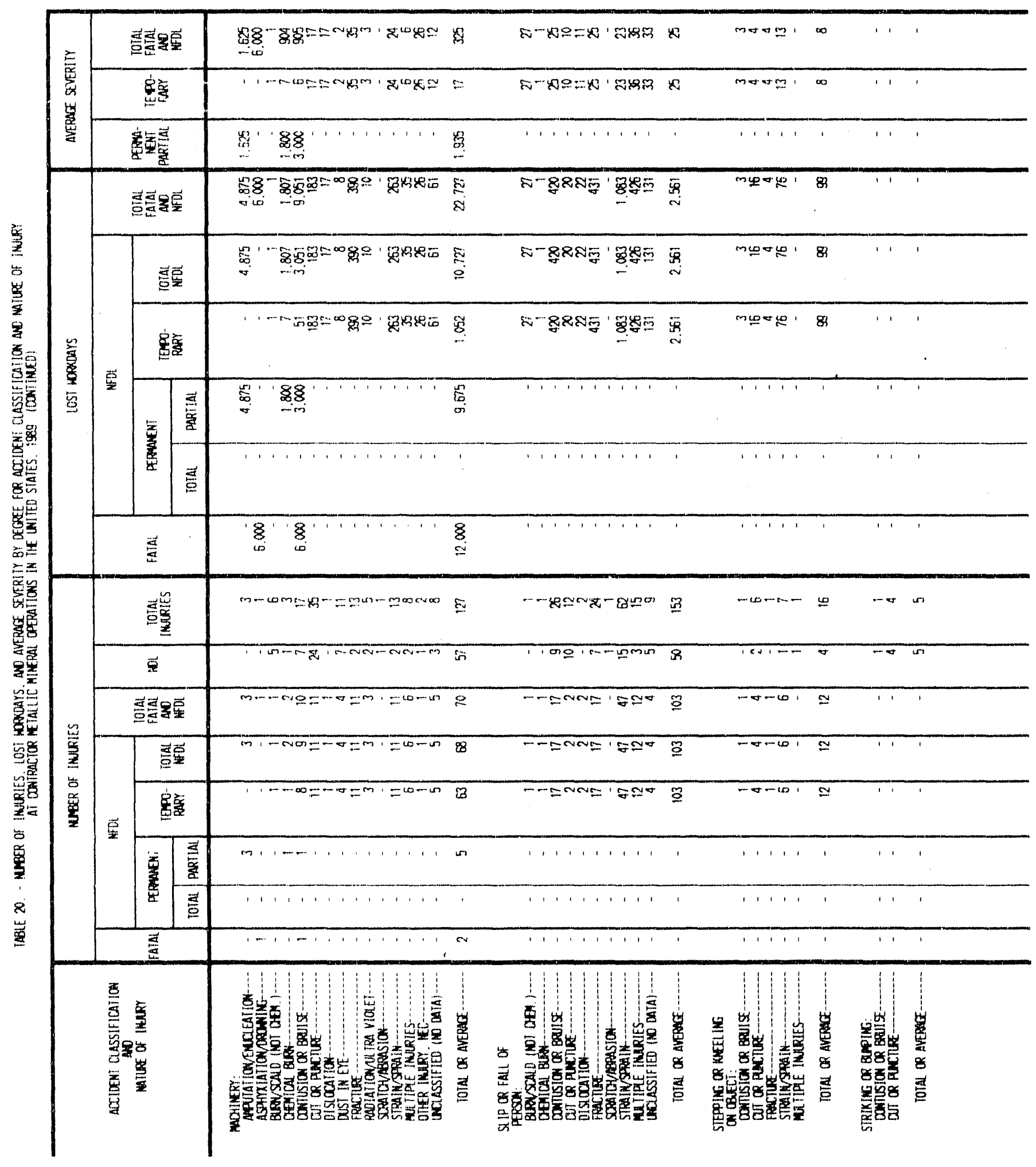




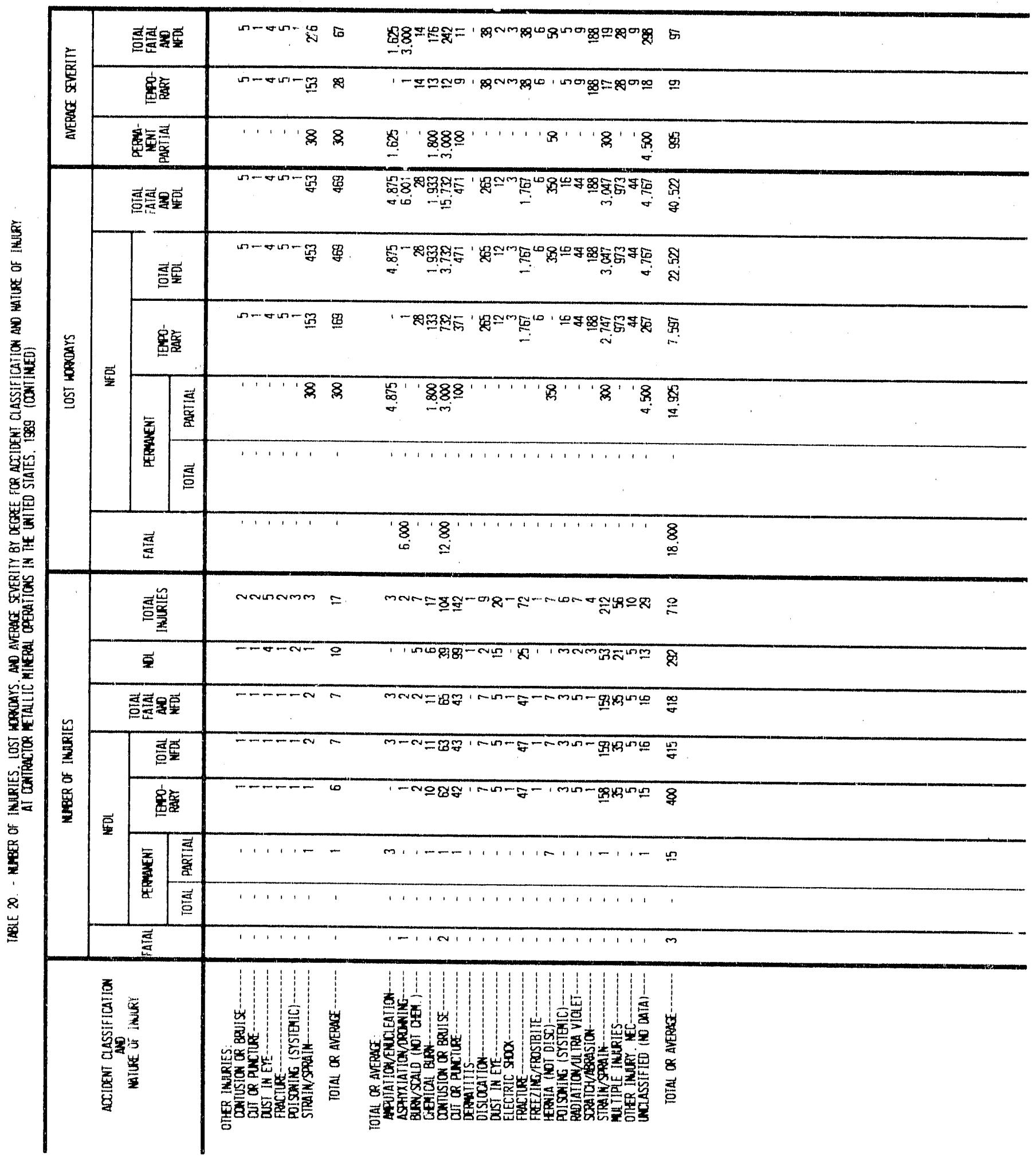




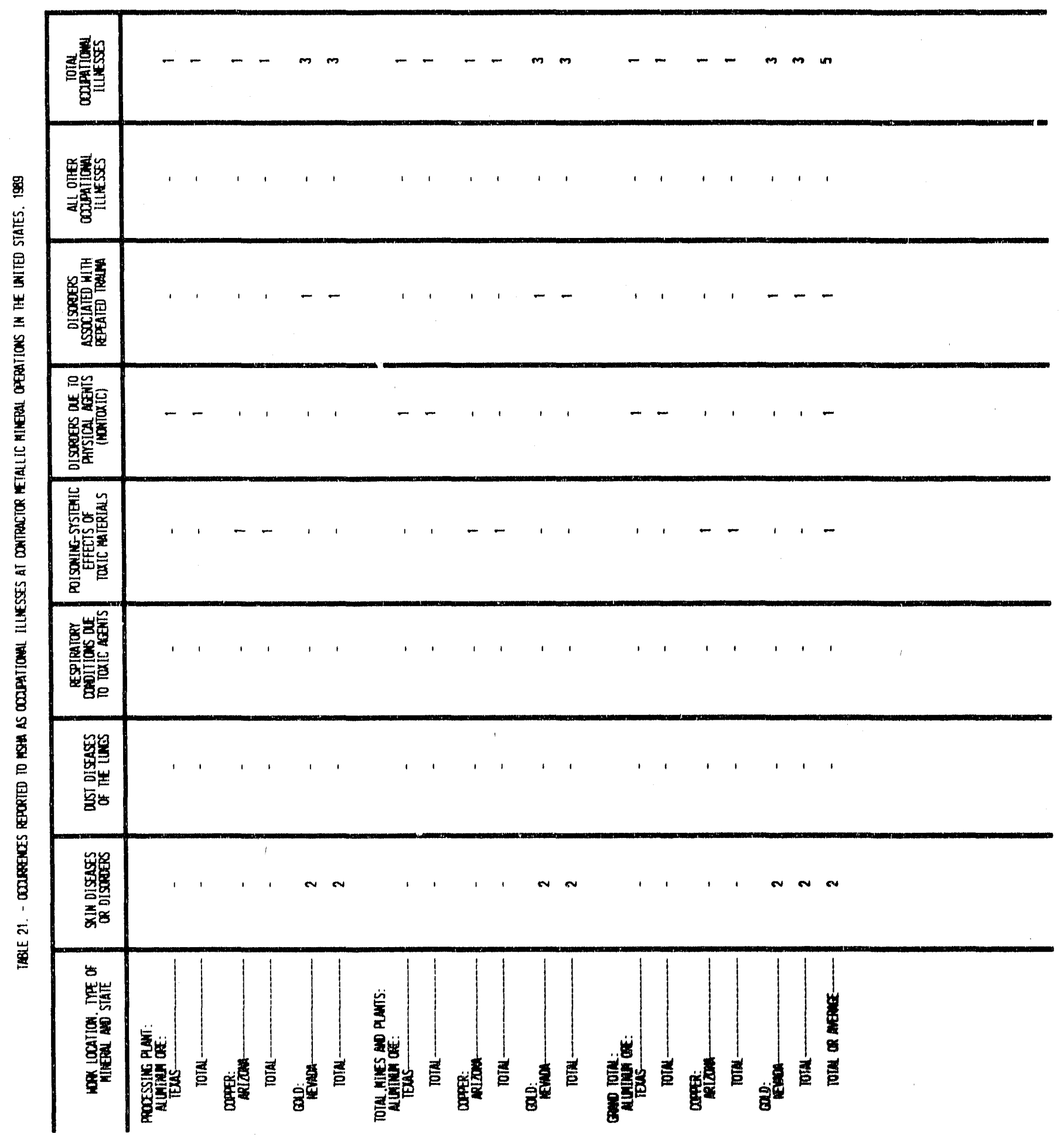




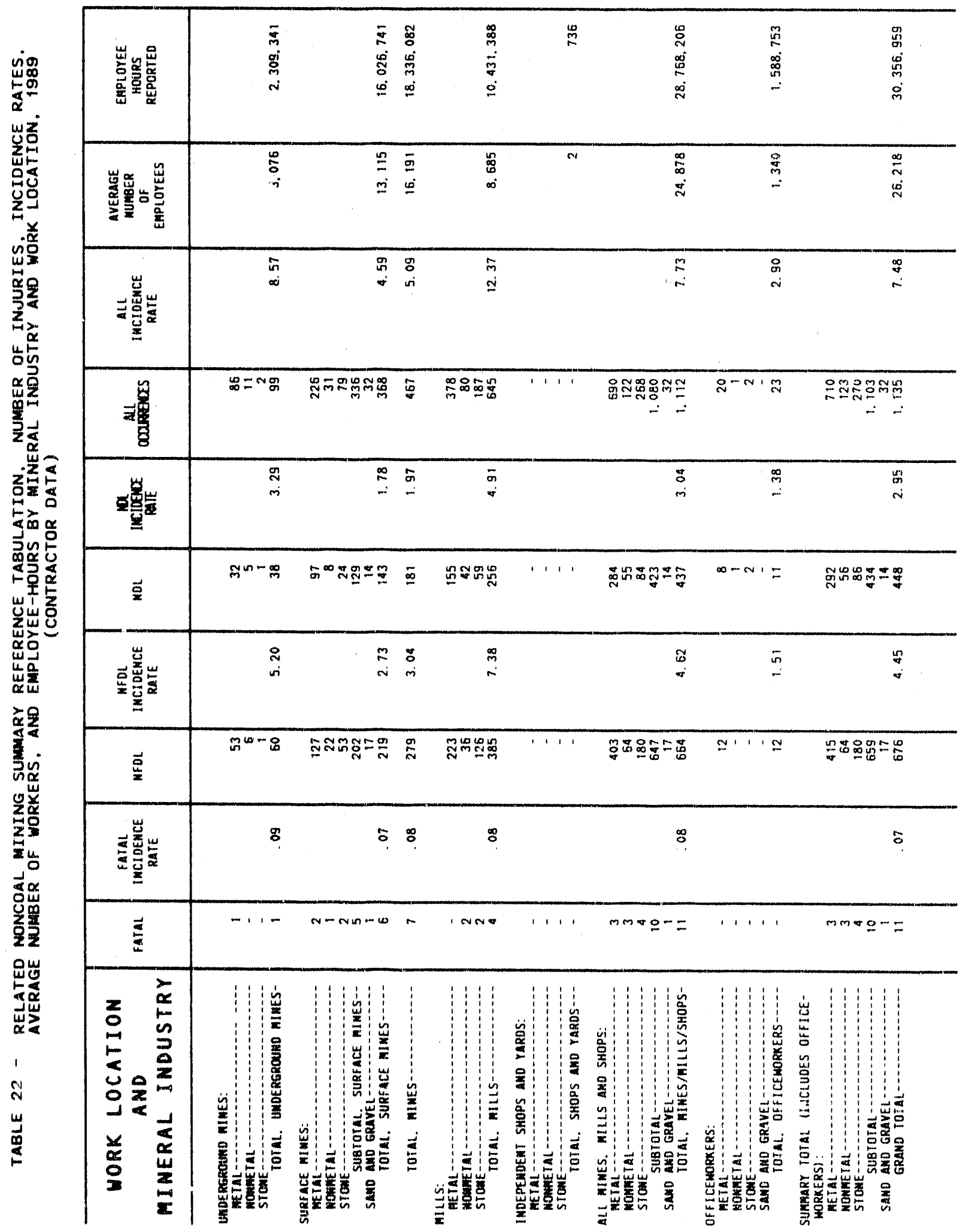




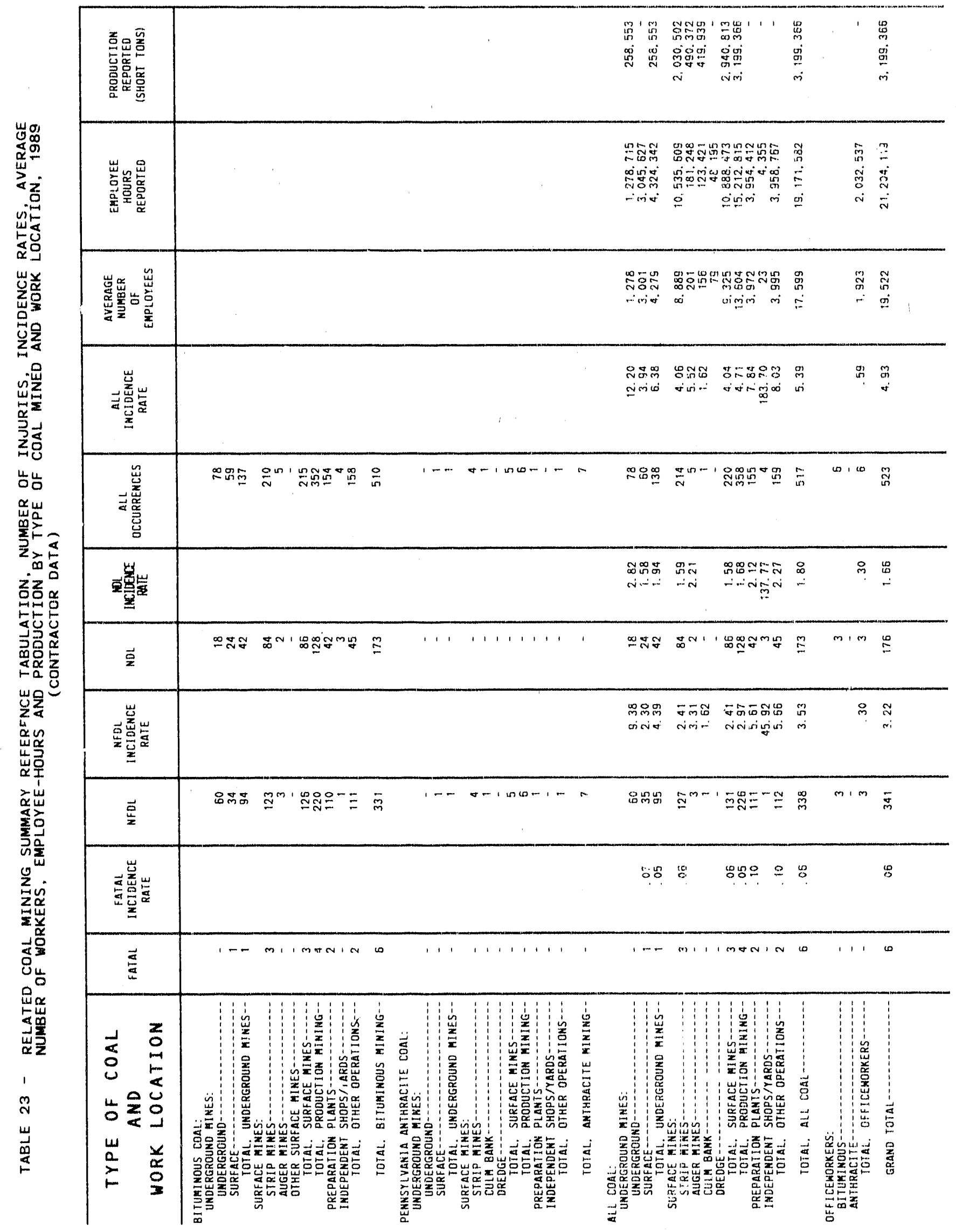



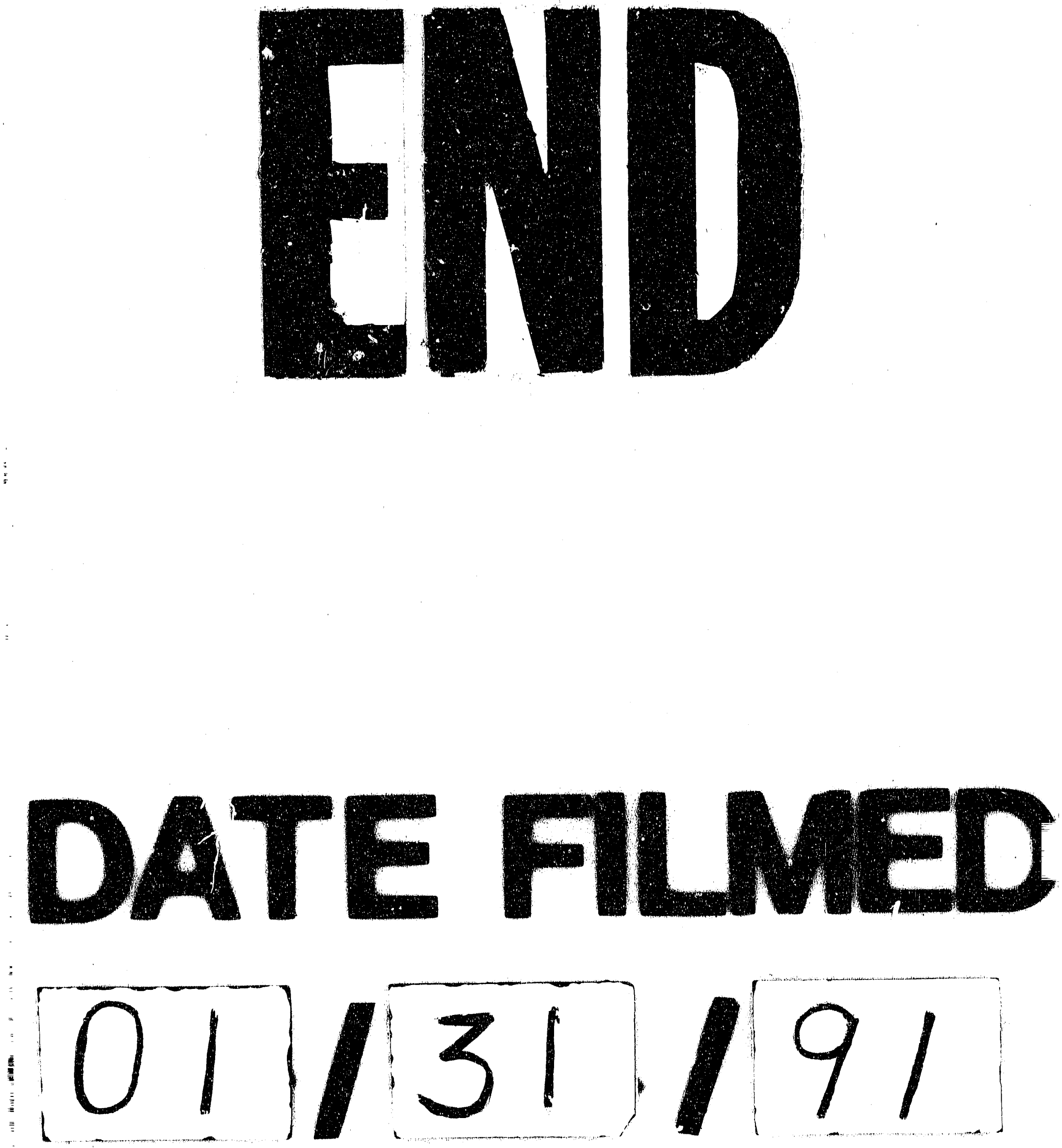
